\title{
ECONOMIC EVALUATION APPLIED TO HEALTH POLICY IN NEW ZEALAND
}

\author{
by \\ William Guy Scott
}

A thesis

submitted to Victoria University of Wellington in fulfilment of the

requirements for the degree of

Doctor of Philosophy in Public Policy

Victoria University of Wellington 


\section{ACKNOWLEDGEMENTS}

The support and help of Robert Stephens and Claudia Scott at Victoria University, and the warm support and encouragement of family and close friends is acknowledged.

I wish to thank the editors of the journals (Journal of Medical Economics, New Zealand Medical Journal, PharmacoEconomics, WP Press) in which copyright subsists, for permission to include the full text of the published papers as an appendix. 


\section{Victoria University of Wellington}

\section{Important Disclaimer}

1: Victoria University of Wellington and its Council, its members, staff, employees, students and agents undertake no duty of care in contract, tort, or otherwise, to users (whether direct or indirect) of this "Economic Evaluation Applied To Health Policy In New Zealand", and make no warranties or representations of any kind whatsoever in relation to any of its contents.

2: The "Economic Evaluation Applied To Health Policy In New Zealand", is only made available on the basis that all users of it, whether direct or indirect, must take appropriate legal or other expert advice in relation to their own circumstances and must rely solely on their own judgement and such legal or other expert advice.

3: Under no circumstances will Victoria University of Wellington and its Council, its members, staff, employees, students or agents be liable in any way whatsoever, whether in contract, tort (including negligence), for breach of any statutory or regulatory duty (to the fullest extent permissible by law), or otherwise, to any user (whether direct or indirect) of this "Economic Eluluation Applied To Health Policy In New Zealand" for any loss or damage whatsoever arising directly or indirectly as a result of the use in any way of this "Economic Evaluation Applied To Health Policy' In New Zealand". 


\section{CONTENTS}

ABSTRACT .

CHAPTER 1: INTRODUCTION TO ECONOMIC EVALUATION APPLIED TO

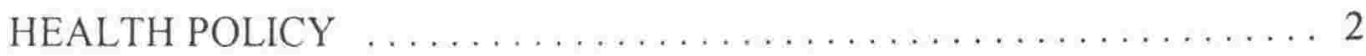

1.1 INTRODUCTION $\ldots \ldots \ldots \ldots \ldots \ldots \ldots \ldots \ldots \ldots \ldots \ldots \ldots \ldots$

1.2 PUBLIC POLICY EVALUATIONS $\ldots \ldots \ldots \ldots \ldots \ldots \ldots \ldots \ldots$

1.2.1 Policy Cycles and Problem Solving $\ldots \ldots \ldots \ldots \ldots \ldots$

1.2.2 Determinants of Health Policy $\ldots \ldots \ldots \ldots \ldots \ldots \ldots$

1.3 SCOPE AND METHOD $\ldots \ldots \ldots \ldots \ldots \ldots \ldots \ldots \ldots \ldots$

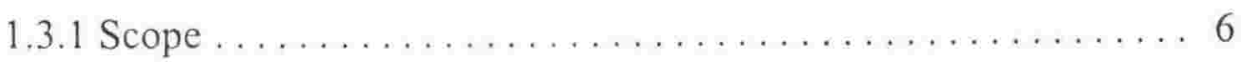

1.3.2 Techniques Reviewed $\ldots \ldots \ldots \ldots \ldots \ldots \ldots \ldots$

1.3 .3 Cases . . . . . . . . . . . . . . . . . . . . . . . 7

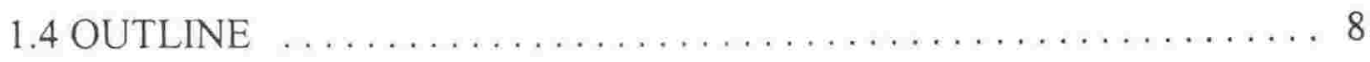

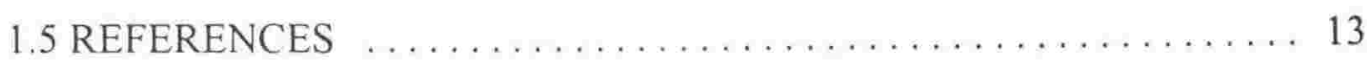

CHAPTER 2: CONCEPTUAL FRAMEWORK FOR ECONOMIC EVALUATION OF HEALTH CARE ............................ 14

2.1 INTRODUCTION ............................ 14

2.2 DETERMINANTS OF HEALTH $\ldots \ldots \ldots \ldots \ldots \ldots \ldots \ldots \ldots \ldots$

2.3 BACKGROUND TO THE NEW ZEALAND HEALTH SYSTEM . . . . 17

2.4 POLICY GOALS AND TRADE-OFFS ................. 19

2.4.1 Efficiency .......................... 20

2.4 .2 Evaluating Welfare $\ldots \ldots \ldots \ldots \ldots \ldots \ldots \ldots \ldots \ldots \ldots$

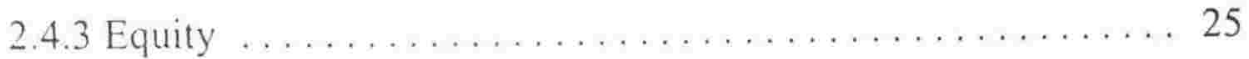

2.4.4 Equity Efficiency Trade-off . . . . . . . . . . . . . . 29

2.5 MARKETS AND GOVERNMENT INTERVENTION ......... 31

2.5.1 The Role of the Market . . . . . . . . . . . . . 32

2.5.2 Market Failure and the Second Best . . . . . . . . . 34

2.5 .3 Government Failure .................. 35 
2.5.4 Technical and Policy Evaluations of Health Care ......... 36

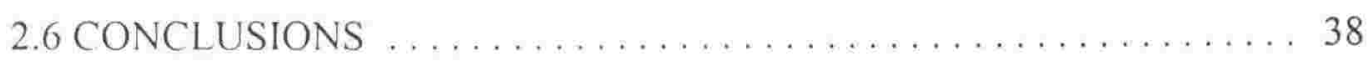

2.7 REFERENCES ............................ 40

CHAPTER 3: VALUING HEALTH OUTCOMES $\ldots \ldots \ldots \ldots \ldots \ldots \ldots \ldots$

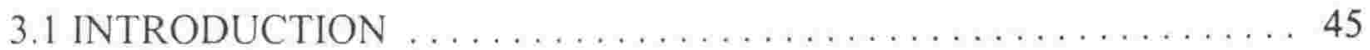

3.1 .1 What is Health? . . . . . . . . . . . . . . . 45

3.1.2 Why We Need to Quantify Changes in Health Outcomes ... . 46

3.1.3 Differences in the Valuations of Health and Health Outcomes . 46

3.2 WAYS OF VALUING CHANGES IN HEALTH OUTCOMES . . . . . . 48

3.2.1 Cost-benefit Analysis . . . . . . . . . . . . . 48

3.2.2 Cost-Effectiveness and Cost-Utility Analysis . . . . . . . . 53

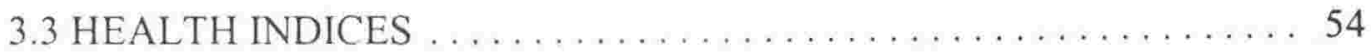

3.3.1 Health Indices as a Subset of Social Indicators . . . . . . . . 54

3.3 .2 Objectives of Health Indices . . . . . . . . . . . 55

3.3 .3 Validated Instruments . . . . . . . . . . . . . 56

3.3.4 Classification of Health Indices $\ldots \ldots \ldots \ldots \ldots \ldots \ldots$

3.3.5 Health Related Quality of Life Indices . . . . . . . . . . . 57

3.4 VALUING HEALTH STATES . . . . . . . . . . . . . . . . 73

3.4 .1 Utility Methods $\ldots \ldots \ldots \ldots \ldots \ldots \ldots \ldots \ldots \ldots \ldots$

3.4 .2 Psychological Scaling . . . . . . . . . . . . . 77

3.5 QUALITY ADJUSTED LIFE YEARS (QALYS or QUALYS) . . . . . 77

3.6 NEW ZEALAND INITIATIVES ................... 82

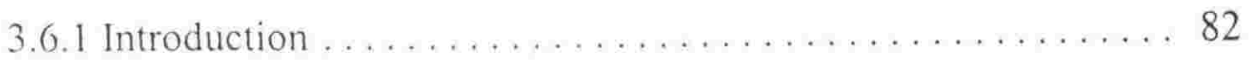

3.6.2 Surgical Priority Scoring System $\ldots \ldots \ldots \ldots \ldots \ldots . \ldots 2$

3.6.3 Health Funding Authority Health Related Quality of Life Instrument

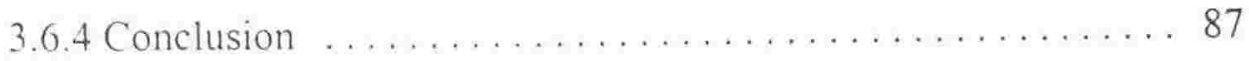

3.7 CONCLUSIONS AND POLICY POINTS .............. 88

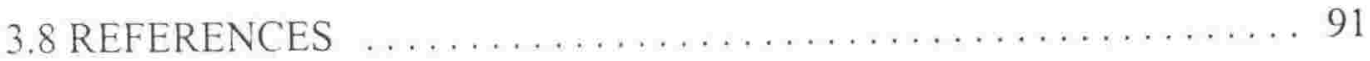


CHAPTER 4: COSTS ............................ 96

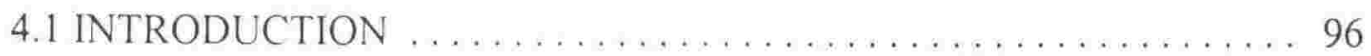

4.2 HISTORICAL PERSPECTIVE . . . . . . . . . . . . . . . 97

4.3 CLASSIFICATION OF COSTS ADOPTED . . . . . . . . . . . . . . 102

4.3.1 Direct Costs . . . . . . . . . . . . . . . . . . . . . 104

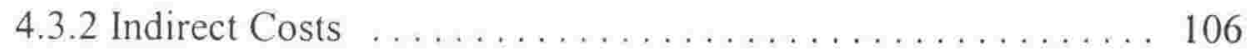

4.3 .3 Intangible Costs . . . . . . . . . . . . . . . . . . . . . . . . 109

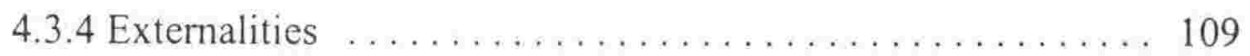

4.4 INCREMENTAL, MARGINAL, AVERAGE AND SUNK COSTS . . 110

4.5 AVOIDING DOUBLE COUNTING OF COSTS ........... 111

4.5.1 Productivity and Health Related Quality of Life ... . . . . . 112

4.5.2 Transfer Payments . . . . . . . . . . . . . . . . . . . 113

4.5 .3 Cost Shifting . . . . . . . . . . . . . . . 113

4.5.4 Capital Costs . . . . . . . . . . . . . . . . . . . 113

4.5 .5 Co-morbidity . . . . . . . . . . . . . . . . . . . 114

4.6 COSTING PROCESS $\ldots \ldots \ldots \ldots \ldots \ldots \ldots \ldots \ldots \ldots \ldots \ldots$

4.7 SENSITIVITY ANALYSIS . . . . . . . . . . . . . . . . 115

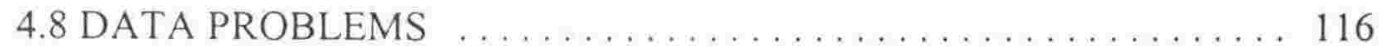

4.9 PERSPECTIVE AND COSTS $\ldots \ldots \ldots \ldots \ldots \ldots \ldots \ldots \ldots \ldots$

4.10 CONCLUSION ......................... 118

4.11 REFERENCES ......................... 120

CHAPTER 5: DISCOUNTING ....................... 125

5.1 INTRODUCTION . . . . . . . . . . . . . . . . . 125

5.2 MECHANICS OF DISCOUNTING ................ 128

5.3 APPROACHES TO DISCOUNTING . . . . . . . . . . . . . . . . . 129

5.3.1 Social Rate of Time Preference (SRTP) . . . . . . . . 130

5.3.2 Opportunity Cost or Social Opportunity Cost (SOC) . . . . . 130

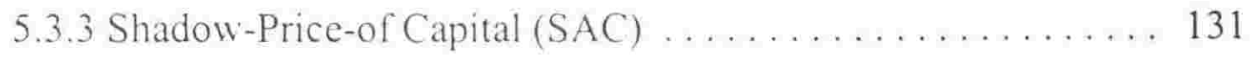

5.3.4 Diminishing Marginal Utility . . . . . . . . . . . . 132

5.3 .5 Uncertainty and Risk . . . . . . . . . . . . . . . . . 132

5.4 LOOKING BACK AT PAST INVESTMENT DECISIONS . . . . . . 132 
5.5 DISCOUNTING COSTS AND CONSEQUENCES . . . . . . . . . 133

5.6 REAL AND NOMINAL RATES .................. 134

5.7 DISCOUNT RATES IN USE ... . . . . . . . . . . . . . 136

5.8 CONCLUSIONS AND RECOMMENDATIONS ... . . . . . . 138

5.9 REFERENCES ......................... 140

CHAPTER 6: ECONOMIC EVALUATION .................. 143

6.1 INTRODUCTION $\ldots \ldots \ldots \ldots \ldots \ldots \ldots \ldots \ldots \ldots \ldots \ldots \ldots \ldots$

6.2 ECONOMIC EVALUATION AND HEALTH . . . . . . . . . . . . . . . 144

6.3 TYPES OF ECONOMIC EVALUATION $\ldots \ldots \ldots \ldots \ldots \ldots \ldots . . \ldots$

6.3.1 Cost-Benefit Analysis (CBA) . . . . . . . . . . . . . . 148

6.3.2 Economic Evaluations Other Than CBA ............ 154

6.4 THE PROBLEM OF CLASSIFYING ECONOMIC EVALUATIONS . 163

6.5 CONCLUSION ............................ 164

6.6 REFERENCES ......................... 167

CHAPTER 7: MODELS $\ldots \ldots \ldots \ldots \ldots \ldots \ldots \ldots \ldots \ldots \ldots \ldots \ldots \ldots$

7.1 INTRODUCTION $\ldots \ldots \ldots \ldots \ldots \ldots \ldots \ldots \ldots \ldots \ldots \ldots \ldots$

7.2 BACKGROUND ............................. 170

7.2.1 History and Development . . . . . . . . . . . . 170

7.2.2 Purpose of Modelling . . . . . . . . . . . . 171

7.2.3 Criticisms of Modelling $\ldots \ldots \ldots \ldots \ldots \ldots \ldots \ldots \ldots \ldots$

7.3 TYPES OF MODELS ...................... 172

7.4 WHY MODELS ARE USEFUL . . . . . . . . . . . . . . . . . . . . . . 174

7.4.1 Why Use Models . . . . . . . . . . . . . . . . . . . . . . . . . . . 174

7.4.2 Situations Where Models Are Useful . . . . . . . . . . . . . 174

7.5 THE MODELLING PROCESS ................. 178

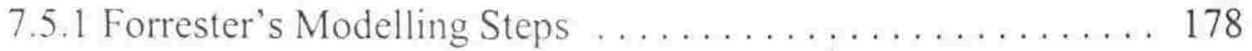

7.5.2 Generalised Modelling Steps ............... 178

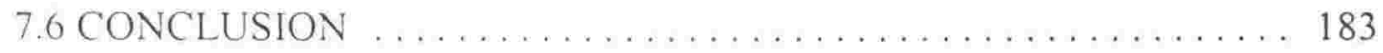

7.7 REFERENCES ........................ 185 
CHAPTER 8: CARE WITH DATA $\ldots \ldots \ldots \ldots \ldots \ldots \ldots \ldots \ldots \ldots \ldots \ldots$

8.1 SUMMARY AND INTRODUCTION .............. 186

8.2 BACKGROUND ........................ 187

8.3 CASE ONE: HEART FAILURE . . . . . . . . . . . . . . . . . . . 189

8.4 CASE TWO: BENIGN PROSTATIC HYPERPLASIA (BPH) ... . . 191

8.5 DISCUSSION ............................. 195

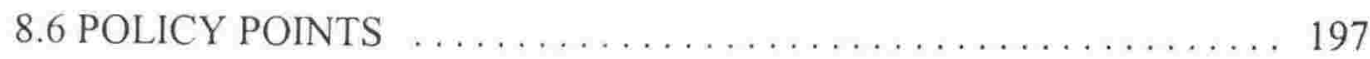

8.7 REFERENCES ......................... 198

CHAPTER 9: ANTIBIOTIC COSTING $\ldots \ldots \ldots \ldots \ldots \ldots \ldots \ldots \ldots \ldots \ldots$

9.1 SUMMARY AND INTRODUCTION ............... 201

9.2 METHOD ............................... 202

9.2.1 Target Population, Perspective and Overall Method ....... 202

9.2.2 Investigative Steps . . . . . . . . . . . . . . . . 203

9.3 RESULTS AND DISCUSSION .................... 206

9.4 POLICY POINTS .......................... 209

9.5 DATA COLLECTION INSTRUMENTS $\ldots \ldots \ldots \ldots \ldots \ldots \ldots \ldots$

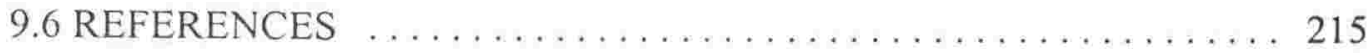

CHAPTER 10: BENIGN PROSTATIC HYPERPLASIA . . . . . . . . . . 217

10.1 SUMMARY AND INTRODUCTION $\ldots \ldots \ldots \ldots \ldots \ldots \ldots \ldots$

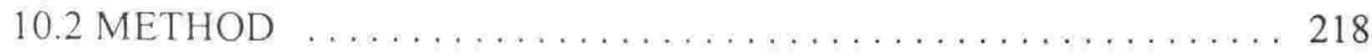

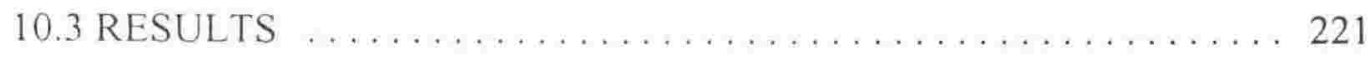

10.4 DISCUSSION ............................. 224

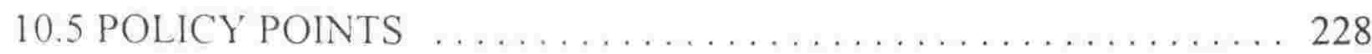

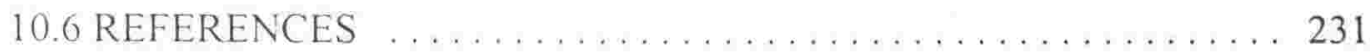

CHAPTER 11: HYSTERECTOMY .................... 233

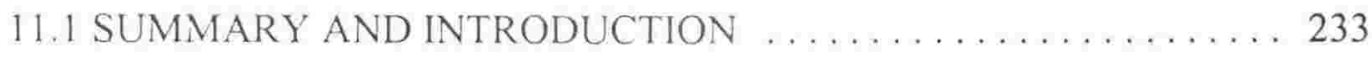

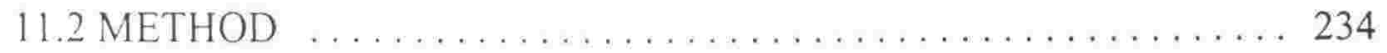

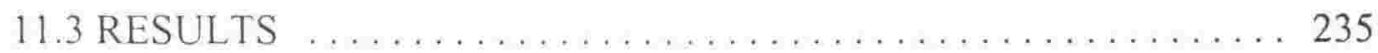

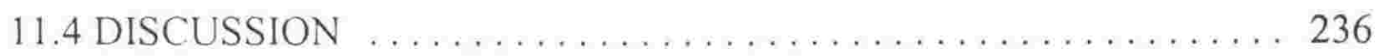


11.5 CONCLUSIONS ............................ 239

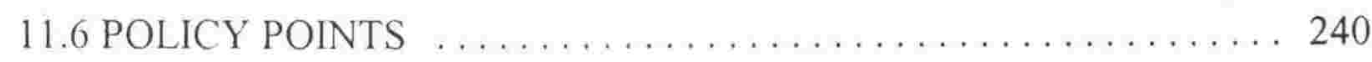

11.7 REFERENCES .......................... 242

CHAPTER 12: LOWER RESPIRATORY TRACT INFECTION . . . . . . . 243

12.1 SUMMARY AND INTRODUCTION $\ldots \ldots \ldots \ldots \ldots \ldots \ldots \ldots$

12.2 METHOD ................................ 244

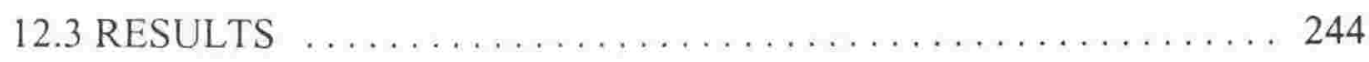

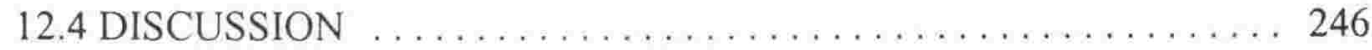

12.5 CONCLUSIONS .............................. 249

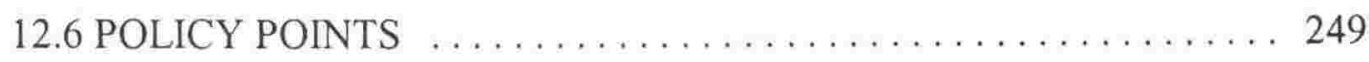

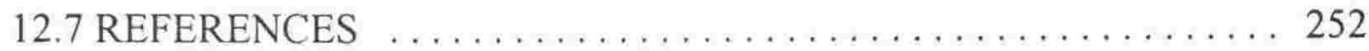

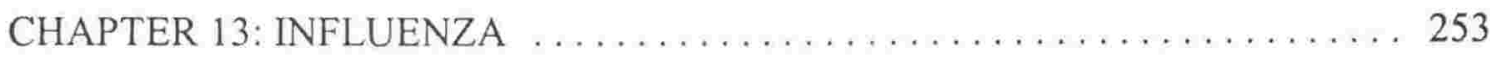

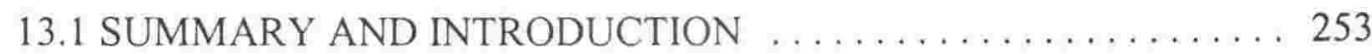

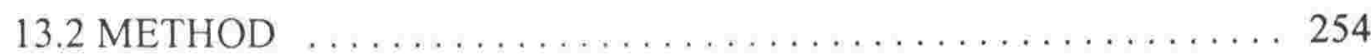

13.2.1 Vaccine Cost ...................... 256

13.2.2 Utilisation of Health Care Resources If No Vaccination Given 256

13.2.3 Medical Costs Avoided . . . . . . . . . . . . 257

13.2.4 Health Care Resource Unit Costs . . . . . . . . . . . . 257

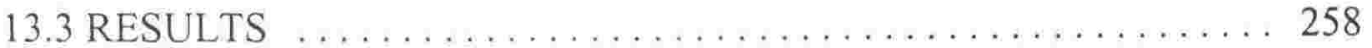

13.4 DISCUSSION ............................ 260

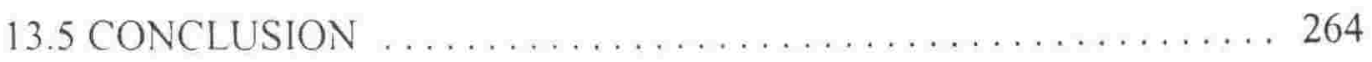

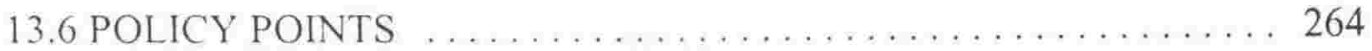

13.7 REFERENCES .......................... 266

CHAPTER 14: CRITICAL LEG ISCHAEMIA . . . . . . . . . . . . 269

14.1 SUMMARY AND INTRODUCTION ... . . . . . . . . . 269

14.2 METHOD .............................. 270

14.3 RESULTS ............................. 272

14.4 DISCUSSION ............................ 274 
14.5 CONCLUSIONS ........................ 276

14.6 POLICY POINTS . . . . . . . . . . . . . . . . . 277

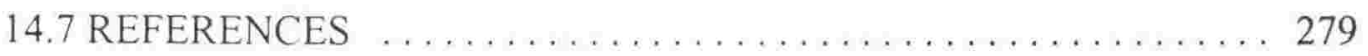

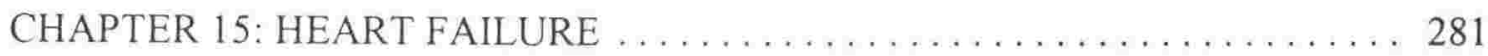

15.1 SUMMARY AND INTRODUCTION .............. 281

15.2 METHOD ................................ 282

15.3 RESULTS ............................ 285

15.4 DISCUSSION ........................... 287

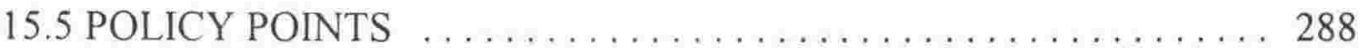

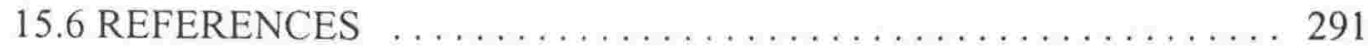

CHAPTER 16: ISCHAEMIC STROKE . . . . . . . . . . . . . . . . 293

16.1 SUMMARY AND INTRODUCTION ................. 293

16.2 COST OF ISCHAEMIC STROKE . . . . . . . . . . . . . . . . . . 294

16.2.1 Introduction and Objectives . . . . . . . . . . . . 294

16.2.2 Method ............................ 295

16.2.3 Results . . . . . . . . . . . . . . . . . . . . . . . . . . 298

16.2.4 Discussion/ Conclusions . . . . . . . . . . . . . . 301

16.3 COST-EFFECTIVENESS OF ALTERNATIVE METHODS OF ISCHAEMIC

STROKE PREVENTION $\ldots \ldots \ldots \ldots \ldots \ldots \ldots \ldots \ldots \ldots . \ldots . \ldots . \ldots$

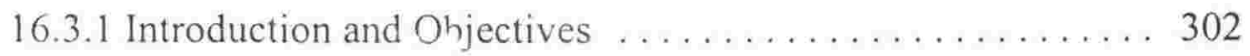

16.3.2 Method ........................ 302

16.3 .3 Results . . . . . . . . . . . . . . . . . . . . . . . . . 304

16.3.4 Discussion/ Conclusions . . . . . . . . . . . . . . . 306

16.4 COMPARISONS BETWEEN ALTERNATIVE METHODS OF EVALUATION (CEA, CUA AND CBA) ........... 308

16.4.1 Introduction and Objectives ... . . . . . . . . . 308

16.4.2 Method ........................... 308

16.4.3 Results . . . . . . . . . . . . . . . . . . . . . . 308

16.4.4 Discussion/ Conclusions ..................... 309

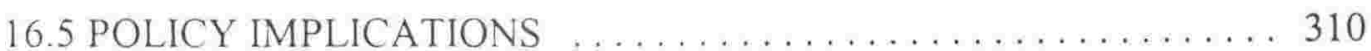


16.6 REFERENCES ......................... 314

CHAPTER 17: ASTHMA ......................... 315

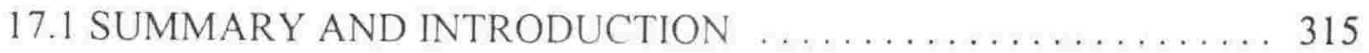

17.2 BACKGROUND AND OVERALL OBJECTIVES . . . . . . . . 316

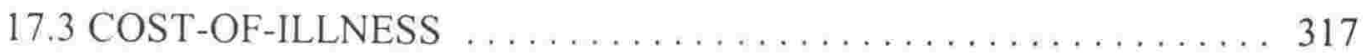

17.3.1 Background and Specific Objectives ........... . 317

17.3.2 Methods and Results .................... 317

17.3.3 Discussion/ Conclusion . . . . . . . . . . . . . . . . . 322

17.4 COST OF ALTERNATIVE TREATMENT STRATEGIES . . . . . . 323

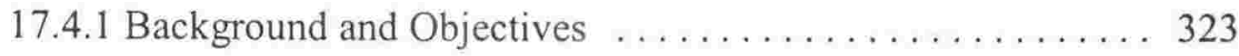

17.4.2 Method ........................... 324

17.4.3 Results . . . . . . . . . . . . . . . . . . . . . . . . 325

17.4.4 Discussion and Conclusion ................ 327

17.5 DECISION ANALYTIC MODEL . . . . . . . . . . . . . . . 329

17.5.1 Background and Objectives .................. 329

17.5.2 Method ............................. 329

17.5.3 Results and Discussion ................ 331

17.6 POLICY POINTS $\ldots \ldots \ldots \ldots \ldots \ldots \ldots \ldots \ldots \ldots \ldots \ldots \ldots \ldots \ldots$

17.7 REFERENCES ........................... 336

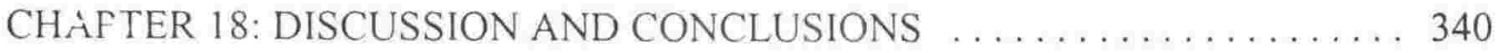

18.1 INTRODUCTION ............................. 340

18.2 ECONOMIC EVALUATION PROCESS ............... 340

18.2.1 Problem Definition ..................... 341

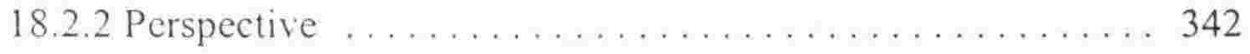

18.2.3 Target Population .................... 344

18.2.4 Alternatives/ Comparators ... . . . . . . . . . . 344

18.2.5 Time Horizon $\ldots \ldots \ldots \ldots \ldots \ldots \ldots \ldots \ldots . \ldots \ldots$

18.2.6 Technical Evidence Check ... . . . . . . . . . . 346

18.2.7 Constraints to Implementation . . . . . . . . . . . . 346

18.2.8 Identify an Appropriate Methodology . . . . . . . . . . . 347 
18.2.9 Identification of Costs and Outcomes (or Benefits) . . . . . 348

18.2.10 Evaluating Costs and Outcomes ............. 351

18.2.11 Choice Criterion $\ldots \ldots \ldots \ldots \ldots \ldots \ldots \ldots \ldots \ldots \ldots \ldots \ldots$

18.2.12 Sensitivity Analysis . . . . . . . . . . . . . . 352

18.2.13 Equity Consequences $\ldots \ldots \ldots \ldots \ldots \ldots \ldots \ldots \ldots \ldots \ldots \ldots$

18.2.14 Evaluate the Results . . . . . . . . . . . . . . . . 353

18.2.15 Monitor the Decision Taken ................ 353

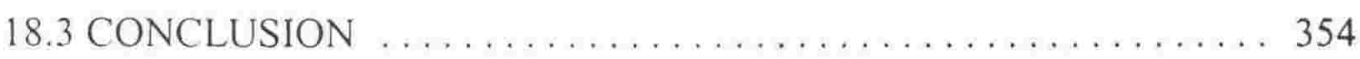

18.4 REFERENCES ........................ 356

APPENDIX: FULL TEXT OF THE ORIGINAL PAPERS DISCUSSED IN THE CASES

CASE 1: CARE WITH DATA

CASE 2: ANTIBIOTIC COSTING

CASE 3: BENIGN PROSTATIC HYPERPLASIA

CASE 4: HYSTERECTOMY

CASE 5: LOWER RESPIRATORY TRACT INFECTION

CASE 6: INFLUENZA

CASE 7: CRITICAL LEG ISCHAEMIA

CASE 8: HEART FAILURE

CASE 9: ISCHAEMIC STROKE

CASE 10: ASTHMA 


\section{ABSTRACT}

Background: New Zealand health reforms implemented in the 1990's split the purchaser from the provider and placed greater emphasis on contracting and competition. This was not sufficient in itself to solve the twin problems of achieving society's goals of equity and efficiency within the health sector. To improve decision making at an operational level microeconomic studies are required to highlight potential changes in both efficiency and equity. Furthermore, the analytical process must be easily understood and applied, transparent and theoretically grounded if these studies are to be used widely in the health sector often by non-economists.

Problem: How can economic theory be applied to health care to improve decision making with respect to the efficiency of resource use in New Zealand?

Objectives: To: (1) provide policy decision makers with the criteria and techniques by which the decision-making process in relation to resource allocation within the health sector can be improved to achieve greater efficiency and more equitable outcomes; and (2) present a range of practical examples that illustrate appropriate methodologies and provide suggested solutions to a range of problems that may be encountered by decision makers in health care.

Method: The underlying theory to economic evaluation of health policy options is discussed and a series of different types of economic and policy evaluations (cases) relating to a range of health care resource allocation questions are used to illustrate the application of the theory to actual health policy issues in New Zealand.

Results and conclusion: The cases presented indicated that although a common overall approach may be applied to the policy questions no one recipe could be applied in all cases. The policy question and the data available dictated the methodology. Using these findings, a checklist was developed as a guide for conducting policy evaluations and assessing efficiency gains in the health care. 


\section{CHAPTER 1: INTRODUCTION TO ECONOMIC EVALUATION APPLIED TO HEALTH POLICY}

\subsection{INTRODUCTION}

This chapter outlines the objectives, background and scope of the study. Public policy evaluations, determinants of health policy, policy questions, types of evaluation, the rationale for public policy intervention and the need for policy evaluation are discussed.

The focus of the study is encapsulated in the following question: "How can economic theory be applied to health care to improve decision making with respect to the efficiency of resource use in New Zealand?" The specific objectives of the investigation were to; (1) provide policy decision makers with the criteria and techniques by which the decisionmaking process in relation to resource allocation within the health sector can be improved to achieve greater efficiency and more equitable outcomes; and (2) present a range of practical examples that illustrate appropriate methodologies and provide suggested solutions to a range of issues that may be encountered by policy decision makers in health care.

\subsection{PUBliC POLICY EVAlUATIONS}

In most developed countries health care is predominantly funded by the state ${ }^{\prime}$ which means that governments must make policy decisions on what to fund, how it should be provided, and who should receive the funded services. Improving efficiency and equity of resource use thus requires evaluation of existing and future public policies.

I In 1995 OECD publically funded expenditure as a percentage of total health expenditure ranged from over $90 \%$ in Luxembourg to just under $50 \%$ in the US (N.Z. Ministry of Health, 1998: 31). 


\subsubsection{Policy Cycles and Problem Solving}

All policy decision makers are faced with an array of policy problems and policy instruments. Policy instruments in a mixed market economy range from the voluntary (little or no state intervention) to the compulsory (high level of state intervention). A mix of instruments will normally be used. Government intervention is necessary to correct for market failure, but even where markets are efficient, state involvement may be necessary to achieve society's equity goals. Where the state is subsidising or providing a service, economic evaluation should be applied to investigate the efficiency consequences of policies.

Economic evaluation provides one set of information for policy formulation, decision making, and monitoring but it cannot set the policy agenda, make policy decisions or implement policy. Figure 1.1 shows the relationship that economic evaluation has within the policy cycle. Similar ideas have been presented by Howlett and Ramesh (1995) and Bardach (1995).

Figure 1.1: Economic Evaluation and the Policy Cycle

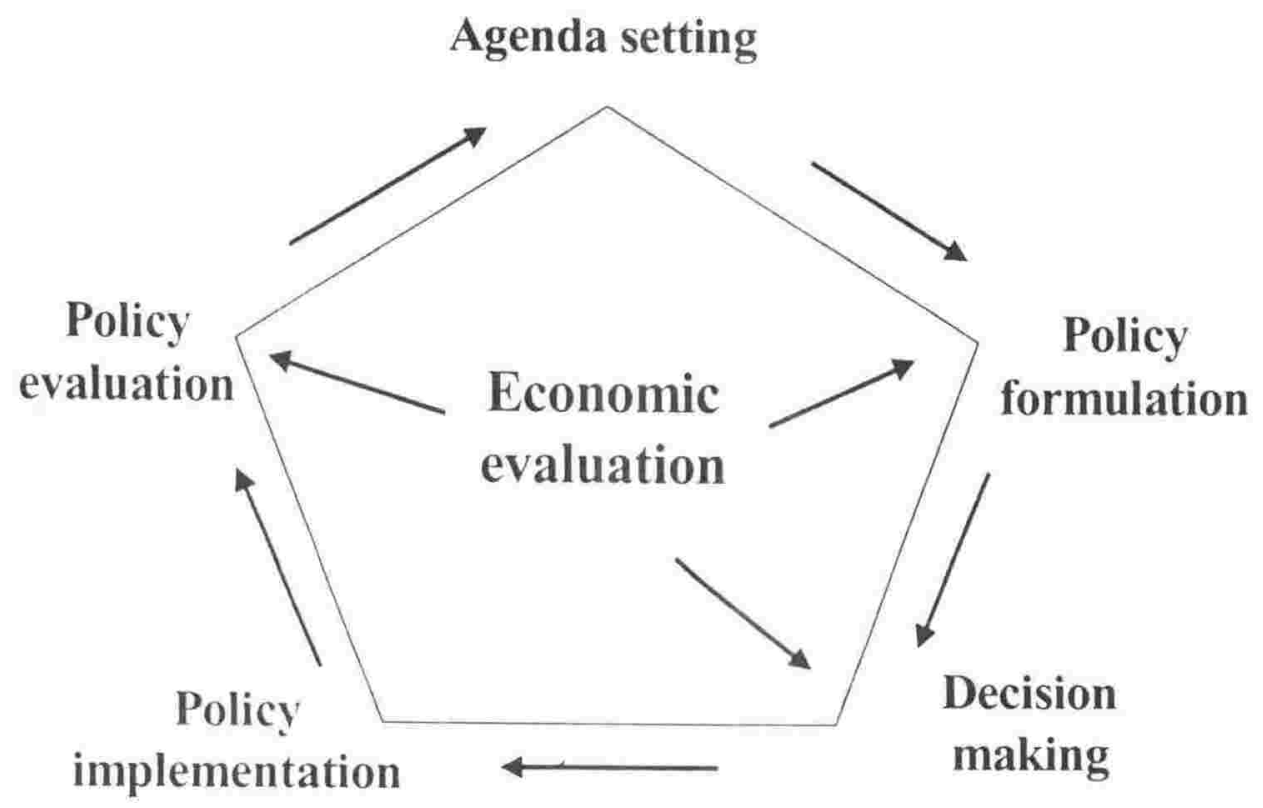


The cycle does not have a fixed beginning or end as a policy may evolve and re-enter the cycle. In practice policy evaluations may not conform exactly to the steps outlined, and the process is more likely to be iterative rather than sequential.

Economic evaluation as applied to public policy evaluation of health care is a systematic approach to gathering and analysing information about costs and consequences (or benefits) of different health care programmes, policy changes or treatment options. It is concerned with providing decision makers with knowledge about potential improvements in resource usage. In essence, the evaluation procedure involves the identification and measurement of all relevant costs and health related outcomes connected with the options under investigation.

\subsubsection{Determinants of Health Policy}

Policy determinants fall into two categories; environmental factors that cannot be influenced by health policy, and those factors over which health policy has some control.

- Environmental factors that health policy cannot change: Power and political structure, budget and funding constraints.

- Factors which health policy may influence: Society's values and beliefs, available supply of resources, rationing methods and systems, health care delivery systems, demand and need for health care and the population to be provided with health care.

All of these determinants of health policy (shown diagrammatically in Figure 1.2) are relevant and should be taken into account if public policies are to be successful and objectives achieved. The diagram is simplified in that not all the interactions are shown.

\section{Environmental Factors That Health Policy Cannot Change}

In a democratic society the power and political structures are exogenous to public policy but will determine the acceptability and success of policies. Social policies are not independent of economic policies as they cannot be implemented without resources and funding. Although funding allocations to various government expenditure options are a 
part of fiscal policy, they are exogenous to health policy.

Factors Which Health Policy May Influence

A key element of public health policy is to alter the values, attitudes and beliefs within society toward a more healthy lifestyle. Examples of public health initiatives designed to change values and beliefs include campaigns to convince the public that smoking is unhealthy, and education programmes designed to limit the spread of AIDs.

The available supply of health care resources, (such as labour and capital), both influence and are altered by health policy. The population to be provided with health care is under the control of health policy but the characteristics of this population are not. Delivery systems, and rationing methods are a part of government health policy. Rationing may be achieved by the price system or by administrative means such as waiting and booking lists and/ or criteria based on assessed need or by a combination of these.

Figure 1.2: Determinants of Health Policy

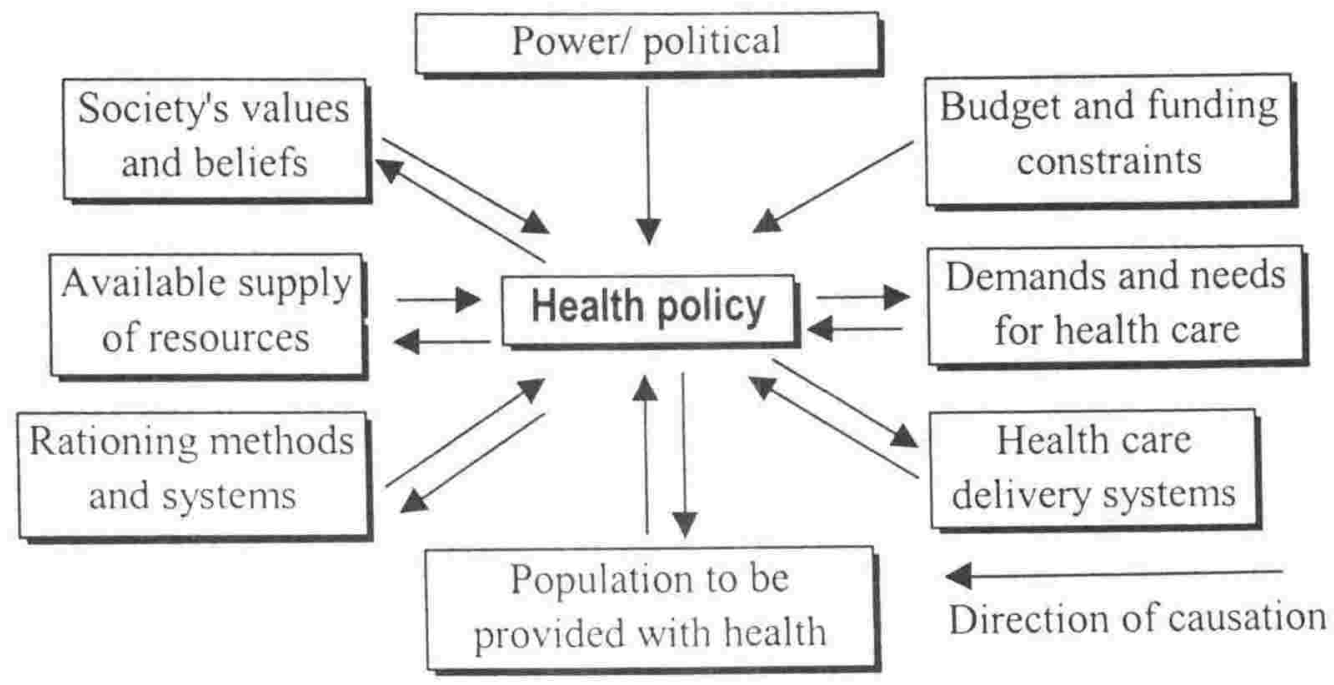

Perceived needs by consumers may be quite different from assessments of need made by health care professionals and policy makers. Cullis and West (1979) isolate three types of need: (1) normative need, the minimum standard of health care assessed by experts, (2) felt need, reflecting individuals views of their own wants, and (3) expressed need, felt need 
converted into demand for health care.

The Grossman (1972: 224) model argues that demand for health care (perceived need) by consumers is derived from the demand for good health. People desire good health rather than health care. Demand for good health is derived from the desire to reduce pain and suffering and achieve a higher quality of life (consumption component) and to have more time for work and recreational activities (investment component). Demand in this context is not the economic concept of willingness and ability to pay for a service but rather the actual seeking of health care from, for example, the public health system.

\subsection{SCOPE AND METHOD}

\subsubsection{Scope}

This thesis is concerned with providing evidence on the economic consequences of health care decisions. The study is intended to improve decision making in health by providing some basic guidelines for economic evaluation of health together with examples illustrating the application of economic evaluation methods to real New Zealand situations.

A comprehensive review of the current literature relating to economic evaluation as it applies to health and health policy was undertaken. A series of cases relating to original research is presented to illustrate the common economic evaluation techniques. The cases were prepared as a part of thesis development and sent to journals for peer review and publishing prior to completion of the thesis. The thesis was conceived as combining these pieces of original research into an integrated whole. An overview of each case is presented and policy implications, methodological issues and new and original findings highlighted. The process from research proposal to final publication of each case ranged between 2 and 4 years.

Although a wide range of methods and concepts are discussed, the focus is on practical application of theory relating to economic evaluation. Policy analysts and managers in the 
health sector face a wide range of different resource allocation problems. No one methodology or theoretical concept can be uniformly and universally applied. To answer the research question, "How can economic theory be applied to health care to improve decision making with respect to the efficiency of resource use in New Zealand?" it was necessary to discuss a wide range of techniques, and show in a series of cases, how economic evaluation may be applied in a variety of situations. No one case, technique, or segment of economic theory is discussed in minute detail.

\subsubsection{Techniques Reviewed}

The analytical techniques reviewed include:

- Cost-benefit analysis where costs of the programme and consequences or intended benefits are measured in money units.

- Cost-utility analy sis where costs are measured in money units and consequences in utility values.

- Cost-effectiveness analysis where costs are quantified in money units and consequences in natural or clinical units.

- Cost-minimisation analysis where costs are measured in money units and the consequences are assumed to be identical.

- Cost-of-illness (burden of illness) analysis and cost-of-treatment analysis where as many as possible of the costs of an illness or its treatment are measured in money terms (and those that cannot be measured in money terms are quantified or described in some other way).

- Computer based decision analytic modelling which incorporates elements of many of the above techniques into an interactive management and policy analysis framework.

\subsubsection{Cases}

The cases illustrate economic evaluations of health policy. Each case is discussed in a manner that demonstrates the application of the technique/s to different problems encountered in health policy analysis. Most of the cases described are concerned with 
medical interventions (for example, a surgical technique or a drug). The underlying principles are equally applicable to diagnostic tests, equipment, accident prevention and rehabilitation programmes, health promotion, illness prevention and protection programmes.

All the cases presented are extended summaries of published papers. It was considered that for clarity and focus a synopsis of each paper should be included in the body of the thesis and full text versions in an appendix. Because sufficient detail needed to be supplied, portions of the text and some of tables in the body of the thesis are similar to the published papers.

All cases discuss how a policy maker could use or has used the results. Some of the examples relate to only one perspective or decision maker, but others consider the policy implications from a number of viewpoints.

\subsection{OUTLINE}

This section outlines the content chapter by chapter. Chapter 2: Conceptual Framework for Economic Evaluation of Health Care, sets the scene for the thesis and discusses a range of issues relating to public policy in general and government health policy in particular.

Economic evaluations involving resource allocation decisions in health care require reliable outcome (output) and cost (input) measures. Health, ill-health and health related quality of life are concepts that are difficult to define and quantify with the precision required for economic evaluation. The next wo chapters discuss the quantification of outputs and inputs.

Chapter 3: I'aluing Health Outcomes, covers definitions of health, ways of valuing health outcomes, health indices, valuing health states, quality adjusted life years, validated instruments and current New Zealand initiatives. Cost concepts and measurement techniques are discussed in Chapter 4: Costs. Costs involving money outlays (explicit 
costs) are frequently recorded for accounting purposes and may often be isolated and measured relatively easily. But as economic costs include both implicit (do not involve money payments) and explicit costs, adjustments to accounting costs will frequently be necessary to estimate economic costs.

Costs and consequences that occur at different times must be adjusted to a common base before comparisons are made. Chapter 5: Discounting, discusses why discounting is necessary and examines the selection of an appropriate discount rate. A theoretical discussion of the various economic evaluation techniques is to be found in Chapter 6: Economic Evaluation. The specific evaluation technique is determined by the policy question, the nature of the data, and the time and budget available for the analysis. Chapter 7: Models, discusses the historical and theoretical foundations of models and their use in health and public policy analysis. Different types of model are outlined and the modelling process discussed. Models assist in integrating data from a number of sources and in the consideration and testing of a range of scenarios.

The cases illustrating different techniques and problems are summarised in Chapters 8 to 17. The general approach to discussing these cases is as follows. The background, research question, health and policy issues are first addressed and then the analytical methods and data, results, conclusions and the public policy points illustrated and/ or identified by the case are covered.

Chapter 8: Care With Data, uses two cases (Heart Failure and Benign Prostatic Hyperplasia) to illustrate a number of problems associated with aggregating and using secondary data. It presents a strategy to avoid such problems. New and previously unknown evidence on the differences between Māori and non-Mãori hospital admission rates for benign prostatic hyperplasia was found. Paradoxical and previously unexplained differences between aggregated gender specific heart failure prevalence rates and age specific gender rates were isolated and explained.

Chapter 9: Antibiotic Costing, uses micro costing techniques to quantify costs associated with antibiotic regimens for serious infections in three New Zealand public hospitals. A 
decision analytic model suitable for costing any antibiotic regimen was constructed and tested. The costing data were based on observation rather than the more usual self recorded diary entries. The model developed in this study provided a new tool for hospital administrators and clinicians to more accurately compare the full costs of antibiotic regimens.

Chapter 10: Benign Prostatic Hyperplasia, describes a cost-of-treatment study that integrated data from a wide range of sources; surgical treatment audits, hospital discharges, and prices. New information on the economic costs of treated benign prostatic hyperplasia was published. The research highlighted the hitherto hidden intangible costs of the side effects of surgery and was instrumental in prompting clinicians to investigate the differences in treatment rates between Māori and non-Māori. The research used hospital discharge unit record data (with patient identifiers removed) which enabled filtering and detailed analysis. This was the first published New Zealand study that used newly available unit record discharge data for economic policy analysis. Earlier research could access aggregated data only, which did not permit filtering and detailed analysis.

Chapter 11: Hysterectomy, discusses a cost-of-illness study and highlights issues of equity and efficiency. For the first time in New Zealand, incidence rates and costs of hysterectomy were isolated for women between the ages of 15 and 55 years undergoing hysterectomy for non-malignant conditions. Unit record hospital discharge data were filtered and used to estimate incidence rates and costs. This study was one of those cited by the National Health Committee (1998) in their Guidelines for the management of heavy menstrual bleeding.

Chapter 12: Lower Respiratory Tract Infection, presents a cost-effectiveness analysis that was conducted alongside a clinical trial in a general practice setting. This was the first such New Zealand economic study. It was particularly significant because the clinical results were published only in the economic paper. The results provided evidence on costeffectiveness enabling better informed funding decisions to be made relating to general practitioner antibiotic prescribing. 
Chapter 13: Influcnza, combined overseas clinical findings with New Zealand cost information to evaluate the cost-effectiveness of vaccinating the 65 years and older age group in New Zealand. The published results of this study provided essential costeffectiveness evidence and was used by the N.Z. Ministry of Health (Letter, 1996) to evaluate and design their subsidised vaccination programme.

Chapter 14: Critical Leg Ischaemia, describes a cost-effectiveness analysis that compared surgery with a pharmaceutical intervention. Application of breakeven analysis was used to evaluate the situations in which the hospital cost of drug treatment would be costeffective compared with surgery.

Chapter 15: Heart Failure, uses a decision analytic model to integrate New Zealand cost data with clinical evidence from an international randomly controlled trial. The model assessed the cost-effectiveness of alternative drug treatment options. Application of the results provided new cost-effectiveness information vital for government decision making when negotiating prices and in preparing treatment guidelines.

Chapter 16: Ischaemic Stroke, uses the findings of a cost-of-illness study and evidence from an international clinical trial to conduct a cost-effectiveness analysis of stroke prevention. The cost-effectiveness analysis of two drug treatment options would not have been possible without the cost-of-illness study results. This case has been used to illustrate the differences between cosi-effectiveness analysis, cost-utility analysis and cost-benefit analysis.

Chapter 17: Asthma, illustrated the use of an administrative database to provide information for cost-of-illness and cost-effectiveness studies and for a decision analytic model. This study analysed general practice records to investigate differences in direct costs between different asthma medication delivery devices. The published results of the cost analysis study were used to construct a computer-based decision analytic model. This model was used with general practice fund holders and Regional Health policy makers to demonstrate cost differences between alternative asthma treatments and permitted users to assess the budgetary impact of different asthma treatment policy options. 
Chapter 18: Discussion and Conclusions, presents a suggested procedure and checklist for policy and economic evaluation as applied to health. Economic evaluation tends to be poorly understood and applied by non-economist policy makers, managers and clinicians. A workable economic evaluation checklist was developed to provide policy decision makers and managers with the criteria and techniques by which the decision-making process in relation to resource allocation within the health sector can be improved.

Figure 1.3 summarises the structure of the thesis and shows how the chapters relate to each other.

Figure 1.3: Thes is Structure

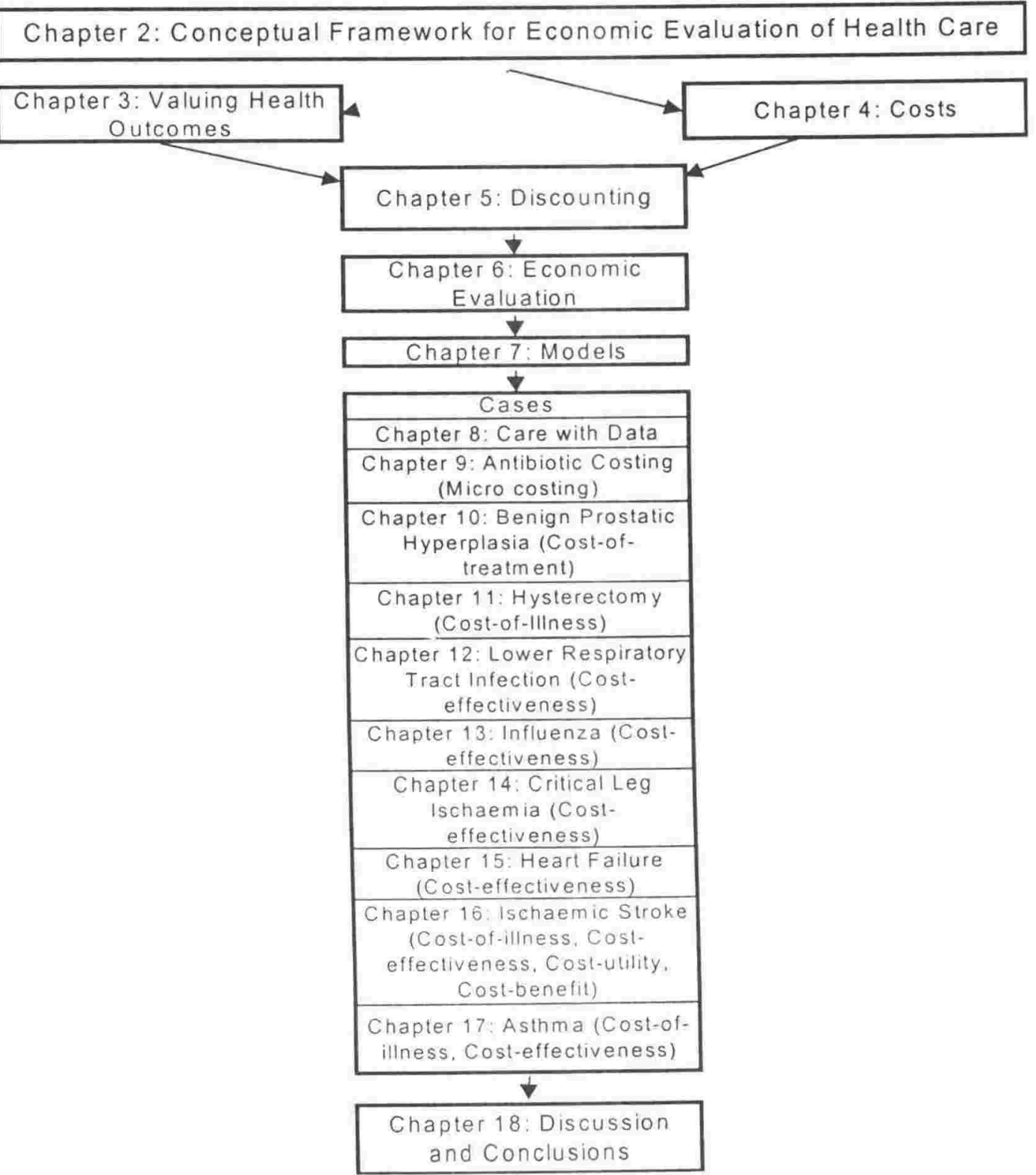

Chapter 2 discusses the conceptual framework for the study. 


\subsection{REFERENCES}

Bardach, E. Policy analysis: a handbook for practice. Washington: Cascade Centre for Public Service, 1995.

Cullis, J. G., and P. A. West. The economics of health. Oxford: Martin Robertson, 1979.

Grossman, M. "On the concept of health capital and the demand for health." Journal of Political Economy 80 (1972): 223-55.

Howlett, M., and M. Ramesh. Studying public policy: policy cycles and policy subsystems. 1st ed. Toronto: Oxford University Press, 1995.

National Health Committee. Guidelines for the management of heavy menstrual bleeding. Wellington: The Committee, 1998.

N.Z.Ministry of Health letter 18 June 1996

N.Z. Ministry of Health. Health expenditure trends in New Zealand 1980-97. Wellington: Sector Analysis Section, N.Z. Ministry of Health, 1998. 


\section{CHAPTER 2: CONCEPTUAL FRAMEWORK FOR ECONOMIC EVALUATION OF HEALTH CARE}

\subsection{INTRODUCTION}

The purpose of this chapter is to discuss the rationale for public policy interventions in the New Zealand health care market. The determinants of health are first considered. Technical and policy evaluation methods are considered as are the concepts of efficiency, welfare and equity. Health care market shortcomings are reviewed to establish the need for government intervention in the market to achieve society's goals of equity and efficiency.

\subsection{DETERMINANTS OF HEALTH}

Health care is but one of the many determinants of the health status of individuals and populations. Wildavsky (1977) notes that the medical system (doctors, drugs, hospitals) is responsible for about $10 \%$ of a nation's health status. "More available medical care does not equal better health." (Wildavsky, 1977: 105). Arrow (1963: 491) observed that "...the causal factors in health are many, and the provision of medical care is only one. Particularly at low levels of income, other commodities such as nutrition, shelter, clothing, and sanitation may be much more significant."

Grossman (1972) described inputs into a production function for an individual's stock of health with an output of healthy time. Production of health was dependent upon many environmental variables (including; health care, education, housing, income, employment status, and nutrition), the most important of which was education. It was assumed that higher levels of education would improve the efficiency of health production. Figure 2.1 is a diagrammatic representation of Grossman's model based on Donaldson and Gerard (1993: 180). Evans and Stoddart (1990: 1359) also presented a similar model showing the causal relationships between; social environment, physical environment, genetic 
endowment, individual response, behaviour, biology, diseases, health care, health and function, prosperity and well-being.

Figure 2.1: The Grossman Model

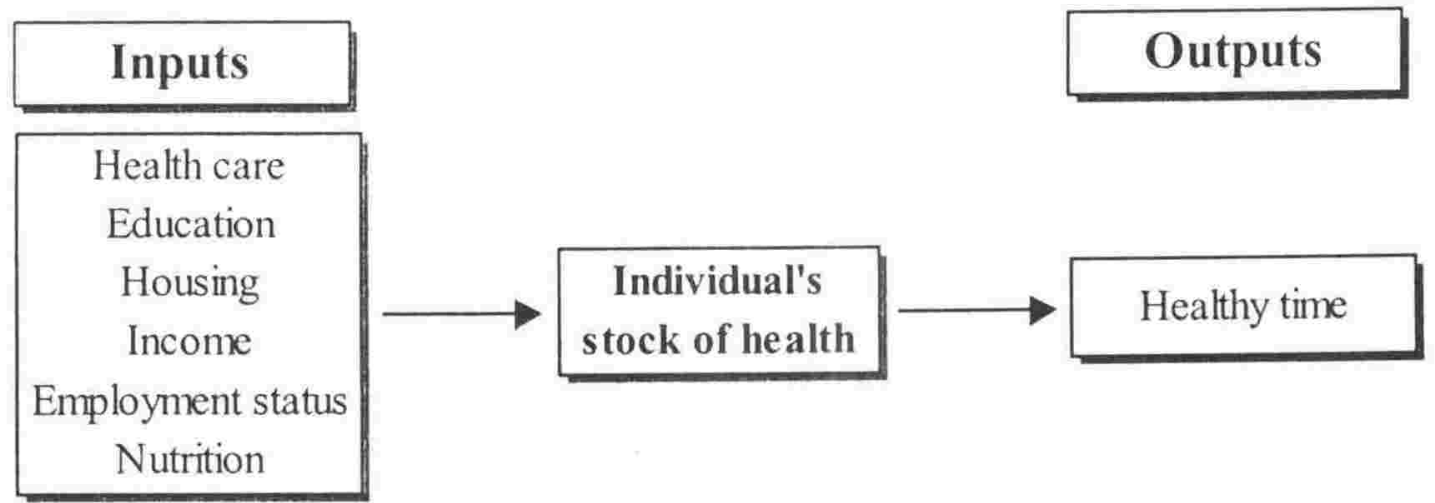

The determinants of good health, or lack of it, are discussed under the following groupings:

- Factors that cannot be controlled by public policy: Genetic endowment.

- Factors that are difficult for public policy to influence: Values and beliefs, lifestyle, diseases, demographics and the physical environment.

- Factors that are controllable by public policy: Health care resources, access to health care and health promotion.

Figure 2.2 summarise the main determinants of health and to show the dominant directions of causation. The diagram is a simplification of reality in that not all the interactions between the exogenous variables and health status are shown.

Factors That Cannot Be Controlled by Public Policy

The genetic endowment of individual citizens influences health and the need for health care, but cannot be changed by public policy. 
Values, beliefs and lifestyle affect health status. Disease prevalence and incidence, and demographics both influence and are modified by health status. (In this context demographics embrace a wide range of factors, including age, gender, marital status, education, occupation, religion, ethnicity, family size, income, employment status and geographic location.) Aspects of the physical environment, including clean water and clean air, rubbish disposal, good drainage and sewage, satisfactory living and working conditions, and diseases, may be affected by public policy.

\section{Figure 2.2: Determinants of Health}

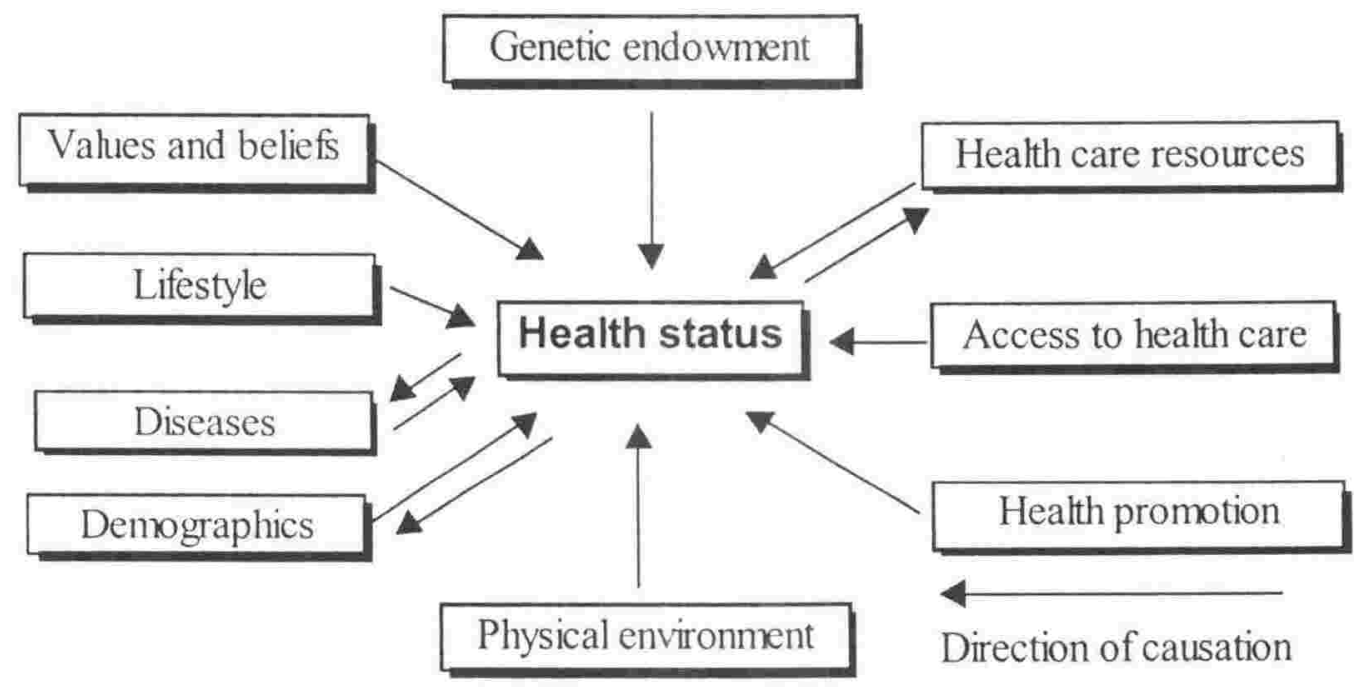

Factors That Are Controllable by Public Policy

Specific health policies can change the levels of health care resources and access to health care for some population groups. Improved health status may influence health care resources through increased productivity. Health promotion policies may have some success in altering behaviour, which in tum may alter health status. For example, advertising campaigns and taxes aimed at reducing the prevalence of smoking may lower the incidence and prevalence of lung cancer and heart disease. 


\subsection{BACKGROUND TO THE NEW ZEALAND HEALTH SYSTEM}

This section looks at the historical development of the New Zealand health system and places the discussion of efficiency and equity in perspective within the current policy settings and trade-offs.

Although there was some public provision of health care in New Zealand prior to the Social Security Act of 1938, the Act heralded the birth of the modern day New Zealand public health system. Benefits (ranging from hospital care to pharmaceuticals and artificial aids) were progressively introduced from April 1939 (New Zealand Royal Commission on Social Policy, 1988). Although the intention of the legislation was that all New Zealanders should have access to health care regardless of their ability to pay, the system that developed was never entirely publically funded.

The Report of the Health Benefits Review (1986:1) comments: "Despite these good intentions, a fair distribution of health care may not have been realised. Access to health care is easier for the affluent than for the disadvantaged, and there is a tendency for a proportionately greater share of services to go to those people who have less need." The report provides a number of examples (ranging from general practitioner services to pharmaceuticals) to support these claims.

In the 1980s public funding of health care came under financial pressure for a number of reasons including; slow economic growth, an aging population requiring more health care, new technology ${ }^{2}$ which was expanding treatment options, and growing consumer expectations for health care. In addition, the health system's structure and management style had not evolved rapidly enough to keep pace with changes in technology, society's wants, and the cconomic and political environments.

New technology is a double edged sword, in that it provides benefits by opening up new treatment options (which add to cost) but may reduce treatment time (which lowers costs) and improve health outcomes. 
The Report of the Hospital and Related Services Task force (Gibbs, 1988) considered that there were problems of access to public hospital care and that hospitals were inefficient. This Report concluded that the main problem facing public hospitals was poor management. There was a lack of accurate and relevant information, and no incentive to obtain and use such information. As hospitals were bulk funded, the value of their specific outputs was unknown to managers.

A recommendation of the "Gibbs" report was to separate government's role of purchaser and provider of hospital services and create a market in which prices would be set by modified competition between hospitals. It was considered that this limited market could achieve a 30\% to 50\% increase in technical efficiency (Gibbs, 1988: 26). The intention was that Regional Health Authorities (RHAs) would purchase hospital services from Area Health Boards, private hospitals, and voluntary organisations. Some of these ideas in the Gibbs report (1988) were also discussed in the "Green and White Paper" (Upton, 1991) and implemented in part.

The main health sector problems perceived at the time of the "Green and White Paper" (Upton, 1991) included; growing health expenditure, inefficiencies, lack of accountability, lack of choice and responsiveness of services for consumers, fragmented funding, and lack of integration between primary and secondary care. In essence these may be summarised as problems in achieving the implicit societal targets of efficient allocation of resources and equity.

The New Zcaland health reforms implemented the purchaser/ provider split and contracting, but have not achieved the efficiency gains quoted in the "Gibbs" report. As New Zealand health care is still predominantly government funded (77.3\% of all funding in 1996:97 (N.Z. Ministry of Health, 1998: 25)) public policy influences both the demand and supply sides of the market.

The initial post reforms funding of the Regional Health Authorities and Crown Health Enterprises (CHEs) was "...based on unrealistic expectations about how fast and to what degree CHEs could reduce costs through efficiency gains, and about how much growth in 
open-ended expenditure could be controlled."(N.Z. Ministry of Health, 1996: 5). Although the reforms sharpened the focus on evidence-based practice, information within the health system, and improved accountability and integration of services, public concem about the health system continued (N.Z. Ministry of Health, 1996: 5).

Stated goals of the New Zealand's public health policy are to; improve people's health and independence (improving the quality of life, not just its length), protect consumer rights and safety, and ensure access to services based on need and ability to benefit (N.Z. Ministry of Health, 1996: 9). To achieve these goals public policy decisions should be directed towards improving allocative efficiency and the distribution of output. As these two goals may conflict with each other, there will be a need to consider potential trade-offs between them.

The National Advisory Committee on Core Health and Disability Support Services (1994: 10) advanced the following criteria as a guide to specifying health and disability support services that are publically funded:

- "the treatment or service provides benefit

- the treatment or service is value for money

- the treatment or service is fair use of public funding

- the treatment or service is consistent with communities' values"

The first two criteria concern aspects of economic efficiency while the next two criteria relate to equity and judgements of fairness and worth. Where there are multiple and conflicting policy goals trade-offs will be necessary.

\subsection{POLICY GOALS AND TRADE-OFFS}

Important goals of public policy are to increase social welfare by improving efficiency and achieve an equitable distribution of income. This inevitably involves trade-off between efficiency and equity objectives. Although the concepts of efficiency and welfare are intertwined it is convenient to discuss them under discrete headings. 


\subsubsection{Efficiency}

This section discusses production efficiency, consumer efficiency and allocative efficiency.

\section{Production Efficiency}

The terms; production efficiency, technical efficiency and x-efficiency are frequently used synonymously. Production efficiency is achieved when the output from each combination of inputs is maximised, that is, there is not an input combination which could produce the same output at a lower input cost (Gravelle and Rees, 1985). Maximum output is realized only when the "best" possible use is made of existing technology, and all resources are fully employed. Points on the production possibilities frontier (defining different output mixes) are efficient while points within the frontier are inefficient.

\section{Consumer Efficiency}

Consumer efficiency (embracing both exchange and product mix) is the efficient distribution of commodities between consumers to maximise their utility. Exchange efficiency occurs when for a given basket of goods (not necessarily produced efficiently) it is not possible by reallocating the goods between people to make anyone better off without making someone worse off. All consumers have equal marginal rates of substitution $^{3}$ (for any pair of goods). No one can be made better off through trade and exchange. (Stiglitz, 1999). Product mix efficiency ensures that the basket contains the mix of goods most desired by consumers, that is, producers' marginal rate of transformation ${ }^{4}$

The marginal rate of substitution (MRS) is the ratio of the marginal utilities of two goods and is measured by the slope of the consumer's indifference curve between the two goods. Utility for a consumer is maximised when the ratio of the marginal utilities is equated with the price ratios. At this point, the benefits a consumer gains from purchasing one more unit of a product are just equal to the benefits foregone from purchasing one less unit of the other product.

4 The marginal rate of transformation (MRT) is the ratio of the marginal costs of producing two goods and is measured by the slope of the production possibilities frontier (PPF). The PPF for two goods defines the rate at which the production of one good may be transferred into the production of the other. 
is equal to consumer's marginal rate of substitution.

\section{Allocative Efficiency}

Allocative efficiency is a general equilibrium concept relating to the entire economy and all markets. Resources are allocated efficiently between different uses if the greatest output of the mix of wanted goods is produced from available resources and technology and marginal benefits of all goods and services are equal to their marginal costs of production. For allocative efficiency, there must be simultaneous efficiency in production, and consumption.

An allocation of resources generated as a result of general equilibrium of a perfectly competitive economy is Pareto efficient (discussed in section 2.4.2) and any Pareto efficient allocation of resources can be achieved by the solution to a general equilibrium in a competitive economy (Boadway and Bruce 1984: 3). If the conditions for perfect competition are broken market failure occurs (discussed in section 2.5).

Different types of economic evaluation are concerned with different aspects of efficiency. Cost-benefit analysis is concerned with allocative efficiency while cost-minimisation, costeffectiveness and cost-utility analysis are concerned with production efficiency. If we had a utility measure that would permit comparisons among all goods and services (including leisure) then cost-utility analysis could be used to assess allocative efficiency. Chapter 6 : Economic Evaluation discusses these concepts.

\subsubsection{Evaluating Welfare}

\section{What is Welfare?}

As economic policy aims to maximise social welfare subject to constraints of tastes, technology and resources (Barr, 1987: 70, 73), evaluation of welfare changes is important. The welfare of society represents the aggregated satisfaction of all its members and "Welfare comparisons would be simple if it were possible to aggregate the utilities of 
individuals into a single utility function." (Henderson and Quandt, 1958: 201). However, creation of such a societal utility function would require cardinal utility and comparability among household utilities (Boadway and Bruce 1984: 61). Pareto provided a workable, albeit partial, solution to this question.

\section{Pareto Concept}

The basic premise underlying welfare economics is the Pareto principle (Pareto, 1927) which states that a change will result in an improvement in community welfare if the change increases the utility of at least one individual (in their opinion) but does not decrease the utility of another (Price 1977, Baumol 1965).

There are two fundamental theorems of welfare economics which provide some guidance in assessing welfare according to the Pareto criteria. First (the direct theorem) may be stated as; "Under certain assumptions, a state (allocation of goods and factors) resulting from a competitive general equilibrium is Pareto optimal." Second (the converse theorem) says that; "Under certain assumptions, every Pareto optimum state (allocation of goods and factors) can be realized as the outcome of a competitive equilibrium given the distributional claims on income." (Boadway and Bruce, 1984: 64). Underlying assumptions are; that markets are efficient, households aim to maximise utility, and firms are profit maximising.

In practice most resource allocation charges make some individuals better off and others worse off. Pearce and Nash (1981:2) maintain that imagining a policy change that will not harm someone is difficult, particularly as economic evaluations consider costs and benefits over time.

The compensation principal (potential Pareto or Kaldor-Hicks Criterion) enunciated separately and in different forms by Kaldor (1939) and Hicks (1939) is adopted in costbenefit analysis. Under the Kaldor version of the compensation principal, if it is possible for the gainers to compensate the losers for their loss, the project is desirable. Baumol (1965: 378) states the Kaldor criterion as "A change is an improvement if those who gain evaluate their gains at a higher figure than the value the losers set upon their losses.". The 
Hicks version of the compensation principle requires that the potential losers be able to bribe the gainers to maintain the status quo. Thus, if the benefits exceed the costs of a project and if compensation is costless then the project is desirable. A comparison between the situations with and without the programme (counterfactual) provides a check that the compensation test is met (Battiato, 1993:27). This does not mean that the gainers actually compensate the losers. They simply have the potential to do so out of their gains. Thus, the compensation criterion tells us nothing about equity changes.

Scitovsky (1941: 86) pointed out that changing from one situation to another will generally affect the distribution of welfare by "...shifting relative prices...boosting some industries and depressing others...". In applying the Kaldor criterion it is possible that a change in one direction could be regarded as an improvement as could a movement back to the original point. To overcome this paradox Scitovsky proposed a two-part test: first, apply the Kaldor criterion to evaluate if a change is an improvement, and second, use the Kaldor criterion to check that the return back to the original situation is not an improvement. The proposed change would be an improvement only if both parts of this test were satisfied (Baumol, 1965: 379; Scitovsky, 1941). As most health care projects are not large enough to substantially influence relative prices the Scitovsky reversal criteria has little practical application in the evaluation of health care programmes.

Potential Pareto improvements should not be the only criteria considered when evaluating a decision. A free market is unlikely to ensure that the "winners" will compensate the "losers" thus policy makers must consider equity changes. Kaldor (1939:551) commented that it was "...quite impossible to decide on economic grounds what particular pattern of income-distribution maximises social welfare." Atkinson and Stiglitz (1980:6) comment that a Pareto optima "...does not ensure that the distribution that emerges from the competitive process is in accord with the prevailing concepts of equity (whatever these may be)."

The strength of the Pareto criteria is that societal welfare can be evaluated without the need for utility comparisons (or weightings) between households. However, this is also its major weakness in that it tells us nothing about distributional equity of alternative Pareto 
efficient outcomes.

There is an infinite number of general equilibrium possibilities (efficient outcomes), each reflecting a different distribution of income and there are many possible Pareto efficient outcomes given any particular distribution of income. We cannot tell which Pareto efficient outcome (equivalent to allocative efficiency) is the "best" for society because this would involve changing the income distribution as well as the relative quantities of each good and service.

Provided a social welfare function could be constructed, it would be possible to define a situation that reflects "... simultaneous determination of the efficient output mix and its distribution among consumers." Musgrave and Musgrave (1982: 67). This may be illustrated by figure 2.3 , where the vertical axis represents the welfare or utility of $Y$ while the $\mathrm{X}$ axis denotes X's utility. Social indifference curves are plotted. These curves identify combinations of utility of two individuals, $Y$ and $X$, which yield equal levels of utility to society (as defined by the social welfare function) and describe how society would evaluate utility trade-offs between $Y$ and $X$. Finally a utility possibilities frontier that traces the maximum utility that $Y$ can obtain for a given level of utility of $X$ (and vice versa) is constructed. Society's preferred point "bliss point", is where the highest indifference curve is tangential to the utility possibilities frontier. Both points A and B are allocatively efficient. In practice, policy makers cannot use such a model to evaluate utility trade-offs between individuals because they are unable to assign relative weights to levels of welfare of each member of society. 
Figure 2.3: Distribution Choices

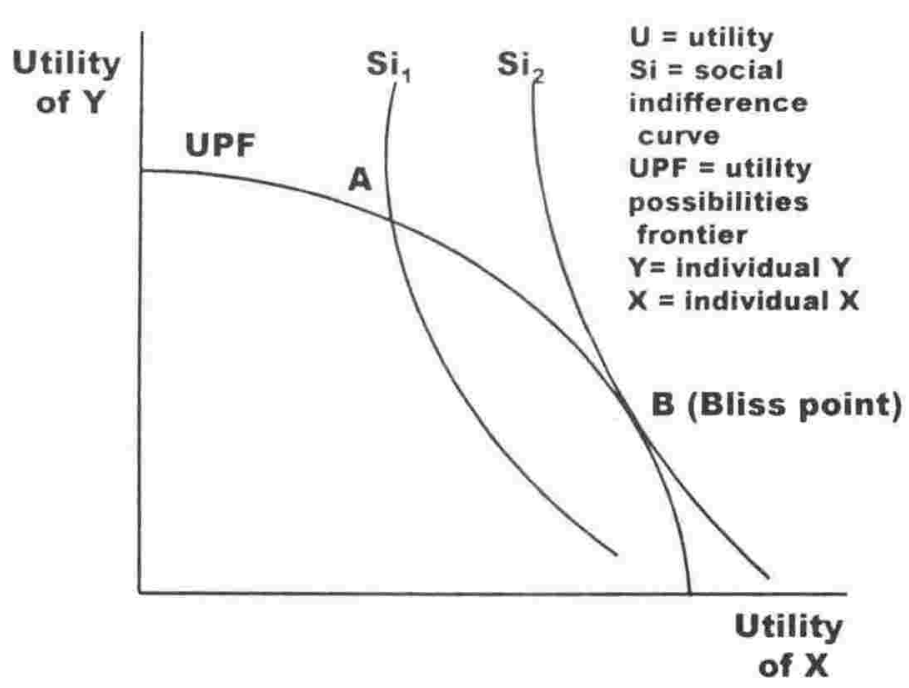

Pareto provided us with the criteria to guide us to the utility possibility frontier but policy makers must decide how to trade-off utility of different individuals in society. This leads to a discussion of equity.

\subsubsection{Equity}

This section is not intended to be a philosophical discussion of distributive justice or ethics but to introduce some of the issues underlying equity concepts. A more detailed discussion of social justice in economics may be found in Schokkaert (1992) and a discussion of equity in health care in Wagstaff and Van Doorslaer (2000) and Williams and Cookson (2000).

Equity is concemed with fairness and justice and involves the distribution of income or wealth, and access to goods and services. Defining equity with precision is difficult, as it is concemed with value judgements, and values differ between individuals, groups and societies, and may vary over time. Economic theory provides no guidance in evaluating moral or ethical issues. 
All concepts of equity are concerned with equalising something and because many things of relevance to heal th could be equalised, this leads to many possible definitions of equity. The implications and interpretation of equity depend upon what is being equalised. Equity is evaluated by comparing a specific feature of one citizen with the identical aspect of others. Sen alludes to the wide range of variables upon which equity could be judged. “... the judgement and measurement of inequality is thoroughly dependent on the choice of the variable (income, wealth, happiness, etc.) in terms of which comparisons are made..." (Sen, 1992: 2).

Le Grand (1982: 14) identifies five criteria that have been used as objectives for the distribution of government expenditure. These are, equalizing: (1) public expenditure, (2) final income, (3) use, (4) cost/ access, and (5) outcome. Public expenditure on a particular service or good could be allocated equally to all relevant citizens. Expenditure could be allocated so that the same quantum of service is used by all relevant individuals. Government funds could be spent so that their final incomes, private earnings plus transfers, are the same. Equalizing cost or access was described as all individuals facing the same out of pocket cost. However, equal access could be regarded as a separate category. Individuals may face equal cost, but access to services may be quite different, as other factors such as education and knowledge of how to obtain the service may differ. Equalizing outcome refers to final results of the service which could be an equal state of health.

"Equity in health care is the subject of much debate...most health care systems seem to define their equity goals in terms of equal access, normally equal access for equal need..." (Mooney, 1996: 100). Culyer and Wagstaff (1993: 431) considered four equity propositions of relevance to health and health care. These propositions related to; equalizing utilization, distribution according to need, equal access and equalizing health. They asserted that these four concepts were incompatible. Equity, it was suggested, should be based on equalizing health. Thus, equity in health care should involve "...distributing care in such a way as to get as close as feasible to an equal distribution of health...". 
It is convenient to consider two types of equity, that is, horizontal equity and vertical equity. Horizontal equity is the identical treatment of identical people, that is, equal access for equal need. For example, the provision of universal benefits for all elderly, or for all people under a specified income level. The provision of minimum standards of, or equality of access to health care for all citizens are also examples of horizontal equity. Most health care systems are primarily concerned with horizontal equity.

Vertical equity is the different treatment of unequals to reduce the differences between them (Atkinson and Stiglitz, 1980). Examples of vertical equity include; targeted benefits, redistribution of income from rich households to poor households, the provision of wheel chairs to some disabled and walking sticks to others (depending upon need). Policies aimed at closing the gap between Māori and non-Māori health status also have a vertical equity focus.

Mooney discusses vertical equity in relation to Aboriginal health in Australia and suggests that if vertical equity principals are to be applied to health care, then community preferences to weight the health gains of different groups in society (with different health status) must be established (Mooney, 1996: 102). Findings from a survey of Australian health care decision makers suggested that Australians value health gains differently for different groups that have different health states ( Mooney et al. 1995). The survey also discovered that people were prepared to express preferences relating to the allocation of health gains.

Other concepts relating to equity and distributive justice are discussed because they are of relevance to public policy decision making in health care. These ideas are; utilitarianism, Rawls' theory of justice, Sen's capacity to achieve, Williams "fair innings" notion, the inverse care rule and the rule of rescue.

Utilitarianism defined societal welfare as the sum of the welfare of all citizens. "The utilitarian objective is to maximize the sum-total of utility irrespective of distribution, but that requires the equality of the marginal utility of everyone..." (Sen, 1982: 354). Sen (1997) asserts that utilitarianism is unconcerned with inequalities in individual utilities "All 
that matters in the utilitarian view is the sum total of these utilities representing the respective individual advantages, independently of their distribution." This approach "...takes no account of the total utility enjoyed by a person - only the impact on utility at the margin, so that a person who is much worse off in terms of overall well-being or utility receives no particular consideration for that reason." (Sen, 1997:110). The inability to focus on total well being of individuals makes practical application of this criterion socially unacceptable as a means of evaluating societal equity.

Rawls (1972) accepts inequality provided that it is impossible to improve the situation for the worse off. He argues that if individuals make decisions behind a "veil of ignorance" they will automatically choose (if they are risk averse) to improve the situation of the worst off because they fear that the circumstances could apply to them.

An alternative view was tendered by Sen (1992: 148) who argues that a person's relative situation should be judged in terms of capacity to achieve rather than actual achievements. People are diverse. Variations in factors such as age, gender and genetic makeup mean that different individuals have very different capacities to benefit from an intervention.

Williams (1997a, 1997b, 1997c), discusses the notion of the "fair innings" as a way of considering intergenerational equity and rationing health care by age. Rationing using the "fair innings" approach implies "... that healthcare resources should be allocated so as to reduce the inequalities in people's lifetime experience of health..." (Williams, 1997a:1499). Everyone should be "...entitled to some 'normal' span of health..." (Williams, 1997b: 117). The "...preferred concept would be the number of quality adjusted life years a person had enjoyed..." rather than a specific age in years (Williams, 1997c: 822). Thus a "fair innings" for a person experiencing a low quality of life would be a greater length of time than for someone else living a healthy life free of pain or suffering.

The inverse care rule states "...the availability of good medical care tends to vary inversely with the need of the population served. ... The force that creates and maintains the inverse care law is the operation of the market..." Hart (1971: 412). In other words, those with greatest need are least able to access health care in a market driven health system. Hart 
advocates removing as much of health care as possible from the market.

The rule of rescue, (Luce, 1994; Morreim, 1994; Hadorn, 1991) that compels people to ignore the cost and help those in immediate need, creates a dilemma. It is argued that "To rescue someone with interventions beyond his health care package thwarts the democratic process and cheats all other citizens whose care remains within the designated limits, or whose entitled care must now be abridged to accommodate the drain on resources..." (Morreim, 1994: 466).

While ethics or justice criteria do not bound utilitarianism, Rawls' "veil of ignorance" makes certain that any application of justice is impartial. Williams' "fair innings" idea, and Sen's "capacity to achieve" concept are both constrained by a sense of justice. Both Williams and Sen attempt to address inequalities in health over a lifetime or period of time rather than inequalities at one specific time. The rule of rescue is strongly based on ethical criteria. While the inverse care rule is a factual statement about income, burden of illness and access to health care, its ethical underpinning could be derived from those who believe that the market distribution of income is fair.

Because definitions of different types of equity may be interpreted differently by different people, and equity values differ between individuals, clear explanations and descriptions of equity goals and consequences of a policy are essential.

\subsubsection{Equity Efficiency Trade-off}

Moving from one allocative efficient solution to another is not costless. Arrow (1963:943) states "... if the allocation mechanism in the real world satisfies the conditions for a competitive model, then social policy can confine itself to steps taken to alter the distribution of purchasing power...". However, in a footnote (Arrow (1963: 943, note 2) comments, "....in practice, it is virtually impossible to find a set of taxes and subsidies that will not have an adverse effect on the achievement of an optimal state...". If the allocative and distributional effects of a policy cannot be separated "... the traditional economic method, in which competitive forces are allowed to prevail and distribution is only done 
afterward, is not necessarily appropriate..." (Rice, 1998:37).

The efficiency equity trade-off may be demonstrated by use of a utilities possibilities frontier and social indifference curves. In figure 2.4 point $\mathrm{A}$ is not efficient but is more equitable while point $\mathrm{B}$ is efficient but less equitable. If there is a shift from point $\mathrm{B}$ to point $\mathrm{A}$, some societal utility is lost, equity is enhanced, individual $\mathrm{Y}$ gains utility and individual $\mathrm{X}$ experiences a reduction in utility.

Figure 2.4: Equity Efficiency Trade-off

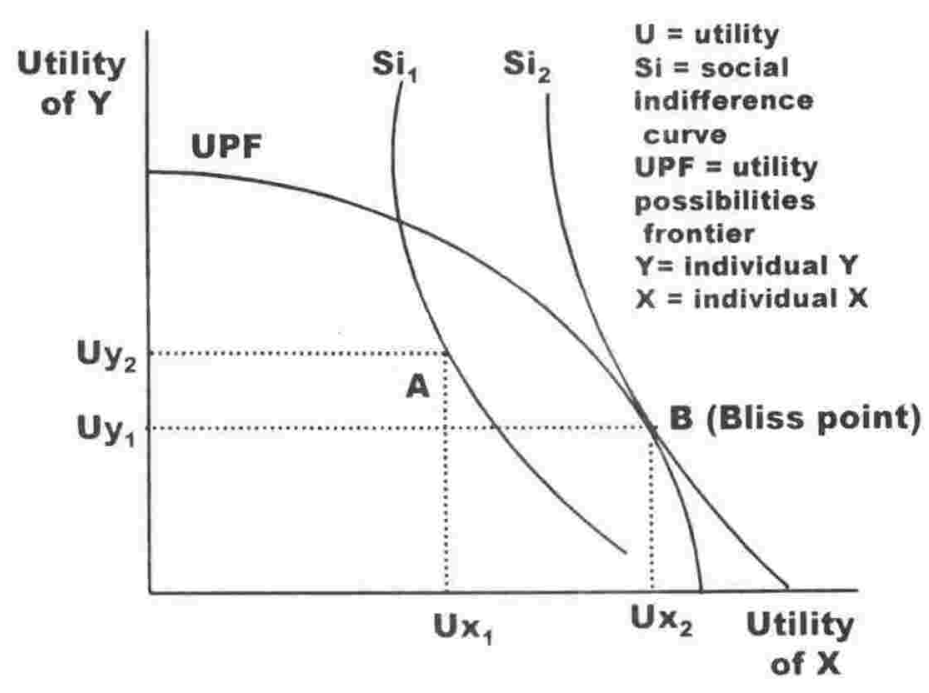

Two examples of efficiency and equity trade-off are as follow. Taxation and redistribution of income (except lump-sum taxes and transfers which are not feasible) to redress equity may cause inefficiencies; “... any practicable system of taxation may restrict factor supply, reduce output and cause inefficiency in production and/or product mix." Barr (1987: 77). If a rural hospital is closed, productive efficiency may be increased at the expense of equity of access to hospital services for rural citizens.

Efficiency/ equity trade-off in public policy decision making can be avoided only if:

- Aggregated net benefits of the policy are positive and the poor are net gainers and the wealthy are net losers, or

- It is possible to ascribe weighting factors to the net benefits of different groups in society and thus obtain a weighted societal net benefit for the policy. A social 
welfare function would be necessary for such an analysis.

The policy maker will often be forced to trade off some efficiency to achieve a policy outcome in accord with society's equity values. Accordingly, equity and efficiency consequences should be considered in parallel when evaluating public policies. Community preferences to weight health gains of different groups in society are necessary if equity goals are to be addressed (Mooney (1996: 102). Research is required to derive such usable societal preference weights for defined population groups.

\subsection{MARKETS AND GOVERNMENT INTERVENTION}

Most policy objectives may be accomplished using a range of policy instruments (Howlett and Ramesh, 1995) which have varying degrees of government involvement. Voluntary policy instruments involve little or no government intervention and include private markets, family, and voluntary and community organisations. At the other end of the spectrum there are compulsory instruments such as direct government provision of goods and services.

Evans (1997: 428-9) provides some management advice and a set of social objectives relevant to health care. "Market like mechanisms within publically funded health care systems constitute a particular set of management tools that might be used along with other more established mechanisms to promote the following generally accepted social objectives:

a Effective health care, efficiently provided and equitably distributed across the population according to need;

b Fair but not excessive reimbursement of providers; and

c Equitable distribution of the burden of contributions according to ability to pay; within

d An overall expenditure envelope that is consistent with the carrying capacity of the general econony, or rather its members' collective willingness to pay."

Figure 2.5 shows how the interaction of supply, demand (market forces) and government spending on health influences resource utilization. This is a simplified diagram in which 
lagged feedback loops are not shown (for example, utilization of resources that are intended to alter health status may in turn alter needs).

As the New Zealand health care system is predominantly government funded, public policies have a major impact on utilization of health care resources. Succeeding sections discuss the role of the market and failure of the markets and governments.

Figu re 2.5: Utilization of Health Care and the Interaction of Supply and Demand

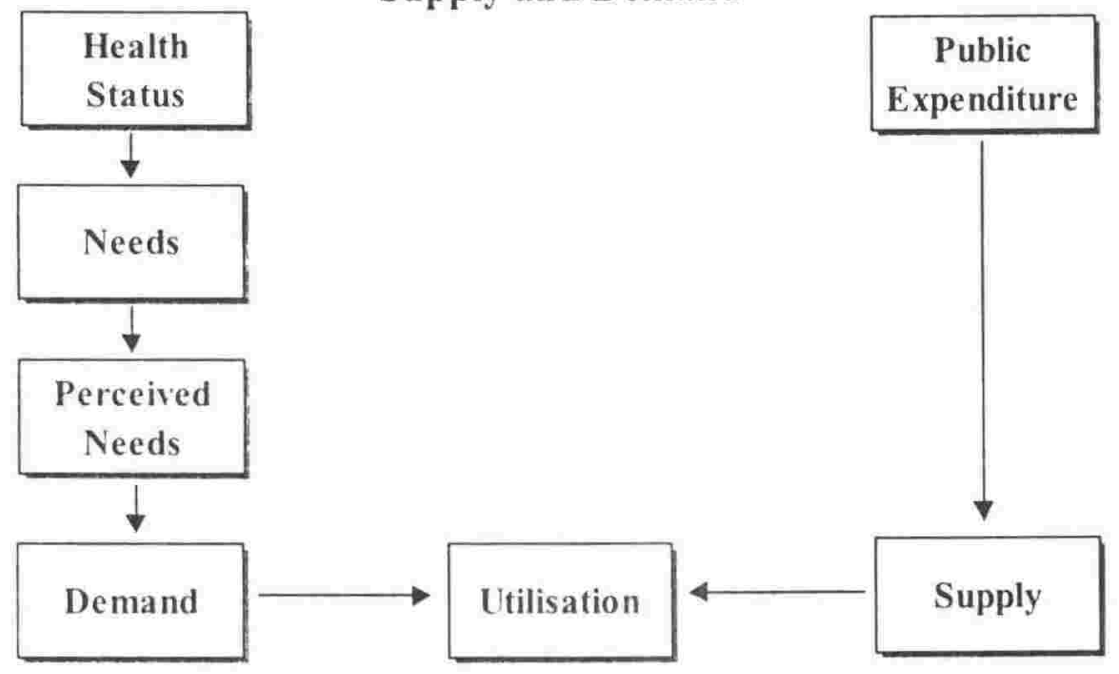

\subsubsection{The Role of the Market}

Although the application of economic evaluation techniques is of value in both free and unregulated markets a discussion of markets and market failure is provided because many public policies are introduced either to correct for market failure or to address equity issues. These public health interventions frequently complicate economic evaluation. Prices charged may no longer be an accurate reflection of production costs. Regulation may impose limited direct explicit (or financial) costs but require substantial compliance and generate considerable implicit costs. In both cases, the economic evaluation must ensure that all costs and benefits are captured with the true economic value incorporated. Accordingly, policy decision makers should understand markets; what they can and cannot achieve, how they function, and when they fail. 
Where there is market efficiency (in all markets) and if markets exist for all goods (including leisure time), the "invisible hand" of the market place ensures that marginal social benefit and marginal social cost are equated. The price system functions automatically. Every individual in pursuing her/his selfish interests is led as if by an "invisible hand" to achieve the best good for all of society (Smith, 1776). Sellers cannot sell goods or services the market does not want nor can they charge more than their competitors for identical goods. Perfectly competitive markets produce the goods and services that society wants at the lowest cost.

Evans (1977: 428) notes "There is in health care no "private, competitive market" of the form described in the economics textbooks, anywhere in the world. There never has been, and inherent characteristics of health and health care make it impossible that there ever could be. Public and private action have always been interwoven." Enthoven (1988) comments that health care markets are not naturally competitive and "deregulation" will not make them so. Rice (1997) also notes that although market forces may have a prominent role in allocating resources in health care, economic theory does not show them to be necessarily a superior approach to health care policy.

Uncontrolled and unregulated free markets may fail to produce the optimal outcome for society (with respect to society's goals of efficiency and equity). That is, the market solution may not result in outcomes that are in accord with society's goals.

When there is market failure, or if the market solution is not congruent with society's equity goals government may wish to intervene and attempt to correct for these shortcomings. Gibbs (19SS: 5) noted that "One of the central reasons for governments becoming involved in health care is the concem for faimess and the possible outcomes that would otherwise result."

For market efficiency a number of criteria must be met. Consumers and sellers have perfect and certain knowledge of the market, there are no externalities or public goods, there are large numbers of sellers and consumers with no power to influence the market. Market failure occurs when any of these efficiency criteria are violated. (Boadway and Bruce, 1984:13-15). 
Registration of medicines and health care providers is necessary because most consumers would have insufficient information (market failure) to enable them to make informed decisions on the safety of medicines and competence of providers. An example of a policy to address an equity issue is the provision of additional health subsidies to welfare beneficiaries and those on low incomes.

\subsubsection{Market Failure and the Second Best}

Public policy interventions aiming to maximise social welfare are often concerned with attempting to correct for market failure by endeavouring to fulfill as many of the Pareto efficiency conditions as possible. Given the difficulties of determining when a Pareto efficiency is attained, and of satisfying all the conditions for Pareto efficiency (first choice or first best) the concept of second best is important. If a Pareto efficient outcome cannot be attained, is it best to satisfy as many of the Pareto conditions as possible or would the second best be attained in some other way?

The theory of the second best, first ${ }^{6}$ described by Lipsey and Lancaster (1956), contends that, if in any given situation, it is not possible (or not desirable) to achieve a first best solution it may be better to diverge equally from all the efficiency conditions than to attempt to satisfy as many as possible of the efficiency conditions.

For example, it may be better to counter monopoly power of sellers with monopsony power of consumers rather than attempt to shift the market conditions and structure towards perfect competition. It could be argued that this is the situation relating to the pharmaceutical market in New Zealand where monopoly power of the pharmaceutical industry is matched by the monopsony power of the Pharmaceutical Management Agency (PHARMAC).

6. The concept of the second best was first formalised by Lipsey and Lancaster but the ideas had been discussed in the literature earlier (McKee \& West, 1981 and Streissler \& Neudeck, 1986) 
It is important to note the following:

- In general, nothing can be said about the direction or the magnitude of the secondary departures from optimum efficiency conditions made necessary by the original non-fulfilment of one condition (Lipsey and Lancaster, 1956: 12).

- There is no way of determining if one second best outcome is better or worse than another.

- $\quad$ "Second-best policy recommendations typically require some knowledge of where on the utility possibility frontier we want to be or in what direction we want to go. In this sense, policy recommendations cannot be distribution free and one cannot divorce efficiency from equity to the degree that one might have thought possible after studying first-best environments." (Blackorby 1990: 749).

- In many situations the second best solution would be so complex that they would not be usable or able to be translated into workable policies.

- The theory of the second best provides little practical guidance of how to achieve the second best (Price, 1977).

Although the theory of the second best is a useful concept, it raises as many questions as it answers. In practice, policy makers are virtually always attempting to reach the second best or some other "best" choice.

\subsubsection{Government Failure}

Market inefficiency or inequity does not mean that government intervention will improve the outcome (Atkinson and Stiglitz,1980 and Price,1977). Government intervention to correct for failure to achieve society's goals of efficiency and equity may not always be desirable. Governments may also fail to maximise social welfare (Weimer and Vining, 1989) and some market failures and distributional goals are too costly to correct. Stiglitz (1999) identifies four causes of government failure; limited information, limited control over private market responses, limited control over bureaucracy and limitations imposed by political processes. 


\subsubsection{Technical and Policy Evaluations of Health Care}

Having discussed policy analysis in general, this section targets policies relating specifically to health. Policies for investigation may enter the policy agenda in a number of ways; by evolution from existing policies, by transplantation from other countries, from developments in clinical and business management, and from scientific discoveries. Although the focus is on how new health related public policy strategies that emerge from basic scientific research are evaluated, the procedure is generic in that it may be applied to any new technology or policy.

Basic scientific research may suggest possible new interventions and policies. Applied research must then be employed, to ask, in an ideal situation, does the science work (efficacy) and, if so, could it improve economic efficiency? If the answers to these questions are yes, then a second set of questions follow; does it work in the real world, will it be cost-effective, is it equitable and ethically acceptable? If the programme is to be introduced, how will the patients waiting for treatment be selected? Selection or rationing options could include the price system, various assessed needs based methods (normative need, or the minimum standard of health care as assessed by experts), or by assessing quality of life gain. Finally, once a new policy is introduced, it should be monitored to check that it continues to work in a technical sense, and to satisfy equity, ethical, and efficiency criteria.

Table 2.1 illustrates the sequence in which new or existing health care interventions are evaluated from basic scientific research to final application in society. The evaluations listed will rarely provide a definitive "accept/ reject" result. More commonly, changes in efficiency and equity will be indicated with varying degrees of confidence. The central theme of this thesis is economic evaluation in the real world as highlighted by the italic text in Table 2.1. 
Table 2.1: Technical and Policy Evaluations of Health Care

\begin{tabular}{|c|c|c|c|}
\hline $\begin{array}{l}\text { Technical } \\
\text { question }\end{array}$ & Technical evaluation & Policy question & $\begin{array}{c}\text { Policy evaluation } \\
\text { method }\end{array}$ \\
\hline Is it possible? & Basic research & & \\
\hline$\nabla$ & $\nabla$ & & \\
\hline Could it work? & Applied research & & \\
\hline$\nabla$ & $\nabla$ & & \\
\hline $\begin{array}{l}\text { Does it work in an } \\
\text { ideal setting? }\end{array}$ & $\begin{array}{c}\text { Efficacy: The extent to which a } \\
\text { health care intervention achieves } \\
\text { beneficial health outcomes in an } \\
\text { ideal situation. Eg Therapeutic } \\
\text { outcomes determined in a } \\
\text { randomised clinical trial. } \\
\end{array}$ & $\begin{array}{l}\text { Could it improve } \\
\text { economic } \\
\text { efficiency? }\end{array}$ & $\begin{array}{c}\text { Economic evaluation in } \\
\text { an ideal situation or } \\
\text { setting. }\end{array}$ \\
\hline$\nabla$ & $\nabla$ & $\nabla$ & $\nabla$ \\
\hline \multirow[t]{2}{*}{$\begin{array}{l}\text { Does it work in } \\
\text { everyday practice } \\
\text { in the real world? }\end{array}$} & $\begin{array}{c}\text { Effectiveness: The extent to } \\
\text { which a health care intervention } \\
\text { achieves beneficial health } \\
\text { outcomes in a real world } \\
\text { situation. Eg Therapeutic } \\
\text { outcomes determined by a } \\
\text { controlled clinical trial in a real } \\
\text { word setting. }\end{array}$ & Is it cost-effective? & $\begin{array}{l}\text { Economic evaluation } \\
\text { in a real world setting } \\
=\text { Cost-effectiveness. }\end{array}$ \\
\hline & & $\nabla$ & $\nabla$ \\
\hline \multirow[t]{2}{*}{$\nabla$} & $\nabla$ & $\begin{array}{l}\text { Is it fair, where do } \\
\text { the costs and } \\
\text { benefits fall? Is it } \\
\text { ethically } \\
\text { acceptable? If the } \\
\text { programme is } \\
\text { introduced, who } \\
\text { will receive the } \\
\text { treatment? }\end{array}$ & $\begin{array}{l}\text { Evaluation of equity } \\
\text { changes and ethical } \\
\text { issues and analysis of } \\
\text { rationing options. }\end{array}$ \\
\hline & & $\nabla$ & $\nabla$ \\
\hline $\begin{array}{c}\text { Does it continue } \\
\text { to work? }\end{array}$ & Technical monitoring & $\begin{array}{l}\text { Does it remain } \\
\text { cost-effective/ } \\
\text { equitable? }\end{array}$ & $\begin{array}{c}\text { Monitoring of } \\
\text { economic and equity } \\
\text { effects. }\end{array}$ \\
\hline
\end{tabular}




\subsection{CONCLUSIONS}

The health reforms of the 1990s (Upton 1991) that separated the funder from the purchaser and increased competition were insufficient on their own to deliver the efficiency and equity goals of our society. Public policies are necessary adjuncts to the market process.

A variety of resource allocation decisions must be made at many levels within society and within the health sector. These decisions relate to efficiency and equity, and the trade-off between these two objectives. Government, for example, must apportion budgets between Votes, rationing funds between Vote: health, education, or social welfare. Within the health budget, services to fund from the public purse must be selected. The range and level of this publicly provided health care or "core health services" are matters of political debate and decisions will be different in different societies and will change over time. Resource allocation decisions may also need to be made between different diseases, such as, coronary artery bypass grafts compared with hip replacements. Finally, a choice may need to be made of the most cost-effective treatment option for a particular patient.

Public sector policy analysts and managers in health care enterprises make day to day resource allocation decisions and prepare long term plans and budgets that have spillover effects on the rest of society (externalities). Not only do their decisions impact on their own organisation, but they have efficiency and equity implications for society as a whole.

Economic evaluation (from a societal perspective) assists in the policy decision making process by providing information on efficiency. The technique considers all costs and benefits irrespective of whether or not a monetary payment is involved or who pays or benefits. Howerer, ethical implications and safety issues must be considered separately (before, during or after the economic investigation). The public policy decision making process must be transparent, and where efficiency equity trade-offs are recommended, the consequences of the choices should be explicit. 
Although much of the theory underlying health economics and resource allocation from a public policy perspective is well developed there are a number of practical difficulties in applying economic theory and techniques to actual problems. Succeeding chapters of this thesis focus on the practical application of economic theory to everyday policy problems. Chapter 3 considers ways in which health outcomes may be valued. 


\subsection{REFERENCES}

Arrow, K. J. "Uncertainty and the welfare economics of medical care." American Economic Review 53 (1963): 941-73.

Atkinson, A. B., and J. E. Stiglitz. Lectures on public economics. London: McGraw-Hill, 1980.

Barr, N. The economics of the welfare state. 1st ed. London: Weidenfeld and Nicolson, 1987.

Battiato, S. E. "Cost-benefit analysis and the theory of resource allocation." In Efficiency in the public sector: The theory and practice of cost-benefit analysis. 1st ed., edited by A. Williams and E. Giardina. Aldershot: Edward Elgar Limited, 1993.

Baumol, W. J. Economic theory and operations analysis. 2nd ed. Englewood Cliffs: Prentice-Hall, 1965.

Blackorby, C. "Economic policy in a second-best environment." Canadian Journal of Economics 23 (1990): 748-71.

Boadway, R., and N. Bruce. Welfare economics. Oxford: Blackwell, 1984.

Culyer, A. J., and A. Wagstaff. "Equity and equality in health and healthcare." Journal of Health Economics 12 (1993): 431-57.

Donaldson, C., and K. Gerard. Economics of health care financing: The visible hand. 1st ed. London: Macmillan, 1993.

Enthoven, A. C. Theory and practice of managed competition in health care finance. Vol. 9. Ansterdam: North-Holland, 1988.

Evans, R. G. "Going for gold: The redistributive agenda behind market-based health care reform." Joumal of Health Politics, Policy and Law 22 (1977): 427-65. 
Evans, R. G., and G. L. Stoddart. "Producing health, consuming health care." Social Science and Medicine 31 (1990): 1347-63.

Gibbs, A. Unshackling the hospitals: Report of the Hospital and Related Services Taskforce. Wellington: Hospital and related services taskforce, 1988.

Gravelle, H., and R. Rees. Microeconomics. 1st ed. New York: Longman, 1985.

Grossman, M. The demand for health: A theoretical and empirical investigation. Occasional Paper 119. New York: National Bureau of Economic Research, 1972.

Hadorn, D. C. "Setting health care priorities in Oregon. Cost-effectiveness meets the rule of rescue." Journal of the American Medical Association 265 (1991): 2218-25.

Hart, J. T. "The inverse care rule." The Lancet (1971): 405-12.

Health Benefits Review. Choices for health care: Report of the Health Benefits Review. Wellington: Health Benefits Review, 1986.

Henderson, J. M., and R. E. Quandt. Microeconomic theory a mathematical approach. 1st ed. New York: McGraw-Hill, 1958.

Hicks, J. R. "The foundations of welfare economics." Economic Journal 49 (1939): 696-712.

Kaldor, N. "Welfare propositions of economics and interpersonal comparisons of utility." Economic Joumal 48 (1939): 549-52

Le Grand, J. The strategy of equality. Ist ed. London: George Allen and Unwin, 1982.

Lipsey, R. G, and K. Lancaster. "The general theory of second best." The review of economic studies 24 (1956): 11-32. 
Luce, J. M. "The changing physician-patient relationship in critical care medicine under health care reform." American Journal of Respiratory and Critical Care Medicine 150 (1994): 266-70.

McKee, M., and E. G. West. "The theory of the second best: a solution in search of a problem." Economic Inquiry 19 (1981): 436-48.

Mooney, G., S. Jan, and V. Wiseman. "Examining preferences for allocating health care gains." Health Care Analysis 3 (1995): 261-65.

Mooney, G. "And now for vertical equity? Some concerns arising from Aboriginal health in Australia." Health Economics 5 (1996): 99-103.

Morreim, E. H. "Of rescue and responsibility: Learning to live with limits." The Journal of Medicine and Philosophy' 19 (1994): 455-70.

Musgrave, R. A., and P. G. Musgrave. Public finance in theory and practice. 3rd ed. London: McGraw-Hill, 1982.

National Advisory Committee on Core Health and Disability Support Services. Core Services for 1995/96. Wellington: National Advisory Committee on Core Health and Disability Support Services, 1994.

N.Z. Ministry of Health. Healthy New Zealanders: Briefing papers for the Minister of Health. Vol. 1 Key policy issues. Wellington: N.Z. Ministry of Health, 1996.

N.Z. Ministry of Health. Health expenditure trends in New Zealand 1980-97. Wellington: Sector Analysis Section, N.Z. Ministry of Health, 1998.

New Zealand Royal Commission on Social Policy. Report of the New Zealand Royal Commission on Social Policy: Wellington: The Commission, 1988. 
Pareto, V. Manuel d'Economie Politique. 2nd ed. Paris: Girard, 1927. Translated by

Schwier, A. S. and edited by Schwier, A. E. and Page, A. N. New York: MacMillan Press, 1971.

Pearce, D. W., and C. A. Nash. The social appraisal of projects: a text in cost-benefit analysis. 1st ed. London: Macmillan Press, 1981.

Price, C. M. Welfare economics in theory and practice. London: Macmillan, 1977.

Rawls, J. A theory of justice. Oxford: Oxford University Press, 1972.

Rice, T. "Can markets give us the health system we want?" Journal of Health Politics, Policy and Law 22 (1997): 383-423.G13

Rice, T. The economics of health reconsidered. Chicago: Health Administration Press, 1998.

Scitovsky, T. D. E. "A note on welfare propositions in economics." The review of economic studies 9 (1941): 77-88.

Sen, A. Choice Welfare and Measurement. Oxford: Blackwell, 1982.

Sen, A. Inequity reexamined. 1st ed. ox ford: Oxford University Press, 1992.

Sen, A. On economic inequality, Oxford: Clarendon Press, 1997; Sen, A. 1997.

Smith, A. The wealth of nations. London: Dent, 1981(reprinted from the original 1776)

Streissler, E., and W. Neudeck. "Are there interllectual precursers to the idea of second best optimization?" Jounal of economics Zeitschrift fur nationalokonomie Supplement 5 (1986): 227-41.

Stiglitz, J. E. Economics of the public sector. NY: Norton, 1999. 
Schokkaert, E. 1992. The economics of distributive justice. Justice: Interdiscliplinary Perspectives. Editor K. Scherer, 65-113. Cambridge: Cambridge Unuversity Press.

Upton S. Your health and the public health: a statement of government health policy. Wellington: Minister of Health, 1991. (The Green and White Paper)

Weimer, D. L., and A. R. Vining. Policy analysis: concepts and practice. NJ: Englewood Cliffs, 1989.

Wagstaff, A., and E. Van Doorslaer. 2000. Equity in health care financing and delivery. Handbook of health economics. 1 ed., Editors A. J. Culyer, and J. P. Newhouse, 1803-62. Amsterdam: Elsevier.

Willians, A. "Rationing health care. Can a "fair innings" ever be fair?" British Medical Journal 14 (1997a): 1499.

Williams, A. "Intergenerational equity: an exploration of the 'fair innings argument'." Health Economics 6 (1997b): 117-32.

Williams, A. "The rationing debate: Rationing health care by age: The case for." British Medical Journal 314 (1997c): 820-22.

Williams, A., and R. Cookson. 2000. Equity in health. Handbook of health economics. 1 ed., Editors A. J. Culyer, and P. Newhouse, 1863-910. Amsterdam: Elsevier.

Wildavsky, A. "Doing better and feeling worse: The political pathology of health policy." Daedalus 106 (1977): 105-24. 


\section{CHAPTER 3: VALUING HEALTH OUTCOMES}

\subsection{INTRODUCTION}

To evaluate economic efficiency, it is necessary to quantify both inputs and outputs. Economic evaluation in health care focuses on the relationship between changes in resource usage and changes in health status and it is therefore necessary to measure changes in both resource cost and health outcomes.

This chapter discusses the problems of valuing health and the methods used to quantify health related outcomes (the measurement of inputs is covered in Chapter 4: Costs.) A brief history of quality of life measurements with respect to health is outlined, and some examples of the instruments that are in use are described. There is a large and increasing array of such measures which are continually evolving. Individual instruments will not be discussed in detail as this chapter is intended to provide an overview of developments in the measurement of health outcomes. However the Rosser index, because of its historical significance, and the EuroQol and Short Form 36, because of their wide use, have been discussed in greater depth.

\subsubsection{What is Health?}

Health and good health are ill-defined and multi dimensional concepts. The World Health Organisation (WHO) defines health as "a state of complete physical, mental and social well-being, and not merely absence of disease or infirmity" (World Health Organization, 1948). This concept of health is central to the development of methods to measure patient well-being or health related quality of life (HRQOL). Bulpitt (1997: 613) defines health related quality of life as "... the degree of subjective well-being attributable to or associated with lack of symptoms, psychological state and activities pursued."

Only where problems of high premature mortality are no longer a pressing social concern does it become relevant to think of health in WHO terms (McDowell and Newell, 1996: 
12). At low levels of income, mortality is likely to highly influenced by deficiencies in factors such as nutrition, shelter, clothing, and sanitation. In developed countries with growing incomes and an aging population, quality of life becomes increasingly important particularly as the cost of extending the life of an individual with a poor quality life can be very high. Cooper (1980: 4) comments "...death is extremely cheap and survival often very expensive as a faulty body has to be maintained until death claims it by the same or another means...".

\subsubsection{Why We Need to Quantify Changes in Health Outcomes}

As beneficial health related outcomes (prolonged life or a reduction of pain and suffering) are not traded on markets, other approaches must be employed to value them. The problem of valuing non-market goods and services is not unique to health outcomes, and economists have developed a number of ways of obtaining implicit values. This is equally applicable to costs. Even where market prices exist it is often necessary to apply non-market valuation methods if there is market failure.

\subsubsection{Differences in the Valuations of Health and Health Outcomes}

Valuations of health differ, depending upon the perspective taken and the individuals in the population group surveyed. This section discusses these differences and the consequences for valuing health related outcomes.

The evaluation of health and health related quality of life is highly dependent on value judgements which will differ according to whose values are considered (patients, clinicians, hospital managers, the Minister of Health, the Minister of Finance). Individuals have different preferences and accordingly they may value health status and quality of life differently. Variations may result from differences in age, income or cultural background. Of particular concem are the potential differences in values derived from expert opinion compared with those from individuals. 
If the philosophy of consumer sovereignty is followed, then the values and preferences of the consumers of health care should be paramount. Economists since the writings of Adam Smith in his 1776 book Wealth of Nations have recognised the importance of consumer sovereignty (Smith, 1981:155).

Gold et al. (1996: 99) assert that if a societal perspective is adopted for decision making, the most accurate indicator of society's preferences for a particular health state would be preferences obtained from informed members of the community. As "... a rational public decides what is the best course of action when blind to its own self-interest, aggregating the utilities of persons who have no vested interest in particular health states seems most appropriate." (Gold et al., 1996: 100). This is referred to as decision making under a "veil of ignorance" (Garber et al., 1996: 35).

However, there are even problems in using preferences from informed members of the general public or even what the term "informed" means. People who are actually experiencing a particular health state may be better able to give a valuation of that state. Some health states impose externalities onto family and friends of the sufferer. To overcome this lack of information and experience, values may be obtained by expert opinion from clinicians and nurses familiar with the health state.

Another complication is that the interests of society are not necessarily the same as those of the individual and the sum of individual preferences do not necessarily reflect those of society. Weinstein et al. (1980) illustrate this by means of an example of two individuals who each stand to benefit from medical treatment. If each acted in their own interests each would seek all health care from which they expect to benefit (assuming no time or other costs). Assume patient A stands to gain one year of life from the treatment and patient B one and a half year, treatments are mutually exclusive and that there are sufficient resources to provide treatment to one but not both patients. Each individual (without information about the other) would choose treatment for themselves. However an independent adjudicator acting on behalf of society (principal agent) and having knowledge of both patients may allocate treatment to the patient with the greatest potential benefit (with respect to changes in health related quality of life). This conflict is mirrored in the 
perspectives of policy makers (society or budget perspective where patients are not individually known to them) versus clinicians (where individual patients are known to them).

A similar paradox relating to individual values and social choice is embodied in Arrow's (1951) impossibility theorem, which says that under a number of plausible assumptions no aggregation procedure exists that will always result in collective choices that are consistent with choices of the individuals making up the group concerned. This implies that (even if each individual's preferences were known) it may not be possible to aggregate individual preferences into a meaningful societal measure.

\subsection{WAYS OF VALUING CHANGES IN HEALTH OUTCOMES}

Because of the difficulties in measuring changes in health related outcomes a number of evaluation techniques have developed. As each of these economic analysis methods copes with the quantification of health outcomes in different ways. With cost-benefit analysis, both changes in resource usage and in health outcomes are measured in dollars. As costeffectiveness measures the costs in dollars and the health outcome in physical units, it avoids some of the valuation difficulties. In cost-utility analysis, costs are measured in dollars and health outcomes in a utility measure (see section 3.3 Health Indices).

\subsubsection{Cost-benefit Analysis}

Many costs and benefits are not traded on markets, and even where markets exist, market failure may mean that prices do not provide accurate societal values. In cost-benefit analysis (CBA) a number of methods have been used to assign money values for non market goods and services and establish non market or shadow prices. These techniques fall into two main groups, that is, non-demand curve or demand curve methods, and may be further classified as shown in Figure 3. 1 ((Tumer et al., 1994: 115, Box 8.3) provided some of the basic ideas for this diagram). 
Figure 3.1: Valuation of Non-Market Goods and Services

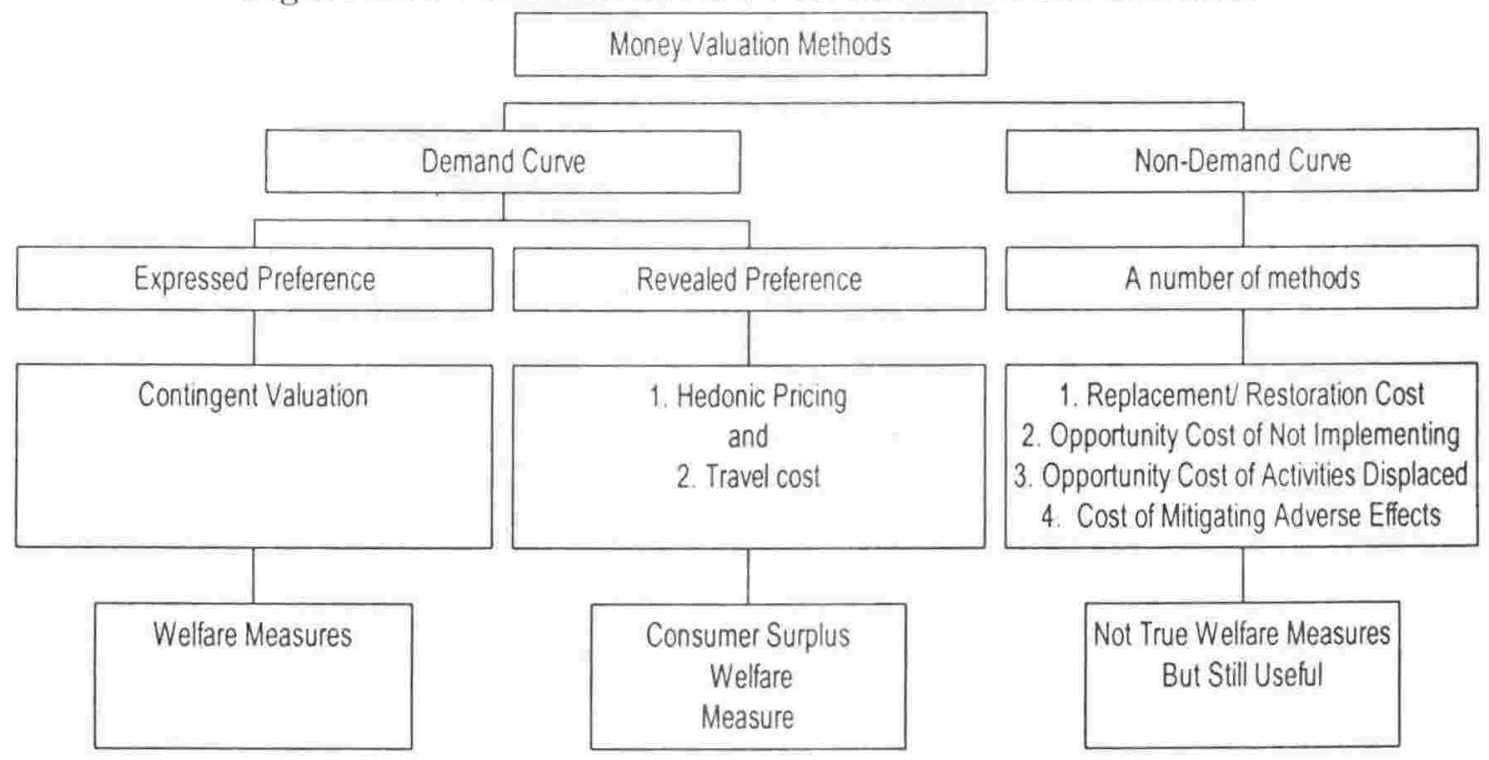

\section{Non-market Valuation Methods}

Although non-demand curve methods are more relevant to environmental economics they will be briefly discussed. Health economics and environmental economics have many similar problems and both disciplines can learn from each other. Health economics can apply the experience gained in cost-benefit analyses of the environment, while environmental economists may profit from experience with cost-effectiveness and costutility analyses in health. Non-demand curve methods discussed are; replacement cost, opportunity cost and the cost of mitigating adverse effects.

\section{Replacement Cost for Valuing Health Facilities}

The replacement cost method considers the cost of replacing or restoring assets that will be damaged by the project being implemented. Upgrading hospital facilities could necessitate destruction or loss of car parking space which could be replaced by a parking building. The loss of the existing parking space would be measured by the cost of constructing a parking building to provide the same number of parking spaces. 
Opportunity Cost

Opportunity costs of not undertaking the project involve estimating the benefits of the project to determine the magnitude of compensation for costs imposed on others. The building of a hospital may impose negative externalities of noise and dust on adjacent homes and businesses and potential compensation costs could be calculated. Alternatively the value of the project may be evaluated by considering the opportunity costs of the activities displaced. Building a new hospital may result in the closure of a factory and opportunity cost of earnings foregone to society could be assessed by estimating the value of the lost factory output.

\section{Mitigating Behaviour}

Mitigating behaviour relates to activities designed to mitigate damage or costs imposed by the programme. An example of mitigating behaviour would be the installation of sound insulation in housing adjacent to a city ambulance base.

\section{Demand Curve Methods}

Demand curve evaluation methods are discussed in more detail as they have direct relevance to public policy issues in health. These methods attempt to measure willingness to pay for a good or service. The economic basis is that the total willingness to pay (WTP) for a health outcome is the purchase price $(\mathrm{P})$ multiplied by the amount purchased $(\mathrm{Q})$ plus the consumer surplus $(\mathrm{S})$. WTP $=\mathrm{P} \times \mathrm{Q}+\mathrm{S}$ (Dasgupta and Pearce, 1980: 45). The idea behind the consumer surplus approach is that some consumers would have been prepared to pay more than the market price to obtain the good. These consumers' preferences are measured by the triangle " $\mathrm{S}$ " (if the demand curve is linear) denoted by the area under the demand curve and above the market price (Figure 3.2). This assumes that it is a competitive market with no externalities. Figure 3.2 illustrates total consumer surplus but it is the change in consumer surplus induced by the project that is more relevant to health programmes. 
Figure 3.2: Consumer Surplus

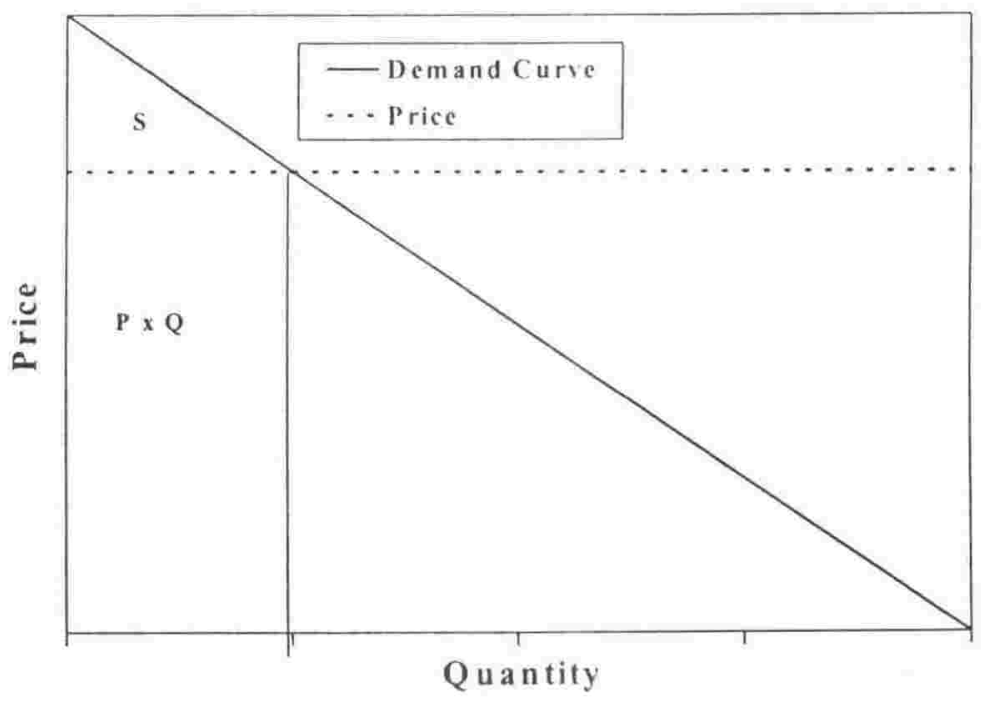

Expressed (Stated) Preference Or Contingent Valuation

Expressed (stated) preference or contingent valuation methods use marketing research techniques to measure willingness to pay for the good or service in question (or some related good or service). The willingness to pay measure so derived is based on preferences expressed in hypothetical surveys not on actual decisions made. Many economics studies have utilised these techniques, but the validity of their application to valuing health outcomes is not universally accepted because the relationship between hypothetical payments and actual money payments is unclear (Johannesson, 1996). There is a considerable body of literature (for example, Zikmund, 2000 and Sekaran, 1992) on survey methods applied to social sciences and business research but this will not be reviewed.

Although it is not the purpose of this chapter to discuss the multitude of ways in which contingent valuation questions could be constructed, administered, and analysed, one example will be mentioned. Johamnesson (1996: 92) describes the use of contingent valuation with respect to drug therapy for hypertension. A mail-out survey was conducted. Contingent valuation was used to estimate how much patients would be prepared to pay out of pocket for their treatment. The willingness to pay question was worded as follows:

"Would you choose to continue your treatment for high blood pressure if 
your fees for treatment were SEK 500 per month?

YES, DEFINITELY

YES, PROBABLY

NO, PROBABLY NOT

NO, DEFINITELY NOT

DON'T KNOW"

The bid was varied between SEK 50 and SEK 1500 in 15 subsamples to generate data that could be used to define a demand curve. Regression analysis (explanatory variables included gender, age, income, education and household size) was used to estimate a demand curve.

\section{Revealed Preference}

Revealed preference methods evaluate willingness to pay by observing actual choices concerning risk to health or life. In their everyday activities people make decisions and take actions that influence the probability of their dying, having an accident, or falling ill. The additional cost of purchasing and driving a large car compared with a small car will lower the probability of the driver being seriously injured in an accident. A low animal fat diet is generally considered to reduce cardio-vascular disease risk factors but may cost more than a diet high in saturated fats. Protective clothing costs more than everyday street-wear. Additional remuneration must be paid to induce workers to do a risky job. In making these choices, consumers and employees implicitly reveal their preferences (subject to income constraints and imperfect markets). Two techniques are used in economics, they are, the travel cost and hedonic pricing methods. In applying these methods, an implicit assumption is perfect information and "rational" behaviour. Data should also be collected on factors such as income, gender, education and age as these will also influence the decisions.

Travel costs have been used to define the demand curve for (and hence the value of) recreational assets such as lakes or forests. This involves estimating the cost of visiting the site by asking visitors how far they have travelled. To my knowledge this method has not been applied to health care but could be used to measure some of the benefits of 
maintaining a rural hospital.

Hedonic or implicit pricing involves econometric or statistical techniques to estimate price or wage/ risk trade-offs. Hedonic pricing equations could be used to assess willingness to pay to avoid risk of death or injury from fire by purchasing a smoke detector. The demand curve (the maximum willingness to pay) for safety from the purchase of safety products could be evaluated by observing the difference in willingness to pay over a sample of individuals. Johannesson (1996) describes the use of hedonic pricing in wage-risk studies and consumer evaluations of safety. In New Zealand the value of a statistical life has been estimated for transport decision making (Miller and Guria, 1991) (See Chapter 4: Costs section 4.3.2: Indirect Costs). Although hedonic pricing analysis is not commonly used, it could be applied to a wide range of health care interventions.

\section{Conclusion}

Although these techniques have not been widely used in health economics, contingent valuation methods are likely to be used more frequently in the future as experience and knowledge of methods used in other branches of economics, marketing and business is transferred to health.

\subsubsection{Cost-Effectiveness and Cost-Utility Analysis}

These methods are discussed in greater detail in Chapter 6: Economic Evaluation.

Cost-Effectiveness Analysis (CEA)

Cost-effectiveness analysis avoids allocating monetary values to outcomes but if there is more than one health related outcome of interest this method becomes difficult to use. If a single measure could be used for all health related outcomes, it would then be possible to relate resource costs to this measure. This would involve first identifying, then quantifying and weighting each outcome indicator to derive a single number to describe and quantify the health related state. 
Cost-utility Analysis (CUA)

Cost-utility analysis relates cost to outcomes measured by a HRQOL indicator. Utility as used in CUA is not necessarily the utility as understood by economists. Utility (as used in CUA) refers to the preferences individuals or society have for a specified set of health outcomes or health state. Drummond et al. (1987: 15) provide an elegant example demonstrating that a health outcome is different from the utility of a health outcome. Two twins identical in every way except that one is a sign painter and the other a translator. If they were asked to rank having a clinically identical broken arm on a health utility scale of 0 (dead) to 1 (perfect health) their rankings may well differ because arm use may affect their occupations differently.

\subsection{HEALTH INDICES}

\subsubsection{Health Indices as a Subset of Social Indicators}

According to Culyer et al. (1972) social indicators are needed to measure the output of social policies and to indicate how that output could be increased. In this context, health indices are a subset of social indicators. Culyer classifies health indicators as: state-ofhealth, need-for-health and effectiveness indicators. State of health indicators are used to quantify the output of a programme or policy. Need for health indicators attach social values to these outputs and thus allow judgements to be made as to the social desirability of increasing or decreasing this output. Effectiveness indicators measure how effective the policy has been at delivering health care. Table 3,1 lists the type of indicator and the task it has to perform. 


\section{Table 3.1: Health Indicator Tasks and Types}

\begin{tabular}{|c|c|c|}
\hline Task & & Indicator \\
\hline • & $\begin{array}{l}\text { Measure quantity of output of } \\
\text { health policies }\end{array}$ & - $\quad$ State of health \\
\hline - & $\begin{array}{l}\text { Provide social valuations on the } \\
\text { outputs of health policies }\end{array}$ & Need for health \\
\hline - & $\begin{array}{l}\text { Measure the technical possibility } \\
\text { of increasing output }\end{array}$ & Effectiveness \\
\hline
\end{tabular}

The first documented use of a health index applied to hospital management is attributed to Florence Nightingale in 1863. She considered the outcome of hospital care should be classified "dead, relieved or unrelieved" (Rosser, 1983:52). Rosser notes that "this primitive classification is a little more sophisticated than those in routine use in our hospitals today!"

\subsubsection{Objectives of Health Indices}

The objective of health indices or indicators is to quantify health status of a defined population group, or an individual with a specific disease. Health indices may be used to track health trends over time, for international comparisons, to investigate determinants of health status, or to provide information for resource allocation.

Time series analysis of health status is a vital check on the effectiveness of programmes such as control of infectious diseases. If the notifications of water borne disease increased it could indicate a failure of water treatment methods. Comparisons of disease incidence in New Zealand with similar populations in other countries can indicate how effective New Zealand prevention and treatment programmes compare with those in other countries or they could indicate differences in causal factors. Within New Zealand, knowledge of health indicators relating to different populations (defined by geographic location, ethnicity, age, gender or sociocconomic measures) could provide a means of allocating health resources according to assessed need. Reliable health indices would thus facilitate public policy formulation, evaluation and monitoring. Uhde, 1983:110-111 comments on the need for such indices. 


\subsubsection{Validated Instruments}

McDowell and Newell (1996: 8-9) provide a useful checklist of questions to ask when evaluating a health related quality of life instrument or method.

- Is the method suitable for the intended use and target population?

- Is the method broad enough to capture health changes?

- What is the underlying concept and is the theoretical basis valid and well established?

- Will there be problems with administration? How long will the questionnaire take? Is the data readily available? What will it cost?

- How can the scoring method be statistically analysed and interpreted?

- What degree of change can be detected, that is, how sensitive is the instrument?

- How strong is the evidence for reliability and validity? How many other instruments has it been compared against?

Bulpitt (1997: 616) has 6 recommendations for using quality of life as a outcome measure."The measure must be relevant, scientifically sound, have been extensively tried and tested, and include a profile and a summary statistic." These questions and recommendations make a useful checklist and illustrate the need for careful selection of an appropriate index at the planning stage of a project.

Policy makers need to be aware that considerable effort is needed to test an instrument for reliability, responsiveness and validity. The EuroQol has taken decades to develop and is still evolving. It is usually difficult to justify the cost of inventing and validating a new index for a specified study when internationally validated instruments exist. Using a validated index in common use also facilitates the comparison of findings with other studies.

\subsubsection{Classification of Health Indices}

There are a vast and growing number of health indices and as these indices are continually evolving, only a few selected examples only will be discussed. The purpose of this brief 
review is to highlight some common features, and applications of such in indices.

Health indices refer to a wide range of indicators of health status ranging from general measures such as mortality and morbidity to health related quality of life indices (Hansen, 1991). These may be broadly classified as either general or health related quality of life measures.

Three classifications are in common use; functional, descriptive and methodological (McDowell and Newell, 1996: 12). The functional classification describes how the index is used, the descriptive classification is based upon the scope of the index, while methodological classifications consider technical aspects of the index.

The descriptive classification of health indices presented in Figure 3.3 is for illustrative purposes, other authors may use different classifications.

Figure 3.3: Descriptive Classification of Health Indices

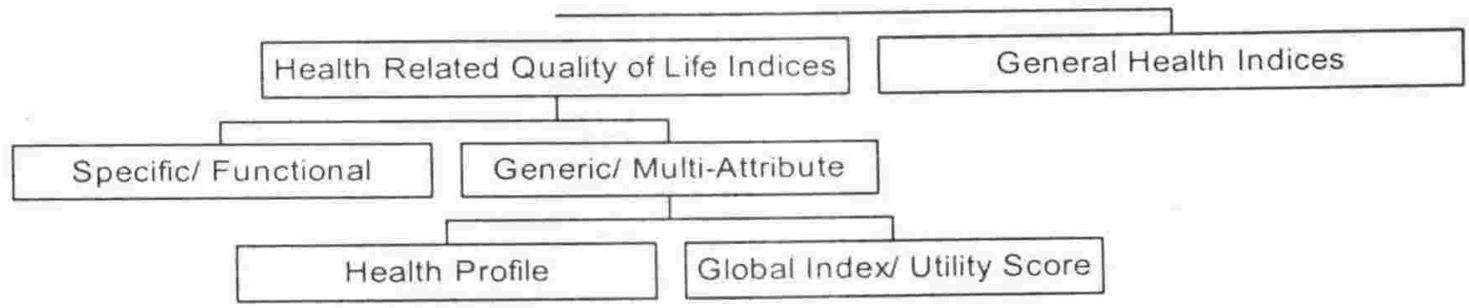

Each of these types of index will be briefly discussed in the following sections.

\subsubsection{Health Related Quality of Life Indices}

Quality of life (QOL), health related quality of life (HRQOL), functional status and health status are frequently used interchangeably. Some (pharmaceutical researchers) refer to quality of life as "outcomes research" (Strom and Melmon, 1994: 450). Part of this confusion has occurred because health indices have been developed by a number of different disciplines.

Although QOL and HRQOL are often used interchangeably they refer to different concepts. Quality of life indicators encompass all physical, social and emotional aspects (including 
items such as housing, education, job satisfaction and the environment) of an individual's well-being. Health related quality of life (HRQOL or HRQL) indicators exclude domains that are unlikely to be influenced by health status. However this distinction is not always relevant, for example, Guyatt et al. (1993: 622) comment "Clinicians focus on HRQL, although when a patient is ill or diseased, almost all aspects of life can become health related."

Bulpitt (1997: 614) provides a listing of " ... a rough current consensus on what should be included under the heading of qualityof life and those areas that are usually not considered...". As this catalogue is a good illustration of the scope of health related quality of life it has been reproduced as Table 3.2 (Bulpitt, 1997: 614). The health related quality of life domains of interest have been assembled into three groups; areas that should be assessed, areas that are not usually measured because they are stable in the short term, and those considered too difficult to quantify.

Table 3.2: Quality of Life

\section{Areas to be assessed}

- $\quad$ physical well-being measured by absence of symptomatic complaints: to include pain, system functioning (eg, respiratory, digestive, neurological) sleep, sexual functioning

- $\quad$ psychological well being: to include depression, anxiety, cognitive functioning

- activities: to include work, leisure, social functioning, fulfilment of roles (eg, mother, carer, etc)

Areas uswally aroided owing to low probahility of change in the short term

- financial security

- marital status

- freedom

- personality

Areas thought to he difficult to evaluate

- happiness

- ambition, expectation

- aggression, criminal activities

- burden

- religious experience, shame 
Items assessed were; physical well-being (pain, system functioning, sleep, sexual functioning), psychological well-being (depression, anxiety, cognitive functioning), and activities (for example, work, leisure, social functioning). These areas are important indicators of health related quality of life.

Areas usually avoided because they are not expected to change greatly in the short term included; financial security, marital status, freedom and personality. This does not necessarily mean that they have a low correlation with health status, but simply that they are relatively stable over the short term. The third group of factors included the following; happiness, ambition, expectation, aggression, criminal activities, a burden to others, religious experience and shame. These are important dimensions of quality of life but are considered too difficult to evaluate.

Two approaches to quality of life assessment are used. Generic/ multi-attribute instruments provide a summary of HRQOL (Guyatt et al., 1993). Specific/ functional instruments focus on problems associated with single diseases, patient groups, functionality or symptoms. Each type of instrument has its strengths and weaknesses and may be suitable in different situations.

It is often desirable to use both a disease specific and a generic instrument in combination so that (1) treatment options for specific diseases may be compared and (2) utility evaluated across disease states. The EuroQol Group (1996: 2) comment that the EuroQol (a generic instrument) complements condition specific measures. Bulpitt (1996) in comparing the performance of generic or disease specific instruments, notes that, when considering a particular disease, a specific instrument is likely to be more sensitive than a generic index.

Furthermore Bulpitt (1996: 613) makes a similar point in recommending that "...both a health profile and a summary statistic such as a Health Status Index should be measured". The global index will measure differences in health related quality of life while the profile will provide detail on how and why health related quality of life has changed. "The clinician and researcher require to see precisely the areas of quality of life that are affected, while the policy-maker is interested in the cost-effectiveness of the treatment in 
standardised units." Bulpitt (1996: 616). A health status index is a global index using the terminology in this chapter.

\section{Specific/ Functional Indices}

These indices focus on specific diseases or disabilities and are used to measure health related quality of life changes brought about by health interventions for specific diseases. Specific indices are more responsive in detecting health related quality of life changes brought about by treating specific conditions. As they are useful in evaluating alternative treatments for specific diseases but not for making comparisons across disease states, they are often used in conjunction with generic measures.

These instruments will not be discussed in detail as they may be viewed as a subset of generic indices. However, a selected number are listed simply to illustrate the range of specific aspects of health or disease covered.

The 5-Year Survival Rate is concerned with survival and cancer, Symptom Free Days describes disease symptoms, the Functional Living Index-Cancer (FLIC) focuses on quality of life in relation to every day living of cancer patients, the New York Heart Association Classification considers various levels of severity of heart disease, the Profile of Mood States (POMS) is used in mental health, and the McGill Pain Questionnaire (MPQ) provides a means of quantifying body pain.

Arthritis Impact Measurement Scales: These scales have been isolated for more detailed discussion to show how this class of indices may be used. The Arthritis Impact Measurement Scales (AIMS) (McDowell and Newell, 1996) is specific to arthritis symptoms. These scales measure physical, social, and emotional well-being and focus on the outcome of care for arthritis patients. Most of the questions relate to experiences over the last month, the instrument is self administered and can be completed in 15 minutes. 
The specific domains covered by the Arthritis Impact Measurement Scales are:

Domain

- Mobility*

- Physical activity*

- Dexterity*

- Household activity*

- $\quad$ Social activity

- Activity of daily living

- $\quad$ Pain*

- Depression*

- Anxiety
Number of items per domain

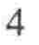

5

5

7

4

4

4

6

6

A total health score may be obtained by adding the values from 6 of the domains, that is, mobility, physical and household activities, dexterity, pain, and depression (marked with an *).

The arthritis impact measurement scales may be used to compare the effectiveness of different arthritis treatment methods and to track the severity of the disease in defined patients. For example, a new analgesic could be tested against aspirin using the total score from the arthritis impact scales as a measure of the effectiveness of each medicine in each patient group.

\section{Generic/ Multi-Attribute Indices}

Generic indices are intended to be used across a multitude of disease states and patient groups and cover a number of health related quality of life domains. A health related quality of life domain (also called a dimension, attribute or state) refers to an aspect of health related quality of life, behaviour, experience or attribute that the analyst wishes to quantify. These indicators are used in a variety of different situations, for example, to consider changes in health status across disease types, to evaluate health care interventions, and to compare changes in health states and quality of life dimensions in defined populations. 
Generic indices may be further broken down into either health profiles or a global index, and will be discussed under these headings. The health status index describes overall quality of life while the health profile focuses on how and why quality of life has changed (Bulpitt, 1997). A global index may indicate that a new arthritis medicine is more effective overall in treating arthritis but if a clinician wanted to evaluate which aspect of quality of life had changed then it would be necessary to consider the health profiles of the patients, dimension by dimension.

\section{Health Profiles}

Health profiles are questionnaires made up of a number of domains which are in turn composed of a number of items or questions. No attempt is made to amalgamate the scores from each domain into a summary score. Although they were not designed for use in cost-utility analysis (because a single index number is not possible) they are often used in parallel with generic indices.

Some of the earliest health profiles focussed on disability, scoring a number of activities (such as feeding, bathing, dressing and walking) by degrees of disability. Examples of general health profiles are the Nottingham Health Profile, Short Form 36, Sickness Impact Profile and the General Heath Questionnaire (GHQ). It is not intended to discuss these in any detail but as they have been widely used in clinical trials as measures of the effectiveness profile of a medical intervention and provide good examples of such profiles they will be mentioned in brief.

Nottingham Health Profile: The Nottingham Health Profile was developed in the early 1980 s and has been used extensively in the United Kingdom. It was originally designed for use in primary medical care but has been used in surveys to assess need or demand for health care in defined populations. The instrument is self administered and focuses on the respondent's subjective evaluation of their health state and takes between 10 and 15 minutes to complete (McDowell and Newell, 1996). This profile has particular application with moderate to severe illness but is less sensitive in measuring quality of life in groups with mild illness severity (Anderson et al., 1996). 
The profile in its refined form consists of 6 health domains covering 38 items:

- Physical mobility, 8 items

- Pain, 8 items

- Sleep, 5 items

- $\quad$ Energy, 3 items

- Social isolation, 5 items

- Emotional reactions, 9 items

There are also 7 domains of 1 item each relating to different activities:

- $\quad$ Paid employment

- Looking after the house

- Social life

- Home life

- Sex life

- Hobbies and interests

- Holidays

Short Form 36 (SF 36): The second example is the Short Form 36 health profile developed in the US and commonly used in clinical trials as a measure of effectiveness (McDowell and Newell, 1996; Brazier 1995). The SF36 questionnaires have been adapted for use in New Zealand and Australia (Medical Outcomes Trust, 1994). The profile is constructed from a self-administered questionnaire.

Although the SF36 was not developed for economic evaluation it is suitable for use with cost-minimisation where the health outcomes are identical. In this case preference weighting would not be necessary because all that is required is a method of determining if the outcomes are the same between treatment options. O'Brien et al. (1999) used the SF36 in this way to check for differences in quality of life between two drug treatments for thrombosis when conducting an economic evaluation.

The questionnaire contains thirty-six items that cover the eight dimensions and is self administered taking between 5 and 10 minutes to complete. The scores from each dimension cannot be aggregated because no preference weights have been developed. 
However the instrument is being converted into a preference weighted instrument (Tobias, 1998). If the transformation is successful the SF 36 could become a standard quality of life measure for cost-utility analysis.

Eight health dimensions are covered by the SF36:

- Physical functioning

- $\quad$ Role limitations (physical problems)

- Pain

- General health perceptions

- Vitality

- Social functioning

- Role limitations (emotional problems)

- Mental health

In addition there is a question related to changes in health status compared to a year ago, but this is not counted in the scoring for the eight dimensions.

First, each item contained (within each dimension) is scored on a Likert ${ }^{7}$ scale which has a range of options. Item responses are then recorded on to an equal interval scale and scores computed for each dimension. Dimensional scores range from 1 to 100 (worst health to best health). These dimensional scores are not measures of utility and are not aggregated to provide an overall single score of health.

\section{Global Index Or Utility Scores}

Global indices weight and aggregate scores for each health dimension to produce a single score to describe health related quality of life. Although the score typically ranges from one (healthy) to zero (dead), there are states considered to be worse than dead, hence raising the

A Likert scale "A measure of attitudes ranging from very positive to very negative designed to allow respondents to indicate how strongly they agree or disagree with carefully constructed statements relating to an attitudinal object." (Zikmund, 2000: GL-8) 
possibility of negative values. The existence of states worse than death is supported by Rosser and Kind (1978:348) who note the occurrence of suicide, requests to withdraw from life saving procedures, and the euthanasia debate as evidence of states worse than death.

In constructing health indices all aspects (domains) of health must be addressed and each must be measured. Then each of these domains must be weighted to derive one number. Common steps in constructing a global index are as follows;

- choose the health related quality of life domains and the items within these domains,

- describe the health related quality of life domains and devise a way of quantifying them, and

- $\quad$ select or devise a weighting method to enable the domains to be aggregated into a single summary score.

The domains must be capable of describing the aspects of health related quality of life of relevance to the intended use of the instrument. The method of quantifying them must be capable of identifying differences across populations and disease states. The weighting method should reflect the values and preferences of consumers of health care.

In practice there are two methods of assigning preference weights to health states (Gold et al., 1996). Individuals may be asked questions to enable the researcher to locate their health state on a classification matrix containing preference weights. The EuroQol uses this method. The second way is to employ techniques such as the standard gamble or time trade-off to have patients rate their health state directly on to a scale such as $1=$ perfect health to $0=$ dead.

Two examples only will be discussed in detail, the EuroQol because it is in common use and the Rosser index for historical reasons.

EuroQol: An example of a global or generic index is the EuroQol (The EuroQol Group 1996). This index has been validated in a number of countries and provides information that is useful for both clinical and economic evaluation. EuroQol complements condition- 
specific measures. Originally the EuroQol had six health dimensions or domains, but the latest version, EQ-5D, has five domains. The EQ-5D questionnaire has 2 parts, part 1 is a 5 dimensional health state classification and part 2 a visual analogue scale in the form of a thermometer.

The 5 dimensions (earlier version 6 domains) evaluated in part 1 are as follows:

- Mobility

- Self-care

- Main activity

\}combined to 'Usual activities' (EQ-5D)

- Family/ leisure activity

\}

- Pain/ discomfort

- Anxiety, depression

Each of these domains has one item with 3 levels of severity:

- $\quad$ No problem

- Some or moderate problem/s

- Unable or extreme problem

In the second part of the questionnaire respondents are asked to indicate their current health state by use of a scale (like a thermometer) running from 0 "worst imaginable health state" to 100 "the best imaginable health state". The respondent marks a score on this 0 to 100 scale and the score is then converted to a decimal on a 0 to 1 scale.

The questionnaire is designed to be self-completing either in a postal survey, in a clinic or in face to face interviews. Full instructions for respondents are embedded within the questionnaires. Completing the instrument takes about 2 minutes.

Information derived from the EQ-5D may be used in a number of ways. Responses on the 5 dimensions may be presented as profile or as a weighted index (using preferences established from surveys) while the results from the analogue scale can be used to provide a self-rated value. 
As a profile the self reported health states are presented as \% numbers of patients reporting problems or reported as a dimension scores. For example, the case study Chapter 10: Benign Prostatic Hyperplasia could have evaluated health related quality of life before and after prostatectomy using EQ-5D. This would have provided information on the change in quality of life in addition to the change in cost.

The procedure to obtain a health profile based on EQ-5D is as follows:

- Have respondents place a tick in the box that best describes their health state for each dimension, that is 1 tick per dimension

- Do this before and three months after surgery

- Analyse by counting the ticks in each box of the questionnaires to obtain a total for the survey before and after surgery.

- Present the results as tables and/ or graphs.

The example, Table 3.3, relating to changes in self reported health status by domain for patients before and after prostatectomy shows the differences in the proportion of patients recording "any" problems.

Table 3.3: EQ-5D as a Profile, Change in Health Status for Patients Before and

\begin{tabular}{lcc}
\hline & \multicolumn{2}{c}{ \% of patients reporting "any" problems } \\
\cline { 2 - 3 } Dimension & 30 & 3 months after surgery \\
\hline Mobility & 12 & 25 \\
Self care & 32 & 11 \\
Usual activity & 61 & 29 \\
Pain discomfort & 29 & 41 \\
Anxicty! & & 15 \\
depression & & \\
\hline
\end{tabular}

These results could have enabled clinical managers to better evaluate resource allocation options with respect to a particular quality of life dimension or dimensions. For example 
if a clinical manager was particularly concemed with decreasing the numbers of elderly patients who were reliant on nursing staff for care, the percentage of patients reporting problems with "self care" would be an important consideration when comparing treatment options.

If the objective was to maximise utility (as measured by health related quality of life a single score) summarising utility would be of more use. A global index could also have been calculated and this together with the cost data would have permitted a cost-utility study to be undertaken to better evaluate cost-effectiveness of hospital expenditure.

Using EQ-5D as a global index involves the following steps:

- Have respondents place a tick in the box that best describes their health state for each dimension, that is, one tick per dimension

- Level 1 is recorded as 1 , level 2 as 2 , level 3 as 3

- The questionnaire may then be coded as a 5 digit number, one digit for each domain, the digits ranging between 1 and 3. A number such as 13221 may result, from a tick in box 1 for mobility, 3 for self-care, 2 for usual activities, 2 for pain/ discomfort and a tick in box 1 for anxiety and depression.

- Go to the table of scores and look up this number and convert it to a score, for 13221 the score is 0.28

- Conduct a survey before and after surgery

- Analyse all questionnaires in this way

- Calculate mean scores for the survey before and after surgery

- Present the results as tables and/ or graphs.

The mean weighted score of respondents may have fallen from 0.29 before surgery to 0.15 after surgery indicating that treatment was not successful in improving quality of life.

The "thermometer" may be used to first generate a "thermometer score" which is then used in the same manner as the weighted score. The "thermometer" score for a patient before surgery may have been 0.55 and after surgery 0.65 indicating treatment was successful in improving quality of life. Scores may be averaged for populations under investigation. The 
advantage of the thermometer score over the weighted score is that it is easier and faster to derive and calculate but it lacks specificity and precision. The thermometer score may also be used as a quick cross check on the weighted scores derived.

Figure 3.4: Indices Derived From the EuroQol

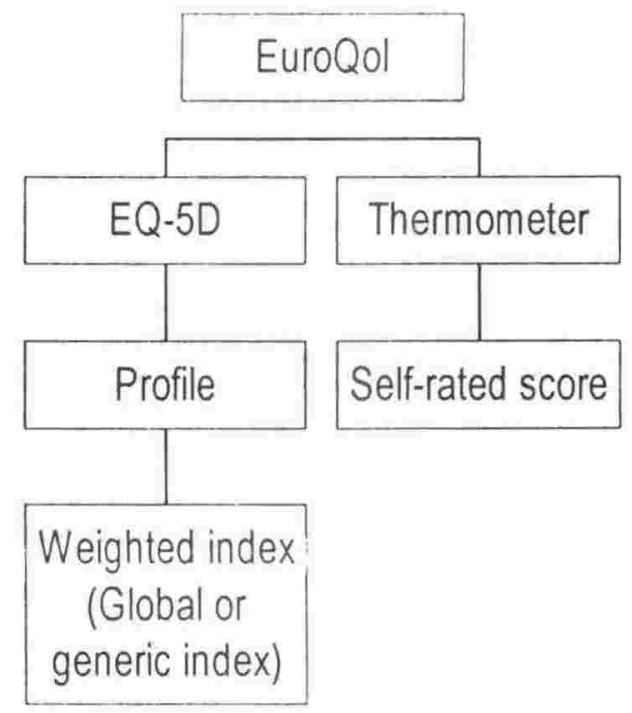

In summary, the EQ-5D may be used in clinical trials as an effectiveness measure, for costutility analysis at one time (prevalence or cross-section studies), or to compare changes in health related outcomes over time (longitudinal or time-series). Figure 3.4 (The EuroQol Group 1996) illustrates the different health related quality of life indices that can be derived from the EQ-5D and "thermometer".

Rosser Index: As the Rosser Index is a well-known and pioneering example of a global index it is discussed in more detail than the other indices. The Rosser Index was developed in South London in the early 1970's by Rosser and Watts (Rosser and Watts, 1972). Initially the index describing states of illness was used in evaluating if hospital treatment had improved patient's health status. Subsequently (Rosser and Kind, 1978) developed a scale of well-being for more general use.

Rosser and Watts (1972) developed a methodology to measure the effectiveness of hospital treatment by comparing disability and distress states of patients on admission and then at discharge from hospital. The Rosser and Watts model used eight classes of disability (ranging from (1) no disability to (8) unconscious) combined with four levels of distress 
(ranging from (1) no distress to (4) severe). Classification was undertaken by clinicians seeing the patients. Results were reported as a matrix ( 8 disability states by 4 distress states with cells containing the percentage of patients falling within each cell). One matrix represented the results on admission and the second matrix the results on discharge. The two matrices were compared to determine the effectiveness of hospital treatment.

The authors stated that the results would be of much greater interest if they could be scaled. Rosser and Kind (1978) later developed a method of assigning a score for each of the health states as defined by the matrix. They derived a matrix of 29 "states of illness" by combining the 8 states of "objective disability" with the 4 states of "subjective distress." Their objective was to value each of these states according to subjects' perceived relative degree of undesirability.

Rosser descriptions of illness and disability states are as follows:

1. No disability.

2. Slight social disability. (Slightly disabled but performance of normal work not impaired.)

3. Severe social disability and/ or slight impairment of performance at work. Able to do all housework except very heavy tasks.

4. Choice of work or performance at work very severely limited. Housewives and old people able to do light housework only, but able to go out shopping.

5. Unable to undertake any paid employment. Unable to continue any education. Old people confined to home except for escorted outings and short walks and unable to do shopping. Housewives able only to perform a few simple tasks

6. Confined to chair or to wheelchair or able to move around the house only with support from an assistant.

7. Confined to bed.

8. Unconscious. 
Rosser distress ratings are:
A. No distress
B. $\quad$ Mild
C. Moderate
D. Severe

Rosser obtained values on a scale of $0=$ death to $1=$ good health, in a detailed interview lasting between 1.5 and 4.5 hours. Psychometric scaling, and magnitude estimation methods, were used to produce a set of valuations for each state. The sample was made up of 70 subjects with different experiences of illness; 10 patients from medical wards, 10 psychiatric in-patients, 10 experienced state registered nurses, 10 experienced state registered psychiatric nurses, 20 healthy volunteers and 10 experienced specialist doctors having a membership or fellowship in at least one Royal College. This original sample was not a random selection from the general population.

The Research Group on the Management and Valuation of Health at the University of York (Williams, 1995) applying a slightly different methodology used Rosser's descriptive system and re-estimated the valuation weights by interviewing 140 members of the general public. Different weights were obtained and are reported in Table 3.4. These re-estimated weights should better reflect the values of the general public and society as a whole and should accordingly be more appropriate for cost-utility analysis.

The best known use of the Rosser index has been in the calculation of quality adjusted life years (QALY's), first described by Williams (1985) in an evaluation of coronary artery bypass surgery. QALYS are discussed in greater detail later in this chapter. Rosser's index, and the application of it by Williams to calculate QALYS had a major impact on the development of health related quality of life measures. As the Rosser index has now been overtaken by other indices, detailed analysis of the revised weights compared with the original weights will not be discussed. 
Table 3.4: Rosser Matrix Revised Weights

\begin{tabular}{c|cccc}
\hline \multirow{2}{*}{ Disability } & \multicolumn{4}{|c}{ Distress } \\
\hline 1 & $\mathrm{~A}$ & $\mathrm{~B}$ & $\mathrm{C}$ & $\mathrm{D}$ \\
\hline 2 & 1.00 & 0.89 & 0.89 & 0.67 \\
3 & 0.89 & 0.81 & 0.78 & 0.56 \\
4 & 0.70 & 0.63 & 0.57 & 0.44 \\
5 & 0.63 & 0.56 & 0.51 & 0.40 \\
6 & 0.44 & 0.43 & 0.44 & 0.22 \\
7 & 0.44 & 0.44 & 0.34 & 0.22 \\
8 & 0.38 & 0.40 & 0.33 & 0.20 \\
\hline
\end{tabular}

Fixed points: Healthy $=1 \quad$ Dead $=0$

(QWB) Quality of Well-Being Scale (formerly the Index of Well-Being): This scale combines mortality with estimates of the quality of life among survivors. A "Well-Year" is a year of completely well life. The QWB is broad in scope being the only instrument considering mortality, symptoms, and problems together with functional levels (McDowell and Newell, 1996).

This index classifies patients by use of 3 domains; mobility ( 3 items), physical activity ( 3 items) social activity (5 items). The analyst also records the presence of symptoms or problem complexes (27 items). Each item has a preference weight that permits an aggregated score to be calculated. The overall scores range from one (complete well-being) to zero (death), although the scoring does permit values below zero to represent a state worse than death.

Health L'tilities Index (HUI): This index was developed by Torrance and colleagues at McMlaster University Canada and has been used in economic evaluations, clinical trials and population health surveys. As it was based in part on QWB it is not discussed in detail. The latest version of this index (HUI:3) has eight domains; vision, hearing, speech, ambulation, dexterity, emotion, cognition and pain. Each of these domains has five to six 
levels of severity (Gold et al., 1996).

\subsection{VALUING HEALTH STATES}

Methods used to value or assign preference weights to health states have been derived from expected-utility theory, and psychological scaling techniques (Gold et al., 1996: 113). Weights are usually reported as a number on a scale with two fixed anchor points 0 and 1 (but negative values are permitted).

\subsubsection{Utility Methods}

Utility describes the satisfaction derived from the consumption of a product or service. In the context of measuring health outcomes, utility, preference, and value are frequently used interchangeably. When referring to health related quality of life; von NeumannMorgenstern utilities, health status, functional status and health related quality of life are used synonymously in the literature (Gold et al., 1996).

The two most commonly applied utility methods are the standard gamble and time tradeoff. These procedures have evolved from economic utility theory. Economists favour the standard gamble method over the time trade-off method as it has stronger links to utility theory (Gold et al., 1996: 114). Neither method results in utility values as defined in the economic literature. Utility values in this sense relate only to health related quality of life not to the utility derived from the consumption of any good or service.

A third method in common use is the application of psychological scaling. The three methods of assigning preferences to health states may yield different results. Where the three techniques have been compared the standard gamble scores are consistently higher than scores from the other two methods which suggests that most respondents are risk averse (Torrence et al., 1995: 506). This means that the results of studies using different scoring methods cannot be easily compared. 
In using these indices for policy evaluation the analyst makes an implicit assumption that individuals' utilities may be aggregated to represent societies utility. But this is not so when health resources are limited, see section 3.1.3 and Weinstein et al. (1980: 230).

\section{Standard gamble}

An expected utility index was developed by von Neumann and Morgenstern. Baumol (1965) describes the construction of such an index by reference to a lottery. Two necessary assumptions in developing such a cardinal index are that consumers have consistent preferences and make rational choices between various qualities of life and death.

But this is not necessarily so. For example, the rule of rescue (Luce, 1994: 269) assumes that society will always favour life saving interventions over those that improve quality of life. This "rule" is not necessarily rational and it implies that quality of life cannot be traded off against death.

Baumol (1965) describes the use of standard gamble technique in deriving utility values. However, in the example described and in health economics, utility indices for health states are more correctly preference indices of health. In valuing health states the standard gamble is used to determine preferences between health states, but is not taken to the next step of determining a utility value of the health state. In the context of health indices the term utility is being used interchangeably or instead of preference.

The standard gamble approach may be applied to health related outcome states as shown in Figure 3.5

The steps involved in valuing any health state are as follows:

- First, construct an artificial or standard lottery (lottery 1 ) with two extreme prizes full health $\mathrm{H}=1$ probability $\mathrm{p}$, and $\mathrm{D}$ death $=0$ probability $\mathrm{I}-\mathrm{p}$. This is often referred to as a standard gamble. The probability of winning prize $\mathrm{H}$ is $\mathrm{p}$ while the probability of winning prize $D$ is $1-p$. If $p=1$ then the outcome is a healthy state with certainty but if $\mathrm{p}=0$ then the outcome is death with certainty. The probability 
is not defined and is allowed to vary.

- Second, introduce an ordinary lottery (lottery 2 ) with prize $i$.

- The probability $\mathrm{p}$ (that relates to winning both lotteries) is then varied until the individual is indifferent between buying a ticket in lottery 1 or lottery 2 .

Figure 3.5: The Standard Gamble

\begin{tabular}{|l|l|}
\hline $\begin{array}{l}\text { Sealthy }(\mathrm{H}) \\
\text { (p) }\end{array}$ \\
$\begin{array}{l}\text { Stcps } 1 \text { \& } 2 \text { are repeated for different } \\
\text { health states in lottery } 2,(\text { Lottery } 1 \\
\text { unchanged). In this manner the utility of } \\
\text { different health states are obtained. }\end{array}$ \\
\hline
\end{tabular}

To calculate the utility of any particular outcome it is necessary to either observe or interview respondents to determine the probability at which they are indifferent between the two lotteries. If the standard lottery has a prize of perfect health with a utility 1 , or death with a utility 0 , then the utility of any other lottery may be calculated by varying the odds of winning until the gambler is indifferent between the two lotteries. It is usual, but not essential, to use death $=0$ and perfect health $=1$ as fixed anchor points.

\section{Time Trade-off}

Torrence and others in 1992 developed the time trade-off method as a simple and convenient way to derive scores that would be comparable to scores based on the standard gamble (Drummond et al., 1997: 154).

Time trade-off asks subjects to trade-off an existing health state for a specified time period followed by death against a shorter time in a healthy state followed by death (Zweifel and 
Breyer, 1997: 46). Time trade-off has its origins in decision theory and as such has a strong following from decision analysts and operational researchers.

Figure 3.6 illustrates that being in health state $\mathrm{H}_{1}$ for a time period $\mathrm{T}_{1}$ is equivalent to being in health state $\mathrm{H}_{2}$ for a time period $\mathrm{T}_{2}$. The procedure is equivalent to the situation encountered in QALY analysis where years spent in a health state of less than perfect health are transformed into quality adjusted life years.

Figure 3.6: Time Trade-off

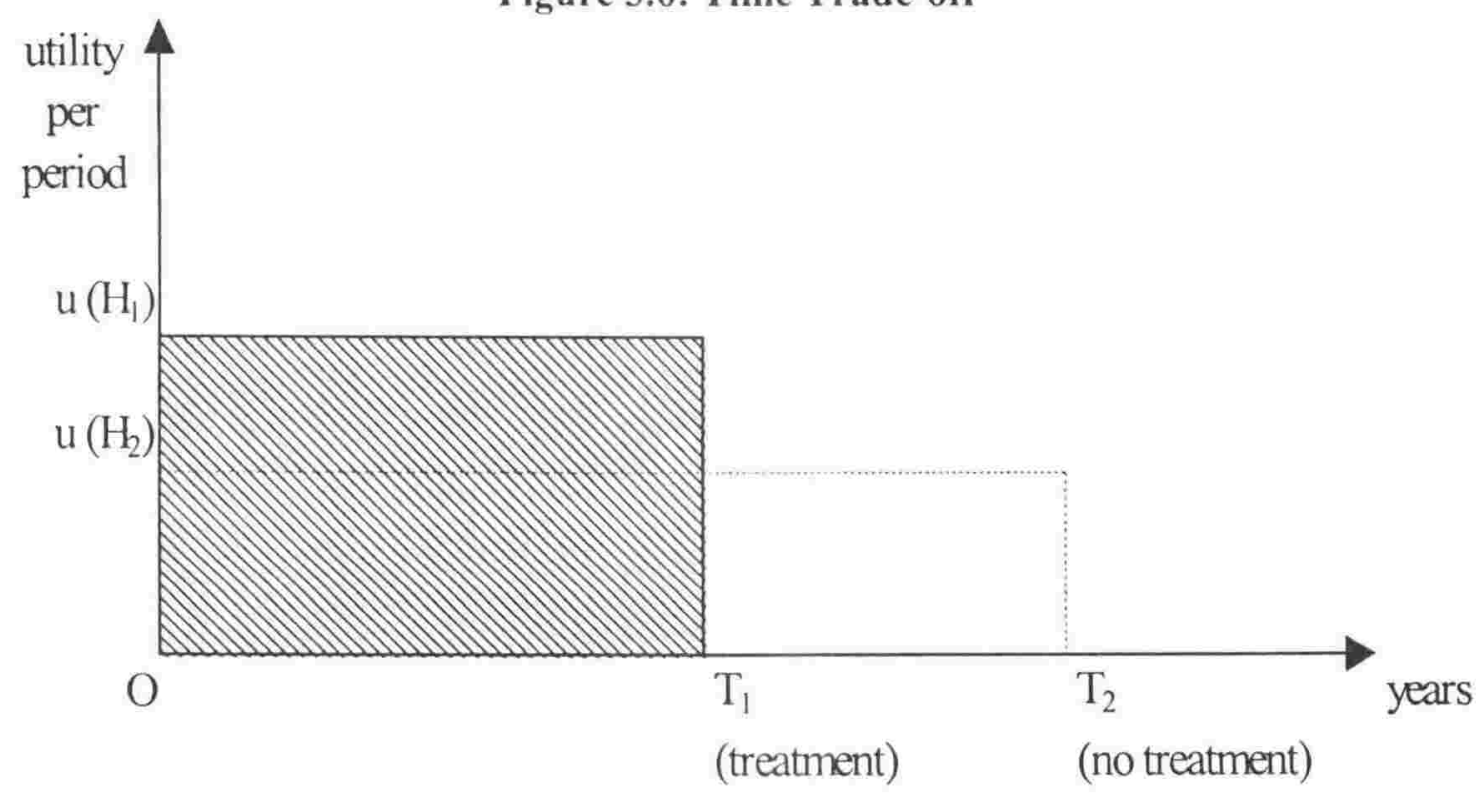

Standard Gamble Versus Time Trade-off

Zweifel and Breyer (1997: 48) comment that the standard gamble and time trade-off methods will not necessarily lead to the same result. However, they also demonstrate that if respondents expected that total utility is a linear function of duration and utility weights of health states, time trade-off and standard gamble methods give the same result. In practice the two methods may lead to different results, if for example, the health states are not interpreted by all respondents in the same way. The standard gamble approach has theoretical support because of its links with von Neuman-Morgenstern utility theory.

Material to assist researchers conduct standard gamble and time trade-off trials to generate 
preference scores are available (Gudex, 1994a; Gudex, 1994b). This material is very useful because it not only describes how to undertake such studies but also provides the questionnaires and props needed for execution.

\subsubsection{Psychological Scaling}

Although Williams (1995) provides some useful examples of the application of category rating, pairwise comparisons and magnitude estimation, these valuation methods will not be discussed in detail as the focus of this study is economic.

There are three variants of category rating. Subjects are asked to score states by means of an (i) analogue graphical scale or, a series of (ii) numbered or (iii) labelled boxes with descriptions of the quality of life/ health states. Responses may then be converted to a scale ranging from $0=$ worst imaginable health state to 1 the best imaginable health state. Pairwise comparisons involve comparing various health states with each other while in magnitude estimation subjects are required to judge each state in terms of severity against a benchmark of no disability and no distress. An example of the application of magnitude rating is the Rosser index.

\subsection{QUALITY ADJUSTED LIFE YEARS (QALYs or QUALYs)}

The objective of QALYS measurement is to obtain a single value that summarises all relevant aspects of health related quality of life and combines this quality score with the time spent in this health state. If QALYs are aggregated for defined populations or patient groups and compared with net intervention costs they may be used to conduct cost-utility analysis for policy decisions and clinical management.

The idea behind QALYs is that the utility of a year of additional life with reduced health is not as high as a year of full health. That is, individuals would choose to trade-off a longer life of reduced health for a shorter life of superior health status. For example, two years of additional life with a score of 0.5 is equivalent to 1 year of healthy life with a score 
of 1 . In both cases there would be a gain of 1 QALY.

There is lack of agreement on how to measure and interpret QALYs. There are differences of opinion on the scaling of health related quality of life states and the weighting of these states used in the QALY. QALYs may be interpreted in terms of individual utility, or individual utility may be side stepped and the QALY used directly as a measure of social value (Nord, 1994).

QALYs may be calculated as follows:

- Categorise individuals into the appropriate Rosser category, for example, 2 patients in cell $2 \mathrm{~A}$ [score 0.89 ] and 4 in cell $5 \mathrm{C}$ [0.44] each patient spending one year in that state.

- Multiply the numbers of patients in each cell by the appropriate Rosser score and by the time spent in that state to obtain the number of QALYs, for example $2 \times 0.89 \times 1=1.78$ and $4 \times 0.44 \times 1=1.76$.

- QALY gains in future years should be discounted to present values.

- Add the QALYs to obtain a total number of QALYs for the treatment, for example, $1.78+1.76=3.54$ or an average gain of $3.54 / 6=0.59$ of QALYs.

In this example Rosser categories and weights were used to calculate QALYs, but a variety of generic health indices may be used to define and weight the health states. Of the scales that provide estimates of QALYs, McDowell and Newell (1996) consider that the Quality of Well-Being Scale (formerly the Index of Well-Being (QWB) is the most widely used. However other authorities (Paul Kind, University of York, personal communication, July 1998 ) consider the EuroQol (EQ5D) and the Health utilities index (HUI) the two most commonly used indices.

QALY's may be used to compare quality of life gains from treatment across different diseases and within diseases by use of cost-utility analysis. Application of a net resource cost per QALY provides a method of comparing the treatment cost with the health related quality of life gained from different interventions within and across diseases. QALY league tables result from the compilation of the data for these comparisons. Such league tables 
provide a means of evaluating health care treatments with respect to resource allocation decisions within the health sector.

Implicit in the QALY calculation is that factors other than those measured by the QALY instrument such as income, are held constant. Income is a confounding factor as income levels are highly correlated with health and quality of life. This means that increasing quality of life could have occurred from factors other than the health care intervention being assessed.

Comparing costs per QALY calculated in different studies is complicated by variations in the:

- Range of health related quality of life states included

- Quantification and weighting of states

- $\quad$ Range of costs included and valuations of those cost items.

The spread of health related quality of life states varies enormously between generic instruments, and different preference weights mean that all QALYs are not equal. Including a different range of costs and measuring costs differently adds to the problems. Thus, when comparing different studies that have used QALYs, it is important to check that the same generic instrument has been employed and that costs have been identified and quantified in the same manner. Comparisons cannot be made unless all costs and QALYS are calculated in the same way.

Gerard and Mooney (1993) consider that when calculating the cost per QALY only those costs falling on the health sector should be considered. They argue that if a societal perspective is taken costs should be valued in terms of all benefits foregone of which health related benefits are merely a subset. This implies that QALYS can be used to evaluate health sector resource allocations with a fixed health budget but not to evaluate resource allocations across all society. Another point is that if indirect costs are included in costs and if the health related quality of life includes a dimension that relates to ability to work then there will be an element of double counting. 
A partial solution to the problem of comparability and the range of costs to include when calculating costs per QALY' may be resolved as follows:

- Use incremental net direct medical resource costs to calculate the cost per QALY for league tables, for decisions relating to a health sector perspective.

- Use a wider definition of costs if direct medical and /or, indirect costs are important and the decision maker wishes to consider a wider perspective.

Drummond (1997: 268) notes that "...it has become fashionable..." to use QALY league tables as an aid to prioritising resource allocations within a health budget. There are two ways in which they may be used. First, if a qualifying price per QALY could be established then all programmes that had a cost per QALY less than this price should be implemented. Second, provided all relevant health care interventions have been ranked in terms of cost per QALY, then programmes could be implemented sequentially until the budget is exhausted.

A problem with both these approaches is that cost per QALY will differ between patient sub groups because the characteristics of the individuals making up the group may differ. This means that a vast and potentially unmanageable number of defined populations by intervention would need to be evaluated.

QALYS are not necessarily equivalent to utilities and it is not necessarily valid to sum utilities or QALYS for different individuals or indeed for the same individual at different times. Economists generally agree that if a societal perspective is adopted, health states should be valued by members of the public. However, health states are frequently scored by different groups of individuals on behalf of the patient or public (Weinstein et al., 1980).

QALY's are implicitly assumed to be equivalent across all demographic groups. Health states may be valued by health professionals, patients with the condition or members of the general public, and each may provide different preference values. Hadorn and Uebersax (1995: 608) noted that there was some evidence that preferences for a specific health state may vary based on people's experience with those states. Some authorities argue that as the general public do not have sufficient knowledge, clinicians or nurses with a clear 
Economic Evaluation Applied To Health Policy In New Zealand 


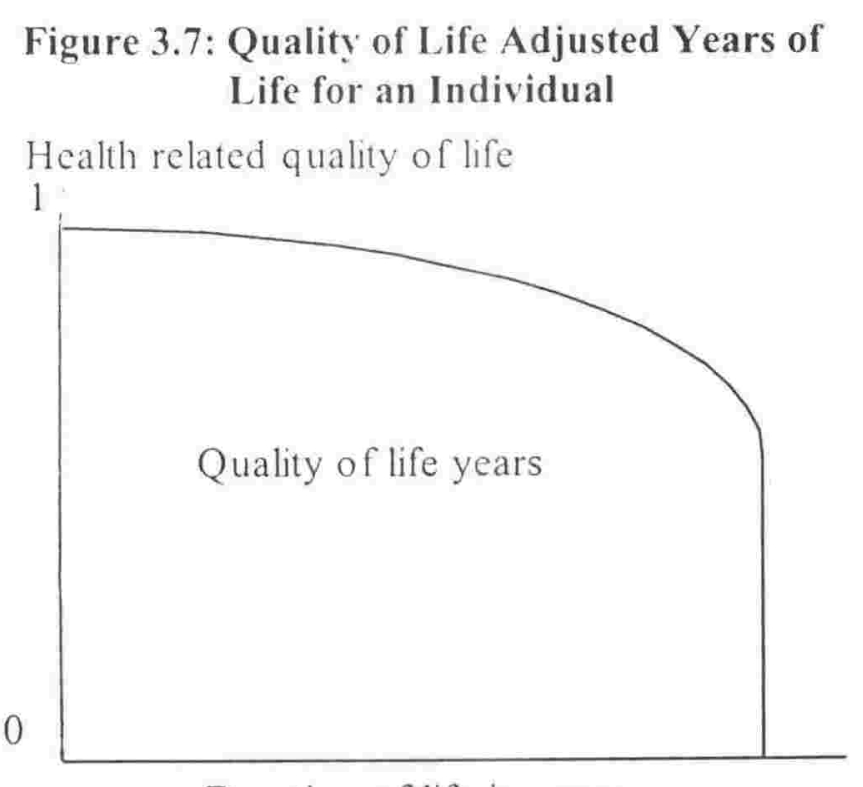

Duration of life in years

Healthy year equivalents (HYE) is an altemative to the QALY and has been debated extensively in the literature (Drummond et al., 1997) but will not be discussed in this thesis.

\subsection{NEW ZEALAND INITIATIVES}

\subsubsection{Introduction}

Two New Zealand approaches to valuing outcomes; the surgical priority scoring system and the suggested Health Funding Authority health related quality of life instrument will be briefly discussed.

\subsubsection{Surgical Priority Scoring System}

The surgical priority scoring system was introduced to the New Zealand public hospital system to replace waiting lists with a booking system as a rationing device. The generic surgical priority criteria (GSPC) were introduced first into Auckland Hospital in June 1997. Surgeons placing patients on waiting lists had to complete a GSPC form. 
The scoring system encompassed five health dimensions; suffering, disability, clinical cost of delay, degree of improvement anticipated and likelihood of improvement. The first three dimensions describe clinical severity and add to a maximum score of 20 . The last two dimensions (maximum score of five) describe the capacity to benefit from the surgery. The total score is found by multiplying the score for clinical severity by the score for capacity to benefit, hence a maximum possible score for a patient is $100(20 * 5=100)$.

This method, although crude, is theoretically better than the waiting list system it replaces as it is transparent and attempts to combine clinical severity with capacity to benefit across a range of surgical procedures. However, the final result may well be identical because the same resources are available and decision makers who prioritised the waiting list will be scoring patients for the booking system.

The scoring system can only be applied to conditions requiring surgery and is not as objective as it appears. Experience in using a prioritisation scoring system specifically designed for cataract surgery found that scores awarded to the same subjects by different clinicians varied as much as 26 points out of 100 points (Halliwell, 1998).

\subsubsection{Health Funding Authority Health Related Quality of Life Instrument}

The Health Funding Authority (HFA) and the Pharmaceutical Management Agency Ltd (PHARMAC, a wholly owned subsidiary of the HFA that manages pharmaceutical subsidies), are considering applying cost-utility analysis using QALYs. Each agency has issued discussion papers (PHARMAC, 1998; Prioritisation Team, 1998). The focus of the HFA paper is more general than PHARMAC's paper which is specific to drugs. As the methodologies presented in both of these papers are essentially the same, the HFA paper has been chosen for more detailed comment.

The HFA adrocate applying cost-utility analysis to the evaluation of services with “...aggregate costs exceeding \$1 million or with major social or political implications" (Prioritisation Team, Appendix A, 1998: 2). The stated goals of the paper were "... to provide a rigorous, explicit, ethical and transparent process based on an agreed set of 
principles to guide the setting of priorities and resources." (Prioritisation Team, 1998: 10). Further, this process was to relate to "...prioritisation for publically funded health and disability services which is applicable to all levels of the health system..." (Prioritisation Team, 1998: 1).

The HFA acknowledges the difficulties in defining health and health outcomes, reject the WHO definition of health (and thus changes in health based on this measure) as being too broad. They define "health gain" as "...improvements in suffering, limits on activities, and life-expectancy produced by services or programmes for defined kinds of patients." (Prioritisation Team, 1998: 6). Important considerations of the group were that prioritisation should be explicit, transparent, and the public (to date the public have not been involved) should be involved (Prioritisation Team, 1998: 8-9).

The report recommends that five factors be considered when "...setting health care priorities:

- Effectiveness

- Cost

- Equity

- Māori health

- Acceptability" (Prioritisation Team, 1998: 24)

A disturbing aspect (from a public policy viewpoint) of the HFA stated objectives is the limitation of perspective to publically funded services. If the HFA is an agent for the New Zealand government and if government is an agent representing the interests of its citizens then a societal perspective should be taken.

Although the criteria employed to select a suitable generic health index (Table 3.5 presented without comment) represent an acceptable way of selecting and designing a generic health related quality of life instrument, the HFA do not explain in any detail how their selected instrument meets these criteria. 
Table 3.5: Health Funding Authority Selection Criteria for A Generic Health Related Quality of Life Instrument

"In selecting a generic health state measure, a number of a priori features can be considered necessary to meet the HFA's needs for prioritising services, including:

1. a manageable number of mutually exclusive, collectively exhaustive health states;

2. the dimensions measured must be relevant to aspects of life that health services can be expected to improve;

3. low interdependence/ correlation between dimensions to prevent double counting and unnecessary complexity;

4. sensitivity to change within dimensions;

5. health states calibrated by empirically-derived values;

6. use of societal preferences to empirically derive health states' values;

7. response methods (ie how values/preferences are elicited) are conceptually robust, preferably using choice-based methods rather than direct scaling. Choicebased methods include paired comparison, time trade-off and person trade-off approaches;

8. response methods are intuitive, easily understandable by the average citizen. This comprises pairwise comparison and direct scaling rating methods;

9. face validity, i.e. results must seem to be intuitively plausible;

10. good construct validity (both convergent and discriminant validity), ie the measure "behaves" as it is expected or hypothesised to behave with respect to other measures. Construct validity should be demonstrated by accepted methods such as the multitrait multimethod (MTMM) technique or Cronbach's alpha; )

11. good inter-rater and test-retest reliability;

12. simplicity of use (feasibility); and

13. use and acceptance elsewhere, as an indicator both of reliability/validity and to aid any comparison with other health systems."

(Prioritisation Team, 1998 Appendix A: 6). 
The proposed health related quality of life instrument consists of 2 domains; "suffering (physical and/or psychological)" and "limits to independent daily activities". Each of these domains has 1 item with 11 levels of severity (ranging from 0 to 10). This instrument is based on the Quality of Life and Health Questionnaire (QLHQ) developed by Hadorn and Uebersax (Hadorn and Uebersax, 1995).

Hadorn and Uebersax wished to develop "....a standard, very brief, generic quality-of-life questionnaire that is calibrated according to empirically derived public preferences." (Hadorn and Uebersax, 1995: 608). The questionnaire tested consisted of three domains; physical suffering, emotional/ outlook on life, and daily activities each with 4 levels of severity; none, mild, moderate and severe. In addition, participants were asked to score their overall quality of life on a numeric scale from 0 , worst possible quality-of-life to 10 , best possible quality-of-life.

The HFA have used the Hadorn and Uebersax questionnaire and collapsed the three domains into 2 and expanded the levels of severity from 4 to 11 . The HFA paper does not make it clear how these modified scores from the Quality of Life and Health Questionnaire were developed.

PHARMAC (1998) advocated using the same dimensions or domains but instead of scoring levels of severity on a 0 to 10 scale they propose using a 4 level scale. The preference ratings for this instrument are described in Table 3.6.

The HFA (and PHARMACs modification) scheme has the attributes of simplicity and there is minimal danger of double counting but it is difficult to envisage how it will capture all relevant aspects of health related quality of life. In addition, there are dangers in using an unvalidated and New Zealand specific instrument, especially since there are well tested and freely available generic instruments. For example, the EuroQol is freely available with manuals and questionnaires, is used in many countries, and in a wide range of health economic evaluations and clinical trials. Developing a new instrument is costly, takes a long time and there is no guarantee that it will perform satisfactorily. 
However, after consultation with a wide range of interested parties and those with expertise in health economics, PHARMAC (1999) recommended using the EuroQol to evaluate health related quality of life.

Table 3.6: Quality of Life and Health Questionnaire Preference Scores

\begin{tabular}{ll|ccccc}
\hline & & \multicolumn{5}{|c}{ Dimension: Limitations to daily activities } \\
& & none & mild & moderat & severe & dead \\
& & & & $\mathrm{e}$ & \\
\hline \multirow{2}{*}{$\begin{array}{l}\text { Dimension: } \\
\text { Suffering }\end{array}$} & mone & 1.00 & 0.85 & 0.66 & 0.41 \\
(physical and & moderate & 0.65 & 0.74 & 0.59 & 0.38 \\
mental) & severe & 0.41 & 0.38 & 0.33 & 0.18 & \\
& dead & & & & & 0.38 \\
\hline
\end{tabular}

PHARMAC (1998: 21)

Quite appropriately (from a public policy perspective) the HFA state that where significant externalities exist, the preferences of the immediate family/ care givers should be considered but do not explain how this will be achieved. The proposed method of assigning patients to health states using "...whatever information is available to decision makers." (Prioritisation Team, 1998 Appendix A: 3) is low cost but has dangers in that decision makers could manipulate the instrument to suit their own objectives. This cuts across the requirement for transparency, may not be ethically sound, and may not satisfactorily evaluate efficiency and equity consequences of decision.

\subsubsection{Conclusion}

Theoretically the proposed new HFA instrument could replace the surgical priority scoring system and would be more appropriate as a health resource rationing device because all health care interventions would be considered. 
Both the booking system and the HFA's cost-utility approach have inherent dangers in their application. These dangers are: sole reliance on either one for decision making, a uniform methodology is not suitable for all situations, and the perspective taken is not that of society. With respect to the booking system, there is no distinction between whether the delay in surgery will lead to disability or death, for example, both malign and malignant conditions are equally likely to score the same number of points (Dennett and Parry, 1998).

The major potential advantages of the system are an increased transparency and uniform treatment across all hospitals and patients. This uniformity has ethical and equity advantages but uniformity can only be achieved if the system does not involve judgement (the issue raised earlier). As the booking system is based on an absolute score and does not consider cost it cannot be used in cost-effectiveness or cost-utility studies as these compare changes in the utility score with changes in cost.

\subsection{CONCLUSIONS AND POLICY POINTS}

Cost per QALY gained was once seen as the ultimate answer to health resource allocation problems. However, QALYs have a number of potential difficulties. They do not discriminate by age of patient, for example, a one QALY gain for a 90 year old would be identical to that of a one QALY gain for a 20 year old mother of a young child. There is no standard agreed international generic health state classification system and method of eliciting preferences. While QALYs assist with decisions relating to resource allocation within the health budget they cannot consider resource allocation across the whole economy. QALYs assume that length of life can be traded off against quality of life, that individual utilities may be aggregated for policy purposes and a gain of 0.2 to 0.3 is assumed to be identical to a gain of 0.8 to 0.9 . These trade-offs and aggregations may not be valid.

From the wide and confusing array of health indices, two appear to dominate (Short Form 36, a health profile and EQ-5D a global or generic index). Both of these have been validated in a range of countries. While the EQ5-D is suitable for CUA, the SF36 is not. However, the SF36 has been used to check that health related outcomes are identical in 
CMA studies ${ }^{8}$, and has been used to value health states in CUA studies 9 . It is difficult to justify inventing a new instrument when internationally tested and validated instruments exist. There is no consensus on the best single measure to use in all studies (Gold et al., 1996: 121).

It is often valuable to use a health profile as well as a generic and/or disease specific health status index as it gives more specific detail on how and why the quality of life as measured by the summary statistic in the index has changed. From a clinical perspective the information provided by a health profile would be useful but an index is essential for costutility analysis.

Policy makers need to be aware of the large and growing number of ways of quantifying changes in health related outcomes. The appropriate way of measuring changes in health outcomes will be determined by the policy question, the time available for the analysis, the budget, and the expected value of the information. The following points should be helpful to policy decision makers.

- Do not construct your own health related quality of life instrument but use an existing instrument.

- If using health related quality of life instruments, where possible use a validated instrument with an extensive body of literature that enables judgements to be made about its validity, reliability and its use in other studies.

- As health outcome measures do not consider important non health components of quality of life they can only be used to make resource allocation decisions within the health sector.

- For policy decision making, a generic health related quality of life instrument should be used in conjunction with either a health profile or a specific index.

\& For example, in an economic evaluation of treatments for thrombosis, (O'Brien et al., 1999)

9 For example, in a study of alternative treatments for arthritis, (Kristiansen et al., 1999) 
- Health related quality of life instruments should be used in conjunction with a range of policy decision making tools such as CEA.

Resource allocation decisions require measures of both the change in health outcomes and cost. Chapter 4 focuses on cost identification and quantification. 


\subsection{REFERENCES}

Anderson, R. T., N. K. Aaronson, M. Bullinger, and W. L. Mcbee. "A review of the progress towards developing health-related quality-of-life instruments for international clinical studies and outcomes research." PharmacoEconomics 10 (1996): 336-55.

Arrow, K. J. Social choice and individual values. New York: Wiley, 1951. (2 ${ }^{\text {nd }}$ ed 1963)

Baumol, W. J. Economic theory and operations analysis. 2nd ed. Englewood Cliffs: Prentice-Hall, 1965.

Brazier, J. "The short-form 36 (SF-36) health survey and its use in pharmacoeconomic evaluation." PharmacoEconomics 7(5) (1995): 403-15.

Bulpitt, C. J. "Quality of life as an outcome measure." Postgraduate Medical Journal 73 (1997): 613-16.

Cooper, M. H. Resource allocation in the health services sector. Economics discussion paper No 8104, University of Otago, 1980.

Culyer, A. J., R. J. Lavers, and A. Williams. "Health indicators." In Social indicators and social policy: 1st ed., edited by A. Shonfield and S. Shaw, 94-118. London: Heinemann Educational Books, 1972.

Dasgupta, A. K., and D. W. Pearce. Cost-Benefit Analysis Theory and Practice. London: Macmillan, 1980.

Dennetl, E. R., and B. R. Parry: "Generic surgical priority criteria scoring system: the clinical reality." New Zealand Medical Joumal 111 (1998): 163-66. 
Drummond, M. F., B. J. O'Brien, G. L. Stoddart, and G. W. Torrance. Methods for the economic elaluation of health care programmes. 2nd ed. New York and Toronto: Oxford University Press, 1997.

EuroQol Group. EQ - 5D user guide: a measure of health-related quality of life. Rotterdam: EuroQol Group, 1996.

Garber, A. M., M. C. Weinstein, G. W. Torrence, and M. S. Kamlet. "Theoretical foundations of cost-effectiveness analysis." In Cost-Effectiveness in health and medicine, edited by M. R. Gold, J. E. Siegel, L. B. Russell, and M. C. Weinstein, 25-53. New York: Oxford University Press, 1996.

Gerard, K., and G. Mooney. "QALY league tables: handle with care." Health Economics 2 (1993): 59-64.

Gold, M. R,, D. L. Patrick, G. W. Torrance, D. C. Fryback, D. C. Hadorn, M. S. Kamlet, N. Daniels, and M. C. Weinstein. "Identifying and valuing outcomes." In Cost-effectiveness in health and medicine. 1st ed., edited by M. R. Gold, J. E. Siegel, L. B. Russell, and M. C. Weinstein, 82-134. New York and Oxford: Oxford University Press, 1996.

Gudex, C. ed Standard gamble user manual: props and self-completion methods.York: University of York, Centre for Health Economics, 1994a. Occasional Papers; Occasional Papers.

Gudex, C. ed Time trade-off user mamual: props and self-completion methods. York: University of York, Centre for Health Economics, 1994b. Occasional Papers.

Guyatt, G., H, D. Feeny H, and D. Patrick L. "Measuring health related quality of life." Annals of Intemal Modicine 118 (1993): 622-29 
Hadom, D. G., and J. Uebersax. "Large-scale health outcomes evaluation: how should quality of life be measured? Part I - Calibration of a brief questionnaire and a search for preference subgroups." Jowmal of Clinical Epidemiologr 48 (1995): 607-18.

Halliwell, T. H. "How fair is cataract prioritisation?" New Zealand Medical Journal 111 (1998): 405-7.

Hansen, R. W. "Health Status indices and quality of life assessment." In Principles of pharmacoeconomics, 1st ed., edited by J. L. Bootman, R. J. Townsend, and McGhan, 5171. Cincinnati, OH: Harvey Whitney Books Company, 1991.

Johannesson, M. Theory and methods of economic evaluation of health care. 1st ed. Dordrecht: Kluwer Academic Publishers, 1996.

Kristiansen, I. S., T. K. Kvien, and E. Nord. "Cost effectiveness of replacing diclofenic with a fixed combination of misoprostol and diclofenic in patients with rheumatoid arthritis." Arthritis \& Rheumatism 42 (1999): 2293-302.

Luce, J. M. "The changing physician-patient relationship in critical care medicine under health care reform." American Journal of Respiratory and Critical Care medicine. 150 (1994): 266-70.

McDowell, I., and C. Newell. Measuring health: a guide to rating scales and questionnaires. 2nd ed. New York: Oxford University Press, 1996.

Medical Outcomes Trust. Australia/ New Zealand SF-36. Boston, MA: The Health Institute, 1994 .

Miller, T., and J. Guria. The value of statistical life in New Zealand. Wellington: Ministry of Transport, 1991.

Mooney, G. Key issues in health economics. London: Harvested Wheatsheaf, 1994. 
Nord, E. "The QALY - a measure of social value rather than individual utility." Health Economics 3 (1994): 89-93.

O'Brien, B., M. Levine, A. Willian, R. Goeree, S. Harley, G. Blackhouse, and M. Gent. "Economic evaluation of outpatient treatment with low-molecular-weight heparin for proximal vein thrombosis." Archives of Internal Medicine 159 (1999): 2298-304.

PHARMAC. A prescription for pharmaceutical analysis. Wellington: PHARMAC, 1998.

PHARMAC. A prescription for pharmaceutical analysis. Wellington: PHARMAC, 1999.

Prioritisation Team. How shall we prioritise health and disability services? Wellington: Health Funding Authority, 1998.

Rosser, R. "A history of the development of health indicators." In Measuring the social benefits of medicines. 1st ed., edited by G. T. Smith, 50-62. London: Office of Health Economics, 1983.

Rosser, R., and V. Watts C. "Measurement of Hospital Output." International Journal of Epidemiology 1 (1972): 361-68.

Rosser, R., and P. Kind. "A scale of valuations of states of illness: is there a social consensus?" International Journal of Epidemiology 7 (1978): 347-58.

Sekaran, U. Research methods for business: A skill-building approach. 2nd ed. New York: John Wiley and Son, 1992.

Smith, A. The wealth of nations. London: Dent, 1981 (reprint of 1776 work).

Strom, B. L., and K. L. Melmon. "The use of pharmacoepidemiology to study beneficial drug effects." In Pharmacoepidemiology. 2nd ed., edited by B. L. Strom, 448-67. Chichester: Wiley and Sons Ltd., 1994. 
Tobias, M. "Measuring health outcomes: SF36". HRC Health Economics Research Workshop, Centre for Health Services Research and Policy, University of Auckland, 27 November 1998.

Torrance, G. W., W. Furlong, D. Feeny, and M. Boyle. "Multi-attributive preference functions health utilities index." PharmacoEconomics 7(6) (1995): 503-20.

Turner, R. K., D. Pearce, and I. Bateman. Environmental economics. 1st ed. Hemel Hempstead: Harvester Wheatsheaf, 1994.

Uhde, A. "The need for health indicators." In Health indicators, edited by A. J. Culyer, 110-16. Oxford: Martin Robertson, 1983.

Weinstein, M. C., H. V. Fineberg, A. S. Elstein, H. S. Frazier, D. Newhauser, R. R. Neutra, and B. J. McNeil. Clinical decision analysis. 1st ed. Philadelphia: BW Saunders Company, 1980.

Williams, A. "Economics of coronary artery by-pass surgery." British Medical Journal 291 (1985): 326-29.

Williams, A. The measurement and valuation of health: a chronicle. Discussion Paper 136, Centre for Health Economics, York Health Economics Consortium, NHS Centre for Reviews \& Dissemination, 1985.

World Health Organisation. Official records No 2 (1948): 100. Cited in Mooney, G. H. "Values in health care." In Economics and health planning. 1st ed., edited by K. Lee, 23-44. London: Groom Heln, 1979.

Zikmund, W.G. 2000. Business Research Methods. Gth ed, Dryden Press. Fort Worth. 


\section{CHAPTER 4: COSTS}

\subsection{INTRODUCTION}

Economic evaluation of health care requires information on both changes in costs and changes in health outcomes. In this chapter, the following specific aspects relating to costs and costing are addressed; the historical perspective, classifications of costs, incremental, marginal, average and sunk costs, double counting traps, the costing process, sensitivity analysis, data problems and perspectives.

Health policy makers require information on costs if they are to establish priorities and set budgets. Such data may be provided by cost-of-illness and cost-of-treatment studies. The results of cost-of-illness and cost-of-treatment studies are essential inputs for costeffectiveness, cost-utility and cost-benefit studies.

There is some confusion over the terminology used to define costs and this has been noted by Balas et al. (1998: 54) "...the assessment of efficiency is confused by diverse and conflicting interpretations of the term cost...". It is important that costs and costing methods be described in sufficient detail to permit users to assess the validity of the estimates (Rice, 1994).

Programme or intervention costs are more correctly termed net costs, that is, costs incurred less costs avoided or saved. Costs may be either positive or negative. A cost avoided is a negative cost and could be regarded as a benefit. Outcomes of medical interventions are intended to be beneficial, accordingly, they are often regarded as benefits. As there is no universally accepted rule that determines if an item should be classified as a cost or a negative benefit care is needed when interpreting cost/ benefit ratios of different studies. (See Chapter 6: Economic Evaluation). 
Health outcomes and resource use changes caused by medical intervention may be evaluated explicitly by the market or implicitly by means of the opportunity cost of the resource. Market valuations will not reflect the frue value to society if market failure is present, or there is no market for the health outcome produced by the intervention.

\subsection{HISTORICAL PERSPECTIVE}

Confusion arises from the use of different systems of categorising costs and from terminology being interpreted differently by different disciplines. Accounting and economic definitions of costs may differ. Explicit or financial costs ( also referred to as accounting costs.) are those costs identified by money transactions. Implicit costs do not involve money payments. Economic costs include all costs, both implicit and explicit. This section outlines common cost classification systems and defines some commonly used nomenclature. The terminology used to describe and classify costs in health economics may be traced back to the language of cost-benefit analysis from which it evolved.

Historically, cost-benefit analysis (CBA) distinguished three cost types; direct, indirect, and intangible costs. Direct (primary) costs represented resources directly consumed by a project (for example, one-time fixed costs, investment costs and recurring or operating costs). Indirect (secondary, spillover or external) costs fell upon third parties not directly involved with the project. (Other definitions and approaches to measurement of indirect costs specifically used in health economics are discussed later in this section.) Intangible costs are either impossible or very difficult to quantify in money terms. Dasgupta and Pearce (1980), Dunn (1981) and Havenan and Weisbrod (1977) provide an overview of CBA.

Cullis and West (1979: 172) classified health care costs (and benefits) in a two by two matrix (Table 4.1). This classification stems directly from cost-benefit analysis nomenclature. Costs were classified as direct or indirect, and as tangible or intangible. Direct costs are those that are closely related to the objectives (for example, medical expenses) of the project while indirect costs are not closely related to the project objectives 
(for example, loss of leisure time). Tangible costs can be valued by the market and intangible costs cannot.

Table 4.1: Classification of Health Care Costs and Benefits (After Cullis and West, 1979: 172)

Tangible $\quad$ Intangible

Direct Closely related to the project objective Can be valued in the market Not valued in the market Indirect Not closely related to the project objective Can be valued in the market Not valued in the market

Drummond et al. (1987: 22) defined costs as (a) direct (organising and operating costs within the health sector and costs borne by patients and their families), (b) indirect (time lost from work, (c) psychic costs) and (d) costs external to the health sector and their families (Table 4.2).

Table 4.2: Classification of Health Care Costs (After Drummond et al., 1987: 22)

(a) Direct Organizing and operating costs within the health sector (Health professionals time, supplies, equipment, power, capital)

Costs borne by patients and their families (excluding time lost from work and psychic costs which is recorded in indirect costs)

(b) Indirect Time lost from work (also borne by patients and their families)

(c) Psychic costs (also borne by patients and their families)

(d) Costs borne externally to the health sector, patients, and their families

In another section of their book, Drummond et al. (1987:2) define costs as C1 direct costs, $\mathrm{C} 2$ indirect costs (production loss) and C 3 intangible costs. 
Donaldson (1990: 345) classified costs in a similar way to the second method described in Drummond et al. (1987), but excluded intangible costs. Direct costs related to health care resources (staffing, consumables, overheads, capital), other related services (community services, ambulance services, voluntary services, patients and family caregivers) and costs incurred by clients and their families (inputs to treatment, out-ofpocket expenses). Indirect costs were time lost from work. Costs borne externally by health and welfare services were included as a third category. Donaldson comments that these external costs should be mentioned for completeness but are rarely included in health economic evaluations (Table 4.3). The externalities are difficult to isolate and quantify and frequently the perspective adopted is that of a purchaser or provider in which case they are not relevant.

\section{Table 4.3: Classification of Health Care Costs (After Donaldson, 1990: 345)}

Direct Health care resources: staffing, consumables, overheads, capital Other related services: community, ambulance, voluntary

Indirect Time lost from work

Costs borne externally by the health and welfare services

Both Drummond et al. (1987) and Donaldson (1990) classifications typify those found in the health economics literature at that time, and exhibit a strong link with traditional CBA terminology.

Balas et al. (1998: 56) attempt to fit all cost items into an accounting or financial framework from the perspective of a provider (Table 4.4). This management accounting classification is helpful (for non accountants) in that it lists accounting expense categories where data may be obtained from which to estimate economic costs. It is not useful as a standard for recording and reporting economic costs because the focus is on sources of data rather than an output or sector classification. Furthermore, accounting costs include only those costs recorded in money values and exclude implicit costs (except for depreciation). 
Table 4.4: An Accounting or Financial Classification of Costs

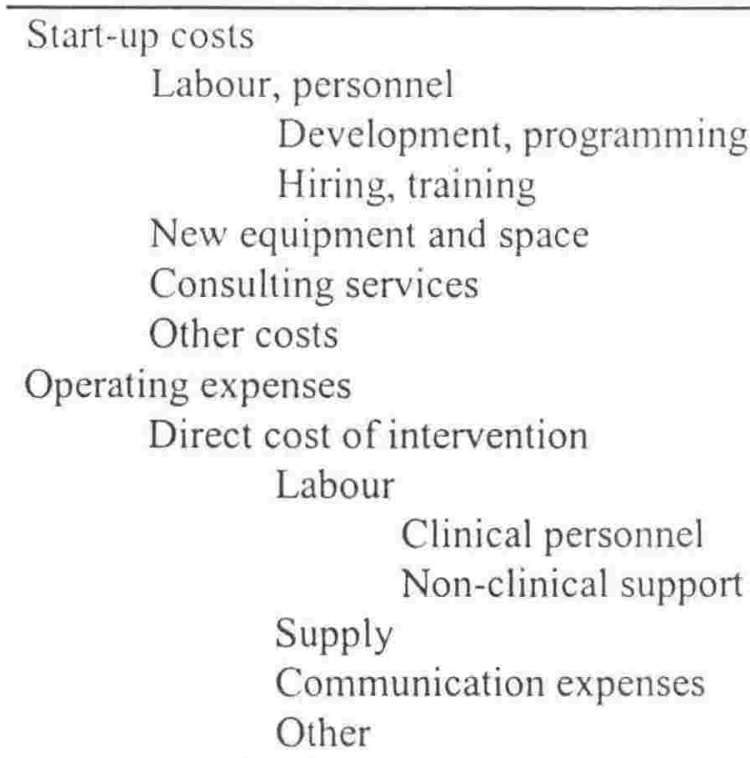

General overhead

Administration

Occupancy (rent, utilities, and telephone)

Total cost

The current standard American text (Luce, B. R. et al., 1996) classifies resource costs as direct or productivity (Table 4.5).

\section{Table 4.5: American "Standard"}

\section{Direct costs}

Direct health care Changes in use of health care resources

\section{Direct non health care}

Changes in use of non health care resources

Informal caregiver time

Patient time for treatment

Productivity (recommend capturing in QALY rather than recording as a cost)

Morbidity

Mortality 
Direct health care costs represent the changes in health care resources brought about by the intervention, for example, drugs, hospital costs and diagnostic tests.

Direct non health care costs include transport, special foods, clothing, or furnishings. Informal caregiver time includes, family, friends or volunteers time costs in providing care such as home nursing. Patient time cost is the time a patient spends seeking care or participating in or undergoing treatment, recuperation and convalescence.

Productivity costs (loss of production, also called indirect costs) relate to changes in the use of patient time associated with the ability to work or engage in leisure time activities because of morbidity or loss of output from premature death. Luce et al. (1996) recommend measuring productivity in the QALY rather than a cost. If it is considered important to isolate productivity costs, these may be measured in money values and reported separately but not included in the cost-effectiveness ratio.

This classification requires that treatment time be separated from morbidity time. Treatment, recuperation and convalescence time will be captured under patient treatment time while morbidity time will be found in the QALY. The separation of such time will of necessity be somewhat arbitrary. "As a general rule, when time could be categorized equally well as treatment time or as morbidity time we suggest that analysts consider it to be morbidity time and incorporate it in the denominator..." Luce et al. (1996: 182). The denominator is a quality of life measure. Under this system, productivity costs (morbidity and mortality, that is changes in life expectancy) are more often captured in the QALY. If, however the quality of life measure specifically excludes the effects of time, productivity changes should be included as a cost. Both alternatives are technically correct but shifting costs from the numerator to the denominator (or vice versa) of a $\mathrm{C} / \mathrm{E}$ ratio will alter the value of the ratio. Intangible costs are not included as it is assumed these costs will be captured in the health related outcome measure.

Drummond et al. (1997: 32) in their latest book present yet another classification of costs. Costs are defined according to sector, health care, patient and family, and other sectors (Table 4.6). 


\section{Table 4.6: Sector Classification of Costs}

Health care sector

Patient and family

Other sectors

This system is easy to apply and functions well when one perspective (health care, patient or family or other sectors) only is considered. If however an analyst wishes to set up a matrix and cross-tabulate cost categories by perspective she/ he will encounter trouble because in this system the categories are in fact perspectives. For example, some health sector costs will be borne by the patient and family but the Drummond et al. (1997) classification does not allow for this. Other commonly used categorisations of costs avoid this problem.

As the language describing costs is evolving differently in Europe and America analysts should clearly define the cost categories used in their reporting.

\subsection{CLASSIFICATION OF COSTS ADOPTED}

Although there are slight differences, the most common breakdown of costs in the health economics literature is; direct (direct medical and direct nonmedical), indirect and intangible. Luce and Elixhauser (1990) use three categories; direct, indirect and intangible, while Eisenberg et al. (1994) categorise costs as direct medical, direct nonmedical, indirect and intangible. Table 4.7 presents the breakdown of costs used in this thesis. The range of costs and their valuations will depend upon the perspective adopted. 
Table 4.7: Current Generally Accepted Classification of Costs (or Benefits) in Health Economics

Direct: These are the opportunity costs associated with goods and services consumed or altered by the health care intervention and its adverse events.

Direct medical: These costs relate to medical goods and services. They fall directly on the health sector but may be funded from a number of different sources. Examples include:

Community: Medical professionals' time, drugs, diagnostic laboratory tests and imaging.

Hospital: These costs are ustally captured in aggregate as a DRG cost Ward nursing, medical, pathology, imaging, theatre, drugs, critical care, allied health and pharmacy, medical and surgical supplies, catering, allocated overhead, other cost centres.

Direct nonmedical: These costs are generally borne by the patient, family and friends and relate to non health care or nonmedical goods and services. Examples include:

Transport: Ambulance, private motor vehicle, taxi, public transport.

Other: Special foods, clothing, alterations to house, additional housekeeping costs, hotel costs if patient travels away from home for treatment.

Indirect, productivity or time: Indirect costs are concerned with the opportunity cost of patients' and informal caregivers 'time. Time costs relate to paid work, unpaid work and leisure-time activities. The patients' time may either be estimated as a cost or captured in a health related quality of life outcome measure.

Morbidity: Lost or reduced ability to work or engage in leisure-time activities.

Mortality: Lost production because of death.

Unpaid or informal caregivers: Lost work or reduced ability to work or engage in leisure-time activities.

Intangible: Intangibles do not involve opportunity costs but people may be willing to pay to avoid discomfort. They may be valued using willingness to pay criteria or captured in a health related quality of life outcome measure. Examples include:

Quality of life, pain and suffering.

Externalities: These costs are not borne by the heallh sector, the patient or family.

Costs to the food or hospitulity industry in preventing food borne disease.

Increased cost of sawn timber because of a safety programme for workers.

Costs avoided in water treatment from the elimination water borne diseases.

Monitoring and testing food and water samples.

Enforcement of health legislation. 


\subsubsection{Direct Costs}

Direct costs are related to treating or preventing a specific illness and the side effects of such treatment or prevention. These costs are frequently broken down still further into direct medical, and direct nonmedical costs. Direct medical costs may be either community and/ or hospital based.

Direct medical costs are those associated with medical treatment and include medicines, general practice consultations and hospital stays. These costs fall directly within the health sector but may be funded from a range of sources. Adopting different analytic perspectives may result in different valuations. As services provided by voluntary organizations have an opportunity cost they should be included. Community costs could include medical professionals' (clinician, nurse, physiotherapist, chiropractor) time, diagnostic imaging and laboratory tests.

For convenience and comparability, hospital costs are normally recorded as diagnosisrelated group (DRG ) costs or in Australia and New Zealand an Australian DRG (ANDRG) cost. This aggregate DRG cost may be broken down into sub groupings such as: ward nursing, medical, pathology, imaging, theatre, drugs, critical care, allied health and pharmacy, medical and surgical supplies, catering, allocated overhead and other cost centres (Casemix Development Program, 1995).

Capital costs are relevant to both community and hospital service providers. Capital items include; land, buildings, fixtures, fittings, vehicles, machinery and equipment. If new equipment is to be set up in a hospital where land, buildings and other infrastructures already exist and if those facilities are currently not in use, they should not be included in the cost of the project. Where existing buildings are currently in use (for example, laboratory, reception office or ward) and would need to be moved, the costs of such re establishments are a part of the project cost. 
Calculating total annual costs of a project facilitates management understanding and the comparison of alternatives having different economic lives (Richardson and Gafini, 1983). Provision can be made for capital by a notional "rent" to cover the cost of benefits foregone (opportunity costs) of the use of existing buildings. A one-time capital cost may be converted into an annual equivalent. Subtracting the present value of the salvage (or scrap) value of the asset at the end of its economic life from the purchase price and dividing the result by the appropriate annuity factor produces an annual equivalent cost. The annuity factor is dependent upon the number of years the asset is to be used and the discount rate. Alternatively, the appropriate spreadsheet financial function may be used by inputting the discount rate, the economic life, the acquisition cost, and the expected salvage value.

Richardson and Gafini (1983) note that the literature abounds with incorrect methods adopted to evaluate annual equivalent costs. Incorrect methods include the straight line depreciation and depreciation opportunity cost methods. The straight line depreciation method sets the annual costs equal to the yearly straight-line depreciation cost (purchase cost less the salvage value divided by the number of years). If the discount rate is greater than zero the method underestimates the annual equivalent cost as no account is taken of the time value of money. The second method calculates the annual straight line depreciation cost and adds to this an opportunity cost calculated as the product of an interest rate and the purchase cost. This method overestimates the annual equivalent cost of capital.

Direct nonmedical costs are those costs incurred as a result of the illness or the need to seek health care and are generally bome by patients and their friends and families. These costs relate to non health care or nonmedical goods and services and may include; transport (ambulance, private motor vehicle, taxis and public transport) and other additional costs as a result of the illness or intervention (special foods, clothing, wheel chairs, walking sticks, hearing aids, glasses, prostheses, housekeeping, modifications to home or motor vehicle, and hotel costs if the patient must travel away from home for treatment). 


\subsubsection{Indirect Costs}

Indirect costs are concerned with time costs as a result of reduced capacity to work or engage in leisure-time activities arising from illness, or lost production to society through death. They are external to the health sector and fall on patients and the rest of society. Production loss relates to those in paid and unpaid employment. Excluding these costs from analyses undertaken from a societal perspective will cause a systematic bias towards interventions and programmes that have the greatest impact on the health sector and a discrimination against those that have productivity implications.

It is convenient to divide indirect costs into the following categories; morbidity, unpaid or informal caregivers' time, and mortality costs. These are the opportunity costs of lost or reduced ability to work or engage in leisure-time activities. Mortality costs are concerned with lost production as a result of death from the illness (years of production lost).

Rice (1994: 1520) recommends calculating morbidity costs as "...(a) mean earnings an individual would have accrued had he or she not been affected by the disease, disaggregated by age and gender; and (b) the number of days lost from work for the employed population or days lost in performing their main activity for those keeping house. ...Mortality costs are the product of the number of deaths from the disease and the expected value of an individuals's future earnings with gender and age taken into account."

Indirect costs of patients and informal caregivers may be quantified by using average eaming rates as a proxy for the value of production and leisure-time loss. If different rates are applied to different population groups (employed, unemployed, age, gender, ethnic groups, and high versus low earners), it can result in differing levels of importance being placed on specific population groups. This has important consequences for evaluation of policy options that are gender and age specific. If the objective of the economic analysis is to provide information for policy decisions that are intended to be gender and age neutral, an economy-wide average wage rate should be applied to all time related costs (Weinstein 1996: 1255; Scott and Scott, 1993; Scott, McKendry and Scott, 1992). 
Until the early 1990s most cost-of-illness and cost-effectiveness studies estimated the potential productivity cost changes. Koopmanschap and van Ineveld (1992) introduced the frictional cost method of measuring the value of production lost to society as a result of disease. Using this method, production losses are confined to the period needed to replace the sick worker. The length of this interval, the friction period, depends on the conditions in the labour market and level of unemployment. In a later study (Koopmanschap and Rutten, 1994) investigated eight health care programmes. They found, (not unexpectedly), that the impact of indirect costs as measured by the friction cost method varied considerably across programmes and were greater if absence from work was a major effect of the programme, and if a high proportion of the target population was employed when they benefited from the programme.

The idea behind the friction cost method is that in the short-run, losses in production as a result of an absent worker can be made up by colleagues working harder and unimportant tasks not being undertaken. In the long-run the sick worker will be replaced from the unemployed. Thus, the production loss should be confined to the period required for the enterprise to reorganise production by hiring and training a replacement. The potential cost method and the frictional cost method may give widely divergent results and should be investigated in sensitivity analysis.

Two methods (human capital and willingness to pay) may be used to measure mortality costs. The human capital approach, the earliest method used, calculates the value of an individual's life by discounting expected future earnings (Rice, Hodgson, and Kopstein 1985). Rice and Hodgson (1982: 536) note that while the human capital method provides reliable and consistent values for future earnings lost, it underestimates the impact of death because it excludes non-market activities and pain and suffering.

Rice (1994) adrocates using market earnings (including an imputed value for housekeeping services), together with, life expectancy, and specific labour force participation rates for different age and gender groups. If the perspective taken is that of society, the individual's lifetime consumption should not be deducted from the present 
value of future earnings as it is the total contribution to national output that is relevant (Sorkin, 1984: 116).

A weakness of the human capital approach is that it places a lower value on the lives of children (if future earnings are not considered), older people, and those unemployed or not in the workforce and may also discriminate against gender and ethnic groups. The method gives no consideration to the well-being of the individual or to the individual's family and friends. A good example of the consequences of gender differences in paid labour participation and different wage rates may be found in (Rice, 1967: 433).

The second method uses willingness to pay criteria and values life according to the amount that an individual would be willing to pay to reduce the risk of illness or death by a given amount. Willingness to pay estimates provide higher values for life than the human capital approach (Rice, 1994). Statistical lives rather than particular lives are used, as the value placed on a particular identified life is likely to be higher than a statistical life (Viscusi, 1986: 194). In addition, a wealthy individual would probably be willing to pay more to save his/her life than a poor individual.

Rice (1994) and colleagues refined the human capital approach while the willingness-to-pay method, was first proposed by Schelling (1968) and Mishan (1971). Willingness to pay techniques may be used in public policy to value health and life and the avoidance of pain and suffering. The Ministry of Transport and Transit NZ (Miller and Guria, 1991) have valued a life at \$2 million (in 1989/90 dollars). This value was arrived at by asking people what they would be prepared to pay to reduce the risk that they would die on the roads. The question was posed for both family members and strangers.

Very few cost-benefit analysis health public policy studies have been conducted using willingness to pay values for human life. If cost-benefit analysis is to be applied and used with confidence by policy decision makers more tenable values and methods will need to be developed for the health sector. Conjoint analysis, a technique derived from marketing research, may offer scope for obtaining credible valuations of healthcare benefits. The 
technique has been used in marketing to evaluate the importance of specific features of a product on consumer preferences (Cattin and Wittink, 1982; McDaniel, 1996; Davis, 1999). If cost is included as an attribute of a health outcome, willingness to pay can be evaluated using conjoint analysis without having to ask a direct open ended question (Ryan, 1996).

\subsubsection{Intangible Costs}

Intangible costs are concerned with reduced quality of life as a result of the illness. These intangible costs do not involve opportunity costs of resources used and some economists working in the non health sector would not regard these as true economic costs. Never-theless it would be expected that people would be willing to pay to avoid them. These costs are relevant if a cost-benefit analysis is being undertaken and are captured in the health related outcome measure in a cost-utility analysis.

\subsubsection{Externalities}

External costs are not borne by the health sector, the patient or family and they are not usually measured in a cost-of-illness study. However, if a societal perspective is adopted there is no valid reason for excluding externalities provided they can be clearly identified and quantified. Examples of externalities include; costs to the food or hospitality industry incurred in preventing food and water borne diseases. Johannesson (1994: 676) refers to two types of externality; consumption externality, and caring externality. Consumption externalities are the impact on consumption by those other than the recipients of health care. Caring externalities do not involve resource opportunity costs and are defined as "...the altruistic value of changes in other people's health status...". Johannesson (1994: 681) notes there is an argument against including caring externalities under some circumstances, for example, "...if altruism concems the utility of other individuals, these altruistic values would cancel out at the welfare optimum..." 


\subsection{INCREMENTAL, MARGINAL, AVERAGE AND SUNK COSTS}

As there is some confusion (by non economists) between incremental, marginal, average and sunk costs, these are defined and the situations in which they are used are discussed.

Marginal cost (MC) is the addition to total cost resulting from the production of one more unit of output. Variable Costs (VC) are related to the volume of output, they increase as output rises and are zero at zero output. Fixed Costs (FC), do not change as output changes and are incurred even at zero output. Total Cost(TC), is the sum of variable cost plus fixed cost. Average total cost is total cost divided by the units of output. Sunk costs (Sloan 1996: 65) are costs that have already been incurred.

The incremental cost of a decision is the additional cost incurred less the costs avoided. If a cost will be altered by a decision (within the time horizon of the programme) it is relevant but if the cost item will not be altered, or has already been incurred, it is not relevant. For example, the historical cost of building a hospital is a sunk cost that will be unchanged by the activity or decision under investigation and is therefore not relevant. In the long-run, decisions should be based upon long-run marginal or incremental costs but in the short-run, short-run costs are applicable.

All costs and benefits should be caiculated incrementally. Incremental analysis takes into account only the differences in costs and the differences in benefits between the alternatives being considered, within the time horizon of the project. Alternatives could include various treatment options and could encompass such valid options as doing nothing (maintaining the status quo or the counterfactual).

Economic efficiency implies that marginal social cost equals marginal social benefit. A real project is unlikely to operate at this margin. Potential improvements in economic efficiency (with respect to the distribution of resources) implies incremental changes not marginal changes in costs and benefits. Often the terms marginal and incremental are used interchangeably. However, incremental costs may relate to both the short-run or long-run 
(depending on time horizons and plant capacity) and are defined as the change in total cost resulting from a decision. Marginal cost is a short-run concept and excludes fixed cost.

Although average costs (total costs per unit of output) and marginal costs may not be appropriate for calculating differences in costs between options, analysts will be forced to use them if it is impossible to obtain more appropriate incremental cost data. If this is likely to bias the results some comment should be made.

For example, two additional hospital patients will not add to the overhead costs of a hospital but 500 more patients may necessitate the hiring of additional staff and the building of additional facilities. Where marginal costs do not change markedly, they may be used to evaluate changes that involve a small number of additional patients. Average cost (which include overheads) may provide a better guide in evaluating larger changes (such as a new hospital ward) but not when there is unused capacity or where the change in the number of patients is small.

\subsection{AVOIDING DOUBLE COUNTING OF COSTS}

If costs are counted more than once or counted when they should have been excluded a programme's costs will be overestimated and the programme may be rejected when it should have been accepted. Productivity costs and transfer payments are frequently counted more than once or counted inappropriately. It is important that policy makers and managers be aware of these potential problems.

In this section the following will be discussed:

- Productivity costs and quality of life

- Transfer payments

- Cost shifting

- Capital costs

- Co-morbidity 


\subsubsection{Productivity and Health Related Quality of Life}

Cost-utility analysis (Chapter 6: Economic Evaluation) compares the cost-of-treatment with a health related quality of life measure. Double counting can occur as changes in productivity may be captured in both the health related quality of life measure and in the cost measure. This confusion can also lead to changes in productivity not being captured in either costs or effects. A solution suggested to this problem is that when quality of life values are being obtained from subjects, respondents be told to assume that all health care costs and income losses are fully reimbursed (Johannesson, 1997). Productivity (output) losses may also be overestimated if a money payment from loss of earnings, insurance, and lost earnings are counted as costs.

There is no generally accepted convention of where to record productivity. The Washington Panel on Cost-effectiveness in Health and Medicine consider that productivity losses should be excluded from the costs of cost-utility analyses and included in the utility measure (Garber et al., 1996). The Panel note that if production loss for the individual is captured in the utility measure then only those productivity costs external to the individual should be included as a cost. Examples of these externalities are the frictional costs to an employer. If, however, respondents to the utility questionnaire are specifically asked to exclude loss of income in their evaluation of health related quality of life states then productivity loss should be evaluated as a cost.

The Washington Panel (Garber et al., 1996: 39) state that..." under conditions of perfect markets, the cost-effectiveness method leads to the same decision rules for allocating health resources..." no matter whether time costs are captured in the cost component or in the effectiveness measure. Brouwer, Koopmanschap and Rutten (1997: 253) argue that "...only direct health related effects on quality of life that cannot be meaningfully monetarized should be considered as health effects." Brouwer et al., note that the patient's perspective is useful for quality of life assessment but not for evaluating productivity from a societal perspective. 


\subsubsection{Transfer Payments}

As transfer payments involve the redistribution of money they do not represent real costs from the perspective of society (Luce et al., 1996). In New Zealand, the goods and services tax (GST) is a cost from the perspective of an individual patient but is a transfer payment if the perspective of all society is taken. Similarly, unemployment benefits are relevant if the perspective is that of the government budget but are irrelevant from the perspective of society as a whole. The costs of collecting taxes, distributing benefits and any costs resulting from reduced market efficiency are relevant but would be very difficult to measure. Government welfare benefit payments used to pay for medical treatment could be counted both as a medical subsidy and as a medical cost. Taxes could be multiple counted if income tax losses to government were added to losses of eamings of individuals and again if the tax revenues were then used for public health care which had been counted as a health cost (Rice, 1967: 429).

\subsubsection{Cost Shifting}

Cost shifting, occurs when a budget holder shifts costs out of their budget into another budget. If mentally ill patients were discharged from hospital to be treated in the community, but funding for community care not increased, cost shifting would occur. With a societal perspective, all costs are captured and cannot be shifted elsewhere.

\subsubsection{Capital Costs}

Interest on borrowed funds is irrelevant if a societal perspective is adopted, but is relevant if the perspective taken is that of an individual enterprise. However, for an enterprise, deducting interest costs from a cash flow and then discounting to a present value will underestimate present values. 


\subsubsection{Co-morbidity}

The presence of multiple diseases creates difficulties in isolating the costs and consequences of each condition may result in multiple counting or omissions (Rice, 1976:429; Sorkin, 1984:115). The problem of joint or shared costs is well understood in economics but easy solutions remain elusive.

\subsection{COSTING PROCESS}

The costing process may be described in the same way irrespective of the type of economic evaluation in which the costs will be used. The first step in estimating costs is to prepare an itemised list of resources that will be altered by the illness or intervention. A unit cost for each resource item should be obtained. However, unit costs and shadow prices frequently need to be imputed where there is market failure and where policies have been introduced to correct for inequity. Prices paid by purchasers may not necessarily reflect economic costs, different prices may be charged to different consumers for the same product, and a market price for the product or service may not exist because it may not be traded on a market. Resource costs in money units may then be derived by multiplying each resource utilisation by its respective unit cost. Finally, where shadow prices and other uncertainties are involved sensitivity analysis is required.

It is desirable to report all resource costs both in volume units and money values to facilitate sensitivity analysis and allow updating if unit costs change. Provided unit costs and total costs in money values are presented, resource utilisations (volumes) may be calculated.

Micro-costing is the most accurate method of costing and involves deriving a unit cost for each resoure cost component. For example, a unit cost could be derived for imaging, diagnostic tests and ward stays. Activity-based costing involves breaking a process down into a series of activities and micro-costing each of these activities. For example, antibiotic treatment may be broken down into preparation of drug, administration of the drug, and 
monitoring. Resource utilisations (for example, drugs, nurse time) for each of these activities may then be measured. Other costing methods are less accurate. Unit costs per diagnosis related group, or hospital average daily costs may be used to cost hospital admissions. Drummond et al. (1997) discuss costing methods in detail.

\subsection{SENSITIVITY ANALYSIS}

Sensitivity analysis allows the soundness of economic models to be examined through changes in variables of these models (Agro et al., 1997). Effects of uncertainty in costs and other estimates may be investigated and taken into account. The degree of influence that each factor has on the outcomes of a model may also be evaluated using sensitivity analysis. It is recommended in guidelines (for example, Ontario Ministry of Health, Canadian Coordinating Office for Health Technology Assessment, Australian Commonwealth Department of Health Housing and Community Services) for conducting such studies, but no set method is outlined.

Sensitivity analysis may be either; single parameter (univariate, one way) or various forms of multiple parameter analysis. The latter include scenario analysis such as best and worst cases and plausible future scenarios.

In single parameter analysis variables or parameters are examined one at a time and the impact on the final results recorded. Variables are often changed plus or minus the same set percentage. This approach is quite useful as it that allows the critical parameters in a model to be determined. Single parameter analysis should be conducted alongside some sort of scenario analysis as investigating at only one parameter at a time can provide an incomplete and optimistic estimate of total uncertainty.

Multiple parameter sensitivity analysis, where more than one variable is changed, can provide a more complete understanding of uncertainty. Best and worst-case scenario analysis combines the low or high range of variables to determine the worst or best case. "In most cases, multiple sources of uncertainty will generate a less "certain" result than a one-at-a-time, single parameter sensitivity analysis would indicate. ...combining the 
extreme values of parameters to gain an "overall" best or worst estimate can overstate the uncertainty. Ignoring the correlations among terms or the presence of interactions could have the same effect" (Manning, Fryback, and Weinstein, 1996: 252).

In threshold analysis, a parameter (such as a drug price) is altered over a range to determine when major changes in conclusions are warranted. This analysis estimates the values of a parameter or number of parameters to isolate the point at which a different decision should be made. Breakeven analysis is a simple example of threshold analysis. Threshold analysis becomes difficult to use with multiple variable changes (Agro et al., 1997).

Sometimes the diverse range and quality of the data preclude using the standard statistical tests. Sensitivity analysis may then be used as an alternative means of testing the robustness of the data. As it is possible for a value to be statistically different from zero yet so close to zero to be of little consequence for policy or clinical decision making (O'Brien and Drummond, 1994), sensitivity analysis may also be required in addition to statistical tests of significance.

The following texts provide valuable discussion of sensitivity analysis; (Gold et al., 1996; Haddix et al., 1996; Sloan ,1996).

\subsection{DATA PROBLEMS}

Frequently, policy analysts have limited control over their statistical data. Time and budget constraints mean that information must often be drawn from secondary sources. Data may consist of administrative records, with the variables defined by other people, and with the records collected in different situations for different purposes. Data available in the public domain are frequently aggregated to preserve confidentiality (for commercial, ethical and legal reasons) and to reduce a database to a manageable size. It is essential that a clear definition and description be obtained for each variable and a clear understanding of how the data have been collected recorded and aggregated. 
Researchers should study the data-set in the most disaggregated form available and check how data have been defined, collected and recorded, before preparing summary tables and graphs. Additional research or data from another source may be needed to clarify findings. Unit record data may not be available, and it may be necessary to use tabulations provided by others. Data should be analysed using cross-tabulations which involve disaggregation to the lowest possible level. Variables that are found not to influence the outcomes may be aggregated to produce tables that highlight the important determinants. Increasing availability of unit-record data sets, and software tools to manage and analyse them, makes such detailed analysis practicable. For example data available from PC INFOS (Statistics New Zealand) have already been aggregated but Health Statistics New Zealand hospital discharge data are available as unit records. Software packages such as SPSS (Norusis, 1997) permit data exploration, aggregation and analysis. Chapter 8: Care With Data discusses these data problems in more depth.

Where costing data have been derived from clinical trials, adjustments may be required to take account of "protocol driven costs" (Coyle and Lee, 1998). Protocol driven costs arise because the trial design may require certain diagnostic tests that would not normally be required. This results in clinical management of the illness that differs from everyday practice. Problems of this sort may be overcome by modelling (Chapter7) and sensitivity analysis.

\subsection{PERSPECTIVE AND COSTS}

The analytic perspective is the viewpoint from which a study is conducted. Most policies may be viewed differently by different groups in society and implementation of policies will affect different sectors of society in different ways. Perspective is used in two ways; (1) to indicate the scope or boundary of the costs, and (2) to indicate the viewpoint from which costs are measured. Direct medical costs bounded within the health sector may be measured from the perspective of a private household (patient) or from the perspective of a health care purchaser.

Different cost items will be relevant to different perspectives and the same cost item may 
be need to valued differently for different view points. For example, GST is a transfer payment from the perspective of society or a business enterprise but is a cost from the perspective of a single household.

It is vital for policy makers to consider the impact of decisions from a number of different angles and failure to do so may result in failure of the policy to achieve its objectives. The perspectives analysed should be wide enough to permit all stakeholders' interests to be considered. The widest possible perspective is that of society. A societal perspective encompasses the broadest possible range of costs and measures them with respect to their impact on society as a whole.

A central government decision maker, if acting as an agent on behalf of society, should be interested in a societal perspective. A manager in a hospital would be primarily concerned with costs relevant to the hospital perspective, that is, explicit costs rather than implicit $\operatorname{costs}^{10}$ will be relevant to the hospital manager. Some policies may shift costs from one sector of society to another.

\subsection{CONCLUSION}

Cost categories and terminology describing costs should be clearly defined and explained as terms may be interpreted diffeisnily by different disciplines and there are different systems of classifying costs. If the analysis is intended to provide information for gender and age neutral policy making, an economy-wide average wage rate should be applied to all time related costs. Care should be taken not to count costs more than once, to include all costs relevant to the perspectives adopted, and to recognise the consequences of joint costs and co-morbidities. The perspectives chosen should be wide enough to enable the effects of a programme on all stakeholders to be assessed.

Accounting costs may provide a basis for estimating economic costs where the bulk of 
inputs and products produced are traded on efficient competitive markets; in situations like these market prices will reflect unit costs. However, accounting costs are measured from the perspective of a financial entity whereas public policy makers should be concerned with a societal perspective. Prices charged for goods and services do not necessarily reflect economic costs and in many cases unit costs and shadow prices must be imputed for use in the economic evaluations.

The following points are reiterated:

- The perspective should dictate the relevant range of costs and how they should be valued.

- Clearly define all cost categories used.

- Estimate costs incrementally as this will help to avoid traps of inclusion (sunk costs, double counting) and exclusion (overheads that will change).

- Value the costs in a way that is relevant to the research question and the perspectives adopted.

- $\quad$ Present as wide a range of costs as possible

- Consider converting a one-time capital cost into an annual equivalent.

- Present costs in resource units and in dollars.

- Apply sensitivity analysis.

This chapter has focussed on costs in isolation. Succeeding chapters discuss how cost data are used in combination with effectiveness measures to formulate policy advice. Chapter 5 considers discounting of future costs and effects. 


\subsection{REFERENCES}

Agro, K. E., C. A. Bradley, N. Mittmann, M. Iskedjian, A. L. Ilersich, and T. R. Einarson. "Sensitivity analysis in health economic and pharmacoeconomic studies." PharmacoEconomics 11 (1) (1997): 75-88.

Balas, E. A., R. A. C. Kretschmer, W. Gnann, D. A. West, S. A. Boren, R. M. Centor, M. Nerlich, M. Gupta, T. D. West, and N. S. Soderstrom. "Interpreting cost analysis of clinical interventions." Journal of the American Medical Association 279 (1998): 54-57.

Brouwer, W. B., M. A. Koopmanschap, and F. F. Rutten. "Productivity costs measurement through quality of life? A response to the recommendation of the Washington Panel." Health Economics 6 (1997): 253-9.

Canadian Coordinating Office for Health Technology Assesment. Guidelines for economic evaluation of pharmacenticals: Canada. 2 nd ed. Ottawa: Canadian Coordinating Office for Health Technology Assesment, 1997.

Casemix Development Program. Report on the development of AN-DRG Version 3 cost weights. Canberra: Commonwealth Department of Human Services and Health, 1995.

Cattin, P., and D. R. Wittink. "Commercial use of conjoint analysis: a survey." Journal of Marketing 46 (1982): 44-53.

Commonwealth Department of Human Services and Health. Report on the development of AN-DRG version 3 cost weights. Canberra: Commonwealth Department of Human Services and Health, 1995.

Coyle. D., and K. M. Lee. "The problem of protocol driven costs in pharmacoeconomic analysis." PhamacoEconomics 14 (1998): 357-63. 
Cullis, J. G., and P. A. West. The economics of health. Oxford: Martin Robertson, 1979.

Davis, D. Business research for decision making. 5th ed. Pacific Grove: Duxbury, 1999.

Dasgupta, A. K., and D. W. Pearce. Cost-Benefit Analysis Theory and Practice. London: Macmillan, 1980.

Donaldson, C. "The state of the art of costing health care for economic evaluation." Community Health Studies 14 (4) (1990): 341-56.

Drummond, M. F., B. J. O'Brien, G. L. Stoddart, and G. W. Torrance. Methods for the economic evaluation of health care programmes. 2nd ed. New York and Toronto: Oxford University Press, 1997.

Drummond, M. F., G. L. Stoddart, and G. W. Torrance.Methods for the economic evaluation of health care programmes. Oxford: Oxford University Press, 1987.

Dunn, W. Public policy analysis: an introduction. Englewood Cliffs (NJ): Prentice-Hall Inc., 1981.

Eisenberg, J. M., K. A. Schulman, H. Glick, and H. Koffer. "Pharmacoeconomics: economic evaluation of pharmaceuticals." In Pharmacoepidemiology. 2 nd ed., edited by B. L. Strom. England: John Wiley and Sons Ltd., 1994.

Garber, A. M., M. C. Weinstein, G. W. Torrence, and M. S. Kamlet. "Theoretical foundations of cost-effectiveness analysis." In Cost-Effectiveness in health and medicine, edited by M. R. Gold, J. E. Siegel, L. B. Russell, and M. C. Weinstein, 25-53. New York: Oxford Lniversity Press, 1996.

Gold, M. R., S. E. Siegel, L. B. Russell, and M. C. Weinstein. Cost-effectiveness in health and medicine. New York: Oxford University Press, 1996. 
Haddix, A. C., S. M. Teutsch, P. A. Shaffer, and D. O. Dunet, eds. Prevention effectiveness; a guide to decision analysis and economic evaluation. 1st ed. New York: Oxford University Press, 1996.

Haveman, R. H., and B. A. Weisbrod. "Defining benefits of public programs: some guidance for policy analysts." In Public expenditure analysis. 2nd ed., edited by R. H. Haveman and J. Margolis, 135-60. Chicago: Rand McNally College Publishing Company, 1977.

Johannesson, M. "The concept of cost in the economic evaluation of health care: a theoretical enquiry." International Journal of Technology Assesment in Health Care 10 (1994): 675-82.

Johannesson, M. "Avoiding double-counting in pharmacoeconomic studies." PharmacoEconomics 11 (5) (1997): 385-88.

Koopmanschap, M. A., and B. M. van Ineveld. "Towards a new approach for estimating indirect costs of disease." Social Science and Medicine 34 (1992): 1005-10

Koopmanschap, M. A., and F. F. Rutten. "The impact of indirect costs on outcomes of health care programmes." Health Economics 3 (1994): 385-93.

Luce, B. R., W. G. Manning, J. E. Siegel, and J. Lipscomb. "Estimating costs in costeffectiveness analysis." In Cost-effectireness analy sis in health and medicine. 1 st ed., edited by M. R. Gold, J. E. Sicgel, L. B. Russell, and M. C. Weinstein, 176-213. Oxford: Oxford University press, 1996.

Luce, B. R., and A. Elixhauser. "Estimating costs in the economic evaluation of medical technologies." International Jounal of Technology Assessment in Health Care 6 (1990): $57-75$. 
McDaniel, C. D., and R. Gates. Contemporary marketing research. 3rd ed. Minneapolis: West Publishing Company, 1996;

Manning, W. G., D. G. Fryback, and M. C. Weinstein. "Reflecting uncertainty in costeffectiveness analysis." In Cost-effectiveness in health and medicine. 1st ed., edited by M. R. Gold, J. E. Siegel, L. B. Russell, and M. C. Weinstein. New York and Oxford: Oxford University Press, 1996.

Miller, T., and J. Guria. The value of statistical life in New Zealand. Wellington: Ministry of Transport, 1991.

Misham, E. J. "Evaluation of life and limb; a theoretical approach." Journal of Political Economy 79 (1971): 687-705.

Norusis, M. J. SPSS Guide to data analysis. Upper Saddle River: Prentice Hall, 1997.

O'Brien, B. J., and M. F. Drummond. "Statistical versus quantitative significance in the socioeconomic evaluation of medicines." PharmacoEconomics 5 (1994): 389-98.

Rice, D. P., T. A. Hodgson, and A. N. Kopstein. "The economic cost of illness: a replication and update." Health care Financing Review 7 (1985): 61-80.

Rice, D. P. "Cost-of-illness studies: Fact or fiction?" The Lancet 344 (1994): 1519-21.

Rice, D. "Estimating the cost of illness." American Joumal of Public health 57 (1967): $424-40$.

Rice, D. P., and T. A. Hodson. "The value of human life revisited." American Journal of Public health 72 (1982): 536-37.

Richardson, A. W., and Gafni A. Treatment of capital costs in evaluating health-care programs. Cost and Management (1983); November-December: 26-30. 
Ryan, M. Using consumer preferences in health care decision making: the application of conjoint analysis. London: Office of Health Economics, 1996.

Schelling, T. C. "The life you save may be your own." In Problems in public expenditure analysis, edited by S. B. Chase. Washington, DC: Brookings Institution, 1968.

Scott, W. G., C. G. McKendry, and H. M. Scott. Methodologies for economic evaluation of high technology in health care. Wellington: , 1992.

Sloan, F. A. Valuing health care: costs, benefits, and effectiveness of pharmaceuticals and other technologies. Cambridge: Cambridge University Press, 1996.

Sorkin, A. "The costs and benefits of health programs." In Health economics: an introduction. 2nd ed., edited by A. Sorkin. Lexington: DC Heath and Company, 1984.

Statistics New Zealand. Personal computer information network for official statistics: a computerised database of official statistics for New Zealand and programme for manipulation and analysis of statistical series (PC INFOS). Wellington, 1999.

Viscusi, W. K. "The valuation of risks to life and health: Guidelines for policy analysis." In Benefits assessment: The state of the art, edited by J. D. Bentkover, 193-209. Dordrecht: D Reidel Publishing Company, 1986.

Weinstein, M. C., J. E. Siegel, M. R. Gold, M. S. Kamlet, and L. B. Russell. "Recommendations of the panel on cost-effectiveness in health and medicine." Journal of the American Medical Association 270 (1996). 


\section{CHAPTER 5: DISCOUNTING}

\subsection{INTRODUCTION}

This chapter discusses methods for setting the discount rate with a particular focus on public policy evaluations of health care in New Zealand. The mechanics of and rationale for discounting and the appropriate magnitude of the rate are discussed. Discount rates are examined with respect to the study perspective. Current thinking on the discounting of outcomes or effects measured in non-money units is reflected upon. Real versus nominal rates are described, as are discount rates that have been or are currently being used in the public sector. Suggestions and recommendations for New Zealand policy evaluations of health care are outlined.

There are two relevant questions. (1) what is the most appropriate rationale for discounting and hence the most appropriate method of choosing the discount rate, and (2) given the method, what magnitude or numeric value should be selected for the discount rate? Although there has been much argument by policy analysts over discounting, the debate has focused on the most appropriate numeric value of the rate to use rather than the underlying rationale for discounting (Goodin, 1982).

Discounting is used in economic evaluation to adjust costs and benefits that occur in different time periods to a common base. Frequently costs are incurred before benefits accrue from a programme. Essentially discounting means that costs and benefits occurring in the future are assigned a smaller weight than those closer to the present. The weighting applied to a future cost or benefit to convert it into a present value is the discount factor. The magnitude of the weight depends upon the discount rate and the timing when the cost or benefit occurs.

Discounting is necessary because society places a higher value on goods and services received earlier rather than later. A dollar today may be invested to earn interest in the future. Accordingly, societal discount rates may be based on the social rate of time preference or the social opportunity cost of capital. Goodin (1982) outlines four reasons for 
discounting: (1) psychological or pure time, (2) uncertainty and risk, (3) diminishing marginal utility, and (4) opportunity costs.

Often discounting is not necessary as many health policy investigations are prevalence ${ }^{\text {II }}$ or cross-sectional studies and consider incremental or total costs during one time period (usually one year). Discounting will usually be required if the costs and benefits or health related outcomes are derived from longitudinal or time series studies that follow events over a number of time periods.

While there is general agreement on the need to discount future costs and consequences (health related outcomes), the selection of an appropriate method of determining the discount rate for public sector investments is open to debate. The choice of different ways of estimating the discount rate can lead to different rates and thus different present values of costs and benefits.

The choice of the weighted cost of capital or cost of borrowing as an appropriate private sector discount rate is less contentious. As it is appropriate to take a societal perspective when evaluating public policy options, the social discount rate should be used to discount costs and benefits occurring in the future. Other perspectives may require the use of alternative discount rates. For example, a private hospital capital budgeting evaluation could use the cost of capital for that project, while a government department with a budget perspective may apply the capital chat ge rate.

Baumol (1968: 788) comments that the social rate of discount should measure: "The opportunity cost of postponement of receipt of any benefit yielded by a public investment." Further he asserts that the opportunity cost represents the welfare foregone by not receiving the benefits immediately. The discount rate used to evaluate public projects should accurately reflect this opportunity cost. Although this concept elucidated by Baumol (1968)

11 The prevalence of an illness is the number of cases in a population at one point in time while the incidence of a disease is the number of new cases occurring over a specified time period. That is, a still photograph or snapshot compared with a movie. For example, most of the cases discussed in this thesis were prevalence based and costs and effects did not require discounting. 
may appear clear and straight forward there is considerable misunderstanding and debate about the method of selecting the rate, its magnitude, and the application of the discount rate in practice.

The social rate of discount does not necessarily equate either with the cost of funds to the government or to the private sector. The weighted cost of capital to an entity (public or private) is appropriate solely to that entity. If there is market failure in any market then the social rate of discount will not equate with the marginal rate of return on investment. In all (or virtually all) societies there are varying degrees of market failure.

Arrow (1985: 382-3) isolates five problems in using market rates as proxies for social discount rates in a mixed economy. (1) There is a spectrum of market rates rather than one single rate. (2) Price anticipations are not necessarily accurate. (3) The future is risky. (4) Government investment is often concerned with the provision of public goods. (5) Governments have a responsibility for the future above and beyond private individuals or firms. Three of Arrow's problems are important. (1) There is a range of market rates posing a problem of choice. (2) Government investment is often undertaken to correct for market failure in which case market discount rates are not necessarily be valid, and (3) government has additional responsibilities for the future. Thus the choice of public sector discount rates is often more involved than the selection of a discount rate for private sector investments.

Private sector discount rates are commonly based on the weighted average cost of capital to the firm. Public sector discount rates differ from those applicable to individuals and the private sector for a number of reasons. (1) Human entities have a limited lifespan, while society is made up of many individuals and continues indefinitely. Because of this, society has a more distant time horizon for returns on investments than individuals. Accordingly, an individual would place less value on benefits occurring in the future than would society as a whole. (2) There is an argument for using a lower discount rate for society than for individuals as the lower the discount rate the more importance is placed on future returns. However there is a danger that if there is a lower public sector discount rate than the private sector, inferior projects in the public sector could divert investment away from superior 
private sector projects. (3) Another important factor for consideration is the lower cost of borrowing by government compared with the private sector. The private sector pays a risk premium over that of government, because with government there is less risk of default in debt repayment than with the private sector. Governments also have a greater opportunity to spread risk over a wider range of projects than the private sector.

\subsection{MECHANICS OF DISCOUNTING}

Although spreadsheet software and financial calculators have taken the drudgery out of discounting it is useful to review the underlying methodology.

The net present value (NPV) of each alternative may be calculated by applying the following formula:

$$
N P V=\frac{B_{0}-C_{0}}{(1+r)^{0}}+\frac{B_{1}-C_{1}}{(1+r)^{1}}+\frac{B_{2}-C_{2}}{(1+r)^{2}}+\ldots+\frac{B_{t}-C_{1}}{(1+r)^{t}}+\ldots+\frac{B_{n}-C_{n}}{(1+r)^{n}}
$$

Where NPV is the net present value, $B_{1}$ the expected benefit of the programme in period $t$, $\mathrm{C}_{1}$ the expected cost of the programme in period $\mathrm{t}, \mathrm{n}$ the total number of time periods, and $r$ the discount rate. If the values of the costs and benefits are known, this formula can be evaluated easily using spreadsheets, business calculators or tables. The formula assumes costs and benefits for period t occur at the end period t.

Present values are inversely proportional to the magnitude of the discount rate and the time horizon of the costs and benefits. For example, $\$ 100$ in one year's time discounted at $10 \%$ has a present value of $\$ 90.91$ (that is $\$ 90.91$ could be invested at $10 \%$ and yield $\$ 100$ in a year's time) while $\$ 100$ in a year's time discounted by $5 \%$ would have a present value of $\$ 95.24$. In another example, $\$ 100$ in 10 years' time discounted at $10 \%$ has a present value of $\$ 3 \$ .55$ while $\$ 100$ in five years' time discounted by $10 \%$ yields a present value of $\$ 62.09$ (see Table 5.1).

The programmes described in Table 5.1 all have identical total present values if discounted at $0 \%$ (undiscounted). Programme $A$ records a present value of $\$ 72,170\left(A_{1}\right)$ when a discount rate of $5 \%$ is adopted but shows a negative present value of $\$ 85,540\left(\mathrm{~A}_{2}\right)$ when 
discounted at $10 \%$. The effect of different time patterns of net benefits is demonstrated by programmes $\mathrm{B}$ and $\mathrm{C}$. Programme $\mathrm{C}$ has a higher total present value than programme $\mathrm{B}$ because positive net benefits occur earlier.

Table 5.1: Effect of Different Discount Rates and Different Time Patterns of A Programme's Net Benefits on the Net Present Value (NPV)

Period Programme $\mathrm{A}_{1} \quad$ Programme $\mathrm{A}_{2} \quad$ Programme $\mathrm{B}$ Programme $\mathrm{C}$ Net benefit $\$(000)$ Net benefit $\$(000)$ Net benefit $\$(000) \quad$ Net benefit $\$(000)$

\begin{tabular}{lrrrrrrrr}
\multicolumn{1}{c}{ CV } & \multicolumn{1}{c}{ PV } & \multicolumn{1}{c}{ CV } & PV & CV & PV & \multicolumn{1}{c}{ CV } & \multicolumn{1}{c}{ PV } \\
\hline 0 & $(700.00)$ & $(700.00)$ & $(700.00)$ & $(700.00)$ & $(700.00)$ & $(700.00)$ & $(700.00)$ & $(700.00)$ \\
1 & 100.00 & 95.24 & 100.00 & 90.91 & 1.00 & 0.95 & 489.00 & 465.71 \\
2 & 100.00 & 90.70 & 100.00 & 82.64 & 2.00 & 1.81 & 256.00 & 232.20 \\
3 & 100.00 & 86.38 & 100.00 & 75.13 & 4.00 & 3.46 & 128.00 & 110.57 \\
4 & 100.00 & 82.27 & 100.00 & 68.30 & 8.00 & 6.58 & 64.00 & 52.65 \\
5 & 100.00 & 78.35 & 100.00 & 62.09 & 16.00 & 12.54 & 32.00 & 25.07 \\
6 & 100.00 & 74.62 & 100.00 & 56.45 & 32.00 & 23.88 & 16.00 & 11.94 \\
7 & 100.00 & 71.07 & 100.00 & 51.32 & 64.00 & 45.48 & 8.00 & 5.69 \\
8 & 100.00 & 67.68 & 100.00 & 46.65 & 128.00 & 86.64 & 4.00 & 2.71 \\
9 & 100.00 & 64.46 & 100.00 & 42.41 & 256.00 & 165.02 & 2.00 & 1.29 \\
10 & 100.00 & 61.39 & 100.00 & 38.55 & 489.00 & 300.20 & 1.00 & 0.61 \\
\hline Discount rate per & $5 \%$ & & $10 \%$ & & $5 \%$ & & $5 \%$
\end{tabular}

period

\begin{tabular}{lllllllll} 
Total & 300.00 & 72.17 & 300.00 & $(85.54)$ & 300.00 & $(53.44)$ & 300.00 & 208.45 \\
\hline
\end{tabular} ( ) denotes a negative benefit $=$ a cost, $\mathrm{CV}=$ current or nominal value, $\mathrm{PV}=$ present value

\subsection{APPROACHES TO DISCOUNTING}

Liscomb et al. (1996:218) assert that three approaches to discounting costs and benefits from the perspective of society have gained prominence in recent years. These are: (1) the social rate of time preference (SRTP), (2) the social opportunity cost (SOC), and (3) the shadow-price-of-capital (SPC).

Arrow considered the discount rate to be determined by the interaction of time preferences and diminishing marginal utility. He described the relationship as follows:

(1) utility time preference $+(2)$ growth-of-consumption decrease in marginal utility $=$ time preference in goods $=$ interest rate (Arrow, 1976: 123) 


\subsubsection{Social Rate of Time Preference (SRTP)}

SRTP is the rate at which society is prepared to trade off present consumption for future consumption. Accordingly, the social discount rate should reflect the opportunity cost to society of alternative benefits forgone. It is assumed that individuals in society would rather have a benefit now, while putting off a cost as far into the future as possible. Time preference is the marginal rate at which individuals within society are prepared to forego present consumption for future consumption. Goodin (1982) argues that human psychology should not necessarily be applied to public policy.

This rate will not necessarily coincide with government borrowing rates, private sector discount rates or the marginal rate of return on capital (unless perfect competition exists in all markets). Methods used to select the SRTP range from the real rate of return on safe investments (government stock) to market interest rates. These estimation procedures are outside the main focus of this study and accordingly will not be discussed.

\subsubsection{Opportunity Cost or Social Opportunity Cost (SOC)}

\section{Displacing Private Sector Investment}

The rationale underlying SOC is that if too low a rate is used, public investment could "crowd out" or displace more "profitable" private investment. The SOC is equivalent to the private sector marginal before ${ }^{12}$ tax return on investment. This rate is a measure of the opportunity cost of resources consumed by the project under investigation. It is the weighted average rate of return that could have been achieved from the projects displaced by the investment. Baumol (1977: 163) states, “... the correct discount rate for the evaluation of a government project is the percentage rate of return that the resources utilized would othenvise provide in the private sector."

Taxes are a transfer payment from a societal perspective. The before tax return is appropriate because this is a measure of the output accruing to society. Part of a firm's earnings goes to society as taxes and part in the form of profits (Arrow, 1976: 127), 
SOC involves calculating the weighted average rate of return on all investments. However, the weighted average rate of return on all investments is unknown and for all practical purposes not possible to estimate with accuracy. It would only be valid to equate government discount rates with the private sector if all the government funding came from the same sources as corporate funding (Arrow, 1976:125). Some government funds are derived from taxation and the opportunity cost of this funding is more correctly related to private consumption and investment opportunities foregone and the deadweight loss of taxation. Many investments available to individual small savers yield lower returns than those from corporate investment opportunities.

\section{Opportunity cost of future consumption}

A variant of the opportunity cost argument is explained by Johannesson (1996: 127) who notes that a reason for discounting is that resources consumed in the present could instead be used to increase future output, that is, the discount rate should relate to the real return on investments. Goodin (1982) contends that the opportunity cost argument only applies to investments and not consumption. However, in my opinion, this argument fails to recognise the link between investment and output in terms of goods and services available for consumption made possible by the investment.

\subsubsection{Shadow-Price-of Capital (SAC)}

Liscomb et al.(1996:218) assert that the opportunity cost method has been transcended by the shadow-price-of-capital technique. This procedure first converts programme costs into consumption losses caused by the investment. Programme benefits are then converted into consumption gains generated by the project. These consumption losses and gains are then discounted to present values using the social rate of time preference. SAC in effect means that the social rate of time preference (SRTA) is preferred as the discount rate because the aim of all investment activity should be increased consumption for society. While this may have a sound theoretical basis, difficulties in undertaking the appropriate estimates make its practical application difficult (or impossible) for most policy makers and analysts. 


\subsubsection{Diminishing Marginal Utility}

This argument relies on the premise that economies continue to expand and consumption continues to increase. The law of decreasing marginal utility means that a unit of consumption will yield more utility in the present than in the future (as total consumption will be higher in the future). As not all economies grow and as the rate of growth may be variable over time the discount rate need not be positive or constant over time (Goodin, 1982: 57).

\subsubsection{Uncertainty and Risk}

Frequently discount rates are increased to provide a margin to cover risk. Goodin (1982: 56) agrees that expected values should be adjusted for risk but points out that this is technically and philosophically different from discounting. Risk does not necessarily increase over time and even if it did why should it increase at a constant rate?

\subsection{LOOKING BACK AT PAST INVESTMENT DECISIONS}

Policy managers should be aware that the discount rate used to appraise an investment in the planning stages need not necessarily be identical with the rate used to value the investment in hindsight. One could argue that a rate used before the investment is undertaken may include a margin to take account of possible over-optimism or risk. However, when evaluating the performance of already implemented investment programmes a more appropriate rate might be the actual growth in real output in the economy over the period as this is the opportunity cost or the return that could have been achieved by the resources displaced by the programme or project (Wells (1985: 11). When looking back and evaluating the performance of past investment decisions processes (as opposed to investments themselves) it is important to consider the information that was available at the time decisions were being made. 


\subsection{DISCOUNTING COSTS AND CONSEQUENCES}

Non-tradable goods such as human lives introduce trade-off problems. This does not necessarily mean that a policy that costs lives cannot be justified (Goodin, 1982: 63). Trading one life for another can be justified within a programme (for example, closing a rural hospital may cost lives but this could be traded off against lives saved through expansion of an emergency flying doctor service). It is possible to justify trading lives for lives, but it is difficult to justify trading lives for more of some unrelated good such as new carpet in government buildings. Although one cannot necessarily say that all lives are equivalent, the use of such measures as quality adjusted life years or healthy year equivalents, are useful ways of comparing outcomes or effects induced by health care interventions or programmes. These topics were discussed more fully in Chapter 3: Valuing Health Outcomes.

There is general (but not universal) agreement that future costs and consequences (outcomes or effects) measured in non-money units should be discounted at the same rate. Viscusi (1996: 144) notes that health effects should be discounted but "... Because there are no markets for explicitly trading health status across time, the choice of the appropriate discount rate for health status has remained a substantial subject of debate." Drummond, Stoddart and Torrance (1987: 81-82), Drummond et al. (1997: 107-109), Gold et al., (1996), Weinstein (1996) and Weinstein and Stason (1977) discuss this concept and generally agree that costs and effects should be discounted using the same rate for both. For example, effects such as QALYs (quality adjusted life years) gained as a result of a health programme should be discounted in the same way as money-quantified costs and benefits.

Weinstein and Stason (1977: 720) state that they have not assumed a year of life in the future is worth less than one in the present, or that it is possible to invest a life year in the present to obtain more life years in the future. They argue that if a cost-effectiveness analysis is conducted life years are being valued relative to dollars. Dollars can be invested to yield more dollars in the future thus a life year in the future should be discounted. 
However, Goodin (1992: 60) argues that unless everything is tradable in terms of something else then the argument for discounting all costs and consequences by the same rate is weakened.

Some studies in cost-effectiveness analysis and cost-utility analysis have used lower discount rates for effects or have not discounted effects at all. Keeler and Cretin (1983: 300 ) discuss the issue of discounting nonmonetary effects in cost-effectiveness analysis and comment "...if the ability to produce the nonmonetary effect does not diminish too quickly over time, failure to discount benefits implies that programmes are always improved by delay." Although this statement lacks precision it nevertheless highlights a potential issue in a number of public policy areas in addition to health. If the stream of future costs is discounted at a higher rate than the flows of future benefits then the net present benefit of the programme will be increased. As an example, the present value of a $\$ 100$ cost in ten years time discounted at 10 percent is $\$ 38.55$, while the present value of a benefit of $\$ 100$ occuring at the same point in time without discounting is still $\$ 100$. This gives a net present benefit of $\$ 61.45(\$ 100-\$ 38.55)$, while if the time period was halved the net present value would be lower $(\$ 100-\$ 62.09=\$ 37.91)$.

Most studies employ a uniform rate over all time periods and categories of goods. Although Goodin (1982: 67) challenged this practice and states that there is no reason to assume that the same discount rate is appropriate over all time periods or to all categories of goods, he advanced no practical alternative.

These issues are equally relevant to other areas of public policy analysis, for example, an engineering project may save lives or work place legislation may increase safety and reduce accident rates.

\subsection{REAL AND NOMINAL RATES}

Discounting has nothing to do with inflation. (Discounting future benefits and costs accounts for the opportunity cost of not incurring those costs or receiving the benefits in 
the present). Costs and benefits may be adjusted for inflation and discounted using a real rate, or they may be evaluated in nominal terms and discounted using a nominal rate. Consistency is vital. If nominal costs and benefits are discounted by real rates or if real costs and benefits are discounted by nominal rates, incorrect results will be obtained (Florio, 1990: 109 and Sugden and Williams, 1978: 36-39). N.Z. Treasury (1996:4) recommend that "If future costs are estimated in nominal dollars then they need to be converted into real dollars before being discounted by a real discount rate." N.Z. Treasury also note that this procedure requires an estimate of expected inflation.

In practice most evaluations are conducted using real or inflation adjusted values with a real discount rate. It is important to note that if a real rate is used to discount, with costs and benefits measured in current prices and unit costs, there is an implicit assumption that the various components of costs and consequences will inflate at the same rate in the future. This is not necessarily so, but unless there is clear evidence to the contrary it is usually a reasonable assumption to make. N.Z. Treasury (1997: 8) indicate that the consumer price index may be used as the expected rate of inflation. The GDP deflator may in some cases be better than the consumer price index as it covers a wider range of goods and services.

Frequently a nominal rate will need to be converted into a real rate to use with future costs and benefits in real dollars.

The following formula may be used to convert a nominal rate to a real rate (NZ Treasury, 1997: 8):

Where:

$$
r_{r}=\frac{1+r_{n}}{1+i}-1
$$

$r_{r}=$ real discount rate

$=\frac{r_{n}-i}{1+i}$

$r_{n}=$ nominal discount rate

$\mathrm{i}$ = expected rate of inflation

An approximation commonly used in finance is to simply subtract the inflation rate from the nominal rate to derive a real discount or interest rate. This is a good approximation at low rates of inflation but will introduce increasing errors as the price inflation rises (see Sugden and Williams, 1978: 37). 


\subsection{DISCOUNT RATES IN USE}

Viscusi $(1996: 144)$ recommends that though in most situations medical decisions will not be sensitive to reasonable variations in the discount rate, sensitivity analysis should be conducted using real discount rates ranging from $1 \%$ to $7 \%$.

Although the cost of funding to government enterprises for investment purposes is not usually equivalent to the cost to society it is never-the-less useful to consider government capital charging costs and rates.

The New Zealand Treasury in a guide on estimating the cost of capital for Crown entities (N.Z. Treasury, 1997, Appendix 1: 29) comment on the wide divergence of practices internationally for discount rates for capital charging and capital budgeting. The UK government uses a risk free rate for public health capital charging to the National Health System. The US Central Budgeting Office also apply a risk free rate. Both the UK and US rates are equivalent to a private sector rate adjusted to a risk free rate. The Australian Government Department of Finance apply a private sector rate with an allowance for risk.

In the past, in New Zealand, a common real discount rate was used for all public sector investment (from the perspective of the entity) and economic evaluations from the perspective of society. Departments are now required to pay a return on capital to the Government, which is called a capital charge. In a N.Z. Treasury guide book (1991: 32) it is stated "Departments should make investment decisions which generate sufficient return for them to pay a return on capital to the Government". Treasury state that "While the capital charge for each department may be used as the discount rate when evaluating investment, different rates may be more appropriate for specific investments. The rate chosen will depend on the riskiness of the investment." The capital charge rate reflects government financial costs of capital not the economic cost to society which is measured by the social rate of discount. The capital charging and social rate of discount may well be different. 
In the mid 1960's the New Zealand public sector real discount rates in common use were around $5 \%$ and rising to $7 \%$ in the late 1960 s. These rates were comparable to then current govermment borrowing rates. In 1971 Treasury instructed departments to use a real discount rate of $10 \%$. The selection of this $10 \%$ rate was probably influenced by US official public sector rates in the late 1960's. (See Copeland and Rose, 1975: 15; Wilkinson, 1982: 1).

The UK and US governments encouraged the use of a public sector real discount rate of $10 \%$ in the mid 1960s (Goodin, 1982: 59). Arrow (1976: 126) considered that the 10\% rate reflected the high rate of technological change in the previous two decades and maintained that as the rate of change in technology had fallen a lower rate of $6-7 \%$ (Arrow, 1976: 139) would be more appropriate.

N.Z. Treasury (1996: 4) note that while a real discount rate of $10 \%$ should be used in N.Z. Treasury economic evaluations, the capital charge rate (then $11.5 \%$ nominal) should be used for financial analysis. Future costs in nominal dollars should be converted into real dollars before being discounted by the real rate.

Liscomb et al. (1996:231-2) make the following comparisons of recommendations for and usage of discount rates by a range of organisations.

- The US Office of Management and Budget guidelines for cost-effectiveness analysis recommend using the real Treasury borrowing rate applicable to the time horizon of the project being evaluated. Recent rates range between $2.1 \%$ for a 3 year term to $2.8 \%$ for 30 years. The Office recommends using a real rate of $7 \%$ (reduced from 10\% in October 1992) for cost-benefit analysis.

- The US Center for Disease Control and Prevention (CDC) suggest using a baseline real rate of $5 \%$ with sensitivity analysis between $0 \%$ and $8 \%$.

- The UK National Health Service (NHS) advise using a real rate of $6 \%$.

- The World Bank in 1993 decided to use a real rate of $3 \%$ for discounting outcome measures in cost-effectiveness studies in health.

- A survey of the international literature reporting economic evaluation studies in health indicates that $5 \%$ is a commonly used real discount rate. 
Liscomb et al., (1996:233) advocate using rates of $3 \%$ and 5\% with sensitivity analysis in the range of $0 \%$ to $7 \%$. They contend that current evidence of real economic growth supports a base real riskless rate of $3 \%$ but international comparisons require $5 \%$ to be used. (See also, 5.4 Looking Back At Past Investment Decisions)

Turning now to the health sector. Drummond et al. (1997: 73) offer the following advice; (a) costs and consequences should be presented undiscounted and in a form that allows others to evaluate the impact of applying different discount rates, (b) use for the base case either the locally accepted discount rate or real rates of $3 \%$ and $5 \%$, (c) perform sensitivity analysis at rates of 0,3 and $5 \%$, and (d) highlight the discount rate used where it has an major influence on the results. Drummond et al. (1997: 107-109) also note that although there is some divergence of opinion as to whether future health effects should be discounted, the weight of the argument is for discounting.

Although Treasury advocate using a discount rate of $10 \%$ for economic evaluations, the most common rate used internationally and in published health economic evaluations is $5 \%$. Accordingly, it is recommended that $5 \%$ be used as the base case and sensitivity analysis be performed using discount rates ranging from $0 \%$ to $10 \%$.

\subsection{CONCLUSIONS AND RECOMMENDATIONS}

Although there is a large body of literature on the social discount rate much of it is contradictory and or of little practical value to the policy analyst and public sector manager when making actual decisions. A review of the relevant literature and current practice indicates that the following practical recommendations would be appropriate for public policy evaluations of health care in the current New Zealand context.

- Discount all expected future costs and benefits (whether measured in money terms or in some other way) to present values using a common rate of discount for all 
categories of goods and for all time periods (discounting is not required when all costs and benefits occur in the same time period.).

- Present all costs and benefits in manner that allows readers to apply discount rates of their own choice.

- Use a real discount rate with real values of costs and benefits to simplify analysis.

Use a base case real discount rate that reflects the locally accepted rate or rates of 3 and $5 \%$ with sensitivity analysis ranging from no discounting ( $0 \%$ to $10 \%$, that is, $(0,3,5$ and $10 \%$ are suggestions see page 135$)$.

- International comparisons would be facilitated by using 0,3 and $5 \%$ as real discount rates.

- Be aware of the consequences of the selection of a discount rate. If, for example, a high rate $(10 \%$ rather than $5 \%)$ is chosen this will reduce the number of potential projects able to be undertaken and will also decrease the viability of projects with long-term rather than short-term benefits.

This chapter has discussed discounting of costs and effects, health outcomes the next chapter covers the range of economic evaluation techniques of relevance to policy analysis. 


\subsection{REFERENCES}

Arrow, K. J. "The rate of discount for long-term public investment." In Energy and the environment: A risk-benefit approach, edited by H. Ashley, R. L. Rudman, and C. Whimple, 113-84. New York: Pergamon, 1976.

Arrow, K. J. "The social discount rate." In Collected papers of Kenneth J Arrow: Production and capital. I st ed., edited by K. J. Arrow . Vol. 5. Cambridge, Mass: Belknap Press of Harvard University Press, 1985.

Baumol, W. J. On the social rate of discount. American Economic Review 58 (1968): 788-802.

Baumol, W. J. "On the discount rate for public projects." In Public expenditure and policy analysis. 2nd ed., edited by R. H. Haveman and J. Margolis, 591. Chicago: Rand McNally College Publishing Company, 1977.

Copeland, M. C., and W. D. Rose. The determination of a public sector discount rate. Wellington: A paper prepared for Treasury by the Institute of Economic Research, 1975.

Drummond, M. F., B. J. O'Brien, G. L. Stoddart, and G. W. Torrance. Methods for the economic evaluation of health care programmes. 2nd ed. New York and Toronto: Oxford University Press, 1997.

Drummond, M. F., G. L. Stoddart, and G. W. Torrance, eds. Methods for the economic evaluation of health care programmes. Oxford: Oxford University Press, 1987.

Florio, M. "Cost benefit analysis and the control of public expenditure: an assessment of British experience in the 1980s." Jommal of Public Policy. 10 (1990): 103-31.

Goodin, R. E. "Discounting discounting." Journal of Public Policy 2 (1982): 53-72. 
Johannesson, M. Theory and methods of economic evaluation of health care. 1st ed. Dordrecht: Kluwer Academic Publishers, 1996.

Keeler, E. B., and Cretin S. Discounting of life-saving and other nonmonetary effects. Management Science 1983;29(3):300-6.

Lally, M. The cost of capital for government entities: an evaluation of the New Zealand Government's capital charge model. Accounting Research Journal 8 (1995): 15-26.

Lipscomb, J., M. C. Weinstein, and G. W. Torrance. "Time preference." In Cost-effectiveness in health and medicine. 1st ed., edited by M. R. Gold, J. E. Siegel, and M. C. Weinstein, 214-46. New York: Oxford University Press, 1996.

Sugden, R., and A. Williams. The principles of practical cost-benefit analysis. 1st ed. Oxford: Oxford University press, 1978.

N.Z. Treasury. Estimating the cost of capital for crown entities and state-owned enterprises a handbook prepared for the N.Z. Treasury. Wellington: N.Z. Treasury, 1997.

N.Z. Treasury. A guide to the management of departmental fixed assets. Wellington: N.Z. Treasury, 1991.

N.Z. Treasury. Guidelines for costing policy proposals. Office minute 1996/B90.

Viscusi, W. K. "Discounting health effects for medical decisions." In Valuing health care: costs, benefits and effectiveness of pharmaceuticals and other medical technologies. 1st paperback ed., edited by F. A. Sloan, 125-47. Cambridge: Cambridge University Press, 1990.

Weinstein, M. C., and W. B. Stason. "Foundations of cost-effectiveness analysis for health and medical practices." New England Journal of Medicine 296 (1977): 716-21. 
Weinstein, M. C., J. E. Siegel, M. R. Gold, M. S. Kamlet, and L. B. Russell. "Recommendations of the pannel on costeffectiveness in health and medicine." Journal of the American Medical Association 276 (1996): 1253-58.

Wells, G. "Choosing the right path towards real rates of return." National Business Review 5 August (1985): 11. 


\section{CHAPTER 6: ECONOMIC EVALUATION}

\subsection{INTRODUCTION}

This chapter discusses the theory underlying economic evaluation and different techniques (cost-minimisation (CMA), cost-effectiveness (CEA), cost-utility (CUA) and cost-benefit analysis (CBA)) used in health policy analysis.

Table 6.1 presents a classification system for health policy evaluations and illustrates how economic evaluation fits into this scheme. Economic evaluations consider both costs and health related outcomes and/or compare two or more health care interventions, programmes or policy altematives. The objective of economic evaluation is to assist with policy decisions relating to resource allocation. Partial evaluations consider either costs or health related outcomes in isolation or do not compare options (see Drummond et al., 1987: 8; Drummond et al., 1997: 10).

\section{Table 6.1: Types of Health Care Intervention/ Policy Evaluations}

\begin{tabular}{|c|c|c|}
\hline Type of Analysis & Objective/ use & \\
\hline \multicolumn{2}{|c|}{ Purtial eraluations (Consider costs alone or, health outcomes alone and/or no comparator) } & \\
\hline Cost-of-illness & Identify and quantify costs of an illness. & $\frac{\overrightarrow{\tilde{\omega}}}{\frac{\pi}{0}}$ \\
\hline Outcome analysis & Identify and quantify health related outcomes. & ఏิ \\
\hline Cost-of-treatment & Identify and quantify costs of treating an illness. & $\frac{\delta}{\tilde{z}}$ \\
\hline \multicolumn{2}{|c|}{ Economic eraluations (Compare costs and outcomes of two or more alternatives) } & $\stackrel{4}{4}$ \\
\hline \multirow[t]{2}{*}{ Cost-minimisation } & Allocation of resources between treatments for the same condition. & \\
\hline & Outcomes are identical in all respects. & \\
\hline \multirow[t]{2}{*}{ Cost-eflectiveness } & Allocation of resources between treatments for the same condition. & \\
\hline & Outcomes are in same dimension but achieved to differing degrees. & \\
\hline \multirow[t]{2}{*}{ Cost-utulity } & Allocation of resources across different treatments for over a range of & $\overline{\check{~}}$ \\
\hline & conditions within the health sector. & $\sum_{\downarrow}^{\infty}$ \\
\hline Cost-benefit & Allocation of resources between different uses across a whole economy. & \\
\hline
\end{tabular}


Economic evaluation is now considered in more detail. Health related outcome analysis was discussed in Chapter 3: Valuing Health Outcomes and costs of relevance to an illness or its treatment were covered in Chapter 4: Costs.

\subsection{ECONOMIC EVALUATION AND HEALTH}

The purpose of economic evaluation of health care is to assess the economic efficiency consequences of health care programmes or interventions. Economic evaluation may be simplified by regarding all changes in resource usage as costs or the causes, and all changes in health related outcomes as benefits or effects of the intervention (see Donaldson, 1990). If this approach is adopted, a health care intervention or programme may be likened to a production process where outputs and health related outcomes are generated from inputs. The intervention consumes inputs of resources to produce outputs of health care goods and services intended to alter health outcomes. This is shown in Figure 6.1.

Figure 6.1: Health Intervention Or Programme As a Production Process

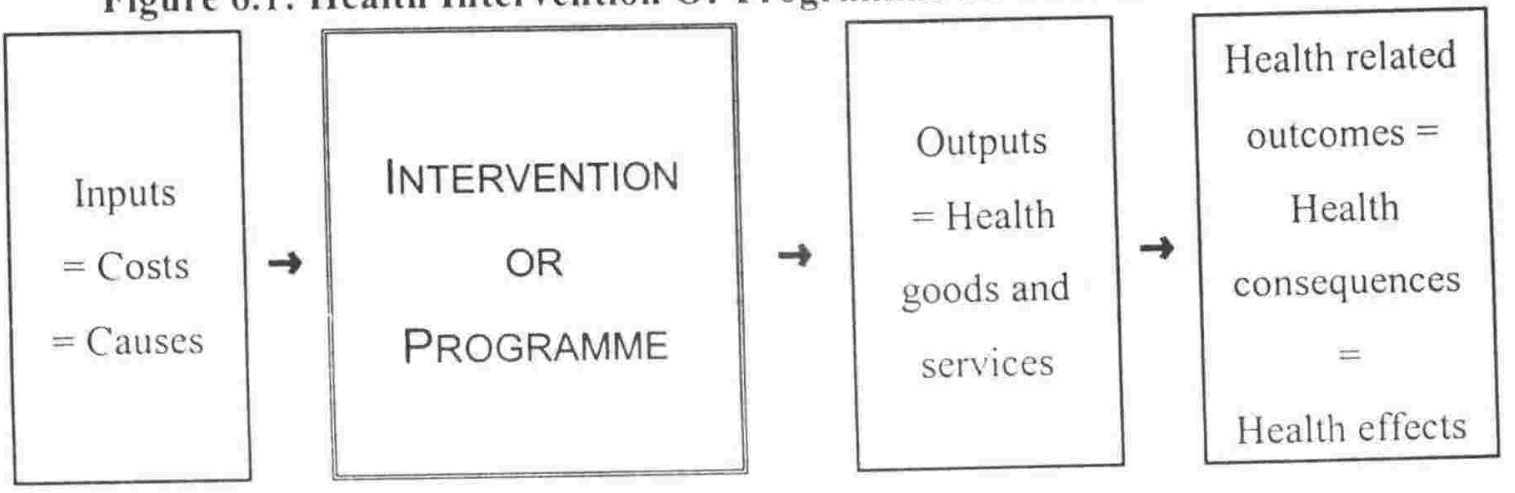

Direction of causation $\rightarrow$

Inputs or opportunity costs altered by the intervention represent the changes in resource utilisation caused by the health care intervention or policy. That is, the intervention may consume or create resources. The opportunity cost of resources used is the value of those resources in their next best alternative use or the value of the goods and services they could 
have produced. (Costs are discussed in chapter 4). As resource opportunity costs may be either incurred or avoided, they should be more correctly termed net ${ }^{13}$ costs.

Outputs are the goods and services produced which are intended to, but may not, cause changes in health related outcomes. Health related outcomes represent the consequences or effects of the intervention on health status. Although the changes in health status are intended to be beneficial or positive, interventions may also have adverse or negative effects.

As an intervention results in changes in resource usage and is intended to alter health related outcomes economic analysis may be further simplified as shown in Figure 6.2.

Figure 6.2: Interventions Change Costs and Outcomes

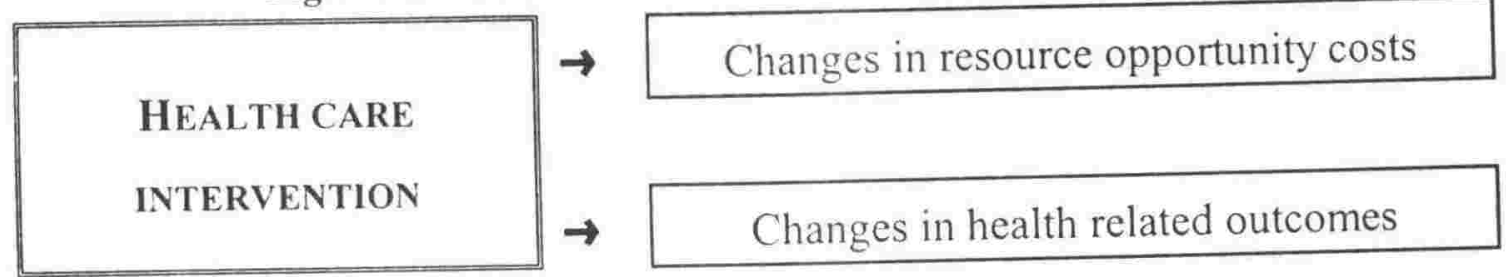

Economic analysis of treatment options involves comparing these changes in resource opportunity costs with the changes in health related outcomes produced by the treatment or intervention.

Incremental analysis is used to isolate and measure opportunity costs and health outcomes that are changed by the intervention. A cost or a health outcome unchanged by the intervention, policy, or illness, is irrelevant. Costs already incurred (sunk costs) are not applicable as they cannot be altered by any decision made in the future. See Chapter 4: Costs. 
Differences $^{14}$ between costs and health related outcomes are compared between comparators; illness versus no illness, one treatment option versus another, treatment compared with no treatment. Adverse effects of treatment must be set off against positive health outcomes.

\subsection{TYPES OF ECONOMIC EVALUATION}

Different techniques of economic evaluation will be appropriate for different situations.

The methodology chosen will be determined by;

- the research question,

- the analytic perspective,

- relevant costs and outcomes,

- availability of data,

- the methods used to measure costs and health related outcomes,

- the research budget, and

- the time available for the study and when the results are required.

The research question or problem to be addressed dictates the perspective/s that should be adopted. For example, does the problem relate to resource allocation decisions within the health sector or across the whole of society?

Perspectives may include one or more of the following; society, health care provider, health care purchaser, private individual. The analytic perspective determines the range of resource costs (see Chapter 4: Costs) and health related outcomes that should be considered. If viewed as a continuum, CBA is at one end (widest perspective, most comprehensive) and cost-minimisation is at the other end (narrowest perspective, least comprehensive).

14 Change in costs = costs incurred less costs avoided; change in benefits/ outcomes = benefits/outcomes gained less outcomes/benefits foregone. 
Data availability will determine which costs and health outcomes can be measured and their method of quantification. Study design may also be influenced by the size of the research budget. For example, very few cost-utility studics (discussed later in this chapter) have been undertaken in New Zealand. They are more complex and costly to conduct than costeffectiveness studies (discussed later in this chapter) and cost-effectiveness studies may yield sufficient information for decision makers' purposes.

As the aim of a health care intervention is to alter health-related outcomes, efficiency is more usefully evaluated by comparing net resource cost against net health outcomes rather than against outputs of health care goods and services (Weinstein and Stason, 1977). One treatment option is more efficient than another if more of the beneficial health-related outcomes of interest can be produced with the same quantity of inputs or if the same quantity of health outcomes can be achieved using less inputs.

The ways in which health related outcomes(discussed fully in Chapter 3: Valuing Health Outcomes.) are measured or taken into account provides a means of classifying economic evaluations. Table 6.2 shows that for all techniques costs are measured in money values. Cost-minimisation assumes health related outcomes of comparators are identical. Costeffectiveness analysis considers alternatives that produce the same health effect but to varying degrees. For example, two alternative treatments may both reduce blood pressure, but they may differ in effectiveness. With cost-utility analysis, the outcomes are measured in utility values. Cost-benefit analysis measures both costs and health related outcomes in money units. 
Table 6.2: Economic Evaluations May Be Classified According to the Method of Measuring Health Outcomes (Effects)

\begin{tabular}{llll}
\hline Technique & $\begin{array}{l}\text { Cost } \\
\text { measurement }\end{array}$ & $\begin{array}{l}\text { Health related } \\
\text { outcome } \\
\text { measurement }\end{array}$ & Perspective \\
\hline Cost-minimisation & Money values & $\begin{array}{l}\text { Assumed to be } \\
\text { identical }\end{array}$ & Decision maker \\
Cost-effectiveness & Money values & Clinical/ physical & Decision maker \\
Cost-utility & Money values & Utility measures & Societal \\
Cost-benefit & Money values & Money values & Societal \\
\hline
\end{tabular}

\subsubsection{Cost-Benefit Analy'sis (CBA)}

The discussion commences with CBA because (in my opinion), all other types of evaluation may be regarded as subsets of CBA. Many of the ideas and concepts embodied in CBA underpin all other economic evaluation types considered in this chapter.

As cost-benefit analysis (CBA) takes a full societal perspective the widest possible implications of the use of resources is considered. All relevant societal costs and benefits are taken into account and measured in money values. A benefit may be gained or lost (a negative benefit is a cost) and a cost may be incurred or avoided (a negative cost is a benefit). CBA is also referred to as social CBA or as BCA.

The goals of public policies can be grouped into wo broad categories; allocative efficiency, and distributional equity. As Cost-benefit analysis focuses on allocative efficiency it could be regarded as a model for public sector decisions that would be made if private markets functioned satisfactorily (Haveman and Weisbrod, 1977: 136-7). Dasgupta et al. (1972) considered the main reason for undertaking CBA was to ensure that 
a consistent set of rules were used to evaluate public projects. Further they stated that CBA is not a technique but an approach to evaluating social choices.

Because CBA is concerned with the potential improvement in the efficient allocation of society's resources it can compare a range of diverse outcomes. For example, costs and benefits of medical treatments can be compared with costs and benefits of protecting the environment or extending an airport runway (provided all costs and benefits can be isolated and valued and that equity changes are also considered).

Succinct summaries of the historical evolution of cost-benefit analysis are provided in Pearce (1971) and Pearce and Nash (1981). The underpinning theory of cost-benefit analysis can be linked to nineteenth century welfare economic theory. French economist Dupuit (1844) is regarded as the first to describe the maximisation of a project's net benefits (cost-benefits) in terms of the maximisation of societal welfare or utility.

The first practical application of cost-benefit analysis was concerned with US water resources in the 1930's. Pearce (1971) notes that the US Flood Control Act of 1936 embodied "the principle of comparing benefits to whomsoever they may accrue with the estimated costs". However according to Pearce (1971) the precise meaning of benefit was unclear and different US government agencies applied different interpretations of the costbenefit criteria. Attempts to standardise the methodology were made by the Federal InterAgency River Basin Committee in their Green Book of 1950 and in a Bureau of Budget's Budget Circular A-47 in 1952 (Pearce, 1971).

Cost-benefit analysis in the UK began to be used in the 1950's to evaluate transport investment but it was not until the 1970's that the methodology was extended to water resources. A 1967 UK government white paper reviewing the economic and financial objectives of nationalised industries was the first acknowledgement that cost-benefit analysis had relevance to the evaluation of these industries. The first comprehensive text on cost-benefit analysis was published in 1971 by the UK economist Mishan (1988, 1st edition 1971) and in 1972 the United Nations published a set of guidelines for project evaluation (Dasgupta et al., 1972). 
Cost-benefit analysis is often criticised as lacking objectivity because value judgments cannot be avoided. "Cost-benefit analysis can never replace the need for sheer political decision."(Self, 1970: 9). Self also comments that the logic of CBA requires social or political judgements at every stage of CBA. He expresses an extreme view that cost-benefit analysis is "nonsense on stilts" and "... its use is blocking more prosaic but more rational planning methods." He states many values and estimates are notional, cannot be adequately validated by actual exchanges and are arbitrary in that a "...very wide range of values can be plausibly predicted, depending upon innumerable opinions and assumptions". In addition, Self was concerned about the possibility that important items may have been excluded.

Drummond (1993: 108) in discussing CBA in health and health care comments that analysts should consider other social objectives besides efficiency. The analysis becomes more of an interchange between the analyst and the social decision maker, and a forum for making the decision maker's values explicit." Drummond calls this the decision-making approach to CBA. Pearce and Nash (1981:9) comment that “... a CBA will be sensitive to the value judgements underlying it." All value judgements and assumptions should be transparent and the implications of changing them should be provided.

Shadow prices are used when market prices do not reflect the social value of the inputs and outpus of a project, or when no market price exists. Consumer sovereignty dictates that shadow prices should be based on willingness to pay criteria. Where an output or an input cannot be quantified the items should be listed and described. Many health-related outcomes do not have market prices and it is often difficult to estimate values on a willingness to pay basis.

If willingness to pay criteria are used to evaluate benefits then it is likely that decisions will favour high income and wealthy individuals at the expense of low income and poor individuals. The marginal utility of money is higher for low income individuals. Thus, low income individuals would not be willing to pay as much for a particular benefit as a high 
income individual. Hicks (1939: 700) discusses the difference in marginal utilities of money between rich and poor.

Another kind of equity change can result from individuals having divergent tastes and preferences for the same change in health effect and thus valuing the same health effect differently. A similar situation arises where different people place different values on different dimensions of health-related quality of life. This would result in different rankings and trade-offs for health care interventions when different aspects of quality of life are affected. Pauly (1996: 107-9) discusses consequences arising from individuals having different incomes, tastes and preferences.

For the practical application of cost-benefit analysis the basic choice criterion is the potential improvement in allocative efficiency (the potential Pareto improvement, KaldorHicks or compensation criterion). If social benefits exceed social costs, the gainers from the programme could (but need not do so) compensate the losers, and there would still be net gains to society. This rule can be applied by calculating the present value of the net benefits using an appropriate discount rate and choosing those programmes which have the highest net present value. Equity and ethical consequences should also be considered.

In practice, decision-makers face different choices. Where there is a set of independent options, the decision-maker must decide which, if any, ought to be undertaken. If there are no constraints on the number of programmes which may be undertaken all programmes that have net present benefits greater than zero should be implemented. However, if a specific input is scarce, the feasible programmes should be implemented in the combination which exhausts the input which is scarce and yields the highest total NPV. The process of economic evaluation is discussed in Chapter 18: Discussions and Conclusions.

If some options are mutually exclusive (that is, undertaking one prevents implementation of one or more of the others), the decision-maker must choose between them. Where there are resource constraints or where programmes are mutually exclusive, some analysts advocate applying the benefit-cost ratio. The project which has the greatest ratio of discounted benefits to discounted costs is chosen. However, this can lead to wrong choices 
being made as the numeric value of the ratio depends on how costs and benefits are classified in each project. Is a cost treated as a benefit forgone or a benefit treated as a cost avoided? If the former, the benefit-cost ratio is reduced; if the latter, it is increased.

Interpretation of the cost-benefit ratio where there are cost offsets requires care. Consider a programme with a present value of $\mathrm{B}-\mathrm{C}+\mathrm{K}$ and a $\mathrm{C} / \mathrm{B}$ ratio of either $\mathrm{C} /(\mathrm{B}+\mathrm{K})$ or $(\mathrm{C}-\mathrm{K}) / \mathrm{B}$, where $\mathrm{C}=$ costs, $\mathrm{B}=$ benefits and $\mathrm{K}=$ a cost offset or a negative cost. Shifting $\mathrm{K}$ between denominator and numerator will not reverse the sign of the ratio but the relative rankings will alter. Evans (1984: 244 and Culyer (1989: 218) discuss this problem. Table 6.3 illustrates how the cost/benefit ratio may be altered by changing the classification of costs and benefits.

Table 6.3: Costs and Benefits and the Cost/ Benefit Ratio of A Health

\begin{tabular}{|c|c|c|c|}
\hline Method A & SMillion & Method B & SMillion \\
\hline Additional costs incurred & 100 & Additional costs incurred & 100 \\
\hline Less costs avoided & 55 & Plus benefits foregone & 60 \\
\hline$=$ Incremental costs & 45 & $=$ Programme costs & 160 \\
\hline Additional benefits & 200 & Additional benefits & 200 \\
\hline Less benefits foregone & 60 & Plus costs avoided & 55 \\
\hline$=$ Incremental benefits & 140 & $=$ Progranme benefits & 255 \\
\hline Net benefits $=$ & 95 & Net benefits $=$ & 95 \\
\hline incremental benefits & & Progranme benefits & \\
\hline Less incremental costs & & Less programme costs & \\
\hline Cost benefit ratio & $0.321+3$ & Cost/benefit ratio & 0.62745 \\
\hline
\end{tabular}

The net benelits will be identical under both methods of calculation but a cost/benefit ratio calculated using method $\mathrm{A}$ differs from that calculated using method $\mathrm{B}$.

Where projects are mutually exclusive and have the same life (if they did not have the same life, proceeds from the project with the shorter life could be reinvested in another project that has different characteristics from the original project) but are of different scale in terms 
of cost (if the net present value of the costs is identical there is no conflict between the two criteria), the cost-benefit ratio selects project $\mathrm{A}$, whereas the net present value criterion selects project $B$. Use of the internal rate of return (IIR) to compare mutually exclusive projects results in the same dilemma.

The project with the smallest cost-benefit ratio may not have the greatest NPV. Table 6.4 illustrates this problem. Project $\mathrm{A}$ has the lowest $\mathrm{C} / \mathrm{B}$ ratio and also the lowest NPV. The C/B ratio criteria would choose project A but the NPV criteria would select project B.

Table 6.4: The Selection Criteria NPV and C/B Ratio May Result in Different Choices Being Made

\begin{tabular}{ccccc}
\hline $\begin{array}{c}\text { Project } \\
\text { costs }\end{array}$ & $\begin{array}{c}\text { Present value of } \\
\text { benefits }\end{array}$ & $\begin{array}{c}\text { Present value of } \\
\text { Cost-benefit ratio }\end{array}$ & $\begin{array}{c}\text { Net present } \\
\text { value }\end{array}$ \\
\hline A & 300 & 675 & 0.44 & 375 \\
B & 600 & 1200 & 0.50 & 600 \\
C & 900 & 1350 & 0.67 & 450 \\
\hline
\end{tabular}

The choice criteria for CBA may be summarised as follows (Pearce and Nash, 1981: 46-7):

- Accept or reject for a single project: Accept the project if the net present value (NPV) is greater than zero, and reject if less than or equal to zero.

- Ranking: If an entity has unlimited funds all projects with a NPV greater than zero should be implemented. If working within a finite investment budget, projects should be ranked in order of desirability and selected in order of this list until the budget is exhausted. It is important to rank projects in order of desirability (another value judgement) not on the NPVs,

- Mutually exclusive projects: Select the project with the highest NPV.

Although CBA is not perfect, it is not so bad compared with the alterative (Williams, 1977: 544). Williams notes that CBA is imperfect, prone to misunderstanding and misapplication by the uninitiated and unscrupulous. CBA should not be abandoned on the grounds that it is imperfect, costly, difficult to apply and could be misused or misunderstood. CBA provides a logical framework for public policy analysis, and if assumptions and estimation procedures are transparent and clearly stated will provide 
valuable information about potential economic efficiency. At best, CBA assists in decision making, it cannot make the decisions. Equity and ethical issues must also enter the public policy decision process.

In summary, the following should be discussed by analysts when presenting results of CBA studies; the alternatives or comparators evaluated, the costs and benefits included or and excluded and the reasons for inclusion or exclusion, the valuation methods, the discount rate, and distributional changes.

Difficulty in assigning money values to all health-related outcomes led to the development of cost-effectiveness analysis (which avoids this problem), and contingent valuation techniques to measure willingness to pay for improved health (Johannesson, 1996: 2). In the health sector cost-effectiveness analysis (various types) has dominated CBA.

\subsubsection{Economic Evaluations Other Than CBA}

These economic evaluation techniques compare changes in costs in money values with changes in health related outcomes. Health related effects or outcomes are assumed to be identical, quantified in clinical or physical units, or measured in utility or quality of life terms. These techniques focus on potential improvements in efficiency of resource use within the health care sector or within a fixed budget. They cannot be used to compare resource allocations between health and other non health uses. For example, the use of resources for hip replacement surgery cannot be compared with the use of resources for motorway construction.

Unlike CBA, where the perspective should always be that of society, the perspective taken in other economic evaluations may range from that of society to narrower perspectives such as that of the purchaser or provider of health care services. Frequently, the objective is given and the decision maker wishes to determine the most cost effective way of achieving that goal. Sometimes economic evaluations other than CBA are referred to as decision maker approaches. A major danger with these forms of evaluation is that the 
health related outcomes of different programmes being considered may not be directly comparable.

Mishan (1988: 110) comments that, "... the analysis of cost-effectiveness can be described as a truncated form of cost-benefit analysis:...", "... while it is true that a knowledge of cost-benefit techniques more than suffices for any cost-effectiveness calculation, it is important to make explicit the difference in political constraints involved in the latter and, therefore, their prescriptive significance." Further, Misham contends that although CEA is useful, $\mathrm{CBA}$ is theoretically superior. Because CEA focuses on one objective, the technique may provide misleading information for public policy decision making.

Cost-minimisation analysis (CMA), cost-effectiveness analysis (CEA), and cost-utility analysis (CUA) can consider only resource allocation questions for a particular disease or at most across different diseases encountered within the health sector because the full range of societal costs are not assessed.

The terminology relating to CEA and CUA in the US is different from that used in UK/ Canada. The current US approach categorises CUA as a sub-set of CEA and avoids using the term CUA (Gold et al,, 1996) but in the UK and Canada a distinction is drawn between CEA and CUA (Drummond et al., 1997). I have chosen to follow the UK and Canadian approach as this is more clearly understood by New Zealanders.

\section{Cost-Minimisation Analysis (CMA)}

Cost-minimisation analysis (CMA) is the narrowest type of economic evaluation where costs alone are used to compare health care treatment or policy options. CMA is also called cost-identification which is not an ideal term as it may be confused with the cost identification or cost enumeration steps in the economic evaluation process (Chapter 18: Discussion And Conchusions).

Because costs are not compared with outcomes, valid comparisons are possible only if the treatments result in identical (or almost identical) outcomes. Problems in interpreting the 
results of CMA investigation occur if the outcomes are not identical. The results of CMA are presented as cost per unit of health care service such as, the cost per patient treated, or the cost per patient treatment day.

For example, the case, critical limb ischaemia, considered costs per amputation with costs per patient treated with the drug Iloprost (Chapter 14: Critical Leg Ischaemia). In this case the patient outcome measure was lives saved by the treatment which would classify the analysis as CMA. However consideration of a different set of health related outcomes such as quality adjusted years would reclassify the study as cost-utility analysis.

\section{Cost-Effectiveness Analysis (CEA)}

CMA and CEA are the most commonly used type of study for health and policy evaluation. In his early text on CBA Mishan (1988: 6) says CEA may be used to evaluate the costs of alternative politically desirable programmes and provides an example of costs averted by eliminating a disease (expenditures on medical care, production loss and pain and discomfort).

In health care, CEA allows comparisons to be made between different ways of treating or preventing a particular disease, for example, heart failure. CEA cannot provide robust information on resource allocation between different diseases unless there ... "is a common effect of interest (eg lives saved)" (Drummond, O'Brien, et al. 1997: 97). For example, it would not help in determining whether resources should be directed away from treating heart failure to that of hip replacement surgery.

Costs are measured in money values and health outcomes are quantified in clinical measures or physical units (for example, cost per clinical cure or deaths avoided). CEA is appropriate when outcomes are in the same dimension (for example, lives saved) but are achieved to differing extents. Comparisons are made between identical treatment objectives but the outcomes may be achieved to varying degrees. Examples of outcome measures are; lives saved, life years gained, adverse events avoided, patients successfully 
treated, symptom free days or pain-free days. The treatments may be more or less effective in achieving these outcomes. The outcome could also be an intermediate clinical measure such as, percentage cholesterol or blood pressure reduction, or cost per patient treated. Unless the economic evaluation is being conducted alongside a clinical trial, the clinical outcomes are determined from the literature or from surveys of expert opinion.

The cost-effectiveness ratio is the change in cost resulting from the intervention divided by the change in the health-related outcome of interest. The outcome of interest is the effectiveness measure.

\section{Changes in resource opportunity costs}

Cost-effectiveness ratio (C/E ratio)
$\$(\Delta C)$

Changes in health related outcome (effectiveness measure)

$(\Delta \mathrm{E})$

As an example of CEA, the net cost of medical treatment for hypertension might be compared with millimetres of mercury reduction in blood pressure achieved. Hypertension may be treated by two different medicines with the objective of lowering blood pressure but one medicine may reduce blood pressure to a greater extent. In another example the results of a comparison between two antibiotics with differing clinical efficacy could be reported as a cost per clinical success.

Cases relating to lower respiratory tract infection (Chapter 12), influenza (Chapter 13) and critical limb ischaemia (Chapter 14), do not satisfy all the criteria for CEA. However they illustrate a range of possible outcome measures and thus extend beyond CMA and also illustrate the difficulty of measuring health related outcomes. 
Both CMA and CEA frequently consider:

- Direct medical costs from the perspective of the health sector

- Direct medical costs plus direct nonmedical from the perspective of the health sector

- Direct medical costs plus direct nonmedical from the perspective of the health sector plus indirect costs from the perspective of society.

The decision criteria and rules for CEA

\section{For all programmes:}

- Programme costs and effects should be measured incrementally compared with "do nothing" or placebo. Costs and effects are relevant only if they would be changed by the programme.

For independent programmes:

- Rank according to the average $\mathrm{C} / \mathrm{E}$ ratios and implement them in order of $\mathrm{C} / \mathrm{E}$ ratio until the budget is exhausted.

- If there is a maximum acceptable or cutoff price per effectiveness unit then all programmes with a $\mathrm{C} / \mathrm{E}$ ratio below or equal to the cutoff price should be implemented.

For mutually exclusive programmes:

- Rank in order of effectiveness.

- Calculate incremental C/E ratios.

- Exclude dominated programmes and recalculate incremental $\mathrm{C} / \mathrm{E}$ ratios.

- Continue this process.

- If there are only two options, incremental $\mathrm{C} / \mathrm{E}$ ratios do not need to be calculated as the average $\mathrm{C} / \mathrm{E}$ ratios can be used to select the most cost effective option. 
The incremental $\mathrm{C} / \mathrm{E}$ ratio is the difference in cost between two alternatives divided by the difference in effects. For example, (Costs of programme A - costs of programme B) divided by (Effects of programme A - effects of programme B). A programme is said to be dominated if the incremental $\mathrm{C} / \mathrm{E}$ falls for the next (ranked in order of effectiveness) programme with higher effectiveness. Provided there are constant returns to scale more units of effectiveness are always gained by excluding the dominated programme.

There is often a distinction drawn between average and incremental cost-effectiveness ratios. Confusion may arise because the costs are always measured incrementally from either no treatment or a comparator treatment. The following definitions will clarify this point:

- The average cost of a programme is the cost of the programme compared with the cost of the placebo or of doing nothing.

- The average effect of a programme is the effect of the programme compared with the effect of the placebo or of doing nothing.

- The cost-effectiveness ratio is the net change in cost [C] resulting from the intervention divided by the net change in the health-related outcome (health related effect) $[\mathrm{E}]$ of interest, $\mathrm{CE}$ ratio $=\mathrm{C} / \mathrm{E}$.

- The average cost-effectiveness ratio is the $\mathrm{C} / \mathrm{E}$ of the programme compared with no intervention or the "do nothing" or placebo option. The average ratio can be used to rank independent programmes.

- The incremental cost-effective ratio is the (net costs of programme A less net costs of programme B) divided by (net effects of programme A less net effects of progranme B)

Table 6.5 summarises the results of a cost-effectiveness analysis of two antibiotics. Direct and indirect costs are considered from the perspective of society. Costs that were identical for both treatments were not evaluated. Costs that are identical between options disappear when differences between options are taken. Examples of such identical costs are medical consultations and diagnostic tests that would be undertaken for both antibiotics. Because the outcomes (clinical success rates) for each medicine are different, this is a CEA study, but had the clinical success rates proved to be identical the analysis would have been 
classified as CMA. However, there would have been absolutely no difference in the way in which the economic evaluation was conducted.

Table 6.5: Direct Medical Costs of Antibiotic A Compared with Antibiotic B: An Example of Costs and Outcomes in A Cost-effectiveness Analysis.

\begin{tabular}{|c|c|c|c|c|c|c|}
\hline \multirow[t]{2}{*}{ Cost } & \multicolumn{2}{|c|}{ Medicine A } & \multicolumn{2}{|c|}{ Medicine B } & \multicolumn{2}{|c|}{$\begin{array}{c}\text { Incremental } \\
\text { costs of } \\
\text { medicine A less } \\
\text { medicine B }\end{array}$} \\
\hline & Cost $\$$ & $\mathbf{N}$ & Cost $\$$ & $\mathbf{N}$ & Cost $\$$ & $\mathbf{N}$ \\
\hline Antibiotic acquisition cost & 3,300 & 100 & 2,500 & 100 & 800 & 0 \\
\hline $\begin{array}{l}\text { Medicines to treat adverse } \\
\text { events of the antibiotic }\end{array}$ & 500 & 20 & 700 & 30 & -200 & -10 \\
\hline $\begin{array}{l}\text { Diagnostic tests for adverse } \\
\text { events or treatment failure }\end{array}$ & 50 & 2 & 75 & 3 & -25 & -1 \\
\hline Clinical successes & & 98 & & 87 & & 11 \\
\hline Total Direct medical cost & 3,850 & & 3,275 & & 575 & \\
\hline $\begin{array}{l}\text { Total direct medical cost per } \\
\text { clinical success }\end{array}$ & 39.29 & & 37.64 & & 52.27 & \\
\hline $\begin{array}{l}\text { Indirect costs (production } \\
\text { loss) }\end{array}$ & 200 & $\begin{array}{c}1 \\
\text { week }\end{array}$ & 500 & $\begin{array}{c}2.5 \\
\text { weeks }\end{array}$ & -300 & $\begin{array}{c}-1.5 \\
\text { weeks }\end{array}$ \\
\hline Total cost & 4,050 & & 3,775 & & 275 & \\
\hline $\begin{array}{l}\text { Total cost per clinical } \\
\text { success }\end{array}$ & 41.33 & & 43.39 & & 25.00 & \\
\hline
\end{tabular}

Note: A negative cost is a benefit, $\mathrm{N}=$ number of patients

Medicine A costs more per clinical success than medicine B in terms of direct medical costs, but if indirect costs are considered, medicine $A$ is the most costeffective alternative.

Table 6.5 shows that on the basis of treating 100 patients, the incremental cost of antibiotic A was $\$ \$ 00$ higher than antibiotic B. Medicine A generated fewer adverse events and avoided $\$ 225$ in related costs compared with medicine B. Total direct medical cost was $\$ 575$ higher for medicine A. Indirect cost of time off work was $\$ 300$ lower for medicine A. Total cost (direct $\$ 575$ plus indirect \$-300) was $\$ 275$ more per 100 patients treated for 
medicine A. The clinical success rate was 98 patients per 100 treated for medicine A and 87 per 100 for medicine $B$, a difference of 11 .

Average cost-effectiveness ratios (\$ per clinical success) were calculated by dividing total direct and total costs by the number of successfully treated patients. Direct cost per clinical success were $\$ 39.29(3,850 / 98)$ for medicine $A$ and $\$ 37.64(3,275 / 87)$ for medicine $B$ while total cost-effectiveness ratios were $\$ 41.33$ (4,050/98) for medicine A and $\$ 43.39(3,775 / 87)$ for medicine B. Incremental cost-effectiveness ratios were calculated by dividing the incremental costs by the incremental effects. The direct incremental cost-effectiveness ratio was $\$ 52.27(575 / 11)$ while the ratio using total incremental costs was $\$ 25(275 / 11)$.

Luce et al. (1996: 209) describe a health care intervention as altering costs and health effects, but exclude productivity changes conferred on the recipients of health care. Their rationale for this opinion is that if the measurement of changes in health effects is comprehensive enough, it will capture these changes in productive output. Luce et al. (1996) are forced into this stance because they do not draw a distinction between costeffectiveness analysis and cost-utility analysis (defined and discussed later in this chapter). Double counting of productivity changes and utility changes as a result of a change in health related status would occur in a CUA study.

Drummond et al. (1997) suggest that a way out of this impass is to report productivity changes separately. Users of the study may then make their own decisions as to whether or not to include them. This would seem to be a workable solution to the different opinions held. Drummond et al. (1997) also draw attention to the possibility of double counting productivity changes in cost-utility studies. Double counting traps are discussed in Chapter 4: Costs.

\section{Cost-Utility Analysis (CUA)}

Cost-utility analysis (CUA) is often regarded as a subset of CEA (for example, Gold.et al., 1996) or as a halfway house between CEA and CBA. Unlike CBA, CUA avoids the necessity of placing a money value on human life and the quality of life. Outcomes are 
measured as utility (satisfaction, pleasure, well-being). The costs of alternative medical procedures may be compared with increased quality adjusted life years (QALYs) resulting from treatment given. Whereas CEA can measure only one dimension of outputs (for example, lives saved), CUA can combine outcome categories (for example, lives saved and symptom free days) by assigning utility values to each output category.

CUAs are usually conducted from a societal perspective. Costs evaluated may range from direct medical alone, through to direct medical plus direct nonmedical plus indirect costs. It is common to use direct treatment costs less indirect benefits of gained production to derive a net resource cost per QALY or healthy year equivalents ( HYE) gained as a result of treatment. Accordingly, the treatment of different medical conditions may be compared. Quality of life measures have been discussed more fully in Chapter 4: Costs.

A major obstacle faced by a researcher conducting this type of analysis is that of obtaining suitable utility values for various health states being evaluated. Utility values for health states may be derived from the health economics and medical literature, calculated from surveys of patients with the disease state, or by asking healthy respondents to imagine they had a particular health status and describe their utility for that state. Gold et al. (1996) consider that indirect costs should be excluded as the quality of life measure should capture the impact on lost productivity. Chapter 3: Valuing Health Outcomes, discusses disease specific and general or generic quality of life specific instruments in more detail.

Ideally the influenza and critical limb ischaemia cases (Chapters 13 and 14) should have been evaluated in terms of cost per utility measure because quality of life was different. This was not possible because of lack of readily available data and budget constraints that did not allow the researchers to apply quality of life instruments.

\section{Cost-consequence Analysis}

Cost-consequence analysis is (in my opinion) a variant of CEA and CUA where sensitivity analysis is conducted and a number of different perspectives and associated costs and 
outcomes are considered. It is an extension of the idea covered when the desirability of adopting a range of perspectives was discussed. In this type of analysis costs and consequences are not aggregated and ratios of cost-effectiveness are not calculated. Costs and outcomes are listed and it is left to the user of such studies to calculate the aggregates and ratios that interest them. For example, instead of calculating a cost per QALY, a vector of all clinical outcomes and relevant costs may be given. (Torrance et al., 1996:59; Detsky, 1994: 284)

\subsection{THE PROBLEM OF CLASSIFYING ECONOMIC EVALUATIONS}

In real life, it may be difficult to classify an economic evaluation technique into a particular type as the categories grade into each other. It is often not possible or sensible to classify an investigation until it is completed because the analyst will not necessarily know exactly which costs and outcomes are relevant and how they can be quantified. If the economic evaluation is being conducted alongside a clinical trial, the results of the clinical trial will determine whether or not the outcomes of two treatments are different.

The appropriate type of analysis will be determined by the problem under investigation, the availability of data and the research budget. The decision maker should be more concerned with obtaining the most appropriate information and analysis for their purposes than the classification of the study. Classifying methodologies into different types is however convenient to discuss the concepts of economic evaluation.

If the cost of two antibiotic treatment regimens is being compared, a suitable way of reporting the results of such a study could be cost per patient successfully treated. Costminimisation is appropriate if the patient outcomes are identical. However, if one regimen has a greater rate of adverse events then the patient outcomes are not identical. These adverse events could range from the minor such as a temporary skin rash to non life threatening but serious disabilities such as deafness or life threatening kidney failure. If all the costs (direct, indirect and intangible) of primary treatment and the treatment of adverse 
events were enumerated and quantified in money values then the study could be classified as cost-benefit analysis. Depending upon the definition of success and the range of costs evaluated the study could either be classified as cost-minimisation or cost-effectiveness.

Health related outcomes are frequently identical or similar in the clinical sense but the quality of life aspects may differ. For example, a patient could be treated either by surgery or by drugs at home to produce the same clinical outcome, but for the patient the pain and suffering, the treatment setting, time off work, and leisure time activities could be very different. This problem is highlighted in the critical limb ischaemia case study (Chapter 14). Pain and/ or gangrene of a leg or foot could either be prevented by drug therapy to increase the blood supply to the limb or the amputation of the limb. In both treatment options the individual's life may be saved and pain relieved but quality of life for the patient would be different under each treatment option. Successful drug treatment would avoid the loss of a limb or part thereof and the quality of the patient's life would be higher compared with the surgical option. It could be argued that both studies were costminimisations, because the clinical outcomes as defined (avoidance of death) were identical, but there are differences in quality of life. These outcomes were discussed although not quantified.

\subsection{CONCLUSION}

This chapter has discussed the various types of economic evaluation. Although policy decision makers are likely to be most concerned with CEA and CUA they need to be aware of the full range of economic evaluation techniques, their strengths and weaknesses and where they can and cannot be used. Cost-benefit analysis has not been widely applied in health policy analysis where variants of cost-effectiveness analysis (including; cost-utility, cost-minimisation and cost-consequences analysis) have dominated. As experience with, and development of health outcome measures progresses, cost-utility analysis is likely to grow in importance. 
Table 6.6 summarises the distinguishing features of the different types of economic evaluation discussed in this chapter.

The following points are reiterated:

- The study design (type of evaluation) should be driven by the policy analysis objectives, data, time, research budget and expected benefits of the research. Accordingly, a range of techniques are likely to be applied in public policy analysis of health.

- It may not be possible to categorise the type of evaluation until the study is completed.

Table 6.6: Economic Evaluation Categories: Distinguishing Features

\begin{tabular}{|c|c|c|c|c|c|}
\hline $\begin{array}{l}\text { Type of } \\
\text { analysis }\end{array}$ & $\begin{array}{l}\text { Range } \\
\text { of costs }\end{array}$ & $\begin{array}{l}\text { Health } \\
\text { outcome } \\
\text { measure }\end{array}$ & Perspective & $\begin{array}{l}\text { Choice } \\
\text { criteria }\end{array}$ & Use \\
\hline $\begin{array}{l}\text { Cost- } \\
\text { minimisation } \\
\text { CMA }\end{array}$ & $\begin{array}{l}\mathrm{C} 1 \text { or } \\
\mathrm{C} 2 \text { or } \\
\mathrm{C} 3\end{array}$ & $\begin{array}{l}\text { Assumed } \\
\text { identical }\end{array}$ & $\begin{array}{l}\text { C1 \& } 2 \\
\text { Health } \\
\text { sector': } \\
\text { C3 Socicty }\end{array}$ & $\begin{array}{l}\text { Cost per } \\
\text { outcome }\end{array}$ & $\begin{array}{l}\text { Comparing treatment options where } \\
\text { outcomes are identical and a given } \\
\text { quantity of resources or expenditure }\end{array}$ \\
\hline $\begin{array}{l}\text { Cost- } \\
\text { effectiveness } \\
\text { CEA }\end{array}$ & $\begin{array}{l}\mathrm{Cl} \text { or } \\
\mathrm{C} 2 \text { or } \\
\mathrm{C} 3\end{array}$ & $\begin{array}{l}\text { Clinical } \\
\text { or } \\
\text { physical } \\
\text { units }\end{array}$ & $\begin{array}{l}\mathrm{C} 1 \& \mathrm{C} 2 \\
\text { Health } \\
\text { sector }^{1} \text {; } \\
\text { C3 Society }\end{array}$ & $\begin{array}{l}\text { Cost per } \\
\text { outcome }\end{array}$ & $\begin{array}{l}\text { Comparing treatment options for the same } \\
\text { disease and a given quantity of resources } \\
\text { or expenditure }\end{array}$ \\
\hline $\begin{array}{l}\text { Cost-utility } \\
\text { CUA }\end{array}$ & $\begin{array}{l}\mathrm{C} 1 \text { or } \\
\mathrm{C} 2 \text { or } \\
\mathrm{C} 3 \\
\text { (possible } \\
\text { double } \\
\text { counting } \\
\text { if } \mathrm{C} 3 \text { ) }\end{array}$ & Utility & $\begin{array}{l}\mathrm{Cl} \& \mathrm{C} 2 \\
\text { Health } \\
\text { sector }^{1} \\
\text { C3 Society }\end{array}$ & $\begin{array}{l}\text { Cost per } \\
\text { utility } \\
\text { measure }\end{array}$ & $\begin{array}{l}\text { Comparing treatment options for the same } \\
\text { disease and resource allocation across } \\
\text { different diseases from a given quantity of } \\
\text { resources or expenditure }\end{array}$ \\
\hline $\begin{array}{l}\text { Cost-benefil } \\
\mathrm{CBA}\end{array}$ & $C+$ & $\begin{array}{l}\text { Money } \\
\text { values }\end{array}$ & Society & $\begin{array}{l}\text { Net } \\
\text { present } \\
\text { value }\end{array}$ & $\begin{array}{l}\text { Resource allocation for the whole of } \\
\text { society }\end{array}$ \\
\hline
\end{tabular}

$\mathrm{Cl}=$ Direct Medical Costs

C2 = Direct Medical Costs Plus Direct Nonmedical Costs

C3 $=$ Direct Nedical Costs Phas Direct Nonmedical Costs Plus Indirect Costs

$\mathrm{C} 4=$ Direct Medical Costs Plus Direct Nonmedical Costs Plus Indirect Costs Plus Intangible Costs

${ }^{T}$ The health sector may be dissected into funders/ purchasers (eg government, insurance companies and patients) and providers (eg hospitals, general practitioners).

${ }^{2}$ Both the internal rate of return and the cost benefit ratio have been omitted because where the options are mutually exclusive these two criteria can lead to incorrect choices being made.

Notes: A negative cost $=\mathrm{a}$ benefit. A negative benefit $=\mathrm{a} \cos t$ 
The next chapter considers modelling and its application to public policy evaluations in the health sector. Of particular importance is the ability of modelling techniques to integrate data from a variety of diverse sources, compare costs with outcomes and to conduct sensitivity and scenario analysis. 


\subsection{REFERENCES}

Culyer, A. J. "A glossary of the common terms encountered in health economics." In Compendium of English course syllabi and textbooks in health economics. Copenhagen: WHO, 1989.

Dasgupta, P., A. Sen, and S. Marglin. Guidelines for project evaluation. New York: United Nations, 1972.

Detsky, A. S. "Using cost-effectiveness analysis for formulary decisions." PharmacoEconomics 6(4) (1994): 281-88.

Donaldson, C. "The state of the art of costing health care for economic evaluation." Community Health Studies 14 (4) (1990): 341-56.

Drummond, M. F., G. L. Stoddart, and G. W. Torrance, eds. Methods for the economic evaluation of health care programmes. Oxford: Oxford University Press, 1987.

Drummond, M. F. "Cost-benefit analysis in health and health care: fine in practice but does it work in theory?" In Efficiency in the public sector: The theory and practice of cost-benefit analysis. 1st ed., edited by A. Williams and E. Giardina. Aldershot: Edward Elgar Publishing Limited, 1993. N.

Drummond, M. F., B. J. O'Brien, G. L. Stoddart, and G. W. Torrance. Methods for the economic evaluation of health care programmes. 2 nd ed. New York and Toronto: Oxford University Press, 1997.

Dupuit, J. H. "De la mesure de lutilite des travaux publics. Annals des Ponts et Chaussees 18+4. On the measurement of utility of public works." Translated by Barback, RH. International Economic Papers 17 (1952): 83-110. 
Evans, R. G. "Evaluating health care programs: efficiency, effectiveness, and cost." In Strained mercl: the economics of Canadian health care, edited by R. G. Evans. Toronto: Butterworth and Co., 1984.

Gold, M. R., S. E. Siegel, L. B. Russell, and M. C. Weinstein. Cost-effectiveness in health and medicine. New York: Oxford University Press, 1996.

Haveman, R. H., and B. A. Weisbrod. "Defining benefits of public programs: some guidance for policy analysts." In Public expenditure analysis. 2 nd ed., edited by R. H.

Haveman and J. Margolis, 135-60. Chicago: Rand McNally College Publishing Company, 1977.

Hicks, J. R. "The foundations of welfare economics." Economic Journal 49 (1939): 696-712.

Johannesson, M. Theory and methods of economic evaluation of health care. 1st ed. Dordrecht: Kluwer Academic Publishers, 1996.

Luce, B. R., W. G. Manning, J. E. Siegel, and J. Lipscomb. "Estimating costs in cost-effectiveness analysis." In Cost-effectiveness analysis in health and medicine. 1 st ed., edited by M. R. Gold, J. E. Siegel, L. B. Russell, and M. C. Weinstein, 176-213. Oxford: Oxford University press, 1996.

Mishan, E. J. Cost-henefit analysis: an informal introduction. 4th ed. London: Unwin Hyman, 1988.

Pauly, M. V. "V'aluing health care benelits in money terms." In Valuing health care; costs, benefits, and effectiveness of pharmaceuticals and other medical technologies, edited by F. A. Sloan, 99-124. Cambridge: Cambridge, 1996. 
Pearce, D. W. Cost-benefit analysis. 1st ed. Vol. 1. London: Macmillan, 1971.

Pearce, D. W., and C. A. Nash. The social appraisal of projects: a text in cost-benefit analysis. 1st ed. London: Macmillan Press, 1981.

Self, P. "Nonsense on stilts: the futility of Roskill." New Society 16 (1970).

Sugden, R., and A. Williams. The principles of practical cost-benefit analysis. 1 st ed. Oxford: Oxford University press, 1978.

Torrance, G. W., J. E. Siegel, and B. R. Luce. "Framing and designing the cost-effectiveness analysis." In Cost-effectiveness in health and medicine. 1st ed., edited by M. R. Gold, J. E. Siegel, L. B. Russell, and M. C. Weinstein, 54-81. New York: Oxford University Press, 1996.

Weinstein, M. C., and W. B. Stason. "Foundations of cost-effectiveness analysis for health and medical practices." New England Journal of Medicine 296 (1977): 716-21.

Williams, A. "Cost-benefit analysis: bastard science? And/or insidious poison in the body politick?" In Public expenditure and policy analysis. 2 nd ed., edited by R. H. Haveman and J. Margolis, 519-45. Chicago: Rand McNally College Publishing Company, 1977. 


\section{CHAPTER 7: MODELS}

\subsection{INTRODUCTION}

Models describe and mimic relevant aspects of reality and may be used to simulate real situations. A definition by a group of modelling pioneers described models as "... an ordered set of assumptions about a complex system." (Meadows et al., 1972: 20). This modelling definition is still relevant today.

All economic analyses and policy studies involve a model of some sort. This chapter discusses a more formal approach to models that use computers and computer software. Policy analysts can make use of modelling but models are equally useful for policy makers who may wish to test options.

This chapter discusses the use of models and modelling methodology, with application to health care policy. A history of models and their recent use in the health sector, types of models, how models are used, and the modelling processes are presented. A growing number of studies in health economics that have used models and modelling are being published $^{15}$.

\subsection{BACKGROUND}

\subsubsection{History and Development}

The origins of computer-based modelling for decision making and forecasting purposes can be traced back to the work by Forrester and Meadows in the 1960's and 1970's (Forrester,

A MEDLIXE (Healthworld, 1999) search of articles published for the years 1990 to 1997 inclusive using the keyword, "model?" found 148,767 articles, using the key words" model?" and "cost?" 1,702 articles, and "model?" and "economic?" 154 articles. Trends using the key word "model?" were as follows:1980-84, 39,$679 ; 1985-89,54,769 ; 1990-92,50,446 ; 1993-95,69,085$ and 1996-97 (incomplete year) 29,236 articles. 
1961; Meadows et al., 1972). Forrester and Meadows modelled world population and resources to investigate the limits to growth. Meadows et al. (1972:23) concluded "If the present trends in world population, industrialization, pollution, food production, and

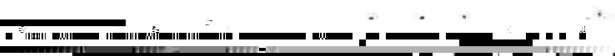


Modelling is increasingly being used as a tool for decision making in business and its application to health policy is no exception to this trend. Models and modelling assist in the decision making process by helping to assemble and analyse relevant information but cannot make decisions for clinicians, managers and policy makers. This helps in clarifying the problem, and in comparing and evaluating options in a simulated environment. For effective use, the policy decision maker must have some understanding of the structure and operation of the model and be aware of its limitations.

Economic evaluation requires information on costs and health related outcomes. Models assist in integrating this information and in the calculation of net benefits and other decision criteria such as cost-effectiveness ratios. This information must then be used to develop workable policies. Models allow policy makers to test and evaluate policy options by simulating situations in a model rather than in the real world.

\subsubsection{Criticisms of Modelling}

Common criticisms of models and modelling are that they are imperfect, oversimplify actual situations, or are incomplete and unfinished. However, many decisions cannot be postponed until perfect models are developed and a full understanding of the problem is achieved (Meadows et al., 1972: 21-2). Some researchers who are familiar and skilled at data acquisition from controlled trials, experiments and observation feel uneasy with the fact that models may draw data from a variety of sources and may use estimates and assumptions. However, this is a strength as a model can integrate data from a number of actual experiments with observations, estimates and data on file or in databases.

\subsection{TYPES OF MODELS}

Models normally have a mathematical basis and may be split into two main types; deterministic or stochastic. Each of these main divisions may be further subdivided. In practice, a particular model may have features of a number of varieties, making precise classification difficult. The types of models of relevance to health policy and health economics include the following non-mutually exclusive categories: 
- Deterministic

- $\quad$ Stochastic

- Decision analytic

- Longitudinal cohort or incidence

- Cross-sectional population or prevalence.

\section{Deterministic and Stochastic models}

Deterministic models give the same result each time the model is run provided the same input data are used. Inputs and outputs are point estimates. Stochastic models are based on probability distributions and the same inputs will not give identical results for each run (if replicated and run many times a distribution of results is obtained). Outputs are probability distributions rather than point estimates.

\section{Decision Analytic Models}

Decision analytic models based on decision trees track a sequence of events and branching options over time (Drummond et al., 1997: 242). Each pathway up through the branches of the tree represents one sequence of events. Chapter 15: Heart failure and Chapter 17: Asthma provides examples of models that are in part decision analytic deterministic models. However, both these models mentioned also have features of some of the model types that follow.

\section{Longitudinal and Prevalence Models}

Longitudinal cohort or incidence models consider expected outcomes for groups of people over time. A subset of this model classification is the Markov or state transition model. These models allot members or groups of the study population to distinct states or categories over time. Population units may switch from one category to another as time progresses. An example of a Markoy model was used to evaluate alternative ways of treating heart failure (Chapter 15: Heart Failure). 
Cross-sectional population or prevalence models consider a population at one point in time. Chapter 17: Asthma considers the costs of treating asthmatics over the course of one year. Deterministic prevalence-based models are valuable for assessing the annual costs and benefits of various policy options.

(See also Drummond et al., 1997: 242-6; Keeler; 1996; Mandelblatt et al., 1996: 151-6; and Sheldon, 1996)

\subsection{WHY MODELS ARE USEFUL}

\subsubsection{Why Use Models}

Models can make the process of integration of data from a variety of primary and secondary sources explicit and transparent. Modelling of costs and health outcomes enables the assessment of many factors simultaneously. Users are able to evaluate the effects of changing data and assumptions about unit costs, risks, prevalence, and treatment options. Models may be based on actual clinical practice rather than randomised controlled clinical trials. Thus, models are tools to be used in evidence-based medicine and management decisions relating to actual health policy and treatment protocols.

\subsubsection{Situations Where Models Are Useful}

Models are useful in the following situations:

- Integrating different types of data from a variety of sources

- Comparing costs with health related outcomes

- Translating and applying clinical trial results to actual clinical practice

- Scenario and sensitivity analysis

- Applying the findings from one setting to another

- Linking intermediate endpoints with final health related outcomes

- Extending the time horizon of a study 


\section{Integrating Different Types of Data From A Variety of Sources}

Obtaining and integrating data with different characteristics and from a plethora of sources is often one of the most difficult processes in policy analysis. Usually data for an economic or policy analysis must be obtained from a variety of diverse sources. Examples of these include; clinical trials, surveys, administrative data bases and literature searches. Clinical trials provide effectiveness data on new treatment options. Surveys of various kinds, interviews, expert panels, focus groups and Delphi groups are frequently necessary to fill information gaps where data are unavailable from secondary sources. Often information collected for administrative purposes may be accessed and processed. For example, reimbursement and accounting data relating to government subsidised prescription medicines may be useful in estimating the volume of prescriptions for different illnesses. Patient record data could be used to obtain information on disease prevalence and incidence. Literature searches provide data in a wide range of forms both qualitative and quantitative.

Often these data will be presented in a variety of forms including; frequency distributions, index numbers, summarised tables, rates and percentages, unit record and verbal statements. For example in public policy evaluations in the health sector, clinical effectiveness evidence may be presented as frequency distributions, prices as index numbers, and prevalence as rates and summarised tables. Without some sort of integrating device much of this information cannot be analysed and interpreted by policy analysts.

\section{Comparing Costs with Health Related Outcomes}

The changes in costs of resources consumed by an intervention or policy must be compared with the changes in health related outcomes produced. A model facilitates both the integration of all cost and health related outcome data and in the calculation of appropriate summary statistics. Unit costs of resources it $\mathrm{cm}$ by item (dollars), changes in resource utilisations (volume) and changes in health related outcomes must be integrated and compared. 


\section{Translating and Applying Clinical Trial Results to Actual Clinical Practice}

Policy makers and managers are more interested in the operational implications of new clinical evidence than the evidence itself. Information derived from artificial and controlled situations are not (without further analysis and/or research) sufficient for developing and implementing workable policies. Clinical trials that are designed to evaluate the efficacy of treatment options are highly controlled and participants are carefully selected according to the trial protocol. Additional clinical monitoring and diagnostic procedures will usually be undertaken than in normal clinical practice. Accordingly, trials will probably not reflect actual practice in terms of patients selected, treatment protocols, patient compliance with treatment prescribed, monitoring and follow up. A model provides a means of taking these differences into account through inclusion of a range of assumptions and different data.

\section{Scenario And Sensitivity Analysis}

It is useful to simulate policy and treatment protocol options and conduct "what if analysis" by changing input data and model assumptions. Time is often a critical constraint and undertaking a new and separate analysis to evaluate another set of different policy options is often not practicable. Running a validated model with different input data and assumptions facilitates this type of analysis. Implementing a new and untested public health policy has a greater probability of failure than one that has been tested in a simulation model.

\section{Applying the Findings From One Setting to Another}

Results from a hospital-based study may be applied to a general practice setting. A multicentre international study or a trial conducted in one country can be transported to other countries. (Drummond et al., 1997: 275-80). Replicating an international study in New Zealand or in another setting is not usually cost effective and may not be an option because of time and budget constraints. Care must be exercised to take account of differences in demographics, health systems, treatment protocols and unit costs when transferring findings from one setting to another. It is not possible to translate findings from one country to 
another simply by adjusting only for exchange rates and population differences as this would not adjust for all unit costs, differences between health systems, and other factors such as demographic profiles and income levels.

Chapter 15: Heart Failure discusses a cost-effectiveness analysis using a decision analytic model based on data derived from an overseas clinical trial. New Zealand specific unit costs and epidemiological data were used in the model to investigate the cost-effectiveness of introducing the new drug into the New Zealand health system.

\section{Linking Intermediate Endpoints with Final Health Related Outcomes}

Intermediate endpoints are of limited value for policy analysis. It is more valuable to have information on the final health-related outcome: Population models link intermediate endpoints determined from clinical trials with health outcomes of a population. For example, the effects of altering blood pressure may be related to changes in final outcomes such as death or ischaemic heart attack (Drummond et al., 1997: 244-5). Disease specific outcomes as measured in a clinical trial may also be extrapolated into utility scales (see Chapter 3: Valuing Health Outcomes).

\section{Extending the Time Horizon of A Study}

Most clinical trials have a short duration compared with the life expectancy of a patient or the normal progression of a disease. Extrapolation models project beyond the time horizon of a clinical trial, for example 30 days, to perhaps a number of years (Drummond et al., 1997: 244). A policy analyst who wishes to track the policy consequences of an intervention to reduce heart attacks over a number of years but who has only actual clinical trial data relating to six months, may be able to use a Markov model to extend the time horizon. 


\subsection{THE MODELLING PROCESS}

\subsubsection{Forrester's Modelling Steps}

Many of the steps and processes of modern modelling techniques for business and industry stem from Forrester (1961). His book 'Industrial Dynamics' has survived the test of time and is still relevant. Forrester listed and elaborated on the following modelling steps:

- "Identify a problem.

- Isolate the factors that appear to interact to create the observed symptoms.

- Trace the cause-and-effect information-feedback loops that link decisions to action to resulting information changes and to new decisions.

- Formulate acceptable formal decision policies that describe how decisions result from available information streams.

- Construct a mathematical model of the decision policies, information sources, and interactions of the system components.

- Generate the behaviour through time of the system as described by the model (usually with a digital computer to execute the lengthy calculations).

- Compare results against all pertinent available knowledge about the system.

- Revise the model until it is acceptable as a representation of the actual system.

- Redesign, within the model, the organizational relationships and policies that can be altered in the actual system to find the changes that improve system behaviour.

- Alter the real system in the directions that model experimentation has shown will lead to improved performance." (Forrester, 1961: 13)

\subsubsection{Generalised Modelling Steps}

Building a model is a cyclical process and the steps presented are not intended to be definitive, but rather indicative of the key elements. Some of these steps overlap or are synonymous with the economic evaluation steps described in Chapter 18: Discussion and Conclusions.

Although there is a common thread, the steps considered relevant by different practitioners 
depend on the analyst's perspective and specific purpose of the model. For example, Snider et al. (1996) describe the basic steps in decision analysis with a focus on prevention effectiveness analysis (where decision tree models dominate) as: structuring the problem, developing a decision tree, estimating probabilities, valuing consequences, calculating expected values and interpreting results.

The development of the model requires skills of a number of professionals (for example, economists, clinicians, statisticians) and thus mirrors the multi disciplinary nature of health economics. It is important these people are involved from the first step of problem identification to the end if confidence is to be gained with end users.

The process of modelling is iterative with feed back loops rather than linear. The simplified process showing the circular nature and major links between steps is illustrated in Figure 7.1. For the purpose of this discussion on modelling, the process has been generalised and reduced to the following steps:

- Problem description and definition

- Isolation of relevant factors

- $\quad$ Structural design

- Construction

- $\quad$ Testing and validation

- Sensitivity tests and scenario analysis

- Recommendation for action in the real world

\section{Problem Description and Definition}

A description of the problem with a clear problem statement and set of objectives is a necessary first step. This step will require some qualitative pre-research and literature search. Structuring the problem involves determining the key issues involved in the decision, deciding upon the analytic perspective and time horizon and deciding upon the real or practicable alternative courses of action. Unless the problem is clearly defined further analysis is pointless. 
Figure 7.1: Modelling Process in the Form of A Flow Chart with Feedback Loops

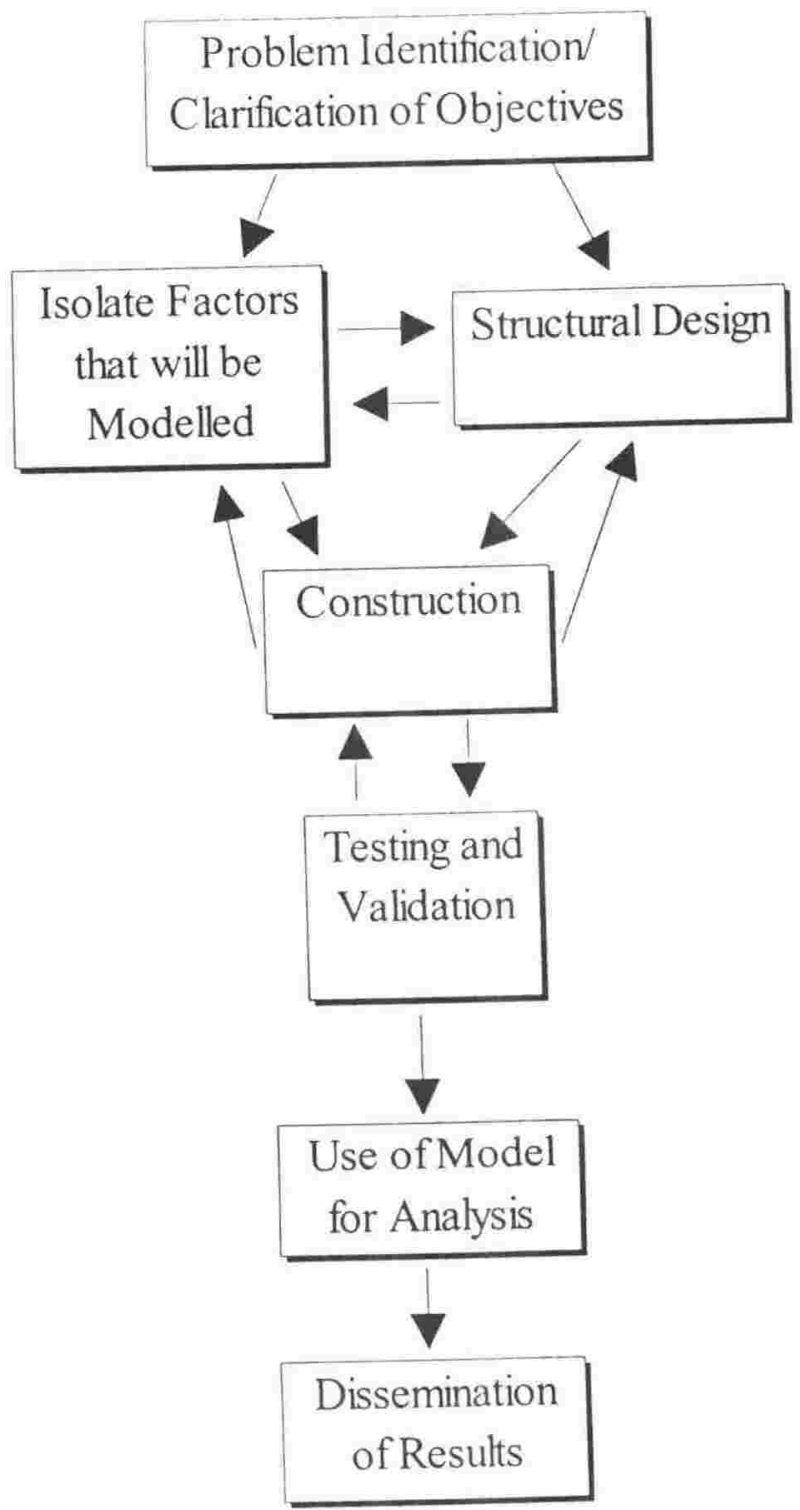

Isolation of Relevant Factors and Structural Design

The steps of isolating relevant factors and the structural design of the model are closely linked, and often culminate in a decision tree. During construction of the model, absence of data to quantify a structure or parameter does not mean that it should be excluded from the model. Simulation tests will help to refine the value of a parameter within plausible limits (Forrester, 1992: 60). 
Throughout the modelling process, simplicity, transparency, ability to modify and change, and flexibility of use are of underlying importance when building the model. Other design criteria are that a model should be able to be used easily with minimal training, and be able to run on a wide range of computing platforms.

\section{Decision Trees}

A decision tree can form the basis for model construction, and is particularly useful in health care for showing treatment pathways and outcomes. An example of a decision tree can be found in Chapter 17: Asthma. Developing a decision tree involves preparing a graphical representation of how the possible choices impact on the final outcome. At each node there are a number of branches representing choices among options (decision node) or a number of branches representing chance events (chance node). Figure 7.2 illustrates vaccination against influenza choices and outcomes. Those at risk may be vaccinated or not, and those vaccinated may contract influenza or not, and so on.

Estimating probabilities is concerned with assigning probabilities to each chance node along an event pathway in the decision tree. The sum of all the probabilities at each chance node must equal one. Valuing consequences is the quantification of the outcomes at the end of each event pathway. The consequences may be evaluated in money terms, natural units, clinical values or a health related quality of life measure.

The first step in calculating expected values using the decision tree involves calculating the numbers of patients passing along each event pathway. Then the number of patients reaching the end of each pathway is multiplied by the expected cost per patient for that path to obtain the total cost for the path. The same process is applied to each event pathway to derive a total cost.

Using the "do not vaccinate do not contract influenza" branch, the expected cost is (probability of no vaccination multiplied by the cost of no vaccination plus the probability of not contracting pneumonia multiplied by the cost of not contracting pneumonia $(0.80 \mathrm{x}$ $\$ 0.00$ plus $0.80 \times \$ 0.00=\$ 0.00$ ). Adding up the expected values of all branches gives the 
expected value for the tree. $(\$ 2.24+\$ 14.40+\$ 0.00+\$ 4.00=\$ 20.64)$. The decision tree provides the cost per at risk population member of a vaccination programme with the defined probabilities and costs as illustrated in Figure 7.2.

\section{Figure 7.2: Decision Tree Diagram}

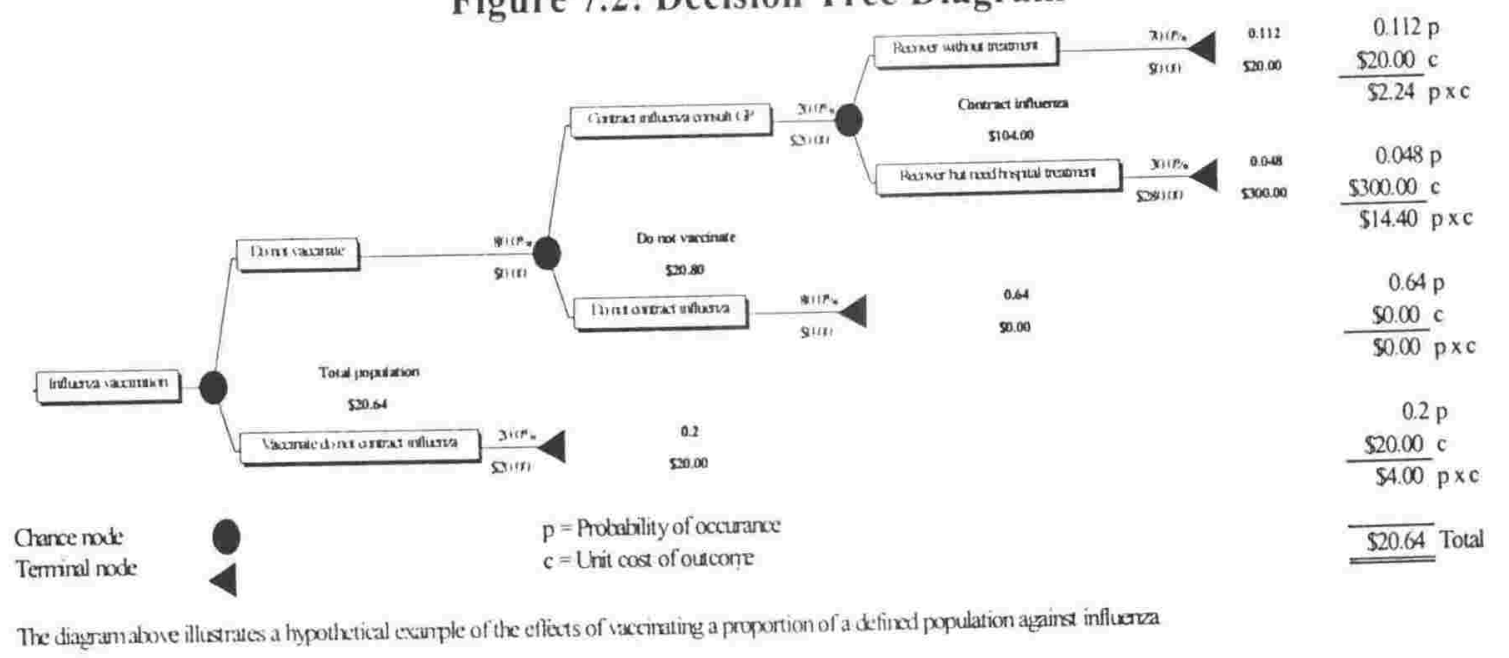

\section{Testing and Validation}

After the model is constructed, it must be tested and validated, and accuracy of data ensured. Documentation should take place at this stage to facilitate use by parties not directly involved in the design and construction process. Validation of the model can take many forms. Common methods involve comparing model results with historical data (predictive validity) and evaluation by experts in the study area. Often it is difficult to validate a model directly because if accurate information relating specifically to the model's outputs were available then there would have been no need to develop a model. "Thus face validation of a model may have to rest solely on evaluating the inherent reasonableness of model assumptions as a representation of reality." (Mandelblatt et al., 1996: 161).

\section{Sensitivity Tests and Scenario Analysis to Obtain Results}

After validation, sensitivity and scenario analysis must be undertaken. This often serves the dual purposes of linking back to validation, and revealing hidden insights and effects with regard to the problem investigated. 
Interpreting the results includes undertaking various types of sensitivity analysis where numerical values of input probabilities and other estimates are changed and the expected values of the model outcomes tracked. Threshold analysis involves altering model input values until the point that a different decision should be made is reached. Best and worst case scenario analysis applies worst and best estimates to the model. Sensitivity analysis was discussed in Chapter 4: Costs.

\section{Recommendation for Action in the Real World}

Finally results found need to be reported and important policy options and implications outlined. The modelling process is not mechanical or blind. Judgement is vital in determining how to simplify the real world, and how far the process of simplification should proceed. When the model is used to draw conclusions and make recommendations for action, wisdom and insight are also needed.

\subsection{CONCLUSION}

Models in the health sector may combine data from clinical trials, health databases, and qualitative information from clinicians involved in treatment. Models are particularly valuable aids in combining quantitative, and qualitative information. It is this wide range of information sources that necessitates a multi-disciplinary approach. To ensure the model built has the confidence of all potential users, is realistic, and of value, the potential users should also be involved during the final steps of testing. Thus it can be seen that a model permits a policy maker to simulate and evaluate potential policy options and thereby minimise the risk of making potentially costly mistakes and introducing policies that are likely to fail.

The way in which models are used to integrate and aggregate a range of cost data from a variety of sources is shown by the asthma case. Chapter 17: Asthma, presents a spreadsheet-based deterministic, prevalence decision-analytic model. The model described in this case allows the user to evaluate the full costs of alternative treatments, or by entering different values in the input screen, to look at asthma treatment costs from the 
viewpoint of a patient, a general practitioner, a hospital, or a defined population. The design is sufficiently flexible to take account of changing treatment options and guidelines. A user is able to calculate total costs, compare data through bench marking, undertake sensitivity analysis, or consider a defined population, different patient categories, or appropriate medication use.

The heart failure case (Chapter 15: Heart Failure) illustrates the use of a decision analytic model that combines international clinical trial and cost data to conduct a cost effective analysis using New Zealand cost and epidemiological data.

The following Chapters ( 8 to 17) present the results of cases designed to illustrate the methodologies discussed. 


\subsection{REFERENCES}

Drummond, M. F., B. J. O'Brien, G. L. Stoddart, and G. W. Torrance. Methods for the economic evaluation of health care programmes. 2nd ed. New York: Oxford University Press, 1997.

Forrester, J. W. "Policies, decisions and information sources for modelling." European Journal of Operations Research 59 (1992): 42-63.

Forrester, J. W. Industrial dynamics. 1st ed. New York: MIT Press and Wiley, 1961.

Keeler, E. "Decision trees and Markov models in cost-effectiveness research." In Valuing health care: Costs, benefits, and effectiveness of pharmaceuticals and other medical technologies, edited by F. A. Sloan, 185-205. New York: Cambridge University Press, 1996.

Mandelblatt, J. S., D. G. Fryback, M. C. Weinstein, L. B. Russell, M. R. Gold, and D. C. Hadorn. "Assessing the effectiveness of health interventions." In Cost-effectiveness in health and medicine, edited by M. R. Gold, J. E. Siegel, L. B. Russell, and M. C. Weinstein. New York: Oxford University Press, 1996.

Meadows, D. H., D. L. Meadows, J. Randers, and W. W. Behrens III. The limits to growth; a report for the Club of Rome's project on the predicament of mankind. New York: Universe Books, 1972

MEDLINE; http://www.healthworld.com/library/search/medline.htm; 19 January 1999

Sheldon, T. A. "Problems of using modelling in the economic evaluation of health care." Health Economics 5 (1996): 1-11.

Snider, D. E., D. R. Holtgrave, and D. O. Dunet, "Decision analysis." In Prevention effectiveness: a guide to decision analy sis and economic evaluation. 1st ed., edited by A.C. Haddix, S. M. Teutsch, P. A. Shaffer, and D. O. Dunet, 27-45. New York: Oxford University Press, 1996. 


\section{CHAPTER 8: CARE WITH DATA}

\subsection{SUMMARY AND INTRODUCTION}

\section{Summary}

Two cases (Heart Failure and Benign Prostatic Hyperplasia) are used to illustrate a number of problems associated with aggregating and using secondary data. The chapter also presents a strategy to avoid such problems. New and previously unknown evidence on the differences between Māori and non-Māori hospital admission rates for benign prostatic hyperplasia was found. Paradoxical and previously unexplained differences between aggregated gender specific heart failure prevalence rates and age specific gender rates were isolated and explained.

\section{Introduction}

This chapter discusses problems that may occur when data are aggregated (Scott, Camden \& Scott, 1998). These problems, if unrecognised, (by either analysts and/ or decision makers) have the potential to mislead policy makers into making decisions that are not in accord with their objectives. Economic and policy evaluations often use secondary processed data from administrative records, and from experimental and observational studies. Unit records for a population or study group may be aggregated to form cross tabulations of counts for subgroups. These counts are commonly used to create rates (number observations per total number in the category). Often the unit record data are not available to the researchers. Problems can arise from the definition of the original data, with aggregation into summary tables and with the formation of rates. Examples of such problems include confusion over the definitions and meanings of variables, Simpson's paradox, and the ecological fallacy. 


\subsection{BACKGROUND}

Demographic or epidemiological rates or indices are important summary statistics in the study of community health, environmental health, health economics and policy analysis. Rates are formed by taking the number of occurrences (incidence or prevalence) of some event, dividing the number of these events by the population "at risk" and multiplying by a convenient constant (for example 1000). If the population is divided or "disaggregated" into groups (for example by age) a set of "specific" rates is derived, which may behave differently from the aggregated or "crude" rate. Researchers are often forced to use aggregated data and aggregate data themselves, but are frequently unaware of the possible consequences of doing so.

Statisticians consider their discipline to be a scientific enquiry process (Pfannkuch, 1996) which involves care with data quality at all stages. The total statistical process (Lipson and Jones 1996) includes posing the right questions, collecting the right data and examining it with care and an eye for the unexpected. The exploratory approach, where the data is examined carefully with an open mind, is recognised as an important alternative to the "confirmatory" approach of statistical inference. When a research project is based on administrative records, this exploratory approach is almost always the most appropriate. When using secondary data sources, the definitions and meanings of variables are not always explicit or clear and this may result in confusion and misinterpretation.

Basing policy advice on the results of macro studies is recognised as a potential problem by journal editors and by some government agencies. The British Medical Journal (Drummond et al., 1996) has published a checklist for referees and authors of health economic studies (Chapter 18: Discussion and Conclusions). British Medical Journal, checkpoint number 32 relates to aggregation and states that major outcomes should be presented in disaggregated as well as in an aggregated form. The US Public Health Service in a report on the conduct of cost-effectiveness analysis (Luce, 1996:210) recommends (where possible) using micro-costing in preference to gross costing using aggregated data (Chapter 4: Costs). 
Two illustrations from published original research are used to demonstrate a number of problems and their resolution. The cases relate to New Zealand health economics studies: heart failure (Scott and Scott, 1996) and benign prostatic hyperplasia (BPH) (Scott and Scott, 1993). Additional information relating to methodology, data, results and conclusions may be found in these two papers and in Chapters 10: Benign Prostatic Hyperplasia and Chapters 15: Heart Failure.

The first example (Heart Failure) discussed in this chapter illustrates Simpson's paradox, (Wild and Seber, 1996: 561-3 and Simpson, 1951). The paradox involves a three-way cross-classification table, where one of the three factors is a categorical variable (such as gender or the presence or absence of disease) with values presented as percentages or rates. Collapsing (that is, aggregating) the table and considering only the two (remaining) factors of interest in isolation can be very misleading.

Frazier $(1993 ; 28)$ discusses Simpson's paradox in relation to US educational attainment. In this article Simpson's paradox was described as showing "... that an average can change in a direction opposite from all subgroups if the proportion of the total represented by the subgroup changes". The educational statistics described revealed that although every ethnic or racial population subgroup maintained or improved its average Scholarship Aptitude Test (SAT) score, there was a decline in the combined average score. This decline arose because more students in the lower percentiles in each class were taking the SAT test. The median test taker fell from the 79 th percentile to the 73 rd percentile within a class. A more fitting interpretation of this data is that educational attainment was rising rather than falling. Education policy and funding decisions based on the macro data may have led to inequitable and inefficient resource allocations.

Another example of Simpson's paradox was raised by Westbrooke $(1996,1997)$ when commenting on a publication by Dunstan et al. (1995). Dunstan and colleagues studied the pool of 2984 people who reported for jury service in New Zealand in a four-week period of 1993. $10.1 \%$ of them were Māori, and the population in the Jury Districts was $9.5 \%$ Māori, which gave a slight over representation. However, when the authors calculated expected numbers of Māori jurors for each of the 13 Jury Districts separately, the actual numbers were less than the expected numbers in 12 Districts (and equal in the 13 th). 
A further phenomenon with its own body of literature is the ecological fallacy (Steel and Holt, 1996). Frequently, unit records are aggregated into groups where the unit record values of a quantitative variable are replaced by the mean values for each group. The ecological fallacy arises when an analysis based on aggregated or grouped data produces different results from an analysis using unit level data. Conclusions based on means may "give conclusions very different from those that would be obtained from an analysis of unit level data, if they were available" (Steel and Holt, 1996: 39). This fallacy is also discussed by Greenland and Robins (1994).

The second example discussed in this chapter (Benign Prostatic Hyperplasia) is about differences between Māori and non-Māori hospital admission for benign prostatic hyperplasia. Health care for Māori is an issue that involves public policy, public debate and research. Pōmare et al. (1995: 29) claim, "It has become apparent during the last two decades that Māori want to define the threats to wellbeing, as well as the possible range of solutions". To succeed in these aspirations, researchers will need data that is appropriately defined, collected and analysed. There are also issues of control and ownership of data, research processes, decision making and delivery of services. Bishop and Glynn (1992: 127) assert "Powerful and rapid changes have occurred in areas like Māori women's health ... since Māori people have been taking control over their own development and resources in these areas." and they discuss (1992: 130) research as "empowerment".

\subsection{CASE ONE: HEART FAILURE}

Heart failure occurs when the heart muscle is damaged, overloaded, or exhausted by such conditions as hypertension, coronary artery disease, damage to cardiac valves, cardiomyopathy, and arrhythmias. The rates for deaths and hospital admissions for heart failure increase with age. Table 8.1 and Figure 8.1 illustrate the age and gender specific death rates for heart failure in 1992 calculated from New Zealand death certificate data. 
Table 8.1: Mortality From Heart Failure in New Zealand 1992: Numbers, Age and Gender Specific Rates

\begin{tabular}{|c|c|c|c|c|c|c|c|c|}
\hline \multirow[t]{2}{*}{$\begin{array}{l}\text { Age } \\
\text { Years }\end{array}$} & \multicolumn{2}{|c|}{$\begin{array}{l}\text { Deaths } \\
\text { Number }\end{array}$} & \multicolumn{2}{|c|}{$\begin{array}{l}\text { Population } \\
\text { Number }\end{array}$} & \multicolumn{2}{|c|}{$\begin{array}{l}\text { Deaths } \\
\text { Rate per } \\
100,000\end{array}$} & \multicolumn{2}{|c|}{$\begin{array}{l}\text { Population } \\
\text { age group as a } \% \text { of the } 59+ \\
\text { total population, by gender }\end{array}$} \\
\hline & Male & Female & Male & Female & Male & Female & $\begin{array}{r}\text { Male } \\
\end{array}$ & Female \\
\hline $60-64$ & 3 & 1 & 69,860 & 69,480 & 4 & 1 & $30 \%$ & $24 \%$ \\
\hline $65-69$ & 3 & 4 & 61,290 & 66,210 & 5 & 6 & $26 \%$ & $23 \%$ \\
\hline $70-74$ & 6 & 7 & 45,270 & 57,320 & 13 & 12 & $19 \%$ & $20 \%$ \\
\hline $75-79$ & 13 & 10 & 31,540 & 46,170 & 41 & 22 & $13 \%$ & $16 \%$ \\
\hline $80-84$ & 53 & 63 & 17,740 & 31,020 & 299 & 203 & $8 \%$ & $11 \%$ \\
\hline $84+$ & 90 & 203 & 9,710 & 23,420 & 927 & 867 & $4 \%$ & $8 \%$ \\
\hline Total $59+$ & 168 & 288 & 235,410 & 293,620 & 71 & 98 & $100 \%$ & $100 \%$ \\
\hline
\end{tabular}

Using aggregated data, (ages 60 years and over, and all ages) the statistics show higher death rates from heart failure for females than for males. However, different patterns emerge when five-yearly age-specific rates for 60 years and over are used. Age-specific mortality rates are higher for males than for females. This is a clear example of Simpson's paradox. The opposite conclusions would be drawn from the two sets of data.

Figure 8.1: Mortality From Heart Failure 1992. Rate Per 100,000 Population

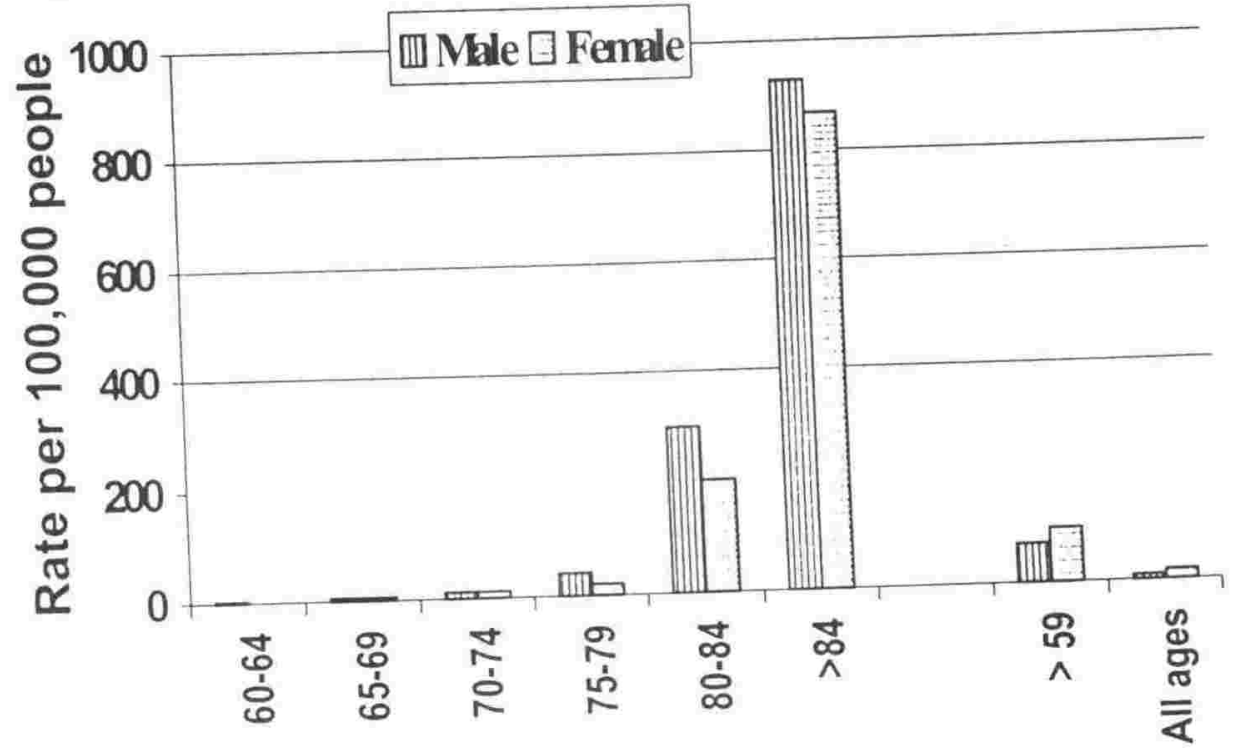

Age group 
The data-set upon which Figure 8.1 and Table 8.1 are based has three category variables relating to the New Zealand population of 1992; two independent variables (gender and age group), and one dependent variable (mortality from heart failure, or not). The mortality variable is strongly related to both the gender variable and the age group variable. However, the two independent variables (gender and age group) are strongly related themselves. If mortality rates are defined by gender alone (aggregated over all ages), or by age group alone (aggregated over all ages), we get an incomplete portrayal of the information from the dataset. Defining the mortality rates by both gender and age group together provides a more complete picture, which reverses the effect of gender. Overall, a male is less likely to die of heart failure than a female ( 71 per 100,000 compared with 98 per 100,000). However a male of a specific age is more likely to die of heart disease than a female of the same age (as shown by higher age specific death rates for males than females). This explains how Simpson's paradox arises.

The heart failure example demonstrates that researchers need to be very careful about what their data really mean: the definitions of variables, and the way these definitions are interpreted when the data are collected. The main conclusion from this case is that health policy decisions made entirely on data aggregated across all age groups would direct resources towards heart disease in females, while decisions made entirely on age specific rates would direct resources towards heart disease in males. In this case age specific rates give a more complete and accurate picture than rates aggregated over all ages.

\subsection{CASE TWO: BENIGN PROSTATIC HYPERPLASIA (BPH)}

Two studies of BPH are discussed. The first is an analysis of administrative records relating to hospital admissions, and the second is a comparative community-based study. $\mathrm{BPH}$ is a nommalignant enlargement of the prostate in men that commonly occurs with ageing. This study is used to discuss the apparent differences in prevalence of BPH between Māori and non-Māori men. 
Statistics on admissions for BPH were derived from hospital discharge data for all of New Zealand. Hospital discharge data includes information on ethnicity, gender, age, diagnosis and surgical procedures. Table 8.2 and Figure 8.2 use this admissions data and combine it with data about the population of New Zealand by age and ethnicity. They present the age and ethnicity specific rates for admissions with $\mathrm{BPH}$ as the primary diagnosis. All-ages hospital admission rate for BPH for Māori is much lower (0.37 per 1000) compared with non-Māori (2.04 per 1000). For males aged 50 and above, the rate for Māori is lower (3.37 per 1000) than for non-Māori (8.71 per 1000). When the data for age $50+$ is disaggregated into age groups, the same feature occurs in each age group. The admission numbers are small (especially outside the 60 - 79 age groups), but the difference in rates is clear and consistent.

Table 8.2: Hospital Admissions 1988 With Primary Diagnosis BPH

\begin{tabular}{|c|c|c|c|c|c|c|}
\hline \multirow{3}{*}{$\begin{array}{l}\text { Age } \\
\text { years }\end{array}$} & \multicolumn{3}{|c|}{ Māori. } & \multicolumn{3}{|c|}{ Non-Māori } \\
\hline & Admissions & Population & Rate & Admissions & Population & Rate \\
\hline & Number & Number & per 1000 & Number & Number & per 1000 \\
\hline $50-54$ & 4 & 5,220 & 0.77 & 87 & 68,400 & 1.27 \\
\hline $55-59$ & 4 & 4,360 & 0.92 & 257 & 68,160 & 3.77 \\
\hline $60-64$ & 10 & 2,940 & 3.40 & 459 & 67,150 & 6.84 \\
\hline $65-69$ & 14 & 1,770 & 7.91 & 645 & 52,370 & 12.32 \\
\hline $70-74$ & 11 & 990 & 11.11 & 603 & 40,180 & 15.01 \\
\hline $75-79$ & 9 & 590 & 15.25 & 525 & 28,200 & 18.62 \\
\hline $80-84$ & 2 & 300 & 6.67 & 304 & 15,110 & 20.12 \\
\hline $84+$ & 1 & 140 & 7.14 & 146 & 7,980 & 18.30 \\
\hline Total $49+$ & 55 & 16,310 & 3.37 & 3026 & 347,550 & 8.71 \\
\hline
\end{tabular}

Clinicians, at the time of the study, considered (because they treated very few Mãori men for $\mathrm{BPH}$ ) that something protected Māori men from BPH. There was a perception that $\mathrm{BPH}$ was not a problem for Māori men and that health care and health promotion resources would be better be directed towards other health problems and conditions.

However, the higher non-Māori hospitalisation rates could have resulted from differences in a number of factors, including: disease prevalence as a result of genetic or lifestyle differences, cultural attitudes to health and health care, ability to access health services, 
knowledge about health and socioeconomic status. The age profiles of the two populations are quite different, that is, there are proportionally fewer Māori in older age groups. As BPH symptoms increase with age, this will affect the total numbers of admissions and allages prevalence and incidence rates but not age specific rates. The disparate age profiles explain most of the very large differences noted in the all-ages rates for admissions. Use of these rates alone for policy decisions, even though they point in the same direction as the age specific rates, could be inappropriate as BPH is an age specific condition.

Figure 8.2: Hospital Admissions 1988 with Primary Diagnosis BPH. Rates Per 1000 Male Population

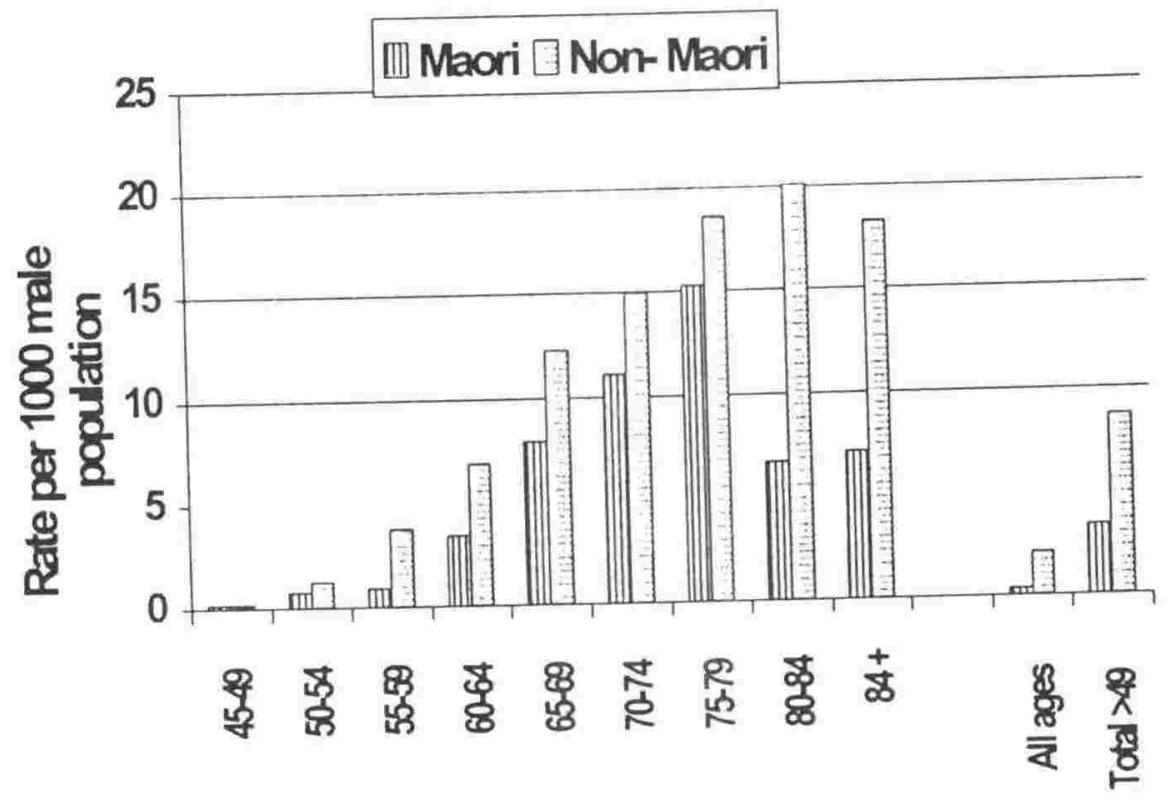

Age group

At the time of this economic study (Scott and Scott, 1993) a clinical investigation (Nacey et al., 1993) was begun. The Nacey study found that no statistically significant difference in prevalence of symptomatic BPH could be demonstrated among European, Mãori and Polynesian men aged 50 years and over. The crucial difference between the Nacey study and the admission data as analysed by Scott and Scott is that the Nacey study took a representative sample of New Zealand men, and asked them about BPH symptoms, whereas the admissions data involved men who had self-selected by seeking treatment.Clearly, in this case, hospital admissions were not a measure of the incidence or prevalence of BPH. 
A conclusion from these two studies considered together is that Māori and non-Māori appeared to have different rates of seeking and obtaining treatment. The most likely explanation for the differences between Māori and non-Māori age specific treatment rates are differences in access to treatment for economic and social reasons rather than an underlying difference in disease prevalence. Durie (1994: 201) claims that "...the biological substrate is only one indicator of health. The others include spiritual, psychological, interpersonal, family, economic and environmental dimensions." Pōmare et al. (1995: 168) claim that "...Māori have difficulty in accessing health care services.", and that mainstream services need to be "...affordable, accessible, appropriate and acceptable".

Interpreting the data at face value could lead to the conclusion that Māori men are not susceptible to developing $\mathrm{BPH}$ and that comparatively fewer health care resources should be directed towards Māori BPH health problems than to the treatment of BPH in nonMāori. A more appropriate policy would suggest that more resources should be directed towards ensuring that Māori men have access to and seek treatment for BPH problems (for example, consultation with Māori groups and specifically tailored and targeted health promotion). Pōmare et al. (1995: 159) note that "Māori use health services differently than non-Māori. When need for health care is taken into account, Māori visit a general practitioner less often than non-Māori."

The BPH example also raises the issue of how well the country's health statistics collection and analysis systems meet the needs of Māori, as the health profiles and needs of the two partner cultures to the Treaty of Waitangi are often very different. In the past much research was designed to meet the needs of the researcher and the dominant group (Bishop and Glynn, 1992: 126). In discussing the importance of the community perspective, Raeburn (1994: 338-9) comments that if research into Māori health is to be "empowering", researchers "...may need to reassess their stance, especially with regard to the "community" and to the voices of those in it." 


\subsection{DISCUSSION}

Researchers may wish to investigate a problem using data at the individual "unit record" or micro level but are often unable to do so. The degree of aggregation is frequently imposed on them by the form in which the data are available. Individual or unit data are often not available because of commercial sensitivity or confidentiality with respect to individuals.

Frequently, published data are in tables that have already been aggregated across important variables, and researchers will not have access to less aggregated forms. However, it is sometimes possible to obtain the underlying raw data and conduct a more disaggregated analysis. For example, Health Statistics New Zealand publishes aggregated data, but unit record data, with the patient identifiers removed, are available in electronic form. If an analysis is based on grouped or aggregated data there should be some analysis and discussion of the grouping procedure or of the consequences of aggregation and/ or, of any major changes in the structure of the underlying population.

Researchers are often confronted with data sets containing variables which they did not define and for which they did not control the data collection. These variables will often not match the research questions precisely. Variables and rates calculated from them must be treated with care.

When working with counts of events (or conditions) from a population with diverse features there are two important factors that should be considered. Firstly features which appear when the data are disaggregated (and expressed as rates) by the groups within the population may disappear (or even be apparently reversed) when the data are aggregated too much or inappropriately. Secondly, rates depend on two sets of numbers; the counts of the events (by group) and the counts for the total numbers in the population (by group). The counting by group of both events and population may be subject to errors, varying interpretations and different definitions. 
Another important point when analysing with subgroups is that there is a greater probability of a significant result being found by chance alone, with increasing numbers of subgroups analysed. In the medical area, subgroups are correctly formed when these groups are defined according to mutually exclusive categories that are observed in baseline attributes. It is difficult, if not impossible, to interpret accurately subgroup treatment differences that may be found when subgroups are defined by characteristics measured after randomization as they are potentially influenced by treatment (Abramson, Kelsey, Safar and SuttonTyrrell, 1992).

This chapter has not discussed the important problems of confounding and multicollinearity in detail because it was considered that these are well understood by researchers and that information is readily available in epidemiology and econometric texts. Both confounding and multicollinearity result in similar problems of interpretation.

Confounding occurs when an independent variable not included in the study is related independently to both an independent variable in the study and the dependent variables. For example a study of risk factors for deafness could find a strong association between developing deafness and being a successful business leader. This is not a causal association but an indirect association, confounded by age as most successful business leaders tended to be older.

Multicollinearity occurs when some or all independent variables in a study are highly correlated. It then becomes difficult to isolate the separate influences of the variables. For example, multicollinearity could occur if in a multiple linear regression using time series data, hospital admissions (as the dependent variable) were regressed on both educational attainment and income (as independent variables). Educational attainment and income are highly correlated. 


\subsection{POLICY POINTS}

Occasionally clear examples of paradoxes, fallacies and misinterpretation of data will arise and be recognised. More often the underlying problems will be present and will cause problems, but will not make themselves evident. This has serious implications in that policy decisions made on faulty information will be flawed.

In health economics and in other disciplines, researchers often have limited control over their statistical data. These data may consist of administrative records, with the variables defined by other people, and with the records collected in different situations. Unit record data may not be available, and the researchers may need to work with cross-tabulations provided by other people. Accordingly, researchers need to take great care at all stages in their analyses, choosing the level of aggregation at which they analyse data with care, and investigating unexpected results.

The following points are presented as a summary. The research question and each variable in the data set must be clearly defined and described. Subgroups of a human population may have different structures, behaviours and needs, and investigators should be aware of these differences. Data involving Māori health should be analysed and controlled in ways that meet the needs of Māori and in partnership with the affected group. Data should be analysed using cross-tabulations which involve disaggregation to the lowest possible level. If some variables are found not to influence the outcomes, these variables can be aggregated to produce tables that highlight the important influencing factors. Increasing availability of unit-record data sets, and software tools to manage and analyse them, makes such detailed analysis practicable. 


\subsection{REFERENCES}

Abramson, N. S., S. F. Kelsey, P. Safar, and K. Sutton-Tyrrell. "Simpson's paradox and clinical trials: what you find is not necessarily what you prove". Annals of Emergency Medicine, December 1992: 1480-82.

Bishop, R., E. Glynn, and Kitea He Kanohi. "Conducting and evaluating educational research". New Zealand Journal of Educational Research 1992; 27:25-135.

Drummond, M. F., and T. O. Jefferson. on behalf of the BMJ Economic Evaluation Working Party. "Guidelines for authors and peer reviewers of economic submissions to the BMJ". BMJ 1996;313:275-83.

Dunstan, S., J. Paulin, and K. Atkinson. Trial by Peers? The Composition of NZ Juries. Wellington. Dept of Justice. 1995.

Durie, M. "Māori perspectives on health and illness". In Social dimensions of health and disease: New Zealand perspectives. Edited by Spicer J, Trlin A and Walton J, Palmerston North: Dunmore Press, 1994.

Frazier, K. 1993. "Perspectives on Education in America: Sandia Study Challenges Misconceptions". Skeptical Inquirer 18:26-31.

Greenland, S., and J. Robins. "Ecological studies - biases, misconceptions and counterexamples". American Joumal of Epidemiology 1994;139:747-60.

Lipson, K., P. and Jones. "Teaching Statistics in the 21 st Century". Proceedings of the 47th Annual Conference of the New Zealand Statistical Association, Wellington, 30 and 31 August, 1996. 
Luce, B. R., W. G. Manning, J. E. Siegel, and J. Lipscomb. "Estimating costs in cost-effectiveness analysis." In Cost-effectiveness analysis in health and medicine. 1 st ed., edited by M. R. Gold, J. E. Siegel, L. B. Russell, and M. C. Weinstein, 176-213. Oxford: Oxford University press, 1996.

Nacey, J. N., P. Morum, and B. Delahunt. "The incidence of benign prostatic hyperplasia in Caucasian, Māori and Polynesian men: a comparative community based study". Poster presented at the 46th Annual Scientific Meeting, Urological Society of Australasia, Hobart, February 28 - March 5, 1993

Pfannkuch, M. "Statistical Interpretation of Media Reports". Proceedings of the 47th Annual Conference of the New Zealand Statistical Association, Wellington, 30 and 31 August, 1996.

Pömare, E., V. Keefe-Ormsby, C. Ormsby, N. Pearce, P. Reid, B. Robson and, N. Watene-Haydon. Hawora: Māori standards of health III. Eru Pōmare Maori Health Research Centre Wellington School of Medicine. Wellington, 1995.

Raeburn, J. "The community in health research and policy". In Social dimensions of health and disease New Zealand perspectives. Edited by Spicer J, Trlin A and Walton J, Palmerston North: Dunmore Press, 1994.

Scott, G., M. Camden, and H. Scott. "Pitfalls in aggregating data and using rates for policy decisions." Wellington Polytechnic Occasional Papers 98/1 (1998): 1-22.

Scott, W. G., and H. M. Scott. "Benign prostatic hyperplasia in New Zealand: a cost of illness study". PharmacoEconomics 1993;4(6):455-68.

Scott, W. .G., and H. M. Scott. "Heart failure: a decision analytic analysis of New Zealand data using the published results of the SOLVD treatment trial". PharmacoEconomics $1996 ; 9(2): 156-67$. 
Simpson, E. H. "The interpretation of interaction in contingency tables". Journal of the Royal Statistical society $1951 ; 13: 387-94$.

Steel, D. G., and D. Holt. "Analysing and adjusting aggregation effects: The ecological fallacy revisited”. International Statistical Review 1996;64(1):39-60.

Westbrooke, I. "Simpson's paradox; an example in a New Zealand survey of jury composition". Research and Analytical Report. Statistics New Zealand, 1997.

Westbrooke, I. "Simpson's paradox in New Zealand data". 47th Annual Conference of the New Zealand Statistical Association, Wellington, 30 and 31 August, 1996.

Wild, C. J., and G. A. F. Seber, eds. Introduction to probability and statistics. Auckland: Dept of Statistics, University of Auckland, 1996. 


\section{CHAPTER 9: ANTIBIOTIC COSTING}

\subsection{SUMMARY AND INTRODUCTION}

\section{Summary}

Micro costing techniques were used to quantify costs associated with antibiotic regimens for serious infections in three New Zealand public hospitals. A decision analytic model suitable for costing any antibiotic regimen was constructed and tested. The costing data were based on observation rather than the more usual self recorded diary entries. The model developed in this study provided a new tool for hospital administrators and clinicians to more accurately compare the full costs of antibiotic regimens.

\section{Introduction}

This chapter discusses and illustrates a cost-of-treatment analysis (see Chapter 4: Costs) in the evaluation of antibiotic treatments used within New Zealand hospitals (Scott et al., 1999). Tightening budgets within the New Zealand health system have increased the need for clinicians and managers in hospitals to consider the cost-of-treatment regimens as well as clinical efficacy and safety when selecting treatment options.

Objectives of the study were to identify and quantify the cost components of antibiotic regimens used in hospitals in the treatment of patients with severe infections. This information was used to construct a decision analytic model. The model would enable hospital clinicians and managers to compare the cost of new and current antibiotic regimens. 


\subsection{METHOD}

\subsubsection{Target Population, Perspective and Overall Method}

This study was collaborative, multicentre and multidisciplinary involving medical and economic elements. The target population was hospital patients with severe polymicrobial infections in intensive care or febrile neutropenia in immunocompromised patients in haematology departments. Patients were eligible for inclusion in the study if they were treated with a particular monotherapy, or could have potentially been treated with this regime. The analytical perspective adopted was that of a hospital rather than society as a whole because the policy question related to the hospital management of serious infections. Approvals for the study were obtained from the relevant ethics committees of the three hospitals. As the study was observational and non-intrusive it did not alter treatment or clinical decision making.

Research was conducted in intensive/ critical care units of three hospitals (Auckland, Christchurch and Palmerston North), and in the haematology departments of two hospitals (Christchurch and Palmerston North). Both Auckland and Christchurch hospitals were major hospitals in a main city in the North and South Islands respectively, and Palmerston North hospital was representative of a large hospital in a provincial city. Christchurch was used as a pilot centre to develop the study protocol and data collection methods. Data collection forms were designed in consultation with hospital staff in each centre, tested in Christchurch then modified. Information was gathered sequentially from each centre commencing in late November 1995 and ending in March 1997.

Data were acquired from a prospective time and motion study (to quantify time taken and materials consumed in various stages of the treatment process), and from a retrospective study of hospital records (chart reviews and laboratory files). Treatment processes were split into a number of activities and the component costs of each activity identified. A clinically qualified observer recorded staff time, drugs and materials used for each activity. A similar approach was used by (Malek et al., 1992; Atkinson et al., 1989). 
Resource utilisation observations for the study were patient treatment days. Data were collected only for complete hospital department day stays per patient. That is, the patient had to be in the department for a complete day of treatment. It was not possible to use data relating to part of a day because the exact fraction could not be determined from patient records.

Labour rate information was derived from the literature (Norton, 1997), and from consultation with health sector managers. All unit costs and prices were in 1997 New Zealand dollars.

\subsubsection{Investigative Steps}

The investigation took the following steps:

- $\quad$ Project setup

- Definition of the research question and overall approach

- Study protocols established

- Ethical approval

- Costing method developed

- Antibiotic treatment protocols defined

- Resources identified

- Data collection instruments designed

- Data collection method trialed

- Data collection method and forms revised

- Resource volumes and unit costs data collected

- Data analysis

- Decision analytic model designed and constructed

- Sensitivity analysis completed 


\section{Setup}

A considerable amount of qualitative research was required to clarify the policy question, the research objectives and general approach. The health care staff of the hospitals were familiar with clinical research, but not with economic and policy analysis, while the economist team members were not familiar with the clinical treatment processes. Before the methodology could be developed it was necessary to embark on a process of discovery and find out about the complex treatment processes involved. This process was not unidirectional. The policy question, research objectives and an overview of economic costing methodologies were discussed with the clinical staff who would be collecting the data. Establishing the study design and protocol required detailed interviews with hospital medical, nursing and pharmacy staff.

Because the research involved observing and recording the resources used in treating hospital patients it was necessary to prepare a research proposal for approval by the ethics committees of the three hospitals.

\section{Costing method}

A micro-costing analysis of antibiotic treatment regimes used for serious infections was undertaken. Micro-costing is the most precise and detailed method of hospital costing (see Drummond, O'Brien, Stoddart, et al., 1997; Luce et al., 1996, and Chapter 4: Costs). The following list from Kerr, Barr, Smyth, et al. (1992) is an example of the activities that can be costed in such a study; insertion of intravenous cannula, drug delivery, drawing of blood, sharps disposal, and delivery of blood to the laboratory. The following were costed; drug preparation, administration and monitoring in detail. Similar listings of cost items can be found in Davis and Bryson (1994) and Atkinson et al. (1989). 
Drug treatment regimens were defined first by clinicians and subsequently verified by a retrospective analysis of patient records. Treatment processes were broken down into a series of distinct activities, (1) preparation and administration and (2) monitoring, that could be observed and costed.

Relevant resources specifically required or altered by a treatment regimen were identified and listed. Costs incurred, irrespective of the antimicrobial treatment regime used, or costs that would not be changed by the selected treatment, were not applicable to the study. For example, the insertion of a central intravenous line would not be relevant if all patients irrespective of the treatment received required such a line.

When the various activities and resources were identified, the data collection instruments for obtaining resource utilisations and unit costs were developed, trialed and refined. Drug preparation and administration used two instruments; one for obtaining information on the antibiotic and consumables (Figure 9.1: Antibiotic and Consumables) and another for measuring staff labour (Figure 9.2: Drug Administration Time). Monitoring resource utilisations and unit costs required a further two instruments, one for consumables (Figure 9.3: Consumables and Laboratory Charges), and another for labour (Figure 9.4: Drug Monitoring Time). Unit costs were obtained from each of the hospitals involved in the study.

\section{Data analysis}

Resource volumes measured by the micro-costing method and unit costs applicable to each hospital were used to convert resource utilisations into costs measured in monetary values. Volume and unit cost data were recorded separately for each centre. An implicit price per resource unit was calculated by dividing the average cost per patient day data by the average volume per patient day for each different type of unit used. This gave a weighted average of prices over the three geographic centres and was used in the decision analytic model. 
Administration and preparation times were combined because of the difficulties encountered with overlap and merging of these activities. Infusion times derived from protocols were not measured by the time and motion study. Analysis was conducted on groups of items; for example, syringe sizes from 5 to $20 \mathrm{ml}$ were summed and analysed in aggregate to derive mean resource cost and resource volumes used. However, within these aggregates the actual price for each syringe size was used. Accordingly, implicit unit costs for syringes differ between the different drugs analysed because different mixes of types of syringes were used. This was also done for fluid additives, needles (sharps and nonsharps), and fluid bags. This analysis produced weighted average costs and volumes of items unique to each drug regime.

One drug only was monitored and the rate of monitoring was obtained from a chart and laboratory record review within each of the study hospitals. Monitoring rate was the total number of monitoring events divided by the total number of patient treatment days for the drug. A micro-costing study determined labour and consumables required per monitoring event. The resource units per monitoring from the micro-costing study were multiplied by the rate of monitoring to derive the monitoring resource utilisation per patient treatment day.

A deterministic decision analytical model (Chapter 7: Models) was constructed and used for single and multiple parameter sensitivity and scenario analysis (Chapter 4: Costs). This modelling approach permitted the costs of treatment using any antibiotic to be evaluated. Inputs to the model were unit costs and volumes of resources per patient treatment day. The output screen provided information on total treatment cost.

\subsection{RESULTS AND DISCUSSION}

A total of 411 patient days for drug preparation and administration were collected from 94 hospital admissions for treatment. Final analysis of aggregated drug data was confined to those drugs for which 20 or more observations or patient days were recorded. Thus, the study analysed 83 patients for whom a total of 360 treatment days were recorded. 
The study focus was on evaluating treatment costs. Neither the severity of the infection, nor patient outcomes concerning efficacy or adverse events were considered. A full economic study (Chapter 6: Economic Evaluation) would require the evaluation of patient outcomes, drug acquisition, preparation and administration costs, monitoring, length of hospital stay, and the cost of adverse events (see Beringer et al., 1998; Birmingham et al., 1997).

While the cost of adverse events is acknowledged as important in the treatment of such patients, the evaluation of these events is difficult for the following reasons. Very large numbers of patients are required to detect infrequently occurring adverse events. There are obvious ethical impediments to setting up traditional randomised placebo controlled clinical trials to collect such information. It is also difficult to attribute an adverse event to a particular drug where a combination of drugs is used, or in a situation in which regimens are continually changing. As most patients were seriously ill there would have been difficulties in determining if an adverse event was a result of the treatment or the underlying condition of the patient. Other investigators have also omitted evaluating the cost of adverse effects (Kerr et al., 1992; Malek et al., 1992).

Although there were differences between the three hospitals in the prices of antibiotics and other materials used, most of this could be explained by differences in timing of purchases and negotiated price. Commercial sensitivity precluded disclosing price data from individual hospitals. This problem is not peculiar to New Zealand, other investigators (Malek, Lynch, Wells, et al., 1992) have encountered similar problems with commercially sensitive data in the UK.

Administration and preparation times were combined in the analysis because it was difficult to differentiate between the two tasks. There was little variation in preparation and administration times between hospitals and departments and the differences that did occur may be explained by some staff not being as familiar with routine procedures as other more 
experienced staff or by difficulties with a particular patient. For example, a child who may require coaxing before a medication is given.

A hospital's financial costs (monetary outlays) with respect to nursing staff will not normally be lowered by reducing the labour component in the administration of drugs because these patients frequently require one-to-one nursing. There is however, an opportunity cost as the nurse could be providing other care to the patient (Atkinson, Chambers, McGinlay, 1989). Another point is that advances in technology could alter the one-to-one nursing requirement.

Wastage of antibiotics, defined as the unused portion from a pack, occurred with only two drugs. While wastage may indicate that an inappropriate pack size has been selected or that the appropriate pack size was unavailable, wastage may not necessarily add to costs. For example, it may be more time consuming for staff to use a number of smaller packs, and/or a combination of smaller pack sizes may be more expensive than one larger pack.

Drug protocols are continually evolving and during the course of the study there was a move to once-a-day administration and a greatly reduced frequency of monitoring. However, the model was designed so that protocol changes such as this could be taken into account.

The drug regimen chosen is determined by known adverse reactions, drug resistance and the patient's severity of illness. The study did not take into account the spectrum of cover of the various antibiotics and the susceptibility of micro-organisms to specific antibiotics. Many of the recently introduced antibiotics have a broader spectrum of cover than the older products. Narrow-spectrum antibiotics are often combined (for example, triple combination therapy) to provide the same spectrum of cover provided by a single broadspectrum agent. When comparing costs of antibiotic combinations offering the same coverage, the additive costs of a combination should be taken into account and compared with the cost of the single agent. 


\subsection{POLICY POINTS}

The study was multicentred which in itself created issues for data collection, consistency and quality control. Clear protocols for the study had to be set up and efforts made to ensure the protocols were interpreted in the same manner and uniform data collection methods used. Each centre was monitored and the data carefully checked to assure accuracy and consistency.

The multicentre nature of the study and that fact the data was collected by persons in addition to their normal work meant the data collection period took longer than planned. When antibiotics in a hospital are being administered there is much activity, and recording consumables used posed some problems. These were resolved by collecting ail packaging of consumables in a container to be recorded later.

Recording labour times raised issues of staff management as people were not comfortable in being monitored in their work. Staff were very suspicious of any time and motion study and careful explanation had to be given that this was not a cost cutting management exercise.

The information gained from this observational study can be considered superior to that gained from a self-recording diary approach. Staff are busy and would not be able to accurately record activities in a diary until after the event. Micro-costing is in essence a time and motion study with a new name, but it has in recent times been neglected by policy analysts who have tended to use data recorded primarily for other purposes or from diary records.

Although the study results and model could be used for benchmarking and comparisons between hospitals commercial sensitivity precluded this. Commercial sensitivity is a major barrier to the gathering of data necessary for informed policy evaluation by people other than the financial officers of a hospital. This could be overcome by a data warehousing approach where data is passed to a central source and released to users in an anonymous form. 
Some costs, such as nurse labour although fixed in the short run could be variable as protocols and technology change over the long run. Although administrators may not in the short run have the ability to control some fixed costs, knowledge of these costs is vital for long term planning and policy analysis. The patients in our study had serious infections and protocols dictated that each patient have a nurse allotted to them exclusively; the nurse was thus unable to undertake other duties.

The Gibbs Report (1988) considered that the main cause of technical inefficiency within the public hospital system was the lack of good quality management information and the lack of incentives to use and acquire such information. This study has shown that the methods developed may be used to estimate the full costs of an antibiotic regimen. The model developed enables managers within public hospitals to be better informed on total treatment costs (not just drug purchase prices).

Different policy decision makers could use the results of this study in different ways. Business unit managers and clinicians could jointly use such a model to evaluate and design new antibiotic treatment protocols and monitor the costs of existing policies. Drug purchasing managers and health funding agencies could use the cost comparisons between different antibiotics as a means of obtaining better information for negotiating prices with suppliers. Hospital general management could use the model in benchmarking to compare costs calculated in a uniform manner between different business units within a hospital and between hospitals. 


\subsection{DATA COLLECTION INSTRUMENTS}

Figure 9.1: Antibiotic and Consumables

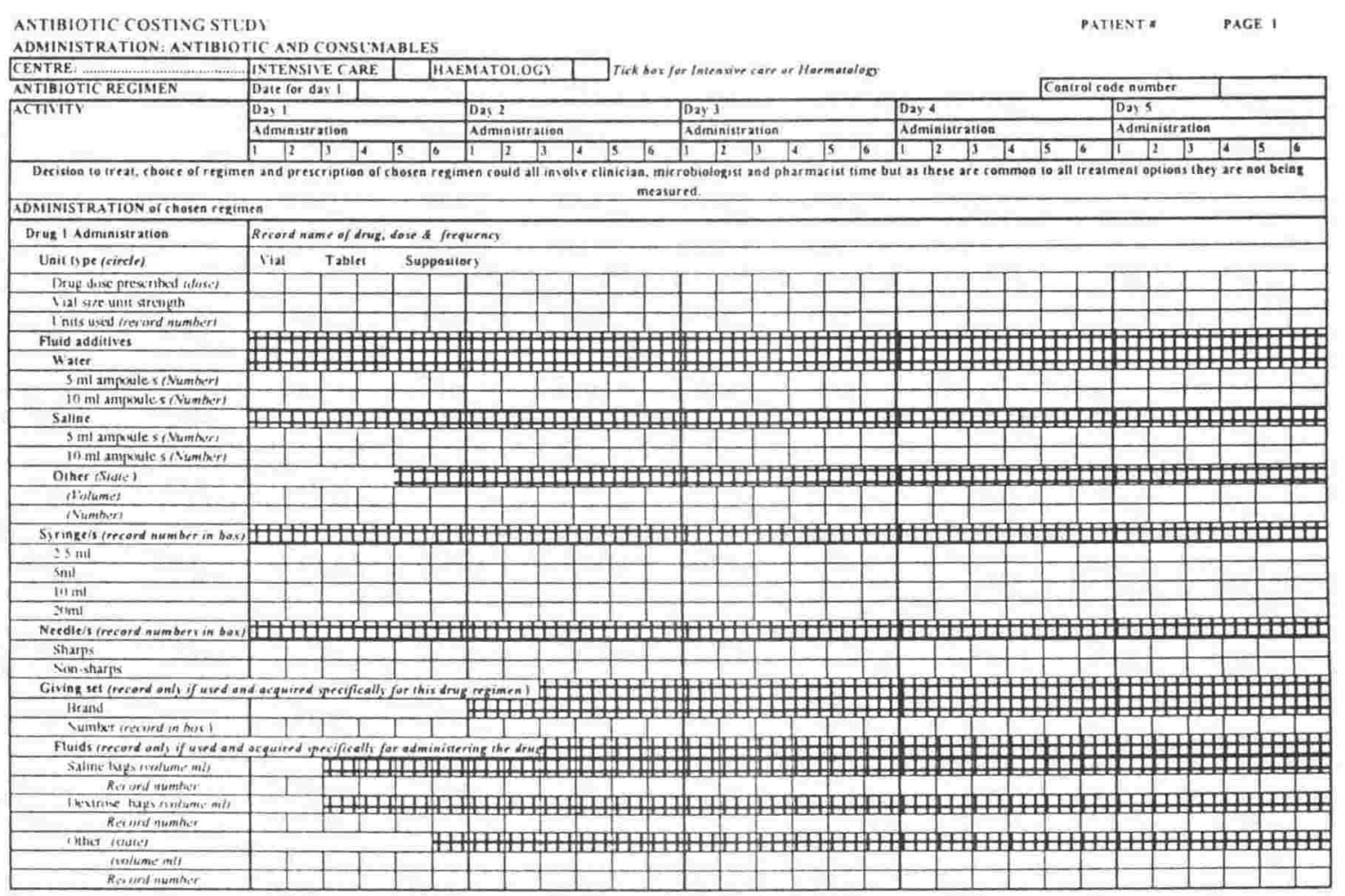


Figure 9.2: Drug Administration Time

ANTIBIOTIC COSTING STUDY

STOPWATCH FORM: DRUG ADMINISTRATION

CENTRE:

INTENSIVE CARE

HAEMATOLOGV

Tick

HAEMATOLOGY

One form per drug

Drug.

\begin{tabular}{|c|c|c|}
\hline \multirow[t]{2}{*}{ Observation } & \multicolumn{2}{|c|}{ Nurse time } \\
\hline & $\begin{array}{l}\text { Preparation } \\
\text { Minutes }\end{array}$ & $\begin{array}{r}\text { Administration } \\
\text { Minutes }\end{array}$ \\
\hline 1 & & \\
\hline 2 & & \\
\hline 3 & & \\
\hline 4 & & \\
\hline 5 & & \\
\hline 6 & & \\
\hline 7 & & \\
\hline 8 & & \\
\hline 9 & & \\
\hline 10 & & \\
\hline 11 & & \\
\hline 12 & & \\
\hline 13 & & \\
\hline 14 & & \\
\hline 15 & & \\
\hline 16 & & \\
\hline 17 & & \\
\hline 18 & & \\
\hline 19 & & \\
\hline 20 & & \\
\hline
\end{tabular}


Figure 9.3: Consumables and Laboratory Charges

ANTIBIOTIC COSTING STUDY

MONITORNG: consumables and laboratory charges

\begin{tabular}{|c|c|c|c|c|c|c|c|c|c|c|c|c|c|c|c|c|c|c|c|c|}
\hline CENTRE: & INT & NSI & EC & & & IHAF & $11 \mathrm{AT}$ & LOO & & & Tick & box ft & Int & insive & care o & or $\mathrm{Ha}$ & emato & $\log y$ & & \\
\hline Drug: Record name of drug $b$ & ing $m$ & nito & & & & & & & & & & & & & & & & & & \\
\hline Observation $\longrightarrow$ & 1 & 2 & 3 & 4 & 5 & 6 & 7 & 8 & 9 & 10 & 11 & 12 & 13 & 14 & 15 & 16 & 17 & 18 & 19 & 20 \\
\hline & Excl & dea & rou & te te & sthe & noul & bes & iden & ken & all & iatien & s rege & irdles & sof th & edrug & gadm & rinister & & & \\
\hline Blood collection & 䎴 & & & & & & & & & & & & & & & & & & & \\
\hline Syringe's (record number) & 曲 & & & & & & & & & & & & & & & & & & & \\
\hline $2.5 \mathrm{mll}$ & & & & & & & & & & & & & & & & & & & & \\
\hline Sml & & & & & & & & & & & & & & & & & & & & \\
\hline $10 \mathrm{ml}$ & & & & & & & & & & & & & & & & & & & & \\
\hline $20 \mathrm{ml}$ & & & & & & & & & & & & & & & & & & & & \\
\hline Needles (record number) & & & & & & & & & & & & & & & & & & & & \\
\hline Sharps & & & & & & & & & & & & & & & & & & & & \\
\hline Non-sharps & & & & & & & & & & & & & & & & & & & & \\
\hline Tubes (record number) & & & & & & & & & & & & & & & & & & & & \\
\hline Transpont iTick hot helow on & if ifo & lorh & on & $m$ & $n n$ & whe's. & (с) & 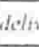 & Th & & & & & & & & & & & \# \\
\hline Transpert fritch bot) & & & & & & & & & & & & & & & & & & & & \\
\hline Lab charge 5 & & & & & & & & & & & & & & & & & & & & \\
\hline
\end{tabular}


Figure 9.4: Drug Monitoring Time

ANTIBIOTIC COSTING STUDY

STOPIVATCH FORM: DRUG MONITORING

CEYTRE:

\begin{tabular}{|l|l|}
\hline INTENSINE CARE & \\
\hline HAEMATOLOGY & bick \\
\hline
\end{tabular}

One form per drug

Drug.

\begin{tabular}{|c|c|c|c|c|c|c|}
\hline \multirow[t]{2}{*}{ Observation } & \multicolumn{2}{|c|}{ Blood collection } & \multicolumn{2}{|c|}{ Obtaining results from lab \# } & \multicolumn{2}{|c|}{ Interpretation of results } \\
\hline & $\begin{array}{l}\text { Doctor } \\
\text { Minutes }\end{array}$ & $\begin{array}{l}\text { Nurse } \\
\text { Minutes }\end{array}$ & $\begin{array}{l}\text { Doctor } \\
\text { Minutes }\end{array}$ & $\begin{array}{l}\text { Nurse } \\
\text { Minutes }\end{array}$ & $\begin{array}{l}\text { Doctor } \\
\text { Minutes }\end{array}$ & $\begin{array}{c}\text { Pharmacist } \\
\text { Minutes }\end{array}$ \\
\hline 1 & & & & & & \\
\hline 2 & & & & & & \\
\hline 3 & & & & & & \\
\hline 4 & & & & & & \\
\hline 5 & & & & & & \\
\hline 6 & & & & & & \\
\hline 7 & & & & & & \\
\hline 8 & & & & & & \\
\hline 9 & & & & & & \\
\hline 10 & & & & & & \\
\hline 11 & & & & & & \\
\hline 12 & & & & & & \\
\hline 13 & & & & & & \\
\hline 14 & & & & & & \\
\hline 15 & & & & & & \\
\hline 16 & & & & & & \\
\hline 17 & & & & & & \\
\hline 18 & & & & & & \\
\hline 19) & & & & & & \\
\hline 20 & & & & & & \\
\hline
\end{tabular}

\# Record outh if a specific offort made to contact lab for results 


\subsection{REFERENCES}

Atkinson, H.C., S. T. Chambers, and A. M. McGinlay. "Antibiotic therapy costs". NZ Med $J 1989 ; 102: 409-11$.

Beringer, P.M., and A.Wong-Beringer, and J. P. Rho. "Economic aspects of antibacterial adverse effects". PharmacoEconomics 1998;13(1):35-49.

Birmingham, M. C., J. M. Hassett, J. J. Schentag, and J. A. Paladino. "Assessing antibacterial pharmacoeconoimes in the intensive care unit". PharmacoEconomics $1997 ; 12(6): 637-47$.

Davis, R., and H. M. Bryson. "Ceftriaxone; a pharmacoeconomic evaluation of its use in the treatment of serious infections". PharmacoEconomics 1994;6(3):249-69.

Drummond, M. F., B. J O'Brien, G. L. Stoddart, and G. W. Torrance. Methods for the economic evaluation of health care programmes. 2nd ed. New York: Oxford University Press, 1997.

Gibbs, A. Unshackling the hospitals: report of the hospital and related services taskforce. Wellington: Hospital and Related Services Task force, 1988.

Kerr, J. R., J. G. Barr, E. T. M. Smyth, and J. O'Hare. "Technique for calculation of the true costs of antibiotic therapy". European Journal of Clinical Microbiology and Infectious Diseases 1992;11(9):823-7.

Luce, B. R., W. G. Manning, J. E. Siegel, and J. Lipscomb. "Estimating costs in cost-effectiveness analysis". In Cost-effectiveness analysis in health and medicine. 1st ed., edited by M. R. Gold, J. E. Siegel, L. B. Russell, and M. C. Weinstein, 176-213. Oxford: Oxford University press, 1996. 
Malek, M., W. Lynch, N. Wells et al. "Antimicrobial practice; a comparison of the costs of ceftazidime therapy and gentamicin combinations in three UK hospitals". Journal of Antimicrobial Chemotherapy' 1992;29:207-17.

Norton, A. "Nursing pay rates continue to widen". Nursing New Zealand 1997;3(8):12.

Scott, W. G., H. M. Scott, S. Henderson, A. Inder, J. Sanders, R. Spearing, C. McArthur, J. Judson, B. Baker, P. Hicks, and P. Cotterell. "Comparative cost of antibiotic therapies for serious infections: a New Zealand 3-hospital study." PharmacoEconomics 16 (1999): 183-92. 


\section{CHAPTER 10: BENIGN PROSTATIC}

\section{HYPERPLASIA}

\subsection{SUMMARY AND INTRODUCTION}

\section{Summary}

This cost-of-treatment study integrated data from a wide range of sources; surgical treatment audits, hospital discharges, and prices. New information on the economic costs of treated benign prostatic hyperplasia was published. The research highlighted the hitherto hidden intangible costs of the side effects of surgery and was instrumental in prompting clinicians to investigate the differences in treatment rates between Māori and non-Māori. The research used hospital discharge unit record data (with patient identifiers removed) which enabled filtering and detailed analysis. To my knowledge this was the first New Zealand study that used newly available unit record discharge data for economic policy analysis. Earlier research work could access only published aggregated data which did not permit filtering and detailed analysis.

\section{Introduction}

The objective of the cost-of-illness analysis, which had a societal perspective, was to estimate the economic cost of treated benign prostatic hyperplasia (BPH). Little attention has been focussed on the cost of BPH because it has been regarded as part of the normal aging process in males. However if attitudes were changed by a health education or health promotion programme, and more men sought treatment, demands on the public health budget would increase. Aspects of this study were discussed in Chapter 8: Care With Data. Detailed information on the methodology and findings can be found in Scott and Scott, (1993). 
$\mathrm{BPH}$ is a nonmalignant enlargement of the prostate that commonly occurs with aging (Barry, 1990). Enlargement of the prostate may cause a variety of urinary problems ranging from acute urinary retention to recurrent urinary tract infections (Christensen and Bruskewitz, 1990). BPH frequency of occurrence increases with age, $70 \%$ of men by 80 years of age will have significant symptoms of BPH (Cockett, 1992). It is uncommon in men under the age of 50 but most men of over 60 years will have some symptoms of prostate enlargement (Griffiths, 1992).

BPH manifests itself in the following symptoms; the sensation of incomplete bladder emptying, a hesitant urinary stream, slow urinary flow, urgency, a need to push or strain to begin urinating, and nocturia (McConnell, 1992). Over a period of several years one-third of patients will spontaneously inprove, one-third will remain stable while one-third will deteriorate. Appropriate treatment options are watchful waiting, surgery or medicines (Denis, 1992).

A patient in New Zealand with symptomatic BPH will usually consult a general practitioner who will normally refer the patient to a specialist urologist in a public hospital or in private practice. If assessed as needing surgery (scoring enough points, see Chapter 3: Valuing Health Outcomes) the patient would be given a booking for public hospital surgery. If not booked for surgery in a public hospital the patient would either be referred back to their general practitioner for watchful waiting or booked for surgery in a private hospital.

\subsection{METHOD}

\section{Range of Relevant Costs}

The costs of BPH from the perspective of New Zealand society may be classsified as follows:

- Direct medical costs

Ambulance

Hospital ward 
Operations

Outpatient and specialist consultations

Ancillary hospital staff (e.g. district nurse)

General practitioner consultations

Medicine prescribed (hospital and community) and associated markups and dispensing charges

Diagnostic procedures and laboratory tests (hospital and community based)

Medical research

- Direct nonmedical

Transport costs of patient, family and friends

Community and voluntary organisations

Additional laundry costs and protection aids

Additional costs associated with premature entry into residential care

- Indirect costs (time related)

Production loss by patients

Production loss by unpaid care-givers

Leisure time loss by patients

Leisure time loss by unpaid care-givers

- Intangible costs

Quality of life of patient, family and friends

Ideally for a cost-of-illness study all of these costs should be evaluated but data covering the full set were not available. For those costs that could be quantified, the latest available resource utilisation (volume) data (at the time of data analysis) were combined with March quarter 1992 prices to calculate total economic costs. All unit costs were net of GST which is a transfer payment from the societal perspective adopted in this study.

\section{Hospital Ward and Surgery Costs}

Public and private hospital admissions and numbers of operations performed for the year ending December 1988 were obtained as unit records on computer diskette from Health Statistics Services (HSS) of the Department of Health. 
Lacking reliable public sector costs, prices from the private sector were used for direct medical costs. At the time of the study, reliable and detailed public sector costing data in New Zealand were not readily available for economic research, and in many instances the data did not exist. Such data, if they existed, were regarded by most hospitals and Regional Health Authorities as commercially sensitive.

Competition between hospitals means that supernormal profits in the private sector are likely to be small, and offset by higher costs in the public sector, where hospitals may have a teaching component and be more likely to treat "at risk" and therefore more costly cases. Public hospitals usually have more back-up facilities such as intensive care units.

The Southern Cross schedule of fees for surgical and anaesthetic procedures current in the March 1992 quarter, was used for prices in relation to preoperative consultation fees, surgery and anaesthetics. Wakefield Hospital in Wellington provided theatre and ward unit costs.

\section{Medical Consultations and Prescriptions}

Without any national data at general practitioner or specialist level it was assumed that each patient admitted to hospital would require two specialist consultations and these were valued using the Southern Cross Medical Care Society schedule of specialist fees. Survey data relating to general practitioner consultations for primary diagnosis BPH were obtained from IMS (1992). These data together with an average consultation fee of \$27.56 (Health Benefits Letter 4, 1991) were used to calculate the cost of consultations.

Medicines prescribed for BPH at general practitioner level were derived from IMS survey data (IMS, 1992). Wholesale and retail markups (Pricing Schedules 1991) were added to the cost at manufacturer selling price (IMS 1992) and the number of prescriptions was multiplied by the average dispensing fee and container allowance (Pricing Schedules, 1991). No data were available on medicines prescribed by specialists or at hospital level. 


\section{Production and Leisure-time Foregone}

The combined value of leisure-time and production (both paid and unpaid) was calculated using the average hourly earnings for males and an estimated two weeks for patients undergoing surgery and one week for hospital patients not requiring surgery. This time lost from normal activities was estimated from in-depth interviews with a number of urologists. Average hourly earnings for males was used as a proxy for the value of all leisure and work time.

\section{Quality of life}

The study attempted to collect information on aspects of the quality of life of patients with BPH. A literature search was undertaken to see if there were any patient support groups within New Zealand and find studies (both published and unpublished).

\subsection{RESULTS}

Estimates of the total direct medical costs of BPH in New Zealand were $\$ 14$ million, and production loss and leisure time loss by patients $\$ 4$ million (Table 10.1). Direct medical costs accounted for almost $80 \%$ of total quantified costs.

In New Zealand the most important operation done for BPH is transurethral prostatectomy (TURP). If this operation was performed in a public hospital, patients on average stayed 8.5 days, while those in private hospitals stayed 4.3 days. The mean age of these public hospital patients was 71 years compared with 67 years for those in private hospitals. 
Table 10.1: Summary Of Quantified Costs For BPH

\begin{tabular}{|c|c|c|c|}
\hline Cost (1991 resource volumes in 1992 dollars) & $\begin{array}{l}\text { Low } \\
\$ \\
\text { million }\end{array}$ & $\begin{array}{l}\text { Base case } \\
\$ \\
\text { million }\end{array}$ & $\begin{array}{l}\text { High } \\
\$ \\
\text { million } \\
\end{array}$ \\
\hline \multicolumn{4}{|l|}{ Direct medical costs } \\
\hline Hospital ward & 6.510 & 7.552 & 8.760 \\
\hline Operations & 4.742 & 5.501 & 5.501 \\
\hline Outpatients and specialists & 0.285 & 0.331 & 0.496 \\
\hline General practitioners & 0.234 & 0.234 & 0.234 \\
\hline $\begin{array}{l}\text { Medicines, dispensing fees and container } \\
\text { allowances }\end{array}$ & 0.152 & 0.152 & 0.152 \\
\hline Laboratory tests & 0.189 & 0.219 & 0.219 \\
\hline Diagnostic procedures & 0.144 & 0.167 & 0.167 \\
\hline Total direct medical costs & 12.256 & 14.156 & 15.529 \\
\hline \multicolumn{4}{|l|}{ Indirect costs } \\
\hline $\begin{array}{l}\text { Production loss by patients under } 65 \text { years } \\
\text { (unadjusted for labour participation and } \\
\text { unemployment) }\end{array}$ & 1.082 & 1.255 & 1.255 \\
\hline Leisure time lost by patients 65 years and over & 2.566 & 2.977 & 2.977 \\
\hline Total Indirect costs & 3.648 & 4.232 & 4.232 \\
\hline Total quantified costs & 15.904 & 18.388 & 19.761 \\
\hline
\end{tabular}

HSS data reveal an overall 16.2\% increase in admissions for BPH during the period 1988 to $1991^{16}$. Public hospitals recorded a $14 \%$ increase and private hospitals recorded an increase of $20 \% \mathrm{in}$ admissions. Accordingly, all 1988 volumes that were dependent upon hospital admissions were increased by $16 \%$ to obtain a 1991 volume estimate - the base case estimate of $\$ 18.388$ million. (Direct costs of $\$ 14.156$ million, indirect costs of $\$ 4.232$ million).

A high estimate was obtained by increasing the base case estimate of hospital ward costs

16 Between 1988 (hospital unit record data used to estimate costs) and 1991 (latest published volume data on hospital admissions available at time of finalising the paper) the volume of admissions for principal diagnosis BPH to all hospitals increased by $16 \%$. 
by $16 \%$ (to take account of higher daily ward costs as indicated by Wellington and Christchurch data), and outpatient and specialist consultations by $50 \%$ (based on survey data), to give a total of $\$ 19.761$ million. (Direct costs of $\$ 15.529$ million, indirect $\$ 4.232$ million).

Hospital admission information in New Zealand are recorded at time of discharge or death. In 1988 there were $1.9 \mathrm{BPH}$ patients per thousand males of all ages admitted to all hospitals. The equivalent rates for males 50 years and over were; Mãori 3.4, non-Māori 8.7, and all males 8.5. The data indicate that the prevalence of BPH among the Māori population could be lower than that for non-Māori. Chapter 8: Care With Data for a discussion of the observed differences between Māori and non-Māori.

Admission data (BPH Diagnosis A) for public hospitals were analysed with respect to type of admission and source (comparable data were unavailable from private hospitals). Fiftynine percent of admissions came from waiting lists, $27 \%$ were acute admissions and $14 \%$ were arranged (by general practitioners, specialists or outpatient departments). Some patients with acute urinary retention present directly at accident and emergency departments of public hospitals. Most hospitals admit directly to the appropriate ward but as some major hospitals admit all patients through the accident and emergency department very little can be read into this manner of admission.

Thirty-seven percent of patients ware referred for admission by specialists, $32 \%$ by outpatient departments, $23 \%$ by general practitioners, $5 \%$ by accident and emergency, and $2 \%$ by other hospitals. Analysis of admission data suggested a greater degree of monitoring by general practitioners in small centres and rural areas at some distance from a urologist.

Analysis of admission and discharge dates for BPH Diagnosis A (all types of surgery not only TURP) showed that the mean days stay was higher for public hospital patients; (public) 8.9 days compared with (private) 4.6 day's (for all ages).

From a total of 79 patients (survey of urologists in both private practice and public hospitals undertaken by the authors) 33 patients presented with perioperative complications (within 
30 days of surgery) and 25 patients presented with late postoperative complications (over 30 days). The complication rate for perioperative complications was $42 \%$ and $32 \%$ for postoperative complications. All 79 patients had a TURP.

The survey also indicated that the following laboratory tests were routinely undertaken for all patients in both public and private settings; urine culture, prostatic acid phosphatase, prostate specific antigen, complete blood count, electrolytes, group and hold blood (half of the patients), and post-discharge urine culture (half of the patients). The total cost of these tests was $\$ 0.189$ million.

Although the survey showed that the following diagnostic procedures were undertaken the data were insufficient to extrapolate for all specialist consultations. The procedures undertaken were; intravenous polygram, plain abdominal X-ray, chest X-ray, abdominal ultrasound, urinary flow rate, and electrocardiogram. It was clear that virtually all patients had an X-ray of some sort.

The following costs were either unquantified or not isolated because of inadequate data; ambulance, ancillary hospital staff, medicine prescribed at hospital and specialist levels, production loss and loss of leisure time by unpaid care-givers, transport costs of patient, family and friends, services provided by community and voluntary organisations, medical research, additional laundry costs and protection aids, premature entry into residential care, auditional home help when a patient is partially incapacitated, pain and suffering for the patient and their effects on the family, quality adjusted life years lost.

\subsection{DISCUSSION}

A complication rate of $2.02 \%$ was recorded for TURP in public hospitals but surveys (conducted by the researchers) in both public and private settings suggested that the complication rate is substantially under-recorded. This could have a major impact on reducing the quality of life status of patients undergoing surgery but this could not be quantified. 
The direct medical costs of $\mathrm{BPH}$ account for almost $80 \%$ of the total quantified costs. Hospital ward and operation costs account for just over $90 \%$ of the direct medical costs. However, ward costs are underestimated in the study as BPH diagnoses B, C, and D were excluded (it was impossible to isolate ward costs attributed solely to BPH where admission was primarily for another condition, (see joint costs in Chapter 4: Costs). As most BPH patients in New Zealand are over 65 years of age, paid production loss is relatively unimportant in relation to total costs.

Hospital ward stays and operations are the 2 areas where savings would have maximum impact on total costs. A substantial reduction in costs could be achieved if the length of stay in hospitals could be reduced. Public hospital patients stay on average 8.9 days compared with 4.6 days for private hospital patients (all types of surgery for BPH). If the public hospital average ward stay could be reduced to that of private hospitals then total ward costs would fall by $37 \%$, total direct costs would fall by $21 \%$ and total quantified costs by $16 \%$. This would implicitly assume that the patient outcomes and patient profiles are similar. Not withstanding these qualifications, the differences in length of stay are worthy of investigation.

Reasons were sought for the disparity in the length of stay between public and private hospital patients. The case mix of public and private hospitals is likely to differ in that older, at risk, and emergency patients are more likcly to be admitted to public hospitals which are more able to cope with complications, and have a more intensive level of care and monitoring.

Factors were investigated that could possibly affect length of stay and that could be examined using HSS data. Age and discharge type (covering classifications of dead, routine, self discharge, and transfers to other types of institutional care) were analysed. The average length of stay for all age cohorts in private hospitals was about half that of public hospitals. There was little difference in discharge type with $97 \%$ of patients in public hospitals and $99 \%$ of all patients in private hospitals recorded as routine discharges. It was thought that had there been substantially more patients in the public system returning to 
nursing homes or other hospitals this could explain a longer hospital stay as presumably such patients would require a more intensive level of care. As the private hospital data base covers only the principal diagnosis and principal operation it was impossible to determine if there was a difference in health status between the public and private hospital patients by reference to multiple diagnoses and operations. The identified differences in case mix do not fully explain why patients stay twice as long in public hospitals as patients in private hospitals.

Surgery was the most common treatment option for most patients with symptomatic BPH. Watchful waiting and/or treatment with medicines was relatively unimportant at the time of the study but should either of these two options assume greater importance there will be follow-on costs in areas of laboratory and diagnostic investigations, specialist consultations and medicine prescribed. But if treatment with medicine could avoid the need for surgery or lessen the hospital stay this treatment option could substantially reduce costs. If, however, surgery is merely postponed rather than avoided, surgery costs are simply moved forward in time, and the medicine costs become an additional cost. Moving the cost on in time reduces the net present cost. However, the patient may enjoy an improved quality of life and older patients may die from unrelated causes before surgery is needed.

Laboratory tests and diagnostic procedures were under-reported in this study as data at general practitioner level were unavailable. In addition, although the urologist audits recorded tests undertaken, the sample sizes were considered to be too small to allow rates to be applied to all patients except for the major tests carried out on most patients. Both the tests and the procedures were conservative estimates of the true costs.

Laboratory tests and diagnostic procedures are areas in which changes are occurring and a measure of the impact of potential changes can be gained by consideration of possible additions or deletions to the tests costed. The prostate specific antigen test is likely to replace the cheaper prostatic acid phosphatase test as it is regarded as being more sensitive as a tumour marker for cancer of the prostate. Both tests were included in the study, but if the phosphatase test is deleted the measured costs would fall by only \$0.019 million. 
Changes in tests and procedures with the introduction of new technology could increase costs but may have little impact on the overall cost of medical treatment as this area forms only a small percentage of the total direct medical costs. It is realistic to assume that each patient may in the future receive an abdominal ultrasound which would increase the cost of diagnostic procedures by $157 \%$. While this would more than double the total cost of diagnostic procedures it would only increase the direct medical costs by $1.7 \%$. Conversely, confining chest X-rays ${ }^{17}$ to only those patients over 65 years would lower the cost of diagnostic procedures by $23 \%$ and lower total direct medical costs by $0.3 \%$. However, as the current cost of diagnostic procedures is $1 \%$ of direct medical costs none of these changes will make a meaningful difference to the costs of $\mathrm{BPH}$.

Complications recorded for surgery in this study came from three different sources, each at a different stage of treatment for BPH. Hospital data recorded complications within the duration of the patients' hospital stay, the survey collected data within 30 days of surgery, and after 30 days of surgery, and Gilling's study (1988) interviewed patients at a minimum of 6 months following surgery (sexual dysfunction only was studied).

Hospital staff may regard some problems/complications associated with surgery as normal occurrences and therefore may not record them as the survey indicated a higher complication rate. Recording compliance is likely to be higher in a survey, and as the survey covered a post-discharge period a higher complication rate would also be expected but the difference between the hospital data and the audits was sufficiently large to assume that under-reporting is occurring within hospitals. (Under-reporting of complications will not have resulted in an underestimate of hospital ward costs as these were fully captured if the patient was admitted under BPH diagnosis A).

There may also be an inadequate appreciation of postoperative problems facing BPH patients in New Zealand if patients do not volunteer information on what they may regard as a sensitive subject. Although most of the complications of the treatment for BPH are not

17 Required to check for underlying conditions that could increase the risks from surgery. 
life threatening and add little to direct medical costs they are important in terms of quality of life. Society may not fully appreciate the magnitude of the reduced quality of life resulting from symptomatic BPH and its possible treatment complications for the patient. As information on this topic were not available from other sources, more research would be required to quantify the quality of life costs.

A possible lower prevalence of BPH among Māori may be explained by the fact that the Māori age cohort for those 50 years and over has a lower ratio of older people than the same age group in the non-Mäori population. Furthermore, as small numbers are involved this apparent difference in rates should be interpreted with caution. A reluctance to use the health system for social and economic reasons may in part explain the lower number of Māori presenting for treatment. A clinical investigation (Nacey, Morum and Delahunt, 1993) found that no statistical difference in rates could be demonstrated between European, Māori or Polynesian males aged 50 and over. (See Chapter 8: Care with Data)

Papers presented at the International Consultation on BPH (1992) produced evidence that suggested that the rate of BPH in the community is under-reported. Many people consider $\mathrm{BPH}$ to be part of the normal aging process of males and tend to accept attendant problems. If these attitudes change and BPH symptoms become less acceptable, more focus will be placed on intangible costs. Society may be prepared to accept higher direct medical costs to achieve improved quality of life. As the New Zealand population ages the prevalence of BPH will increase.

\subsection{POLICY POINTS}

Ward costs were underestimated in the study as diagnoses B, C, D were excluded as costs could not be isolated from the primary diagnosis. This is a common problem when attempting to apportion shared or joint costs, typically overheads. (Chapter 4: Costs discusses joint costs and related co-morbidities). 
This study used male wage rates to evaluate production and leisure time foregone but lower cost estimates would have been obtained had the male and female combined rate been used. Using the combined rate is important if policy makers are to allocate funds in a gender neutral manner. This means that published results in the literature may need to be adjusted to take this into account as the publication may not be targeting policy makers. (See Chapter 4: Costs)

Under-reporting of complications may have arisen as some clinical conditions were regarded as normal occurrences and therefore may not have been recorded as complications. Because the complications of surgery have the potential to greatly reduce the quality of life of patients, they are important when making policy choices from a societal perspective. Further research would be desirable to quantify quality of life changes as this could have a major impact on policy recommendations.

Apparent differences in disease prevalence between Māori and non-Māori were probably a result of Māori not accessing health services. Data on hospital admissions needs to be interpreted with care if inferences are to be made about disease prevalence and allocation of resources. See Chapter 8: Care With Data.

If societal attitudes change from a condition considered to be normal to a disease that is not acceptable, provision of more medical intervention may be needed. Although this would increase health care resource utilisation and impose greater pressure on health budgets it must be weighed up against quality of life gains. As the population ages this particular disease will assume greater importance. The cost of increased health resource utilisation should be fully accounted for when considering the costs and benefits of a health education or promotion programme.

This case study illustrated the need to assemble and integrate data from a wide range of sources. The process of integrating data requires transparent careful reporting so that policy makers may make fully informed decisions. This is a particular problem when the results of such a study are presented to decision makers with clinical or accounting backgrounds where data is usually derived from a single source (clinical trials or financial 
records).

Particular problems arising out of this study were the difficulties of obtaining any data other than hospital admissions and surgery costs and the need to cross-check data using different sources and studies. Policy makers could use the results of the study to estimate the costs of "hidden" illness in the community. This "hidden" illness would surface if government, support groups, clinicians or private firms embarked on educational and health promotion policies and campaigns.

In summary the results of this study could be used in the following ways by:

- Ministry of Health policy makers to assess the potential cost consequences of an educational campaign that provides information about BPH and treatment options,

- hospital health funding agencies in setting up contracts with hospitals. 


\subsection{REFERENCES}

Barry, M. J. Epidemiology and natural history of benign prostatic hyperplasia. Urologic Clinics of North America 17(3): 495-508, 1990

BPH data relating to 1988 (D-base 3 format on 3.5 disk). Health Statistical Services, N.Z. Department of Health, Wellington, 1991

Christensen, M. M, and R. C. Bruskewitz. Clinical manifestations of benign prostatic hyperplasia and indications for therapeutic interventions. Urologic Clinics of North America 17(3): 509-516, 1990

Cockett, A. T. K. General introduction to BPH and the International Consensus. The International Consultation on BPH, Paris, March 6, 1992. World Health Organization, Paris, 1992

Denis, L. Treatment of BPH. The International Consultation on BPH, Paris, March 6, 1992. World Health Organization, Paris, 1992

Griffiths, K. Hormonal treatment of BPH. The International Consultation on BPH, Paris, March 6, 1992. World Health Organization, Paris, 1992

Health Benefits Letter 4: 29 August 1991, 4:17 October 1991 (N.Z. Department of Health)

IMS (N.Z.) Limited. Data relating to general practitioner consultations and prescriptions. 1992

McConnell, J. How is BPH diagnosed? How will the guidelines help? The International Consulution on BPH, Paris, March 6, 1992. World Health Organization, Paris, 1992 
Nacey, J. N., P. Morum, and B. Delahunt. The incidence of benign prostatic hyperplasia in Caucasian, Māori and Polynesian men. A comparative community based study. Poster presented at the 46th Annual Scientific Meeting, Urological Society of Australasia, Hobart, February 28 - March 5, 1993

Pricing Schedules 1 August 1991. (Medicine dispensary fees, container allowances and markups) (N.Z. Department of Health)

Scott, W. G., and H. M. Scott . 1993. Annual costs of Benign Prostatic Hyperplasia in New Zealand. PharmacoEconomics, 4 (6): 455-468.

Southern Cross Medical Care Society. Schedule of fees for surgical and anaesthetic procedures. 17 th rev. The Society, Auckland, 1991 


\section{CHAPTER 11: HYSTERECTOMY}

\subsection{SUMMARY AND INTRODUCTION}

\section{Summary}

This chapter discusses another cost-of-illness study and issues of equity and efficiency. For the first time in New Zealand, incidence rates and costs of hystersctomy were isolated for women between the ages of 15 and 55 years undergoing hysterectomy for non malignant conditions. Prior to this study, published data related to total hysterectomies. In this study unit record hospital discharge data were filtered and used to estimate incidence rates and costs.

\section{Introduction}

This chapter presents the results from a cost-of-illness study (Scott and Scott, 1995) (see also Chapter 4: Costs). The analysis sought to establish the annual hospital and indirect costs to New Zealand society of hysterectomy for nonmalignant menstrual conditions. The target population was women between puberty and menopause. Comparison of New Zealand's rates of hysterectomy with those of other developed countries within the OECD was also undertaken.

Accurate cost data is essential if alternative interventions that could reduce the need for, or the economic cost of surgical procedures, are to be compared with existing surgical treatments. Policy options evaluated could include alternatives to surgery and different hospital management systems. Economic evaluations for policy analysis require both cost and health related outcome data. Cost-of-illness studies provide the costing data required for policy analysis. This information would also assist both funders and providers to negotiate contract prices. 


\subsection{METHOD}

Costs were measured incrementally from the perspective of society. Public hospital costs were estimated by multiplying the public hospital Diagnostic Related Groups (DRG) unit cost by the number of patients for each operation. A similar calculation was made for private hospitals using private hospital cost data.

The combined total of production and leisure-time loss was calculated by multiplying the average number of weeks incapacitated by surgery by the average weekly earnings (as a proxy for the value of production and leisure time forgone). Discussions with nurses and patients suggested that a conservative estimate of the time a patient was incapacitated could be made by using three times the hospital stay.(a literature review did not uncover any more definitive data). Numbers of surgical procedures for 1991 were estimated from unit record data in electronic format (N.Z. Department of Health, 1993). This data encompassed all New Zealand hospitals, both public and private.

Data were sorted and a subset made which excluded patients with indications of malignancy, infections, and congenital abnormalities. Patients under the age of 15 years and over the age of 54 years were also excluded from the subset. This was done because the focus of the study was hysterectomy for nonmalignant conditions in women between the ages of 15 and 54 years.

The public hospital unit cost of $\$ 4,736$ was derived from DRG 359 uterine and adnexa procedures for non-malignancy age less than 70 without co-morbidities and/ or complications (National Advisory Committee on Core Health and Disability Support Services, 1992). Private hospital unit costs from the Southern Cross Medical Care Society (1991), and Wakefield Hospital, Wellington (1992) were also used as an alternative method

for evaluating costs in the private sector. Costs of the surgeon and the anaesthetist were taken from the Society, and the ward and theatre costs were provided by Wakefield Hospital, Wellington. 
Public hospital costs were estimated by multiplying the public hospital DRG unit cost by the number of patients for each operation. Private hospital costs were calculated using private hospital cost data. Daily ward cost multiplied by the total number of days in the hospital was added to the unit cost of the operation (excluding ward costs) multiplied by the number of operations.

\subsection{RESULTS}

The all ages all conditions hysterectomy rate per 100,000 women was 365 in New Zealand in 1991 compared with 431 in 1980. This rate for New Zealand is similar to that of Australia, lower than Canada and the US but is much higher than in Japan and some European countries. Time series data showed that rates in most OECD countries (including New Zealand) have been falling since the late 1970's (OECD, 1993; Office of Health Economics, 1992).

In 1991, 6,331 hysterectomies (for all diagnoses, all operations and all ages) were undertaken in New Zealand ( $50.5 \%$ of these operations were carried out in public hospitals, and $49.5 \%$ in private hospitals). Both the numbers of all hysterectomies in public and private hospitals and the rate per 100,000 females are shown in Table 11.1. Hysterectomies for nonmalignant menstrual conditions $(4,390)$ represent $69 \%$ of the total hysterectomies undertaken in New Zealand in 1991. The hysterectomy rate per 100,000 females (15 to 54 years) was 446 .

Table 11.1: Hysterectomies 1991

\begin{tabular}{|c|c|c|c|c|c|}
\hline \multirow[t]{2}{*}{ Operation } & Public & Private & All & Female & Ratel \\
\hline & hospitals & hospitals & hospitals & population & 100,000 \\
\hline All hysterectomies, all ages & 3,199 & 3,132 & 6,331 & $1,733,700$ & 365 \\
\hline \multicolumn{6}{|l|}{ Hysterectomies, 15 to 54 years, } \\
\hline for nonmalignant conditions & 1,981 & 2,409 & 4,390 & 984,120 & 446 \\
\hline$\%$ of all Hysterectomies & $61.9 \%$ & $76.9 \%$ & $69.3 \%$ & & \\
\hline
\end{tabular}

Note: Because of rounding individual items may not add exactly to the totals shown. 
Table 11.2 shows that public hospital patients are about 1 year younger than private hospital patients, and stay on average one day longer in a hospital. Private hospitals conduct $55 \%$ of the hysterectomy operations for this group of women, but generate $45 \%$ of the cost.

Table 11.2: Hospital Cost of Hysterectomies for Nonmalignant Conditions Patients Aged 15 to 54 Years: 1991

\begin{tabular}{lrrr}
\hline & Public & Private All hospitals \\
\hline Number & 1,981 & 2,409 & 4,390 \\
$\%$ of all hospital number & $45 \%$ & $55 \%$ & $100 \%$ \\
Age in years & 41.5 & 42.3 & \\
Days stay & 6.5 & 5.3 & \\
Total cost $\$(000)$ & 9,382 & 7,601 & 16,983 \\
$\%$ of all hospital total cost & $55 \%$ & $45 \%$ & $100 \%$ \\
Total cost per hysterectomy $\$$ & 4,736 & 3,155 & 3,868 \\
\hline
\end{tabular}

Table 11.3 summarises the cost of hysterectomies for nonmalignant conditions by cost category. Annual hospital costs of $\$ 17$ million accounted for $73 \%$ of total cost while production and leisure time foregone was estimated to be $\$ 6$ million or $27 \%$ of total costs. Hospital cost per hysterectomy was $\$ 4,390$ while work and leisure time costs were $\$ 1,400$.

Table 11.3: Summary of $1991 \mathrm{NZ}$ Cost of Hysterectomies for Nonmalignant Disorders

\begin{tabular}{lrrr}
\hline Cost & Number/ \$ & \$Million & \multicolumn{1}{c}{$\%$} \\
\hline Hospital cost & & 17 & 73 \\
Production/leisure cost & & 6 & 27 \\
Total cost & & & 100 \\
Number of hysterectomies (N) & 4,390 & & \\
Hospital cost per hysterectomy & 3,868 & & \\
Production/leisure cost per & 1,400 & & \\
Total cost per hysterectomy & 5,268 & & \\
\hline
\end{tabular}

\subsection{DISCUSSION}

This study selected patients between puberty and menopause experiencing menstrual problems of a nommalignant nature. It did not include women of 55 years and older as it 
was assumed that most women would be post-menopausal at that age. Although most women begin menstruating before 15 years of age it may be several years, and as a last resort, before a patient with menstrual problems undergoes a hysterectomy.

Average total weekly earnings for males and females combined, as at June 1991 (Statistics New Zealand, 1993), were used as a proxy for the value of production and leisure-time loss. Earning rates relating specifically to women were rejected because it was considered that health decisions should be gender neutral. If a lower rate is used for valuing women's output and leisure, it can result in less importance being placed on women's health when comparing the costs of different diseases and the benefits to society of treating them (See Chapter 4 : Costs). This has important consequences for evaluation of policy options that are gender and age specific. If the objective of the economic and policy analysis is to provide information for decisions where the outcome is intended to be gender and age neutral then an economy-wide average wage rate should be applied to all time related costs.

Deductions were not made for unemployment in the paid workforce as it was considered that unpaid work and leisure-time were as relevant as paid work for this age group. This also takes account of the situation where, if a mother of a family is incapacitated, her partner may lose some time in paid employment or may have to employ home help to run the household (discussed in Chapter: 4 Costs in the section covering the frictional method of estimating productivity loss).

The total New Zealand rate for hysterectomy for all ages is lower than comparable rates in USA and Canada, similar to rates in Australia and the Netherlands, but higher than rates in England and Wales, and Ireland. It is unlikely that any physiological difference between women in different countries could account for the variation in rates. The differences are more likely to result from different health systems, medical trends, income levels, and social pattems between countries. Possible explanations for the discrepancies include, differences in the preparedness to put up with a reduced quality of life, an increase in women's knowledge of themselves and diseases, treatment options, a higher rate of uterine/pelvic disease, a sterilisation procedure that can be legitimised as medically necessary, and the availability and access to surgeons and hospital facilities. 
The recent decline in the number of hysterectomy procedures in some developed countries may be explained by controversy over unnecessary operations, patient pressure to avoid hysterectomy, awareness of new treatments (for example endometrial ablation, pharmacotherapy), and the costly nature of a long convalescence.

Lower numbers of hysterectomies undertaken in women of the younger age groups will partially reflect a desire by both the patient and the clinician to avoid or delay surgery until the patient no longer wishes to have children. Up to this time it is considered that watchful waiting and the treatment with medicines and/ or ablative surgery will be preferred to a hysterectomy (Session conducted at RACOG Annual Scientific Meeting, 1994).

The 4,390 hysterectomies for nonmalignant conditions in New Zealand represented $69 \%$ of the total hysterectomies conducted $(62 \%$ of the public and $77 \%$ of the private hospital hysterectomies). In private hospitals some hysterectomies for malignant conditions may not have been recorded under a specific diagnoses but in the more general ICD 621 disorders of uterus, not elsewhere classified, and therefore may not have been filtered from the data.

The age of patients was similar in both public and private hospitals. Public hospital patients were on average 41.5 years and private hospital patients were 42.3 years. Public hospital unit cost was higher $(\$ 4,736$ public unit cost, $\$ 3,155$ private unit cost), there were fewer patients, and these patients stayed on average a day longer compared with private hospitals. Actual fees for the surgeon and anaesthetist are often higher than those derived from the Southern Cross Schedule (1991) which would increase the estimates of private hospital unit costs. The higher cost and longer stays in public hospitals compared with private hospitals may not indicate that public hospitals are less efficient. Public hospitals' case mix may be biased towards patients likely to require a greater degree of care and whose stay is therefore likely to be longer.

For the age cohort studied and the conditions analysed, private hospitals recorded a greater number of hysterectomies than public hospitals. A possible explanation for this may be that many women in this working age group (15-54 years) may have health insurance and prefer 
to plan surgery to suit business and family commitments. Surgery in a public hospital is usually scheduled by a waiting list/booking system over which the patient has little control.

If New Zealand women are opting for private surgery for the above reasons then such surgery is likely to be price and income sensitive. For example, if women in the working age group have less disposable income or lower rates of insurance cover there may be a drop in the number of hysterectomies in private hospitals, and a corresponding increase in the number of hysterectomies in public hospitals. The decision made to have a hysterectomy in a private hospital at a time convenient to the patient may be the best option for these patients if the cost of treatment is traded off against lost production and reduced quality of life.

Some important costs, such as, medical costs after discharge, voluntary and community care, care provided by family and friends, and intangible costs relating to quality of life could not be evaluated because of a lack of robust data. Costs of treatment and production and leisure time lost before surgery for hysterectomy were not quantified as the intention of the study was to measure only those costs incurred following the decision to operate.

\subsection{CONCLUSIONS}

Further reduction in the rate of hysterectomy in New Zealand may save hospital costs but these savings should be offset against the costs of any other treatment options chosen. For example, watchful waiting could incur continuing costs relating to general practitioner and/ or specialist consultations, analgesics, protective tampons and pads, loss of output and reduced quality of life. Although it was found that unit costs of public hospitals were higher than those of private hospitals the case mix for the two types of hospital may differ and public hospital cost reductions may not be possible.

At the time of this study the unit costs of public hospitals were higher than those of private hospitals. Accordingly, if the public hospital unit costs fell to that of the private hospitals a reduction of $18 \%$ in total hospital costs could occur. Subsequent changes to the New 
Zealand health system under which hospitals must compete for funding may make cost reductions of this order possible. But if the case mix is different, with public hospitals treating more complicated cases, this may not be possible. Further research on the case mix of public and private hospital patients would be needed to obtain the data with which to evaluate relative efficiency.

Data for decision making would be enhanced if research were undertaken to detect the reasons why New Zealand women elect to have hysterectomies, and why most choose a private hospital. Information should also be collected before and after hysterectomy to evaluate changes in productivity, out of pocket expenses (such as analgesics and protective clothing), and quality of life for these patients.

\subsection{POLICY POINTS}

Although most policy issues have already been discussed in the body of this chapter, the key issues are highlighted below.

Hysterectomy is an intervention for a gender and age specific condition. Valuing productivity losses using the lower female wage rates would provide misleading information to policy makers (Ministry of Health) if the objective is to implement gender and age neutral policies.

Public hospital costs were higher than private hospital costs implying that if hospital stays could be shortened in public hospitals considerable savings could be made. This may not be possible if case mix is different.

Women with greater disposable income and/ or with health insurance may opt for private surgery to circumvent public hospital waiting lists. Further research would be needed to establish this. 
Quality of life is an important element in this condition but was not taken into account because sufficiently robust data did not exist and could not be obtained within the research budget. Economic evaluation of alternative treatment options (from a societal perspective) would be improved if all relevant costs (direct, indirect and intangible) were known.

A similar comparison between public and private hospitals would be more difficult now because private hospitals can tender for publicly funded surgery and there is a more intense focus on costs within the public hospital sector.

This was another study in which data other than hospital admissions and surgery were unavailable. If effective public policy evaluations are to be undertaken, community care and morbidity data need to be collected and made available for policy analysis. Evaluation of indirect costs is particularly difficult as there are no national surveys of lost production and leisure time as a result of specific illnesses.

Health funders for the first time had information on volume and cost of hysterectomies for nonmalignant conditions and could consider separate funding options and policies for malignant and nonmalignant conditions. This paper was one of those used and cited by the National Health Committee (1998) in their Guidelines for the management of heavy menstrual bleeding. 


\subsection{REFERENCES}

Endometriosis. Session conducted at RACOG Annual Scientific Meeting, Sydney 20th 22nd April, 1994

National Advisory Committee on Core Health and Disability Support Services. First report. Wellington: the Committee, 1992. Appendix 6.

N.Z. Department of Health, Health Information Services. Unit record data 1991. Wellington, 1993.

OECD Health data. version \# 1.5. Paris: OECD, 1993.

Office of Health Economics (OHE). Compendium of health statistics. 8th ed. London: OHE, 1992.

Scott, H. M., and W. G. Scott. "Hysterectomy for non-malignant conditions: cost to New Zealand society." New Zealand Medical Journal 108 (1995): 423-26.

Southern Cross Medical Care Society. Schedule of surgical and anaesthetic procedures. 17 th revision. Auckland: the Society, 1991.

Statistics New Zealand. Information Network for Official Statistics (INFOS): Online computerised official statistics for New Zealand. Wellington: Statistics New Zealand, 1993.

Wakefield Hospital, Wellington. Personal communication, (1992). 


\section{CHAPTER 12: LOWER RESPIRATORY TRACT INFECTION}

\subsection{SUMMARY AND INTRODUCTION}

\section{Summary}

This chapter presents a cost-effectiveness analysis that was conducted alongside a clinical trial in a general practice setting. This study was (to the author's knowledge) the first published economic analysis conducted along side a clinical trial that attempted to mirror actual general practice treatment in New Zealand. It was particularly significant because the clinical results were published only in the economic paper. The results provided hard data on cost-effectiveness to enable informed decisions to be made with respect to the funding of different antibiotics for general practitioner prescribing.

\section{Introduction}

The study that is summarised and discussed considers the policy implications of an economic analysis undertaken to compare the effectiveness and the direct medical costs of two antibiotics used in the treatment of lower respiratory tract infection(LRTI). Additional detail can be found in Scott et al., (1993).

LRTIs account for between $1.5 \%$ and $2.6 \%$ of general practice consultations in New Zealand, and antibiotics are prescribed in the majority of cases. Roxithromycin was compared with cefaclor. Roxithromycin was a new oral macrolide antibiotic with an improved pharmacokinetic profile over earlier generation macrolides, a broad spectrum of antibiotic activity and a long half life (Acar, 1992). Cefaclor, is one of the antibiotics most commonly used for treating LRTIs and as such represents a standard against which other treatments should be evaluated. 


\subsection{METHOD}

A multicentre, randomised, double-blind controlled clinical trial, comparing the efficacy, tolerance and cost-effectiveness of roxithromycin $150 \mathrm{mg}$ twice daily with cefaclor $250 \mathrm{mg}$ 3 times per day for the treatment of LRTI, was undertaken in 8 centres throughout New Zealand. 240 patients with a diagnosis of LRTI were enrolled. Ethical approval was obtained to conduct the trial. The sample size was chosen to reliably detect a difference of $15 \%$ between the two medicines, with $80 \%$ power, $5 \%$ risk and assuming $90 \%$ efficacy for roxithromycin. Data for economic evaluation was obtained for 238 patients (roxithromycin 120 patients, cefaclor 118 patients) with 2 patients being lost to follow-up.

Diagnosis was based on community practice standards and treatment was for a minimum of 7 days, with provision for a further 7 days therapy if clinically indicated. Clinical assessment was made at baseline and at withdrawal or completion of treatment. Patients were eligible for selection if they were clinically diagnosed as suffering from lower respiratory tract infection.

The economic evaluation considered only the direct medical costs, and all prices relate to the last quarter of 1991. General practitioners were given forms on which to record details of resource utilisations. The data collected are detailed in the results section.

\subsection{RESULTS}

The two randomly selected treatment groups were comparable at commencement of the trial with respect to age (mean 51 years), gender and underlying disease. Results of clinical effectiveness (are shown in Table 12.1).

There was a statistically significant difference in side-effects, withdrawal rates and extra courses of treatment in favour of roxithromycin compared with cefaclor. In other words, the Roxithromycin group of patients experienced fewer withdrawals (usually for untolerated side-effects effects) and required fewer extended courses of treatment (for 
unresponsive treatment). Treatment failures, withdrawals or side-effects incurred additional costs for $11 / 120(9 \%)$ patients on roxithromycin and $19 / 118(16 \%)$ patients on cefaclor. Additional antibiotics and general practitioner visits (for treatment failures, withdrawals, side-effects) were required more often and cost more for cefaclor treated patients than for those receiving roxithromycin.

Table 12.1: Results of Clinical Effectiveness

\begin{tabular}{lccc}
\hline & Roxithromycin & Cefaclor & Significance \\
\hline Effectiveness & $115 / 120$ & $110 / 118$ & No sig dif \\
& $(95.8 \%)$ & $(93.2 \%)$ & \\
Number of patients requiring extended & 33 & 50 & $\mathrm{p}<0.05$ \\
course of treatment & & & \\
Average treatment duration & 8.9 days & 9.7 days & \\
$\begin{array}{l}\text { Number patients withdrawn from study } \\
\text { Side-effects considered to be related to }\end{array}$ & 6 & 15 & $\mathrm{p}<0.05$ \\
treatment & & 24 & $\mathrm{p}<0.05$ \\
Number of patients generating additional & $11 / 120$ & $19 / 118$ & \\
costs (based on treatment failure, & $(9 \%)$ & $(16 \%)$ & \\
treatment withdrawal or adverse effects) & & & \\
\hline
\end{tabular}

The economic analysis of this study assessed primary medicine cost, the cost of additional medicine to treat side-effects and therapeutic failures, additional general practitioner consultations and additional diagnostic tests required, over and above that required as part of the study protocol. 
Table 12.2: Summary of Direct Medical Costs of Roxithromycin Over Cefaclor

\begin{tabular}{lcc}
\hline $\begin{array}{l}\text { Direct incremental medical costs/ (benefits) of } \\
\text { roxithromycin over cefaclor }\end{array}$ & $\begin{array}{c}\text { Per patient in the } \\
\text { trial }\end{array}$ & $\begin{array}{c}\text { Per } 70,000 \\
\text { patients } \\
\$ 1,000\end{array}$ \\
\hline Additional primary medicine acquisition cost & 0.61 & 43 \\
Less: Other medicines acquisition cost avoided & $(1.98)$ & $(139)$ \\
Less: Dispensing \& container costs avoided & $(0.44)$ & $(31)$ \\
Less: General practitioner consultation costs & $(5.42)$ & $(379)$ \\
avoided & & $(2.14)$ \\
Less: Diagnostic test costs avoided & $(9.37)$ & $(656)$ \\
$=$ Net Cost (Benefit) &
\end{tabular}

Table 12.2 summarises the difference in cost between the two antibiotics. Although the medicine acquisition cost was greater for roxithromycin than for cefaclor additional drugs, general practitioner consultations and diagnostic tests for the treatment of LRTI and the side-effects of treatment were less for roxithromycin. Total direct medical cost per patient treated with roxithromycin was $\$ 9.37$ lower than for the cefaclor treated group despite a higher primary medicine cost. An estimate of $\$ 0.656$ million per year in total savings in direct medical costs could be made in New Zealand if roxithromycin was to replace all cefaclor prescriptions in the treatment of LRTI.

\subsection{DISCUSSION}

In contrast with many trials, the study on which this economic analysis was based was specifically designed to emulate general practice diagnostic and assessment methods ${ }^{18}$. However, the variation in treatment length between the protocol of this trial and day-to-day clinical experience should be noted.

18 A literature search using MEDLINE at the time of the study did not reveal any other such trials. Other studies were either not designed to emulate actual practice or were in a different setting. 
Direct medical cost savings to the country were based on the actual trial usage of the primary antibiotics by general practitioners but the numbers of additional medications and consultations and diagnostic tests may have been higher in the trial than in day to day medical practice. This is a common difficulty in applying economic analysis to clinical trial data. Bias is minimised by the use of incremental analysis as most of the additional costs are common to both the studied and control groups and cancel out when comparisons are made. The economic analysis took into consideration only those additional diagnostic tests, general practitioner visits, and pharmaceutical costs which resulted from inadequate treatment of the initial infection.

It would have been desirable to have included indirect and intangible costs and benefits in this study but information on such costs and benefits (non-medical and intangible costs and benefits, such as travel costs, increased production, and the effect of treatment on quality of life) were not available. The savings calculated therefore represent an underestimate to society as there were fewer side-effects and treatment failures at seven days with roxithromycin than with cefaclor.

In the trial, a minimum treatment duration of 7 days was set to ensure that cases of acute on chronic bronchitis and pneumonia would receive an appropriate treatment length (Mandell, Douglas and Bennett, 1990; Conte and Barriere, 1988). In actual practice prescribing length would have been shorter.

A minimum of 2 general practitioner visits were required for evaluation of each case whereas in routine clinical practice a patient would most likely only return if treatment failed or if there were significant side-effects. The 2 haematological and biochemistry investigations carried out as an assessment of comparative safety would not normally contribute to the routine management of LRTI in general practice. Chest X-rays and microbiological tests were undertaken whenever possible and the fact of the study bearing the cost of these investigations would have encouraged greater use than normal. All of these costs, however, were incurred equally for patients on roxithromycin and cefaclor and therefore cancel out in an economic comparison of treatments. 
By calculating primary medicine costs based on the national average treatment duration for each antibiotic for the indication of LRTI, a fairer assessment of the overall comparative costs, nationally, of the 2 treatments would be achieved. This was not undertaken because data on prescribing practice was unavailable to the researchers.

It could be argued that cases of acute bronchitis (which represent a significant proportion of the study patients) are viral in aetiology and do not require antibiotic therapy (Billas, 1990). Three percent of the cases included in the intent to treat analysis of clinical efficacy in the study were deemed viral in origin by serology. On the other hand, others (Dunlay et al., 1987) have demonstrated a benefit from antibiotic therapy for acute bronchitis, and in this study practitioners selected patients whom they believed clinically to have a bacterial LRTI which would benefit from antibiotic therapy. Results are thus representative of actual general practice usage patterns as compared to restrictive analyses based on infection proven by sputum culture, a tool widely regarded to lack sensitivity and specificity in this setting (Lentino and Lucks 1987).

The recommended dose of cefaclor for general practice LRTI varies internationally ranging from $250 \mathrm{mg}$ twice daily to $500 \mathrm{mg} 3$ times per day for mild to moderate infections. However, as with most antibiotics there has been a trend to reduce the dosage to the lowest effective level associated with the most favourable side-effect profile. The dose of $250 \mathrm{mg}$ 3 times per day is that recommended for mild to moderate infections in the official New Zealand prescribing information for cefaclor. It is possible that a small number of patients may have been more effectively treated with a higher dose of cefaclor, however, efficacy was already $93 \%$ and one-half of the treatment failures observed with cefaclor were due to early withdrawal necessitated by intolerable side-effects, rather than insufficient efficacy. Any small increase in efficacy would therefore be offset by substantially increased direct medicine cost, and in all probability increased other medicine costs, consultations and diagnostic tests required to treat and assess an increase in side-effects and resultant treatment withdrawals. 


\subsection{CONCLUSIONS}

The observed statistically significant difference in side-effects, withdrawal rates and extra treatment courses in favour of roxithromycin in this study is translated into actual cost savings. Theoretically, if roxithromycin were to be used in place of cefaclor for the estimated 70,000 prescriptions for cefaclor filled each year in New Zealand for LRTI, savings in excess of $\$ 0.656$ million could result, in spite of roxithromycin having a greater unit cost. This analysis demonstrates that economic data can and should be obtained from clinical trials and used to evaluate costs and benefits. If the an economic component is not included in a clinical trial then decisions that effect the efficiency of resource use will continue to be made without robust cost data.

\subsection{POLICY POINTS}

Most clinical trials take place in an artificial setting and the results are therefore difficult to apply to everyday usage. This trial was designed to emulate the general practice environment and actual treatment as far as possible. Policy analysts and managers must make decisions in the real world and for this they need real world data.

A common but often flawed criticism of applying economic evaluation based on clinical trial data is that this is an artificial situation and costs evaluated using trial resource utilisations will be biased upwards. Although the trial setting and protocols were as close to reality as possible, there was more intense monitoring and diagnostic evaluation of patients than in actual practice. The study protocol applied the same rules and conditions to both the drug under investigation and the comparator. Incremental analysis was used to minimise the bias introduced by the trial. Thus, when differences between test drug and comparator were calculated all the cost increases resulting from the trial protocol will cancel out. In addition, general practitioners were specifically asked to record and note any prescribing, consultations and diagnostic tests over and above that set down in the trial protocol. 
Economists are usually forced into using published clinical trial results to conduct an expost evaluation. In this instance, the economic data was collected alongside the clinical data. Even so some desirable data could not be obtained. Data collected relating to direct non medical or indirect costs were considered to be too unreliable for analysis as general practitioners were unable to question patients in sufficient detail on non medical aspects of their illness. Attempts to obtain such information using nurse research assistants may be more successful as the nurses would be able to devote more time and may be more interested in collecting the non clinical data required for economic analysis. It was not possible to calculate levels of statistical confidence for the economic differences observed because the sample size was set to achieve sufficient statistical power to detect clinical differences rather than economic differences. See Chapter 4: Costs where risk, uncertainty and sensitivity analysis is discussed.

As the clinical results for these trials had not previously been published in a specialised peer reviewed clinical journal it was necessary to describe them in more detail than is usual in an economic paper. The study was interesting in that although there was no significant difference between the effectiveness of the two drugs and while cefaclor had a lower acquisition cost, when the average length of treatment and the side-effects were taken into account roxithromycin was more cost-effective.

There is no set formula that can be mechanically applied to all economic evaluations and it is usually not possible to classify a study until it has been completed. Although the original intention was to conduct a standard cost-effectiveness analysis, no significant differences were detected in the clinical outcomes measured. The results were thus presented as incremental cost differences rather than cost-effective ratios because this was considered to be of more use for policy evaluation and budget cost management decisions. As there were differences in the side effect profile between the drugs tested it would have been possible to conduct a cost-utility study (had the budget allowed for the collection of this additional information). The information collected and the type of analysis was however quite sufficient for the intended use. A cost-utility analysis would not have added to the usefulness from a decision maker perspective. 
The study findings allow policy analysts to better compare the net costs differences between two treatment options and to evaluate the budgetary impact of the policies. When a government agency such as PHARMAC is negotiating prices and selecting drugs to fund the full treatment cost of each drug should be taken into account and not just the drug price in isolation. It is possible that a low priced drug, in comparison with a higher priced drug, could generate higher health spending because it may have more side effects and treatment failures necessitating additional patient consultations, drugs and diagnostic tests.

Similar methodologies as used in this study could be applied to the evaluation of a wide range of policies involving new or existing technology. Undertaking an economic evaluation alongside a clinical trial is expensive and probably necessary only for high expenditure medicines. 


\subsection{REFERENCES}

Acar, J. F. Foreword. Diagnostic Microbiolog! and Infectious Disease. 15 (4): (1992) 61S-62S

Billas, A. "Lower respiratory tract infections". Primary Care 17: 8 (1990) 11-824.

Conte, J.E., and S. L. Barriere. Manual of antibiotics and infectious diseases, 6th ed., Lea and Febiger, New York, 1988.

Dunlay, J., R. Reinhard, and L. Donn Roi. "A placebo controlled, double-blind study of erythromycin in adults with acute bronchitis". Journal of Family Practice 25: (1987) 137 141.

Lentino, J. R., and D. A. Lucks. "Non value of sputum culture in the management of lower respiratory tract infections". Journal of Clinical Microbiology 25: (1987) 758-762.

Mandell, G. L., R. G. Douglas, and J. B. Bennett. Principles and practice of infectious diseases. 3rd ed. Churchill, London, 1990.

Scott, W. G., M. W. Tilyard, S. M. Dovey, B. Cooper, and H. M. Scott. "Roxithromycin versus Cefaclor in lower respiratory tract infection. A General Practice pharmacoeconomic study." PharmacoEconomics 4(2) (1993): 122-30. 


\section{CHAPTER 13: INFLUENZA}

\subsection{SUMMARY AND INTRODUCTION}

\section{Summary}

This case combined overseas clinical findings with New Zealand cost information to evaluate the cost-effectiveness of vaccinating the 65 years and older age group in New Zealand. The published results of this study provided essential cost-effectiveness evidence and was used by the Ministry of Health to evaluate and design their subsidised vaccination programme (Ministry of Health letter 18 June 1996).

\section{Introduction}

The objective of this cost-benefit analysis was to evaluate the costs and benefits of influenza vaccination for the population aged 65 years and over, from the perspectives of individuals, health insurers, government, and society. This chapter uses a study (Scott and Scott, 1996) that had some influence on public health policy changes. At the time of the study, influenza vaccinations were not subsidised by government. Since publication government policy has been changed and influenza vaccinations are now paid for by government for those 65 years and over.

Influenza is regarded world wide as an important and highly infectious viral illness and is most severe for the very young and the elderly. The likelihood of being hospitalised or dying from influenza increases with age and the number of underlying medical conditions present in the patient (Wiselka, 1994). The benefits of influenza vaccination are dependent upon age, health status, the rate of vaccination, and the efficacy of the vaccine. Although influenza vaccination is considered to be safe, effective vaccination rates are low in New Zealand and vaccination is regarded as being under-utilised (Tobias M, 1991; Jennings, 1994). 
There are four groups targeted for influenza vaccination in New Zealand. The first group are those at high risk of complications, for example, adults and children with chronic debilitating diseases, persons aged over 65 years, residents of rest homes, geriatric hospitals and other chronic care facilities, and immune compromised individuals. Group two are those individuals who may spread infection to those at high risk, and group three are those engaged in essential community services. The fourth group covers children with specific health conditions (N.Z. Department of Health, 1993).

\subsection{METHOD}

The incremental direct medical costs and benefits of influenza vaccination were evaluated using New Zealand health care resource usage and unit cost data for 1992 applied to clinical trial results reported in the literature (Nichol et al., 1994).

Direct medical costs are those involved in providing treatment and include the purchase of vaccines, medical practitioner consultations and hospital costs. Direct nonmedical costs incurred by the illness and treatment could include transport to receive treatment and the cost of special food as a result of the illness. Indirect costs encompass work output lost as a result of morbidity and mortality while intangible costs are concerned with loss of quality of life, pain and suffering rather than changes in resource opportunity costs (Eisenberg, Schulman, Glick and Koffer, 1994).

The net benefit/ net cost of vaccination was compared against no vaccination. At risk adults under 65 years of age and children with chronic debilitating diseases are evaluated as an adjunct to the sensitivity analysis. Together these two at risk groups account for about $20 \%$ of the total New Zealand population (Tobias, 1991). If vaccination were to be extended to the houschold contacts and care givers of these people the numbers would double (Tobias, 1991), but insufficient data exist to allow quantification of the costs and benefits for these people. 
International Classification of Diseases (ICD) diagnosis codes relating to influenza and pneumonia, other respiratory conditions, and heart failure as described by Nichol et al. (1994) were used as the basis for calculating health care resource volumes in New Zealand.

As all costs related to one year (prevalence study) discounting to present values was not necessary. All unit prices and costs used in the study were in 1992 dollars and net of goods and services tax (GST).

Table 13.1 summarises the steps followed to estimate the incremental net costs of vaccinating the 65 year and over age group.

Table 13.1: Steps in Estimating the Incremental Costs and Benefits of Influenza Vaccination

1 Estimate the cost of vaccination (C)

2 Estimate utilisation of health care resources if no vaccination given (Up)

3 Calculate the change in volume of resources (increased vaccinations and reductions in other health care resources utilisations) if vaccination given (Uc)

4 Apply unit costs (P) to the change the volume of resources used (Uc) to estimate the costs avoided (benefits) of vaccination $(B),(B=U c \times P)$

5 Calculate the incremental net costs or benefits $(\mathrm{NC}),(\mathrm{NC}=\mathrm{C}-\mathrm{B})$

6 Conduct sensitivity analysis

The current vaccination rate of $20 \%$ (Tobias, 1991) for the 65 years and over, (together with the base case values for health resource utilisation reductions) were used in the base case. Sensitivity analysis was used to evaluate the impact of changes to the key variables. The per vaccination cost relating to the older group (excluding the costs of heart failure and adjusting the hospital costs to take account of different Diagnostic Related Group (DRG) hospital costs for those under 65 years) was applied to the younger group as no specific data on health resource utilisation changes were available. 


\subsubsection{Vaccine Cost}

The potential number of vaccinations was based on the number of people who fell within the age group 65 years and older (Statistics New Zealand INFOS) who accounted for $11.5 \%$ of the total population in 1992. Those under 65 years at risk (for the sensitivity analysis) accounted for $9.3 \%$ of the total population (Tobias, 1991). Influenza vaccine acquisition cost to general practitioners was $\$ 12.96$ (information supplied by Rhône-Poulenc Rorer NZ Ltd, and CSL NZ Ltd). General practitioners charged a total fee of $\$ 17.78$ (fee plus vaccine) for an influenza vaccination (mean charge from an informal telephone survey of Wellington general practitioners). This fee was unchanged from that reported in 1991 (Tobias, 1991).

\subsubsection{Utilisation of Health Care Resources If No Vaccination Given}

Tobias (1991) considered that based on influenza vaccine sales in New Zealand, about 20\% of the elderly population and $10 \%$ of the younger at risk groups were currently being vaccinated. A number of researchers (Karalus et al., 1991; Nichol et al., 1994; Fedson et al.,1993) have found that vaccinated individuals utilise fewer health care resources than unvaccinated individuals. Accordingly, to establish the counterfactual, it was necessary to rate upwards the actual recorded utilisation data to take account of the estimated $20 \%$ rate of influenza vaccination and for the consequent reduction in utilisation of health resources per vaccination for this group. This procedure was necessary to establish the counterfactual case so that comparisons could be made.

Where:

$$
L^{\prime} p=\frac{U_{a}}{1-\left(1 r \times L_{c}^{\prime}\right)}
$$

$L_{p}=$ posential urilisation volume (rolume had there been no vaccinations)

L'a = actual urilisation rolume

Ir = vaccination rate as a dicimal

$L^{\prime} c^{\prime}=$ proportional reduction as a decimal for urilisation volume as a result of vaccination

Data (broken down by ICD code) on the numbers of general practitioner consultations and prescription items dispensed were averaged over the years 1991, 1992 and 1993. These data were obtained from International Medical Statistics (NZ) Ltd. The number of 
prescriptions for those aged 65 years and over was estimated from the ratio of prescription items written per general practitioner consultation for all ages multiplied by the number of consultations for those aged 65 years and over. Hospital admissions by ICD code were derived from public hospital separation data averaged over the three years 1990, 1991 and 1992 (the latest three years available at the time of study, N.Z. Ministry of Health, 1993).

\subsubsection{Medical Costs Avoided}

The potential reduction in the volume of health care resource use was the mean of the percentage reductions in hospitalisations over three influenza seasons for patients aged 65 years and over who were vaccinated compared with unvaccinated individuals (Nichol et al., 1994). These percentage reductions were obtained for pneumonia and influenza, other acute and chronic respiratory conditions (all chronic and acute respiratory conditions less pneumonia and influenza), and congestive heart failure. The comparisons between vaccinated and unvaccinated groups were adjusted for differences in patients' baseline characteristics which could alter outcome variables (Nichol et al., 1994). In the absence of resource specific data from randomised clinical trials or case controlled studies these percentage reductions were employed to calculate the potential reductions in all health care resource utilizations in our study.

Base case savings in the volume of health care resources used were: influenza and pneumonia $52 \%$, other respiratory conditions $28 \%$, and heart failure $24 \%$. The incremental cost of influenza vaccination in volume terms was the percentage reduction in utilisation of New Zealand health care resources multiplied by the volume of resources used assuming no vaccination. The incremental resource utilisations volume, multiplied by New Zealand unit costs gave the incremental cost in dollars.

\subsubsection{Health Care Resource Unit Costs}

General practitioner consultation costs used in the study were based on the mean fee actually charged of \$23.20 (W Guy Scott and Associates Ltd, 1992), inclusive of the general practitioner subsidy [(general medical services benefit (GMS)] but exclusive of 
GST. The GMS used was the rate applicable to beneficiaries, \$13.33 as at February 1992 (McKendry and Muthumala, 1994). The average government subsidised cost of a medical prescription used for the calculations was \$31.16 applicable to the elderly at June 1992 (Health Benefits Ltd., Health Benefits Centre,1993). The patient co-payment was $\$ 4.44$, applicable to beneficiaries, and chronically ill and high user groups at February 1992 (Health Benefits Ltd,, Health Benefits Centre,1993).

Hospital costs were based on DRG costs (National Advisory Committee on Core Health and Disability Support Services, 1992). The DRG codes most closely relating to the specific age group concerned and to the diagnoses codes were used. A weighted average (by volume) was derived for pneumonia and influenza $(\$ 5,501)$ and for other acute and chronic respiratory conditions $(\$ 4,700)$. Weighted average hospital unit costs for the at risk age group under 65 years of age were similarly estimated $(\$ 1,787)$. For Heart failure the DRG unit cost was $\$ 4,963$.

\subsection{RESULTS}

Costs and benefits to society as a result of vaccinations (at the current rate of $20 \%$ ) for the 65 years and older age group were estimated to be; the additional direct medical costs of vaccination $\$ 1.42$ million, ( $\$ 17.78$ per vaccination) direct medical costs avoided of $\$ 5.35$ million ( $\$ 67.18$ per vaccination) and net benefits of $\$ 3.93$ million ( $\$ 49.40$ per vaccination). Direct medical costs avoided per dollar cost of vaccination were; $\$ 1.04$ for individuals, $\$ 4.69$ for the goremment budget perspective and $\$ 3.78$ for society as a whole. If the vaccination uptake for this group is increased in $20 \%$ increments, the net benefit to society increased by a further $\$ 3.93$ million at each step. If the economic evaluation is extended to include vaccination of those at risk individuals under 65 years of age net benefits to society increase by $15 \%$.

Considering the additional costs from a societal perspective. Additional direct medical costs of vaccination amounted to $\$ 1.42$ million ( $\$ 17.78$ per vaccination), direct medical costs avoided were $\$ 5.35$ million ( $\$ 67.18$ per vaccination) with net benefits of $\$ 3.93$ 
million ( $\$ 49.40$ per vaccination). Because the majority of costs fall on the individual (out of pocket) and most of the costs avoided accrue to government, there is a small net benefit to individuals of \$0.19 per vaccination and a comparatively much larger net benefit to government of $\$ 49.22$ per vaccination. Direct medical costs avoided per dollar vaccination cost were; $\$ 1.04$ for each dollar spent by individuals, $\$ 4.69$ for each dollar spent by government and $\$ 3.78$ for society.

Turning now to benefits from a societal perspective. The medical costs avoided as a result of reduced calls on health resources for various diagnoses were estimated to be $\$ 1.43$ million (\$18.00 per vaccination) for pneumonia and influenza, other respiratory conditions, $\$ 2.40$ million ( $\$ 30.13$ per individual), and heart failure $\$ 1.52$ million (\$19.05 per individual).

Sensitivity analysis was undertaken for the societal net costs/benefits for the 65 years and over age group. The analysis considered the percent vaccinated, and the maximum and minimum reported changes in health care resource utilizations. Changing the vaccination cost and subsidy rates could not be effectively evaluated as the price elasticity of demand for vaccinations with respect to out of pocket payments was unknown. If the percentage of those vaccinated (in the age group 65 years and over) was increased in steps of $20 \%$ the net benefit rose in increments of $\$ 3.93$ million. The ratio of costs avoided per dollar cost of vaccination indicates that the vaccination cost (vaccine plus administration) could rise by a multiple of 3.78 before net benefits fall to zero.

The at risk population under 65 years of age was also considered as an additional adjunct to the sensitivity analysis. If this younger at risk population is considered, the societal net benefits increase by $\$ 0.58$ million or $15 \%$ over the base case. The ratio of costs avoided per dollar cost of vaccination for the younger at risk group was $60 \%$ lower than the older age group (1.51 compared with 3.78 ).

Analysis using the reported minimum reductions in health resource usage as a result of vaccination (pneumonia and influenza 19\% (Karalus et al., 1991), other respiratory 
conditions $20 \%$ (Nichol et al, 1994) reduced the net benefit to society by $81 \%$ and the direct medical costs saved per dollar of vaccination cost by $\$ 2.24$ to $\$ 1.54$. Using the maximum reported resource savings (Nichol et al., 1994) of 57\% for pneumonia and influenza, $34 \%$ for other respiratory conditions, and $38 \%$ for heart failure, increased the net benefits to society by $42 \%$ and the direct medical costs saved per dollar of vaccination cost by $\$ 1.18$ to $\$ 4.96$. The results of the sensitivity analysis from the perspective of society ( 65 years and over) are presented in Table 13.2, while the sensitivity analysis for those under 65 years of age is presented in Table 13.3.

Table 13.2: Sensitivity Analysis From the Perspective of Society. Net Cost (Benefit) SMillion Population 65 Years and Over

\begin{tabular}{cccc}
\hline $\begin{array}{l}\text { \% of population } \\
\text { vaccinated }\end{array}$ & \multicolumn{3}{c}{ Change in health care resource usage } \\
\cline { 2 - 4 } & Base case & High & Low \\
\hline Base case $=20$ & -3.93 & -5.6 & -0.76 \\
40 & -7.87 & -11.2 & -1.52 \\
60 & -11.8 & -16.8 & -2.27 \\
80 & -15.74 & -22.4 & -3.03 \\
100 & -19.67 & -28 & -3.79 \\
Costs avoided per dollar cost of vaccination & 3.78 & 4.96 & 1.54 \\
\hline
\end{tabular}

Table 13.3: Sensitivity Analysis At Risk Group Under 65 Years of Age

Cost

Perspective

Personal out Government Society

of pocket /

insurance

Total costs

$\$$ million

$\$$ million $\$$ million

Net cost [( ) = net benefit)]

0.08

$-0.66$

$-0.58$

Costs avoided per dollar cost of vaccination

0.71

1.77

1.51

\subsection{DISCUSSION}

Influenza vaccination for the older age group is cost-effective from the perspective of society and government and for individuals. If the vaccination rate for the 65 years and over population in New Zealand could be increased from $20 \%$ to $60 \%$ the net benefits 
reported in this paper would increase by $200 \%$, however, the costs of promotion and education to achieve this would need to be deducted from the net benefits. Strategies to increase the vaccination rate include altering the cost of vaccinations to the individual, intensifying education and promotion programmes, and changing the mode of delivery. Each of these strategies has a resource cost which would need to be offset against the benefits.

Sensitivity analysis indicated that although there is considerable variation when upper and lower ranges in resource savings are applied, the direct medical costs avoided remain greater than the additional costs of vaccination. From a societal perspective changing the co-payment or subsidy rates altered the division of costs that individuals or government paid. Adtering the GMAS subsidy is alco likely to alter the rates of vaccination as the price to the patent would change but this could not be quantified. Sensitivity analysis also indicated that as the uptake of vaccinations rose by increments of $20 \%$ there would be an incremental net societal benefit of $\$ 3.93$ million. To achieve a higher vaccination rate additional costs of education and promotion (targeting at risk groups and health care professionals) would be incurred by either the suppliers of the vaccine, by government, or by both. This additional cost has not been taken into account in our analysis but the results provide the information required to set an upper limit on an educational/promotional budget.

It was found that there was a difference in the breakdown of cost savings by disease category between this study and that reported by Nichol et al. (1994) because the underlying hospital admission rates for pneumonia and influenza in the United States were higher than those recorded in New Zealand and conversely, the New Zealand hospitalisation rate for heart failure was higher than the United States' study. (Although it is beyond the scope of this study to evaluate the reasons for different hospitalisation rates a possible explanation is that the prevalence and mode of treatment of the diseases considered could be different) Although the absolute volumes were different it was considered that it was valid to apply the US percentage reductions in utilizations of health care resources to New Zealand base resource utilisation volumes. 
This analysis used average rather than marginal unit costs. Policy decisions relating to influenza vaccination would involve increments of hundreds or thousands rather than a single individual. Multiplying the marginal net cost of one additional vaccination by the number of vaccinations would provide a false estimate of the incremental net costs/benefits of the programme. The short run marginal net cost of one additional vaccination would ignore overhead costs but the long run incremental net cost of large numbers of additional vaccinations should include those overhead costs that would change if the vaccination rate altered.

As health authorities consider that influenza vaccinations should also be given to at risk groups under 65 years of age the authors applied a similar methodology to estimate the costs and benefits of such vaccinations. The costs of heart failure were excluded from this group, and in the absence of more specific information, the reduction in costs for pneumonia and influenza, and other respiratory conditions were those applicable to the 65 years and over group. Although the percentage reductions in resource utilisation for the under 65 years of age group are unlikely to be identical and the results obtained for this group cannot be accepted with the same confidence as those pertaining to the older age group it was considered that policy makers would find this information useful. It was found that the ratio of net costs avoided per dollar of vaccination cost was substantially less for the younger at risk group than the ratio applicable to the older group. The main reason for this difference was the lower incidence and unit costs for hospitalisations for the younger age group (the average length of hospital stay was less for this age group).

As no specific data were available on general practitioner and prescription utilizations the authors applied the percentage reductions in hospital resource use to general practitioner consultations and prescriptions. It could be argued that influenza vaccination is more efficient in preventing hospitalisations than in avoiding general practitioner consultations and prescriptions. As less than one-third of the total medical costs avoided were nonhospital costs, inaccuracies in estimates of non-hospital resource utilizations would not impose a serious bias to our total cost saving estimate. 
Subsets of the target groups studied could also be researched. For example, in 1992 there were 20,962 beds in rest homes and approximately 20,000 full and part-time employees (Rest Homes Association Inc. Letter 11 January 1995). If it is assumed that the net benefit of vaccinating 20,000 patients is the same as that estimated in this study (rest home residents are a sub set of this), the net benefit to society of vaccinating these full and parttime employees would be $\$ 1.34$ million.

For an individual of working age, the cost of a vaccination would be offset by additional production gains. Riddiough et al. (1983) found that 0.03 work days per vaccination could be gained for an individual aged between 45 and 54 years. In a study of Canterbury Health Laboratory staff it was found that absenteeism from influenza was $39.5 \%$ in the vaccinated group compared with $66.7 \%$ in the unvaccinated group (Schousboe, 1993). It is sometimes argued that short term absence from work is not a cost because there is slack in the economy (unemployment and workers able to cover for absentees) or that work set aside is actioned on the return of the worker (Koopmanshap and Rutten, 1993). Potential production loss, both paid and unpaid, should be estimated on the understanding that actual production loss may be somewhat less. However, in many situations in New Zealand if a worker becomes ill there is no surplus capacity and the output of the absentee worker will be lost. A production loss may also occur when parents or guardians take time from work to care for children who are ill with influenza.

The cost of adverse events relating to the vaccine were not evaluated in this study. Modern influenza vaccines are considered to have few adverse events, and medical practitioners are advised against vaccinating those patients with a known or possible anaphylactoid hypersensitivity to hens' eggs (N.Z. Department of Health, 1993). Although intangible costs were not evaluated, quality of life aspects are of importance for potential sufferers, their family and friends.

New Zealand data for 1992 recorded 1132 deaths from pneumonia and influenza, (N.Z. Ministry of Health, 1994) and for males and females over 75 years of age it was the fourth and third leading cause of death respectively. However, it is considered that influenza is 
responsible for many deaths recorded under categories other than influenza and pneumonia (Fedson et al., 1993; Nicholson, 1990; Sprenger and Van Naelten, 1989). The Manitoba study by Fedson (1993) (covering influenza outbreak periods 1982 to 1983 and 1985 to 1986), found that vaccination was $43 \%$ to $65 \%$ effective in preventing hospital deaths of patients admitted with pneumonia, influenza or other respiratory conditions, and $27 \%$ to $30 \%$ effective in preventing deaths from all causes.

Vaccination, where an infectious disease is involved, confers external benefits to unvaccinated individuals in a community by limiting epidemics. The protection, or herd immunity, afforded to the unvaccinated when others around them are vaccinated has been estimated by Tobias (1991) to be achieved by vaccinating $70 \%$ to $75 \%$ of residents in a rest home or $80 \%$ in open communities. The U.S. Department of Health and Human Services (1991) have recommended a vaccine coverage of $80 \%$ for institutionalised at risk individuals and $60 \%$ for those at risk in the community.

Research into the impact of vaccination on the utilisation of non-hospital resources for the 65 and older age group would be useful, but not essential, as most cost savings relate to hospital costs. In order to formulate a vaccination policy and recommendations for the at risk group of working age further information is required on all costs.

\subsection{CONCLUSION}

The results of this paper demonstrated the economic benefits of vaccination of at risk groups in society. In particular considerable health sector costs could be avoided by increasing the vaccination rate to the over 65 years age group. For those in the working age group the net benefits were less clear.

\subsection{POLICY POINTS}

The vaccination rate in New Zealand is less than is medically desirable (Tobias, 1991) and a higher rate has been demonstrated in this paper to be justifiable in economic terms. 
Public policiy options to increase the vaccination rate include; subsidising vaccinations to reduce the cost to the individual, changing the mode of delivery, and education and promotion programmes. The N.Z. Ministry of Health chose to increase the vaccination rate for the 65 years and over by providing "free" subsidised vaccinations and running a health promotion campaign from February 1997. These efforts raised the vaccination rate in all high risk groups from an estimated 29\% in 1996 to $44 \%$ in 1997 and $46 \%$ in 1998 (Kennedy, 1999) which is still less than optimal.

There are a number of policy initiatives the Ministry of Health can take to increase the vaccination rates. Suggestions include; investigating the reasons why high risk individuals do not seek vaccination, investigating how general practitioners notify their high risk patients that a vaccine is available and would benefit them, and determining what and how effective are their recall systems. A policy maker would then be able to determine where the problem/s lay and perhaps design better information and follow up systems and implement a more focussed health information campaign.

This paper has combined published overseas findings with New Zealand volume and unit cost data. Because of the need to use health care resources efficiently suitable overseas studies should, where appropriate, be applied to New Zealand to evaluate treatment and health policy options for New Zealand. The resources required to replicate overseas studies and generate equivalent New Zealand clinical data may be better devoted to evaluating and applying overseas studies to New Zealand and in providing health care.

Average rather than marginal unit cost data were used for estimating cost changes because policy decisions relating to influenza vaccination would involve increments of hundreds or thousands rather than a single individual and it is important from a policy perspective to consider the long-run rather than the short-run.

The argument for government funding of the vaccination programme is strengthened as vaccination against influenza, confers external benefits to unvaccinated individuals in a community by limiting epidemics. Private provision of vaccinations would result in a sub optimal allocation of resources from a societal perspective. 


\subsection{REFERENCES}

Core health and disability support services for 1993/94. First report of the National Advisory Committee on Core Health and Disability Support Services. Wellington: the Committee, 1992.

Eisenberg, J. M., K. A. Schulman, H. Glick, and H. Koffer. "Pharmacoeconomics: economic evaluation of pharmaceuticals." In Pharmacoepidemiology. 2nd ed., edited by B. L. Strom, 741. England: John Wiley and Sons Ltd., 1994; 469-493.

Fedson, D. S., A. Wajda, J. P. Nicol, G. W. Hammond, D. L. Kaiser, and L. L Roos. "Clinical effectiveness of influenza vaccination in Manitoba." JAMA 270 (1993): 19561961.

Health Benefits Ltd., Health Benefits Centre. Cash and Accrual Report for Regional Health Authority - Combined. Monthly reports for the year ended 1993. Wanganui: Health Benefits Centre, 1993.

Jennings, L. C. Towards the control of influenza in New Zealand. Paper presented to the Public Health Commission and the Communicable Diseases Advisory Committee, Wellington, 8 December 1994.

Karalus, N. C., R. T. Cursons, R. A. Leng, C. B. Mahood, R. P. G. Rothwell, B. Hancock, S. Cepulis, M. Wawatai, and L. Coleman. "Community acquired pneumonia: aetiology and prognostic index evaluation." Thorax 46 (1991): 413-418.

Kennedy, E. "UK outbreak a 'reminder that fly can be fatal'." The Dominion 19 January (1999)

Koopmanshap, M. A, and F. F. H. Rutten. "Indirect costs in economic studies: confronting the confusion." Pharmacoeconomics 4 (1993): 446-454. 
McKendry, C. G., and D. Muthumala. Health expenditure trends in New Zealand; update to 1993. Wellington: N.Z. Ministry of Health.1994. (Appendix 5).

N.Z. Department of Health. Immunisation handbook. Wellington: , 1993.

N.Z. Ministry of Health. Mortality and demographic data 1992. Wellington: Health Information Service, 1994

N.Z. Ministry of Health. Hospital and selected morbidity data 1992. Wellington: Health Information Service, 1993.

Nichol, K. L., K. L. Margolis, J. Wuorenma, and T. Von Sternberg. "The efficacy and cost effectiveness of vaccination against influenza among elderly persons living in the community." New England Journal of Medicine 331 (1994): 778-784)

Nicholson, K. G.. "Influenza vaccination and the elderly". BMJ 310 (1990): 617-618.

Riddiough, M. A., J. E. Sisk, and J. C. Bell. "Influenza vaccination; cost-effectiveness and public policy." JAMA (1983): 3189-3197.

Schousboe, M.. The evaluation of influenza vaccination of Canterbury Health Laboratory Staff. Internal report. Christchurch: Canterbury Health Laboratories, 1993.

Scott, W. G., and H. M. Scott. "Economic evaluation of vaccination against influenza in New Zealand." PharmacoEconomics 9 (1996): 51-60.

Sprenger, M. J. W., M. A. M. G. Van Naelten, P. G. H. Mulder, N. Masurel. "Influenza mortality and excess deaths in the elderly, 1967-82." Epidem. Inf 103 (1989): 633-641.

Statistics New Zealand. INFOS (Information Network for Official Statistics) Population statistics for 1992. 
Tobias, M.. "Influenza vaccination still underutilised." New Ethicals September (1991): 23-30.

U.S. Department of Health and Human Services. Healthy people 2000: national health promotion and disease prevention objectives. U.S. Department of Health and Human Services. Washington DC: the Department, 1991.

W Guy Scott and Associates Ltd. Survey of general practitioners adult consultation fees 1992. Wellington, 1992.

Wiselka, M.. "Influenza; diagnosis, management, and prophylaxis." BMJ 308 (1994): $1341-1345$. 


\section{CHAPTER 14: CRITICAL LEG ISCHAEMIA}

\subsection{SUMMARY AND INTRODUCTION}

\section{Summary}

Chapter 14: Critical Leg Ischaemia, describes a cost-effectiveness analysis that compared surgery with a pharmaceutical intervention. A variant of breakeven analysis was used to evaluate the situations in which the hospital cost of drug treatment would be cost-effective compared with surgery. Prior to this study the full cost of surgical intervention was unknown and therefore public hospital administrators and clinicians were unable to compare the cost of alternative treatments.

\section{Introduction}

The economic analysis set out to establish the incidence of surgical amputation for critical leg ischaemia in New Zealand. Standard therapeutic alternatives (surgery plus prostheses) were compared, in economic terms, with a new pharmaceutical treatment option, Iloprost. Background information and more detail may be found in (Scott and Scott, 1994).

Iloprost is a prostacyclin analogue with a half life of 30 minutes (Grant and Goa, 1992) (natural prostacyclin has a half life of 2 to 3 minutes). The analogue inhibits platelet aggregation and induces vasodilation. This provides a non surgical treatment option for patients with critical leg ischaemia where revascularisation is not possible or has failed and the next procedure would be amputation.

Critical leg ischaemia is ischaemia (inadequate flow of blood) that endangers the leg or part of a leg. Existing data on the prevalence of critical leg ischaemia have been based on a proportion of the leg amputation procedures undertaken or on the numbers of referrals to limb-fitting centres. The information available indicates that the prevalence of peripheral arterial disease is $5 \%$ in males over 50 years of age, but fewer than $10 \%$ of these will 
develop critical leg ischaemia (Dormandy and Stock, 1990). Incidence rates from studies in Western Europe and USA, have been estimated to fall between 500 and 1000 new cases per year per million population (European Working Group on Critical Leg Ischaemia 1991). Patients with diabetes are five times more likely to develop critical leg ischaemia than nondiabetic patients (European Working Group on Critical Leg Ischaemia 1991).

For patients with critical leg ischaemia who present with rest pain and/or ischaemic ulcers the first choice of treatment is revascularisation by surgical bypass or percutaneous angioplasty but almost $40 \%$ of such patients are not suitable for reconstructive surgery (U.K. Severe Limb Ischaemia Study Group, 1991). The last resort is amputation (Norgren et al., 1990) which is regarded as a high risk procedure.

\subsection{METHOD}

The study was retrospective, using unit record patient admission data for 1991 for both public and private hospitals. Unit costs for 1991 were applied to the volume data of patients and procedures to derive total costs. A societal perspective was adopted and costs were estimated incrementally. The incremental analysis measured costs that would change as the result of the decision to amputate or to use a pharmaceutical treatment. It was assumed that the costs up to the point of amputation would be identical for both treatment alternatives studied. The option of no treatment was not evaluated as the authors considered this would be ethically and socially unacceptable in present day New Zealand society.

Patients who undenwent vascular reconstructive surgery followed by amputation were included in the analysis but costs were evaluated for the amputation only. Hospital discharge data relating to $\mathrm{DRG}^{10}$ codes 113 and 285 and ICD procedure code 841 were

14. DRGs (Diagnosis Related Groups) are a classification scheme relating the type of patients that a hospital treats (case mix) to the costs incurred by the hospital in their treatment. That is, DRGs are groupings of diagnoses according to both their clinical similarities and treatment costs. DRG 113 Amputation for circulatory system disorders except upper limb and toe, DRG 285 Amputation of lower limb for 
obtained for all New Zealand public and private hospitals (N.Z. Department of Health, 1991). Data are recorded at the time of discharge or death. Any patient readmitted for the same condition within the period studied was recorded as a separate admission which could have resulted in the annual costs per patient undergoing surgery to be underestimated. Data were analysed with respect to age and gender groupings.

Two methods were used to isolate patients undergoing leg amputations; the first method selected records by ICD procedure code (the procedure code method, and the second method selected records by DRG code the DRG method). The two methods were used as a cross-check on data extracted.

Amputation data, were also used to calculate the cost of providing patients with artificial limbs (unit costs were supplied by the Artificial Limb Board, Wellington, 1993) after below knee, the knee and above knee procedures. It was assumed that half of these patients would be fitted with artificial limbs (European Working Group on Critical Leg Ischaemia, 1991).

Production loss was evaluated using the total average weekly earnings for males and females combined (15 to 64 years inclusive), (N.Z. Department of Statistics, 1991) and assumed a week off work for every day in hospital (informal survey of physiotherapists and surgeons).

The standard treatment with the pharmaceutical, Iloprost was for the patient with critical leg ischaemia to be hospitalised and the medicine administered intravenously for 6 hours per day for 20 days at a cost of $\$ 185$ per day.

All unit costs relate to the 1991 year and are exclusive of goods and services tax (a societal transfer payment).

endocrine, nutritional. and metabolic disorders. ICD = International Classification of Diseases, ICD 841 Amputation of lower limb. 


\subsection{RESULTS}

The data analysed using ICD procedure codes resulted in a total of 688 records ( 628 records in the public hospital file, and 60 in the private hospital file). These records were used to estimate hospital costs and calculate incidence rates. The incidence of amputations for critical leg ischaemia was 202 per million.

Major amputations (involving more than digits only) were almost exclusively undertaken in public hospitals. The analysis of the data with respect to gender and age found fewer female patients, both in total and in the working age group, but found little variation in the mean age between males and females. A third of ICD 841 patients in public hospitals were classified as having been readmitted for the same condition but not necessarily within the same year which may mean that annual costs per surgical patient were underestimated.

The total cost of prostheses was $\$ 0.370$ million when estimated by the ICD procedure method, and $\$ 0.341$ when estimated by the DRG method. Production loss for those of working age amounted to $\$ 2.764$ million. Using the procedure method, 218 patients fell within the working age group (15-64 years inclusive), that is, $32 \%$ of the total number of amputations for critical leg ischaemia. Males made up 67\% (145) and females 33\% (73) of this working age group. Under the DRG method, 90 patients (or 25\%) of the total number of critical leg ischaemia amputees were of working age. Males made up $72 \%(65)$ and females $28 \%$ (25) of this group and together incurred a production loss of $\$ 1.901$ million.

The procedure cost estimates were: hospital costs of $\$ 12.7$ million, prosthesis $\$ 0.4$ million, and lost production of $\$ 2.8$ million; while the DRG estimates were: hospital costs of $\$ 6.3$ million, prosthesis \$0.3 million, and lost production of \$1.9 million (Table 14.1). 
Table 14.1: Summary: Costs of Amputations for Critical Leg Ischaemia

\begin{tabular}{lcc}
\hline Total and average costs & $\begin{array}{c}\text { Procedure } \\
\text { method }\end{array}$ & $\begin{array}{c}\text { DRG } \\
\text { method }\end{array}$ \\
\hline Total costs & S million & $\$$ million \\
\hline Hospital (all costs) & 12.716 & 6.267 \\
Prostheses & 0.370 & 0.341 \\
Subtotal (quantified direct medical & 13.086 & 6.608 \\
costs) & & \\
Production loss & 2.764 & 1.901 \\
Total quantified costs & 15.850 & 8.509 \\
\hline Number of procedures & 688 & 353 \\
\hline Average cost per procedure & $\$$ & $\$$ \\
\hline Quantified direct medical costs & 19,020 & 18,720 \\
Production loss & 4,017 & 5,385 \\
Total cost & 23,038 & 24,105 \\
\hline
\end{tabular}

If Iloprost is administered for 20 days at a pharmaceutical cost of $\$ 185$ per day in a hospital setting at a ward cost of $\$ 330$ per day (Scott and Scott, 1993) the total medical cost per patient would be $\$ 10,300$ compared with a cost of $\$ 19,020$ (procedure method) per critical leg ischaemia patient undergoing an amputation. Medical cost savings per amputation avoided were $\$ 8,720$. If the medical treatment fails (Iloprost) then this pharmacotherapy cost of $\$ 10,300$ becomes an additional cost. There need to be 1.2 amputations avoided, that is, successful iloprost treatments to pay for 1 failure. In other words, for every 10 treatment failures with the drug there have to be 12 successes of the drug treatment. Thus the success rate must be $0.55(12 /(12+10)$ to breakeven on costs

If Iloprost is administered for 24 days (an average of the treatment length reported in European studies) the treatment cost rises to $\$ 12,360$ and the savings fall to $\$ 6,660$ per amputation avoided. There have to be 1.9 amputations avoided, that is, successful treatments to pay for 1 failure (for every 10 treatment failures there have to be 19 successes). Thus the success rate must be $0.66(19 /(19+10)$ to breakeven on costs. 


\subsection{DISCUSSION}

This study presented new information, hitherto unavailable, on the incidence and annual economic cost of amputations for critical leg ischaemia in New Zealand. Although the study focussed on hospital costs for amputations, artificial limb costs, Iloprost costs, and production loss for amputees of working age, other costs are important in evaluating the cost-of-illness but could not be quantified using the data available. Such costs include the quality of life, reduced mortality, community care costs, and production loss of those not in the work force.

The estimates of loss of production of those in the working age group would have been higher had a separate rate been used for males and females, as the male earning rate is higher and there were more males in the study. However, the estimates of lost production would have been lower had adjustments been made for unemployment and labour force non-participation. Lost production of those not in the paid workforce (although of no less importance to society) was not evaluated.

The procedure method of selecting patient records gave higher numbers than the DRG method. This was not unexpected as DRG code 113 related specifically to amputations for circulatory disorders, but if a patient had an additional and more costly procedure they would have been categorised under the more expensive DRG code relating to that procedure (and thus lost to DRG code 113). It was considered that the procedure method gave the best estimate of incidence and costs but the DRG method provided an absolute minimum estimate and was calculated as a cross-check and for sensitivity analysis.

Hospital unit costs used in the paper were the averages of 4 Area Health Boards. The greatest single group of records removed from the data base related to amputations as a result of accidents of all types including medical misadventure $(23 \%)$. Thus measuring the incidence of critical leg ischaemia from total amputation data would result in an overestimation of costs. 
The incidence of amputations for critical leg ischaemia estimated in this study was 202 (procedure method) per million people. If it is assumed that $25 \%$ of critical leg ischaemia patients have amputations (European Working Group on Critical Leg Ischaemia, 1991), the estimate of the incidence of critical leg ischaemia in New Zealand is 808 per million people. Rates in other developed countries ranged between 500 and 1,000 per million people (European Working Group on Critical Leg Ischaemia, 1991).

As one third of patients are readmissions, the prevalence of critical leg ischaemia if derived from amputations and/or admissions will be overestimated (as some but not all patients with readmission indicators will be readmitted during the course of a year). However, this will not result in costs being overestimated as costs were calculated using all admissions over the period of one year.

There were fewer female patients and little variation in average age between males and females but females had longer average hospital stays which may indicate possible inadequate early treatment of female patients, or if the condition develops in females, it is more severe.

The costs per amputation represent a weighted mean taking into account the mix of different types of amputation and requirements for artificial limbs, and as such, allow true comparisons to be made between treatment options. To compare the treatment costs of Iloprost with surgery it was assumed that the only difference in usage of medical resources between each mode of treatment was the hospital, surgery, prosthesis and Iloprost cost. In other words, costs up to the time of intervention either by amputation or treatment with Iloprost, would have been identical.

Treatment with Iloprost has been shown to lower mortality, reduce amputation rates (U.K. Severe Limb Ischaemia Study Group, 1991), heal ischaemic ulcers and to relieve rest pain (Dormandy, 1991). By avoiding or postponing amputation (Grant and Goa, 1992) Iloprost improves quality of life for the patient and reduces amputation costs. If amputation is simply postponed the patient may still have an improved quality of life and the present value of amputation costs will be reduced as the costs will have been moved forward in 
time. These benefits must be offset against the additional cost of the pharmacotherapeutic treatment.

If Iloprost is administered for 20 days there have to be 1.2 amputations avoided, that is, successful treatments to pay for 1 failure. The success rate must be $55 \%$ to breakeven on costs. However, if Iloprost is administered for 24 days there have to be 1.9 amputations avoided, that is, successful treatments to pay for 1 failure. The success rate must be $66 \%$ to breakeven on costs.

Iloprost has the potential to either reduce hospital costs if amputation can be avoided, or increase them if drug treatment fails and amputation is subsequently carried out. This is dependent upon the patients chosen for drug treatment, but at the time of the study clear selection criteria were not available. Iloprost as an alternative to amputation would also become more cost effective if it was able to be administered orally or in a lower cost hospital setting such as a day clinic (Dormandy and Stock, 1990) or at a time convenient to the patient thus minimising disruption to work and loss of production. Additional cost savings for successful pharmacotherapy would accrue if production loss avoided was included as a benefit.

\subsection{CONCLUSIONS}

Amputation for critical leg ischaemia is costly and has a high mortality. If it is possible to target those patients most likely to benefit from Iloprost (that is, avoidance of amputation and reduction of rest pain) the maximum benefit would be obtained from this pharmacotherapeutic option. For Iloprost treatment to be cost effective in a New Zealand hospital setting patients must be targeted and a success rate of $55 \%$ achieved.

Hospital policy makers were presented with two choices; remove a limb or part or a limb and save the patient's life, or attempt to save the limb and the patient's life with expensive drug treatment with the chance that this may fail and the patient would still require surgery and lose the limb. The cost of using the drug was $\$ 10,300$ which became an additional cost 
if the treatment failed and the limb subsequently had to be amputated. However, as the societal value for a limb or portion of a limb, or precise information on incremental effectiveness of the two treatment options was not available, the results were presented as breakeven ratios.

\subsection{POLICY POINTS}

The study may be regarded as a form of cost-effectiveness analysis in that both treatments are intended to save lives, but with surgery a limb or portion of a limb is amputated while drug treatment may save the limb. Outcomes with respect to quality of life are quite different, thus the preferred technique would be a cost-utility analysis but sufficiently robust data were not available for this to be undertaken.

The study used individual hospital records from a national database rather than data in aggregated or summarised form. This meant that the researchers had control over the methods used to summarise and analyse the data (see Chapter 8: Care With Data).

Two methods of estimating hospital resource utilisations were used as a cross-check. Both methods produced similar results and differences were in accord with expectations. Hospital unit costs were also authenticated from two different sources. Obtaining data from a number of sources and estimating results in different ways is a useful check on reliability and accuracy.

As clinical effectiveness information was uncertain, a breakeven technique was applied rather than the normal CBA approach. This method estimated a breakeven effectiveness ratio which was useful for selecting target groups rather than calculating costs per unit of effectiveness. Quality of life, although important for this condition, could not be evaluated because of paucity of data. Before robust policy guidelines for treatment can be developed more clinical and quality of life outcome evidence is necessary. This study presented policy makers with new policy inputs that would enable them to develop treatment policy guidelines. If the surgeon considered that there was a better than $55 \%$ success for a 
particular patient then it was cost effective to use the drug treatment. If the hospital policy makers considered that it was worth spending $\$ 10,300$ in an attempt to save a limb then drug treatment should be used for all such patients. The Health Funding Authority should take these factors into account when setting funding levels and contracting service volumes. In this case contracts should be set on the basis of achieving a desired outcome, leaving hospitals and medical staff to make choices as to the most appropriate treatment which may be either surgery or drug therapy. 


\subsection{REFERENCES}

Dormandy, J. "Use of the prostacyclin analogue Iloprost in the treatment of patients with critical limb ischaemia". Therapie 46: 319-322, 1991.

Dormandy, J. A., and G. Stock eds. Critical leg ischaemia. Its pathophysiology and management. Springer-Verlag, Berlin, 1990.

European Working Group on Critical Leg Ischaemia. Second European Consensus Document on Chronic Critical Leg Ischaemia. Circulation 84 (Suppl. IV): IV - 1 - IV - 26, 1991.

Grant, S. M., and K. L. Goa. Iloprost. "A review of its pharmacodynamic and pharmacokinetic properties, and therapeutic potential in peripheral vascular disease, myocardial ischaemia and extracorporeal circulation procedures". Drugs 43 (6): 889-924, 1992.

N.Z. Department of Health. Health Statistical Services. Unit record data relating to public and private hospitals for 1991. (National computerised data base of individual patient records)

N.Z. Department of Statistics. INFOS. (Information Network for Official Statistics) Total average weekly earnings for males and females, June 1991.

Norgren, L., A. Alwmark, K. A. Angquist, B. Hedberg, and D. Bergquist et al. "A stable prostacyclin analogue (Iloprost) in the treatment of ischaemic ulcers of the lower limb. A Scandinavian-Polish placebo controlled, randomised multicenter study". European Journal of Vascular Surgerv 4: 463-467, 1990.

Scott H. M., and W. G. Scott. 1994. "Critical Leg Ischaenia in New Zealand. Economic cost of amputation versus intravenous Iloprost". PharmacoEconomics 6 (2): 149- 154. 
Scott, W. G., and H. M. Scott. "Benign prostatic hyperplasia in New Zealand: a cost of illness study." PharmacoEconomics 4(6) (1993): 455-68.

U.K. Severe Limb Ischaemia Study Group. "Treatment of limb threatening ischaemia with intravenous Iloprost: a randomised double-blind placebo controlled study". European Journal of Vascular Surgery 5: 511-516, 1991. 


\section{CHAPTER 15: HEART FAILURE}

\subsection{SUMMARY AND INTRODUCTION}

\section{Summary}

A decision analytic model was used to combine New Zealand cost data with clinical evidence from an international randomly controlled trial. The model assessed the costeffectiveness of alternative drug treatment options. Paradoxical and previously unexplained differences between aggregated gender specific heart failure prevalence rates and age specific gender rates were isolated and explained. Application of the results provided new cost-effectiveness information vital for government decision making when negotiating prices and in preparing treatment guidelines.

\section{Introduction}

A cost-effectiveness analysis using a decision analytic model (Scott and Scott, 1996) evaluated the changes in direct medical costs, and life years gained or lost by adding the pharmaceutical, enalapril, to conventional treatment for heart failure using the drugs digoxin and diuretics.

Heart failure occurs when the heart muscic is damaged, overloaded, or exhausted by such conditions as hypertension, coronary artery disease, damage to cardiac valves, cardiomyopthy, and arrhythmias. The rates of hospitalization and mortality among such patients are high. About one-third of those suffering from congestive heart failure are hospitalised each year (The SOLVD Investigators, 1991). The one-year mortality ranges from 15\% among relatively unselected patients to 50\% among those in New York Heart Association functional class IV (The CONSENSUS Trial Study Group, 1987). 
In 1992 there were 456 people aged 60 years and over (168 males and 288 females) who died of heart failure. That is, a rate of 86 per 100,000 ( 71 for males, 98 for females). Mortality age specific rates rise with increasing age. Almost all (88.3\%) of the total heart failure deaths occur in those aged 80 years and over, with only $1.5 \%$ of deaths occurring in people under 60 years of age. As there are more females than males aged 80 years and over there are more deaths recorded for females than for males $(65.0 \%$ of all heart failure deaths were females). Chapter 8: Care With Data discusses these differences with respect to Simpson's paradox.

Heart failure is a high cost generator, incurring direct costs of primary medical care, hospitalisation, pharmaceutical treatment, indirect costs of lost production (lost output or productivity), and intangible costs of a reduced quality of life, and death. As the population ages and survival rates for cardiovascular events continue to improve increasing numbers of people will suffer frorm heart failure and as a result, medical costs from this condition will increase.

\subsection{METHOD}

The study did not evaluate indirect and intangible costs. A societal perspective was adopted and transfer payments were excluded from the analysis. All unit costs and prices were in 1993 dollars (either as recorded, or adjusted to 1993 using a health sector cost index developed for the study). As there were no reliable New Zealand data on age specific rates for heart failure, age specific rates from the US were applied to the New Zealand population to estimate numbers of heart failure patients. A decision analytic model developed in the US and described in Scott and Scott (1996) was used to integrate data from different sources and to conduct sensitivity analysis on key inputs and assumptions.

Costs and benefits were estimated incrementally. It was assumed that as enalapril was additional to existing treatment there would be no extra medical practitioner consultations. However, an additional dispensing fee and container charge amounting to $\$ 2.60$ per three months or $\$ 0.87$ per month was allocated for enalapril (Pricing schedules, 1993). It was 
also considered that as heart failure patients would be consulting their medical practitioners regularly no additional monitoring would be required. This premise was supported by the published results of SOLVD in which no additional monitoring was reported. The treatment altered the hospital admission rates for myocardial infarction, arrhythmia, and stroke in addition to heart failure and these changes were evaluated with respect to hospital costs.

However, as patients treated with enalapril lived on average two months longer than those patients treated with conventional medicines the incremental routine care costs incurred by enalapril patients during the additional two months were incorporated in to the model (SOLVD Investigators, 1991). It was estimated (from discussions with pharmacists) and subsequently verified (Taylor Nelson Healthcare, 1993) that New Zealand heart failure patients routinely visit their medical practitioner on average once every three months. Consultations are for monitoring and to obtain a new prescription for a further three months supply of conventional medicine. Thus, two-thirds of the 3-month cost (60 days supply) of the following were allocated to give; medical practitioner consultation (\$16.09), conventional therapy (digoxin $\$ 2.91$, furosemide $\$ 2.04$ ), and dispensing charges for each item of conventional therapy (\$3.47) (Pricing Schedules, 1993). The total of these additional non-enalapril drug costs or routine care costs was $\$ 24.51$. These costs were incorporated in to the model as a per monthly cost by dividing by the total undiscounted months of enalapril survival (38 months) to give a value of $\$ 0.65$. Medical costs for treatment of conditions other than for heart failure were not evaluated for these surviving patients.

It was considered that if a death occurred outside hospital, either an ambulance or a general practitioner or both would have been called to attend to the patient. Thus, the cost of an ambulance and the cost of a medical practitioner call-out were used for the estimate of the cost of deaths outside hospital. During the year ending March 1992 the average total cost per patient for the New Zealand Ambulance Board was \$159.74 (New Zealand Ambulance Board. Letter 31 August 1993) and was adjusted (using the health sector cost index) to $\$ 162$ per call-out. A typical call-out fee for certification of death by a general practitioner as at September 1993 was around \$50. Rates varied considerably depending on the day 
(holiday, weekend, week day), the time of day and geographic location (from a telephone survey of a small number of general practitioners in Auckland and Wellington). The base case value was $\$ 212$ (consisting of the ambulance cost of $\$ 162$ plus the typical general practitioner call-out fee of $\$ 50)$.

Data were unavailable in sufficient detail to enable separate costs to be calculated for fatal and non-fatal hospitalisations. It was therefore assumed that the costs for the hospital stay would be identical whether or not a patient was discharged alive or dead. However it is possible that those dying in hospital may have stayed a shorter time and thus incurred lower hospital costs. The limited range of DRG unit cost data available from Core Health (National Advisory Committee on Core Health and Disability Support Services, 1992) were supplemented by ICD unit cost data from individual cost-of-illness studies. As $95 \%$ of all hospital inpatients admitted for heart failure during 1992 were admitted to public hospitals (N.Z. Ministry of Health, 1981-93) the unit costs applicable to public hospitals were used for patients in both public and private hospitals.

With the exception of costs for stroke, the DRG and ICD unit costs fully capture the hospital costs. The possibility that some institutional costs associated with stroke have not been captured was taken into account by using data from a cost-of-illness analysis (Scott and Scott, 1994) in the sensitivity analysis.

Average length of stay in a public hospital in 1992 for acute myocardial infarction (ICD 410) was 8.6 days for males and 8.5 days for females (N.Z. Ministry of Health, 1981-93). An inpatient with myocardial infarction will spend on average two days in a coronary care unit and the remainder of their hospitalisation in a cardiology ward (Scott, White and Scott, 1993). The cost for a patient's total time in hospital was the weighted average of a stay of two days in a coronary care unit at $\$ 1,053$ per day, and 6.5 days in a cardiology ward at a daily rate of $\$ 592$ using prices relevant to 1 January 1994 (Nonresident prices. Green Lane Hospital, Auckland. Letter 22 February 1994). Applying this methodology the unit cost per myocardial infarction patient for their hospital stay was $\$ 5,954$. This cost was deflated 
using the HSCI (Health sector cost index constructed specifically for this study ${ }^{20}$ (Scott and Scott, 1996)) to establish a base case value of $\$ 5,948$ in September 1993 dollars. It was necessary to adjust unit cost items occurring at different times to a common base period with the health sector index.

The public hospital unit costs for cardiac arrhythmia for the year ending June 1991 (National Advisory Committee on Core Health and Disability Support Services, 1992) were divided into DRG 138 age over 69 and/or complicating condition and DRG 139 age under 70 without complicating conditions. A weighted average (by patient numbers) of the unit costs applicable to each of these DRGs was calculated and adjusted (using the price index constructed) to give a base case value of $\$ 2,666$. The public hospital DRG 127 unit cost for heart failure and shock (National Advisory Committee on Core Health and Disability Support Services, 1992) was adjusted to give a base case value of $\$ 5,030$.

\subsection{RESULTS}

Applying overseas disease incidence rates to the New Zealand population provided a base case estimate of 10,000 (rounded to the nearest 1000) for the numbers of people suffering from heart failure (Scott and Scott, 1996).

Potential net cost savings per patient treated over a 4 -year period were $\$ 652$ plus an additional two months of life gained. If enalapril therapy was extended to $100 \%(10,000$ people) of the population with heart failure who are untreated with ACE inhibitors there could be discounted ( $5 \%$ ) costs of $\$ 6.517$ million avoided (over 4 years), and in addition 1,583 undiscounted life years gained ( 19,000 months of life gained). A total of 3,465 hospitalisations could be avoided, and 476 deaths could be postponed beyond the 4-year period of the study. Virtually all the gain in lives saved was made in the first two years of treatment (year one: $69 \%$, year two: $21 \%$, a cumulative total of $90 \%$ ). $86 \%$ of hospitalisations avoided, occurred within year 1 . Over the 4-year period, if 1000 individuals

20 The consumers price index relates to costs facing the average household and was not considered appropriate for deflating health sector prices and expenditure. 
were treated with enalapril, 343 would die, while if 1000 individuals were treated only with conventional medicines 390 would die. In other words, there were 48 fewer deaths for enalapril patients treated over the four year period (because of rounding individual items do not add to the total shown). The model showed that total hospitalisations were reduced by 347 per 1000 individuals over a 4 year period of treatment with enalapril.

The additional costs $(\$ 1,064)$ incurred by a patient treated with enalapril were more than offset by hospital costs avoided and medical costs of death outside hospital avoided $(\$ 1,716)$. Base case estimates for an individual and for the population to whom enalapril treatment could be extended (New Zealand total) are summarised in Table 15.1.

\section{Table 15.1: Base Case Estimates}

\begin{tabular}{lcc}
\hline Estimate & Per individual treated & New Zealand \\
\hline Population treated $^{\text {a }}$ & 1 & 10,000 \\
Undiscounted months of life gained $^{\text {Deaths avoided }}$ & 1.9 & 19,000 \\
Hospitalisations avoided & 0.0476 & 476 \\
Costs & 0.3465 & 3,465 \\
Hospital costs avoided and medical cost of & $\$$ & $\$(000)$ \\
death outside hospital avoided & $1,716.16$ & 17,162 \\
Less Additional drug, dispensing, and medical & $1,064.51$ & 10,645 \\
practitioner costs & & 6,517 \\
$=$ Total net costs avoided & 651.65 & \\
\hline
\end{tabular}

a The population to whom enalapril treatment could be extended

As a result of rounding individual items may not add exactly to the totals shown

All costs discounted to present values (September 1993 dollars)

Sensitivity analysis showed that the results were robust with respect to changes in the input variables. Four factors in particular were found to be major determinants of the potential net cost savings; the population with heart failure to whom treatment could be extended, 
the percentage of this population treated with enalapril, the price of enalapril, and the nonfatal hospital costs.

If either of the first two determinants above is changed by a given percentage from the base case estimates then the total net costs avoided alter by the same percentage. If the enalapril price is altered by $20 \%$ (holding all other input values at their base case levels) then the total costs saved change by $32 \%$. Enalapril price could be increased by $63 \%$ before the total net costs avoided fall to zero. If the non-fatal unit cost for treating patients in hospital is altered by $20 \%$ then the total net costs saved change by $47 \%$.

Altering the discount rate makes very little difference to the results as most of the impact on costs occurs in the first year. It might be argued that a death outside the hospital is costeffective for a health care system and if all deaths (100\%) occur outside hospital the net savings in the model reduce by $25 \%$. However, this situation would not occur as there will always be some patients admitted to hospital who die of heart failure.

\subsection{DISCUSSION}

The study demonstrated that the addition of the ACE inhibitor, enalapril, to the conventional treatment of heart failure was cost effective when compared with conventional medical therapy alone.

The potential cost savings achieved by adding enalapril to conventional treatment for heart failure do not include the value to society of lives saved, productivity gains, or the increase in quality of life of those patients treated. Changes in productivity could be considered to be relatively unimportant as most patients with heart failure are not of working age. The benefit of enalapril was also underestimated as the SOLVD Treatment Trial did not take into account the additional cost for the greater number of patients in the placebo group who required additional therapy in the form of vasodilators to treat worsening congestive heart failure. From the narrow perspective of government budgets, prolonging life could impose additional costs with respect to welfare payments. 
It is unlikely that enalapril treatment could be applied to all patients untreated with an ACE inhibitor because, as with any intervention, a certain proportion of patients will suffer side effects and will have to be placed on an alternative treatment regimen. In addition not all patients (and this may include some patients experiencing side effects) will comply with the treatment regimen. The study was unable to evaluate the costs and benefits for males and females separately, or beyond a time horizon of 4 years as these factors were not evaluated in the original trial. Beyond the four year trial period the surviving patients will incur medical costs thereby lowering the net benefits of enalapril treatment.

Application of the model to 3 other countries has also been reported in the literature and average net cost savings per patient were; Australia \$A1 100, Canada \$Can 574, and France FF8076 (Wilde, Bryson and Goa, 1994). If converted into N.Z. dollars using exchange rates these cost savings would differ because of differing health care costs. Although ACE inhibitors slow the progression of the disease and treated patients will stay longer in less severe heart failure categories requiring less hospitalisation (van Hout, Wielink, Bonsel, et al., 1993) the economic benefits of treating asymptomatic patients with enalapril have not yet been assessed.

\subsection{POLICY POINTS}

The statistics on the prevalence of heart failure are interesting and a challenge to interpret. There are higher age specific death rates from heart failure for older males than for older females but the all ages and aggregated 60 years and over prevalence rates are higher for females. This paradox has important consequences for policy analysis and is discussed in Chapter 8: Care With Data.

Decision analytic modelling of the costs and benefits of treatment options is a valuable aid in the health sector for policy makers, hospital managers and clinicians. These models have the potential to provide valuable cost-effective information for policy analysis and decision making and should be used more often. (See Chapter 7: Models) 
Often decision makers want to apply the results of research in one country to another. Applying the results of a single study to different settings by converting costs and health effects estimated in one country by means of exchange rates and crude population numbers is inappropriate. Application of the SOLVD model in different countries will contribute to the global knowledge of the costs of treating heart failure however, country-specific unit costs and epidemiological data should be used as inputs to such health economic models.

Because prices and unit costs related to different time periods, a health sector price index needed to be constructed to standardise all costs to a common base. Commonly, because a specific price index does not exist analysts will resort to using the consumers price index or the GDP deflator. However it is sometimes possible to construct a more suitable index using a suitably weighted set of available indices.

This case illustrated the importance of public policy makers (if they are acting as agents for society) in taking a societal perspective rather than a narrow government budget holder perspective. There is a danger that a budget holder perspective may show that extending the lives of more people may add to social welfare costs. See Chapter 4: Costs where perspective is covered.

The results of this cost-effectiveness analysis were unusual in that costs were saved and lives extended. Most new treatments incur additional costs to achieve additional health gains. In these cases the policy maker faces an ethical dilemma, not choosing the least cost option means that some patients with other diseases may be denied care because budgets have been spent on heart failure patients, yet choosing the least cost option will mean that more patients will die from heart failure.

Chapter 3: I'aluing Health Outcomes, discusses the concept of QALY league tables where (if cost per QUALY are known for all diseases and population groups) resources could be allocated using the criteria of cost per QALY gain. The policy decision makers (PHARMAC and the Ministry of Health) using the results of such a study would have no 
such ethical dilemma as the findings clearly demonstrate both a saving in health resources and reduced mortality and improved quality of life by changing the drug treatment of heart failure patients. In situations such as this, the Ministry of Health (if it accepted the results) could advise the medical profession on the best prescribing policy to achieve the greatest health gains from available resources. 


\subsection{REFERENCES}

Core health and disability support services for 1993/94. First report of the National Advisory Committee on Core Health and Disability Support Services. The Committee, Wellington, 1992. Appendix 6.

N.Z. Ministry of Health. Hospital and selected morbidity data 1992. Wellington: N.Z. Ministry of Health, 1981- 93.

Pricing schedules: 1 September 1993. Wellington: N.Z. Ministry of Health, 1993: 59.

Scott, W. G., and Scott, H. M. "Ischaemic stroke in New Zealand: an economic study". New Zealand Medical Journal 1994; 107: 443-446.

Scott, W. G., and Scott, H. M. "Heart Failure: a decision analytic analysis of New Zealand data using the published results of the SOLVD treatment trial". PharmacoEconomics 1996; $9(2): 156-167$.

Scott, W. G., White, H. D., and Scott, H. M. "Cost of coronary heart disease in New Zealand". New Zealand Medical Journal 1993; 106: 347-349.

Taylor Nelson Cardiomonitor-New Zealand, Autumn 1993. Epsom, Surrey: Taylor Nelson Healthcare, 1993.

The CONSENSUS Trial Study Group. Effects of enalapril on mortality in severe congestive heart failure: results of the Cooperative North Scandinavian Enalapril Survival Study (CONSENSUS). New England Journal of Medicine 1987; 316:1429-35.

The SOLVD Investigators. "Effect of Enalapril on survival in patients with reduced left ventricular ejection fractions and congestive heart failure". New England Journal of Medicine 325 (August): 293-302, 1991 
van Hoult, B.A., G. Wielink, and G. J. Bonsel, et al. "Effects of ACE inhibitors on heart failure in the Netherlands". PhamacoEconomics, 1993 May; 3 (5): $387-97$.

Wilde, M. I., H. M. Bryson, and L. L., Goa, K.L. "Enalapril: a review of quality-of-life and pharmacoeconomic aspects of its use in heart failure and mild to moderate hypertension". PharmacoEconomics 1994; 6: 155-182. 


\section{CHAPTER 16: ISCHAEMIC STROKE}

\subsection{SUMMARY AND INTRODUCTION}

\section{Summary}

This chapter discusses two studies and how the information from two economic studies was integrated and used for policy evaluation. First a cost-of-illness analysis (Scott and Scott, 1994) was used to estimate the societal costs of ischaemic stroke. Second, the cost data generated from the cost-of-illness analysis were updated and used in combination with effectiveness data from a clinical trial data to conduct a cost-effectiveness analysis (Scott and Scott, 1997). The cost-effectiveness analysis of two drug treatment options for stroke prevention would not have been possible without the cost-of-illness study results. Finally, a comparison of CEA, CUA and CBA was made.

\section{Introduction}

Strokes are caused by an interruption of the flow of blood to the brain. They can occur with the rupture of an artery wall (cerebral haemorrhage: haemorrhagic stroke), blood clotting (thrombus: ischaemic stroke), or when a clot lodges in an artery (embolus: ischaemic stroke). A more formal definition of stroke is that of the World Health Organisation (WHO). WHO describe stroke as a rapid onset of disturbances of cerebral function leading to death or lasting for longer than 24 hours with no apparent cause other than vascular (Hatano, 1973).

Eighty-eight percent of all stroke cases of those aged 60 and above admitted to hospital in New Zealand were ischaemic (Friedman, 1992). For policy evaluation of treatment and prevention options for stroke, it is important to differentiate between these two classes of stroke (ischaemic and haemorrhagic) as their treatment differ. For example, if a haemorrhagic stroke were treated as if it were an ischaemic stroke the patients condition 
would worsen. The cost-effectiveness analysis considers treatment options for ischaemic stroke only.

In New Zealand (and in most other developed countries) stroke is the third most common cause of death and the incidence of stroke increases with age. Internationally, age adjusted incidence rates for "first in a lifetime" stroke range between 2 and 7 per thousand people for the age groups 55-64 and 65-74 years. If recurrent strokes (people who have had a stroke) are included, the incidence rates (new strokes per year) rise by between $20 \%$ and $30 \%$. Prevalence rates (all stroke sufferers) are about 4 to 5 times the incidence rates (Dunbabin, 1992).

Stroke patients are high users of hospital resources, and stroke often results in disablement which has important consequences for rehabilitation, and for long-term and community care costs. Patients with cerebrovascular disease (of which stroke is a subset) account for the greatest bed day usage in public hospitals $(1.5 \%$ of patients and $8.9 \%$ of bed days, (Scott and Scott 1994)). As many of stroke patients when discharged from hospital require costly community support or continuing care, strokes are important generators of direct and indirect costs.

\subsection{COST OF ISCHAEMIC STROKE}

\subsubsection{Introduction and Objectives}

This cost-of-illness study (see Chapter 4: Costs for the theoretical basis of cost analysis) estimated the costs of ischaemic stroke in 1992 on a prevalence basis. (Prevalence of a disease is the number of cases in the population for the period studied. Incidence of a disease is the number of new cases that occur within a given time period.) Both those patients having stroke events during the current year and survivors from past events (who still require treatment or support) contribute to costs, and are covered in the prevalence estimates. The objectives were to quantify and describe the annual cost of ischaemic stroke to New Zealand society during 1992. 


\subsubsection{Method}

The prevalence or cross sectional analysis estimated incremental costs from a societal perspective. All identifiable transfer payments, from the perspective of society, were excluded. Costs were measured in 1992 dollars. The methodology, data sources and estimation procedures are described fully in Scott and Scott (1994).

Unit record discharge data for all hospitals in both public and private sectors for the year ended 1992, and mortality data for the year ended 1990 were investigated. Some longer stay stroke patients could be transferred to another hospital and be recorded as a readmission within the same year. To minimise the potential for double counting, all hospital stroke patients with stays of more than 90 days were excluded from the hospital data. However, these patients were not lost to the study as they were captured in the rest home estimates (but at a lower day rate than acute hospital patients).

The number of ischaemic stroke events occurring annually was estimated from New Zealand hospital admissions and the assumption that $70 \%$ of strokes or 4,707 ischaemic stroke cases were admitted to hospital. This was based on a New Zealand study that found that two-thirds of all stroke patients entered hospital, and UK findings that $75 \%$ of patients with acute stroke are admitied to hospital (Scott and Scott, 1994). From this it was deduced that 2,017 were treated in the community and that there were 6,724 ischeamic strokes in total in 1992 . This method of estimating total strokes was necessary as hospital admissions are the only data recorded. These results are presented in Figure 16.1. 


\section{Figure 16.1: Incidence of Ischaemic Stroke in New Zealand and} Treatment Pathways

\section{Estimated incidence $(6,724 ; 100 \%)$}

\section{Treatment or Death}

\section{Hospital $(4,707 ; 70 \%)$ \\ Community $(2,017 ; 30 \%)$}

Information from which to construct event pathways was obtained from a number of different sources. Although the data was the best available from international sources the information should be regarded as indicative rather than conclusive. Figure 16.2 is a flow chart of event pathways for stroke assuming a $50 \%$ survival rate and that $50 \%$ of the survivors would require continuing support. Of the 6,724 ischaemic stroke cases, after 3 to 5 years, 3,362 will die and of the survivors 1,681 will require continuing support thus generating ongoing costs.

The number of patients requiring continuing care or support was based on stroke events, survival rates of $50 \%$ (at 3 to 5 years after an acute stroke event, most dying within the first month), and the percentages of survivors permanently disabled or needing community support. US data indicated that about half of stroke survivors had major motor problems and up to $30 \%$ of stroke survivors may be permanently disabled.

Forty-eight percent of all ischaemic stroke patients ( 60 years and over) surviving a stroke were discharged to rest homes or geriatric hospitals (Friedman, 1992). This percentage (rounded to $50 \%$ ), with hospital discharge data (live discharges of patients over 60 years) 
were used to estimate the number of patients discharged to rest homes or geriatric hospitals during 1992 (see Figure 16.2).

Figure 16.2: Treatment Pathways for Ischaemic Stroke Patients After 3-5 Years

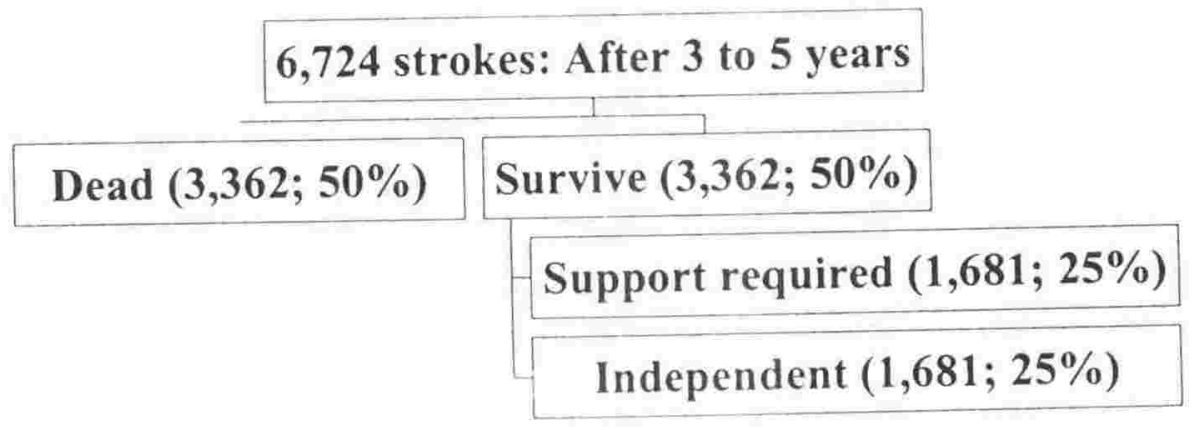

The total cost of general practitioner consultations was estimated from the number of consultations and the average fee charged. The value of medicine prescribed by general practitioners had the appropriate dispensing costs and markups added to the manufacturers selling price to obtain the cost to society. Transport costs of all ischaemic stroke patients admitted to hospital were evaluated using the average cost of an ambulance trip. Hospital costs were estimated, using two methods. The DRG (diagnosis related group) unit cost was multiplied by the number of patients. In the second method hospital patient days were multiplied by the daily rate for public hospitals $(\$ 300.00)$, and for private hospitals $(\$ 284.44)$.

As the costs of continuing care were not available, two estimation techniques were used. The first method evaluated the annual cost of a caregiver (either unpaid or paid) using total average weekly earnings for males and females combined. The second method used as a weekly cost, the average of Stage 1 and Stage 2 rest home subsidy (in general, the subsidy met the full costs). 
Data from Finland (Rissanen, 1992) allowed estimates to be made for the number of stroke patients dependent on outside help, permanently institutionalised as a result of the stroke, and requirements for physiotherapy and speech therapy sessions within the first year after stroke.

Loss of production was valued at the total average weekly earnings for males and females combined. This valuation was made for those in the working age group 15 to 64 years inclusive. A lower bound of 12 weeks off work, and an upper bound of 26 weeks off work was used, (as there was no national information available, these bounds were chosen after discussions with health professionals involved in rehabilitation of stroke). No deduction was made for non-labour force participation or unemployment. It was considered that nonpaid output and leisure time had a value to society and lacking more precise alternatives could be valued in the same manner as paid output.

\subsubsection{Results}

In 1992 there were 6,724 ischaemic stroke patients of whom 4,707 were admitted to hospitals (70\%), and 2,017 were treated in the community (30\%) in New Zealand. Almost all $(98 \%)$ of ischaemic stroke admissions were to public hospitals. There were 18,000 general practitioner consultations for stroke at a total cost of $\$ 0.49$ million. General practitioners prescribed medicinc valued at $\$ 0.46$ million for stroke patients. Transport for patients is a relatively important aspect of this disease because of the large number of acute admissions required for patients, most of whom were elderly. Acute admissions to public hospitals accounted for 3,959 or $86 \%$ of all admissions for ischaemic stroke. For the purposes of this study all admissions to hospital for ischaemic stroke patients were allotted an ambulance trip estimated to be $\mathrm{S} 1$ million in total.

Ischaemic stroke patients' hospital costs amounted to $\$ 28$ million. Calculation of hospital costs using the DRG unit cost gave a higher cost of $\$ 47$ million. The total cost of rehabilitation, that is, physiotherapy and speech therapy for each stroke patient was 
calculated to be $\$ 2$ million. It was estimated that 2,958 ischaemic stroke survivors required continuing care in a rest home as a result of the stroke. The annual cost of this continuing care when valued using the average weekly wage was estimated to be $\$ 90$ million, and when valued using the rest home subsidy rate was estimated to be $\$ 62$ million.

Lost production for the 912 hospital patients of working age ( 15 and under 65 years) who had an ischaemic stroke was valued at \$6 million (using 12 weeks off work), and \$14 million (using 26 weeks off work).

Direct medical costs ranged from $\$ 93$ million to $\$ 140$ million and loss of production was between $\$ 6$ million and $\$ 14$ million. Total quantified costs were estimated to lie between $\$ 99$ million and $\$ 154$ million. Hospital and continuing care costs when combined make up $90 \%$ of all quantified costs. Table 16.1 presents a summary of the costs for ischaemic stroke in New Zealand for 1992.

Table 16.1: Cost Summary - Ischaemic Stroke 1992

\begin{tabular}{lcccc}
\hline Cost & \multicolumn{3}{c}{ Low } & \multicolumn{2}{c}{ High } \\
& S million & $\%$ of total & S million & $\%$ of total \\
\hline General practitioner & 0.49 & 0.49 & 0.49 & 0.32 \\
GP prescribed medicines & 0.46 & 0.46 & 0.46 & 0.30 \\
Transport to hospital & 0.75 & 0.76 & 0.75 & 0.49 \\
Hospital & 27.97 & 28.22 & 47.43 & 30.82 \\
Rehabilitation & 1.17 & 1.18 & 1.50 & 0.97 \\
Continuing institutionalised care & 61.94 & 62.48 & 89.50 & 58.16 \\
Total direct medical & 92.78 & 93.59 & 140.13 & 91.06 \\
Loss of production & 6.35 & 6.41 & 13.75 & 8.94 \\
Total direct and indirect costs & 99.13 & 100.00 & 153.88 & 100.00 \\
\hline Note: Because of rounding, individual items may not add exactly to the totals shown. \\
Results quoted in the text have been rounded to the nearest \$1 million.
\end{tabular}


Some time after publication of the study the authors were asked to conduct a costeffectiveness analysis of ischaemic stroke prevention drug treatment. As numbers of hospital admissions for stroke had not changed greatly, resource utilisation (volume) data from Scott and Scott (1994) was used in conjunction with the latest available unit cost data to update the cost-of-illness study. This was necessary because prices had altered in the intervening period. Table 16.2 shows the how the volumes obtained from the 1992 study were updated using 1996 unit costs. These updated data were used in the cost-effective analysis reported in section 16.3 .

Table 16.2: Ischaemic Stroke - 1992 Volumes, Unit Costs Updated to 1996 Dollars

\begin{tabular}{|c|c|c|c|c|c|}
\hline \multirow[t]{3}{*}{ Event /Cost Item } & & \multirow{3}{*}{$\begin{array}{l}\text { Volume* } \\
1992\end{array}$} & \multirow{3}{*}{$\begin{array}{l}\text { Unit cost } \\
1996 \\
\text { NZ\$ }\end{array}$} & \multirow{3}{*}{$\begin{array}{c}\text { Total cost } \\
\begin{array}{c}1996 \\
\text { NZ\$ }\end{array}\end{array}$} & \multirow{3}{*}{$\begin{array}{c}\text { Cost per } \\
\text { stroke } \\
1996 \\
\text { NZ\$ }\end{array}$} \\
\hline & & & & & \\
\hline & & & & & \\
\hline Number of strokes in 1992 & & 6,724 & & & \\
\hline General practitioner consultations & Number & 18,000 & 29 & 520,000 & 77.33 \\
\hline GP prescribed medicines & & & & 460,000 & 68.41 \\
\hline Primary care & & & & $\underline{980,000}$ & $\underline{145.75}$ \\
\hline Hospital \# & Number & 4,707 & 5,943 & $27,974,000$ & $4,160.32$ \\
\hline Rehabilitation \# & Sessions & 3,694 & 318 & $1,174,692$ & 174.70 \\
\hline Continuing institutional care & Number & 2,958 & 26,217 & $77,549,393$ & $11,533.22$ \\
\hline Transport to hospital \# & Number & 4,707 & 160 & 751,896 & 111.82 \\
\hline Direct medical costs & & & & $\underline{108,429,981}$ & $\underline{16,125.82}$ \\
\hline Loss of production & Weeks & 10,944 & 627 & $6,861,560$ & $1,020.46$ \\
\hline Total direct medical and indirect costs & & & & $\underline{115,291,541}$ & $\underline{17,146.27}$ \\
\hline
\end{tabular}

\section{Unit cost sources}

General practitioner consultations: What do you pay your doctor? Consumer 1996; (April) p24 5

\# Hospital, rehabilitation and transport to hospital: Low estimates from (Table 4, Scott and Scott, 1994)

Continuing institutional care: average of stage 2 and stage 3 rest home care. (Purchasing for your health 1994.95. table 5.3, p116)

Production: Total average weekly earnings for males and females combined, Mar 96. N.Z. Department of Statistics. INFOS 12 weeks per patient

* (Scott and Scott. 1994)

The major cost component was continuing institutional care ( $72 \%$ of direct medical costs) followed by hospital in patient stays $(26 \%)$. 


\subsubsection{Discussion/Conclusions}

Ischaemic stroke affects mainly older people, many of whom are discharged into the community and require costly community support or continuing care. The level of disability for patients after stroke is dependent upon the severity of the stroke. Disability manifests itself as epilepsy, depression, and cognitive losses (for example, in language and perception). Studies of surviving stroke patients have shown reduced social contact with those outside immediate family and diminished leisure activity. Some of the costs associated with these disabilities have been captured in rehabilitation and continuing care costs but none of the reduction in quality of life has been quantified.

As Diagnosis A (the primary diagnosis for which the patient was admitted) was used to select patient records from the hospital unit data both the numbers of ischaemic stroke patients and hospital costs are conservative. Patients admitted to a public hospital for an unrelated Diagnosis A but additionally diagnosed ischaemic stroke in Diagnoses B, C or D were not included in the analysis as it was impossible to separate the additional stroke cost component from the cost of the primary diagnosis using the data available (see Chapter 4: Costs where joint costs and co-morbidities are discussed). Private hospital data are recorded for primary diagnosis only. Patients admitted to one hospital and subsequently transferred to another are recorded as separate records and as such all the hospital costs have been captured but numbers may have been double counted as discussed in section 16.2.2: Method.

It was calculated that in 1992 there were approximately 1,557 patients 60 years and over discharged live to rest homes or geriatric hospitals, but as it was not known how long these patients stayed in care, this estimate could not be used to obtain total costs. Continuing care is an important component of the cost of ischaemic stroke to society.

New Zealand rest home/continuing care total costs were three times the acute hospital total costs. USA data (Adelman, 1981) indicated that nursing home costs were equivalent in 
magnitude to $75 \%$ of inpatient hospital care total costs. This difference between the ratios of continuing care to acute hospital costs between USA and New Zealand may be a result of our decision to exclude long stay patients from the New Zealand hospital data and to capture these costs under rest homes. As this is a major cost (which is "hidden" from policy makers because of difficulty in obtaining data on costs paid for by patients and their families), further research should be undertaken to obtain estimates of the case mix and numbers of patients resident in rest homes and those requiring similar care in private homes. Policy analysts concerned with decisions involving stroke and rest home costs need this data for informed decision making.

\subsection{COST-EFFECTIVENESS OF ALTERNATIVE METHODS OF ISCHAEMIC STROKE PREVENTION}

\subsubsection{Introduction and Objectives}

The cost-effectiveness analysis (see Chapter 6: Economic Evaluation for the theory underlying CEA) used the results from the cost-of-illness study to evaluate cost offsets (costs avoided) as a result of stroke prevention treatment reported by the European Stroke Prevention Study 2 (ESPS-2) (Diener et al., 1996). ESPS-2 compared a new preventative treatment against the standard currently used.

The ESPS-2 trial considered effectiveness but not cost or cost-effectiveness. Accordingly, the aim of this cost-effectiveness analysis study was to compare the cost-effectiveness of alternative drug regimens in the secondary prevention of ischaemic strokes in New Zealand.

\subsubsection{Method}

Comparisons were made between the costs of low-dose acetylsalicylic acid monotherapy and a combination therapy of modilied-release dipyridamole and low-dose acetylsalicylic acid. Differences in undiscounted direct medical costs were calculated over a 2 -year period. New Zealand costs per stroke event (Scott and Scott 1994) were multiplied by the ESPS-2 
incremental reductions in stroke events to derive the costs of strokes avoided.

As the focus of the paper was on direct medical costs the primary perspective adopted was that of a health care provider or funder, but a societal perspective was also considered when indirect costs were evaluated.

The clinical effectiveness evidence was derived from the European Stroke Prevention Study 2 (ESPS-2) (Diener et al., 1996). The two-year trial (the largest to date) involved 6,602 patients who had experienced either a transient ischaemic attack (TIA) (23.7\%) or an ischaemic stroke $(76.3 \%)$ within the previous three months. ESPS-2 patients were randomised to the following treatment regimens: (1) modified-release dipyridamole $200 \mathrm{mg}$ twice a day combined with low-dose acetylsalicylic acid $25 \mathrm{mg}$ twice a day, (2) modifiedrelease dipyridamole $200 \mathrm{mg}$ twice a day, (3) low-dose acetylsalicylic acid $25 \mathrm{mg}$ twice a day, or (4) matched placebo twice a day.

The ESPS-2 study assessed the effects of the treatment regimens in the prevention of ischaemic cerebro-vascular events in those patients who have already had a cerebral ischaemic event. These patients were selected because they were highest risk population group and would derive the most benefit from preventative treatment.

The absolute risk of stroke (fatal or non-fatal events) per 1000 patients treated was reduced in each treatment regimen (compared with placebo): by 26 events for those receiving modified-release dipyridamole, by 29 events for the acetylsalicylic acid group, and by 58 events for those receiving the combination therapy of modified-release dipyridamole and acetylsalicylic acid.

There was greater benefit to the patient (in risk reduction of a stroke) if a combination therapy (modified-release dipyridamole and acetylsalicylic acid) was given rather than either medicine alone. Although each medicine was in itself effective in reducing stroke risk, when given in combination their effects were additive. The analysis compared only the combination therapy against acetylsalicylic acid which was chosen as the comparator 
as it is the drug regimen most likely to be replaced by the combination therapy being the lowest cost and most widely used alternative. Table 16.3 summarises these results which provided the effectiveness evidence for the cost-effectiveness analysis.

The focus of the paper was on incremental costs, thus all resource utilisations (for example, general practitioner consultations) prior to drug acquisition were irrelevant and not evaluated. Differences in undiscounted costs were calculated over a 2-year period from a societal perspective. Cost of stroke data were obtained from a New Zealand cost-of-illness study (Scott and Scott, 1994) updated to 1996 New Zealand dollars.

Table 16.3: Results of the European Stroke Prevention Study 2 Relevant to the Cost Analysis

\begin{tabular}{lc}
\hline Strategy & $\begin{array}{c}\text { Stroke events avoided per } 1000 \text { patients } \\
\text { (relative to placebo) }\end{array}$ \\
\hline DYP + ASP & 58 \\
ASP & 29 \\
Difference ((DYP + ASP) - ASP) & 29 \\
\hline
\end{tabular}

Abbreviations: $\mathrm{ASP}=$ low-dosage aspirin (acetylsalicylic acid); DYP = modified-release dipyridamole.

As very low dose $(25 \mathrm{mg}$ ) acetylsalicylic acid (as used in ESPS-2) was unavailable in New Zealand, unit costs were based on half a $300 \mathrm{mg}$ tablet. The costs per stroke event were calculated by dividing the total costs by the number of stroke events estimated in the costof-illness paper (Scott and Scott, 1994). New Zealand costs per stroke event were multiplied by the ESPS-2 incremental reductions in stroke events to derive the costs of strokes avoided.

\subsubsection{Results}

For every 1000 at-risk patients treated for ischaemic stroke prevention over two years with modified-release dipyridamole combined with low-dose acetylsalicylic acid replacing acetylsalicylic acid on its own the following differences would occur. Twenty-nine stroke 
events would be avoided, direct costs of $\$ 18,223$ would be avoided and in total, $\$ 40,963$ direct and indirect costs would be saved. Individually, each treatment regimen resulted in direct medical cost savings when compared with the placebo but what is important is the difference between the treatments. Table 16.4, column 3, summarises the costs on a per 1000 patient basis for a 2 -year period.

Table 16.4: Two-year Costs Per Patient Treated for Ischaemic Stroke Prevention: Modified-release Dipyridamole/ Low-dose Acetylsalicylic Acid Combination Compared with Low-dose Acetylsalicylic Acid in 1996 Dollars

\begin{tabular}{|c|c|c|c|}
\hline \multirow[t]{3}{*}{ Effect/ Cost } & \multicolumn{3}{|c|}{ Per patient } \\
\hline & DPY/ASP & ASP & Incremental DPY/ASP less \\
\hline & & & ASP \\
\hline \multirow[t]{2}{*}{ Ischaemic stroke events } & 0.058 & 0.029 & 0.029 \\
\hline & NZ\$ & NZ\$ & NZ\$ \\
\hline \multicolumn{4}{|l|}{ Preventative treatment cost: all patents in } \\
\hline \multicolumn{4}{|l|}{$\underline{\text { treatment arm }}$} \\
\hline Drug acquisition & 965.43 & 11.91 & 953.52 \\
\hline \multicolumn{4}{|l|}{ Costs generated by ischaemic stroke } \\
\hline \multicolumn{4}{|l|}{$\underline{\text { events }}$} \\
\hline Primary care & $(16.91)$ & $(8.45)$ & $(8.45)$ \\
\hline Hospital & $(482.60)$ & $(241.30)$ & $(241.30)$ \\
\hline Rehabilitation & $(20.27)$ & $(10.13)$ & $(10.13)$ \\
\hline Continuing institutional care & $(1,337,85)$ & $(668.93)$ & $(668.93)$ \\
\hline Sub total: Direct medical cost & $\underline{(892.20)}$ & $(916.90)$ & $\underline{24.71}$ \\
\hline Transport to hospital & $(12.97)$ & $(6.49)$ & $(6.49)$ \\
\hline Sub total: Direct cost & $(905.16)$ & $(923.39)$ & $\underline{18.22}$ \\
\hline Loss of production & $(118.37)$ & $(59.19)$ & $(59.19)$ \\
\hline Total direct medical and indirect cost & $(1,023.54)$ & $(982.57)$ & $(40.96)$ \\
\hline
\end{tabular}

\section{Notes:}

Loss of production was evaluated for working age hospital patients' time off work, loss as a result of premature death was not evaluated

$($ ) = negative values, a negative cost $=$ a cost avoided or a benefit Incremental stroke events and costs $=$ DPY/ASP less ASP

DPY/ASP = modified-release dipyridamole/ low-dose acetylsalicylic acid combination ASP $=$ low-dose acetylsalicylic acid

Because of rounding individual items may not add exactly to the totals shown 
Incremental cost effective ratios, the difference in cost divided by the difference in effectiveness (strokes avoided), were calculated. To avoid one additional stroke event by changing therapy, $\$ 1,413$ total net direct and indirect costs would be avoided ( $\$ 40.96 / 0.029$ strokes), $\$ 628$ additional net direct costs would be incurred (\$18.22/0.029 strokes) and $\$ 852$ additional net direct medical costs would be incurred (\$24.71/0.029 strokes). Figure 16.3, shows the incremental cost-effectiveness NZ\$ per stroke event that is avoided.

Figure 16.3: Incremental Cost-Effectiveness NZ\$ per Stroke Event Avoided

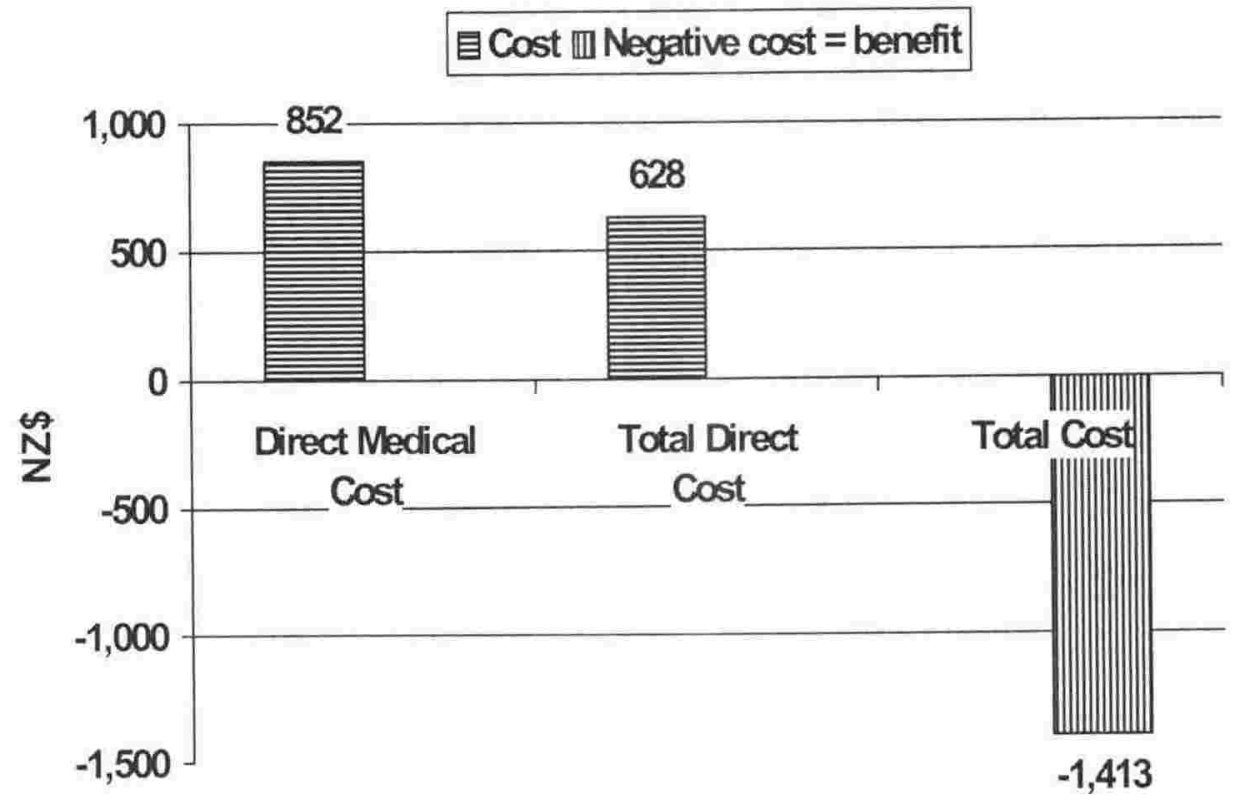

Cost-Effectiveness Ratio

\subsubsection{Discussion/ Conclusions}

The combination therapy was more cost-effective (with respect to total societal costs) compared with acetylsalicylic acid (the comparator). Sensitivity analysis revealed that the main cost drivers were hospital and continuing institutional care volumes and unit costs (Scott and Scott, 1997).

In the ESPS- 2 trial $22 \%$ of the placebo group, $22 \%$ of the acetylsalicylic acid group and 
$29 \%$ of those receiving the combination therapy discontinued treatment for medical or nonmedical reasons. Thus, it was concluded that $78 \%$ of the potential candidates for secondary stroke prevention could be treated with acetylsalicylic acid and $71 \%$ could be treated with the combination therapy of modified-release dipyridamole and acetylsalicylic acid. This would imply that $91 \%$ of patients currently treated with acetylsalicylic acid alone could be changed to the combination therapy.

ESPS-2 demonstrated that with the combination therapy, (although the number of deaths remain unchanged), the number of stroke events are reduced. This means that the number of patients progressing to a state where either continuing care, or more intense care is required would be reduced. However, this assumes that the distribution of severity of stroke events was identical for a!l treatment groups, but if the stroke events of one group are more disabling than those of another group the costs per stroke event would be different.

The weighted average costs for strokes used (Scott and Scott, 1994) covered the costs of all stroke patients, (fatal and non-fatal and those who recovered with varying degrees of incapacity and dependence) rather than just those patients admitted to hospital.

The analysis demonstrated that changing patients from acetylsalicylic acid to a combination therapy of modified-release dipyridamole and acetylsalicylic acid would result in a small rise in incremental direct costs (using our conservative assumptions relating to hospital and continuing institutional care costs). If less conservative unit cost assumptions were adopted (which is a more likely scenario) there would be greater savings in incremental costs. Although the study focused on the cost comparisons between the combination regimen versus acetylsalicylic acid, the combination regimen could replace both single drug therapies. 


\subsection{COMPARISONS BETWEEN ALTERNATIVE METHODS OF EVALUATION (CEA, CUA AND CBA)}

\subsubsection{Introduction and Objectives}

The findings from the cost-effectiveness analysis of ischaemic stroke prevention are used to demonstrate the differences between CEA, CUA and CBA. (Chapter 6: Economic Evaluation discusses the theory underlying CEA, CUA and CBA).

\subsubsection{Method}

The results from the cost-effectiveness analysis and assumptions relating to QALY gain and the value of a QALY to New Zealand society provided the information used to develop the comparisons between the three economic evaluation methods.

Incremental costs and effects were derived from table 16.4. For the purposes of these two illustrative examples it was assumed that 5 QALYs would be gained per stroke avoided, and that the value of a QALY was $\$ 10,000$.

\subsubsection{Results}

In the CUA example, the 0.029 strokes avoided translated into 0.1450 QALYs gained (5 QALYS per stroke avoided $\times 0.029$ strokes avoided $=0.450$ QALYs). The additional direct cost per QALY gained was $\$ 126(\$ 18.22 \div 0.1450=\$ 125.66)$.

For the CBA study, the QALY gain per stroke avoided was converted into an intangible benefit in dollars of $\$ 1,450$ per stroke avoided $(0.1450$ QALYs $x \$ 10,000=\$ 1,450.00)$. When the incremental intangible costs were added to the incremental direct and indirect costs a net incremental cost saving of $\$ 1,491$ per patient treated was obtained. In this example, negative costs represent benefits.

The results of this analysis are presented in table 16.5 . 
Table 16.5: Two-year Incremental Costs Per Patient Treated for Ischaemic Stroke Prevention: Modified-release Dipyridamolel Lowdose Acetylsalicylic Acid Combination Compared with Low-dose Acetylsalicylic Acid in 1996 Dollars: CEA, CUA, CBA: all Costs and Effects Measured Incrementally DPYIASP Less ASP

\section{a) CEA}

\section{Incremental costs (C) and health outcomes (E)}

\begin{tabular}{lc}
\hline Direct costs & $\$ 18.22$ \\
\hline Indirect costs & $-\$ 59.19$ \\
Direct and indirect costs & $-\$ 40.96$ \\
\hline Ischaemic stroke events avoided (E) & 0.029 \\
Incremental cost effectiveness ratio C/E & \\
Direct costs per stroke avoided & $\$ 628$ \\
Total direct and indirect costs per stroke avoided & $-\$ 1,413$ \\
\hline
\end{tabular}

b) CUA

Incremental costs (C) and QALYs (U)

Direct costs

QALY gain $(U)^{1}$

Incremental cost-utility (C/U) ratio: direct costs per QALY gained

(1) Assumed 5 QALYs gained per stroke avoided

\section{c) CBA}

\begin{tabular}{lr}
\hline Incremental costs & \\
\hline Direct & $\$ 18.22$ \\
Indirect & $-\$ 59.19$ \\
Intangible $^{2}$ & $-\$ 1,450.00$ \\
Total incremental cost & $-\$ 1,490.97$ \\
\hline
\end{tabular}

(2) Assumed \$10,000 per QALY

\subsubsection{Discussion/ Conclusions}

Although the original research for the cases did not encompass examples of CUA and CBA it was considered that this case could be used as a hypothetical example to illustrate concisely and clearly the similarity and differences in the data input requirements and output measures between CEA, CUA and CBA studies. Detail on the conduct of CUA and CBA was discussed in chapter 3: Valuing health outcomes and in chapter 6: Economic evaluation. As the CUA and CBA examples are partially based on assumptions, the 
findings quoted were not and should not be used for actual policy decision making.

Results from the CUA demonstrated that if the health sector cut-off price per QALY was $\$ 126$ (of direct costs) or higher, the treatment should obtain funding approval. The CBA study showed that if society valued a QALY at $\$ 10,000$ then the net benefit to society would be $\$ 1,491$ per additional patient treated. These results suggest that (1) resources within the health sector that are not delivering one QALY per \$126 should be transferred towards stroke prevention, and (2) that additional funding be directed into the health budget to allow this treatment to proceed. CBA is used to assist in allocating overall funding levels to health while CUA and CEA assist in allocating resources within the health budget.

Both the CUA and CBA used hypothetical values for the number of QALYs gained per stroke avoided and for the dollar value per QALY gained. The conclusions would be different had different values been used. Further research or literature reviews would be necessary to establish the QALY gain per stroke avoided.

\subsection{POLICY IMPLICATIONS}

Costs of stroke are a major component of total health costs. Stroke prevention is important both in terms of health related outcomes and in the direct and indirect costs generated. Because of the combination of an ageing population and increasing survival rates with constant incidence rates, there will be a substantial rise in the costs of continuing care and community support. Most of these costs are at present obscured because much of the care provided does not involve money payments and/or data are not recorded and collated on a national basis.

Inappropriate policy decisions may be made if the non-hospital costs of stroke are not taken into account. Policy makers frequently base their decisions entirely on the financial impact on their budgets. The results of this could be cost shifting from the public sector to private individuals. Private sector costs that may increase include; paid and unpaid caregiver costs, possible modifications to private homes, loss of quality of life of the patient's family. 
The importance of ignoring costs that would be incurred irrespective of the occurrence of stroke was illustrated by the cost of stroke analysis. This showed that approximately $25 \%$ of stroke patients in institutional care would need this care irrespective of their stroke, although they would probably require a higher level of care as a result of the stroke (see incremental costs, Chapter 4: Costs).

The danger of multiple counting of hospital costs is demonstrated (see the dangers of multiple counting, Chapter 4: Costs). Longer stay stroke patients may be transferred to another hospital and thus be recorded as a readmission within the same year. To minimise the potential for double counting, all hospital stroke patients with stays of more than 90 days were excluded from the hospital data but not lost to the study as they were captured under continuing care.

Accurate cost and prevalence data are essential for the economic evaluation of treatment options, policy changes, and the manner in which an ageing population may affect health costs in hospitals and the community. The cost-of-illness study provided the epidemiological and cost of stroke data necessary for the cost-effectiveness study.

New Zealand national data were non existent or very sparse and posed serious problems which were overcome by obtaining information from panels of experts and from international literature. Results from international multi centre clinical trials and overseas prevalence and resource utilisation studies were combined with New Zealand data to conduct both the cost-of-illness and the cost-effectiveness analyses. Lack of a complete data set is a problem almost invariably encountered when conducting health policy analysis in New Zealand but this should not preclude an analysis being undertaken. Problems with data are discussed in Chapter 8: Care With Data.

New Zealand does not have comprehensive and ready to use cost and effectiveness data. Policy analysts must either derive their own primary cost and effectiveness data or use the best secondary data available. The questions that need to be asked are; will the additional costs of setting up their own studies justify the expected benefits of the data acquisition, 
and will there be time available to obtain the data before the decision must be made? Usually the answer to one of these questions will be "no" and the use of secondary data will be preferred to that of generating primary data.

Cost-of-illness studies provide; (1) essential data for a general overview of the economic importance of a disease, (2) a ready source of costing data for cost-effectiveness analysis and (3) highlight those costs that are of major importance. If a cost-of-illness study needs to be updated it may not be necessary to quantify costs of minor importance.

The results of cost-of-illness evaluations (resource utilisations, unit costs and total costs) should all be reported separately. This facilitates updating and the use of the study findings in modelling (for example, use of the findings in other settings and in other population groups).

The choice of comparator in the cost-effectiveness study was important as both treatments under consideration resulted in cost savings if compared against placebo, but the combination therapy provided a lower cost effective ratio (see Chapter 18: Discussion and Conclusions for a discussion of the selection of comparators).

It could be argued that for an economy with less than full employment, a proportion of the potential loss of output of those of working age should be omitted from the analysis as this would not reduce the economy's measured GDP. However, this approach ignores the opportunity cost of lost unpaid output and the value of leisure time (see Chapter 4: Costs where the frictional cost method is discussed).

Loss of life could have been evaluated using willingness to pay criteria or the human capital approach but because of the age of stroke patients neither method yields satisfactory nor plausible results. The human capital (see Chapter 3: Valuing Health Outcomes where estimating health related outcomes is discussed) method undervalues the lives lost because of the low number of potential working years lost. Given the average age of the patients ( 73 years, and 77 years, for public and private patients respectively) the willingness to pay 
value of \$2 million per life (Millar and Guria, 1991) would result in a total cost that may be considered by some readers to be too high. Age specific values for statistical lives would facilitate analysis of this type.

Hospital and continuing care costs could be saved by reducing the incidence of stroke by using a more cost effective drug regimen. To maximise society's benefits, the decision makers must have information spanning public hospitals, pharmaceuticals, rehabilitation, community care and support, and be prepared to act upon this information.

This published CEA study was used as evidence in negotiations between the government drug buying agency and drug suppliers. Neither a CUA nor a CBA analysis would have have provided any additional information that would have been considered by policy makers.

CEA studies predominate in the health economic evaluation literature because they provide adequate information for decision making within most present policy environments. CBA and CUA are rarely used for health sector policy evaluation because (1) the required data is difficult and costly to obtain and (2) the current decision making processes could not effectively use the results of such studies.

CUA provides information that could potentially be used to compare costs per QALY gained across all health care interventiors. However, neither a set of QALY league tables nor a cut-off price per QALY (that the health sector was prepared to pay) was available. CBA permits comparisons of the net benefits to society from the implementation of any policy or project. The CBA results would not have provided any additional usable information for decision making because New Zealand, government budgeting decisions across Votes are not made using the results of CBA.

Values for the cut off price per QALY for the health sector, and the societal value of a QALY could be estimated by surveying the New Zealand community but the final decisions would have to be political. 


\subsection{REFERENCES}

Adelman, S. M. "Economic impact. National survey of stroke". Stroke 1981; 12 (Suppl. I): $169-78$.

Diener, H. C., L. Cunha, C. Forbes, J. Sivenius, P. Smets, and A. Lowenthal. European Stroke Prevention Study 2. "Dipyridamole and acetylsalicylic acid in the secondary prevention of stroke". Journal of the Neurological Sciences 1996;143:1-13.

Dunbabin, D. "Cost-effective intervention in stroke". PharmacoEconomics 1992; 2(6):468-99.

Friedman, P. J. "Ischaemic stroke and intracerebral haematoma in the Waikato stroke registry". New Zealand Medical Journal 1992;105:171-3.

Hatano, S. Control of stroke in the community - methodological considerations and protocol of WHO stroke register. WHO document no CVD/S73.6 Rev 1, 1973.

Millar, T., and J. Guria. The value of statistical life in New Zealand. Wellington: Ministry of Transport, 1991.

Rissanen, A. Cerebrovascular disease in the Jyrskla region, central Finland. Kuopio: University of Kuopio, 1992 (Department of Neurology Series of Reports No. 23)

Scott, W. G., and H. M. Scott. "Ischaemic stroke in New Zealand: an economic study." New Zealand Medical Joumal 107 (1994): 443-46.

Scott, W. G., and H. M. Scott. "Application of the findings of the European stroke prevention study 2 to a New Zealand ischaemic stroke cost analysis." PharmacoEconomics 12 (1997): 667-74. 


\section{CHAPTER 17: ASTHMA}

\subsection{SUMMARY AND INTRODUCTION}

\section{Summary}

This chapter discusses a research project that was made up of three parts. These components were integrated and published in two papers and in the form of a CD-ROM. An administrative database (general practice records) was used to provide some of the information required for a cost-of-illness study and to investigate differences in direct costs between alternative asthma medication delivery devices. The cost-of-illness analysis and the cost of alternative treatments analysis provided the data used to design and validate the decision analytic model. This model was used with general practice fund holders and Regional Health Authority policy makers to demonstrate cost differences between alternative asthma treatments. The model permitted users to assess the budgetary impact of different asthma treatment policy options.

\section{Introduction}

Three policy evaluation techniques; cost-of-illness analysis (Chapter 4: Costs), an economic evaluation of treatment options (Chapter 6: Economic Evaluation) and decision analytic modelling (Chapter 7: Models) are addressed in this chapter. The techniques are applied to asthma. The cost-of-illness and the asthma inhaler cost comparison analyses provided the data used to design and validate the decision analytic model (Figure 17.1) The discussion draws upon two published papers (Scott et al., 1997, Scott et al., 1998). 
Figure 17.1: Sources of Data for the Asthma Cost Model

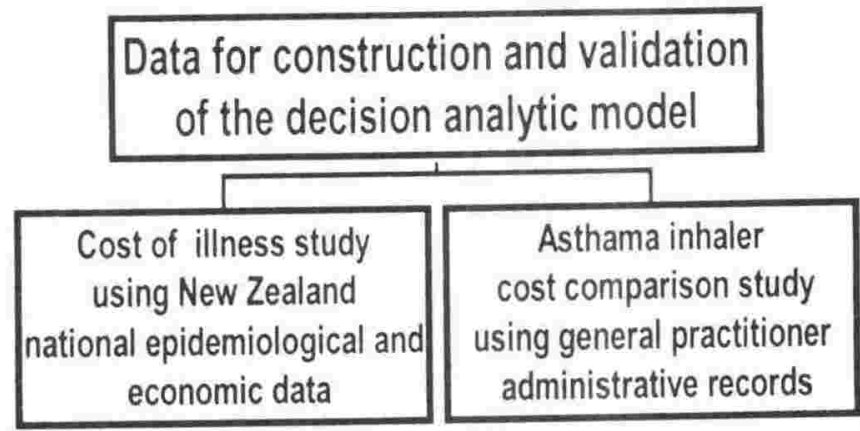

\subsection{BACKGROUND AND OVERALL OBJECTIVES}

Asthma is a chronic inflammatory disease causing airflow obstruction of varying severity and difficulty in breathing. It is an important disease for New Zealand because of the number of people affected and the economic costs, both direct and indirect. It is generally regarded as a chronic disease requiring continuing treatment to prevent symptoms. As such, asthma has considerable associated morbidity and on-going treatment costs which include the exacerbation of existing and other acute illnesses. Accordingly treatment decisions have both important health and cost consequences.

The overall aim of the three integrated studies was to develop a policy and management tool (a decision analytic model) that would enable the costs of alternative asthma treatments to be assessed.

The objectives of each component of this research were: (1) Cost-of-illness: to identify and evaluate the costs of asthma to New Zealand from a societal perspective, (2) Cost of alternative treatment strategies: to compare the direct medical costs of treating asthmatics using altemative corticosteroid inhaler options in a general practice setting, (3) Decision analytic model: to then use this information to develop an asthma treatment cost model that would allow managers and clinical decision makers to evaluate economic costs of treating asthma using different treatment strategies. Each of these objectives are covered in more 
detail when each of the three facets of the research project are discussed.

\subsection{COST-OF-ILLNESS}

\subsubsection{Background and Specific Objectives}

Although asthma is an important disease in New Zealand the information on prevalence and costs are fragmented and incomplete. The objectives of the cost-of-illness study (Scott et al., 1997) were to; (1) demonstrate how data must be gathered from a variety of sources both New Zealand and international, (2) identify and estimate from a societal perspective as many as possible of the costs of asthma to New Zealand and (3) measure resource utilisation volumes and costs in monetary units.

\subsubsection{Methods and Results}

\section{Steps in Estimating Costs}

The following steps were involved. Prevalence of the illness was first estimated to provide information on the numbers of asthmatics in the population. Resource utilisations, unit costs were determined and finally resource costs calculated.

\section{Prevalence of Asthma in New Zealand}

No recorded statistics on asthma prevalence exist but estimates of the prevalence of diagnosed asthma in New Zealand was between 13\% and 14\% of the population, and of treated asthma between $9 \%$ to $11 \%$. Prevalence rates multiplied by total population (Statistics New Zealand 1997) indicate that just under 500,000 people have been diagnosed as asthmatics, about 350,000 are treated with asthma drugs, and there are about 150,000 treated asthmatics in the workforce. Table 17.1 details the prevalence of asthma in New

Zealand in 1996 based on this information. The prevalence estimates were derived from Crane et al. (1994) and A Picture of Health (1993); NRB (1996). 
Table 17.1: Prevalence of Asthma in New Zealand 1996

\begin{tabular}{lrrr}
\hline & $\begin{array}{r}\text { Rate } \% \\
\text { number of } \\
\text { people }\end{array}$ & $\begin{array}{c}\text { Total } \\
\text { employed } \\
\text { workforce }\end{array}$ \\
\hline $\begin{array}{l}\text { Diagnosed asthmatics } \\
\quad \text { Low }\end{array}$ & $13 \%$ & 473,200 & 218,855 \\
$\quad$ High & $14 \%$ & 509,600 & 235,690 \\
& & & \\
Treated asthmatics & $9 \%$ & 327,600 & 151,515 \\
$\quad$ Low & $11 \%$ & 400,400 & 185,185 \\
$\quad$ High & & & \\
& & $3,640,000$ & \\
Total population (mean year ending Dec 1996) & & & $1,683,500$ \\
\hline Total workforce (June 1996) & & & \\
\hline
\end{tabular}

\section{Drug Acquisition}

Asthma medicines and devices accounted for \$104 million (asthma preventive medicines $\$ 72$ million, bronchodilators $\$ 30$ million, and respiratory devices $\$ 2$ million) or $17 \%$ of total Pharmaceutical Schedule expenditure on government subsidised medicines (PHARMAC 1996). A high estimate of $\$ 153$ million for drug cost was obtained by taking $17 \%$ of total public and private expenditure on medicaments (N.Z. Ministry of Health 1997). This includes patient co-payments and premiums (patient out-of-pocket payments where the government subsidy does not cover all of the medicine cost) and over-the-counter (OTC)

medicines. (As different therapeutic groups have different price structures and co-payment amounts and OTC medicine options this estimate must be interpreted with caution.)

\section{General Practitioner}

Asthma is a frequent reason patients consult general practitioners. Studies indicate that asthma accounts for between 2.4\% (Tilyard et al., 1995) and 4.5\% (McAvoy et al., 1994) of general practice encounters. Information from (IMS (NZ) Ltd) showed that consultations for asthma represented $4.0 \%$ of the total number of consultations. 
During the year ending September 1996,791,000 general practitioner consultations were recorded for asthma (IMS (NZ) Ltd) at a cost of $\$ 24$ million. ${ }^{21}$ A low estimate of $\$ 18$ million for expenditure on general practitioner consultations was estimated by multiplying total expenditure on general practitioner consultations (N.Z. Ministry of Health 1997) by $3.5 \%$ (the average of the high and low estimate of encounters).

\section{Specialist Consultations}

The low and base case estimate for specialist consultations was $\$ 3$ million in 1996 (data all specialist consultations $\$ 136$ million (N.Z. Ministry of Health 1997) multiplied by $2.0 \%$ (using the proportion of specialist referrals from general practitioners for asthma) (McAvoy et al., 1994). A high estimate of $\$ 6$ million was obtained ( $\$ 136$ million multiplied by $4.5 \%$, using the proportion of general practitioner consultations as a proxy for the proportion of specialist consultations). (McAvoy et al., 1994). A range ${ }^{22}$ of fees from $\$ 60$ to $\$ 100$ was used to calculate the consultation numbers from total expenditure, giving numbers of between 45,000 to 61,000 consultations (rounded to the nearest thousand).

\section{Diagnostic Imaging and Laboratory Tests}

Diagnostic imaging and tests associated with diagnosis and monitoring could not to be evaluated because of lack of information. However, discussions with clinicians indicated that resource utilisation in this area was very small.

21 The total cost was derived by multiplying the number of consultations by the average adult consultation fee of $\$ 30$ (rounded to the nearest whole dollar) obtained from the Consumers Price Index (Consumers Price Index 1996) (November 1996) and checked against a consumer survey that gave similar results (Consumer 1996).

22 If it is assumed that a specialist consultation fee is double that of a general practitioner, a fee of $\$ 60$ is obtained but if relativity with accident and emergency consultations is assumed a fee of $\$ 100$ would result. 


\section{Emergency Medical Treatment}

As New Zealand national data on emergency treatment was not available data from a UK study (National Asthma Campaign 1996) was used. They found that $13 \%$ of asthmatics had attended a hospital accident and emergency department in the last 12 months. Applying this finding to the number of treated and diagnosed asthmatics, and assuming a unit cost of $\$ 100$ per visit (Asthma and Respiratory Foundation of New Zealand (Inc) 1997), the following costs were obtained. Base case and low estimate (base case treated asthmatics x $13 \%$ x $\$ 100) \$ 4$ million, and high estimate (base case diagnosed asthmatics x $13 \%$ x \$100) \$6 million.

\section{Hospital Inpatient Treatment}

In 1994 there were 10,558 (5,171 male and 5,387 female, ICD (International Disease Category) 493) asthma inpatient discharges from publicly funded hospitals representing 38,009 days stay in hospital (N.Z. Ministry of Health 1996a). The estimated cost of these hospital discharges was $\$ 13$ million (calculated by applying 1994/95 DRG (diagnosis related group) unit prices, DRG codes bronchitis and asthma 096, 097 and 098) (N.Z. Ministry of Health 1996b) to the number of asthma inpatients). Asthma accounted for $1.7 \%$ of all inpatient discharges or $0.8 \%$ of all public hospital patient days in 1994 . A high estimate of \$16 million for hospital costs was made using 1990/91 DRG prices paid to hospitals (National Advisory Committee on Core Health and Disability Support Services 1993) (27\% higher than unit costs in 1994/95) to provide a high estimate of inpatient hospital costs.

\section{Transport Cost}

Transport costs were estimated to fall between \$14 and \$18 million with a base case estimate of $\$ 18$ million. This evaluation, used a conservative unit cost of $\$ 20$ a round trip, on the basis of one round trip per consultation and hospital visit or admission. An informal 
survey ${ }^{23}$ of ambulance service providers indicated a unit cost of about $\$ 100$ would be justified if an ambulance was used for transport.

\section{Indirect Costs}

Indirect costs attributable to lost productivity of those in the paid workforce was estimated to be $\$ 95$ million $^{24}$. The assumptions were; each treated asthmatic in the workforce will lose 5 days or one week of work through asthma (Mellis et al., 1991), the total employed workforce was 1,683,500 (June 1996) (Statistics New Zealand 1997), and the average weekly wage was $\$ 628$ (June 1996) (Statistics New Zealand 1997). If the average weekly wage is applied to the high estimate of employed treated asthmatics a high value of $\$ 116$ million is obtained. Travel and waiting time (at least one hour per consultation) to attend consultations was assumed to be included in the 5 days lost output.

If productivity loss was evaluated on the basis of the total labour force (employed and unemployed) the cost would be $6.3 \%$ higher than our estimates. Calculations made using the working age population (employed, unemployed and those not seeking paid employment) would be $62.0 \%$ greater. Reduced productivity of asthmatics while at work, or asthmatics unable to work, was not evaluated because of insufficient data. Similarly, productivity loss by unpaid caregivers or family members unable to work because they were caring for a child or other family member was not evaluated.

\section{Mortality}

In 1993 there were 126 deaths attributed to asthma which accounted for $0.5 \%$ of all recorded deaths (N.Z. Ministry of Health 1995). These lives would be valued at $\$ 252$ million using a willingness to pay for a statistical life (Miller and Guria, 1991), but considerably less than this if valued using a human capital and lost productivity approach. 
Table 17.2 summarises the costs of asthma to New Zealand society as a total cost, and cost per treated asthmatic. Total costs ranged from \$25I million to \$340 million, direct medical \$142 million to \$205 million, direct non-medical \$14 million to \$18 million, and productivity loss of \$95 million to $\$ 116$ million (See Table 3 in (Scott et al., 1997)).

Table 17.2: Summary of the Base Case Annual Costs of Asthma From the Perspective of New Zealand Society 1996

\begin{tabular}{lcc}
\hline Cost & $\begin{array}{c}\text { Total cost } \\
\text { Cost per } \\
\text { treated } \\
\text { asthmatic } \\
\text { NZ\$ }\end{array}$ \\
\hline Drug acquisition (ingredients, markup and dispensing) & 104 & 318 \\
Plus Primary care-giver (GP and specialist consultations) & 26 & 81 \\
Plus Secondary care (hospital inpatient and accident and emergency) & 17 & 52 \\
= Direct medical & 148 & 451 \\
Plus Direct non medical (transport) & 18 & 54 \\
= Total Direct & 166 & 505 \\
Plus Indirect (Lost productivity) & 95 & 291 \\
= Total Direct and indirect & 261 & $\mathbf{7 9 6}$ \\
\hline Notes & & \\
Because of rounding individual items may not add exactly to the totals shown & \\
Goods and services tax (GST) of 12.5\% (a transfer payment from the perspective of society) & \\
All costs are in 1996 New Zealand dollars exclusive of GST & \\
\hline
\end{tabular}

\subsubsection{Discussion/ Conclusion}

The Asthma and Respiratory Foundation of New Zealand (Inc.) (Asthma and Respiratory Foundation of New Zealand (Inc), 1997) have higher estimates than the study results (see Table 17.2); direct costs were $\$ 160$ million, indirect costs $\$ 216$ million and total societal costs $\$ 376$ million per annum in 1996 (Crane, 1997). The Foundation did not exclude transfer payments (see Chapter 4: Costs section 4.5.2) from costs, and their drug cost data was from an earlier period when drug prices were higher. They used diagnosed rather than treated asthmatics for their prevalence estimates; this would overestimate the number of asthmatics as many people may have been diagnosed as asthmatic at some stage in their lives but no longer be asthmatic. 


\subsection{COST OF ALTERNATIVE TREATMENT STRATEGIES}

\subsubsection{Background and Objectives}

Treatment with a corticosteroid inhaler is considered the primary or first line treatment of asthma (The British Guidelines on Asthma Management 1995 Review and Position Statement, 1997). The delivery system (inhaler device) is an important factor influencing the efficacy of inhaled steroids (Barnes et al., 1995). In New Zealand the general practitioner is regarded as the gate-keeper to the health care system, and hence the decisions they make are major health care cost drivers. Increasingly, the emphasis is on evidencebased medicine which encompasses both the economic and health effects of clinical practice.

Accordingly this study (Scott et al., 1998) focussed on differences in cost outcomes of a choice of corticosteroid inhaler by general practitioners. The objectives were to determine the cost consequences of a general practitioner's choice of inhaler device for the delivery of corticosteroid preventer medication for asthma.

There are two main types of inhaler device ${ }^{25}$ : aerosol (AUTO, MDI), and dry powder (DISK and TURB) inhalers. Dry powder inhalers (DISK, TURB) deliver active ingredient as a powder and require inspiratory effort for drug delivery. Aerosol inhalers may be breath or manually activated. Breath actuated inhaler devices such as AUTO overcome coordination problems commonly associated with MDI use, eliminate the need for spacers, and provide improved medication delivery to the lungs and improved efficacy for patients unable to correctly use MDIs (McFadden, 1995; Newman et al., 1991). 


\subsubsection{Method}

Data were analysed by prescribed corticosteroid inhaler. The setting for the study was general practice, and the analytic perspective taken was that of the funder of health care. Treatment options investigated were treatment of asthma with four corticosteroid inhaler drug delivery systems (Autohaler ${ }^{\mathrm{MM}}$ [AUTO], Diskhaler ${ }^{\mathrm{m}}$ [DISK], metered dose inhaler [MDI] and Turbuhaler ${ }^{\mathrm{IM}}$ [TURB]) in a general practice setting.

As the research was retrospective and observational it recorded (without altering or influencing) actual general practitioner prescribing practice, and because it used an extensive data-base a large population was able to be studied. Utilisation of health care resource volumes (drugs, general practitioner consultations and numbers of prescriptions for the year ending 30 June 1995 were obtained from the Royal New Zealand College of General Practitioners Research Unit, University of Otago (RNZCGP).

Data were obtained from computerised clinical records of 28 general practices covering 128,585 consulting patients (Figure 17.2). The data set is of a standard suitable for research and administrative purposes and is considered to be representative of the nature of general practice (Tilyard et al., 1995) in New Zealand and a valid research tool (Dovey and Tilyard, 1996).

Figure 17.2: Sample Breakdown

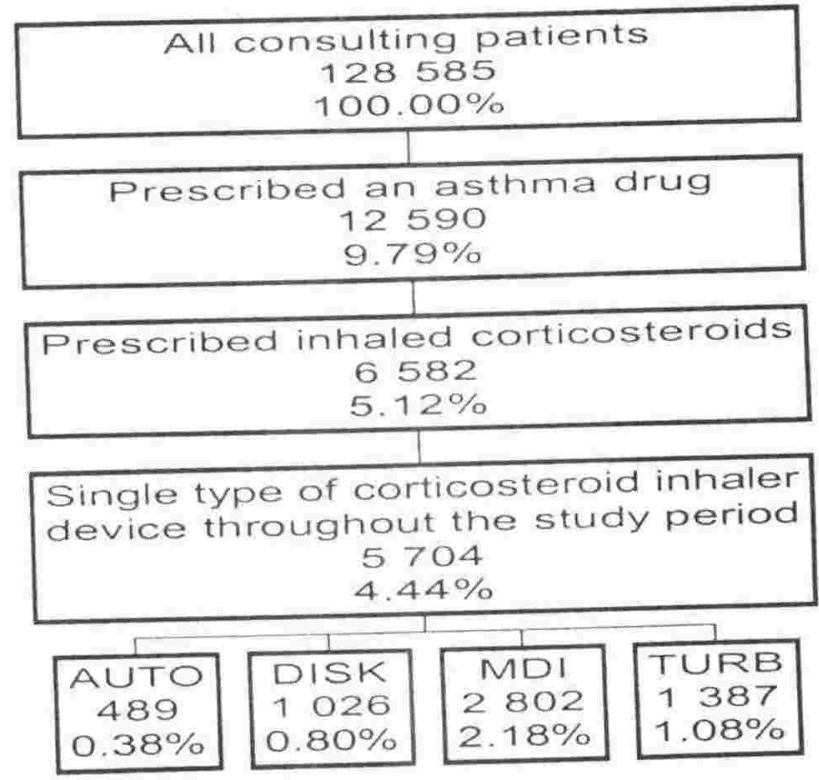


All prescribing records (from the 128,585 consulting patients) were electronically searched to identify those patients using AUTO, DISK, MDI or TURB as the sole inhaler device for corticosteroids (5,704 patients) over the 12 month study period (Figure 17.2). These records for patients prescribed inhaled corticosteroids were further examined to identify all beta agonist and oral steroid prescriptions and consultations.

Prices for the drugs prescribed, retail and wholesale markups, and dispensing fees and charges were obtained from the New Zealand Pharmaceutical Schedule (1996), (Respiratory system and allergies, Dispensing fee plus container charge $\$ 2.60$, wholesale markup $10 \%$, retail markup $11.28 \%$.). General practitioner consultation fees were obtained as described in section 17.3.2. All unit costs and prices were exclusive of Goods and Services Tax (GST). The average daily dose of inhaled corticosteroids was calculated from the prescription information.

\subsubsection{Results}

The results of the cost-of-treatment study are presented in the form of Tables; 17.3, 17.4, and 17.5. More in depth commentary on the results can be found in (Scott et al., 1998), along with statistical analysis of the data and confidence intervals. As the data did not conform with the normal distribution, the Kruskal-Wallis method was applied to calculate confidence intervals (Siegel S and Castellan, 1988). 
Table 17.3: Sample Breakdown by Age and Corticosteroid Inhaler Device

\begin{tabular}{|c|c|c|c|c|c|c|c|c|c|c|}
\hline \multirow{2}{*}{$\frac{\frac{\text { Age }}{\text { Years }}}{<6}$} & \multicolumn{10}{|c|}{ Corticosteroid inhaler device ${ }^{a}$} \\
\hline & 19 & $399^{\circ}$ & 82 & $8.0^{n}$ & 347 & $12.4 \%$ & 71 & $5.1 \%$ & 519 & $9.1 \%$ \\
\hline $6-18$ & 1.46 & $299^{\circ} \circ$ & 509 & $490^{\circ} \%$ & 359 & $12.8 \%$ & 325 & $23.4 \%$ & 1339 & $23.5 \%$ \\
\hline 19. 49 & 162 & $33.1 \%$ & 190 & $18.5 \%$ & 1250 & $44.6 \%$ & 492 & $35.5 \%$ & 2094 & $36.7 \%$ \\
\hline 495 & 162 & $33.1 \%$ & 245 & $23.9 \%$ & $8+6$ & $30.2 \%$ & 499 & $36.0 \%$ & 1752 & $30.7 \%$ \\
\hline All ages & 489 & $1000^{\circ}$ \% & 1026 & $1000^{\mathrm{n}} \%$ & 2802 & $100,0 \%$ & 1387 & $100.0 \%$ & 5704 & $100.0 \%$ \\
\hline Male & 198 & $40.5 \%$ & $49+$ & $48.1 \%$ & 1311 & $46.8 \%$ & 602 & $43.4 \%$ & 2605 & $45.7 \%$ \\
\hline Female & 291 & $59.5 \%$ & 532 & $51.9^{\circ} \%$ & 1491 & $53.2 \%$ & 785 & $56.6 \%$ & 3099 & $54.3 \%$ \\
\hline Mean age (years) & 37 & & 28 & & 36 & & 39 & & 35 & \\
\hline$\%$ of all patients & $8.6 \%$ & & $18.0 \%$ & & $49.1 \%$ & & $24.3 \%$ & & $100.0 \%$ & \\
\hline
\end{tabular}

a Corticosteroid inhaler device: $A$ LTO $=$ Autohaler; DISK $=$ disk inhaler; $M D I=$ metered dose inhaler; $T U R B=$ Turbuhaler

Table 17.4: General Practitioner Consultations (Mean Number Per Year Per Patient) by Corticosteroid Inhaler Device

\begin{tabular}{|c|c|c|c|c|c|c|c|c|c|c|}
\hline \multirow{2}{*}{$\begin{array}{l}\text { Consultations } \\
\text { Asthma }\end{array}$} & \multicolumn{10}{|c|}{ Corticosteroid inhaler device ${ }^{a}$} \\
\hline & 3.59 & $43.5 \%$ & 3.96 & $45.8 \%$ & 4.37 & $45.0 \%$ & 4.05 & $40.6 \%$ & 4.15 & $43.9 \%$ \\
\hline All consultations & 8.26 & $100.0 \%$ & 8.65 & $100.0 \%$ & 9.72 & $100.0 \%$ & 9.97 & $100.0 \%$ & 9.46 & $100.0 \%$ \\
\hline
\end{tabular}

a Corticosteroid inhaler device: $A U T O=$ Autohaler; DISK = disk inhaler; $M D I=$ metered dose inhaler; TURB = Turbuhaler

Table 17.5: Yearly Resource Utilisation and Cost Per Patient Treated for

\section{Asthma Summary}

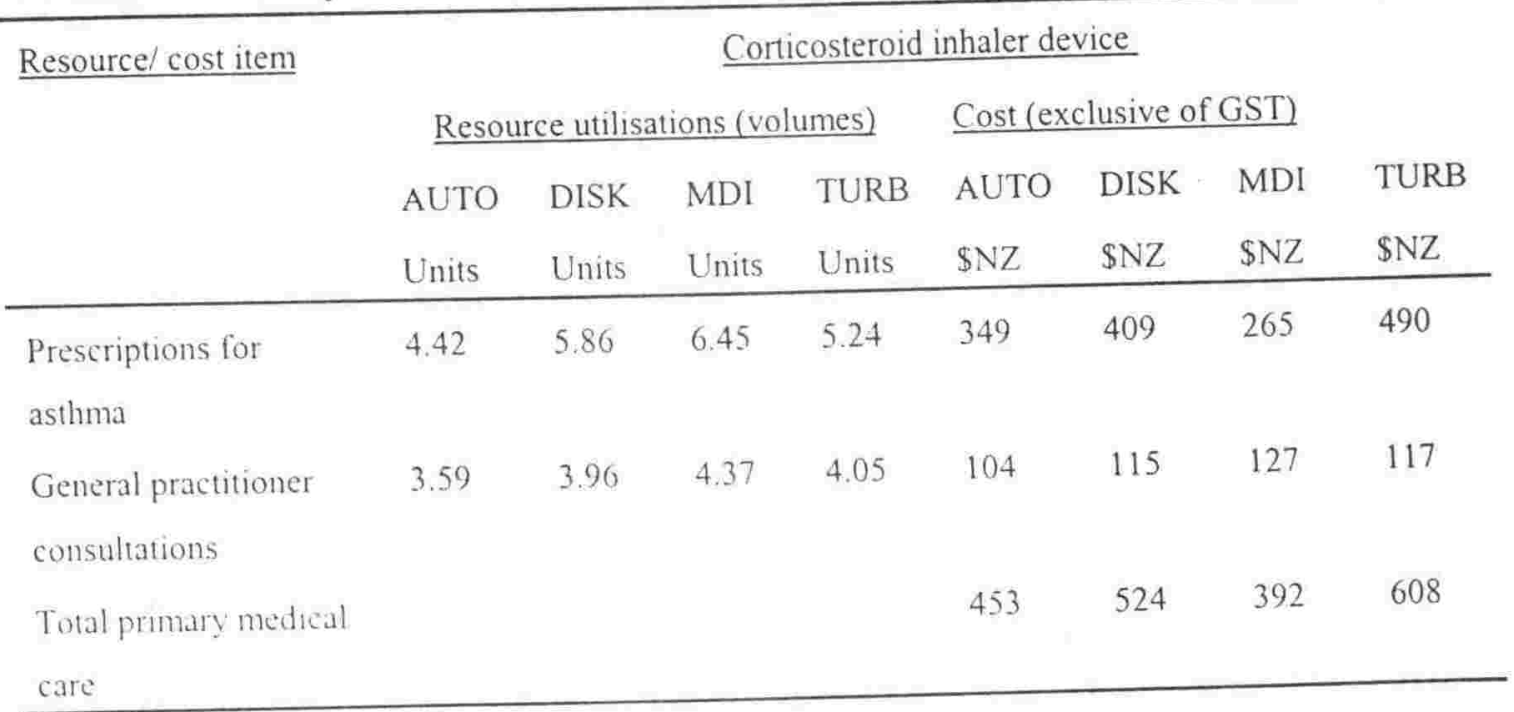




\subsubsection{Discussion and Conclusion}

The largest single cost item identified in this study was that of corticosteroid inhalers which ranged from $41 \%$ of total primary care cost for MDI to $52 \%$ for the TURB group. Total drug acquisition cost (inclusive of dispensing costs and wholesale and retail markups) was highest for TURB at $\$ 490$ and lowest for MDI at $\$ 265$, a difference of $85 \%$. There was less variation in general practitioner consultations for asthma with total costs ranging from $\$ 104$ for AUTO to $\$ 127$ for DISK. Total primary care costs were lowest for MDI at \$392 per annum and highest for TURB at $\$ 608$, a difference of $55 \%$. Conclusions relating to cost differences between the treatment groups were robust, in all but one instance TURB remains the highest cost group.

Not all records in the data-base used allowed clear identification of the reason for the consultation and thus some asthma-related consultations may not have been counted. As data were actual general practitioner prescribing records and not pharmacy dispensing records, failure to present or collect a prescription from the pharmacist was not measured. Estimates for non-dispensing vary but Gardner et al, in a recent study (1996) found that the non-dispensing rate for both bronchodilators and corticosteroids was $10.5 \%$.

A number of factors may cause wastage of asthma drugs; some patients do not comply with treatment, and inhaler delivery devices may be test fired and used incorrectly. Estimates of patient noncompliance with prescribed medication vary widely. Noncompliance depends upon the individual patient, the nature of the disease being treated, and the type of medication prescribed. The study did not attempt to measure noncompliance but never-theless the impact on prescriptions and general practitioner consultations has been accounted for in the study. Over-use would have been measured in additional prescriptions and general practitioner consultations. Under-use would have been accounted for by subsequently fewer prescriptions and fewer general practitioner consultations. If under-use resulted in an asthma attack, additional prescriptions and additional consultations may have been generated and measured in the study. 
Wastage as a result of test firing, incorrect activation (poor co-ordination) or incorrect usage was not specifically measured in the study but is accounted for as it is included in the volumes of medication prescribed. Gebbie (1971) reported from a survey of asthmatics that test firing was an important cause of wastage of bronchodilator aerosols in pressurised containers (MDIs). Test firing is usually a problem associated with MDIs and is considered to be less of a problem for breath-actuated inhalers.

King et al. (1991) found that $68 \%$ of patients were unable to use their MDI inhalers successfully. Errors in use fell into two main categories; failure to synchronise deep inspiration with depressing the canister (a problem of co-ordination), and failure to hold the breath after inspiration. In a review of asthma literature Shrestha et al. (1996) reported that approximately $20 \%$ to $40 \%$ of asthma patients used MDIs correctly and this has important implications for resource usage, morbidity and mortality. McFadden (1995) reviewed the English language literature (20 papers) and found that the error rate per patient in the use of MDIs ranged from $12 \%$ to $89 \%$ with an average of $38 \%$. The proportion of patients misusing MDIs in this review was $36 \%$. A French study of the literature came to similar conclusions (Chinet and Huchon, 1994).

Faulty inhaler technique has both clinical and economic consequences in the treatment of asthma. Cost-effectiveness of the treatment decreases as costs rise and effectiveness falls. Costs rise because of the additional volume of medication used, and because asthma is not as well controlled there is an increase in general practitioner and hospital.

As explanatory variables for asthma severity and risk were not sufficiently documented in the database, further investigation such as multivariate analysis was not considered appropriate. However, comment has been made on differences in age between treatment groups.

This study differed from most randomised controlled clinical trials in that it involved a large study population and recorded (without altering or influencing) actual general practitioner prescribing practice. Clinical trial protocols are frequently different from actual practice and patients tend to be carefully selected to meet specific criteria. 


\subsection{DECISION ANALYTIC MODEL}

\subsubsection{Background and Objectives}

Asthma treatment is complex and clinicians and health sector managers make decisions with important cost and health care consequences. As data for decision making are fragmented, and decision makers are frequently under considerable time constraints, a method of both integrating data and allowing scenario analysis is urgently required.

The objectives of the study were to design and evaluate a model capable of; (1) integrating data from a wide variety of sources, (2) allowing a health sector administrator or clinician to evaluate the economic costs of treating asthma using different therapeutic strategies and (3) permitting decision makers to change data inputs assumptions and analytic perspective.

\subsubsection{Method}

A deterministic decision analytic spreadsheet based model was designed and evaluated the following healthcare costs:

- $\quad$ Drug acquisition cost (ingredients, markup and dispensing charge)

- Plus General practitioner, specialist and other health care professional consultations

- Plus Diagnostic tests (e g. X-ray)

- Plus Secondary care cost (hospital)

$=\quad$ Direct medical (health sector costs)

- Plus Direct non medical cost (non health sector costs: transport to seek treatment and other household costs [e.g. special bedding] of treating or preventing asthma)

$=\quad$ Total direct cost

- Plus Indirect cost (Lost productivity and leisure time activities of patient family and friends. That is, time costs of the illness or its treatment)

$=\quad$ Total direct and indirect cost 
New Zealand epidemiological, resource utilisations and unit cost data (as discussed in the previous sections) were used to construct and test the model. Where New Zealand data was unavailable a literature search yielded Australian and UK information that could be adapted for New Zealand. This was possible as treatment guidelines for asthma are similar in these countries.

The model was constructed using a series of different screens or pages. Welcoming pages contained instructions for running the programme, highlighted cells in which data could be entered, background on asthma prevalence and information about the pre-loaded base case values and assumptions underlying the model. There was a section for inputs another page for, calculations. The outputs were in the form of tables and charts. All pre-loaded values, assumptions and sources were documented within the model.

The flow of the model was as follows:

Inputs $\rightarrow$ Calculations $\rightarrow$ Output table $\rightarrow$ Chart data $\rightarrow$ Output charts

Pathways the model follows to assign the number of asthmatics to each corticosteroid inhaler device are as shown in Figure 17.3. The model calculates the number of asthmatics and assigns them to treatment groups. Resource costs are calculated by multiplying numbers of patients by the input unit costs and by the resource volumes for each treatment option.

The inputs to the model are shown in Table 17.6. Output costs are presented (in both tabular and graph form) by inhaler device. Costs are described in terms of dollars per treated patient, per 1000 patients, and per study population (Table 17.7: is a schematic listing of the cost outputs). 
Figure 17.3: Model Pathways

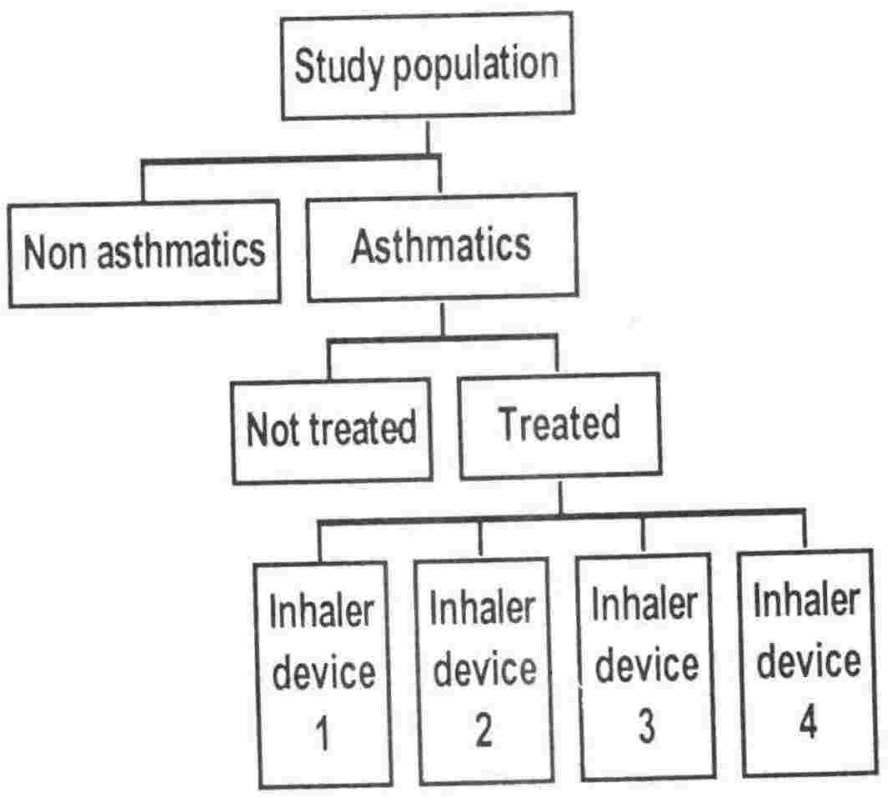

\subsubsection{Results and Discussion}

The base case preloaded values yield identical results to those presented in the earlier sections of this chapter (cost-of-illness and cost-of-treatment studies). Medicine prices and volumes were described in monetary units per milligram rather than price per pack. This was done to enable the model to cope with changing prescribing patterns and so that it could be used in other countries. Managers and policy makers can use the model to evaluate different price scenarios, switching patients from one delivery device to another. Different perspectives can be considered by changing the input unit costs.

\subsection{POLICY POINTS}

There are several policy related issues illustrated by this case; the difficulty of obtaining data for policy analysis, differences in data estimation methods, and the use of a model to integrate data from different sources and conduct scenario analysis from a number of different perspectives. 


\section{Obtaining Data for Policy Analysis}

Lack of country specific data often necessitates the integration of data from a range of New Zealand and international sources; the cost-of-illness study illustrated this process. Absence of primary data on effectiveness and resource utilisations of different treatment options may often be overcome by obtaining and processing secondary data that has been collected for other purposes such as clinical and administrative records. The use of an administrative data-base of general practitioner patient records is an example of an alternative data source that can be used instead of data from a randomised trial to estimate the cost-of-treatment.

\section{Differences in Estimation Methods}

The study highlighted the importance of investigating differences in estimation methods and the need to adjust the findings to take account of this when using secondary data from different sources. If this step is not undertaken inappropriate policies may be implemented. For example, the implications of overestimating the cost of asthma compared with other illnesses are that health resources may be misdirected towards asthma and away from other priorities within the health system. Policy makers will often be presented with conflicting information such as this and must decide how to use such data.

\section{Use of A Model to Integrate Data and Conduct Scenario Analysis From A Number of Different Perspectives}

The model and its construction and testing demonstrated the practical application of integrating data from a cost-of-illness analysis and a cost-of-treatment analysis. It was designed as a management and policy tool to be used by any manager who is able to navigate from sheet to sheet and from cell to cell in a spreadsheet and insert data into designated cells. Decision makers are able to consider a range of costs from any perspective simply by entering the appropriate resource utilisations and unit costs. 
A health purchaser should be making decisions based on the total direct medical costs of an intercention strategy and not simply on the price of the primary medicine. A lower priced inhaler may generate higher total direct medical costs than a higher priced inhaler. Policy makers within funder and purchasing organisations could use such information in negotiating prices with suppliers of asthma inhalers. It would be possible for the funder to calculate breakeven prices for inhalers such that total direct medical costs were equal.

As data for the model were derived from actual general practice (everyday community treatment) it provides a realistic way of making evidence based decisions. Policy makers within fundholder health care provider organisations could use this information to select the most cost effective asthma treatment regimens and clinical guidelines, to design treatment strategies, and to more effectively negotiate contracts with funders to maximise their profits.

The model has been used with general practice fund holders and health sector funders to demonstrate cost differences between alternative asthma treatments and has permitted users to assess the budgetary impact of different asthma treatment policy options. 
Table 17.6: Asthma Treatment Cost Model Inputs

Cost items by annual volumes and unit costs

Drugs

- Corticosteroid inhalers (assuming no wastage)

- Beta-agonist inhalers (assuming no wastage)

- $\quad$ Spacers

- Other drug 1

- Other drug 2

- Corticosteroid inhaler over/ under use and test-firing

- Beta-agonists over/ under use and test firing

- Wholesale/ retail markups and dispensing fees

Primary care

- General practitioner consultations

- Retraining: additional general practitioner consultations

- Specialist consultations

- Physiotherapist consultations

- Nurse consultations

- Retraining: additional nurse consultations

- Diagnostic tests

Secondary cure

- Emergency medical treatment

- Hospital outpatient specialist consultations

- Hospital admissions for inpatient treatment

Direct non medical costs

- Transport to seek treatment

- Household costs

Indirect costs

- Paid and unpaid production

- Education

- Leisure time activities

Epidemiological and demographic data

- Population at risk / study population

- $\%$ of treated asthma patients

- $\%$ of treated patients on each inhaler device 
Table 17.7: Asthma Treatment Cost Model Outputs

Corticosteroid inhalers

Beta-agonist inhalers

Spacers

Other eg Prednisone

Sub total Ingredients

Wholesale and retail markup

Dispensing

Total Drug acquisition (A)

General practitioner consultations

Specialist consultations

Physiotherapist consultations

Nurse consultations

Diagnostic tests

Total Primary care consultations plus diagnostic tests (B)

Total Primary care $(C=A+B)$

Emergency medical care

Hospital inpatient

Total Secondary care (D)

Total Direct medical $(E=C+D)$

Transport to seek treatment

Household costs

Total Direct non medical (F)

Total Direct medical and non medical $(G=E+F)$

Lost paid and unpaid production

Lost education

Lost leisure time activities

Total Indirect $(\mathrm{H})$

Total Direct and indirect $(I=G+H)$ 


\subsection{REFERENCES}

A Picture of Health. 1st ed. Wellington: Statistics New Zealand and N.Z. Ministry of Health, 1993.

Asthma and Respiratory Foundation of New Zealand (Inc). "New Zealand asthma bill: \$376 million per year". Media release 30 March 1997.

Barnes, P. J., and S. Pedersen. "Efficacy and safety of inhaled steroids in asthma." American Review of Respiratory Disease 148 (1993): S1-26.

Chinet, T., and G. Huchon. "La mauvaise utilisation des aerosols-doseurs pressurises dans le traitement des maladies bronchiques: Frequence et consequences cliniques." Ann Med Interne 145 (1994): 119-24.

Consumer. "General practitioner consultations: what do you pay your doctor?" Consumer April (1996): 24-5

Consumers Price Index. "Consumers price index weighted average retail prices of selected items in the CPI: general practitioners' services, consultation adult without community services card." Hot Off the Press September and December quarters (1996).

Crane, J., S. Lewis, T. Slater, L. Crossland, B. Robson, W. D'Souz, N. Pearce, G. Town, J. Garrett, and R. Armstrong. "The self reported prevalence of asthma symptoms amongst adult New Zealanders." New Zealand Medical Joumal 107 (1994): 417-21.

Crane, J. "Asthma costs $\$ 376$ million a year - report." The Dominion May 7 (1997): 8.

Dovey, S. M., and M. W. Tilyard. "The computer research network of the Royal New Zealand College of General Practitioners: an approach to general practice research in New Zealand." British Journal of General Practice 46 (1996): 749-52. 
Gardner, T. L., S. M. Dovey, and W. M. Tilyard. "Differences between prescribed and dispensed medications." New Zealand Medical Journal 109 (1996): 69-72.

Gebbie, T. "Aerosols in generalised airway obstruction (Letter)." New Zealand Medical Journal 74 (1971): 115-16.

King, D., S. M. Earnshaw, and J. C. Delaney. "Pressurised aerosol inhalers: the cost of misuse." British Journal of Clinical Practice 45 (1991): 48-9.

McAvoy, B., P. Davis, A. Raymont, and B. Gribben. "The Waikato Medical Care (WaiMedCa) Survey 1991-1992." New Zealand Medical Journal 107 Suppl 986 Part 2 (1994): 387-432.

McFadden, S. R. "Improper patient techniques with metered dose inhalers: Clinical consequences and solutions to misuse." J Allergy Clin Immunol 96 (1995): 278-83.

Mellis, C. M., J. M. Peat, A. E. Bauman, and A. J. Woolcock. "The cost of asthma in New South Wales." Medical Journal of Australia 155 (1991): 522-28.

Miller, T., and J. Guria. The value of statistical life in New Zealand. Wellington: Ministry of Transport, 1991.

N.Z. Ministry of Health. Mortality and demographic data 1993. Wellington: Health Information Service, N.Z. Ministry of Health, 1995.

N.Z. Ministry of Health. Hospital and selected morbidity data 1994. Wellington: Health Information Service, 1996a.

N.Z. Ministry of Health. Purchasing for your health: 1994/95. A performance report on the second year of the Regional Health Authorities and the Public Health Commission. Wellington: N.Z. Ministry of Health, 1996b. 
N.Z. Ministry of Health. Health expenditure trends in New Zealand 1980-1996. Wellington: Sector Analysis Section N.Z. Ministry of Health, 1997.

National Advisory Committee on Core Health and Disability Support Services. Core Services for 1994/95. Wellington: National Advisory Committee on Core Health and Disability Support Services, 1993.

National Asthma Campaign. "The impact of asthma." Asthma Journal (1996): 32-33.

Newman, S. P., A. W. B. Weisz, N. Talaee, and S. W. Clarke. "Improvement of drug delivery with a breath actuated pressurised aerosol for patients with poor inhaler technique." Thorax 46 (1991): 712-16.

NRB. Research Report; Environmental tobacco smoke study. Prepared for the The N.Z. Ministry of Health, July 1996.

PHARMAC. Anmual review for the year ended 30 June 1996. Wellington: Pharmaceutical Management Agency Limited, 1996.

Respiratory system and allergies. New Zealand Pharmaceutical Schedule 3 (1996): 151-8.

Scuit, W. G., H. M. Scott, and G. D. Frost. "Pharmacoeconomic evaluation of asthma treatment costs." British Journal of Medical Economics 11 (1997): 87-101.

Scott, W. G., H. M. Scott, A. Penrose, G. D. Frost, J. Hall, and D. I. MacKenzie. "Economic evaluation of inhalers used in the treatment of Asthma." Journal of Medical Economics 1 (1998): 1-18.

Shrestha, M., M. F. H. Parupia, B. Andrews, S. W. Kim, T. S. Martin, D. I. Park, and E. Gee. "Metered-dose inhaler technique of patients in an urban ED: prevalence of incorrect technique and attempt at education." Americal Joumal of Emergency Medicine 14 (1996): 380-4. 
Siegel, S., and N. J. Castellan. Non parametric statistics for the behavioural sciences. 2nd ed. New York: McGraw Hill, 1988.

Statistics New Zealand. Personal computer information network for official statistics: a computerised database of official statistics for New Zealand and programme for manipulation and analysis of statistical series (PC INFOS). Wellington, 1997.

The British Guidelines on Asthma Management 1995 Review and Position Statement. Thorax 52 (Suppl.) (1997): S1-S21.

Tilyard, M. W., S. M. Dovey, and G. F. S. Spears. "Biases in estimates from the RNZCGP computer research group." New Zealand Medical Joumal 108 (1995): 118-21. 


\section{CHAPTER 18: DISCUSSION AND CONCLUSIONS}

\subsection{INTRODUCTION}

Although many of the points have been covered in previous chapters it is useful to integrate the ideas discussed and outline the economic evaluation procedure as a series of steps that anyone who works in a public policy position may follow and use. Many policy makers do not have the skills or background knowledge to apply and interpret the results of economic evaluations, or determine the economic consequences of policy interventions.

\subsection{ECONOMIC EVALUATION PROCESS}

This section discusses an evaluation procedure that may be followed irrespective of the type of economic evaluation being conducted. The procedure was developed jointly from the chapters on methodologies and the cases researched in this thesis, and from procedures and check lists created by other authors and agencies including; Australia Commonwealth Department of Human Services and Health (1995), Gold (1996), Canadian Coordinating Office for Health Technology Assesment (1997), Drummond et al., (1996) and Drummond et al., (1997).

These checklists were developed because many health economic studies are used and conducted by non economists. Help is required when evaluating the quality of published research reports and when undertaking economic evaluations. Journal editors considered that guidelines would improve the quality of submitted articles and improve the peer review process. Regulatory and funding agencies wanted technically sound reports in a uniform format to facilitate comparisons study by study. Dangers in adopting such a checklist are that different research questions may require different methodologies and if the process is too prescriptive it may impede methodological development. 
The following steps are relevant to all types of economic evaluation but they should not necessarily be applied in exactly the same manner in all situations and may be iterative rather than linear.

\section{Table 18.1: Economic Evaluation Steps and Checklist}

1. Define the problem, formulate the research question and define the objective/s

2. Decide upon the perspective/s

3. Identify the target population

4. Identify all relevant alternative/s and select comparators for analysis

5 Decide upon the time horizon

6. Check the clinical and technical evidence

7. Identify any constraints to implementation

8. Identify an appropriate methodology

9. Identify relevant costs and health related outcomes for each comparator

10 Value the costs and outcomes

11. Apply the choice criterion for determining the most efficient option/s

12. Conduct sensitivity analysis

13. Investigate equity consequences

14. Evaluate the results, draw conclusions and make recommendations

15. Monitor the decision taken

\subsubsection{Problem Definition}

The problem must be clearly identified and defined. More often than not the health policy analyst is presented with a vague and woolly problem and asked to undertake an economic evaluation and make precise recommendations. It is important to establish the background of the problem and check that the problem exists and that a decision needs to be taken. This will often involve some investigative and qualitative research. The research question must be formulated and the specific objectives of the analysis sharply defined. The research question sets the overall aims but these overall aims can usually be broken down into a number of more specific objectives. These objectives must be precisely stated in terms that will allow them to be measured. Some examples from the case studies will 
illustrate the need for precision.

Chapter 9: Antibiotic Costing had two aims. The first, was to identify and determine the economic costs of the regimens currently used in three New Zealand hospitals in the treatment of serious bacterial infections in haematology patients with febrile neutropenia and in intensive care patients with severe infections. The second aim was to develop a decision analytic model for use by hospital decision makers as an aid in evaluating the comparative cost of drug regimes. Chapter 10: Benign Prostatic Hyperplasia and Chapter 11: Hysterectomy cases aimed to determine the annual costs of treating the conditions in New Zealand so that future new treatments (not at that time in current use) could be easily evaluated. Chapter 12: Lower Respiratory Tract Infection and Chapter 14: Critical Leg Ischaemia cases aims were to determine the cost difference between two alternative treatments. The aim of Chapter 13: Influenza was to evaluate the differences in costs and outcomes of a policy of increasing the rates of vaccination against influenza for at-risk populations. Chapter 16: Ischaemic Stroke case had two aims. First to evaluate the annual cost of ischaemic stroke in New Zealand and second to use these costs in combination with the results of a randomised clinical trial to evaluate the cost-effectiveness of stroke prevention treatments.

\subsubsection{Perspective}

Perspective is used in two ways; to indicate the scope or boundary that captures the costs, and to indicate the viewpoint from which costs are measured. It is important to determine and state the view point or the perspective of the study as this determines the relevant costs and benefits and how they should be valued. The perspective could be that of an individual patient, a hospital, a regional health authority or society as a whole. A full societal perspective encompasses the broadest possible range of costs and benefits and measures them with respect to their impact on society as a whole.

Defining the target audiences and stakeholders will dictate the analytic perspectives. Different decision makers may have distinct viewpoints. Those who may use the study could be several of the following; the patient or patient advocates, health authorities, 
general practitioners, pharmacists, clinicians and hospital administrators. Most user groups can be catered for with either one or more of the following three perspectives:

- Private individuals/ patients/ health insurer

- Health sector or government as a funder/ purchaser (Vote: Health)

- Society as a whole

Failure to take account of the appropriate perspectives is a serious shortcoming in many economic investigations. Most evaluations should try to include a societal perspective or discuss the findings with respect to society. The danger of not including the societal perspective is that some important costs and benefits may be omitted from the analysis or incorrectly valued. Subsequent policy changes may be made on the basis of a partial understanding of the problem. Decision makers (even in a financial analysis) should be encouraged to consider the impact of their actions on society in addition to their own viewpoint.

For policy purposes both a societal perspective and the perspective of the agency concerned are minimum requirements. A government agency may want only its own perspective taken into account because it normally has a responsibility to keep within a fixed budget. However, it (government agency) also acts as an agent for New Zealand citizens and it is therefore essential that the societal perspective is included for full and open advice. If serious externalities are identified then the appropriate decision makers affected by the spillover should be informed and given the opportunity to contribute to the funding of the project, or ensure that other economic instruments are employed to align the agency's perspective with that of society. Unfortunately agencies may refuse to take any cognisance of any other perspective but their own budget. This may indicate that their terms of reference should be changed to reflect the objectives of society.

Chapter 9: Amibiotic Costing, was considered from the viewpoint of a hospital administrator concemed with minimising hospital costs. A health sector perspective was adopted in Chapter 10: Benign Prostatic Hyperplasia, Chapter 11: Hysterectomy, Chapter 12: Lower Respiratory Tract Infection, Chapter 14: Critical Leg Ischaemia, Chapter 15: Heart Failure and Chapter 17: Asthma. A societal perspective was considered in Chapter 
16: Ischaemic Stroke. Chapter 13: Influenza considered the costs and benefits of vaccination from three separate perspectives: an individual, the Government and of society as a whole. Three perspectives were evaluated because the costs and benefits were different when viewed from each these perspectives and because some policy options would shift costs from one group to another.

In each case there was either a single objective or the objectives were sequential. This gave a precise focus to each study and determined the perspective/s.

\subsubsection{Target Population}

The problem to be investigated may be more sharply focussed by clearly identifying the target population. A challenge encountered in defining the problem for Chapter 9: Antibiotic Costing was the vast number of potential treatment combinations and infections. It was therefore decided that the target populations were those patients in haematology and intensive care with severe infections who were or could have been treated with a specific drug.

\subsubsection{Alternatives/ Comparators}

Economic evaluation is concerned with comparing one option with another. All possible options including the option of doing nothing or keeping the status quo should be considered. It is important to identify and list all possible alternatives even if not all alternatives will be evaluated. From the listed options, select the comparators for study.

In evaluating a new therapy the main comparator should be the most commonly used alternative treatment that would be replaced by the new therapy. The results of studies can be biased up or down by selecting the most or least costly or the least effective or most effective altemative treatment. For a breakthrough therapy the comparator could be 'no treatment' or continuing care. There is also a case for considering additional comparators such as the lowest cost treatment, no treatment or treatment as recommended in clinical guidelines. It is possible for a new therapy to be cost effective against the most common 
treatment, but neither the most commonly used treatment nor the new treatment may be cost effective when compared against continuing care or no treatment.

Chapter 9: Antibiotic costing isolated all main treatment options and compared their costs. Chapter 10: Benign Prostatic Hyperplasia and Chapter 11: Hysterectomy compared costs of treatment versus no treatment. Chapter 12: Lower Respiratory Tract Infection compared the treatment costs of a new antibiotic against one in most common use in New Zealand. Chapter 14: Critical Leg Ischaemia studied the options of amputation versus intravenous medication as a treatment for critical leg ischemia. For ethical reasons the no treatment option was not considered. Chapter 13: Influenza measured the costs and benefits of vaccinating or not vaccinating different proportions of the at-risk populations. Chapter 15 : Heart Failure and Chapter 16: Ischaemic Stroke compared a new drug treatment against treatments that would be displaced. Chapter 17: Asthma compared the costs of treatment using four different inhaler devices that were all capable of delivering the same drug.

\subsubsection{Time Horizon}

Prevalence based studies consider all cases and costs in a one year time period while incidence or longitudinal studies isolate new cases and track costs and outcomes over time. Prevalence studies do not require discounting but costs and outcomes in longitudinal analyses occur over a number of years. All of the cases discussed are either prevalence baseù or costs have been converted into annual equivalents. From a policy and budgeting perspective it is usually of more value to consider a "steady state" situation and consider the annual equivalents. Prevalence studies are useful in assessing the total costs of a disease or its treatment in one year, while incidence studies are valuable when considering the treatment of newly diagnosed patients over time.

The time horizon is important in deciding upon the costs that will be altered by a decision. In the long- run all costs are variable and thus relevant but as the time horizon shortens some costs become fixed and thus not relevant to the decision. Setting too short a time horizon has the potential to exclude new technology that may not be cost-effective in the short-run but could be cost-effective in the long-run. 


\subsubsection{Technical Evidence Check}

It may seem obvious, but it is important to check that the clinical and technical evidence for determining the health effects/ outcomes of the treatment options are sound before the economic evaluation is conducted. Some treatments may be clinically unproven or cause more harm than good to patients.

\subsubsection{Constraints to Implementation}

Irrespective of the economic worth of a programme or new treatment, there may be factors that will impede or prevent successful implementation. These constraints must be identified and appraised. It is a waste of resources to conduct an economic evaluation of options that cannot or should not be implemented

Some of the factors which could be of importance are technical feasibility, legal issues, ethical considerations and social aspects. It would be inappropriate to evaluate an option for treating patients that was so hazardous that it would not be implemented on ethical grounds. Exclusion of some groups of the population from treatment, may be regarded as unethical and create such a political fallout that it would be impossible to implement the policy. Certain forms of health promotion may be inappropriate for the Polynesian or Māori population in New Zealand. Different ways of comınunicating may need to be found for different groups.

No technical, ethical or legal constraints were identified in any of the cases discussed. However ethical considerations constrained the choice of comparators that were considered. For example, a policy of no treatment was not an ethically acceptable option for most of the cases analysed and was therefore not evaluated. 


\subsubsection{Identify an Appropriate Methodology}

As discussed in Chapter 6: Economic Evaluation the appropriate method of analysing costs and outcomes is dictated by a number of factors outside the control of the analyst, for example, the research question, data, and available time. It is often not possible to classify an analysis into an economic evaluation category until it has been completed.

Table 18.2: Type of Evaluation

\begin{tabular}{|c|c|c|c|c|}
\hline Perspective & Objective, to investigate: & $\begin{array}{l}\text { Type of } \\
\text { efficiency } \\
\text { evaluated }\end{array}$ & $\begin{array}{l}\text { Economic } \\
\text { evaluation } \\
\text { technique }\end{array}$ & Choice criteria \\
\hline $\begin{array}{l}\text { Clinical } \\
\text { manager, } \\
\text { practice } \\
\text { manager or } \\
\text { hospital } \\
\text { manager }\end{array}$ & $\begin{array}{l}\text { Allocation of resources } \\
\text { between treatments for the } \\
\text { same condition where } \\
\text { outcomes are identical in all } \\
\text { respects }\end{array}$ & $\begin{array}{l}\text { Productive } \\
\text { (Technical) }\end{array}$ & $\begin{array}{l}\text { Cost- } \\
\text { minimisation }\end{array}$ & $\begin{array}{l}\text { Lowest: Costs / } \\
\text { outcome } \\
\text { ratio }\end{array}$ \\
\hline $\begin{array}{l}\text { Clinical } \\
\text { manager. } \\
\text { practice } \\
\text { manager or } \\
\text { hospital } \\
\text { manager }\end{array}$ & $\begin{array}{l}\text { Allocation of resources } \\
\text { between treatments for the } \\
\text { same condition. Outcomes are } \\
\text { in the same dimension but } \\
\text { achieved to differing degrees }\end{array}$ & $\begin{array}{l}\text { Productive } \\
\text { (Technical) }\end{array}$ & $\begin{array}{l}\text { Cost- } \\
\text { effectiveness }\end{array}$ & $\begin{array}{l}\text { Lowest: Costs/ } \\
\text { effectiveness } \\
\text { ratio }\end{array}$ \\
\hline $\begin{array}{l}\text { Health funder } \\
\text { purchaser and } \\
\text { Ministry of } \\
\text { Health }\end{array}$ & $\begin{array}{l}\text { Allocation of resources across } \\
\text { different treatments over a } \\
\text { range of conditions within the } \\
\text { health sector. Maximise } \\
\text { health benefit for a defined } \\
\text { population from a given } \\
\text { budget }\end{array}$ & $\begin{array}{l}\text { Partly } \\
\text { productive } \\
\text { (Technical) } \\
\text { and partly } \\
\text { allocative } \\
\text { efficiency }\end{array}$ & Cost-utility & $\begin{array}{l}\text { Lowest: Costs/ } \\
\text { utility } \\
\text { ratio }\end{array}$ \\
\hline $\begin{array}{l}\text { Government } \\
\text { Cabinet }\end{array}$ & $\begin{array}{l}\text { Allocation of resources } \\
\text { between different uses across } \\
\text { the whole economy to } \\
\text { maximise societal welfare }\end{array}$ & $\begin{array}{l}\text { Allocative } \\
\text { efficiency }\end{array}$ & $\begin{array}{l}\text { Cost-benefit } \\
\text { analysis }\end{array}$ & $\begin{array}{l}\text { Highest Net } \\
\text { present value }\end{array}$ \\
\hline
\end{tabular}


Table 18.2 shows how perspective, objectives, efficiency and the type of evaluation are linked. This was done to assist policy analysts choose an appropriate technique. For example, a health purchaser may wish to conduct a cost-utility analysis because this provides the best information for the decisions they have to make but they may be forced to make do with a cost-effectiveness analysis if the appropriate quality of life data are not available or not obtainable within the time and budget constraints for the analysis. Similarly, a clinical manager may wish to undertake a cost-effectiveness analysis but is restricted to a cost-minimisation study because effectiveness data is unobtainable.

\subsubsection{Identification of Costs and Outcomes (or Benefits)}

All relevant costs and outcomes should be identified, listed and described. This may be facilitated by constructing treatment and outcome trees (Chapter 7: Models). The tree diagrams may simply be a device to assist thinking through the options and making certain all possible outcomes and costs are noted, or it may form an integral part of the model used to estimate cost-effectiveness. Chapter 17: Asthma used diagrams to clarify the method used to isolate and measure costs but this diagram did not form part of a model.

Direct medical costs are those associated with medical treatment and include medicines, general practice consultations and hospital stays. These costs fall directly within the health sector. Direct nonmedical costs are those costs incurred as a result of the illness or the need to seek health care and are generally borne by patients and their friends and families. They could include the cost of transport to seek medical care, the need for special food or clothing or modifications to one's home as the result of illness. Indirect costs relate to production loss as a result of death or reduced capacity to work. Indirect costs are external to the health sector and fall on patients and the rest of society. Production loss relates to both those in paid employment and unpaid employment. Intangible costs are concerned with reduced quality of life as a result of the illness. These intangible costs do not involve opportunity costs of resources but people may be willing to pay to avoid pain and suffering. Most of the cases analysed considered direct costs only.

Costs may be either positive or negative. For example, a cost avoided is a negative cost and could be regarded as a benefit. Outcomes of medical interventions are normally positive, thus they are regarded as benefits. Transfer payments should be identified and excluded from the analysis (Chapter 4: Costs). 
Table 18.3 has been developed to shows the relationship between the type of cost, technique, perspective and boundary. This table may be used to determine which types of cost may be measured by each type of analysis and it shows where the costs fall by each perspective. A societal perspective may be adopted to measure costs but only some of society's costs may be selected. A cost-minimisation study may value costs from a societal perspective but may only include direct medical costs. In this case costs are quantified using societal values but the boundary used to capture the costs is the health sector.

Chapter 10: Benign Prostatic Hyperplasia, evaluated the impact on costs of the wider use of new diagnostic tests and found that this did not alter the conclusions. Chapter 14: Critical Leg Ischaemia used a break even analysis as the effectiveness of medication for all patients was uncertain. It was found that there would have to be 1.2 successful treatments to pay for one treatment failure. In other words the success rate had to be $55 \%$ to break even on the medical costs of drug therapy.

Cost-benefit analysis is the only technique that is capable of capturing all of society's costs and benefits. Cost-minimisation is the narrowest range of costs and the narrowest perspective. Table 18.3 runs from the narrowest perspective and boundary (cell A3, health sector perspective and boundary, cost-minimisation) to the widest perspective and boundary (cell C30, full societal perspective, cost-benefit analysis). Row 6 indicates that in a costbenefit analysis that although the perspective adopted is always that of society, the boundary could be set such that health sector costs only are captured.

Cost-minimisations and cost-effectiveness studies almost always consider direct medical costs valued from a health sector perspective but may also value these same costs from the patient and family and from a societal perspective. Usually the boundary will be set at the health sector but may extend beyond this. Cost-utility analyses usually set their boundaries to capture patient, family and societal costs. Cost-utility studies should not evaluate intangible costs as these will be incorporated in the utility measure. If indirect costs are captured by the utility measure these 100 should be excluded. 
Table 18.3: Type of Cost and Technique by Perspective

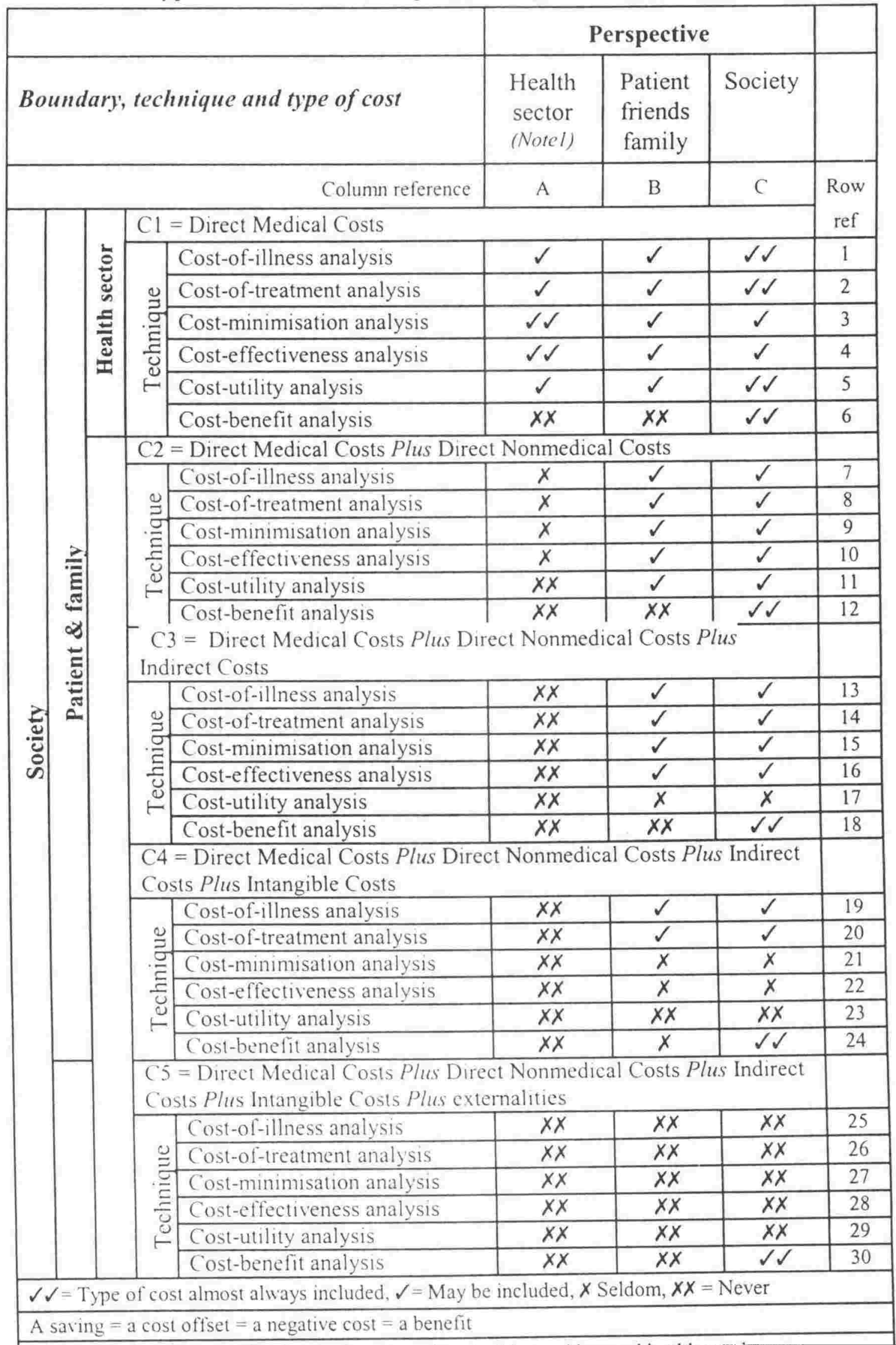




\subsubsection{Evaluating Costs and Outcomes}

Quantifying costs and outcomes was discussed in Chapter 3: Valuing Health Outcomes and Chapter 4: Costs. As economic evaluation looks at differences between comparators, incremental analysis (Chapter 4: Costs) should be used to evaluate costs and outcomes. This simply means that costs and outcomes that are identical between treatment options within the relevant time horizon may be ignored. Only those costs and outcomes that differ between comparators need be measured. Costs and outcomes that were identical for each option would cancel out when comparisons were made between the options. Chapter 14: Critical Leg Ischaemia was designed to provide information that would allow drug versus surgical treatment options to be evaluated. It was assumed that the costs up to the point of deciding to amputate or treat with drugs would be identical but would differ after this point.

Resources used or avoided should be valued in terms of their opportunity cost. The floor space used by the administration in a hospital could be valued by using the rental that office space could earn on the real estate market. Health related outcomes if valued in money terms should be evaluated using willingness to pay criteria (Chapter 3: Valuing Health Outcomes). Where a market does not exist or where there is market failure, contingent valuation methods may be needed to establish shadow prices.

Costs and outcomes that occur at different time periods should be adjusted to present values by means of a discount rate to take account of timing differences. Prevalence based studies that, relate to one time period will not involve discounting. Costs and outcomes estimated from longitudinal studies that track events over time will require discounting to the base year.

Inflation must be taken account of in some consistent way (Chapter 5: Discounting). Costs and benefits are commonly either valued in base period unit costs and prices and discounted using a real discount rate, or valued in dollars of the day and discounted with a nominal discount rate. If some input prices change by different amounts than others, then additional adjustments will have to be made to the future values of these inputs before discounting by a common rate. 
Health economic evaluation is usually undertaken assuming partial equilibrium conditions. The evaluator assumes that the impact of the programmes being evaluated will not alter unit costs and prices. If, however, the changes advocated will alter prices and unit costs, then this must be taken into account when these costs are valued. When evaluating the impact of increasing influenza vaccination (Chapter 13: Influenza) it was assumed that the increased demand for vaccinations would not alter unit costs of general practice consultations or the prices of other drugs.

\subsubsection{Choice Criterion}

In simple terms the best option can be selected by choosing the alternative that provides the highest level of net present benefits or the lowest net present cost per outcome or the lowest cost-effectiveness ratio. Chapter 6: Economic Evaluation discussed the appropriate criteria to use with various types of economic evaluations.

\subsubsection{Sensitivity Analysis}

Sensitivity analysis and uncertainty were discussed in detail in Chapter 7: Models. Sensitivity analysis allows us to evaluate the robustness of the estimates, to determine worst case and best case scenarios and to analyse changes in key estimates. Uncertainty in some of the estimates of costs and outcomes can be taken into account by considering upper and lower bounds for each estimate. Sensitivity analysis of all of these factors should be conducted in all studies.

\subsubsection{Equity Consequences}

Economic evaluations provide information on efficiency of resource allocations but do not evaluate equity changes. Some solutions that are more efficient may not be desirable choices if unacceptable equity changes occur. It will often be necessary to make trade-offs between efficiency and equity. Chapter 2: Conceptual Framework for Economic Evaluation of Health Care provides a more detailed discussion of the concept of equity. 
Chapter 11: Hysterectomy and Chapter 10: Benign Prostatic Hyperplasia are both gender specific conditions and directing resources from one condition to the other would result in gender specific equity changes. Chapter 16: Ischaemic stroke provides but one example of a disease that falls more heavily on the elderly. Shifting resources from asthma treatment (chapter 17: Asthma) to stroke prevention could cause inter-generational equity changes.

The manner in which indirect costs are valued may have a major impact on intergenerational and gender equity where economic evaluation is applied to gender or age specific illnesses. There are usually differences in employment status and market wage rates between genders and ages. Applying gender specific market wage rates to value productivity will not be desirable if policy decisions are intended to be age and gender neutral.

In general the analyst should make explicit all potential winners and losers resulting from a policy change. If equity changes are a concern then a policy maker should clearly specify these concerns in the brief to the analyst.

\subsubsection{Evaluate the Results}

The results of economic evaluation provide information on costs and outcomes and the process provides a logical framework for decision making but cannot make the decisions. Economic evaluation focuses on changes in economic efficiency and is only one of the pieces of information required for decision making. Equity, ethics and other issues must also be considered.

\subsubsection{Monitor the Decision Taken}

If a new policy, programme, or treatment option is introduced, the change should be monitored to check whether the economic and policy analysis has provided accurate information. By monitoring the introduction of new policy it can be evaluated and 
information can be accumulated that will help future policy decision making.

\subsection{CONCLUSION}

Many costs are obscured from policy makers because they do not involve money payments and very little non-hospital cost and morbidity data are collected. The continuous collection of and access to a wide range of data in as dis-aggregated a state as possible is necessary for quality policy analysis. The New Zealand health "reforms" with their emphasis on the commercial model have created difficulties in accessing "commercially sensitive" data, and some loss of continuity in collection coverage. This could be overcome by making mandatory the provision of data to a central data warehouse to be released to users in an anonymous and dis-aggregated form.

Although economic analysis is an integral component in public policy decision making ${ }^{26}$ the theory underlying economic evaluation of health is complex and is not well understood or correctly applied by non economists. The practical cases illustrate the application of the methodologies to a range of policy problems that may be encountered and indicated that although a common overall approach may be applied to the policy questions no one recipe could be applied in all cases. The policy question and the data available dictated the methodology.

The economic evaluation checklist (Table 18.1) that has been developed and the theoretical review (chapters 2 to 8 inclusive) are intended to provide non economist health policy managers with the criteria and techniques by which the decision-making process in resource allocation can be improved. If this checklist is used, many of the common errors in economic evaluation may be avoided.

The economic design should not be predetermined but should be driven by the policy question, perspective, data, time, research budget and expected net benefits of the research.

26. Although it has yet to be determined exactly how New Zealand policy decision makers use economic data. 
Accordingly, a range of techniques is likely to be applied in public policy analysis of health and it may be impossible to categorise the type of evaluation until the study is completed. It is more important to clearly describe the method used than attach a label.

Public sector policy health managers make day to day resource allocation decisions and prepare long term plans and budgets for their organisations that have spillovers with efficiency and equity implications for society as a whole. Public sector decision makers should be encouraged to consider the impact of a programme on all stakeholders in addition to their own viewpoint. The perspectives chosen should be wide enough to enable this to be done. Where efficiency equity trade-offs are recommended, the consequences of the choices on different interest groups should be explicit. 


\subsection{REFERENCES}

Canadian Coordinating Office for Health Technology Assesment. Guidelines for economic evaluation of pharmaceuticals: Canada. 2nd ed. Ottawa: Canadian Coordinating Office for Health Technology Assesment, 1997.

Commonwealth Department of Human Services and Health. Report on the development of AN-DRG version 3 cost weights. Canberra: Commonwealth Department of Human Services and Health, 1995.

Drummond, M. F., T. O. Jefferson, and on behalf of the BMJ Economic Evaluation Working Party. "Guidelines for authors and peer reviewers of economic submissions to the BMJ." British Medical Journal 313 (1996): 275-83.

Drummond, M. F., B. J. O'Brien, G. L. Stoddart, and G. W. Torrance. Methods for the economic evaluation of health care programmes. 2 nd ed. New York and Toronto: Oxford University Press, 1997.

Gold, M. Cost-effectiveness in health and medicine. Washington: US Public Health Service, 1996. 


\title{
ECONOMIC EVALUATION \\ APPLIED TO HEALTH POLICY IN NEW ZEALAND
}

\section{APPENDIX: FULL TEXT OF THE ORIGINAL PAPERS DISCUSSED IN THE CASES}

\author{
by \\ William Guy Scott
}

A thesis

submitted to Victoria University of Wellington

in fulfilment of the

requirements for the degree of

Doctor of Philosophy in Public Policy

Victoria University of Wellington 


\section{CONTENTS}

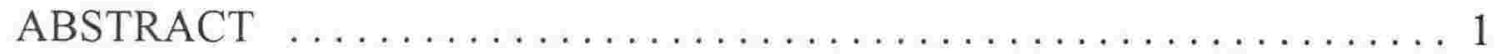

CHAPTER 1: INTRODUCTION TO ECONOMIC EVALUATION APPLIED

TO HEALTH POLICY $\ldots \ldots \ldots \ldots \ldots \ldots \ldots \ldots \ldots \ldots \ldots \ldots \ldots$

CHAPTER 2: CONCEPTUAL FRAMEWORK FOR ECONOMIC

EVALUATION OF HEALTH CARE $\ldots \ldots \ldots \ldots \ldots \ldots \ldots \ldots \ldots \ldots$

CHAPTER 3: VALUING HEALTH OUTCOMES $\ldots \ldots \ldots \ldots \ldots \ldots \ldots 45$

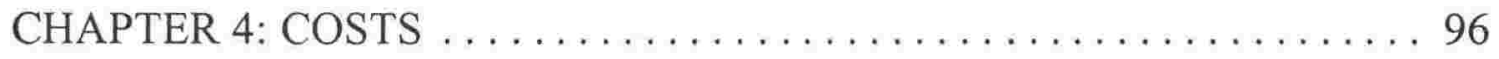

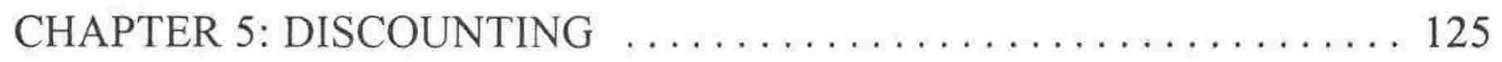

CHAPTER 6: ECONOMIC EVALUATION $\ldots \ldots \ldots \ldots \ldots \ldots \ldots . \ldots \ldots$

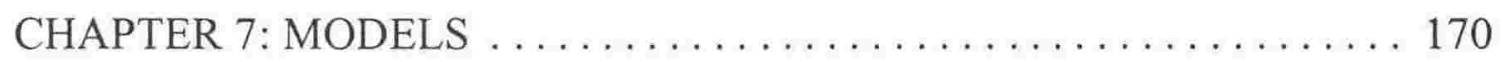

CHAPTER 8: CARE WITH DATA $\ldots \ldots \ldots \ldots \ldots \ldots \ldots \ldots \ldots \ldots$

CHAPTER 9: ANTIBIOTIC COSTING $\ldots \ldots \ldots \ldots \ldots \ldots \ldots \ldots \ldots$

CHAPTER 10: BENIGN PROSTATIC HYPERPLASIA $\ldots \ldots \ldots \ldots \ldots 217$

CHAPTER 11: HYSTERECTOMY . . . . . . . . . . . . . . 233

CHAPTER 12: LOWER RESPIRATORY TRACT INFECTION $\ldots \ldots \ldots 243$

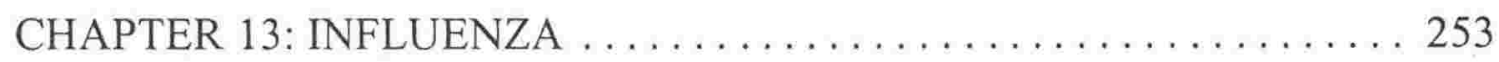

CHAPTER 14: CRITICAL LEG ISCHAEMIA . . . . . . . . . . . . 269

CHAPTER 15: HEART FAILURE $\ldots \ldots \ldots \ldots \ldots \ldots \ldots \ldots \ldots \ldots \ldots$

CHAPTER 16: ISCHAEMIC STROKE . . . . . . . . . . . . . . . 293

CHAPTER 17: ASTHMA .......................... 315

CHAPTER 18: DISCUSSION AND CONCLUSIONS ............ 340 APPENDIX: FULL TEXT OF THE ORIGINAL PAPERS DISCUSSED IN THE CASES 


\section{LIST OF CASES}

CASE 1: CARE WITH DATA (Discussed in Chapter 8)

CASE 2: ANTIBIOTIC COSTING (Discussed in Chapter 9)

CASE 3: BENIGN PROSTATIC HYPERPLASIA (Discussed in Chapter 10)

CASE 4: HYSTERECTOMY (Discussed in Chapter 11)

CASE 5: LOWER RESPIRATORY TRACT INFECTION (Discussed in

Chapter 12)

CASE 6: INFLUENZA (Discussed in Chapter 13)

CASE 7: CRITICAL LEG ISCHAEMIA (Discussed in Chapter 14)

CASE 8: HEART FAILURE (Discussed in Chapter 15)

CASE 9: ISCHAEMIC STROKE (Discussed in Chapter 16)

CASE 10: ASTHMA (Discussed in Chapter 17) 


\section{CASE 1: CARE WITH DATA}

Discussed in chapter 8

\section{Reference}

Scott, G., M. Camden, and H. Scott. "Pitfalls in aggregating data and using rates for policy decisions." Wellington Polytechnic Occasional Papers 98/1 (1998): 1-22. 


\section{Pitfalls in Aggregating Data}

AND USING RATES FOR POLICY DECISIONS

Guy Scott, Mike Camden and Helen Scott 
Date: June 1998

ISBN: $\quad 0.908719-55.8$

Publisher: WP Press

Wellington Polytechnic

PO Box 756, Wellington

New Zealand

Copyright: 1998 Wellington Polytechnic

All rights reserved

ISSN: $\quad 1174-1899$ 


\section{Acknowledgments}

We wish to thank our colleagues for comments on drafts of this paper.

Guy Scott ${ }^{1.2}$, Mike Camden ${ }^{1}$ and Helen Scott ${ }^{2}$

Wellington Polytechnic, ${ }^{2}$ Independent Health Economist 


\section{Contents}

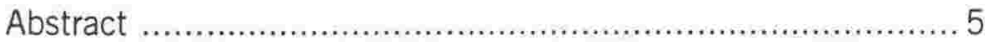

Introduction and Aims ........................................................ 7

Data Care and the Literature .................................................. 8

The Two Cases …............................................................. 10

Case One: Heart Failure ......................................................... 11

Case Two: Benign Prostatic Hyperplasia (BPH) ..................... 14

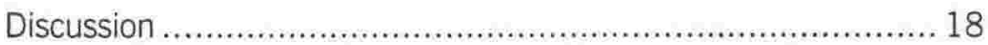

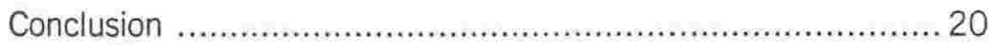

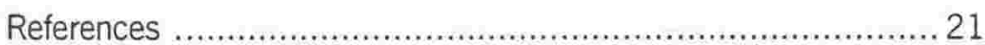

page 4 Pitfalls in Aggregating Data and Using Rates for Policy Decisions - Scott, Camden \& Scott 


\section{Abstract}

Data available in the public domain are frequently aggregated to preserve confidentiality and to reduce a database to a manageable size. Drawing conclusions from such data may lead to inappropriate policy advice.

The aims of this paper are to show how the aggregation of data to form rates may obscure important information and lead to misinterpretation of results. Suggestions are offered on ways in which this problem may be addressed. We also highlight the need to seek additional information in order to clarify findings.

We used a case study approach by drawing on illustrative examples to highlight some problems encountered when using aggregated data about populations. The focus is on health policy.

Two types of problem were discussed in the cases chosen, but a common resolution was appropriate. In the first case policies based on the assumption that hospital admissions equate with disease incidence would be different from policies framed on actual incidence data. In the second, incidence rates changed when they were disaggregated to gender and age-specific rates. Policies formulated from analysis of aggregated data would be different from those based on disaggregated data.

In the cases studied, the variables of gender, age and ethnicity influence incidence rates and must not be ignored. Researchers are recommended to study the data-set in the most disaggregated form available, and to check how data have been 
defined, collected and recorded, before preparing summary tables and graphs. Additional research or data from another source may be needed to clarify findings.

\section{Key words:}

Aggregation, ecological fallacy, economics, epidemiology, health, Maori health, New Zealand, policy, rates, Simpson's paradox, statistics.

\section{Addresses for correspondence:}

Guy Scott, Wellington Polytechnic, Private Box 756

Wellington, New Zealand.E-mail: guy.scott@wnp.ac.nz, m.camden@wnp.ac.nz, helen.scott@xtra.co.nz 


\section{Introduction and Aims}

Economic and policy evaluations often use secondary processed data from administrative records, and from experimental and observational studies. Unit records for a population or study group may be aggregated to form cross tabulations of counts for subgroups. These counts are commonly used to create rates. Often the unit record data are not available to the researchers. Problems can arise with the definition of the original data, with aggregation into summary tables and with the formation of rates.

This paper aims to highlight these problems, suggest some resolutions and show how a closer investigation of the original information may change or even reverse policy conclusions drawn from processed data. The intention is to show how the aggregation of data to form rates may obscure important information and lead to misinterpretation of results.

Demographic or epidemiological rates or indexes are important summary statistics in the study of community health, environmental health, health economics and policy analysis. Rates are formed by taking the number of occurrences (incidence or prevalence) of some event, dividing the number of these events by the population "at risk" and multiplying by a convenient constant (for example 1000). If the population is divided or "disaggregated" into groups (for example by age) a set of "specific" rates is derived, which may behave differently from the aggregated or "crude" rate. Researchers are often forced to use aggregated data but are frequently unaware of the possible consequences of doing so. 


\section{Data Care and the Literature}

When a situation is analysed with less detail than it could be, several problems can arise. These include confusion over the definitions and meanings of variables, Simpson's paradox, and the ecological fallacy.

Statisticians consider their discipline to be a scientific enquiry process (Pfannkuch 1996) which involves care with data quality at all stages. The total statistical process (Lipson \& Jones 1996) includes posing the right questions, collecting the right data and examining it with care and an eye for the unexpected. The exploratory approach, where the data is examined carefully with an open mind, is recognised as an important alternative to the "confirmatory" approach of statistical inference. When a research project is based on administrative records, this exploratory approach is often the most appropriate. When using secondary data sources, the definitions and meanings of variables are not always explicit or clear and this may result in confusion and misinterpretation.

Wild and Seber (1996) illustrate Simpson's paradox (Simpson, 1951). The paradox involves a three-way cross-classification table, where one of the three factors is a yes/no variable presented using percentages or rates. "In situations like this, collapsing (that is, aggregating) the table and looking at the two (remaining) factors of interest in isolation can be very misleading."

Frazier (1993) discusses Simpson's paradox in relation to US educational attainment. In this article Simpson's paradox was described as showing "... that an average can change in a 
direction opposite from all subgroups if the proportion of the total represented by the subgroup changes". The educational statistics described revealed that although every ethnic or racial population sub-group maintained or improved its average Scholarship Aptitude Test (SAT) score, there was a decline in the combined average score. This decline arose because more students in the lower percentiles in each class were taking the SAT test. The median test taker fell from the 79th percentile to the 73rd percentile within a class. A more fitting interpretation of this data is that educational attainment was rising rather than falling. Education policy and funding decisions based on the macro data may have led to inequitable and inefficient resource allocations.

Another example of Simpson's paradox was raised by Westbrooke $(1996,1997)$ when commenting on a publication by Dunstan et al (1995). Dunstan and colleagues studied the pool of 2984 people who reported for jury service in New Zealand in a four-week period of 1993.10.1\% of them were Maori, and the population in the Jury Districts was $9.5 \%$ Māori, which gave a slight over-representation. However, when the authors calculated expected numbers of Mari jurors for each of the 13 Jury Districts separately, the actual numbers were less than the expected numbers in 12 Districts (and equal in the $13 \mathrm{th}$ ). Westbrooke recommended that researchers should take care in choosing the level of aggregation at which they analyse data, and investigate unexpected results.

A further phenomenon with its own body of literature is the ecological fallacy (Steel and Holt, 1996). Frequently, unit records are aggregated into groups where the unit record values of a quantitative variable are replaced by the mean values for each group. The ecological fallacy arises when an analysis based on aggregated or grouped data produces different results from an analysis using unit level data. Conclusions based on means may "give conclusions very different from those that would be obtained from an analysis of unit level data, if they were available" (Steel and Holt, 1996: 39). This fallacy is also discussed by Greenland and Robins (1994). 
Our second case is about differences between Maori and nonMaori hospital admission for a certain condition. Health care for Mari is an issue that involves public policy, public debate and research. Pomare et al (1995: 29) claim, "It has become apparent during the last two decades that Maori want to define the threats to wellbeing, as well as the possible range of solutions". To succeed in these aspirations, researchers will need data that are appropriately defined, collected and analysed. There are also issues of control and ownership of data, research processes, decision making and delivery of services. Bishop and Glynn (1992: 127) assert "Powerful and rapid changes have occurred in areas like Maori women's health... since Maori people have been taking control over their own development and resources in these areas." and they discuss (1992: 130) research as "empowerment".

Basing policy advice on the results of macro studies is recognised as a potential problem by journal editors and by some government agencies. The British Medical Journal (Drummond et al 1996) has published a checklist for referees and authors of health economic studies. Checkpoint number 32 relates to aggregation and states that major outcomes should be presented in disaggregated as well as in an aggregated form. The US Public Health Service in a report on the conduct of cost effectiveness analysis (Gold 1996) recommends (where possible) using micro-costing in preference to gross costing using aggregated data.

\section{The Two Cases}

Two cases drawn from published original research are used to illustrate a number of problems and their resolution. The cases relate to New Zealand health economics studies: heart failure (Scott and Scott, 1996) and benign prostatic hyperplasia (BPH) (Scott and Scott, 1993). Additional information relating to methodology, data, results and conclusions may be found in these two papers. 


\section{Case One: Heart Failure}

Heart failure occurs when the heart muscle is damaged, overloaded, or exhausted by such conditions as hypertension, coronary artery disease, damage to cardiac valves, cardiomyopathy, and arrhythmias. The rates for deaths and hospital admissions for heart failure increase with age. Table 1 and chart 1 illustrate the age and gender specific death rates for heart failure in 1992 calculated from New Zealand death certificate data. Using aggregated data, (ages 60 years and over, and all ages) the statistics show higher death rates from heart failure for females than for males. However, different patterns emerge when five-yearly age-specific rates for 60 years and over are used. Age-specific mortality rates are higher for males than for females. This is a clear case of Simpson's paradox.

The data-set upon which chart 1 and table 1 are based has three category variables relating to the New Zealand population of 1992: two independent variables (gender and age group), and one dependent variable (mortality from heart failure, or not). The mortality variable is strongly related to both the gender variable and the age group variable. However, the two independent variables (gender and age group) are strongly related themselves. 
Table 1: Mortality from heart failure in New Zealand 1992: numbers, age and gender specific rates

\begin{tabular}{|l|cl|ll|ll|ll|}
\hline $\begin{array}{l}\text { Age } \\
\text { Years }\end{array}$ & $\begin{array}{l}\text { Deaths } \\
\text { Number }\end{array}$ & \multicolumn{2}{|c|}{$\begin{array}{c}\text { Population } \\
\text { Number }\end{array}$} & $\begin{array}{l}\text { Deaths } \\
\text { Rate per } \\
100,000\end{array}$ & $\begin{array}{l}\text { Population } \\
\text { age group as a } \\
\% \text { of the 60+ } \\
\text { total population, } \\
\text { by gender }\end{array}$ \\
\hline Male Female & Male & Female & Male & Female & Male & Female \\
$65-69$ & 3 & 1 & 69,860 & 69,480 & 4 & 1 & $30 \%$ & $24 \%$ \\
$70-74$ & 6 & 7 & 61,290 & 66,210 & 5 & 6 & $26 \%$ & $23 \%$ \\
$75-79$ & 13 & 10 & 31,540 & 46,170 & 41 & 22 & $13 \%$ & $16 \%$ \\
$80-84$ & 53 & 63 & 17,740 & 31,020 & 299 & 203 & $8 \%$ & $11 \%$ \\
$84+$ & 90 & 203 & 9,710 & 23,420 & 927 & 867 & $4 \%$ & $8 \%$ \\
\hline Total $59+$ & 168 & 288 & 235,410 & 293,620 & 71 & 98 & $100 \%$ & $100 \%$ \\
\hline
\end{tabular}

Chart 1: Mortality from Heart Failure in New Zealand 1992

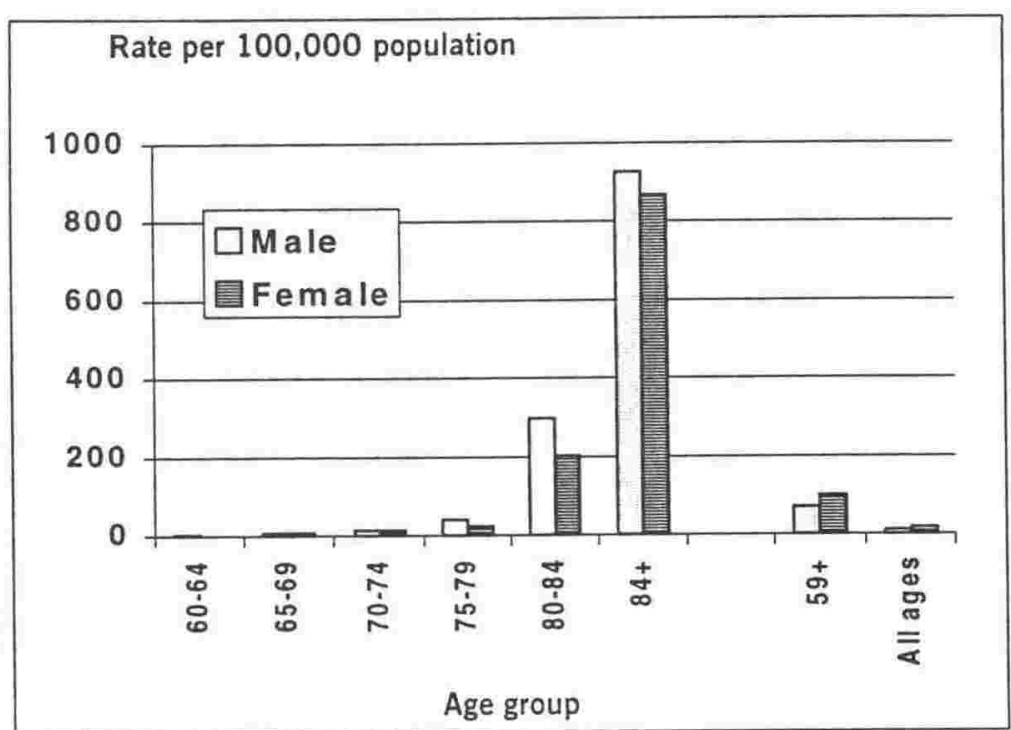

If mortality rates are defined by gender alone, or by age group alone, we get an incomplete portrayal of the information from the data-set. Defining the mortality rates by both gender and age group together provides a more complete picture, which reverses the effect of gender. Overall, a male is less likely to die from heart failure than a female. However a male of a specific age is more likely to fall victim to heart disease than a female of the same age. 
Heart failure is the end result of a number of cardiovascular conditions. Elderly people may have a multitude of health problems, a number of which could cause death or admission to hospitals. Accordingly, the numbers given for people dying from heart failure may be overestimates. This case demonstrates that researchers need to be very careful about what their data really mean: the definitions of variables, and the way these definitions are interpreted when the data is collected.

The main conclusion from this case is that health policy decisions made entirely on data aggregated across all age groups would direct resources towards heart disease in females, while decisions made entirely on age specific rates would direct resources towards heart disease in males. 


\section{Case Two: Benign Prostatic Hyperplasia (BPH)}

Two studies of BPH are discussed. The first is an analysis of administrative records relating to hospital admissions, and the second is a comparative community-based study. BPH is a nonmalignant enlargement of the prostate in men that commonly occurs with ageing.

Prostate enlargement may cause a variety of urinary problems ranging from acute urinary retention to recurrent urinary tract infections. The prevalence of $\mathrm{BPH}$ increases with age.

Symptomatic BPH is uncommon in men under the age of 50 but many men of more than 60 years will have some symptoms. By the time men reach the age of 80 years, $70 \%$ of them will have significant symptoms of BPH. We wish to use this study to discuss the apparent differences in prevalence of BPH between Maori and non-Mãori men.

Statistics on admissions for $\mathrm{BPH}$ were derived from hospital discharge data for all of New Zealand. Hospital discharge data includes information on ethnicity, gender, age, diagnosis and surgical procedures. Table 2 and chart 2 use this admissions data and combine it with data about the population of New Zealand by age and ethnicity. They present the age and ethnicity specific rates for admissions with $\mathrm{BPH}$ as the primary diagnosis. All-ages hospital admission rate for $\mathrm{BPH}$ for Maori is much lower (0.37 per 1000) compared with non-Maori (2.04 per 1000). For males aged 50 and above, the rate for Maori is lower (3.37 per 1000) than for non-Maori (8.71 per 1000). 
When the data for age $50+$ is disaggregated into age groups, the same feature occurs in each age group. The admission numbers are small (especially outside the 60.79 age groups), but the difference in rates is clear and consistent.

\section{Table 2. Hospital Admissions 1988 With Primary Diagnosis BPH}

\begin{tabular}{|c|c|c|c|c|c|c|}
\hline \multirow{3}{*}{$\begin{array}{l}\text { Age } \\
\text { years }\end{array}$} & \multicolumn{3}{|c|}{ Maori } & \multicolumn{3}{|c|}{ Non-Māori } \\
\hline & Admissions & Population & Rate & Admissions & Population & Rate \\
\hline & Number & Number & per 1000 & Number & Number & per 1000 \\
\hline $50-54$ & 4 & 5,220 & 0.77 & 87 & 68,400 & 1.27 \\
\hline $55-59$ & 4 & 4,360 & 0.92 & 257 & 68,160 & 3.77 \\
\hline $60-64$ & 10 & 2,940 & 3.4 & 459 & 67,150 & 6.84 \\
\hline $65-69$ & 14 & 1,770 & 7.91 & 645 & 52,370 & 12.32 \\
\hline $70-74$ & 11 & 990 & 11.11 & 603 & 40,180 & 15.01 \\
\hline $75-79$ & 9 & 590 & 15.25 & 525 & 28,200 & 18.62 \\
\hline $80-84$ & 2 & 300 & 6.67 & 304 & 15,110 & 20.12 \\
\hline $84+$ & 1 & 140 & 7.14 & 146 & 7,980 & 18.3 \\
\hline otal $49+$ & 55 & 16,310 & 3.37 & 3026 & 347,550 & 8.71 \\
\hline
\end{tabular}

\section{Chart 2: Hospital Admissions 1988 With Primary} Diagnosis BPH

\section{Rates per 1000 male population}

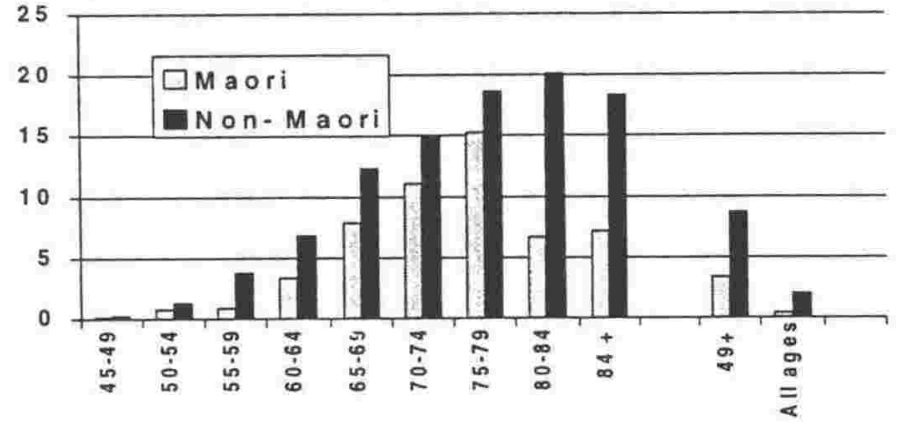

Age group

Clinicians at the time of the study considered (because they treated very few Maori men for BPH) that something protected Maori men from BPH. However, the higher non-Maori hospitalisation rates could have resulted from differences in a number of factors, including: disease prevalence as a result of genetic or lifestyle differences, cultural attitudes to health and health care, ability to access health services, knowledge about 
health and socioeconomic status. The age profiles of the two populations are quite different, that is, there are proportionally fewer Mãori in older age groups. As BPH symptoms increase with age, this will affect the total numbers of admissions and all-ages prevalence and incidence rates but not age specific rates. The disparate age profiles explain most of the very large differences noted in the all-ages rates for admissions. Use of these rates alone for policy decisions, even though they point in the same direction as the age specific rates, could be inappropriate as $\mathrm{BPH}$ is an age specific condition.

At the time of this economic study (Scott and Scott, 1993) a clinical investigation (Nacey et al, 1993) was begun. The Nacey study found that no statistically significant difference in prevalence of symptomatic BPH could be demonstrated among European, Maori and Polynesian men aged 50 years and over. The crucial difference between the Nacey study and the admission data as analysed by Scott and Scott is that the Nacey study took a representative sample of New Zealand men, and asked them about BPH symptoms, whereas the admissions data involved men who had self-selected by seeking treatment. Clearly, in this case, hospital admissions were not a measure of the incidence or prevalence of BPH.

A conclusion from these two studies considered together is that Mari and non-Maori appeared to have different rates of seeking and obtaining treatment. The most likely explanation for the differences between Maori and non-Maori age specific treatment rates are differences in access to treatment for economic and social reasons rather than anderlying difference in disease prevalence. Durie (1994: 201) claims that "...the biological substrate is only one indicator of health. The others include spiritual, psychological, interpersonal, family, economic and environmental dimensions." Pomare et al. (1995: 168) claim that "...Maori have difficulty in accessing health care services.", and that mainstream services need to be "...affordable, accessible, appropriate and acceptable". 
Interpreting the data at face value could lead to the conclusion that Marori men are not susceptible to developing $\mathrm{BPH}$ and that comparatively fewer health care resources should be directed towards Mari $\mathrm{BPH}$ health problems than to the treatment of $\mathrm{BPH}$ in non-Māori. A more appropriate policy would suggest that more resources should be directed towards ensuring that Maori men have access to and seek treatment for $\mathrm{BPH}$ problems (for example, consultation with Maori groups and specifically tailored and targeted health promotion).

Pomare et al (1995: 159) note that "Maori use health services differently than non-Mãori. When need for health care is taken into account, Maori visit a general practitioner less often than non-M arori."

This second case also raises the issue of how well the country's health statistics collection and analysis systems meet the needs of Mãori, as the health profiles and needs of the two partner cultures to the Treaty of Waitangi are often very different. In the past much research was designed to meet the needs of the researcher and the dominant group (Bishop and Glynn 1992: 126). In discussing the importance of the community perspective, Raeburn (1994: 338-9) comments that if research into Maori health is to be "empowering", researchers "...may need to reassess their stance, especially with regard to the 'community' and to the voices of those in it." 


\section{Discussion}

Researchers may wish to investigate a problem using data at the individual "unit record" or micro level but are often unable to do so. The degree of aggregation is frequently imposed on them by the form in which the data are available. Individual or unit data are often not available because of commercial sensitivity or confidentiality with respect to individuals. Frequently, published data are in tables that have already been aggregated across important variables, and researchers will not have access to less aggregated forms. However, it is sometimes possible to obtain the underlying raw data and conduct a more disaggregated analysis. For example, Health Statistics New Zealand publishes aggregated data, but unit record data, with the patient identifiers removed, are available in electronic form. If an analysis is based on grouped or aggregated data there should be some analysis and discussion of the grouping procedure or of the consequences of aggregation.

Researchers are often confronted with data sets containing variables which they did not define and for which they did not control the data collection. These variables will often not match the research questions precisely. Variables and rates calculated from them must be treated with care.

When working with counts of events (or conditions) from a population with diverse features there are two important factors that should be considered. Firstly features which appear when the data are disaggregated (and expressed as rates) by the groups within the population may disappear (or even be apparencly reversed) when the data are aggregated too much or 
inappropriately. Secondly, rates depend on two sets of numbers: the counts of the events (by group) and the counts for the total numbers in the population (by group). The counting by group of both events and population may be subject to errors, varying interpretations and different definitions.

There is computer software currently available and under development that will assist in these procedures. Generalpurpose statistical packages (for example: Data Desk, Minitab and SYSTAT) enable researchers to work from unit-record data-sets (if they are available) to cross-tabulations with many dimensions or factors. Recent releases of these packages facilitate analysis by groups. Spreadsheet software (for example: Excel) makes it very easy for researchers to aggregate tables with many dimensions into simpler ones, and to form rates.

The package Supercross (being developed for Statistics New Zealand), and similar tools will allow clients to specify tabulations from proprietary data-sets (initially the Census data) which are stored in confidential unit record form. A wide range of customised cross-tabulations may be specified for Statistics New Zealand to run on their behalf. Checks are built into the system to ensure that confidentiality will not be violated.

This paper has not discussed the important problems of confounding and multicollinearity because it was considered that these are well understood by researchers and that information is readily available in epidemiology and econometric texts. 


\section{Conclusion}

Occasionally clear examples of paradoxes, fallacies and misinterpretation of data will arise and be recognised. More often the underlying problems will be present and will cause problems, but will not make themselves evident.

In health economics and in other disciplines, researchers often have limited control over their statistical data. These data may consist of administrative records, with the variables defined by other people, and with the records collected in different situations. Unit record data may not be available, and the researchers may need to work with cross-tabulations provided by other people. Accordingly, researchers need to take great care at all stages in their analyses.

The following points are presented as a summary.

- It is essential that each variable is clearly defined and described.

- Subgroups of a human population may have different structures, behaviours and needs, and investigators should be aware of these differences.

- Data involving Maori health should be analysed and controlled in ways that meet the needs of Maori and in partnership with the affected group.

- Data should be analysed using cross-tabulations which involve disaggregation to the lowest possible level. If some variables are found not to influence the outcomes, these variables can be aggregated to produce tables that highlight the important influencing factors.

- Increasing availability of unit-record datasets, and software tools to manage and analyse them, makes such detailed analysis practicable. 


\section{References}

Bishop R, Glynn E. He Kanohi Kitea: conducting and evaluating educational research. New Zealand Journal of Educational Research 1992; 27:25-135.

Drummond MF, Jefferson TO, on behalf of the BMJ Economic Evaluation Working Party. Guidelines for authors and peer reviewers of economic submissions to the BMJ. BMJ 1996;313:275-83.

Durie M. Maori perspectives on health and illness. In Social dimensions of health and disease: New Zealand perspectives. Edited by Spicer J, Trlin A and Walton J, Palmerston North: Dunmore Press, 1994.

Dunstan S, Paulin J, Atkinson K. Trial by Peers? The Composition of NZ Juries. Wellington. Dept of Justice. 1995.

Frazier, K. 1993. Perspectives on Education in America: Sandia Study Challenges Misconceptions. Skeptical Inquirer 18:26-31.

Gold M. Cost Effectiveness in Health and Medicine. Washington: US Public Health Service, 1996.

Greenland S, Robins J. Ecological studies - biases, misconceptions and counterexamples. American Joumal of Epidemiology 1994;139:747-60.

Lipson K, Jones P. Teaching Statistics in the 21st Century. Proceedings of the 47th Annual Conference of the New Zealand Statistical Association, Wellington, 30 and 31 August, 1996.

MINITAB Reference Manual. State College Pennsylvania, MINITAB, 1995.

Nacey JN, Morum P, Delahunt B. The incidence of benign prostatic hyperplasia in Caucasian, Maori and Polynesian men: a comparative community based study. Poster presented at the 46th Annual Scientific Meeting, Urological Society of Australasia, Hobart, February 28 - March 5, 1993 
Pfannkuch M. Statistical Interpretation of Media Reports. Proceedings of the 47th Annual Conference of the New Zealand Statistical Association, Wellington, 30 and 31 August, 1996.

Pomare E, Keefe-Ormsby V, Ormsby C, Pearce N, Reid P, Robson B and Watene-Haydon N. Hauora: Maori standards of health III. Eru Pomare Maori Health Research Centre Wellington School of Medicine. Wellington, 1995.

Raeburn, J. The community in health research and policy. In Social dimensions of health and disease New Zealand perspectives. Edited by Spicer J, Trlin A and Walton J, Palmerston North: Dunmore Press, 1994.

Scott WG, Scott HM. Benign prostatic hyperplasia in New Zealand: a cost of illness study. PharmacoEconomics 1993;4(6):455-68.

Scott WG, Scott HM. Heart failure: a decision analytic analysis of New Zealand data using the published results of the SOLVD treatment trial. PharmacoEconomics 1996;9 (2):156-67.

Simpson EH. The interpretation of interaction in contingency tables. Joumal of the Royal Statistical Society 1951;13:387-94.

Steel DG, Holt D. Analysing and adjusting aggregation effects: The ecological fallacy revisited. Intemational Statistical Review 1996;64(1):39. 60.

Supercross users' guide. Hawthorn East and Wellington, Space-Time Research and Statistics New Zealand, 1994.

SYSTAT 6.0 for Windows Data. Chicago: SPSS, 1995.

Users Guide Microsoft Excel. Microsoft Corporation, 1994.

Velleman P. Learning data analysis with Data Desk. NY, Freeman, 1993

Westbrooke I. Simpson's paradox in New Zealand data. 47th Annual Conference of the New Zealand Statistical Association, Wellington, 30 and 31 August, 1996.

Westbrooke I. Simpson's paradox; an example in a New Zealand survey of jury composition. Research and Analytical Report. Statistics New Zealand, 1997.

Wild CJ, Seber GAF, eds. Introduction to probability and statistics. Auckland: Dept of Statistics, University of Auckland, 1996. 


\section{CASE 2: ANTIBIOTIC COSTING}

Discussed in chapter 9

\section{Reference}

Scott, W. G., H. M. Scott, S. Henderson, A. Inder, J. Sanders, R. Spearing, C. McArthur, J. Judson, B. Baker, P. Hicks, and P. Cotteral. "Comparative cost of antibiotic therapies for serious infections: a New Zealand 3-hospital study." PharmacoEconomics 16 (1999): 183-92. 


\section{Cost Comparison of Antibacterial Therapies for Serious Infections}

\section{A New Zealand 3-Hospital Study}

W. Guy Scott, 1,2 Helen M. Scott, ${ }^{1}$ Seton Henderson, ${ }^{3}$ Alison Inder, ${ }^{4}$ Joanne Sanders, 4 Ruth Spearing, ${ }^{4}$ Colin McArthur, ${ }^{5}$ James Judson, ${ }^{5}$ Bartrum Baker, ${ }^{6}$ Peter Hicks ${ }^{7}$ and Philippa Cotterell ${ }^{7}$

1 W. Guy Scott and Associates Limited, Wellington, New Zealand

2 College of Business, Massey University at Wellington, Wellington, New Zealand

3 Department of Intensive Care, Christchurch Hospital, Christchurch, New Zealand

4 Department of Haematology, Christchurch Hospital, Christchurch, New Zealand

5 Department of Critical Care, Auckland Hospital, Auckland, New Zealand

6 Department of Haematology, Palmerston North Hospital, Palmerston North, New Zealand

7 Department of Anaesthesia \& Intensive Care, Palmerston North Hospital, Palmerston North,

New Zealand

\section{Abstract}

Objective: The first aim was to identify and determine the economic costs of the regimens currently used in 3 New Zealand hospitals in the treatment of bacterial infections in haematology patients with febrile neutropenia and in intensive care patients with severe infections. The second was to develop a spreadsheet-based decision analytic model for use by hospital decision-makers as an aid in evaluating the comparative cost of drug regimens.

Design and setting: The research utilised time and motion and microcosting techniques. The analytical perspective adopted for the study was that of a hospital administrator or clinical manager.

Patients and interventions: Patients were eligible for inclusion in the study if either they were treated with the imipenem/cilastatin monotherapy, or could have been treated with this regimen. The final analysis considered 360 patient-treatment days and 8 antibacterials.

Main outcome measures and results: Drug acquisition cost ranged from 4.52 New Zealand dollars (\$NZ: 1997 values) per patient-treatment day for gentamicin to $\$ N Z 104.81$ for imipenem. The cost per patient-treatment day (when other cost components such as fluid additives, giving sets and needles were added) ranged from \$NZ8.75 for gentamicin to \$NZ129.12 for tazobactam. Drug acquisition cost. as a percentage of total drug preparation and administration cost, ranged from $52 \%$ for gentamicin to $93 \%$ for piperacillin.

Giving sets and intravenous (IV) fluids were found to be important cost items when they were required specifically for the treatment regimen. There was a mean monitoring rate of 0.40 at a cost of $\$ N Z 6.41$ per patient-treatment day for gentamicin. It was estimated that nephrotoxicity could add between $\$ N Z 23$ and $\$ N Z 43$ per day to the cost of aminoglycoside treatment. 
Conclusions: Although the small sample sizes of the study mean that results should be regarded as indicative rather than conclusive, there were sufficient information to construct a working model and show how the total cost of an antibacterial regimen could be evaluated in practical terms. The important cost drivers were found to be drug cost, the use of fluids and giving sets, and monitoring.

Tightening budgets within the New Zealand health system have increased the need for clinicians and managers in hospitals to consider the cost of treatment regimens as well as clinical efficacy and safety when selecting treatment options. Clinical managers and policy decision-makers require timely and relevant information, and budget and time constraints usually preclude the design and execution of randomised controlled clinical trials for this purpose. Other methods of gathering appropriate information should be considered.

There is often limited cost information and a lack of 'user friendly' analytical tools to assist in decision-making. Consequently, many patient treatment cost decisions and selection of preferred protocols may have been made on drug acquisition cost alone rather than on the full direct medical cost of a treatment regimen. Pharmaceutical acquisition cost is easily identifiable but is only 1 component of the total cost of drug therapy. ["]

The overall aim of this research was to identify and determine the direct medical costs of the regimens currently used in 3 New Zealand publicly funded hospitals for the treatment of bacterial infections in immunocompromised haematology patients with febrile neutropenia and in intensive care patients with severe infections. The study objectives were to identify and measure the total direct medical costs of the activities and resources associated with each treatment regimen, and to develop a decision analytic model for use by hospital decision-makers to evaluate the comparative cost of drug regimens.

\section{Methods}

\section{Study Design and Participants}

The study was collaborative, multicentred and multidisciplinary, involving both clinicians and economists. It was not a clinical trial evaluating safety and efficacy but a costing study utilising time and motion and microcosting techniques.

The target population was patients with severe microbial infections in intensive care or immunocompromised patients with febrile neutropenia in haematology departments. Patients were eligible for inclusion in the study if either they were treated with the imipenem/cilastatin monotherapy, or could have been treated with this regimen. It was implicitly assumed by the clinicians that patients would have infections that would respond to either imipenem/cilastatin monotherapy or to the alternative chosen and that the antibacterials were equally effective and produced identical patient outcomes. Imipenem/cilastatin is referred to in this paper as monotherapy because it cannot be broken into its constituents; the components cannot be administered separately. A combination therapy is one in which the components are separate entities and can be administered separately, for example, gentamicin plus piperacillin. The analytical perspective of the study was that of a hospital administrator or clinical manager.

Research was conducted in intensive/critical care units of 3 hospitals (Auckland Public Hospital, Christchurch Public Hospital and Palmerston North Public Hospital), and in the haematology departments of 2 hospitals (Christchurch Public Hospital and Palmerston North Public Hospital). Both Auckland and Christchurch hospitals were major hospitals in the largest cities in the North and South . Islands and Palmerston North hospital was representative of a large hospital in a provincial city.

Approvals for the study were obtained from the Canterbury Ethics Committee of the Southern Regional Health Authority, North Health Ethics Com- 
mittee of the Northern Regional Health Authority, and the Manawatu Wanganui Ethics Committee of the Central Regional Health Authority. Christchurch was used as a pilot centre to develop the study protocol and data collection methods. As the study was observational and nonintrusive, it did not alter treatment or clinical decision-making nor were any drugs or consumables provided.

\section{Resource Utilisation}

Drug treatment regimens were defined by clinicians and verified by a retrospective analysis of patient records. Relevant resources were those specifically required or altered by administering a specific drug.

A microcosting analysis of the antibacterial treatment regimens used for serious infections was undertaken. Microcosting is the most precise and detailed method of hospital costing..$^{[2.3]}$ We costed all activities involved with drug preparation, administration and monitoring. All consumables were itemised [e.g. syringes, needles, giving sets, drugs, fluid additives and intravenous (IV) fluids] and we recorded staff time for preparation, administration and monitoring. Similar listings of cost items can be found in the articles by Kerr et al., ${ }^{[+]}$ Davis and Bryson ${ }^{[5]}$ and Atkinson et al. ${ }^{[1]}$

Costs that would not be changed by the treatment were not applicable to the study. Those costs that would be incurred irrespective of the antimicrobial treatment regimen used, were also not included. For example, the insertion of a central venous line would not be relevant if all patients, irrespective of the treatment received, required such a line. This incremental costing approach was essential to avoid multiple counting of resource items that could occur when the cost of a drug regimen (consisting of a combination of drugs), was calculated by adding together the cost of individual drugs. For example, a giving set may be required for drug A and drug B but 2 giving sets may not be needed for the combination regimen A plus B.

Five data collection instruments (sets of forms) were designed in consultation with hospital staff, and were then tested and modified where necessary. These data collection instruments were; administration (drugs and consumables) form, drug administration staff time form, monitoring (consumables and laboratory charges) form, drug monitoring staff time form and a rate of monitoring form. Information was gathered sequentially from each centre commencing in late November 1995 and ending in March 1997.

Data to complete these forms were acquired from a prospective time and motion study (to quantify time taken and materials used in various stages of the treatment process) and a retrospective study of hospital records (chart reviews and laboratory files). Treatment processes were split into a number of activities and the component costs of each activity were identified. A clinically qualified observer recorded staff time, drugs and materials used for each activity. Resource utilisation unit records for the study were patient-treatment days. Data were collected only for complete hospital department days; incomplete hospital days were excluded from the analysis.

Resource volumes measured by the microcosting method and unit costs applicable to each hospital were used to convert resource utilisations into costs measured in monetary values. These were recorded separately for each centre.

Administration and preparation times were combined because of the difficulties encountered with overlap and merging of these activities. Infusion times derived from protocols were not measured by the time and motion study. Analysis was conducted on groups of items; e.g., syringe sizes from 5 to $20 \mathrm{ml}$ were summed and analysed in aggregate to derive mean resource cost and resource volumes used. However, within these aggregates the actual price for each syringe size was used.

Gentamicin was the only drug in the study that required monitoring. The rate of monitoring was obtained from a chart and laboratory record review within each hospital. The monitoring rate was the total number of monitoring events divided by the total number of patient-treatment days for genta- 
micin. The microcosting study determined labour and consumables required per monitoring event. The resource units per monitoring event from the microcosting study were multiplied by the rate of monitoring to give the resource units used in monitoring per patient-treatment day.

\section{Costs}

Labour rate information relating to nurses, doctors and pharmacists was derived from the literature, ${ }^{[6]}$ and from consultation with health sector members. Commercial sensitivity precluded us from quoting all of the sources or revealing the precise details of the rates obtained. Unit costs for all drugs and consumables were collected from each hospital. All unit costs were prices or charges and were exclusive of goods and services tax (GST) which is a transfer payment. All unit costs and prices are in 1997 New Zealand dollars (\$NZ; $\$ N Z 1=\$ U S 0.5925$, midrate December $1997^{[7]}$ ).

\section{Decision Analytic Model}

A deterministic decision analytical model was constructed and used for sensitivity and scenario analysis. This modelling approach permitted the costs of treatment using any antibacterial or combination of antibacterials to be evaluated. The model consisted of a series of spreadsheets in which the resource items used (drugs, nondrug materials and labour) were analysed. Inputs to the model were mean unit costs and volumes of resources per patient-treatment day. The output screen provided information on total treatment cost.

For the purpose of incorporation into this model, implicit prices per resource unit were calculated by dividing the average cost per patient day by the average volume per patient day for each different type of unit used. This gave a weighted average of prices over the 3 geographical centres.

Univariate sensitivity analysis was undertaken on the major cost components isolated by the data analysis. Sensitivity analysis (conducted using the model), considered 0 labour costs and a $10 \%$ decrease in drug cost.

\section{Results}

Data from a total of 411 patient days for drug preparation and administration was collected from 94 treatment cycles (an average of 4.4 treatment days per treatment cycle). Final analysis of aggregated drug data was confined to those drugs for which 20 or more observations (patient days) were recorded. Thus, the study analysed data from 83 patients, for whom a total of 360 treatment days were recorded. 20 observations was considered to be the minimum number required to provide meaningful information. The sample breakdown of patient-treatment days by centre was as follows; Christchurch 53.6\%, Auckland 29.7\% and Palmerston North $16.7 \%$.

Drugs for which sufficient data were collected were; amoxycillin ( $n=30$ treatment days), cefuroxime $(n=32)$, flucloxacillin $(n=23)$, gentamicin $(n=92)$, imipenem/cilastatin $(n=90)$ [abbreviated to imipenem], metronidazole in minibag form ( $\mathrm{n}=$ 21 ) [abbreviated to metronidazole], piperacillin ( $\mathrm{n}$ =52) and tazobactam/piperacillin $(n=20)$ [abbreviated to tazobactam]. Data relating to 8 drugs were excluded from the analysis because they did not achieve the minimum sample size of 20 observations.

Wastage was recorded only for cefuroxime and gentamicin. Fluid additives, syringes and needles were used for all drugs except metronidazole, while giving sets and IV fluids were not uniformly required by all drug regimens. Metronidazole had the lowest labour cost (2.07 minutes) in terms of administration and preparation time and piperacillin had the highest labour cost (6.7 minutes). Resource utilisation for preparation and administration by drug are presented in table I.

Drug acquisition was the major component of preparation and administration costs. Drug acquisition cost ranged from $\$ N Z 4.52$ per patient-treatment day for gentamicin to \$NZ104.81 for imipenem. The cost per patient-treatment day (when 
the other cost components were added) ranged from \$NZ8.75 for gentamicin to \$NZ129.12 for tazobactam. Drug acquisition cost as a percentage of total drug preparation and administration cost ranged from $52 \%$ for gentamicin to $93 \%$ for piperacillin. Giving sets and IV fluids were important cost items when they were required specifically for the treatment regimen. Both tazobactam and imipenem utilised giving sets and IV fluids to a greater extent than other regimens. Table II presents the preparation and administration costs for each drug regimen.

Imipenem and gentamicin were the 2 drugs used by all 3 hospitals. Imipenem (imipenem/cilastatin) was the only drug used almost exclusively as monotherapy. Gentamicin was the drug most often recorded as part of combination therapy and could be considered as a reference regimen, as many clinicians implicitly relate other antibacterial regimens to this drug.

The major cost component of monitoring gentamicin was the laboratory charge (65\% of total), with labour $(28 \%)$ and consumables $(7 \%)$ making up the balance. Monitoring of gentamicin occurred 116 times out of 289 patient-treatment days. This gave a mean monitoring rate per patient-treatment day of 0.40 . The total cost of monitoring was \$NS6.41 per patient-treatment day. Labour was required for blood collection, obtaining laboratory results and interpretation of these results. Although labour cost data were collected separately for these tasks they have been presented in aggregate. Blood was normally collected by nurses, while obtaining laboratory results and interpretation was primarily undertaken by a clinician. Table III presents the monitoring resource costs for gentamicin (the only drug for which monitoring was recorded).

\section{Sensitivity/Scenario Analyses}

Univariate sensitivity analysis showed that when drug prices were reduced by $10 \%$ (holding all other factors constant), the decrease in total cost for a drug regimen per patient per day ranged from $3 \%$ for gentamicin to $9 \%$ for the piperacillin regi-

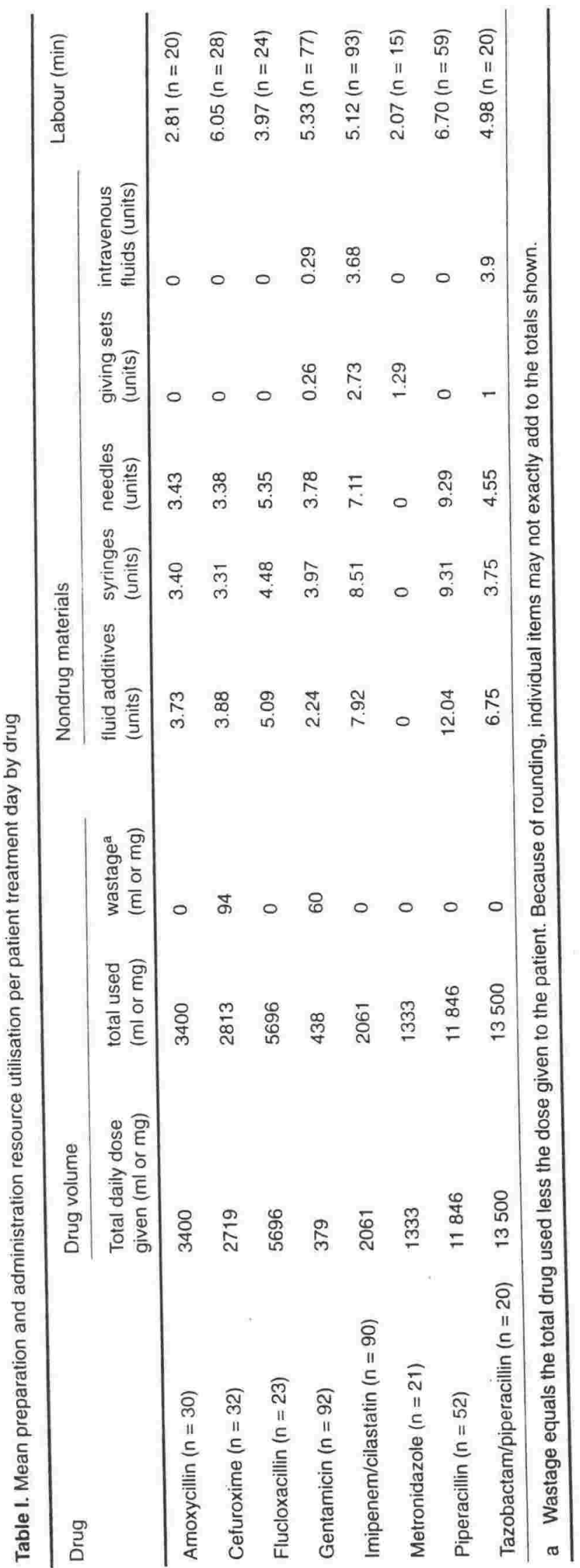

Pharmacoeconomics 1999 Aug; 16 (2) 


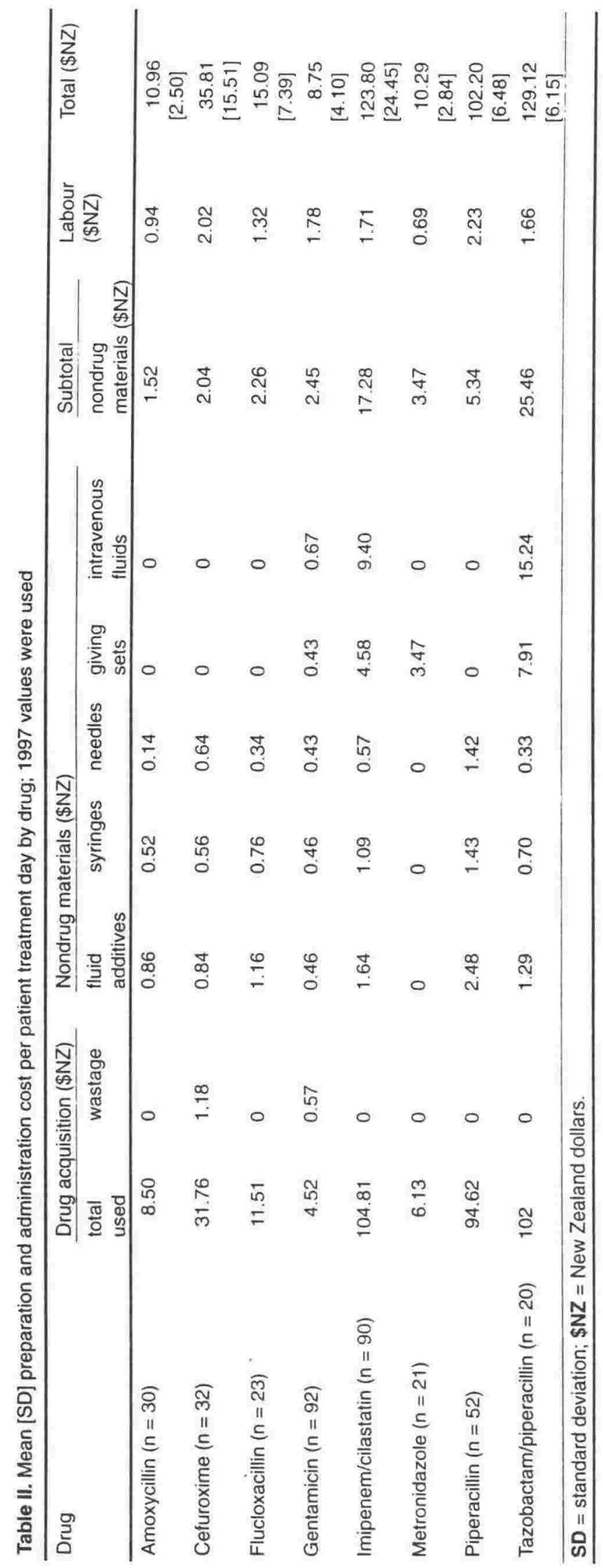

men. As would be expected, the magnitude of change was greater for those regimens in which the drug cost constituted the highest proportion of total cost. Gentamicin included a large cost for monitoring, which is reflected in lower treatment cost changes in response to changes in drug acquisition cost.

As some managers consider labour costs to be fixed, we conducted a sensitivity analysis to determine the effect of eliminating the labour cost (holding all other factors constant). This reduced the total cost of the tazobactam regimen by $1 \%$ and the gentamicin regimen by $22 \%$. These analyses are presented in figure 1.

Nondrug consumables of different regimens were found to be important cost drivers. Drugs administered by IV push do not require a dedicated giving set whereas others do (imipenem, metronidazole and tazobactam). The protocols relating to the use and replacement of giving sets differed both between hospitals and between the departments studied within hospitals with some replacing giving sets with every infusion and others replacing less frequently, but running saline between infusions. Imipenem and tazobactam regimens had nondrug materials (giving sets and IV fluids) amounting to over $\$ N Z 15$ per patient per day.

Some drugs recorded as being used for monotherapy (e.g., amoxycillin/clavulanic acid, cefuroxime, flucloxacillin, gentamicin, imipenem and piperacillin) were also used in combination (more frequently in the intensive care departments) and these combinations included up to 4 antibacterials. The model (used to analyse the results and conduct sensitivity analysis) was also developed so that clinical managers could evaluate the cost of any drug regimen including combination therapies. Table II may also be used to obtain the costs of preparation and administration of combinations of drugs. The combinations that occurred most frequently in the study (and their costs) were: pipercillin plus gentamicin \$NZ117.11 (\$NZ102.34+ \$NZ14.77); tazobactam plus gentamicin \$NZ144.14 $(\$ N Z 129.37+\$ N Z 14.77)$; amoxycillin plus met- 
Table III. Monitoring resource costs for gentamicin per patienttreatment day

\begin{tabular}{|c|c|c|}
\hline \multicolumn{3}{|c|}{ Multiplier for rate of monitoring per treatment day per patient $(T)$} \\
\hline \multicolumn{2}{|c|}{ Monitoring occurences (M) } & 116 \\
\hline \multicolumn{2}{|c|}{ Patient days $(P)$} & 289 \\
\hline \multicolumn{2}{|c|}{ Treatment multiplier $(T=M / P)$} & 0.4014 \\
\hline \multicolumn{3}{|c|}{ Resource costs monitoring } \\
\hline & $\begin{array}{l}\text { Cost/monitoring } \\
\text { (A) [SNZ] }\end{array}$ & $\begin{array}{l}\text { Cost of monitoring per } \\
\text { patient-treatment day } \\
(T \times A)[S N Z]\end{array}$ \\
\hline Consumables & 1.16 & 0.47 \\
\hline $\begin{array}{l}\text { Laboratory } \\
\text { charge }\end{array}$ & 10.38 & 4.17 \\
\hline Labour & 4.42 & 1.77 \\
\hline Total & 15.96 & 6.41 \\
\hline
\end{tabular}

ronidazole minibag plus gentamicin \$NZ36.03 $(\$ N Z 10.96+\$ N Z 10.30+\$ N Z 14.77)$; and amoxycillin plus metronidazole minibag plus gentamicin plus flucloxacillin \$NZ51.01 (\$NZ10.96+ $\$ N Z 10.30+\$ N Z 14.77+\$ N Z 14.98)$.

\section{Discussion}

It was intended that the study findings and model developed would be used by clinical managers as an aid in assembling and evaluating essen- tial information for policy and management decisions rather than clinical decision-making alone.

This was an observational study, with the information recorded at the time of the event by a clinically trained observer. In a busy hospital environment, a diary in which data are self-recorded cannot always be written up at the time events are taking place as clinicians and nursing staff must be primarily concerned with the needs of the patient. Data obtained in this manner may not be as accurate as that derived from observational studies in combination with chart reviews as used in our study.

Although there were differences between the 3 hospitals in the prices of antibacterials and other materials used, most of the variation could be explained by the timing of purchases and differences in negotiated prices. Commercial sensitivity precluded disclosing price and costing data from individual hospitals. Other investigators ${ }^{[8]}$ have encountered similar problems with commercially sensitive data in the UK.

Data on a greater number of drugs were collected in intensive care departments than in haematology departments as patients presented with a

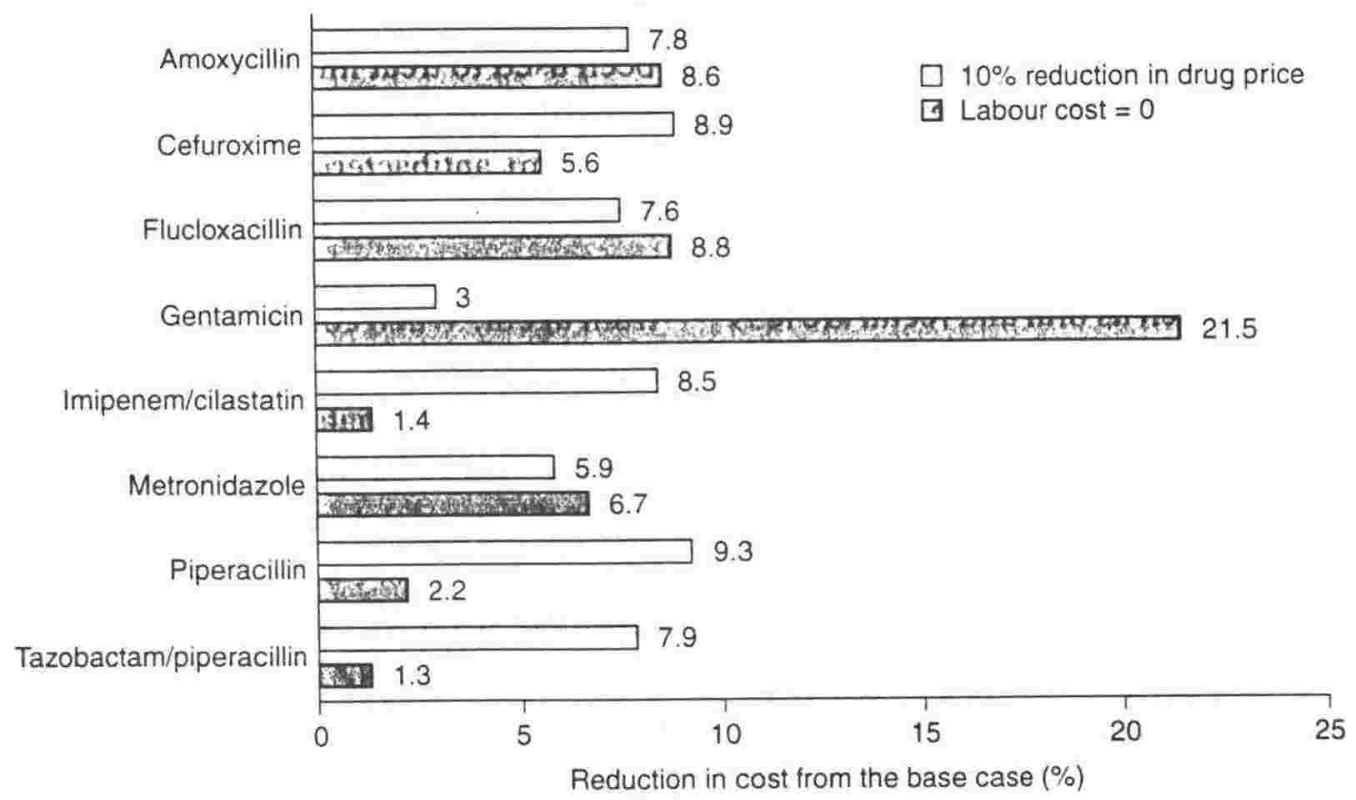

Fig. 1. Sensitivity analysis conducted using a decision analytic model. The model considered 0 labour costs and a $10 \%$ decrease in drug cost. 
greater range of conditions requiring a wider variety of drug treatments. At study outset, the range of antibacterial regimens was not known with certainty and the number of eligible drugs expanded as the study progressed. To achieve our second objective (that of designing an activity costing model that could be used for any antibacterial), we had to ensure that we captured the full range of activities and resources that could be encountered in preparing, administering and monitoring an antibacterial regimen. The drugs excluded from the final analysis (because of insufficient sample size) would not have provided any additional information on activities or consumables.

Wastage of antibacterials, defined as the unused portion of a pack, occurred with 2 drugs only. While wastage may indicate that an inappropriate pack size has been used or that the appropriate pack size was unavailable, wastage will not necessarily add to costs. For example, it may be more time consuming for staff to use a number of smaller packs, and/or a combination of smaller pack sizes may be more expensive than 1 larger pack.

Administration and preparation times were combined in the analysis because in many instances, it was difficult to differentiate between the 2 tasks. There was little difference in preparation and administration times either between hospitals or between departments and the differences that did occur may be explained by some staff not being as familiar with routine procedures as other more experienced staff or by difficulties with a patient.

Delivery systems such as oral and rectal are less intensive on staff time than IV push or infused medications. ${ }^{[1]}$ Although infusion was the method of delivery used in tazobactam, metronidazole and imipenem regimens, the infusion times were not included because it would be possible for nursing staff to perform some other tasks concurrently. From the protocols of the regimens evaluated, infusion times range between 20 minutes and an hour. A hospital's financial costs (monetary outlays) with respect to nursing staff will not normally be lowered by reducing the labour component in the administration of drugs because these patients frequently require one to one nursing. There is, however, an opportunity cost as the nurse could be providing other care to the patient. ${ }^{[1]}$ Norrby ${ }^{[9]}$ comments that unless staff numbers can be reduced or a hospital ward can be fully or partially closed, there will not be a financial saving.

Drug protocols are continually evolving and during the course of the study, an important change occurred with respect to gentamicin. There was a move towards once daily gentamicin administration and a reduced frequency of monitoring. Our study found that monitoring was conducted on average every second day. From a cost point of view, this actual practice finding is more important than a protocol best practice. The model is designed so that protocol changes such as this can be taken into account.

The drug regimen chosen by a clinician is determined by known adverse reactions, organism sensitivity and the patient's severity of illness. This study did not take into account the spectrum of cover of the various antibacterials and the susceptibility of micro-organisms to specific antibacterials. Many of the recently introduced antibacterials such as imipenem and tazobactam have a broader spectrum of cover than the older products and have been used to treat infections caused by both grampositive and gram-negative organisms. Narrowspectrum antibacterials are often combined (e.g., triple combination therapy) to provide the same spectrum of cover provided by a single broad-spectrum agent. When comparing costs of antibacterial regimens offering the same coverage, the additive costs of a combination regimen should be taken into account and compared with the cost of a single agent. The model developed enables these comparisons to be made. For example, a piperacillin plus gentamicin combination would cost \$NZ117.11 compared with \$NZ129.12 for tazobactam monotherapy.

A full economic study of a treatment requires the evaluation of patient outcomes, drug acquisition cost, preparation and administration costs, 
monitoring, length of hospital stay and the cost of adverse events. ${ }^{[10,11]}$ A limitation of the study was that it did not consider different patient outcomes.

While the cost of adverse events is acknowledged as important, the evaluation of such events is difficult for the following reasons. Very large numbers of patients are required to detect infrequently occurring adverse events. It is also difficult to attribute an adverse event to a particular component when a combination of drugs is use ${ }^{[12]}$ or in a situation in which regimens are continually changing. There are also problems in determining if an event is a result of the treatment or the underlying condition of the patient.

It is therefore hardly surprising that few studies have identified statistically significant differences in adverse events between antibacterial regimens. Atkinson et al. ${ }^{[1]}$ were unable to evaluate the cost of adverse effects such as nephrotoxicity, superinfection and complications of IV lines. Other investigators, ${ }^{\left[{ }^{4.8]}\right.}$ have also omitted evaluating the cost of adverse effects. However, Eisenberg et al., ${ }^{[13]}$ using a sample size of 1756 patients infected with psecudomonas aeruginosa, estimated that nephrotoxicity added \$US 103 (1987 values) to the average cost of each patient receiving aminoglycosides. Using the rate of nephrotoxicity ${ }^{[13]}$ of $7.3 \%$ and New Zealand hospital costs [\$NZ1297 for Australian Diagnosis-Related Group (AN-DRG) 587 - other kidney and urinary tract diagnoses without complicating conditions - and \$NZ2484 for AN-DRG 586 - other kidney and urinary tract diagnoses with nonmajor complicating condition $^{[14]}(1996 / 1997$ values $\left.)\right]$, we estimate that the additional cost per patient treated with aminoglycosides could range between \$NZ95 and \$NZ181 (or, if based on the average days per treatment cycle for gentamicin in our study of 4.18 days, nephrotoxicity would add between $\$ N Z 23$ and $\$ N Z+3$ per patient day). For example, if the cost of adverse events of \$NZ23 per day is added to the costs of combination piperacillin plus gentamicin, the cost per day increases from \$NZ117 to \$NZ140.
Univariate sensitivity analysis showed that the total cost of the regimen was inelastic with respect to percentage drug price changes, with the total cost response being less than the percentage change in price. As would be expected, the percentage change in total cost was greatest for those regimens in which the drug cost constituted the highest proportion of total cost. Gentamicin was the only drug requiring monitoring and, as this involves additional labour, the scenario of setting labour costs to 0 had a major impact of reducing this regimen's total cost by $22 \%$.

The nondrug consumables (giving sets and IV fluids) were important components of the total cost for both imipenem and tazobactam regimens. As giving sets and IV fluids made up $14 \%$ and $20 \%$, respectively, of these regimens, any changes to either the unit cost of these consumables or to protocols determining utilisation rates would have important cost consequences.

Incremental costing was applied to all cost items; thus, it is possible to add the individual drug total costs to obtain a total regimen cost. If incremental cost estimation had not been used, there would be serious multiple counting of costs when multidrug regimens were evaluated. It is recommended that clinical managers use such a costing tool at regular intervals or when prices and treatment protocols change, but in doing so, be careful to avoid double counting and being too prescriptive in recommending protocols. As patients differ in the nature and severity of their illnesses, it is important to be able to cost a wide range of treatment protocols from the basic cost components.

Although the sample sizes of the study mean that results should be regarded as indicative rather than conclusive, the study provided sufficient information to construct a working model and show how the full cost of an antibacterial regimen could be evaluated in practical terms. The results can be generalised in that treatment for serious infections is a common occurrence and will be ongoing in the 2 departments studied in all hospitals. The impor- 
tant cost drivers were found to be drug cost, the use of fluids and giving sets, and monitoring.

\section{Conclusion}

The study identified and measured the economic costs of the activities and resources associated with each treatment regimen for serious infections, and developed a decision analytic model for use by hospital decision-makers. The model is a tool to aid in the evaluation of the comparative cost of regimens involving up to 4 drugs and the associated consumables and labour costs for each drug. A copy of the spreadsheet-based model was given to each hospital department involved in the study.

The study found that drug acquisition costs were the most important cost item but the use of giving sets and IV fluids were important cost drivers if required specifically for an antibacterial treatment. Lack of information on treatment outcomes meant that our results, while providing useful costing information, could not be used for cost-effectiveness analysis. Ideally, a large multicentre study is required to provide data on treatment outcomes, particularly adverse events of treatment regimens used in New Zealand.

\section{Acknowledgements}

A research grant was obtained from Merck Sharp \& Dohme to fund this study. The funder has not influenced the design of this study or the reporting of results in this paper. The research team had complete independence in the conduct and analysis of the research results. No drugs were supplied for this study.

We are grateful for the support on statistical aspects of the study from Michael Camden of Wellington Polytechnic. and to Matthew Scott for data entry and assistance with the analysis of this study.

\section{References}

1. Atkinson HC, Chambers ST. McGinlay AM. Antibiotic therapy costs. N Z Med J 1989; 102: 409-11

2. Drummond MF, O'Brien BJ, Stoddart GL. et al. Methods for the economic evaluation of health care programmes. 2 nd ed. New York and Toronto: Oxford University Press, 1997

3. Luce BR. Manning WG. Siegel JE, et al. Estimating costs in cost-effectiveness analysis. In: Gold MR. Siegel JE, Russell LB, et al., editors. Cost-effectiveness analysis in health and medicine. Ist ed. Oxford: Oxford University press. 1996: 176-213

4. Kerr JR. Barr JG, Smyth ETM. et al. Technique for calculation of the true costs of antibiotic therapy. Eur J Clin Microbiol Infect Dis 1992; 11 (9): 823-7

5. Davis R. Bryson HM. Ceftriaxone: a pharmacoeconomic evaluation of its use in the treatment of serious infections. Pharmacoeconomics 1994: 6 (3): 249-69

6. Norton A. Nursing pay rates continue to widen. Nurs N Z 1997; $3(8): 12$

7. Statistics New Zealand. Personal computer information network for official statistics: a computerised database of official statistics for New Zealand and programme for manipulation and analysis of statistical series (PC INFOS). Wellington: Statistics New Zealand, 1996

8. Malek M, Lynch W, Wells N, et al. Antimicrobial practice: a comparison of the costs of ceftazidime therapy and gentamicin combinations in three UK hospitals. J Antimicrob Chemother 1992: 29: 207-17

9. Norrby SR. Pharmacoeconomic studies on antibiotics; current controversies. Pharmacoeconomics 1994; 5 (4): $274-7$

10. Beringer PM. Wong-Beringer A. Rho JP. Economic aspects of antibacterial adverse effects. Pharmacoeconomics 1998; 13 (1): $35-49$

11. Birmingham MC. Hassett JM. Schentag JJ, et al. Assessing antibacterial pharmacoeconoimes in the intensive care unit. Pharmacoeconomics 1997; 12 (6): 637-47

12. Norrby SR. Eriksson M. Ottosson E. Imipenem-cilastatin versus gentamicin-clindamycin: a cost effectiveness study. Scand J Infect Dis 1986; 18: 371-4

13. Eisenberg JM. Koffer H. Glick HA, et al. What is the cost of nephrotoxicity associated with aminoglycosides? Ann Intern Med 1987: 107: 900-9

14. Ministry of Health. Purchasing for your health 1996/97: a performance report on the fourth year of the Regional Health Authorities (appendix eleven: RHA price database 1996/97. average base contract prices paid for AN-DRGs. version 3.1, by the RHAs in 1996/97). Wellington: Ministry of Health. 1998

Correspondence and reprints: Dr Guy Scott, PO Box 14403, Kilbirnie, Wellington, New Zealand.

E-mail: Guy.Scott@xtra.co.nz 


\section{CASE 3: BENIGN PROSTATIC HYPERPLASIA}

Discussed in chapter 10

\section{Reference}

Scott, W. G., and H. M. Scott . 1993. Annual costs of Benign Prostatic Hyperplasia in New Zealand. PharmacoEconomics, 4 (6): 455-468. 


\title{
Annual Costs of Benign Prostatic Hyperplasia in New Zealand
}

\author{
W. Guy Scott and Helen M. Scott
}

W. Guy Scott and Associates Ltd, and Wellington Polytechnic, Wellington, New Zealand

Summary Benign prostatic hyperplasia (BPH) has been regarded as part of the normal aging process in men and little attention has been focused on the cost of the disease in New Zealand. The purpose of this study was to estimate the direct and indirect costs of treating BPH in both the public and the private sectors in New Zealand. The costs of treatment were estimated from public and private hospital data on admissions for BPH, obtained from the New Zealand Department of Health, medical insurance reimbursement schedules, hospital ward costs at one centre, and urology and general practitioner consultation fees. The length of time spent off work, as a measure of indirect costs, during urological investigations or treatment was estimated from interviews with urologists.

The annual 1991 total direct medical costs of treated BPH as primary diagnosis in New Zealand were estimated at $\$ \mathrm{NZ16}$ million (the average of the end-month mid-point exchange rate for the first quarter of 1992 was $\$$ US0.5457 per \$NZ1), and the costs of lost production plus loss of leisure time by patients was estimated at \$NZ4 million (1992 dollars). Patients with a principal diagnosis of BPH stayed on average 8.9 days in a public hospital and 4.6 days in a private hospital. Based on the above costs, if the average length of stay of public hospital patients could be reduced to that of private hospital patients, then hospital ward costs for BPH could fall by $37 \%$ and the total direct medical costs by $21 \%$.

The most commonly performed surgical operation for BPH was transurethral prostatectomy (TURP). For operations performed in a public hospital, patients stayed on average 8.5 days, while patients whose operations were performed in private hospitals stayed 4.3 days. The mean age of these public hospital patients was 71 years compared with 67 years for those in private hospitals.

A complication rate of $2.02 \%$ was recorded for TURP in public hospitals but audits (conducted by the researchers) in both public and private settings indicated that the complication rate was substantially under-recorded.

Benign prostatic hyperplasia (BPH) is a nonmalignant enlargement of the prostate that commonly occurs with aging (Barry 1990). Enlargement of the prostate may cause a variety of urinary problems ranging from acute urinary retention to recurrent urinary tract infection (Christensen \& Bruskewitz 1990). The frequency of occurrence of $\mathrm{BPH}$ increases with age: by 80 years of age, $70 \%$ of men will have significant symptoms of BPH
(Cockett 1992). BPH is uncommon in men under the age of 50 but most men aged over 60 years will have some symptoms of prostatic enlargement (Griffiths 1992).

$\mathrm{BPH}$ manifests itself in the following symptoms: the sensation of incomplete bladder emptying, a hesitant urinary stream, slow urinary flow, urgency, a need to push or strain to start urine flow, and nocturia (McConnell 1992). Over a period of 
several years the condition of one-third of patients will improve spontaneously, that of one-third will remain stable while that of one-third will deteriorate. Appropriate treatment options are watchful waiting, surgery or pharmacotherapy (Denis 1992).

In New Zealand, a patient with symptomatic $\mathrm{BPH}$ will usually present first to a general practitioner who will normally refer the patient to a specialist urologist in a public hospital or in private practice. This treatment profile was established on the basis of qualitative research involving a small number of general practitioners and specialists. At the time of the study the New Zealand population was 3.4 million (Department of Statistics 1992). There were approximately 2300 practising general

Table I. Assignment of costs of benign prostatic hyperplasia in New Zealand

\section{Direct medical costs \\ Ambulance/private transport \\ Hospital ward \\ Surgical operations \\ Outpatient and specialist consultations \\ Ancillary hospital staff (e.g. district nurse) \\ General practitioner consultations \\ Pharmaceutical prescribed (hospital and community) \\ Dispensing fees and container allowances}

Diagnostic procedures and laboratory tests (hospital and community based)

\section{Indirect plus nonmedical costs}

Production loss by patients

Production loss by unpaid care-givers

Leisure time loss by patients

Leisure time loss by unpaid care-givers

Transport costs of patients' family and friends

Services by community and voluntary organisations

Medical research

Additional laundry costs and protection aids

Additional costs associated with premature entry into residential care

\section{Intangible costs}

Quality of life of patient, family and friends

Loss of life practitioners who have a 'gatekeeper' role in the health system and 34 practising urologists based in the main population centres. Urologists may work in public or private practice, or both, and may be involved in teaching and in hospital management. The New Zealand healthcare system is a mixture of both public and private sector providers. Public and private spending on health in New Zealand in 1992 was $7.6 \%$ of gross domestic product (McKendry \& Muthumala 1993). Most hospital care is provided free of patient charges, or at a nominal charge, by public hospitals.

The purpose of this study was to estimate the direct and indirect costs of treated BPH in both the public and the private sectors in New Zealand.

\section{Methods}

\section{Hospital Data}

Public and private hospital data and mortality data for the year ending December 1988 were obtained as unit records from the Health Statistical Services (HSS) of the Department of Health (see Appendix). These official statistics cover all hospital patients and all deaths, for the whole of New Zealand. Subsets of this database were extracted for detailed analysis. HSS diagnoses A, B, C, D and operations 1,2, 3 refer to multiple diagnoses and procedures, respectively, in public hospitals (see Appendix). HSS data for private hospitals record data for only 1 diagnosis and 1 operation per admission.

\section{Audits}

Audits of urology consultations and surgery in both public and private settings were used to collect information on $\mathrm{BPH}$ when this was unavailable from existing data sources. Audits were undertaken in the last quarter of 1991 by 2 consultant urologists and support staff in 2 centres and covered patients in both private and public settings. The audit of consultations $(n=117)$ covered: (a) the place of consultation; (b) the age of the patient; (c) the type of consultation (e.g. first/postoperative visit); (d) the referral source; (e) diagnostic proce- 
dures; (f) laboratory tests; (g) medicines prescribed. The audit of surgical procedures covered: (a) surgical procedures; (b) days in hospital; and (c) perioperative and late postoperative complications. The audit information in this study should be regarded as indicative rather than conclusive, as the sample size was not sufficiently representative to enable statistically precise quantitative conclusions to be drawn.

\section{Costs}

The costs of BPH from the perspective of New Zealand society were classified into 2 categories: direct medical costs and indirect plus nonmedical costs (table I). The latest available patient volume data (at the time of data analysis) were combined with March quarter 1992 prices to calculate total economic costs on an incremental basis. As the perspective taken was that of society, transfer payments ${ }^{1}$ such as taxes, subsidies and welfare benefits were excluded. In the absence of reliable public sector costs, ${ }^{2}$ prices from the private sector were used to estimate direct medical costs, but these private sector prices included profit which was unquantifiable. The impact of including private sector profit is likely to be offset by higher costs in the public sector, where hospitals may have a teaching component and be more likely to treat patients who are at risk of death or complications because of their age and/or health status.

The Southern Cross Medical Care Society ${ }^{3}$ medical insurance schedule of fees for reimbursement for surgical and anaesthetic procedures current in the March 1992 quarter was used to estimate

1 In New Zealand, the goods and services tax (GST) is a cost from the perspective of an individual patient or individual hospital but is a transfer payment if the perspective of all society is taken.

2 Reliable and detailed public sector costing data in New Zealand are not readily available for economic research and in many instances the data do not exist. Such data, if they exist, are regarded by most hospitals as commercially sensitive. Available data are difficult to interpret, as some costs differ by more than $100 \%$ between area health boards, and different items are included in cost breakdowns from different area health boards. prices of preoperative consultation fees, surgery and anaesthetics. The first postoperative visit was included in the surgical fee, but subsequent followup visits were generally charged separately.

\section{Direct Medical Costs}

Not all the costs of the resources used by the healthcare system in caring for BPH patients could be quantified. The coding categories of the New Zealand Ambulance Board statistics were too broad to enable the costs of ambulance transportation for patients with BPH to be evaluated. Data on the numbers or costs of visits by ancillary hospital staff, such as district nurses and social workers, to patients after they were discharged from hospital, were unavailable because of their commercial sensitivity.

\section{Hospital Ward Costs and Operations}

A daily ward cost of \$NZ284.44 was used, based on data from Wakefield Hospital, Wellington (personal communication 1992), cross-checked with the New Zealand Private Hospitals Association survey (1991) and Resource Utilisation System (RUS) ${ }^{4}$ data from Christchurch Hospital, New Zealand. This daily bed rate (which includes the cost of medicines and other consumables used in the ward) was applied to all hospitals.

The ward stay cost per patient per day in a surgical ward of Wellington Hospital was estimated at \$NZ300 (1989 New Zealand dollars)[Stewart et al. 1992]. This ward stay cost was adjusted to first quarter 1992 prices (Scott 1990) to obtain a value of $\$ N Z 330$ which was used for sensitivity analysis and which represents a $16 \%$ increase over \$NZ284.44 (our baseline estimate of ward costs). Adjustments were made by using $80 \%$ of the increase in the Prevailing Weekly Wage Rate Index for central government, and $20 \%$ of the change in

3 The Southern Cross Medical Care Society has operated in New Zealand for over 30 years, owns several hospitals, and has about $80 \%$ of the health insurance market covering one-third of the total population.

4 The RUS is a management database system that allocates all costs that make up the total cost of a patient's stay, except for the cost of buildings and equipment. 
the Producers Price Index for inputs to central government. Weighting factors of $80 / 20$ were used because in a New Zealand hospital the breakdown of expenditure on inputs is approximately $80 \%$ staff costs and $20 \%$ purchases of other goods and services.

Unit prices for BPH operations do not include the ward costs for the hospital stay. Prices in combination with the number of operations were used to derive total costs for all surgical operations.

\section{Outpatient and Specialist Consultations}

As there were no national data it was presumed that each patient admitted to hospital would see a urologist before his admission, in either a private practice or a public hospital outpatient or accident and emergency department. This first consultation was evaluated at $\$$ NZ80 (Southern Cross Medical Care Society 1991). It was also estimated that every patient discharged live from hospital would have a postdischarge consultation valued at \$NZ56 (Southern Cross Medical Care Society 1991). However, the urology audits indicated that the number of consultations could be $50 \%$ higher than this estimate, and this was taken into account in the sensitivity analysis.

\section{General Practitioner Consultations}

Survey data relating to consultations for a primary diagnosis of BPH were obtained from Intercontinental Medical Statistics (IMS) 1992 (see Appendix). These data, together with an average consultation fee of \$NZ27.56 (Health Benefits 1991b) were used to calculate the cost of consultations.

\section{Pharmaceuticals, Dispensing Fees and \\ Container Allowances}

Total sales volumes of pharmaceuticals prescribed for BPH at general practitioner level were derived from New Zealand survey data (IMS 1992). New Zealand wholesale and retail markups (Pricing Schedules 1991) were added to the cost at the manufacturer selling price (IMS 1992). The total cost of pharmaceuticals was obtained by multiplying the number of prescriptions by the average dispensing fee plus container allowance ${ }^{5}$ (Pricing Schedules 1991). No data were available for drugs prescribed by specialists in private practice or in hospitals.

\section{Laboratory Tests and Diagnostic Procedures}

No published national statistics were available for tests and procedures. Volume data from the consultation audits were combined with the fees which the government pays for laboratory diagnostic services (Health Benefits 1991a).

\section{Indirect plus Nonmedical Costs}

On the basis of in-depth interviews with a number of urologists, the loss of production for men of working age ( $<65$ years of age) was estimated at 2 weeks inclusive of surgery for surgical patients, and 1 week for patients who were admitted to hospital but who did not undergo surgery. The assumption was also made that an equivalent amount of leisure time would be lost by men aged 65 years and over. Production and leisure time loss by patients was valued by multiplying the number of days of output forgone by the average male gross weekly earnings for the March quarter 1992 (\$NZ654.14)[Department of Statistics 1992] as a proxy for the economic value of lost output. ${ }^{6}$

The following costs were either unquantified or not isolated because of inadequate data: ambulance; ancillary hospital staff; transport costs of patients, family and friends; services provided by community and voluntary organisations; medical research; additional laundry costs and protection aids; premature entry into residential care; additional home help when a patient is partially incapacitated.

5 Pharmacists are reimbursed by the New Zealand Government for the pharmaceutical ingredients plus markup, plus a dispensing fee, plus an average allowance for the pharmaceutical container.

6 In the absence of more specific information, leisure time for individuals who are not in paid employment was valued on the same basis as production loss for those who are in paid employment. Leisure time for individuals in paid employment was not evaluated. 


\section{Intangible Costs}

Our study attempted to collect information on aspects of the quality of life of patients with BPH. A search was undertaken to ascertain the existence or otherwise of patient support groups within New Zealand and studies on urinary incontinence (both published and unpublished) that may have been conducted. Loss of life was not quantified in monetary terms.

\section{Results}

Hospital admissions in New Zealand are recorded at the time of hospital discharge or death. Table II shows the age-specific discharge rates from both public and private hospitals. In 1988 , for every 1000 men of all ages with BPH, 1.9 either were discharged from, or died in, a hospital. The equivalent rates for men aged 50 years and over were: Maori ${ }^{7} 3.4$, non-Maori 8.7 and all men 8.5. These data suggest that the prevalence of BPH among the Maori population could be lower than that for non-Maori.

Admission data (BPH Diagnosis A) for public hospitals were analysed with respect to admission type (arranged, acute, or waiting list referral) and the referral source of admission (general practitioner, specialist, outpatient, accident and emergency department, another hospital). Comparable data were not available from private hospitals. $59 \%$ of admissions came from waiting lists, $27 \%$ were emergency (acute) admissions and $14 \%$ were arranged by general practitioners, specialists or hospital outpatient departments. Some patients with acute urinary retention present directly at accident and emergency (A \& E) departments of public hospitals. Most hospitals admit patients directly to the

7 Maori made up 9\% of the population in 1990 (Department of Statistics 1992), but had a younger demographic profile than that of the total population. Maori at all ages have higher levels of mortality and morbidity, and lower life expectancy, than do non-Maori citizens. Some of these differences may be explained by different genetic characteristics, by cultural practices relating to health and the use of health services, levels of educational attainment and socioeconomic status.
Table II. Admission rates per 1000 of population (1988) for patients with benign prostatic hyperplasia as primary diagnosis, assessed from rates of discharge from public and private hospitals or death in hospital

\begin{tabular}{lccc}
\hline Age range $(y)$ & All patients & Maori & Non-Maori \\
\hline $0-39$ & 0.003 & $<0.001$ & 0.003 \\
$40-44$ & 0.066 & $<0.001$ & 0.071 \\
$45-49$ & 0.208 & 0.154 & 0.212 \\
$50-54$ & 1.236 & 0.766 & 1.272 \\
$55-59$ & 3.599 & 0.917 & 3.771 \\
$60-64$ & 6.691 & 3.401 & 6.835 \\
$65-69$ & 12.172 & 7.910 & 12.316 \\
$70-74$ & 14.914 & 11.111 & 15.007 \\
$75-79$ & 18.548 & 15.254 & 18.617 \\
$80-84$ & 19.857 & 6.667 & 20.119 \\
$\geq 85$ & 18.421 & 7.143 & 18.296 \\
Totala & 1.888 & 0.369 & 2.042 \\
$\geq 50$ & 8.471 & 3.372 & 8.707 \\
\hline a Individual columns may not add to the totals shown because & \\
\hline
\end{tabular}

appropriate ward, but some major hospitals admit all patients through their A \& E departments. 37\% of patients were referred for admission by specialists, $32 \%$ by outpatient departments, $23 \%$ by general practitioners, $5 \%$ by an A \& E department, and $2 \%$ by other hospitals.

Analysis of admission data indicated a greater degree of monitoring by general practitioners in small centres and rural areas located at some distance from a urologist. Cross-tabulations of general practitioner (referral source) with waiting list patients (admission type) and place of residence showed that all 39 patients thus identified came from small to medium-size towns, cities and country areas having no resident urologist. Similar data cross-tabulations for arranged admissions also showed that the majority of such patients (77) came from small towns and rural areas.

Almost all patient discharges were classed as routine: $97 \%$ from public hospitals, and $99 \%$ from private hospitals. $1 \%$ of public hospital patients and $0.4 \%$ of private hospital patients died in hospital. $2 \%$ of public hospital patients, and almost $1 \%$ 
Table III. Hospital ward costs for all patients with benign prostatic hyperplasia as primary diagnosis (1988 volumes, 1992 prices)

\begin{tabular}{llrlll}
\hline $\begin{array}{l}\text { Hospital } \\
\text { type }\end{array}$ & $\begin{array}{l}\text { Number of } \\
\text { patients }\end{array}$ & $\begin{array}{l}\text { Total } \\
\text { days }\end{array}$ & $\begin{array}{l}\text { Mean } \\
\text { length } \\
\text { of stay }\end{array}$ & $\begin{array}{l}\text { Cost per } \\
\text { day } \\
\text { (\$NZ) }\end{array}$ & $\begin{array}{l}\text { Total } \\
\text { cost } \\
\text { (\$NZ } \\
\text { million) }\end{array}$ \\
\hline Public & 1982 & 17662 & 8.91 & 284 & 5.024 \\
Private & 1128 & 5224 & 4.63 & 284 & 1.486 \\
All & 3110 & 22886 & 7.36 & 284 & 6.510 \\
\hline
\end{tabular}

of private hospital patients, were transferred to other care-giving institutions such as nursing homes or other hospitals. Reasons for the transferrals were not recorded.

\section{Hospital Ward}

Analysis of admission and discharge dates for BPH Diagnosis A showed that the mean length of stay was higher for patients admitted to public than private hospitals ( 8.9 compared with 4.6 days, for patients of all ages; 6.5 days compared with 3.9 days for those patients under 65 years of age). Averaged over all hospitals, the mean length of stay was 5.2 days for patients under 65 years of age compared with 7.4 days for patients of all ages. The total annual hospital ward costs were estimated to be \$NZ6.51 million based on the total number of days in hospital and the cost per patient per day. Table III describes ward costs and how these were calculated.

\section{Surgical Operations}

In New Zealand the operation that is most commonly performed for $\mathrm{BPH}$ is transurethral prostatectomy (TURP). In public hospitals TURP accounted for $91.4 \%$ of all prostatectomies. Retropubic (4.4\%) and suprapubic $(3.6 \%)$ and other prostatectomies $(0.5 \%)$ accounted for the balance (diagnosis A, operation 1). In private hospitals TURP accounted for $98.7 \%$ of all prostatectomies.

The average length of stay for patients having TURP in a public hospital was 8.5 days compared with 4.3 days for private hospital patients. The mean age of public hospital patients was 71 years while the mean age of those in private hospitals was 67 years.

Table IV describes the breakdown of surgical operation unit costs (exclusive of ward costs) for the surgeon, anaesthetist and operating theatre for each operation. Prices in combination with the number of operations were used to derive a total cost for all hospitals of \$NZ4.742 million (\$NZ2.872 million for public hospitals and \$NZ1.871 million for private hospitals). Table V gives the cost by operation for both public and private hospitals.

Complications of surgery, as recorded in diagnosis B, were extracted from HSS public hospital data relating to operation $1, \mathrm{BPH}$ diagnosis $\mathrm{A}$. (HSS data were not available for private hospitals.) Postoperative complications for all TURP procedures (1235 operations), in order of frequency of occurrence, were: haematuria, pneumonia, myocardial infarction, urethral stricture, septicaemia,

Table IV. Prices for surgical operations (\$NZ, March quarter 1992). Sources: surgeon and anaesthetist prices from the Southern Cross Medical Care price schedule, and operating theatre charges from Wakefield Hospital, Wellington, checked against the New Zealand Private Hospitals Association survey data. Cystoscopy and urethroscopy prices were used to cost procedures for WHO code 601

\begin{tabular}{lcccc}
\hline $\begin{array}{l}\text { WHO code and } \\
\text { description }\end{array}$ & Surgeon & Anaesthetist & Theatre & Total \\
\hline $\begin{array}{l}600 \text { Incision of } \\
\text { prostate }\end{array}$ & 516 & 187 & 267 & 969 \\
$\begin{array}{l}601 \text { Diagnostic } \\
\text { procedures on }\end{array}$ & 142 & 93 & 200 & 436 \\
prostate & & & & \\
$\begin{array}{l}602 \text { TURP } \\
603 \text { Suprapubic }\end{array}$ & 1022 & 267 & 444 & 1733 \\
prostatectomy & 1022 & 267 & 622 & 1911 \\
$\begin{array}{l}604 \text { Retropubic } \\
\text { prostatectomy }\end{array}$ & 1022 & 267 & 622 & 1911 \\
$\begin{array}{l}606 \text { Other prosta- } \\
\text { tectomy }\end{array}$ & 1022 & 267 & 444 & 1733 \\
$\begin{array}{l}609 \text { Other opera- } \\
\text { tions on the }\end{array}$ & 516 & 187 & 267 & 969 \\
prostate & & & & \\
\hline
\end{tabular}

Abbreviations: TURP $=$ transurethral prostatectomy; $\mathrm{WHO}=$ World Health Organization. 
Table V. Cost of surgical operations for benign prostatic hyperplasia in New Zealand (1988 volumes, \$NZ, 1992 prices)

\begin{tabular}{|c|c|c|c|c|c|c|c|}
\hline $\begin{array}{l}\text { WHO code and } \\
\text { description }\end{array}$ & Publica & Privateb & All & $\begin{array}{l}\text { WHO code and } \\
\text { description }\end{array}$ & Publica & Private ${ }^{b}$ & All \\
\hline 600 Incision of prostate & & & & 604 Retropubic prosta & ctomy & & \\
\hline number & 3 & 0 & 3 & number & 96 & 4 & 100 \\
\hline cost per operation & 969 & 969 & & cost per operation & 1911 & 1911 & \\
\hline total cost & 2907 & & 2907 & total cost & 183467 & 7644 & 191111 \\
\hline \multicolumn{4}{|c|}{601 Diagnostic procedures on prostate } & \multicolumn{4}{|l|}{606 Other prostatectomy } \\
\hline number & 58 & 6 & 64 & number & 7 & 7 & 14 \\
\hline cost per operation & 436 & 436 & & cost per operation & 1733 & 1733 & \\
\hline total cost & 25262 & 2613 & 27876 & total cost & 12133 & 12133 & 24267 \\
\hline 602 TURP & & & & \multicolumn{4}{|c|}{609 Other operations on the prostate } \\
\hline number & 1457 & 1063 & 2520 & number & 12 & 0 & 12 \\
\hline cost per operation & 1733 & 1733 & & cost per operationc & 969 & 969 & \\
\hline total cost & 2525462 & 1842530 & 4367992 & total cost & 11627 & & 11627 \\
\hline \multicolumn{4}{|c|}{603 Suprapubic prostatectomy } & \multicolumn{2}{|l|}{ All operations } & & \\
\hline number & 58 & 3 & 61 & number & 1691 & 1083 & 2774 \\
\hline cost per operation & 1911 & 1911 & & total cost & 2871702 & 1870654 & 4742356 \\
\hline total cost & 110844 & 5733 & 116578 & & & & \\
\hline
\end{tabular}

a Diagnosis A-D (primary,secondary etc), operations 1-3 (for benign prostatic hyperplasia).

b Diagnosis A, operation 1.

c Same as 600 .

Abbreviations: TURP $=$ transurethral prostatectomy; $\mathrm{WHO}=$ World Health Organization.

cardiac arrest and urinary retention. The complication rate for TURP was $2.02 \%$, and for all prostatectomy operations $2.31 \%$.

From a total of 79 patients in the audits of surgical procedures (in both private practice and public hospitals) 33 patients presented with perioperative complications (within 30 days of surgery) and 25 patients presented with late postoperative complications (over 30 days). The rate for perioperative complications was $42 \%$ and $32 \%$ for postoperative complications and surgical failure. All 79 patients had a TURP.

Perioperative complications recorded were: spinal headache ( 8 patients), clot retention (5), failure to void (4), haemorrhage requiring a transfusion (4), transient impotence (3), urgency or urinary frequency (3), electrolyte imbalance (2), sepsis - urinary infection (1), respiratory insufficiency (1), myocardial ischaemia (1), and transient inconti- nence (1). Late postoperative complications recorded were: retrograde ejaculation (11 patients), partial retrograde ejaculation (3), bladder neck stenosis (3), urethral stricture (2), nocturia (1), urinary tract infection (1), stress incontinence (1), and slight post-void urinary dribbling (1). One patient showed no improvement and one required reoperation.

\section{Outpatient and Specialist Consultations}

The costs of outpatient and specialist consultations were calculated to be $\$ N Z 0.285$ million. Table VI describes how outpatient and specialist consultations were costed.

\section{General Practitioner Consultations}

In 1991 there were 8500 consultations for BPH in New Zealand at a cost of $\$ \mathrm{NZ} 0.234$ million. All these consultations were for patients aged 40 years 
Table VI. Costs of outpatient and specialist consultations for benign prostatic hyperplasia in New Zealand (1988 volumes, 1992 prices)

\begin{tabular}{lcll}
\hline Consultation & $\begin{array}{l}\text { Patient } \\
\text { consultations }\end{array}$ & $\begin{array}{l}\text { Cost per } \\
\text { consultation } \\
\text { (\$NZ) }\end{array}$ & $\begin{array}{l}\text { Total cost } \\
\text { (\$NZ million) }\end{array}$ \\
\hline $\begin{array}{l}\text { Pre-admission } \\
\text { After discharge }\end{array}$ & 63110 & 80.00 & 0.249 \\
Total & 56.00 & 0.036 \\
\hline a & & $\mathbf{0 . 2 8 5}$ \\
\hline & \\
Thitted with BPH diagnosis A, less the 2467 patients who & had BPH diagnosis A and at least 1 operation for BPH, \\
less 6 patients who had no operation and died. This adjust- \\
ment was made because the surgeon's fee normally in- \\
cludes 1 postoperative visit.
\end{tabular}

and over, a rate of 14 per thousand men. Over the same period there were 3612 hospital admissions for $\mathrm{BPH}$, which indicates that for every 10 consultations for BPH at general practitioner level there are 4 hospital admissions.

\section{Pharmaceuticals, Dispensing Fees and}

Container Allowances

The 3351 prescriptions (written by general practitioners), when combined with container allowances of $\$ N Z 0.18$, a dispensing fee of $\$ N Z 2.16$ per prescription and the cost of the drugs at a pharmacy selling price of $\$ N Z 0.144$ million, gave a total of \$NZ0.152 million. Audits of urologist consultations indicate very little prescribing at this level, as the records showed that only 2 pharmaceuticals (phenoxybenzamine hydrochloride and oxybutynin chloride) were dispensed for a total of 117 urologist consultations. Costs of drugs prescribed at hospital and specialist levels were not included because data were unavailable.

\section{Laboratory Tests and Diagnostic Procedures}

The audits indicated that the following laboratory tests were routinely undertaken for all patients in both public and private settings: urine culture; prostatic acid phosphatase; prostate-specific antigen; complete blood count; serum electrolytes; blood group typing and holding blood in storage
(50\% of patients), and postdischarge urine culture (50\% of patients). The total cost of these tests to New Zealand was \$NZ0.189 million. Table VII itemises the laboratory tests routinely undertaken. It shows the number, unit cost, and total cost for each test.

The procedures undertaken were: intravenous pyelogram, abdominal $\mathrm{x}$-ray, chest $\mathrm{x}$-ray, abdominal ultrasonography, urinary flow rate and electrocardiogram (ECG). It was clear that nearly all patients had an $x$-ray. The price of a chest $x$-ray (\$NZ46.22)[Wakefield Hospital 1992] was multiplied by the 3110 patients who received an $\mathrm{x}$-ray, to give a total cost of $\$ N Z 0.144$ million. As the older and 'at risk' patients were more likely to have chest $\mathrm{x}$-rays, ECGs, blood tests, the quantified costs underestimate the cost of diagnostic procedures and laboratory tests. Although the audits showed that these diagnostic procedures were undertaken, the data were insufficient to extrapolate for all specialist consultations.

\section{Lost Production and Leisure Time}

Loss of production during hospitalisation and recuperation for all 850 patients under 65 years of age (BPH Diagnosis A) was estimated to be $\$ N Z 1.082$ million. This estimate was calculated on the basis of 2 weeks off work per patient undergoing surgery (804 patients) and 1 week per patient

Table VII. Laboratory tests (1988 volumes, 1992 prices)

\begin{tabular}{lccl}
\hline Test & Number & $\begin{array}{l}\text { Unit cost } \\
(\$ N Z)\end{array}$ & $\begin{array}{l}\text { Total } \\
\text { cost } \\
(\$ N Z \\
\text { million })\end{array}$ \\
\hline Urine culture & 3110 & 15.11 & 0.047 \\
Prostatic acid phosphatase & 3110 & 6.03 & 0.019 \\
Prostate-specific antigen & 3110 & 12.40 & 0.039 \\
Complete blood count & 3110 & 9.99 & 0.031 \\
Serum electrolytes & 3110 & 6.07 & 0.019 \\
Group and hold blood & 1555 & 6.13 & 0.010 \\
Urine culture & 1555 & 15.11 & 0.024 \\
(postdischarge) & & & \\
Total & & & 0.189 \\
\hline
\end{tabular}


not having surgery (46 patients) and costed at \$NZ654.14 earnings forgone per patient per week. Leisure time loss for patients 65 years and over (1663 patients undergoing surgery, 597 patients no surgery) was estimated similarly at \$NZ2.566 million. Production loss and loss of leisure time by unpaid caregivers were not assessed.

\section{Quality of Life}

The New Zealand literature identified only one relevant article (Gilling et al. 1988). This was a retrospective study of 62 patients interviewed at a minimum of 6 months after TURP to investigate factors associated with sexual dysfunction. Although potency was retained by $89 \%$ of these patients, $68 \%$ reported that their sexual potency had decreased.

There are continence/incontinence clinics for both men and women in several cities but to date the focus has been on women. Literature searches and inquiries found that although the Auckland and Christchurch clinics had undertaken surveys on urinary problems, the focus again had been on women and only the Christchurch survey (Crowder 1991) has been published. The pain and suffering experienced by the patients, and its effects on the family, could not be quantified.

\section{Summary of Quantified Costs}

Total direct medical costs amounted to \$NZ12.256 million and indirect costs were $\$ N Z 3.648$ million (production and leisure time lost) giving a total of quantified costs of \$NZ15.904 million.

Table VIII summarises the quantified costs and the impact of increased admissions, higher ward costs, and greater numbers of outpatient and specialist consultations.

\section{Sensitivity Analysis}

HSS data revealed an overall, nonpopulationstandardised, $16.2 \%$ increase in admissions for BPH during the period 1988 to $1991 .^{8}$ Public hospitals recorded a $14 \%$ increase and private hospi- tals recorded an increase of $20 \%$ in admissions. Accordingly, all 1988 patient volumes that were dependent upon hospital admissions were increased by $16 \%$ to obtain a 1991 volume estimate - the baseline estimate of \$NZ18.388 million (1992 prices)[direct costs \$NZ14.156 million, indirect costs \$NZ4.232 million]. As general practitioner consultations and prescribed medicine costs related to 1991 volumes, it was unnecessary to increase these costs for the sensitivity analysis (table VIII).

A high estimate was obtained by increasing the baseline estimate of hospital ward costs by $16 \%$ (to take account of higher daily ward costs as indicated by Wellington and Christchurch data), and outpatient and specialist consultations by $50 \%$ (based on audit data), to give a total of \$NZ19.761 million (direct costs $\$ \mathrm{NZ15} .529$ million, indirect costs $\$ N Z 4.232$ million).

The production loss would fall by $35 \%$ if adjusted for the labour force participation rate $(73.9 \%)$ and unemployment rate $(11.5 \%)$ for the total male labour force (New Zealand Department of Statistics 1992), but this fall would be offset by a corresponding increase in leisure time lost and would not affect the total indirect costs. The economic cost of paid employment may be overestimated, as short periods of time off work may not result in lower production if co-workers temporarily increase their output to cover the absent worker (see Koopmanshap \& Rutten 1993 for detailed discussion).

\section{Mortality Rates}

In 1988 the total mortality rate for BPH was 0.052 per thousand for men aged 50 years and over (primary cause of death as recorded on death certificates). All 19 men with BPH recorded on death certificates were aged 65 and over, and these deaths were not quantified in monetary terms.

8 Between 1988 (hospital unit record data used to estimate costs) and 1991 [the latest published volume data on hospital admissions available at time of finalising this article (Department of Health 1992)] the volume of admissions for principal diagnosis BPH to all hospitals increased by $16 \%$. 
Table VIII. Summary and sensitivity analysis of quahtified cósts (1992 \$NZ million) for benign prostatic hyperplasia ${ }^{a}$

\begin{tabular}{|c|c|c|c|}
\hline $\begin{array}{l}\text { Cost (1988 and } 1991 \text { volumes in March quarter } \\
1992 \text { prices) }\end{array}$ & $\begin{array}{l}\text { Low } \\
\text { ( } \% \text { of total cost) }\end{array}$ & $\begin{array}{l}\text { Baseline } \\
\text { (\% of total cost) }\end{array}$ & $\begin{array}{l}\text { Highd } \\
\text { (\% of total cost) }\end{array}$ \\
\hline \multicolumn{4}{|l|}{ Direct medical costs } \\
\hline hospital ward & $6.510(40.9)$ & $7.552(41.1)$ & $8.760(44.3)$ \\
\hline operations & $4.742(29.8)$ & $5.501(29.9)$ & $5.501(27.8)$ \\
\hline outpatients and specialists & $0.285(1.79)$ & $0.331(1.80)$ & $0.496(2.51)$ \\
\hline general practitioners $c$ & $0.234(1.47)$ & $0.234(1.27)$ & $0.234(1.18)$ \\
\hline medicines, dispensing fees and container allowances ${ }^{c}$ & $0.152(0.96)$ & $0.152(0.83)$ & $0.152(0.77)$ \\
\hline laboratory tests & $0.189(1.18)$ & $0.219(1.19)$ & $0.219(1.10)$ \\
\hline diagnostic procedures & $0.144(0.91)$ & $0.167(0.91)$ & $0.167(0.85)$ \\
\hline Total direct medical costs & $12.256(77.0)$ & $14.156(76.9)$ & $15.529(78.6)$ \\
\hline \multicolumn{4}{|l|}{ Indirect costs } \\
\hline production loss by patients under 65 years of age & $1.082(6.81)$ & $1.255(6.83)$ & $1.255(6.35)$ \\
\hline leisure time lost by patients $\geq 65$ years & $2.566(16.1)$ & $2.977(16.2)$ & $2.977(15.1)$ \\
\hline Total indirect costs & $3.648(22.9)$ & $4.232(23.0)$ & $4.232(21.4)$ \\
\hline Total quantified costs & 15.904 & 18.388 & 19.761 \\
\hline \multicolumn{4}{|c|}{ a Because of rounding, individual items may not add exactly to the totals shown. } \\
\hline \multicolumn{4}{|c|}{$\begin{array}{l}\text { b } 1988 \text { unit record data for hospitals, and volumes derived from hospital admissions for benign prostatic hyperplasia on which this study } \\
\text { was based. }\end{array}$} \\
\hline \multicolumn{4}{|c|}{ c Low 1988 unit record data rated up to 1991 volumes based on the growth in hospital admissions for BPH, a $16 \%$ increase. } \\
\hline \multicolumn{4}{|c|}{ d Ward costs increased by $16 \%$ and outpatient and specialist consultations increased by $50 \%$. } \\
\hline
\end{tabular}

\section{Discussion}

Our study found that the ratio of direct to indirect costs for $\mathrm{BPH}$ was $80: 20$, and that patients with BPH stayed longer in wards of public than of private hospitals. Our data also suggest that the complication rate for surgery for $\mathrm{BPH}$ was higher than that recorded by public hospitals.

The sensitivity analysis indicated that total direct costs and total indirect costs of $\mathrm{BPH}$ were robust with respect to variations in key parameters such as the type of diagnostic test routinely ordered, numbers of outpatient and specialist consultations, and changes in labour force participation and unemployment rates. The best estimate of 1991 costs (measured in 1992 prices) was considered to be the high estimate which may still be regarded as conservative, as many costs were not quantified in monetary terms, and only those costs relating to treated $\mathrm{BPH}$ were measured.
The direct medical costs of BPH accounted for $79 \%$ of the total quantified costs. Hospital ward and operation costs accounted for $92 \%$ of the direct medical costs. However, hospital ward costs were underestimated in the study as BPH diagnoses B, $\mathrm{C}$, and $\mathrm{D}$ were excluded because it was impossible to isolate ward costs attributed solely to $\mathrm{BPH}$ when the patient was admitted primarily for another condition. As most patients with BPH in New Zealand are over 65 years of age, and retired, production loss was a relatively small component of the total costs. Part of the explanation for the relatively low indirect cost is the short time off work and our assumption that there was no loss of production before surgery was performed. The length of stay in hospital, and the cost of surgical operations (56\% and $35 \%$, respectively, of the direct medical costs) are the 2 areas for which a reduction in costs would have the maximum impact on total costs.

A substantial reduction in costs could be achieved if the length of stay in hospitals could be 
reduced. Public hospital patients stay on average 8.9 days compared with 4.6 days for private hospital patients. If the public hospital average ward stay could be reduced to that of private hospitals then total ward costs would fall by $37 \%$ ( $\$$ NZ3.249 million), total direct costs of BPH would fall by $21 \%$ and total quantified costs for BPH by $16 \%$.

Reasons were sought for the disparity in the length of stay between public and private hospital patients. The case mix of public and private hospitals is likely to differ in that older, at-risk and emergency patients are more likely to be admitted to public hospitals which are more able to cope with complications, and which have a more intensive level of care and monitoring.

We examined factors that could possibly affect length of stay and that could be examined using HSS data. Age and discharge type (covering the classification as dead, routine discharge, self-discharge and transfers to other types of institutional care) were analysed. The average length of stay for all age cohorts in private hospitals was about half that seen in public hospitals. There was little difference in discharge type: $97 \%$ of patients in public hospitals and $99 \%$ of patients in private hospitals had discharges recorded as routine. If substantially more patients in the public system had returned to nursing homes or other hospitals, this could explain a longer hospital stay, as presumably such patients would require a more intensive level of care. As the private hospital database covers only the principal diagnosis and principal operation, it was impossible to determine, by reference to multiple diagnoses and operations, whether there was a difference in health status between the public and private hospital patients. The identified differences in case mix do not fully explain why patients treated in public hospitals stay twice as long as patients treated in private hospitals.

Changes in case management have the potential to reduce hospital costs, particularly if they result in fewer inpatient days. For example, in the public system, patients with acute BPH who require catheterisation and surgery were at one time catheterised and admitted to await surgery, but may now be catheterised and returned to the community until a surgical bed becomes available. However, the reduced inpatient stay should be offset against possible increased costs in the community to the patient and caregivers.

Surgery was the treatment option for most patients with symptomatic BPH. Watchful waiting and/or pharmacotherapy was relatively uncommon at the time of the study, but should either of these 2 options assume greater importance, there will be follow-on costs in areas of laboratory and diagnostic investigations, specialist consultations and pharmaceuticals prescribed. If drug treatment could avoid the need for surgery or lessen the hospital stay, it could substantially reduce costs. If, however, surgery is merely postponed rather than avoided, surgery costs are simply moved forward in time, and the pharmaceutical costs become an additional cost. However, surgery moved forward in time means a resulting saving via deferral of cost. Also, the patient may enjoy an improved quality of life and older patients may die from unrelated causes before surgery is needed, thus saving the cost of surgery for BPH.

Laboratory tests and diagnostic procedures were under-reported in this study, because data at general practitioner level were unavailable. In addition, although the urologist audits recorded the laboratory and diagnostic procedures undertaken, the sample sizes were considered to be too small to allow rates to be applied to all patients, except for the tests carried out on most patients. Total costs for both the tests and the procedures were conservative estimates of the true costs.

Laboratory tests and diagnostic procedures are areas in which changes are occurring, and a measure of the impact of potential changes can be gained by consideration of possible additions to, or deletions of, the tests costed. The prostate-specific antigen test is likely to replace the cheaper prostatic acid phosphatase test, as it is regarded as being more sensitive as a tumour marker for cancer of the prostate (Editorial 1988). Both tests were included in the study, but if the phosphatase test is 
deleted, the measured costs would fall :by \$NZ0.019 million annually.

Changes in tests and procedures with the introduction of new technology could increase costs but may have little impact on the overall cost of medical treatment, as this area forms only a small percentage of the total direct medical costs. It is not unrealistic to assume that each patient may in the future receive an abdominal ultrasound scan which would increase the cost of diagnostic procedures by $157 \%$ (3110 patients $\times \$ N Z 84.44$ per test $=$ $\$ N Z 0.263$ million). Although this would more than double the total cost of diagnostic procedures, it would only increase the direct medical costs for treatment of $\mathrm{BPH}$ by $1.7 \%$. Conversely, confining chest $\mathrm{x}$-rays to only those patients over 65 years of age would lower the cost of diagnostic procedures by $23 \%$ (850 patients $\times \$ N Z 46.22$ per $\times$-ray $=$ $\$ N Z 0.039$ million) and lower total direct medical costs by $0.3 \%$. However, as the current cost of diagnostic procedures is $1 \%$ of direct medical costs, none of these changes will make a meaningful difference to the costs of BPH. Diagnoses may be improved for those patients for whom clinical diagnoses are not definitive, but the impact on total costs was not evaluated.

Complications recorded for surgery came from 3 different sources, each at a different stage of treatment for BPH. Hospital data recorded complications within the duration of the patients' hospital stay, the audits collected data within 30 days of surgery and more than 30 days after surgery, and the study of Gilling and colleagues (1988) interviewed patients at a minimum of 6 months following surgery (sexual dysfunction only was studied).

Hospital staff may regard some problems or complications associated with surgery as normal occurrences, and therefore may not record them, as the audits indicated a higher complication rate than hospital records. Recording $/$ compliance is likely to be higher in an audit than in routine hospital records, and since the audit data covered a postdischarge period, a higher complication rate would also be expected. However, the difference between the hospital data and the audits was sufficiently large to assume that under-reporting is occurring within hospitals. Under-reporting of complications will not have resulted in an underestimate of hospital ward costs, as these were fully captured if the patient was admitted under $\mathrm{BPH}$ diagnosis A.

There may also be an inadequate appreciation of postoperative problems facing $\mathrm{BPH}$ patients in New Zealand if patients do not volunteer information on what they may regard as a sensitive subject. Although most of the complications of the treatment for $\mathrm{BPH}$ are not life threatening, and add little to direct medical costs, they are important in terms of quality of life. Society may not fully appreciate the magnitude of the reduced quality of life resulting from symptomatic BPH and its possible treatment complications for the patient. More research is required to value the cost of changes in quality of life.

It was a commonly held belief that Maori had a lower prevalence of BPH because a lower proportion sought treatment for BPH symptoms than among the rest of the male population. This possible lower prevalence of BPH among Maori may be explained by the fact that mean age of patients in the cohort aged 50 years and over was lower for Maori than for non-Maori patients. Furthermore, as small numbers are involved, this apparent difference in rates should be interpreted with caution. A reluctance to use the health system for social and economic reasons may in part explain the lower number of Maori presenting for treatment. A recent clinical investigation (Nacey et al. 1993) found that no statistical difference in prevalence of BPH could be demonstrated among European, Maori or Polynesian men aged 50 and over.

Papers presented at the recent International Consultation on BPH (1992) produced evidence that suggested that the rate of BPH in the community is under-reported. Many people consider BPH to be part of the normal aging process of men, and tend to accept attendant problems. If these attitudes change and BPH symptoms become less acceptable, more focus will be placed on intangible costs. 
Society may be prepared to accept highẹr direçt medical costs to achieve improved quality of life. As the New Zealand population ages, the prevalence of BPH will increase.

\section{Appendix: Data on Morbidity and Mortality}

\section{New Zealand Department of Health}

Both public and private hospital data and mortality data relating to $\mathrm{BPH}$ for the whole of New Zealand and for all patients were obtained on computer diskettes from HSS, Department of Health. The data supplied related to individual patient records for all New Zealand, with National Master Patient Index numbers removed to protect patient confidentially. At the time of data acquisition (January 1992) the most current data available in this form were for the year ending December 1988. Data for 1989,1990 and 1991 became available after the analysis for this study was completed. Detailed analysis of the latest data in unit record form was not possible because of cost and time constraints. However, published information in tabular form (Department of Health 1992) was used to update some of these data for sensitivity analyses.

Diagnostic data were recorded on up to 4 conditions (A, B, C, D). Primary diagnosis (A) is the diagnosis for which the patient was admitted, and subsequent diagnoses were recorded in order of severity. Similarly, a patient may have had more than one surgical operation (1,2 or 3 ) and these were recorded in chronological order during a patient's hospital stay.

Data collected from private hospitals were not as extensive as those recorded for public hospitals. For example, private hospital data recorded only 1 surgical operation per patient.

\section{Christchurch Hospital, New Zealand}

Cost and volume data relating to Christchurch Hospital were used to validate data from other sources. Data came from the Resource Utilisation System (RUS) Unit, and the Urology Service Delivery Unit publication Business Plan Information Systems, Christchurch Hospital, 1991

\section{IMS (NZ) Limited}

Each calendar quarter the prescribing patterns of 300 general practitioners ( $13 \%$ of general practitioners in New Zealand) are analysed for 7 consecutive days and the results are rated up to the totals for New Zealand.

\section{Acknowledgements}

We would like to thank the staff of Christchurch Hospital, and urologists who provided data for this study. We are very grateful for the assistance of John Nacey who advised on the clinical aspects of the study. This study was supported by a grant from Merck Sharp \& Dohme.

\section{References}

Barry MJ. Epidemiology and natural history of benign prostatic hyperplasia. Urologic Clinics of North America 17 (3): 495-508, 1990

Barry MJ. The epidemiology of BPH. International Consultation on BPH, Paris, 6 March 1992. World Health Organization, Paris, 1992

$\mathrm{BPH}$ data relating to 1988 ('D-base III' format on 3.5 diskette). HSS, Department of Health, Wellington, 1991

Christensen MM, Bruskewitz RC. Clinical manifestations of benign prostatic hyperplasia and indications for therapeutic interventions. Urologic Clinics of North America 17 (3): 509-516, 1990

Cockett ATK. General introduction to BPH and the International Consensus. International Consultation on BPH, Paris, 6 March 1992. World Health Organization, Paris, 1992

Crowder $\mathrm{H}$. Incontinence, the secret problem. New Zealand Nursing Journal, March 1991 (Appendix): 19-21, 1991

Denis L. Treatment of BPH. International Consultation on BPH, Paris, 6 March 1992. World Health Organization, Paris, 1992

Department of Health. HSS: hospital and selected morbidity data 1991. Department of Health, Wellington, 1992

Department of Statistics. Information Network for Official Statistics: Online computerised official statistics for New Zealand, Wellington, 1992

Editorial. Prostatic-specific antigen. Lancet 2: 318-319, 1988

Gilling PJ, Wright WL, Gray JM. Factors associated with sexual dysfunction following transurethral resection of the prostate. New Zealand Medical Journal 101: 484-485, 1988

Griffiths K. Hormonal treatment of BPH. International Consultation on BPH, Paris, 6 March 1992. World Health Organization, Paris, 1992

Health Benefits Letter 3, 29 August, New Zealand Department of Health, 1991a

Health Benefits Letter 4, 17 October, New Zealand Department of Health, 199lb

IMS (NZ) Limited. New Zealand data relating to general practitioner consultations and prescriptions, 1992

International Consultation on BPH, Paris, 6 March 1992. World Health Organisation, Paris, 1992

Koopmanshap MA, Rutten FFH. Indirect costs in economic studies: confronting the confusion. PharmacoEconomics 4: 446-454, 1993

McConnell J. How is BPH diagnosed? How will the guidelines help? International Consultation on BPH, Paris, 6 March 1992. World Health Organization, Paris, 1992

McKendry CG, Muthumala D. Health expenditure trends in New Zealand 1980-1992, pp. 22, 46, Department of Health, Wellington, 1993 
Nacey JN, Morum P, Delahunt B. The incidence of benign prostatic hyperplasia in Caucasian, Maori and Polynesian men: a comparative community based study. Poster presented at the 46th Annual Scientific Meeting, Urological Society of Australasia, Hobart, 28 February-5 March, 1993

New Zealand Official Yearbook, 94th ed., Department of Statistics, Wellington, 1990

New Zealand Private Hospitals Association. Report on the financial survey of private hospitals, $1989 / 90$, p.2, New Zealand Private Hospitals Association, Wellington, 1991

Pricing Schedules. Pharmaceutical dispensary fees, container allowances and markups, New Zealand Department of Health, Wellington, 1 August 1991

Scott WG. Trends in area health and hospital board funding, Occasional Paper No. 1, p.11, Wellington Polytechnic, Wellington, 1990
Southern Cross Medical Care Society. Schedule of fees for surgical and anaesthetic procedures, 17 th revision, Southern Cross Medical Care Society, Auckland, 1991

Stewart RJ, Perry GKS, Bowie RD, O'Dea DJ. An economic evaluation of peritoneal aspiration cytology, Working Paper, Wellington School of Medicine, 1992

Correspondence and reprints: Guy Scott, W. Guy Scott and Associates Limited, 75 Waipapa Road, P.O. Box 14-403, Wellington 3, New Zealand. 


\section{CASE 4: HYSTERECTOMY}

\section{Discussed in chapter 11}

\section{Reference}

Scott, H. M., and W. G. Scott. "Hysterectomy for non-malignant conditions: cost to New Zealand society." New Zealand Medical Journal 108 (1995): 423-26. 


\title{
Hysterectomy for nonmalignant conditions: cost to New Zealand society
}

\author{
Helen M Scott, BA, DipNZLS, ANZLA, Research Consultant; W Guy Scott, MAgrSc, MNZIAS, ANZIM, \\ Independent Economist, and Senior Lecturer and Research Co-ordinator, Wellington Polytechnic, Wellington
}

\begin{abstract}
Aims. The aim of the study was to estimate the annual hospital costs to New Zealand society of hysterectomy for nonmalignant conditions in women between puberty and menopause, and to compare international rates for all hysterectomies.

Methods. The records of patients aged between 15 and 54 years inclusive, who had a hysterectomy for a nonmalignant menstrual condition, were researched. Hospital costs were estimated using specific hospital unit costs and prices.

Results. The 4390 hysterectomies for nonmalignant conditions represent $69 \%$ of the total hysterectomies undertaken in New Zealand in 1991. Estimated costs for all hospitals were $\$ 17$ million or $\$ 3868$ per hysterectomy. The all ages all conditions hysterectomy rate per 100000 women of 365 (368 in 1992) in New Zealand is higher than in some other developed countries.

Conclusions. Further reduction in the rate of hysterectomy, in New Zealand may save hospital costs but these savings should be offset against the costs of any other treatment options chosen. Although it was found that unit costs of public hospitals were higher than those of private hospitals the case mix for the two types of hospital may differ and public hospital cost reductions may not be possible. Economic evaluation of alternative treatment options would be improved if all relevant costs (direct, indirect and intangible) were known. Further research is needed to determine the reasons why New Zealand women elect to have hysterectomies, why most choose a private hospital, and changes (before and after hysterectomy) in productivity, out of pocket expenses, and quality of life for these patients.
\end{abstract}

NZ Med J 1895; 108: $423 \cdot 6$

Published information in New Zealand on hysterectomy does not differentiate between hysterectomy for malignant or nonmalignant conditions. This paper sought to establish the numbers and the direct medical costs of hysterectomy for nonmalignant menstrual conditions and to compare New Zealand's rates of hysterectomy with those of other developed countries within the OECD. Once the costs of hysterectomy are established it then becomes possible to evaluate treatment and hospital management options in economic terms.

Costs were estimated from the perspective of society for those patients (15-54 years inclusive, of menstrual age) who underwent hysterectomies for nonmalignant conditions. The objective of the study was to establish the costs of these hysterectomies from the time of the decision to undergo surgery. Costs already incurred (prior to surgery) or costs that would be incurred (regardless of the treatment option chosen) were thus irrelevant to the study.

\section{Methods}

Data for 1991 were obtained in unit record form from Health Information Services, Department of Health. ${ }^{1}$ Such data were the latest full calendar year available in this format at the time of the study and cover all New Zealand hospitals both public and private. Unit records relating to International Classification of Diseases (ICD) operation codes 683 to 686 for all operations were extracted from the data base (683 sub total abdominal hysterectomy, 684 total abdominal hysterectomy, 685 vaginal hysterectomy, 686 radical abdominal hysterectomy). Subtotal and total refers to the type of procedure and are not numeric aggregates. Private hospital information covers only the principal diagnosis and principal operation. Public hospital data provide up to four diagnoses (A, B, $C, D)$ with primary diagnosis A being the condition for which the patient is admitted.

Data were sorted and a subset made which excluded patients with indications of malignancy, infections, and congenital abnormalities. (Patient records were identified for removal using diagnosis $\mathrm{A}$ of the private hospital file and all four diagnoses categories in the public hospital file.) Patients under the age of 15 years and over the age of 54 years were also excluded from the subset. The data now covered the following ICD codes (International Classification of Diseases. 9th Revision, Clinical Modification [ICD-9CM]) 218 uterine leiomyoma, 219-221 other benign neoplasm of uterus, benign neoplasm of ovary, other female genital organs, 614-616 inflammatory disease of ovary, fallopian tube, pelvic cellular tissue, peritoneum, uterus, cervix vagina, vulva, 617 endometriosis, 620 noninflammatory disease of ovary of fallopian tube broad ligament, 621 diseases of uterus not elsewhere classified, 622-624 noninflammatory disorders of cervix, vagina, vulva, perineum, 625 pain and other symptoms associated with female genital organs, 626 disorders of menstruation, abnormal bleeding from female genital tract.

The public hospital unit cost of $\$ 4736$ was derived from diagnostic related groups (DRG) 359 uterine and adnexa procedures for nonmalignancy age under 70 without comorbidities and/or complications. ${ }^{2}$ Private hospital unit costs from the Southern Cross Medical Care Society, ${ }^{3}$ and Wakefield Hospital, Wellington 1992 were also used as an alternative method for evaluating costs in the private sector. The costs of the surgeon and the anaesthetist were taken from the society, and the ward and theatre costs were provided by Wakefield Hospital, Wellington.

Public hospital costs were estimated by multiplying the public hospital DRG unit cost by the number of patients for each operation. Private hospital costs were calculated using private hospital cost data. The daily ward cost multiplied by the total number of days in hospital was added to the unit cost of the operation (excluding ward costs) multiplied by the number of operations.

Lack of robust data precluded the estimation of some important costs; medical costs after discharge, the value of production and leisure time forgone, voluntary and community care, care provided by family and friends, and intangible costs relating to quality of life. Costs of treatment and production and leisure time lost prior to hysterectomy were not quantified as the intention of the study was to measure only those costs incurred following the decision to operate.

\section{Results}

A comparison was made between hysterectomy rates in 12 OECD countries. Switzerland, Canada and the United States had the highest hysterectomy rates per 100000 females, followed closely by Australia and New Zealand which had substantially higher rates than the lowest group of countries (Ireland, Japan, Norway, Sweden). Time series data indicated that rates in most OECD countries have been falling since the late 1970 's. ${ }^{5}$ The New Zealand rate per 100000 females in 1980 was 431 and the equivalent rate in 1991 was 365 . Table 1 shows the rates of hysterectomies per 100000 women in a range of OECD countries. 
Table 1. - International comparison of hysterectomy rates

\begin{tabular}{lcc}
\hline Country & Year & Rate per 100 000 females \\
Australia(NSW) & 1983 & 376 \\
Canada & 1985 & 483 \\
Denmark & 1982 & 255 \\
England and Wales & 1991 & 292 \\
Ireland & 1989 & 114 \\
Japan & 1980 & 90 \\
Netherlands & 1980 & 381 \\
New Zealand & 1991 & 365 \\
Norway & 1986 & 145 \\
Sweden & 1980 & 145 \\
Switzerland & 1986 & 1661 \\
United States & 1989 & 425 \\
\hline
\end{tabular}

Sources: OECD 1993; ${ }^{-5}$ Dickinson \& Hill 1988 (Australia); OHE $1992^{11}$ (England and Wales).

In 1991 there were in total 6331 hysterectomies (for all diagnoses, all operations and all ages) undertaken in New Zealand $(50.5 \%$ of these operations were performed in public hospitals, and $49.5 \%$, in private hospitals). This total number of hysterectomies is equivalent to a rate of 365 per 100000 females. Both the numbers of all hysterectomies in public and private hospitals and the rate per 100000 females are shown in Table 2.

The study was concerned with hysterectomy within the age cohort 15 to 54 years, for patients with menstrual problems, and where there was no indication for malignancy. Records in the data subset (as described in methods) were selected on operation 1,2 , and 3 for the public sector and operation 1 for the private sector. A total of 4390 hysterectomies was obtained (45.1\% for public hospitals, and $54.9 \%$ for private hospitals). These 4390 hysterectomies represent $69 \%$ of the total hysterectomies performed for all indications. Under the selection criteria (described above) the hysterectomy rate per 100000 females ( 15 to 54 years) was 446 .

Table 3 provides details of the hysterectomy cost calculations for public hospitals for patients 15 to 54 years. $82 \%$ of patients had a total abdominal hysterectomy and as the same unit cost was applied to all hysterectomies in public hospitals (specific cost data was unavailable) this represents $82 \%$ of the estimated hospital cost.

Tables 4 and 5 show how private hospital costs were calculated. Ward, surgeon, anaesthetist and theatre unit costs were used to derive a unit cost for each type of hysterectomy in Table 5 . The weighted average unit cost per hysterectomy in private hospitals was $\$ 3155$. Table 4 shows that $73 \%$ of all hysterectomies undertaken in private hospitals were total abdominal hysterectomies and accounted for $75 \%$ of the total hospital cost.

Table 6 draws together information from Tables 3 and 4 to summarise hospital costs by type of hysterectomy. When specific public and private unit costs were applied to the respective sectors the total hospital costs were $\$ 16.983$ million or $\$ 3868$ per patient.

Table 2. - Hysterectomies 1991

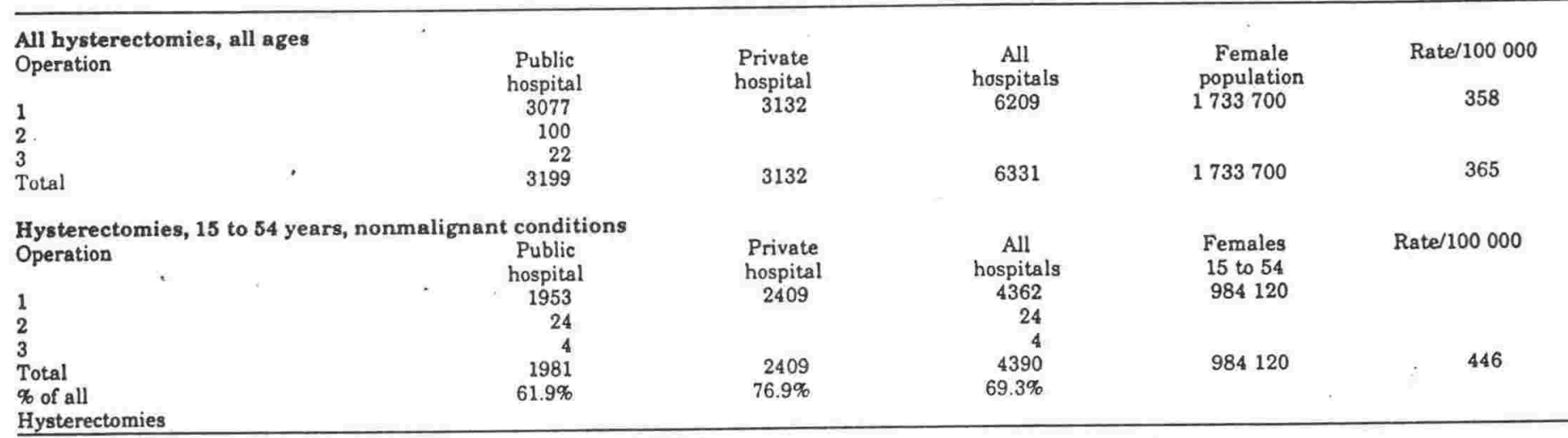

Sources: Unit record data 1991. Health Information Services, Department of Health. Department of Statistics. INFOS. Population statistics.

Table 3. - Public hospitals: cost of hysterectomies for nonmalignant conditions patients aged 15 to 54 years: 1991

\begin{tabular}{|c|c|c|c|c|c|c|}
\hline $\begin{array}{l}\text { ICD } \\
\text { code }\end{array}$ & Operation & $\begin{array}{c}\text { Average } \\
\text { age } \\
\text { years } \\
\#\end{array}$ & $\begin{array}{c}\text { Unit } \\
\text { cost } \\
\$ \\
\mathrm{~A}\end{array}$ & $\begin{array}{c}\text { Number } \\
\text { of } \\
\text { patients } \\
B^{*}\end{array}$ & $\begin{array}{c}\text { Hospital } \\
\text { cost } \\
\$(000) \\
\mathrm{C}=\mathrm{A} \times \mathrm{B}\end{array}$ & $\begin{array}{c}\text { Average } \\
\text { stay } \\
\text { days } \\
\#\end{array}$ \\
\hline 683 & Subtotal abdominal hysterectomy & 44.5 & 4736 & 24 & 114 & 7.4 \\
\hline 684 & Total abdominal hysterectomy & 41.8 & 4736 & 1616 & 7653 & 6.7 \\
\hline 685 & Vaginal hysterectomy & 40.3 & 4736 & 340 & 1610 & 5.5 \\
\hline 686 & Radical abdominal hysterectomy & 34.0 & 4736 & 1 & 5 & 10.0 \\
\hline Total & & 41.5 & & 1981 & 9382 & 6.5 \\
\hline
\end{tabular}

Notes

Because of rounding individual items may not add exactly to the totals shown.

- Operation: operation 1 (the first operation coded).

- All operations (1,2 and 3)

Unit cost: relevant DRG unit cost for the year ending June 1991.

Sources

Unit record data 1991. Health Information Services, Department of Health.

National Advisory Committee on Core Health and Disability Support Services. First report. 1992 (DRG unit costs)

Table 4. - Private hospitals: cost of hysterectomies for nonmalignant conditions patients aged 15 to 54 years: 1991

\begin{tabular}{|c|c|c|c|c|c|c|}
\hline $\begin{array}{l}\text { ICD } \\
\text { code }\end{array}$ & Operation & $\begin{array}{c}\text { Average } \\
\text { age } \\
\text { years }\end{array}$ & $\begin{array}{c}\text { Unit } \\
\text { cost\# } \\
\$ \\
\text { A }\end{array}$ & $\begin{array}{r}\text { Number } \\
\text { of } \\
\text { patients } \\
B\end{array}$ & $\begin{array}{r}\text { Hospital } \\
\text { cost } \\
\$(000) \\
\mathrm{C}=\mathrm{AxB}\end{array}$ & $\begin{array}{r}\text { Average } \\
\text { stay } \\
\text { days } \\
\text { D }\end{array}$ \\
\hline 683 & Subtotal abdominal hysterectomy & 36.0 & 3306.6 & 3 & 10 & 6.0 \\
\hline 684 & Total abdominal hysterectomy & 42.5 & 3268.4 & 1749 & 5716 & 5.5 \\
\hline 685 & Vaginal hysterectomy & 41.6 & 2846.1 & 654 & 1861 & 4.7 \\
\hline 686 & Radical abdominal hysterectomy & 35.3 & 4317.0 & 3 & 13 & 7.3 \\
\hline Total & & 42.3 & & 2409 & 7601 & 5.3 \\
\hline
\end{tabular}


Table 5. - Private hospitals: cost of hysterectomies for nonmalignant conditions patients aged 15 to 54 years: 1991 Unit costs

\begin{tabular}{|c|c|c|c|c|c|c|c|c|c|c|c|}
\hline $\begin{array}{l}\text { ICD } \\
\text { code }\end{array}$ & Operation & $\begin{array}{l}\text { Number } \\
\text { of } \\
\text { patients } \\
\text { A }\end{array}$ & $\begin{array}{c}\text { Average } \\
\text { stay } \\
\text { days } \\
\text { B }\end{array}$ & $\begin{array}{l}\text { Ward cost } \\
\text { per day } \\
\$ \\
\text { C }\end{array}$ & $\begin{array}{c}\text { Total } \\
\text { ward cost } \\
\$(000) \\
D=A x B x C\end{array}$ & $\begin{array}{c}\text { Surgeon } \\
\text { unit cost } \\
\$ \\
\text { E }\end{array}$ & $\begin{array}{c}\text { Anaesthetic } \\
\text { unit cost } \\
\$ \\
\text { F }\end{array}$ & $\begin{array}{c}\text { Theatre } \\
\text { unit cost } \\
\$ \\
\text { G }\end{array}$ & $\begin{array}{c}\text { Surgery } \\
\text { total cost } \\
\$(000) \\
H= \\
(E+F+G) \times A\end{array}$ & $\begin{array}{c}\text { Total } \\
\text { hospital } \\
\text { cost } \\
\$(000) \\
I=H+D\end{array}$ & $\begin{array}{c}\text { Unit } \\
\text { cost } \\
\$ \\
J=L A\end{array}$ \\
\hline 683 & Subtotal abdominal hysterectomy & 3 & 6.00 & 284.44 & 5 & 888.89 & 311.11 & 400.00 & 5 & 10 & 3307 \\
\hline 684 & Total abdominal hysterectomy & 1749 & 5.49 & 284.44 & 2731 & 977.78 & 328.89 & 400.00 & 2985 & 5716 & 3268 \\
\hline 685 & Vaginal hysterectomy & 654 & 4.66 & 284.44 & 867 & 800.00 & 320.00 & 400.00 & 994 & 1861 & 2846 \\
\hline 686 & Radical abdominal hysterectomy & 3 & 7.33 & 284.44 & 6 & 1422.22 & 408.89 & 400.00 & 7 & 13 & 4317 \\
\hline Total & & 2409 & 5.27 & & 3610 & & & & 3991 & 7601 & 3155 \\
\hline
\end{tabular}

Notes for tables 4 and 5

Because of rounding individual items may not add exactly to the totals shown. Operation: operation 1 (the first operation coded).

Unit cost from Table 5 .

Table 6. - All hospitals: cost of hysterectomies nonmalignant conditions patients aged 15 to 54 years

\begin{tabular}{|c|c|c|c|}
\hline $\begin{array}{l}\text { ICD } \\
\text { code }\end{array}$ & Operation & $\begin{array}{l}\text { Number } \\
\text { of } \\
\text { patients }\end{array}$ & $\begin{array}{c}\text { Hospital } \\
\text { cost } \\
\$(000)\end{array}$ \\
\hline 683 & Subtotal abdominal hysterectomy & 27 & 124 \\
\hline 684 & Total abdominal hysterectomy & 3365 & 13370 \\
\hline 685 & Vaginal hysterectomy & 994 & 3472 \\
\hline 686 & Radical abdominal hysterectomy & 4 & 18 \\
\hline Total & & 4390 & 16983 \\
\hline \multicolumn{2}{|c|}{ Total cost per hysterectomy } & . & 3868 \\
\hline
\end{tabular}

Notes and sources: See Tables 3 and 5

Figure 1 shows the age breakdown of the numbers of hysterectomy patients in public and private hospitals. It was found that one third of all such hysterectomies were for patients aged between 35 and 44 years, and two-thirds were for patients aged 35 to 49 years.

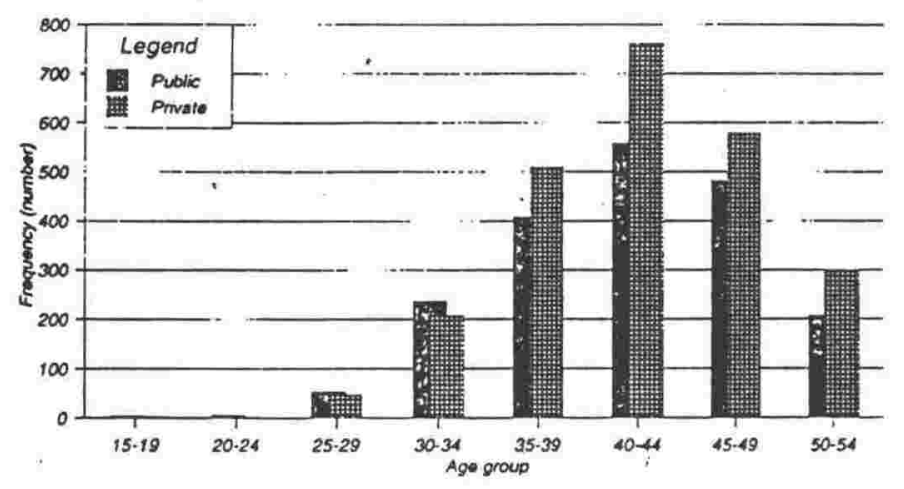

Figure 1. - Number of hysterectomies for nonmalignant conditions in New Zealand by age

\section{Discussion}

The study selected patients between puberty and menopause. Women of 55 years and older were not included as it was assumed that most would be post-menopausal at that age. Although most women begin menstruating before 15 years of age it may be several years, and as a last resort, before a patient with menstrual problems undergoes a hysterectamy.

The 4390 hysterectomies for nonmalignant conditions in New Zealand represented $69 \%$ of the total hysterectomies performed $(62 \%$ of the public and $77 \%$ of the private hospital hysterectomies). In private hospitals some hysterectomies for malignant conditions may not have been recorded under a specific diagnoses but in the more general ICD 621 disorders of uterus, not elsewhere classified, and therefore may not have been filtered from the data. Most public hospital hysterectomies for nonmalignant conditions were classified under ICD $218(31 \%)$ and $626(45 \%)$, while most such hysterectomies in private hospitals were classified ICD $621(66 \%)$ and $626(25 \%)$.

There was little difference in age between public and private hospital patients; public hospital patients were on average 41.5 years and private hospital patients were 42.3 years. Public hospital patients stayed on average 6.5 days, a day longer in hospital than did patients in private
Sources

Unit record data 1991. Health Information Services,

Department of Health.

Southern Cross Medical Care Society, 1991.

Wakefield Hospital, 1992.

hospitals whose average stay was 5.3 days. It is a general belief that public hospitals in New Zealand treat more patients, and that their case mix is biased towards those likely to require a greater degree of care and whose stay is therefore likely to be longer. Thus, the unit cost of public hospitals is likely to be greater than that of private hospitals. The study found that the public hospital unit cost was higher ( $\$ 4736$ public unit cost, $\$ 3155$ private unit cost), there were fewer patients, and these patients stayed on average a day longer. Actual fees for the surgeon and anaesthetist are often higher than those derived from the Southern Cross schedule ${ }^{3}$ which would increase our estimates of private hospital unit costs.

If the average total weekly earnings combined as at June $1991^{6}$ were used as a proxy for the value of production and leisure-time loss and if, for example, a patient was incapacitated to the extent of three times their hospital stay there would be combined loss of production and leisure time of $\$ 6$ million or $\$ 1400$ per patient. As it is important to measure indirect and intangible costs of illnesses ${ }^{7}$ further study is necessary to quantify such costs.

The total New Zealand rate for hysterectomy for all ages is lower than comparable rates in USA and Canada, similar to rates in Australia and the Netherlands, but higher than rates in England and Wales, and Ireland. The difficulties in interpreting the geographic differences in rates of surgery have been discussed in the international literature. , $8-10^{-10}$ Some possible explanations for the discrepancies included an increase in women's knowledge of themselves and diseases, a higher rate of uterine/pelvic disease, a sterilisation procedure that can be legitimised as medically necessary, the availability of surgeons and hospital facilities, differences in payment and insurance cover for health care, supplie ${ }^{r}$ induced demand, patient preferences, referral procedures, differences in income levels and social patterns. The recent decline in the number of hysterectomy procedures in some developed countries may possibly be explained by controversy over unnecessary operations and a desire by both clinicians and patients to avoid hysterectomy where possible.

Although half of all hysterectomies (for all indications and all ages) in New Zealand were undertaken in private hospitals, for the age cohort studied and the conditions analysed, such hospitals recorded a greater number of hysterectomies. A possible explanation for this may be that many women in this working age group (15-54 years) may have health insurance and prefer to plan surgery to suit business and family commitments. Surgery in a public hospital is usually preceded by a waiting list/booking system and an inability to make arrangements other than at short notice. If New Zealand women are opting for private surgery for the above reasons then such surgery is likely to be price and income sensitive. For example, if women in the working age group have less disposable income or lower rates of insurance cover there may be a drop in the number of hysterectomies in private hospitals, and a corresponding increase in the number of 
hysterectomies in public hospitals. The decision made to have a hysterectomy in a private hospital at a time convenient to the patient may be the best option at present for these patients if the cost of treatment, lost production and reduced quality of life before hysterectomy is taken into account.

\section{Conclusions}

Further reductions in the rate of hysterectomy in New Zealand may save hospital costs but these savings should be offset against the costs of any other treatment options chosen. For example, watchful waiting could incur continuing costs relating to general practitioner and or specialist consultations, analgesics, protective tampons and pads, loss of output and reduced quality of life.

At the time of this study the unit costs of public hospitals were higher than those of private hospitals. If the public hospital unit costs fell to that of the private hospitals a reduction of $18 \%$ or $\$ 3$ million in total hospital costs would occur. However, as discussed earlier the case mix for the two types of hospital is likely to be different and cost reductions may not be possible.

It is hoped that the costs and the additional information on the number of hysterectomies for nonmalignant conditions derived in this paper will assist in the economic evaluation of alternative treatment options. Data for decision making would be enhanced if research was undertaken to determine the reasons why New Zealand women elect to have hysterectomies, and why most choose a private hospital. Information should also be collected before and after hysterectomy to evaluate changes in productivity, out of pocket expenses (such as analgesics and protective clothing), and quality of life for these patients.

Acknowledgements. This study was funded in part by a research grant from ICI (NZ).

Correspondence. Helen Scoth, PO Box 14-403 Kilbirnie, Wellington.

1. Department of Health, Health Information Services. Unit record data 1991 Wellington: Department of Health, 1993.

2. National Advisory Committee on Core Health and Disability Support Services. First report. Wellington: the Committee, 1992. Appendix 6.

3. Southern Cross Medical Care Society. Schedule of surgical and anaestheti procedures. 17th revision. Auckland the Society, 1991.

Dickinson JA, Hill AM. The incidence of hysterectomy and its effect on the probability of developing uterine cancer. Community Health Studies 1988; 12:176-81.

OECD Health data. version "1.5. Paris: OECD, 1993.

- Sution New Zealand Infor computerised official statistica for New Zealand. Wellington: Statistic New Zealand,

7. Scott WG, McKendry CG, Scott HM. Methodologies for economic evaluation of high technology in health care. Wellington: Department of Health, 1992.

8. McPherson K. International differences in medical care practices. In: Health Care Financing Review 1989 Annual Supplement; 9-20.

9. Carlson KJ, Nichols DH, Schiff I. Indications for hysterectomy. N Engl J Med 1993; 328: $856-60$

10. Dennerstein L, Wood C, Westmore A. Hysterectomy: advances and new options. 2nd ed. Melbourne: Oxford University Press, 1995.

11. Office of Health Economics (OHE). Compendium of health statistics, 8th ed. London: OHE, 1992.

\title{
IN PRACTICE
}

\section{Hip fracture incidence among older people in Auckland: a population-based study}

\author{
Robyn Norton, PhD, MPH, Director; Meg Butler, ASCT, DPH, Research Officer; Rochelle Currie, DipOT, \\ Research Officer; Trevor Lee-Joe, MEng, Data Manager, Injury Prevention Research Centre, University of \\ Auckland; A John Campbell, MD, FRACP, Professor of Geriatric Medicine, Department of Medicine, University \\ of Otago, Dunedin; lan R Reid, MD, FRACP, Associate Professor, Department of Medicine, University of \\ Auckland; Auckland; Harley Gray, ChM, MMedSci, FRACS, Clinical Director Surgical Services, Middlemore \\ Hospital and Honorary Professor, University of Auckland, Auckland.
}

\section{Abstract}

Aim. To determine the incidence of hip fractures among those aged 60 years or older in the Auckland region.

Methods. Demographic data were obtained on all older people hospitalised with a fracture of the femoral neck in Auckland, between 8 July 1991 and 7 February 1994. Incidence rates were calculated using the 1991 census data. Results. An average incidence of 733 hip fractures or 710 individuals sustaining hip fractures each year was observed. Age-adjusted incidence rates were 571.5 per 100 000 for women and 318.2 per 100000 for men. $97.0 \%$ of the hip fractures were sustained by those identified in the records as European, with the rates for Maori and Pacific Island populations $25-50 \%$ lower than for Europeans. Among Maori and Pacific Island populations, incidence rates were similar for men and women. Overall, $41.8 \%$ of the hip fractures were sustained by those $\geq 85$ years, with $67.0 \%$ sustained by those $\geq 80$ years. Incidence rates increased with age, with the rates continuing to increase in those $\geq 85$ years.

Conclusion. Among Europeans, hip fracture incidence rates in Auckland are similar to those found for other caucasian populations. However, the rates among Maori and Pacific Island populations are among the lowest internationally and are similar for men and women. The increase in incidence rates with age continues in those $\geq 85$ years.
Hip fractures are well recognised as an important cause of morbidity and disability among older people. ${ }^{1,2}$ The New Zealand Health Information Service (NZHIS) routinely collects and publishes data on hospitalisations for hip fracture (ICD code 820 ) in New Zealand, providing information on all discharges for hip fractures among those aged 60 years or over, by five year age groups. ${ }^{3}$ However, these data include hip fractures that are not attributable to either falls or osteoporosis and include data on readmissions for the same hip fracture and transfers between hospitals. Thus the true incidence of hip fracture that may be amenable to preventive interventions is unknown. In addition, in the published data, information on the numbers of hip fractures sustained by those aged 85 years or older are combined, despite the fact that hip fractures sustained by individuals in this age group are shown to comprise over one third of the hip fractures among older people. ${ }^{3}$ Given the preventive, acute and long term management, and cost implications of hip fracture, particularly among the older old, "there is a need to identify the true incidence of hip fracture. This paper, therefore, reports the descriptive epidemiology of hip fracture incidence among the elderly in the Auckland region, using data from a population-based regional surveillance system.

\section{Methods}

Between 8 July 1991 and 7 February 1994, all older people ( $\geq 60$ years) hospitalised with a fracture of the femoral neck in Auckland were identified through the ward registers of Auckland and Middlemore hospitals. Almost all individuals who are hospitalised 


\section{CASE 5: LOWER RESPIRATORY TRACT INFECTION}

Discussed in chapter 12

\section{Reference}

Scott, W. G., M. W. Tilyard, S. M. Dovey, B. Cooper, and H. M. Scott.

"Roxithromycin versus Cefaclor in lower respiratory tract infection. A General Practice pharmacoeconomic study." PharmacoEconomics 4(2) (1993): 122-30. 


\title{
ORIGINAL RESEARCH ARTICLE
}

PharmacoEconomics 4 (2): 122-130, 1993

$1170-7690 / 93 / 0008-0122 / \$ 04.50 / 0$

(c) Adis International Limited. All rights reserved.

PEC1 236

\section{Roxithromycin versus Cefaclor in Lower Respiratory Tract Infection \\ A General Practice Pharmacoeconomic Study}

\author{
W. Guy Scott, ${ }^{1}$ Murray W. Tilyard, ${ }^{2}$ Susan M. Dovey, ${ }^{2}$ Bruce Cooper ${ }^{3}$ and \\ Helen M. Scott ${ }^{1}$ \\ 1 W Guy Scott and Associates Limited, and Wellington Polytechnic, Wellington, New Zealand \\ 2 Royal New Zealand College of General Practitioners Research Unit, Department of General Practice, \\ Otago Medical School, Dunedin, New Zealand \\ 3 Delpharm Limited, Auckland, New Zealand
}

\begin{abstract}
Summary An economic evaluation comparing roxithromycin $150 \mathrm{mg}$ twice daily and cefaclor $250 \mathrm{mg}$ thrice daily in the treatment of lower respiratory tract infections (LRTI) was undertaken as part of a randomised clinical trial in New Zealand general practice. The observed statistically significant difference in adverse events, withdrawal rates and extra treatment courses in favour of roxithromycin in the clinical study was translated into medical cost savings. Treatment failures, withdrawals or adverse events resulted in additional costs for 11 of $120(9 \%)$ patients receiving roxithromycin and 19 of 118 (16\%) patients receiving cefaclor. In these cases (treatment failures, withdrawals, adverse effects) additional antibiotics and general practitioner visits were required 3 times more often and the cost of additional medication for treating failure or adverse effects was 3 times higher for patients treated with cefaclor than for patients receiving roxithromycin. The total direct medical cost per patient treated with roxithromycin was \$NZ9.37 lower (on an incremental basis) than for patients treated with cefaclor, despite a higher drug acquisition cost. An estimate of $\$ N Z 656000$ per year in total savings in direct medical costs could be made in New Zealand if roxithromycin were to replace all cefaclor prescriptions in the treatment of LRTI.
\end{abstract}

Recent studies in Australia (Munro 1989; Teng Liaw 1991) and earlier studies in New Zealand (Reid \& Anyon 1986; Ridley-Smith 1973) confirm that lower respiratory tract infections (LRTI) remain one of the most common reasons for visits to the general practitioner (GP). They account for 1.5 to $2.6 \%$ of consultations in general practice, and antibiotics are prescribed in a large proportion of cases (Tilyard \& Dovey 1992). Because the majority of LRTIs are treated in general practice rather than in a hospital setting, it is important that new therapies are tested in circumstances under which they are intended to be used.
Traditionally, new medications are evaluated and compared with existing therapies using clinical trials which provide information on clinical and bacteriological efficacy, safety and tolerability. Clinical trials seldom include sufficient data for cost-effectiveness analysis. Because of the need to examine economic factors in the provision of health care, economic items were included in the trial evaluation of 2 pharmaceutical treatments. These treatments were compared for safety and efficacy in a randomised double-blind clinical trial.

Roxithromycin is a new oral macrolide antibiotic with an improved pharmacokinetic profile 
compared with earlier generation macrolides, a broad spectrum of antibiotic activity and a long half-life which enables once or twice daily administration (Acar 1992). Clinical studies to date suggest that roxithromycin is at least as effective but significantly better tolerated than commonly used antibiotics such as amoxicillin/clavulanic acid (Dautzenberg et al. 1992), erythromycin (Herron 1987) and doxycycline (Marsac et al. 1988).

Worldwide, the oral cephalosporin, cefaclor, is one of the antibiotics most commonly used for treating LRTIs. It represents a standard against which other treatments should be evaluated. Cefaclor has been proven as a safe and effective therapy (Kammer \& Short 1979) but as yet has not been compared with roxithromycin in a controlled clinical trial. It was therefore neither necessary nor practicable to evaluate all possible treatment options. Medical ethics precluded the evaluation of the no-treatment option.

\section{Methods}

A multicentre, randomised, double-dummy controlled study, comparing the efficacy and tolerability of roxithromycin $150 \mathrm{mg}$ twice daily with cefaclor $250 \mathrm{mg}$ thrice daily for the treatment of LRTI, was undertaken by the Royal New Zealand College of General Practitioners Research Unit in general practice clinics at 8 centres throughout New Zealand. GPs received no payment for taking part in the study.

It was estimated that a sample size of 230 patients was required to reliably discern a difference of $15 \%$ between antibiotics, with $80 \%$ power $(\beta)$ and $5 \%$ risk $(\alpha)$ assuming $90 \%$ efficacy for roxithromycin. After ethical approval for the trial had been obtained from the Otago Area Health Board Ethics Committee, 240 patients with a diagnosis of LRTI were enrolled between June 1990 and June 1991. Data for economic evaluation were obtained for 238 patients (roxithromycin 120 patients, cefaclor 118 patients) with 2 patients being lost to follow-up.

Eligible patients were aged 18 years or older and all gave written informed consent. A clinical diagnosis of bacterial lower respiratory tract infection was required for entry into the study.

Exclusion criteria were: concurrent or immediately prior antibiotic therapy; pregnancy, lactation or inadequate contraception; hypersensitivity to $\beta$ lactams or macrolides; serious liver or renal disease; concurrent use of warfarin. Patients with serious disease, who were likely to require parenterally administered antibiotics or hospitalisation, were also excluded.

Differential diagnoses of acute bronchitis, acute or chronic bronchitis, and typical and atypical pneumonia were assisted by serological, microbiological and radiological examinations in the majority of cases. Diagnosis was based on community practice standards.

The study was conducted and analysed on the basis of intention-to-treat. Absence of a suitable specimen or examination result did not exclude a case from the analysis. Haematology and biochemistry investigations were made on entry and at completion of the study or withdrawal from therapy. Additional tests, outside those required by the study protocol, were not paid by the study.

Treatment was for a minimum of 7 days, with provision for a further 7 days therapy if clinically indicated. This length of therapy was set to ensure that an adequate minimum course of treatment was available for patients with pneumonia and exacerbations of chronic bronchitis (Mandell et al. 1990). Assessment of clinical signs and symptoms was made by the general practitioner at baseline and at withdrawal or completion of therapy. The 'end' of treatment was defined as the time of completion of all study medication, usually at day 14 or earlier if dictated by intolerable adverse events or lack of efficacy of the study compound. 'Treatment failure' was defined as the requirement for further antibiotics, whether at 7 days or treatment end. The trial methods and results of efficacy and tolerability analyses are described in detail elsewhere (Tilyard \& Dovey 1992).

In addition, the number of patients in New Zealand with LRTI who required antibiotic prescriptions and consulted general practitioners was es- 
timated from a study of general practice activities. This data collection was made during February, March and April of 1991 and involved 102 randomly selected general practitioners from throughout New Zealand. Supporting estimates of LRTI incidence and treatment were made from analysis of the total 1991 consultation and prescribing data of 6 general practices (Tilyard et al., unpublished data). From these data it was estimated that approximately 70000 patients would have been treated with cefaclor during the year ending December 1991.

The economic analysis of this study assessed drug acquisition cost, additional support medicine costs, additional general practitioner consultations and additional diagnostic tests required, over and above those required as part of the study protocol.

The principle of incremental analysis was used; only those costs and benefits that changed or differed between the treatment options were relevant to the analysis (Scott et al. 1992). Healthcare costs and benefits that were common to both treatments were not included as they cancelled out when comparisons were made. Direct medical costs and benefits were evaluated from the perspective of New Zealand society, although indirect costs such as those incurred through lost wages or productivity were not evaluated. Not all of the direct medical costs and benefits were captured, because of the artificial constraints of a clinical trial design. As patient copayments in New Zealand (the nonreimbursable portion of government prescription charges payable by the patient) have a different impact on different income and benefit groups, and this information was not available from the trial data, it was impossible to split all the costs and benefits accurately between government and individual patients. Indirect taxes (Goods and Services Tax and copayments) were not included, as these are transfer payments from the perspective of New Zealand society.

Where treatment failure, treatment withdrawal or adverse events occurred, general practitioners used a standardised form to provide data on any additional treatments, investigations and consultations. Although attempts were made to evaluate indirect costs of production loss and the costs of additional patient travel from the trial data collected and from practitioners' records, the information obtained was inadequate (with respect to both quantity and quality) for analysis. The socially sensitive nature of the data relating to employment status and earnings precluded a vigorous questioning by general practitioners to elicit information.

Medicine prices, dispensing fees and container allowance per prescription, and patient premiums on medicine (the portion of the total price of the medicine paid by the patient) for cefaclor and additional therapies were derived from the Department of Health Pricing Schedules, 1 August 1991. Premiums vary between medicine brands, and are included in the cost to society. Roxithromycin pricing negotiations were not finalised at the completion of this study, thus the direct medicine cost was that agreed to by the Department of Health in March 1992. Fees payable in respect of laboratory diagnostic services were obtained from Health Benefits Letter 29, number 3 (1991). The price of a chest $\mathrm{x}$-ray was obtained from Wakefield Specialist Medical Centre, Wellington, New Zealand (November 1991). The charge for a visit to a general practitioner was assumed to be $\$ N Z 27.56$ (Health Benefits Letter 17, 1991). Because of unavailability of cost data, prices were used as a proxy for unit costs, and as such would contain an unquantifiable profit component.

All prices used related to the last quarter of 1991 . An average of mid-point exchange rates for October, November, and December 1991 (INFOS) is appropriate to convert New Zealand values into US dollars $(\$ N Z 1.0000=\$ U S 0.5541)$.

$\chi$-Square and Fisher's Exact (2-tailed) tests were used for between-treatment comparisons. Friedman's test was used for nonparametric data.

\section{Results}

Table I summarises the major findings of the clinical trial. The 2 treatment groups were comparable at baseline for age (mean age 51 years, range 18-90 years for roxithromycin; 51.6 years, range 20- 
Table I. Summary of the clinical findings of the comparison between roxithromycin and cefaclor for treatment of lower respiratory tract infection

\begin{tabular}{|c|c|c|}
\hline Parameter & Roxithromycin & Cefaclor \\
\hline Patients enrolled & 120 & 118 \\
\hline $\begin{array}{l}\text { Treatment failures at } 7 \\
\text { days }^{\text {a }}\end{array}$ & 38 & 53 \\
\hline $\begin{array}{l}\text { Treatment successes at } \\
7 \text { days }\end{array}$ & $82^{*}$ & 65 \\
\hline $\begin{array}{l}\text { Patients requiring } \\
\text { extended therapy }\end{array}$ & $33^{*}$ & 50 \\
\hline $\begin{array}{l}\text { Mean duration of } \\
\text { treatment (days) }\end{array}$ & 8.9 & 9.7 \\
\hline $\begin{array}{l}\text { Patients withdrawing } \\
\text { during treatment }\end{array}$ & $6 *$ & 15 \\
\hline $\begin{array}{l}\text { Patients with treatment- } \\
\text { related adverse events }\end{array}$ & $13^{\circ}$ & 24 \\
\hline $\begin{array}{l}\text { Patients with treatment } \\
\text { failure, adverse events } \\
\text { or withdrawal }\end{array}$ & 29 & 32 \\
\hline $\begin{array}{l}\text { Patients for whom } \\
\text { quantifiable additional } \\
\text { costs were incurred }\end{array}$ & 11 & 19 \\
\hline \multicolumn{3}{|c|}{ 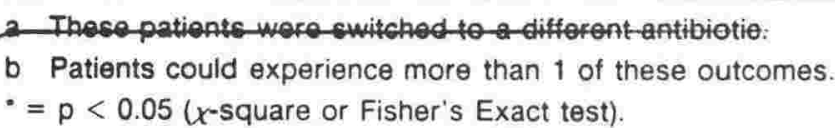 } \\
\hline
\end{tabular}

90 years for cefaclor), gender and underlying disease. Analysis of clinical efficacy (satisfactory or improved response) on the basis of intention-totreat revealed no significant difference between treatments in clinical efficacy at study end $(\mathrm{p}>$ 0.05 ), with 95.8 and $93.2 \%$ efficacy for roxithromycin $(115 / 120)$ and cefaclor (110/118) respectively. An extended course of therapy was required for $50 \%$ more patients randomised to cefaclor than roxithromycin (50 vs $33, \mathrm{p}<0.05)$.

Roxithromycin was significantly more effective than cefaclor, with 82 treatment successes compared with 65 treatment successes for cefaclor after 7 days therapy $(p<0.05)$. The average treatment duration was 9.7 days for cefaclor and 8.9 days for roxithromycin ( $p>0.05$ ). Significantly more patients receiving cefaclor withdrew because of treatment failure or treatment-related adverse events $(15$ vs $6, \mathrm{p}<0.05)$. Adverse events deemed possibly or probably related to treatment were sig- nificantly more common among cefaclor-treated patients ( 24 patients vs 13 patients, $\mathrm{p}<0.05$ ). Four of the 8 treatment failures observed in the first week of treatment with cefaclor were due to early withdrawal necessitated by intolerable adverse events rather than inadequate efficacy.

The difference in costs (apart from the drug acquisition cost) between treatment options was manifest in terms of treatment failure. treatment withdrawal or adverse events. During the study 61 patients (29 receiving roxithromycin and 32 receiving cefaclor) reported adverse events, treatment failure or therapy withdrawal, which potentially could have resulted in additional cost. Of these 61 patients, quantifiable additional costs were actually generated by 30 patients; 11 patients receiving roxithromycin and 19 receiving cefaclor.

\section{Drug Acquisition Cost}

The trial medication cost to New Zealand for roxithromycin was $\$ N Z 2.38$ per patient per day for 8.9 days ( $\$ N Z 21.18$ total) and $\$ N Z 2.12$ per patient per day for 9.7 days for cefaclor ( $\$ N Z 20.57$ total). The incremental primary medical cost for trial medication versus control was $\$ N Z 0.61$ per patient.

\section{Support Medicine Cost}

Four patients receiving roxithromycin required further antibiotic therapy. Five patients were prescribed additional medication to treat adverse events (antifungal, antidiarrhoeal therapies). Two patients required additional medication (antitussive, bronchodilator or decongestant) to treat the infection.

14 patients treated with cefaclor required additional antibiotic therapy. Seven patients required additional medication to treat adverse events (antifungal or antiherpetic therapies or topical steroids). Six patients required additional medication to treat the symptoms, in the form of analgesics or nonsteroidal anti-inflammatory agents (3 patients), oral steroid ( 1 patient) and bronchodilator, antitussive or decongestant (2 patients).

Table II summarises the costs of additional sup- 



\section{Overall Cost Effectiveness}

Table III summarises the overall cost effectiveness of roxithromycin over cefaclor. The net benefit (per patient treated) of using roxithromycin in place of cefaclor was $\$ N Z 9.37$. Although roxithromycin cost more, the roxithromycin-treated patients required fewer prescriptions for support drugs, fewer general practitioner consultations and fewer diagnostic tests than cefaclor-treated patients.

\section{Extrapolation of Results to New Zealand General Practice ${ }^{1}$}

Using an estimate of 70000 patients treated per year with cefaclor for LRTI, the total net benefit to New Zealand of roxithromycin replacing cefaclor for this indication would be $\$ N Z 656000$.

We estimate that the net benefit to New Zealand for 100000 patients would be $\$ N Z 937000$ million, and for 50000 patients would be $\$ N Z 469000$. The reason for applying a smaller estimate is that roxithromycin would not replace all treatments with cefaclor, and the reason for using a higher estimate is that roxithromycin may replace other antibiotic treatment regimens as well as cefaclor. Thus, since roxithromycin has equivalent efficacy but better tolerability than other antibiotics, a similar net benefit could be expected.

\section{Discussion}

In contrast with many trials, the study on which this economic analysis was based was specifically designed to emulate general practice diagnostic and assessment methods. The multicentre, countrywide nature of the study controlled for regional, racial, and climatic differences and the study period of one year allowed for seasonal aetiological

1 The population of New Zealand was estimated to be 3.4 million at the end of 1991 . The predominantly publicly owned and funded system (government currently funds just over $80 \%$ of all health expenditure) has constrained the prices of pharmaceuticals and medical services. Spending on health for the year ended June 1991 was $7.3 \%$ of gross domestic product. Total expenditure on health (both private and public) for the fiscal year ended June 1991 was \$NZ5387 million (McKendry \& Muthumala 1993).
Table III. Summary of incremental medical costs of roxithromycin over cefaclor

\begin{tabular}{lll}
\hline $\begin{array}{l}\text { Incremental direct medical } \\
\text { costs of roxithromycin over } \\
\text { cefaclor }\end{array}$ & $\begin{array}{l}\text { Per patient in } \\
\text { the trial } \\
(1991 \text { \$NZ) }\end{array}$ & $\begin{array}{l}\text { Per } 70000^{\mathrm{a}} \\
\text { patients } \\
(1991 \\
\$ N Z \cdot 1000)\end{array}$ \\
\hline $\begin{array}{l}\text { Additional primary drug } \\
\text { acquisition cost }\end{array}$ & 0.61 & 43 \\
$\begin{array}{l}\text { Support drug costs avoided } \\
\text { Dispensing fees and } \\
\text { container allowances } \\
\text { avoided }\end{array}$ & 1.98 & 1.39 \\
$\begin{array}{l}\text { General practitioner fees } \\
\text { avoided }\end{array}$ & 0.44 & 31 \\
$\begin{array}{l}\text { Diagnostic tests avoided } \\
\text { Net cost avoidance }\end{array}$ & 2.14 & 379 \\
\hline
\end{tabular}

a Estimated number of prescriptions for cefaclor filled each year in New Zealand.

b A small reimbursement paid by the NZ government to pharmacists, for the drug container.

differences. In addition the proportions in the study group of patients with acute bronchitis $(80 \%)$, exacerbations of chronic bronchitis (15\%) and pneumonia (5\%) are consistent with available local epidemiological data on community-acquired LRTI (Tilyard \& Dovey 1992). The results can thus be reasonably extrapolated to actual general practice usage, at least in New Zealand. In generalising these results to other countries it should be noted that $13 \%$ of patients in this study were asthmatic and that New Zealand has a high prevalence of asthma.

For this economic analysis, total direct medical cost savings to the country were calculated based on the actual trial usage of the medications by general practitioners. However, treatment durations and the numbers of consultations and diagnostic tests are often higher in a clinical trial than in day to day medical practice.

In this study, a minimum treatment duration of 7 days was set to ensure that patients with acute or chronic bronchitis and pneumonia would receive an appropriate duration of treatment (Conte \& Barriere 1988; Mandell et al. 1990). The variation in treatment length between the artificial minimum of this trial and day to day clinical ex- 
perience highlights a problem that must be faced when undertaking and interpreting an economic evaluation using clinical trial data. However, this bias is minimised by the use of incremental analysis because most of the additional costs are common to both the active treatment and control groups and cancel out when comparisons are made.

A minimum of 2 general practitioner visits was required for evaluation of each case whereas in routine clinical practice a patient would probably return only if treatment failed or the patients experienced major adverse events. The 2 haematological and biochemistry investigations performed to assess comparative safety would not normally contribute to the routine management of LRTI in general practice. Chest $\mathrm{x}$-rays and microbiological tests were undertaken whenever possible. The fact that the patients were part of a study bearing the cost of these investigations may have encouraged greater use of microbial tests and chest $\mathrm{x}$-rays than would normally be the case. All of these costs, however, were incurred equally by patients receiving roxithromycin and cefaclor, and they cancel out in the economic comparison of treatments.

The economic analysis takes into consideration only those additional diagnostic tests, general practitioner visits, and pharmaceutical costs which resulted either from inadequate treatment of the initial infection at study end or from adverse events of trial treatments. The study did not subsidise these costs, so they are therefore representative of costs which might normally result from the treatments offered. No attempt was made to assume an additional cost for patients who required an extra week of medication, as this was allowed for in the protocol design and it is difficult to extrapolate to routine practice. However, because significantly fewer additional one week treatment courses were required as part of the study for roxithromycin than for cefaclor, it is possible that the difference in favour of roxithromycin has been underestimated.

A fairer assessment of the overall comparative costs, nationally, of the 2 treatments could have been achieved by calculating primary medicine costs based on the national average treatment dur- ation for each antibiotic used to treat.LRTI. This calculation was not undertaken because although data on prescribing practice were available from commercial sources, the researchers were unable to obtain permission to publish calculations based on these data.

It would have been preferable to have included direct nonmedical, indirect and intangible costs and benefits in this study but these could not be evaluated with sufficient accuracy. The savings calculated therefore could represent an underestimate to society, as there were fewer adverse effects and treatment failures at 7 days with roxithromycin.

Accepting the inherent limitations of extrapolation from a prescribing database of 6 general practices for one year and 102 practices over 3 months, an estimated 70000 prescriptions for cefaclor are filled each year in New Zealand for treatment of LRTI's. Theoretically, if roxithromycin were to replace cefaclor, savings in excess of $\$ N Z 656000$ could result, in spite of roxithromycin having a greater unit cost.

It could be argued that cases of acute bronchitis (which represent a significant proportion of the study patients) are viral in aetiology and do not require antibiotic therapy (Billas 1990). Indeed, 3\% of the cases included in the intention-to-treat analysis of clinical efficacy in the study were deemed by serology to be viral in origin. On the other hand, others (Dunlay et al. 1987) have demonstrated a benefit from antibiotic therapy in acute bronchitis, and certainly in this study general practitioners selected patients whom they believed clinically to have a bacterial LRTI that would benefit from antibiotic therapy. The study results are thus representative of actual general practice usage patterns, in contrast with restrictive analyses based on infection proven by sputum culture, a tool widely regarded to lack sensitivity and specificity in this setting (Lentino \& Lucks 1987).

It should be noted that this study was undertaken between epidemic cycles of Mycoplasma pneumonia infection and no cases were detected. Given the demonstrated efficacy of roxithromycin in treating Mycoplasma pneumonia and other 'atypical' pneumonias (Saito 1990), and the inef- 
ficacy of $\beta$-lactam antibiotics such as cefaclor against such pathogens (Mandell et al. 1990), it could be reasonably expected that during the observed cyclic epidemics of such pathogens the cost effectiveness advantage of empirical use of roxithromycin over cefaclor would increase even further.

The recommended dose of cefaclor for general practice LRTI varies internationally from $250 \mathrm{mg}$ twice daily to $500 \mathrm{mg}$ thrice daily for mild to moderate infections. However, as with most antibiotics, there has been a trend to reduce dosage to the lowest effective level associated with the most favourable adverse-effect profile. The dose of $250 \mathrm{mg}$ thrice daily is that recommended for mild to moderate infections in the official New Zealand prescribing information for cefaclor. It is possible that a small number of patients may have been more effectively treated with a higher dose of cefaclor, however, efficacy was already $93 \%$ and one-half of the treatment failures observed with cefaclor were due to early withdrawal necessitated by intolerable adverse events, rather than inadequate efficacy. Any marginal increase in efficacy would therefore be offset by substantially increased drug acquisition cost, and probably increased support medicine costs, consultations and diagnostic tests required to treat and assess an increased frequency of adverse events and resultant treatment withdrawals.

Prices were used as a proxy for unit costs in this study and accordingly there may be an economic profit component in addition to the unit cost of production. However, we consider that excess profits would be minimal because of the competition between providers in New Zealand and the dominant role of government in determining prices. Thus, prices are likely to represent costs.

\section{Therapeutic Implications}

The observed statistically significant difference in adverse effects, withdrawal rates and extra treatment courses in favour of roxithromycin in this study is translated into actual cost savings. Theoretically, if roxithromycin were to be used in place of cefaclor for the estimated 70000 prescriptions for cefaclor filled each year in New Zealand for
LRTI, savings in excess of \$NZ $\$ 656000$ could result, in spite of roxithromycin having a greater unit cost. This analysis demonstrates that economic data can and should be obtained from clinical trials and used to evaluate costs and benefits.

\section{Acknowledgement}

The clinical trial and economic evaluation were supported by a grant from Roussel UCLAF.

\section{References}

Acar JF. Foreword. Diagnostic Microbiology and Infectious Disease 15: 61S-62S, 1992

Billas A. Lower respiratory tract infections. Primary Care 17: 811 824,1990

Conte JE, Barriere SL. Manual of antibiotics and infectious diseases, 6th ed., Lea and Febiger, New York, 1988

Dautzenberg B, Scheimberg A, Brambilla C, Camus P, Godard P et al. Comparison of two oral antibiotics, roxithromycin and amoxycillin plus clavulanic acid, in lower respiratory tract infections. Diagnostic Microbiology and Infectious Diseases 15: 85S-90S, $1992^{\circ}$

Dunlay J, Reinhard R, Donn Roi L. A placebo controlled, doubleblind study of erythromycin in adults with acute bronchitis. Journal of Family Practice 25: 137-141, 1987

Health Benefits Letter No. 3: 29 August 1991. Scale of fees payable in respect of laboratory diagnostic services. New Zealand Department of Health publication, Wellington, New Zealand, 1991

Health Benefits Letter No. 4: 17 October 1991. GMS benefits. New Zealand Department of Health publication, Wellington, New Zealand, 1991

Herron JM. Roxithromycin in the therapy of streptococcus pyogenes throat infections. Journal of Antimicrobial Chemotherapy 20 (Suppl. B): 139-144, 1987

Kammer RB, Shor LJ. Cefaclor - summary of clinical experience. Postgraduate Medical Journal 55 (Suppl. 4): 93-97, 1979

Lentino JR, Lucks DA. Nonvalue of sputum culture in the management of lower respiratory tract infections. Journal of Clinical Microbiology 25: 758-762, 1987

Mandell GL, Douglas RG, Bennett JE. Principles and practice of infectious disease, 3rd ed., Churchill, London, 1990

Marsac J, Akoun G, Balmes P, Butaeye P, Charpin J, et al. Multicentre comparative study of the efficacy and safety of roxithromycin and doxycycline in the treatment of lower respiratory tract infection. British Journal of Clinical Practice 55 (Suppl.): 100-101, 1988

McKendry CG, Muthumala D. Health expenditure trends in New Zealand 1980-1992. New Zealand Department of Health, Wellington, 1993

Munro C. The diagnostic content of general practice. Australian Family Physician 18: 226-244, 1989

Pricing Schedules: 1 August 1991. Drug tariff items. New Zealand Department of Health publication, Wellington, 1991

Reid JS, Anyon CP. What we thought the patients came for: a year's morbidity recording in a New Zealand urban practice. New Zealand Family Physician 13: 145-148, 1986

Ridley-Smith RM. Why the patients came. New Zealand Medical Journal 78: 240-246, 1973

Saito A. Bacteriological and clinical evaluation of roxithromycin 
in pneumonia. Journal of the American Medical Association 6 (Suppl.): 20-23, 1990

Scott WG, McKendry CG, Scott HM. Methodologies for economic evaluation of high technology in health care, Department of Health, Wellington, 1992

Teng Liaw S. The diagnostic profile of a South Australian rural practice. Australian Family Physician 20: 172-183, 1991

Tilyard MW, Dovey SM. A randomized double-blind controlled trial of roxithromycin and cefaclor in the treatment of acute lower respiratory tract infections. Interim report. Diagnostic Microbiology and Infectious Diseases 15 (Suppl.): 97S-102S, 1992 (Final report presented at the 1st International Conference on Macrolides, Azalides and Streptogramins, Sante Fé, New Mexico, January 22-25, 1992)

Correspondence and reprints: Guy Scott, W. Guy Scott and Associates Ltd, PO Box 14403 , Wellington, New Zealand. 


\section{CASE 6: INFLUENZA}

Discussed in chapter 13

\section{Reference}

Scott, W. G., and H. M. Scott. "Economic evaluation of vaccination against influenza in New Zealand." PharmacoEconomics 9 (1996): 51-60. 


\title{
Economic Evaluation of Vaccination Against Influenza in New Zealand
}

\author{
W. Guy Scott and Helen M. Scott \\ Wellington Polytechnic, and Independent Health Economists, Wellington, New Zealand
}

\section{Summary}

The objective of this study was to evaluate the costs and benefits of influenza vaccination for the population aged 65 years and over, from the perspectives of individuals and health insurers, government and society.

The annual incremental direct medical costs and benefits of influenza vaccination (compared with the nonvaccination, or 'do nothing', option) were evaluated using New Zealand healthcare resource usage and unit cost data [in 1992 New Zealand dollars (\$NZ); $\$ N Z 1=\$ U S 0.5458$, June 1992] applied to cohort studies reported in the literature.

The costs and benefits to society as a result of vaccination of people aged 65 years and older ( $20 \%$ of people in this age group are currently vaccinated) were estimated to be: (i) additional direct medical costs of vaccination of $\$ N Z 1.42$ million [\$NZ17.78 per vaccination]; (ii) direct medical costs avoided of \$NZ5.35 million (\$NZ67.18 per vaccination); and (iii) net benefits of \$NZ3.93 million (\$NZ49.40 per vaccination).

The direct medical costs avoided per dollar cost of vaccination were \$NZ1.04 for individuals, $\$ N Z 4.69$ for government and $\$ N Z 3.78$ for society as a whole. If the vaccination uptake for this group is increased in $20 \%$ increments, the net benefit to society increases by a further $\$ N Z 3.93$ million per year at each step. If the economic evaluation is extended to include vaccination of at-risk individuals under 65 years of age, net benefits to society increase by $15 \%$.

Influenza vaccination for people aged 65 years and over is cost effective from the perspective of society, government and the individual. If the vaccination rate for at-risk individuals in New Zealand could be increased to $60 \%$, the net benefits reported in this study would increase by $200 \%$. However, the costs of promotion and education to achieve this vaccination rate would need to be deducted from the net benefits. Strategies to increase the vaccination rate include altering the cost of vaccinations to the individual, intensifying education and promotion programmes, and changing the mode of delivery.
Influenza is regarded worldwide as an important and highly infectious viral illness that is most severe in the very young and the elderly. The likelihood of being hospitalised or dying from influenza increases with age and the number of underlying medical conditions present in the patient." "I The benefits of influenza vaccination are dependent upon age, health status, the rate of vaccination, and the efficacy of the vaccine. Although influenza vaccination is considered to be safe and effective, vaccination rates are low in New Zealand and vaccination is regarded as being underutilised. ${ }^{[2.3]}$ The aim of this study is to assess the cost effectiveness of influenza vaccination from the perspectives of individuals and health in- 
surers, government and society for the at-risk group of patients aged 65 years and older.

Four groups are targeted for influenza vaccination in New Zealand. The first group consists of people described as being at high risk of complications:

- adults and children with chronic debilitating diseases

- persons aged over 65 years

- residents of rest homes, geriatric hospitals and other chronic care facilities, and

- immunocompromised individuals.

The second group comprises individuals who may spread infection to those at high risk, and group 3 consists of people engaged in essential community services. The fourth group covers children with specific conditions (e.g. children who require long term salicylate therapy, and those with sickle cell and other haemoglobinopathies). ${ }^{[4]}$

\section{Methods and Data}

The costs incurred and the costs avoided (benefits) in health economic analyses can be classified as direct medical, direct nonmedical, indirect and intangible. Direct medical costs are those involved in providing treatment, and include the purchase of vaccines, medical practitioner consultations and hospital costs. Direct nonmedical costs incurred by the illness and its treatment could include transport to receive treatment and the cost of special food as a result of the illness. Indirect costs encompass work output lost as a result of morbidity and mortality, while intangible costs are concerned with pain, suffering and loss of quality of life rather than changes in resource opportunity costs. ${ }^{15}$

This study focuses on the incremental direct medical costs of influenza vaccination for people aged 65 years and over. The net cost of vaccination was compared with that of no vaccination. Vaccination of at-risk adults under 65 years of age and children with chronic debilitating diseases was evaluated as an adjunct to the sensitivity analysis. Together, these 2 at-risk groups account for about $20 \%$ of the total (all ages) New Zealand populittion. I I If vaccination were to be extended to the household contacts and caregivers of these people, the numbers would double, ${ }^{[2]}$ but insufficient data exist to allow quantification of the costs and benefits for these individuals.

Table I lists the International Classification of Diseases (ICD) diagnosis codes relating to influenza, pneumonia, other respiratory conditions and heart failure, as described by Nichol et al. ${ }^{[7]}$ These codes were used to define influenza and its complications and as the basis for calculating healthcare resource volumes in New Zealand. The vaccination cost and the direct medical costs avoided for the atrisk group studied (i.e. people aged 65 years and over) were estimated using these diagnosis codes.

Table II summarises the steps followed to estimate the incremental net costs of vaccinating people aged 65 years and over.

Costs were estimated from 3 perspectives: that of the individual (personal, out-of-pocket or health insurance), government and society. Because all costs relate to 1 year (i.e. a prevalence study), discounting to present values was not necessary. All unit prices and costs used in the study were in 1992 New Zealand dollars (\$NZ) and were exclusive of $12.5 \%$ goods and services tax (GST), which is a transfer payment from a societal perspective. The midpoint exchange rate at the end of June 1992 was $\$ N Z 1=\$ U S 0.5458$.

The current influenza vaccination rate of $20 \%$ [2] among people aged 65 years and over, together with the baseline values for health resource utilisation reductions, were used in the base case analysis. Sensitivity analysis was used to evaluate the impact of changes to the key variables in the study. For example. the per vaccination cost relating to the older group ( $\geq 65$ years) [excluding the costs of heart failure and adjusting the hospital costs to take account of different diagnostic related group (DRG) hospital costs for those under 65 years] was applied to the younger group ( $<65$ years) because no specific data on health resource utilisation changes were available. 
Table I. Diagnosis codes taken from the ICD-9CM (International Classification of Diseases, ninth revision, clinical modification) ${ }^{[6]}$ for influenza, pneumonia, other respiratory diseases and heart failure

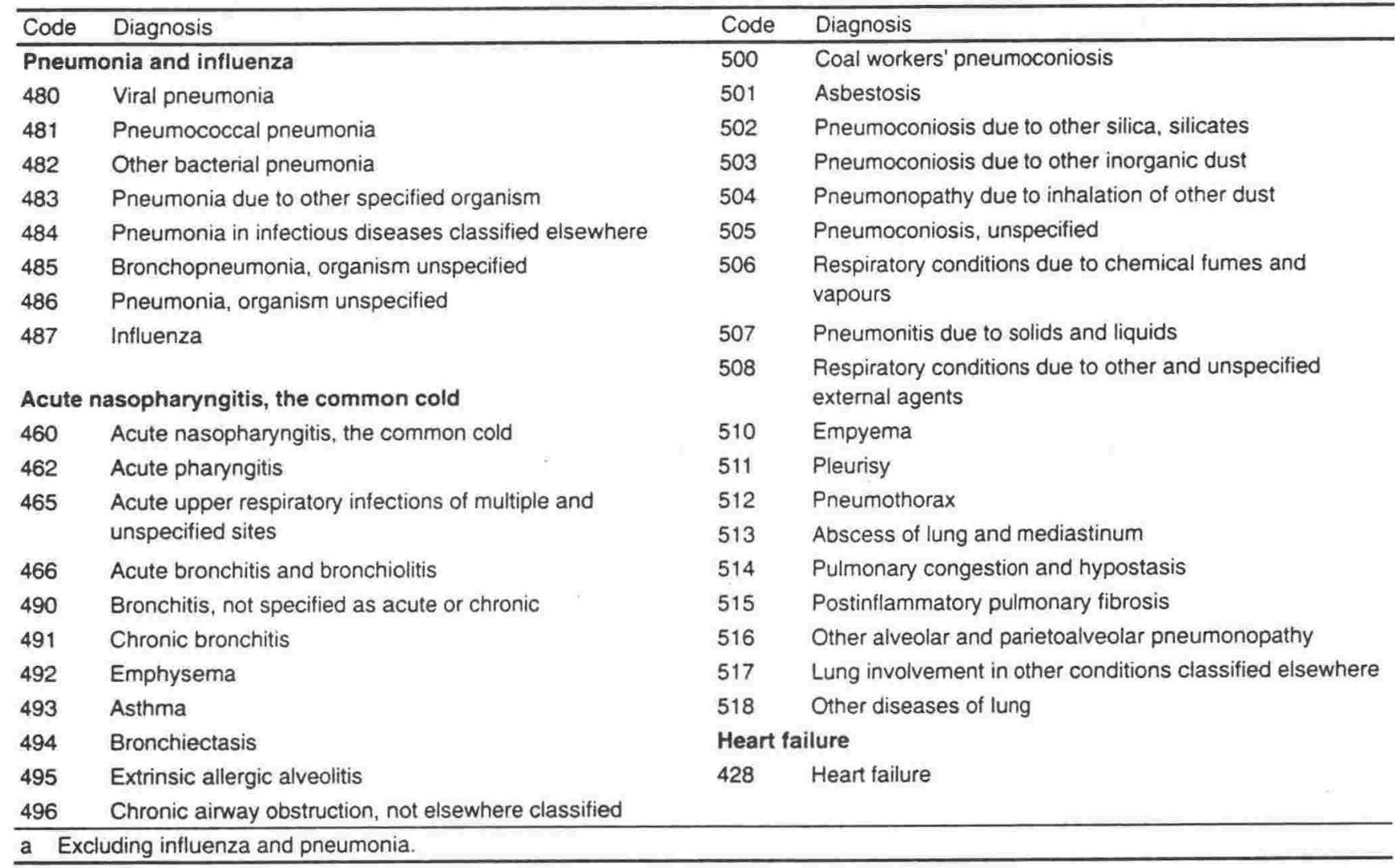

\section{Vaccine Cost}

The potential number of influenza vaccinations was based on the number of people aged 65 years and older, ${ }^{[8]}$ who accounted for $11.5 \%$ of the total population of New Zealand in 1992. Those aged under 65 years and at risk of influenza (for the sensitivity analysis) accounted for $9.3 \%$ of the total population. ${ }^{|2|}$ The influenza vaccine acquisition cost to general practitioners (GPs) was \$NZ12.96 per annual vaccination (information supplied by Rhône-Poulenc Rorer NZ Ltd, and CSL NZ Lid). GPs charged a total fee oi \$NZ17.78 for an influenza vaccination, being the costs of their fee plus that of the vaccine. This figure was the mean charge calculated from an informal telephone survey of approximately 10 GPs in Wellington, New Zealand (own unpublished data). This fee was unchanged from that reported in 1991.121

\section{Utilisation of Healthcare Resources if No Vaccination is Given}

Tobias $^{[2]}$ considered that, on the basis of influenza vaccine sales in New Zealand, about $20 \%$ of the elderly population ( $\geq 65$ years) and $10 \%$ of the

Table II. Steps involved in estimating the incremental costs and benefits of influenza vaccination in New Zealand

\begin{tabular}{|c|c|}
\hline Step & Calculation \\
\hline 1 & Estimate the cost of vaccination (C) \\
\hline 2 & $\begin{array}{l}\text { Estimate utilisation of healthcare resources if no } \\
\text { vaccination given }(U p)\end{array}$ \\
\hline 3 & $\begin{array}{l}\text { Calculate the change in the volume of resources if } \\
\text { vaccination given (Uc) }\end{array}$ \\
\hline 4 & $\begin{array}{l}\text { Apply unit costs }(P) \text { to the change in the volume of } \\
\text { resources used }(U c) \text { to estimate the costs avoided } \\
\text { (benefits: } B \text { ) of vaccination: } B=U c \times P\end{array}$ \\
\hline 5 & $\begin{array}{l}\text { Calculate the incremental net costs or benefits }(N C) \text { : } \\
N C=C-B\end{array}$ \\
\hline 6 & Conduct sensitivity analyses \\
\hline
\end{tabular}


younger ( $<65$ years) at-risk groups are currently being vaccinated. A number of researchers ${ }^{[7.9 .10]}$ have found that vaccinated individuals utilise fewer healthcare resources than unvaccinated individuals. Therefore, to calculate healthcare resource utilisation in the 'no vaccination' scenario, it was necessary to scale up the actual utilisation data to account for the $20 \%$ of the elderly population who are actually being vaccinated. Thus:

$\mathrm{Up}=\frac{\mathrm{Ua}}{1-(\mathrm{Vr} \times \mathrm{Uc})}$

where:

$\mathrm{Up}=$ potential utilisation volume (i.e. the volume had there been no vaccinations)

$\mathrm{Ua}=$ actual utilisation volume

$\mathrm{Vr}=$ vaccination rate expressed as a decimal

$\mathrm{Uc}=$ proportional reduction (expressed as a decimal) for utilisation volume as a result of vaccination.

Data (broken down by ICD code) on the numbers of GP consultations and prescription items dispensed were averaged over the years 1991, 1992 and 1993. These data were obtained from IMS (N.Z.) Ltd. The number of prescriptions for those aged 65 years and over was estimated from the number of prescription items written per GP consultation for all ages multiplied by the number of consultations for those aged 65 years and over. ${ }^{1}$ Hospital admissions by ICD code were derived from public hospital separation data averaged over the 3 years 1990, 1991 and 1992 (the latest 3 years available at the time of the study). ${ }^{[11]}$

\section{Medical Costs Avoided}

The potential reduction in the volume of healthcare resource use was the mean of the percentage reductions in hospitalisations over 3 influenza seasons for vaccinated patients aged 65 years and over, compared with unvaccinated individuals. ${ }^{171}$ These percentage reductions were obtained for pneumonia and influenza, other acute and chronic respiratory

1 These data probably underestimate the number of prescriptions written for those aged 65 years and over. Thus, our costs would be conservative. conditions (i.e. all chronic and acute respiratory conditions excluding pneumonia and influenza; see table I) and congestive heart failure. The comparisons between vaccinated and unvaccinated groups were adjusted for differences in patients' baseline characteristics that could alter outcome variables (e.g. had the patient been diagnosed as having a heart or lung disease before the study). ${ }^{[7]}$

In the absence of resource-specific data from randomised clinical trials, case-controlled studies or cohort studies, these percentage reductions were employed to calculate the potential reductions in all healthcare resource utilisations in our study. The baseline savings in the volume of healthcare resources used were: influenza and pneumonia $52 \%$; other respiratory conditions $28 \%$; and heart failure $24 \%$. The incremental cost of influenza vaccination in volume terms was the percentage reduction in utilisation of New Zealand healthcare resources multiplied by the volume of resources used assuming no vaccination. The incremental cost in dollars was calculated by multiplying the incremental cost in volume terms by New Zealand unit costs.

\section{Healthcare Resource Unit Costs}

GP consultation costs used in the study were based on the mean fee actually charged of $\$ N Z 23.20,[12]$ which is $14 \%$ lower than the listed fee because GPs do not charge all patients the full fee. This figure is inclusive of the GP subsidy [general medical services benefit (GMS)] but exclusive of GST at $12.5 \%$. The GP subsidy (GMS) used was the rate applicable to beneficiaries (i.e. \$NZ13.33 as at February 1992).|13|

The average government-subsidised cost of a medical prescription used for the calculations was the average value of \$NZ31.16 applicable to elderly people ( $\geq 65$ years) at June $1992 .^{[14]}$ The patient copayment was $\$ N Z 4.44$, applicable to beneficiaries, and chronically ill and high user groups, at February $1992 .^{[1+]}$ Medicines available and sold to patients over-the-counter were not evaluated.

Hospital costs were based on DRG costs. ${ }^{[15]}$ The DRG codes most closely related to the specific age group concerned and to the diagnosis codes described in table I were used. A weighted average (by volume) 
Table III. Baseline costs of influenza vaccination versus no vaccination, by perspective. These costs are for a population of people aged 65 years and over, and are in 1992 New Zealand dollars (\$NZ; \$NZ1 = \$USO.5458, June 1992) exclusive of goods and services tax. Calculations assumed that $20 \%$ of this population are vaccinated. Costs were calculated from 3 perspectives: individual, government and societal

\begin{tabular}{|c|c|c|c|c|}
\hline \multirow[t]{2}{*}{ Cost type } & \multirow{2}{*}{$\begin{array}{l}\text { Healthcare } \\
\text { resource volume } \\
\text { change (no.) }\end{array}$} & \multicolumn{3}{|c|}{ Cost by perspective (\$NZ) } \\
\hline & & individual $^{\mathbf{a}}$ & government & society \\
\hline Total costs & & & & \\
\hline \multicolumn{5}{|l|}{ Additional direct medical costs } \\
\hline vaccination at GP & 79632 & 350000 & 1060000 & 1420000 \\
\hline \multicolumn{5}{|l|}{ Direct medical costs avoided (benefits) } \\
\hline GP consultations & 24094 & 240000 & 320000 & 560000 \\
\hline prescriptions & 29494 & 130000 & 920000 & 1050000 \\
\hline public hospital admissions & 745 & & 3740000 & 3740000 \\
\hline Total direct costs avoided (benefits) & & 370000 & 4980000 & 5350000 \\
\hline Net $\cos ^{b}$ & & $(10000)$ & (3920 000) & (3930 000) \\
\hline Costs avoided per dollar cost of vaccination & & 1.04 & 4.69 & 3.78 \\
\hline \multicolumn{5}{|l|}{ Costs per vaccination } \\
\hline \multicolumn{5}{|l|}{ Additional direct medical costs } \\
\hline vaccination at GP & 1 & 4.44 & 13.33 & 17.78 \\
\hline \multicolumn{5}{|l|}{ Direct medical costs avoided (benefits) } \\
\hline GP consultations & 0.30 & 2.99 & 4.03 & 7.02 \\
\hline prescriptions & 0.37 & 1.65 & 11.54 & 13.19 \\
\hline public hospital admissions & 0.01 & & 46.98 & 46.98 \\
\hline Total direct costs avoided (benefits) & & 4.63 & 62.55 & 67.18 \\
\hline Net $\cos t^{b}$ & & $(0.19)$ & $(49.22)$ & $(49.40)$ \\
\hline a Personal, out-of-pocket or insurance. & & & & \\
\hline b Costs in parentheses indicate a net benefit. & & & & \\
\hline Abbreviation: GP = general practitioner. & & & & \\
\hline
\end{tabular}

was derived for pneumonia and influenza (\$NZ5501 for 1 episode) and for other acute and chronic respiratory conditions (\$NZ4700). Weighted average hospital unit costs for at-risk people aged under 65 years were similarly estimated (\$NZ1787). For heart failure, the DRG unit cost was \$NZ4963.

\section{Results}

Tables III and IV summarise the baseline costs by diagnosis and from the perspectives of the individual, government and cociety for the age group 65 years and over. GST is not included in the presentation of results because it is a transfer payment from the perspectives of government, society as a whole and the citizens that make up society. However, from the perspective of an individual as opposed to the sum of all individuals, GST is an additional cost to be paid. If a health insurance company is reimbursing that individual, GST will be included in the payment.

From a societal perspective, additional direct medical costs of vaccination compared with no vaccination amounted to $\$ \mathrm{NZ1} .42$ million ( $\$ \mathrm{NZ17} .78$ per vaccination); total direct medical costs avoided were $\$ N Z 5.35$ million (\$NZ67.18 per vaccination) with net benefits of \$NZ3.93 million (\$NZ49.40 per vaccination) [table III]. Because the majority of costs fall on the individual (out-of-pocket) and most of the costs avoided accrue to government, there is a small net benefit to individuals of \$NZ0.19 per vaccination and a comparatively much larger net benefit to government of $\$ N Z 49.22$ per vaccination (table III). The direct medical costs avoided per dollar vaccination cost were: $\$ \mathrm{NZI} .04$ for each dollar spent by individuals, $\$ N Z 4.69$ for each dollar spent by government and $\$ N Z 3.78$ for society (table III). 
Table IV. Baseline costs of influenza vaccination versus no vaccination, by diagnosis. These costs are for a population of people aged 65 years and over, and are in 1992 New Zealand dollars (\$NZ; \$NZ1 = \$US0.5458, June 1992) exclusive of goods and services tax. Calculations assumed that $20 \%$ of this population are vaccinated, and costs were calculated from 3 perspectives: individual, government and societal

\begin{tabular}{llll}
\hline Cost type & \multicolumn{2}{l}{ Cost by perspective (SNZ) } \\
\cline { 2 - 4 } & individual ${ }^{2}$ & government & society \\
\hline Total costs & & & 1430000 \\
Direct medical costs avoided & & & 2400000 \\
$\quad$ pneumonia and influenza & 30000 & 1400000 & 1520000 \\
$\quad$ other respiratory conditions & 220000 & 2180000 & 5350000 \\
$\quad$ heart failure & 120000 & 1400000 & 18.00 \\
Total direct costs avoided & 370000 & 4980000 & 30.13 \\
Costs per vaccination & & & 19.05 \\
Direct medical costs avoided & & & 67.18 \\
$\quad$ pneumonia and influenza & & 17.56 & 27.40 \\
$\quad$ other respiratory conditions & 0.43 & 17.59 & 62.55 \\
$\quad$ heart failure & 2.74 & 1.46 &
\end{tabular}

From a societal perspective, the medical costs avoided as a result of reduced calls on health resources for various diagnoses were estimated to be \$NZ1.43 million (\$NZ18.00 per vaccination) for pneumonia and influenza, \$NZ2.40 million (\$NZ30.13 per vaccination) for other respiratory conditions, and \$NZ1.52 million (\$NZ19.05 per individual) for heart failure.

Sensitivity analysis was undertaken for the societal net costs for people aged 65 years and over, and the results are presented in table V. The analysis considered the percentage vaccinated, and the maximum and minimum reported changes in healthcare resource utilisation. Changing the vaccination cost and subsidy rates could not be effectively evaluated, because the price elasticity of demand for vaccinations with respect to out-of-pocket payments was unknown. If the percentage of those vaccinated (in the age group 65 years and over) was increased in steps of $20 \%$, the net benefit increased in increments of $\$$ NZ3.93 million (table $\mathrm{V}$ ). The ratio of costs avoided per dollar cost of vaccination indicates that the vaccination cost (vaccine plus administration) of the vaccine could increase by a multiple of 3.78 before net benefits fall to zero.

The at-risk population under 65 years of age was also considered as an additional adjunct to the sen- sitivity analysis; costs of vaccination in this group are shown in table VI. If this younger at-risk population is considered, the societal net benefits of influenza vaccination increase by $\$ N Z 0.58$ million or $15 \%$ over the baseline. The ratio of costs avoided per dollar cost of vaccination for the younger at-risk

Table V. Results of the first sensitivity analysis. The effect of varying the rate of influenza vaccination [from $20 \%$ (baseline) to $100 \%$ ] on healthcare resource utilisation in people aged 65 years and over was studied in this sensitivity analysis. Costs are in 1992 New Zealand dollars (\$NZ; SNZ1 = \$USO.5458, June 1992), are exclusive of goods and services tax, and are calculated from a societal perspective

\begin{tabular}{|c|c|c|c|c|}
\hline \multicolumn{2}{|c|}{$\begin{array}{l}\text { Population vaccinated } \\
(\%)\end{array}$} & \multicolumn{3}{|c|}{$\begin{array}{l}\text { Change in healthcare resource usage } \\
{\text { (millions of } \$ N Z)^{a}}\end{array}$} \\
\hline & & baseline & high $^{b}$ & $10 w^{c}$ \\
\hline \multicolumn{2}{|c|}{20} & $(3.93)$ & $(5.60)$ & $(0.76)$ \\
\hline \multicolumn{2}{|c|}{40} & $(7.87)$ & $(11.20)$ & $(1.52)$ \\
\hline \multicolumn{2}{|c|}{60} & $(11.80)$ & $(16.80)$ & $(2.27)$ \\
\hline \multicolumn{2}{|c|}{80} & (15.74) & $(22.40)$ & $(3.03)$ \\
\hline \multicolumn{2}{|c|}{100} & $(19.67)$ & $(28.00)$ & $(3.79)$ \\
\hline \multicolumn{2}{|c|}{$\begin{array}{l}\text { Costs avoided per } \\
\text { dollar cost of } \\
\text { vaccination (SNZ) }\end{array}$} & 3.78 & 4.96 & 1.54 \\
\hline \multicolumn{5}{|c|}{$\begin{array}{l}\text { a Figures in parentheses indicate net benefits rather than net } \\
\text { costs. }\end{array}$} \\
\hline \multicolumn{5}{|c|}{$\begin{array}{l}\text { Desults assuming that vaccination leads to the maximum } \\
\text { reported reductions in healthcare utilisation. }\end{array}$} \\
\hline \multicolumn{5}{|c|}{$\begin{array}{l}\text { Results assuming that vaccination leads to the minimum } \\
\text { reported reductions in healthcare utilisation. }\end{array}$} \\
\hline
\end{tabular}


group was $60 \%$ lower than the older age group from a societal perspective (1.51 vs 3.78) [tables III and VI].

Running the analysis using the reported minimum reductions in healthcare resource usage as a result of vaccination (i.e. pneumonia and influenza 19\%, ${ }^{[9]}$ other respiratory conditions $20 \%{ }^{[7]}$ and heart failure $0 \%{ }^{[7]}$ ) reduced the net benefit of vaccination to society by $81 \%$, and the direct medical costs saved per dollar of vaccination cost by $\$ N Z 2.24$ to $\$ N Z 1.54$ (table V). Using the maximum reported resource savings ${ }^{[7]}$ (i.e. $57 \%$ for pneumonia and influenza, $34 \%$ for other respiratory conditions, and $38 \%$ for heart failure) increased the net benefits to society by $42 \%$ and the direct medical costs saved per dollar of vaccination cost by $\$ N Z 1.18$ to $\$ N Z 4.96$.

\section{Discussion}

Influenza vaccination is probably more effective in preventing severe complications from influenza than in eliminating minor complications. Thus, the application of the hospital reductions to GP consultations and prescriptions may overestimate this cost saving. However, as the major costs lie within the hospital sector, our final results are unlikely to be seriously biased.

Sensitivity analysis indicated that although there is considerable variation when upper and lower ranges in resource savings are applied, the direct medical costs avoided remain greater than the additional costs of influenza vaccination. From a societal perspective, changing the copayment or subsidy rates altered the division of costs that individuals or government paid. Altering the GMS subsidy is also likely to alter the rates of vaccination because the price to the patient would change, but this could not be quantified.

Sensitivity analysis also indicated that, for each $20 \%$ incremental increase in the uptake of vaccination, there would be an incremental net societal benefit of $\$ N Z 3.93$ million. To achieve a higher vaccination rate, additional costs of education and promotion (targeting at-risk groups and healthcare professionals) would be incurred by the suppliers of the vaccine, government or both. This additional
Table VI. Results of the second sensitivity analysis. The cost of vaccinating at-risk people under the age of 65 years was studied, from the perspectives of the individual, government and society. Costs are in 1992 New Zealand dollars (\$NZ; \$NZ1 = \$USO 5458 , June 1992) and are exclusive of goods and services tax

\begin{tabular}{llll}
\hline Cost type & \multicolumn{3}{l}{ Perspective } \\
\cline { 2 - 4 } & individual $^{\mathrm{a}}$ & government & society \\
\hline Net cost (millions of \$NZ) & 0.08 & $(0.66)$ & $(0.58)$ \\
Costs avoided per dollar & 0.71 & 1.77 & 1.51 \\
cost of vaccination (\$NZ) & & \\
\hline a Personal, out-of-pocket or insurance. & \\
b Figures in parentheses indicate net benefits. & \\
\hline
\end{tabular}

cost was not taken into account in our analysis, but the results provide the information required to set an upper limit on an educational/promotional budget.

It was found that there was a difference in the breakdown of cost savings by disease category between this study and that reported by Nichol et al. ${ }^{[7]}$ because the underlying hospital admission rates for pneumonia and influenza in the US were higher than those recorded in New Zealand. Conversely, the New Zealand hospitalisation rate for heart failure was higher than that in the US study. (Although it is beyond the scope of this study to evaluate the reasons for different hospitalisation rates, a possible explanation is that the prevalence and mode of treatment of the diseases considered could differ between the US and New Zealand.) Although the absolute volumes are different, we consider that it is valid to apply the US percentage reductions in utilisations of healthcare resources to New Zealand base resource utilisation volumes.

In this study, we used average rather than marginal unit cost data to estimate cost changes as a result of vaccination. Policy decisions relating to influenza vaccination would involve increments of hundreds or thousands rather than a single individual. Multiplying the marginal net cost of one additional vaccination by the number of vaccinations would provide a false estimate of the incremental net costs of the programme. The marginal net cost of one additional vaccination would ignore overhead costs, but the incremental net cost of large numbers of additional vaccinations should include those 
overhead costs that would change if the vaccination rate altered. The actual costs associated with policy changes would be greater than marginal costs, but probably less than average costs.

Because health authorities consider that influenza vaccination should also be given to at-risk groups under 65 years of age, we applied a similar methodology to estimate the costs and benefits of such vaccination. The costs of heart failure were excluded from this group. In addition, because more specific information was not available, the reduction in resource volumes for pneumonia, influenza and other respiratory conditions applicable to the 65 years and over group was used in the younger age group.

The percentage reductions in resource utilisation for people under 65 years of age are unlikely to be identical to those for older people. Moreover, the results obtained for people aged $<65$ years cannot be accepted with the same confidence as those pertaining to the older age group. Despite these 2 points, it was considered that policy makers would find this information useful. It was found that the ratio of net costs avoided per dollar of vaccination cost was substantially less for the younger at-risk group than the ratio applicable to the older group. The main reason for this difference was the lower incidence and unit cost for hospitalisation in the younger age group (the average length of hospital stay was shorter for this age group).

As no specific data were available on GP and prescription utilisation, we applied the percentage reductions in hospital resource use to GP consultations and prescriptions. However, it could be argued that influenza vaccination is more efficient in preventing hospitalisations than in avoiding GP consultations and prescriptions. As less than one-third of the total medical costs avoided were nonhospital costs, inaccuracies in estimates of nonhospital resource utilisations would not impose a serious bias on our estimate of total cost savings. Omission of nonhospital costs would detract from the study"s value as a policy analysis tool.

Subsets of the target groups studied could also be researched. For example, in 1992 there were 20962 beds in rest homes and approximately 20000 full and part-time employees. ${ }^{[16]}$ If it is assumed that the net benefit of vaccinating 20000 patients is the same as that estimated in this study (rest home residents are a subset of this), the net benefit to society of vaccinating these full and part-time employees would be \$NZ1.34 million.

For an individual of working age, the cost of a vaccination would be offset by additional production gains. Riddiough et al. ${ }^{[17]}$ found that 0.03 work days per vaccination could be gained for an individual aged between 45 and 54 years. In a study of staff in a New Zealand health laboratory, it was found that absenteeism from influenza was $39.5 \%$ in the vaccinated group and $66.7 \%$ in the unvaccinated group. ${ }^{[18]}$ It is sometimes argued that short term absence from work is not a cost, because there is slack in the economy (unemployment and workers able to cover for absentees), or that work set aside is actioned on the return of the worker. ${ }^{[19]}$ Potential production loss, both paid and unpaid, should be estimated on the understanding that actual production loss may be somewhat less. However, in many situations in New Zealand, if a worker becomes ill there is no surplus capacity and the output of the absentee worker will be lost. A production loss may also occur when parents or guardians take time from work to care for children who are ill with influenza.

The cost of adverse events relating to the vaccine were not evaluated in this study. Modern influenza vaccines are considered to have few adverse effects, and medical practitioners are advised against vaccinating patients with a known or possible anaphylactoid hypersensitivity to hens ${ }^{2}$ eggs. ${ }^{[+1}$ Although intangible costs were not evaluated, quality-of-life aspects are important for potential influenza patients and their families and friends.

New Zealand data for 1992 recorded 1132 deaths from pneumonia and influenza (as a whole), ${ }^{[20]}$ and for men and women over 75 years of age it was the fourth and third leading cause of death, respectively. However, it is considered that influenza is responsible for many deaths recorded under categories other than influenza and pneumonia. ${ }^{|10.21,22|}$ Fedson et al. ${ }^{[10]}$ (which covered the influenza outbreaks of 
1982-3 and 1985-6 in the Canadian province of Manitoba) found that vaccination was 43 to $65 \%$ effective in preventing hospital deaths in patients admitted with pneumonia, influenza or other respiratory conditions, and 27 to $30 \%$ effective in preventing deaths from all causes.

Vaccination, where an infectious disease is involved, confers external benefits to unvaccinated individuals in a community by limiting epidemics. The protection, or herd immunity, afforded to unvaccinated people when others around them are vaccinated has been estimated by Tobias ${ }^{[2]}$ to be achieved by vaccinating 70 to $75 \%$ of residents in a rest home or $80 \%$ in open communities. The US Department of Health and Human Services ${ }^{[23]}$ have recommended a vaccine coverage of $80 \%$ for institutionalised at-risk individuals and $60 \%$ for at-risk community residents. If the vaccination rate for at-risk individuals in New Zealand could be increased to $60 \%$ then the net benefits reported in this study would increase by $200 \%$. As discussed earlier, the costs of promotion and education to achieve this would need to be deducted from the net benefits. In a study of healthy working adults, Nichol et al. ${ }^{[24]}$ found that vaccination against influenza resulted in substantial healthrelated and economic benefits. There was a reduction in days off work and medical costs resulting in net savings (direct and indirect costs) of \$US46.85 per person (1994 dollars).

The results of this study have demonstrated the economic benefits of vaccination of at-risk groups in society. The current vaccination rate in New Zealand $(20 \%)$ is less than is medically desirable, ${ }^{|2|}$ and a higher rate has been demonstrated in this study to be justifiable in economic terms. Strategies to increase the vaccination rate include subsidising vaccinations to reduce the cost to the individual, changing the mode of delivery. and education and promotion programmes.

Research into the impact of vaccination on the utilisation of nonhospital resources for people aged 65 years and older would be useful, but not essential, because most cost savings relate to hospital costs. To formulate a vaccination policy and recom- mendations for at-risk individuals of working age, further information is required on all costs.

This study has combined published overseas findings with New Zealand volume and unit cost data. Because of the need to use healthcare resources efficiently, suitable overseas studies should, where appropriate, be applied to New Zealand to evaluate treatment and health policy options for New Zealand. The resources required to replicate overseas studies and generate equivalent New Zealand clinical data may be better devoted to evaluating and applying overseas studies to New Zealand, and in providing healthcare.

\section{Acknowledgements}

This study was supported in part by a research grant from CSL NZ Ltd and Rhône-Poulenc Rorer NZ Ltd.

\section{References}

1. Wiselka M. Influenza: diagnosis, management, and prophylaxis. BMJ 1994; 308: 134 I-5

2. Tobias $M$. Influenza vaccination still underutilised. $N$ Ethicals 199 I Sep; 28 (9): 23-30

3. Jennings LC. Towards the control of influenza in New Zealand. Paper presented to the Public Health Commission and the Communicable Diseases Advisory Committee. Wellington: 1994 Dec 8

4. New Zealand Department of Health. Immunisation handbook. Wellington: Department of Health. 1993

5. Eisenberg JM. Schulman KA. Glick H. et al. Pharmacoeconomics: economic evaluation of pharmaceuticals. In: Strom BL, editor. Pharmacoepidemiology. 2nd ed. Chichester: Wiley. 1994: $469-93$

6. International Classification of Diseases. 9th rev. ed. Clinical Modification (ICD-9CM). Ann Arbor (MI): WHO, 1978

7. Nichol KL. Margolis KL. Wuorenma J, et al. The efficacy and cost effectiveness of vaccination against influenza among elderly persons living in the community. N Engl J Med 1994; $331: 778-84$

8. Statistics New Zealand. INFOS IIntormation Network for Oificial Stutistics: online computerised official statistics for New Zealand). Population statistics for 1992

9. Karalus NC. Cursons RT, Leng RA, et al. Community acquired pneumonia: aetiology and prognostic index evaluation. Thorax 1991: $46:+13-8$

10. Fedson DS, Wajda A. Nicol JP, et al. Clinical effectiveness of influenza vacination in Manituba. JAMA 1993: 270: 1956-61

11. Neu Zealand Ministry of Health. Hospital and selected morbidity data 1992. Wellington: Health Information Service. $1993^{\circ}$

12. W Guy Scott and Assuciates Ltd. Survey of general practitioners adult consultation fees 1992. Wellington, 1992

13. MeKendry CG. Muthumala D. Health expenditure trends in New Zea!...id: update to 199.3 |appendix 51. Wellington: Ministry of Health. 1994 
14. Health Benefits Lid, Health Benefits Centre. Cash and accrual report for regional health authority - combined: monthly reports for the year ended 1993. Wanganui (New Zealand): Health Benefits Centre, 1993

15. Core health and disability support services for 1993/94: first report of the National Advisory Committee on Core Health and Disability Support Services. Wellington: National Advisory Committee on Core Health and Disability Support Services, 1992

16. New Zealand Licenced Rest Homes Association Inc. An overview of the rest home industry. Wellington: New Zealand $\mathrm{Li}$ cenced Rest Homes Association Inc., 1992

17. Riddiough MA, Sisk JE, Bell JC. Influenza vaccination: costeffectiveness and public policy. JAMA 1983; 249: 3189-97

18. Schousboe $M$. The evaluation of influenza vaccination of Canterbury Health Laboratory staff: internal report. Christchurch: Canterbury Health Laboratories, 1993

19. Koopmanschap MA, Rutten FFH. Indirect costs in economic studies: confronting the confusion. PharmacoEconomics 1993: 4: 446-54
20. New Zealand Ministry of Health. Mortality and demographic data 1992. Wellington: Health Information Service, 1994

21. Nicholson KG. Influenza vaccination and the elderly. BMJ 1990; 310: 617-8

22. Sprenger MJW, Van Naelten MAMG, Mulder PGH, et al. Influenza mortality and excess deaths in the elderly, 1967-82. Epidemiol Infect 1989; 103: 633-41

23. US Department of Health and Human Services. Healthy people 2000: national health promotion and disease prevention objectives. Washington, DC: US Department of Health and Human Services, 199

24. Nichol KL, Lind A, Margolis KL, et al. The effectiveness of vaccination against influenza in healthy working adults. $\mathrm{N}$ Engl J Med 1995: 333: 889-93

Correspondence and reprints: Guy and Helen Scott, P.O. Box 14403, Kilbirnie, Wellington 3, New Zealand. 


\section{CASE 7: CRITICAL LEG ISCHAEMIA}

\section{Discussed in chapter 14}

Reference

Scott H. M., and W. G. Scott. 1994. "Critical Leg Ischaemia in New Zealand. Economic cost of amputation versus intravenous Iloprost". PharmacoEconomics $6(2): 149-154$. 


\title{
Critical Leg Ischaemia in New Zealand Economic Cost of Amputation versus Intravenous Iloprost
}

\author{
Helen M. Scott ${ }^{1}$ and W. Guy Scott ${ }^{2}$ \\ W. Guy Scott and Associates Ltd, Wellington, New Zealand \\ Wellington Polytechnic, Wellington, New Zealand
}

\section{Summary}

Critical leg ischaemia is ischaemia that endangers the leg or part of a leg. Patients with diabetes are 5 times more likely to develop critical leg ischaemia than nondiabetic patients. [1]

Existing data on the prevalence of critical leg ischaemia have been based on all leg amputation procedures undertaken or on all referrals to limbfitting centres. The European Working Group on Critical Leg Ischaemia ${ }^{[1]}$ assumed that virtually all leg amputations were undertaken for ischaemia and that $25 \%$ of patients with critical leg ischaemia would eventually require an amputation. Referrals to limb fitting centres gave an estimate of amputations on the assumption that half of the patients requiring amputation would be referred for a limb fitting. The available information from Western Europe and the US indicates that the prevalence of peripheral arterial disease is $5 \%$ in males over 50 years old, but fewer than $10 \%$ of these will develop critical leg ischaemia. [2] Incidence rates, similarly based on studies in Western Europe and the US, have been estimated to range between 500 and 1000 new cases per million population per year. [1]

For patients with critical leg ischaemia who present with rest pain (i.e. incurring pain while at rest) and/or ischaemic ulcers, the first choice of treatment is revascularisation by surgical bypass or percutaneous angioplasty. However, almost $40 \%$ 
of such patients are not suitable for reconstructive surgery because of the distribution of arterial disease, other medical or anaesthetic considerations, or because previous revascularisation was unsuccessful. ${ }^{|3|}$ The last resort is amputation, ${ }^{14]}$ which is regarded as a high risk procedure.

This study sought to establish the incidence and costs to New Zealand society of critical leg ischaemia patients who had lower limb amputations and to use these costs to evaluate the cost or benefit of treating selected numbers of such patients with iloprost. Thus, both pharmacoeconomic and costof-illness aspects were assessed.

Iloprost is a prostacyclin analogue with an elimination half-life of 30 minutes. ${ }^{\text {[5] Natural prosta- }}$ cyclin has a half-life of 2 to 3 minutes. The analogue inhibits platelet aggregation and induces vasodilation, providing a nonsurgical treatment option for patients with critical leg ischaemia in cases when revascularisation is not possible, or has failed, and the next option would be amputation.

Information on both the economic cost and clinical efficacy of treatment options are essential for cost-effective decision-making relating to individual patients and to hospital management. Accurate clinical and costing data are the starting point for policy analysis and formulation.

\section{Methods}

A retrospective study incorporating incremental analysis was used to estimate costs for critical leg ischaemia patients who had amputations. Incremental analysis is concerned with the measurement of costs that would change as the result of a decision made (in this paper the decision to amputate a limb or to use drug treatment).

Since the objectives of the study were to provide data that would allow drug $v s$ surgical treatment options to be evaluated in economic terms, it was necessary to estimate only those costs and benefits that would differ between these treatments. Costs and benefits that were identical would cancel each other when differences in costs and benefits between treatment options were calculated. It was assumed that the costs up to the point of amputation would be identical for both treatment options studied.

Diagnosis Related Groups (DRGs) is a classification scheme relating the type of patients that a hospital treats (case mix) to the costs incurred by the hospital in their treatment. That is, DRGs are groupings of diagnoses according to their clinical similarities and treatment costs. International Classification of Diseases (ICD) is a classification system (published by the World Health Organization) of diseases, each with a unique code number. $\mathrm{Pa}$ tients who underwent vascular reconstructive surgery followed by amputation were included in the analysis, but costs were evaluated for the amputation only. Individual 1991 patient records (unit record data) relating to (DRG) codes 113 (amputation for circulatory system disorders except upper limb and toe) and 285 (amputation of lower limb for endocrine, nutritional and metabolic disorders), and ICD procedure code 841 (amputation of lower limb) were obtained for all New Zealand public and private hospitals. ${ }^{[6]}$ These data related to all hospital admissions, the details of which are recorded at the time of discharge or death. Any patient readmitted for the same condition within the period studied was recorded as a separate admission. Data were analysed with respect to age and gender groupings.

Records with the following ICD codes were excluded from the analysis: all accident codes, all codes relating to malignant and benign neoplasms likely to result in surgery to the limbs (ICD codes 170 to $173,198,202,213,215,237,238$ ), late effects of injuries to the limbs (ICD codes 905, 906), unspecified disorders of the joint (ICD 719), other disorders of the bone and cartilage (ICD 733), and congenital anomalies of limbs and musculoskeletal deformities (ICD 754 to 757 ).

Two alternative methods were used to isolate patients undergoing leg amputations. The first method selected records by ICD procedure code (the ICD procedure code method) and the second method selected records by DRG code (the DRG method). The 2 methods were used as a cross check on data sorted. ICD procedure code 841 (amputation of the lower limb) was costed using DRG code 
113. ${ }^{[7]}$ The DRG method selected records under DRG codes 113 and 285 (relating to limb amputations), and also those records in DRG codes 110 , 111 and 112 relating to reconstructive vascular procedures where a limb amputation was recorded.

The amputation data, as derived above, were also used to calculate the cost of providing patients with artificial limbs (unit costs were supplied by the Artificial Limb Board, Wellington, 1993) on the basis of below knee, at or above knee procedures. Amputation of toes (ICD procedure 8411 ) was excluded from this costing analysis. It was assumed that one-half of the patients thus identified would be fitted with artificial limbs. ${ }^{[1]}$

Production loss (loss of output or productivity) was evaluated using the total average weekly earnings combined for both males and females (15 to 64 years of age inclusive). ${ }^{\left[{ }^{[8}\right.}$ Estimates of production loss were based on a week off work for every day in hospital. No precise information on time off work was available. However, clinicians and physiotherapists consulted by the authors considered that the method chosen would provide conservative estimates of the loss of production incurred by those of working age given that the recuperation time is likely to be correlated with the length of hospital stay.

For the patient with critical leg ischaemia iloprost is administered as an intravenous infusion for 20 days at a cost of $\$ N Z 185$ per day. The dosage is adjusted according to individual tolerability within the range of iloprost 0.5 to $2 \mathrm{ng} / \mathrm{kg} / \mathrm{min}$ over 6 hours daily. For the first 2 to 3 days, treatment should be started at an infusion rate of $10 \mathrm{ml} / \mathrm{h}$. The dose is then increased at intervals of 30 minutes in steps of $10 \mathrm{ml} / \mathrm{h}$, up to $40 \mathrm{ml} / \mathrm{h}$. Current clinical practice in New Zealand is to administer iloprost for 20 days, although European studies indicate that best results were achieved after treatment periods of 3 to 4 weeks. ${ }^{[9]}$

All unit costs relate to the 1991 year and are exclusive of societal transfer payments such as GST (GST is the New Zealand Goods and Services Tax, a transfer payment from the perspective of society). Individual items may not add exactly to the totals shown because of rounding. The midpoint exchange rate as at June 1991 was $\$ \mathrm{NZ} 1.00$ $=\$$ US 0.579 .

\section{Results}

The data (refined, as in methods) and analysed using ICD procedure codes resulted in a total of 688 records (628 records in the public hospital file, and 60 in the private hospital file). These records were used to estimate hospital costs. Table I shows the total hospital cost of amputations for critical leg ischaemia was \$NZ12.716 million.

The incidence of amputations for critical leg ischaemia was 202 per million. Analysis showed that major amputations (involving more than digits only) were almost exclusively undertaken in public hospitals. The analysis of the data with respect to

Table I. Hospital cost for amputations resulting from critical leg ischaemia, determined by procedure code and DRG estimation methods. Because of rounding, individual items may not add exactly to the totals shown

\begin{tabular}{|c|c|c|c|c|c|}
\hline \multirow[t]{2}{*}{ ICD procedure code or DRG code } & \multicolumn{3}{|c|}{$\begin{array}{l}\text { No. of admissions } \\
\text { (by hospital type) }\end{array}$} & \multirow[t]{2}{*}{$\begin{array}{l}\text { Unit cost } \\
\text { (SNZ) }\end{array}$} & \multirow[t]{2}{*}{$\begin{array}{l}\text { Total cost } \\
\text { (\$NZ million) }\end{array}$} \\
\hline & Public & Private & Total & & \\
\hline \multicolumn{6}{|l|}{ ICD Procedure code estimation method } \\
\hline 841 Amputation lower limb & 628 & 60 & 688 & 18483 & 12.716 \\
\hline \multicolumn{6}{|l|}{ DRG code estimation method } \\
\hline 113 Amputation lower limb except toe & 256 & 2 & 258 & 18483 & 4.769 \\
\hline 285 Amputation lower limb for endocrine disorders & 61 & 0 & 61 & 14254 & 0.869 \\
\hline $\begin{array}{l}\text { Amputation lower limb except toe, recorded under } \\
\text { DRG } 110,111 \text { and } 112\end{array}$ & 34 & 0 & 34 & 18483 & 0.628 \\
\hline Total DRG method & 351 & 2 & 353 & & 6.267 \\
\hline
\end{tabular}


gender and age showed that there were fewer female patients both in total and in the working age group, but little variation existed in average age between males and females.

Using only DRG 113 gave a somewhat restricted sample as additional public hospital patients undergoing leg amputations were also classified under DRGs 110,111,112 and 285. Patients classified under DRG 285 were included and patients classified as DRG 110 to 112 were counted if they had a lower leg except toe amputation. Thus, an alternative estimate of hospital costs of \$NZ6.267 million was obtained, details of which are shown in table I.

Individual patients with critical leg ischaemia may incur considerable hospital costs. It was found that one-third of ICD 841 patients in public hospitals were classified as having been readmitted for the same condition, although not necessarily within the same year.

Estimates of the number of patients with amputations requiring artificial limbs were selected as described in methods, and unit costs applied. The total cost of prostheses was SNZ0.370 million when estimated by the ICD procedure method, and \$NZ0.341 million when estimated by the DRG method. These costs are detailed in table II.

Production loss for those of working age is detailed in table III. Under the procedure method, 218 patients fell within the working age group (15 to 64 years inclusive), i.e. $32 \%$ of the total number of amputations for critical leg ischaemia. Males made up $67 \%$ (145) and females 33\% (73) of this working age group. Lost production amounted to \$NZ2.764 million.

Under the DRG method, 90 patients (25\%) of the total number of patients with critical leg ischaemia amputations were of working age. Males made up $72 \%$ (65) and females $28 \%$ (25) of this group, and together incurred a production loss of $\$ \mathrm{NZ1.901}$ million.

Table IV summarises the ICD procedure and DRG cost estimates. The ICD procedure cost estimates were: hospital costs \$NZ12.7 million, prosthesis \$NZ0.4 million, lost production \$NZ2.8 million. The DRG estimates were: hospital costs \$NZ6.3 million, prosthesis \$NZ0.3 million, lost production \$NZ1.9 million.

If iloprost is administered for 20 days at a drug cost of \$NZ185 per day in a hospital setting at a ward (hotel) cost of $\$ N Z 330$ per day ${ }^{[10]}$ the total medical cost per patient would be $\$ N Z 10300$ compared with a cost of \$NZ19020 (ICD procedure method) per critical leg ischaemia patient undergoing amputation. Medical cost savings per amputation avoided would be $\$ N Z 8720$. If the drug treatment fails, the pharmacotherapy cost of \$NZ10 300 becomes an additional cost. There have to be 1.2 amputations avoided, that is, 1.2 successful treatments to pay for 1 failure. In other words, for every 10 treatment failures there must be 12 successes. Thus, the success rate must be $0.55[12 /(12+10)]$ to break even on the costs of iloprost therapy.

If iloprost is administered for 24 days (an average of the treatment length in European studies) the treatment cost rises to $\$ \mathrm{NZ12} 360$ and the savings decrease to $\$ N Z 6660$ per amputation avoided. There have to be 1.9 amputations avoided, that is, 1.9 successful treatments to pay for 1 failure (for every 10 treatment failures there must be 19 successes). Thus the success rate must be $0.66[19 /(19+10)]$ to break even on the costs of iloprost therapy with this longer regimen.

Table II. Prostheses costs. Because of rounding, individual items may not add exactly to the totals shown

\begin{tabular}{|c|c|c|c|c|c|}
\hline \multirow[t]{2}{*}{ Limb type } & \multirow{2}{*}{$\begin{array}{l}\text { Unit cost } \\
\text { (SNZ) }\end{array}$} & \multicolumn{2}{|c|}{ ICD Procedure method } & \multicolumn{2}{|c|}{ DRG method } \\
\hline & & no. & total cost (SNZ million) & no. & total cost (\$NZ million) \\
\hline Leg: below knee & 1350 & 107 & 0.144 & 110 & 0.149 \\
\hline Leg: knee and above knee & 3467 & 65 & 0.225 & 56 & 0.192 \\
\hline Total & & 172 & 0.370 & 166 & 0.341 \\
\hline
\end{tabular}

Abbreviations: $\mathrm{DRG}=$ Diagnosis Related Group: $I C D=$ International Classification of Diseases. 
Table III. Production loss for those patients of working age (15 to 64 years) undergoing amputation for critical leg ischaemia

\begin{tabular}{lll}
\hline Item & $\begin{array}{l}\text { ICD procedure } \\
\text { method }\end{array}$ & DRG method \\
\hline $\begin{array}{l}\text { Number of patients } \\
\text { Total number of weeks }\end{array}$ & $\begin{array}{l}218 \\
\text { off work }\end{array}$ & 9008 \\
$\begin{array}{l}\text { Average weekly earnings } \\
\text { (\$NZ) }\end{array}$ & 563.22 & 3375 \\
$\begin{array}{l}\text { Total cost (\$NZ million) } \\
\text { Abbreviations: DRG }=\text { Diagnosis Related }\end{array}$ & 2.764 & 563.22 \\
International Classification of Diseases. & \\
\hline
\end{tabular}

\section{Discussion}

This paper presents information. hitherto univailable, on the incidence and annual economic ost of amputations for critical leg ischaemia in New Zealand. Although the paper focuses on hosjital costs for amputations, artificial limb costs. loprost costs, and production loss for patients of vorking age undergoing amputations, other costs re important in evaluating the cost of illness. but could not be quantified with the data available. Such costs include reduced quality of life. mortalty, community care costs, and production losses of Jatients not in the work force.

The estimates of loss of production of those in he working age group would have been higher had i separate rate been used for males and females, secause the male earning rate is higher and there were more males in the study. However, the estimates of lost production would have been lower had adjustments been made for unemployment and abour force nonparticipation. Lost production of patients not in the paid workforce, although of no ess importance to society, was not evaluated.

The ICD procedure method of selecting patient records gave higher numbers than the DRG method. This was not unexpected as DRG code 113 related specifically to amputations for circulatory disorders, but if a patient had an additional and more costly procedure they would have been categorised under the more costly DRG code relating to that procedure (and, thus. lost to DRG code 113). It was considered that the ICD procedure method gave the best estimate of incidence and costs, and that the DRG method provided an absolute minimum estimate.

The hospital unit costs used in the paper were the averages of 4 Area Health Boards. A cross-check with the reported audited data from 1 of these health boards ${ }^{[11]}$ corroborated these averages.

The greatest single group of records (23\%) removed from the data base related to amputations as a result of accidents of all types including medical misadventure. This would indicate that it would be unwise to measure the incidence of critical leg ischaemia based solely on total amputation data as this would result in an overestimation of costs.

The incidence of amputations for critical leg ischaemia estimated in this study was 202 (ICD procedure method) per million people. If it is assumed that $25 \%$ of critical leg ischaemia patients have amputations, ${ }^{[1]}$ the estimate of the incidence of critical leg ischaemia in New Zealand is 808 per million people, which compares with rates found in developed countries of between 500 and 1000 per million people. ${ }^{[1]}$

As one-third of patients are readmitted to hospital, the prevalence of critical leg ischaemia if derived from amputations and/or admissions will be overestimated. This is because some, but not all,

Table IV. Summary of costs of amputations for critical leg ischaemia. Because of rounding, individual items may not add exactly to the totals shown

\begin{tabular}{lcc}
\hline Total and average costs & $\begin{array}{l}\text { ICD procedure } \\
\text { method }\end{array}$ & $\begin{array}{l}\text { DRG } \\
\text { method }\end{array}$ \\
\hline Total costs (SNZ million) & & \\
Hospital (all costs) & 12.716 & 6.267 \\
Prostheses & 0.370 & 0.341 \\
Subtotal (quantified medical & 13.086 & 6.608 \\
costs) & & \\
Production loss & 2.764 & 1.901 \\
Total quantified costs & 15.850 & 8.509 \\
Number of procedures & 688 & 353 \\
Average cost per procedure (SNZ) & \\
Quantified medical costs & 19020 & 18720 \\
Production loss & 4017 & 5385 \\
Total cost & 23038 & 24105 \\
\hline Abbrevatios: DRG
\end{tabular}

Abbreviations: $D R G=$ Diagnosis Related Group; ICD = International Classification of Diseases. 
patients with readmission indicators will be readmitted during the course of a year. However, this will not result in costs being overestimated because costs were calculated on the basis of all admissions over the period of 1 year.

There were fewer female patients and little variation in average age between males and females, but female patients had longer average hospital stays. This may indicate possible inadequate early treatment of female patients.

The costs per amputation represent a weighted average taking into account the mix of different types of amputation and requirements for artificial limbs, and as such, allow true comparisons to be made between treatment options. To compare the treatment costs of iloprost and surgery it was assumed that the only difference in usage of medical resources between each mode of treatment was the hospital, surgery, prosthesis and iloprost cost. In other words, it was assumed that costs. up to the time of intervention either by amputation or treatment with iloprost, would have been identical.

Treatment with iloprost has been shown to lower mortality, reduce amputation rates. ${ }^{[3]}$ heal ischaemic ulcers and to relieve rest pain. ${ }^{[9]} \mathrm{By}$ avoiding or postponing amputation ${ }^{[5]}$ iloprost improves quality of life for the patient and reduces amputation costs. If amputation is simply postponed, the patient may have an improved quality of life and amputation costs will be reduced by being postponed. These benefits must be offset against the additional cost of the pharmacotherapeutic treatment.

If iloprost is administered for 20 days there have to be 1.2 amputations avoided, that is, 1.2 successful treatments to pay for 1 failure. The success rate must be $55 \%$ to break even on costs. However, if iloprost is administered for $2+$ days there have to be 1.9 amputations avoided, that is, 1.9 successful treatments to pay for 1 failure. The success rate must be $66 \%$ to break even on costs. Iloprost has the potential to either reduce hospital costs or increase them depending on the patients chosen for treatment. If it is possible to target treatment to those patients most likely to benefit from it (that is, those who can avoid amputation and who will have a reduction in rest pain) the maximum benefit would be obtained from this pharmacotherapeutic option.

Iloprost as an alternative to amputation would also become more cost effective if it could be administered orally or in a lower cost hospital setting such as a day clinic ${ }^{[2]}$ or at a time convenient to the patient. These changes would minimise disruption to work and loss of production. Additional cost savings for successful pharmacotherapy would accrue if production loss avoided was included as a benefit.

\section{Acknowledgements}

This study was funded by a grant from Schering (N.Z.) Limited.

\section{References}

1. European Working Group on Critical Leg Ischaemia. Second European Consensus Document on Chronic Critical Leg Ischaemia. Circulation 1991: $8+$ (Suppl): IV 1-26

2. Dormandy JA. Stock G editors. Critical leg ischaemia: its pathophysiology and management. Berlin: Springer-Verlag, 1990

3. UK Severe Limb Ischaemia Study Group. Treatment of limb threatening ischaemia with intravenous iloprost: a randomised double-blind placebo controlled study. Eur J Vas Surg 1991: 5: 511-6

4. Norgren L. Alwmark A. Angquist KA, et al. A stable prostacyclin analogue (iloprost) in the treatment of ischaemic ulcers of the lower limb: a Scandinavian-Polish placebo controlled, randomised multicenter study. Eur J of Vasc Surg 1990: 4: $463-7$

5. Grant SM. Goa KL. Iloprost: a review of its pharmacodynamic and pharmacokinetic properties, and therapeutic potential in peripheral vascular disease, myocardial ischaemia and extracorporeal circulation procedures. Drugs 1992; 43: 889-924

6. New Zealand Department of Health. Health Statistical Services. Unit record data relating to public and private hospitals for 1991. (National computerised data base of individual patient records) Wellington

7. Core health \& disability support services for 1993/94. First report of the National Advisory Committee on Core Health and Disability Support Services. National Advisory Committee, Wellington. 1992

8. New Zealand Department of Statistics. INFOS. (Information Network for Official Statistics) Total average weekly earnings for males and females, Wellington, June 1991

9. Dormandy J. Use of the prostacyclin analogue iloprost in the treatment of patients with critical limb ischaemia. Therapie 1991; 46: 319-22

10. Scott WG, Scott HM. Annual costs of benign prostatic hyperplasia in New Zealand. PharmacoEconomics 1993; 4: 455-68

11. Solomon C. van Rij AM. Packer SGK, et al. Amputations in the surgical budget. N Z Med J 1994; 107: 78-80

Correspondence and reprints: Helen Scott, PO Box 14403, Wellington, New Zealand. 


\section{CASE 8: HEART FAILURE}

\section{Discussed in chapter 15}

\section{Reference}

Scott, W. G., and Scott, H. M. "Heart Failure: a decision analytic analysis of New Zealand data using the published results of the SOLVD treatment trial". PharmacoEconomics 1996; 9 (2): 156-167. 


\title{
Heart Failure
}

\section{A Decision Analytic Analysis of New Zealand Data Using the Published Results of the SOLVD Treatment Trial}

\author{
W. Guy Scott ${ }^{1}$ and Helen M. Scott ${ }^{2}$ \\ 1 Wellington Polytechnic, Wellington, New Zealand \\ 2 Health economics consultant, Wellington, New Zealand
}

\section{Summary}

"Increased usage of ACE inhibitors raises the costs of medication, but on the basis of clinical evidence it may be expected that life-years will be saved, quality of life will improve and the frequency of hospital admissions will decrease. "II The purpose of this study was to evaluate the changes in mortality, direct medical costs and life-years gained or lost by adding the ACE inhibitor enalapril to the conventional pharmaceutical regimen (digoxin and diuretics) for heart failure (HF). The published results of the Studies of Left Ventricular Dysfunction (SOLVD) Treatment Trial, ${ }^{[2]}$ and a decision analytical model (Version 3.0) developed by Henry Glick of the University of Pennsylvania in collaboration with investigators from Merck \& Company, were used in combination with New Zealand data to evaluate the possible changes. (Details of the model are described in an unpublished technical appendix available from the developers.) It was appropriate to apply the model to New Zealand because treatment for HF is similar in both the US and New Zealand.

HF occurs when the heart muscle is damaged, overloaded or exhausted by such conditions as hypertension, coronary artery disease, cardiac valve damage, cardiomyopathy and arrhythmias. About one-third of those with HF are hospitalised each year, ${ }^{[2]}$ and the 1-year mortality ranges from $15 \%$ 
among relatively unselected patients to $50 \%$ among those in New York Heart Association (NYHA) functional class IV. ${ }^{[3]} \mathrm{HF}$ incurs direct costs associated with primary medical care, hospitalisation and pharmaceutical treatment, and indirect costs associated with lost production (lost output or productivity), a reduced quality of life (intangible costs) and death. All these costs will increase as the population ages and survival rates for cardiovascular events continue to improve, thus increasing the numbers of people likely to die of $\mathrm{HF}$.

The SOLVD investigators ${ }^{[2]}$ studied the effect of enalapril on mortality and hospitalisation in patients with HF. They found that enalapril treatment substantially reduced morbidity, hospitalisation and mortality. In the SOLVD Treatment Trial arm of the study, the 2569 patients with HF were randomised to receive enalapril or placebo in addition to conventional treatment with digoxin and diuretics. 1284 patients were randomised to placebo, and 1285 were randomised to enalapril ( 2.5 or $5 \mathrm{mg}$ twice daily initially, to a maximum of $10 \mathrm{mg}$ twice daily). The main aim of the trial was to assess the effect of enalapril on mortality, but it was also hoped that additional information would be obtained on hospitalisation and morbidity due to HF.

Patients with HF who were treated with enalapril in addition to conventional therapy showed a significant reduction in mortality of $16 \%$ at the scheduled study end and a $26 \%$ reduction for the combined end-point of mortality or hospitalisation. Although the difference in mortality was still apparent after 4 years of therapy, the difference was greatest in the initial 2 years of the study. The most common cause of death was worsening $\mathrm{HF}$, which accounted for about half of the total number of deaths. More patients in the placebo group required additional therapy in the form of vasodilators to treat worsening $\mathrm{HF}$, but as this was not taken into account in the final analysis of the trial, the benefit of enalapril was conservatively estimated.

The SOLVD investigators'[2] trial results were consistent with other smaller trials of enalapril such as that conducted by the Co-Operative North
Scandinavian Enalapril Survival Study (CONSENSUS) Trial Study Group. ${ }^{[3]}$ However, entry to the CONSENSUS trial and other small studies was restricted to patients with severe $\mathrm{HF}$, whereas the SOLVD study included a broader range of patients with clinically stable HF and a longer trial time period (average 41 months in comparison with an average of a few months in the other studies). The greatest benefit seen in both the CONSENSUS and SOLVD studies was the reduction in mortality from progressive heart disease. These findings have been supported by results from the second Veterans Administration Co-Operative Vasodilator-Heart Failure Trial (V-HeFT II). ${ }^{[4]}$

$\mathrm{HF}$ (as defined by ICD-9 primary diagnosis code $428^{[5]}$ ) in New Zealand accounted for $1.70 \%$ of all deaths, ${ }^{[6]} 0.87 \%$ of all hospital admissions and $1.91 \%$ of all public hospital bed days in 1992. ${ }^{[7]}$ These public hospital bed days equate to approximately 23 million New Zealand dollars (\$NZ) [1.91\% of public surgical and medical hospital expenditure $\left.{ }^{[8]}\right]$. If a broader definition of HF is employed (additional ICD codes and secondary and tertiary diagnoses codes, as used by Doughty et al. ${ }^{[9]}$ ) the numbers of deaths and hospitalisations, and estimated costs, would increase substantially - in the order of 50 to $100 \%$.

In 1992,456 people aged 60 years and over $(168$ men and 288 women) died from HF in New Zealand, a rate of 86 per 100000 within this age group (71 per 100000 for men, 98 per 100000 for women) [table I]. Mortality rates rise with increasing age; almost all (88.3\%) deaths due to HF occur in those aged 80 years and over, with only $1.5 \%$ of deaths occurring in people under 60 years of age. As there are more women than men aged 80 years and over, there are more deaths recorded for women than for men ( $65 \%$ of all people who died from HF were women). Although the number of deaths from HF for those aged 60 years and over has risen by $8 \%$ between 1980 and 1992 , the rate per 100000 people in this age group has fallen from 99 to 86 . Figure 1 shows the rates per 100000 aged 60 years and over dying from HF between 1980 and 1992. 
Table 1. Mortality from heart failure (ICD-9 428 ${ }^{[5]}$ ) in New Zealand (1992 numbers and age-specific rates from death certificate data). ${ }^{[6]}$ As a result of rounding, individual items may not add exactly to the totais shown

\begin{tabular}{|c|c|c|c|c|c|c|}
\hline \multirow[t]{2}{*}{ Age group (years) } & \multicolumn{3}{|c|}{ Rate of deaths per 100000 people } & \multicolumn{3}{|c|}{ Number of deaths } \\
\hline & men & women & total & men & women & total \\
\hline $0-59$ & 0 & 0 & 0 & 5 & 2 & 7 \\
\hline $60-64$ & 4 & 1 & 3 & 3 & 1 & 4 \\
\hline $65-69$ & 5 & 6 & 5 & 3 & 4 & 7 \\
\hline 70-74 & 13 & 12 & 13 & 6 & 7 & 13 \\
\hline 75-79 & 41 & 22 & 30 & 13 & 10 & 23 \\
\hline 80-84 & 299 & 203 & 238 & 53 & 63 & 116 \\
\hline$>84$ & 927 & 867 & 884 & 90 & 203 & 293 \\
\hline$>60$ & 71 & 98 & 86 & 168 & 288 & 456 \\
\hline All ages & 10 & 17 & 14 & 173 & 290 & 463 \\
\hline
\end{tabular}

Abbreviation: ICD-9 = International Classification of Diseases, 9th rev. ed.

The statistics show higher age-specific death ates from $\mathrm{HF}$ for older men than for older women. However, because of the greater number of women and of female deaths in the older age groups, the revalence rates for all ages and for the aggregated group aged 60 years and over are higher for women. This apparent anomaly is known as Simpson's paradox. ${ }^{[10]}$

During 1992, hospital admissions for HF folowed a similar pattern to that of deaths (as described above), with admission rates rising with age.
Hospital admissions, as would be expected, cover a younger age group than those dying. The age cohort 80 years and over accounts for $36 \%$ of hospital admissions for HF, 70 years and over for $71 \%$, and 65 years and over for $82 \%$. Table II illustrates 1992 admissions to all New Zealand hospitals (both public and private), displayed as total numbers and age-specific rates. The admission rates and numbers were calculated at the time of discharge or death; that is, at time of hospital separation. Between 1980 and 1992, hospital admissions for HF

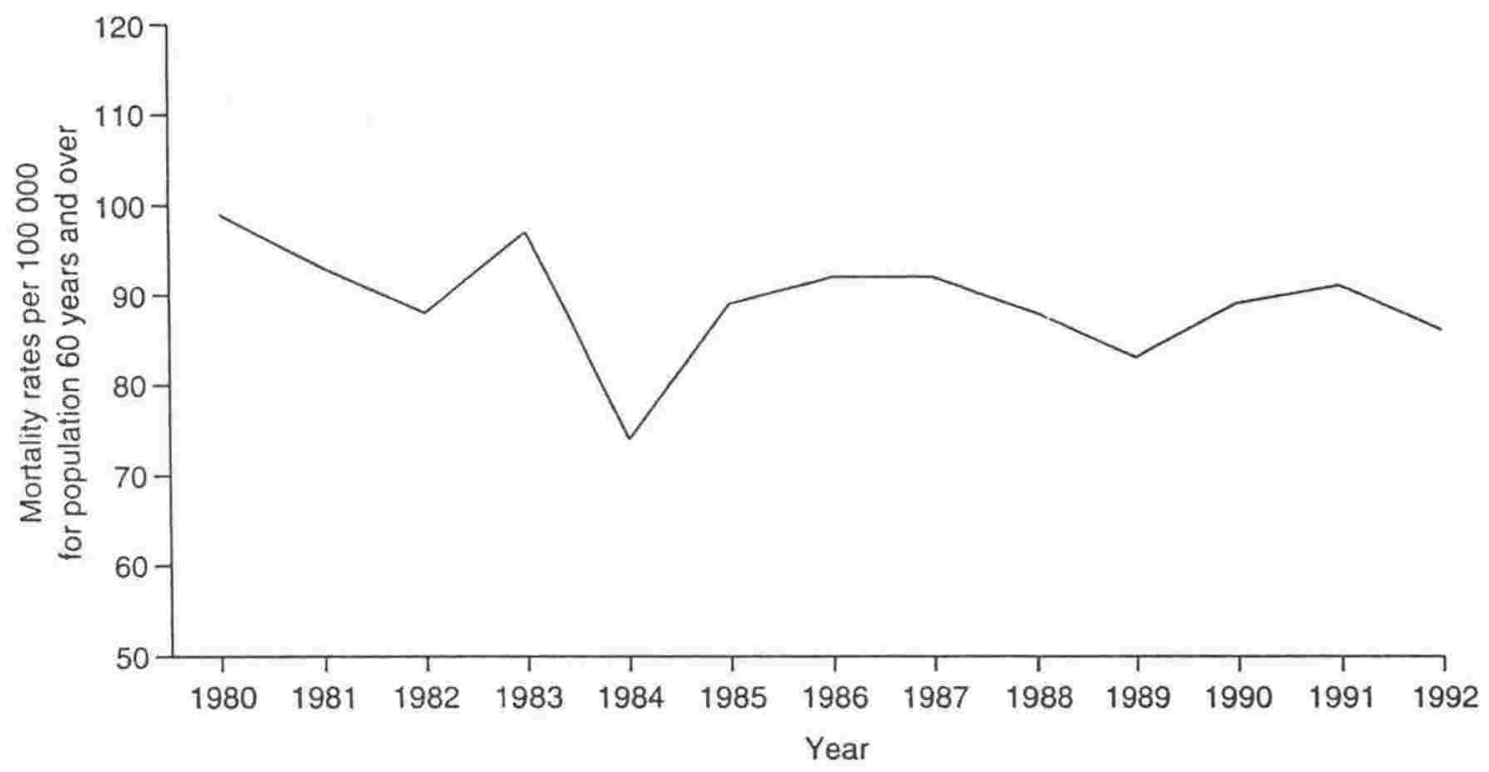

Fig. 1. The mortality rate from heart failure between 1980 and 1992 among the New Zealand population aged 60 years and over. ${ }^{[6]}$ The sharp dip in 1984 was seen in death rates in general and is not specific to heart failure. 
Table II. Hospital admissions for heart failure in 1992 for all New Zealand hospitals, based on hospital separations (i.e. recorded at time of death, live discharge or transfer to another hospital). ${ }^{[7]}$ As a result of rounding, individual items may not add exactly to the totals shown

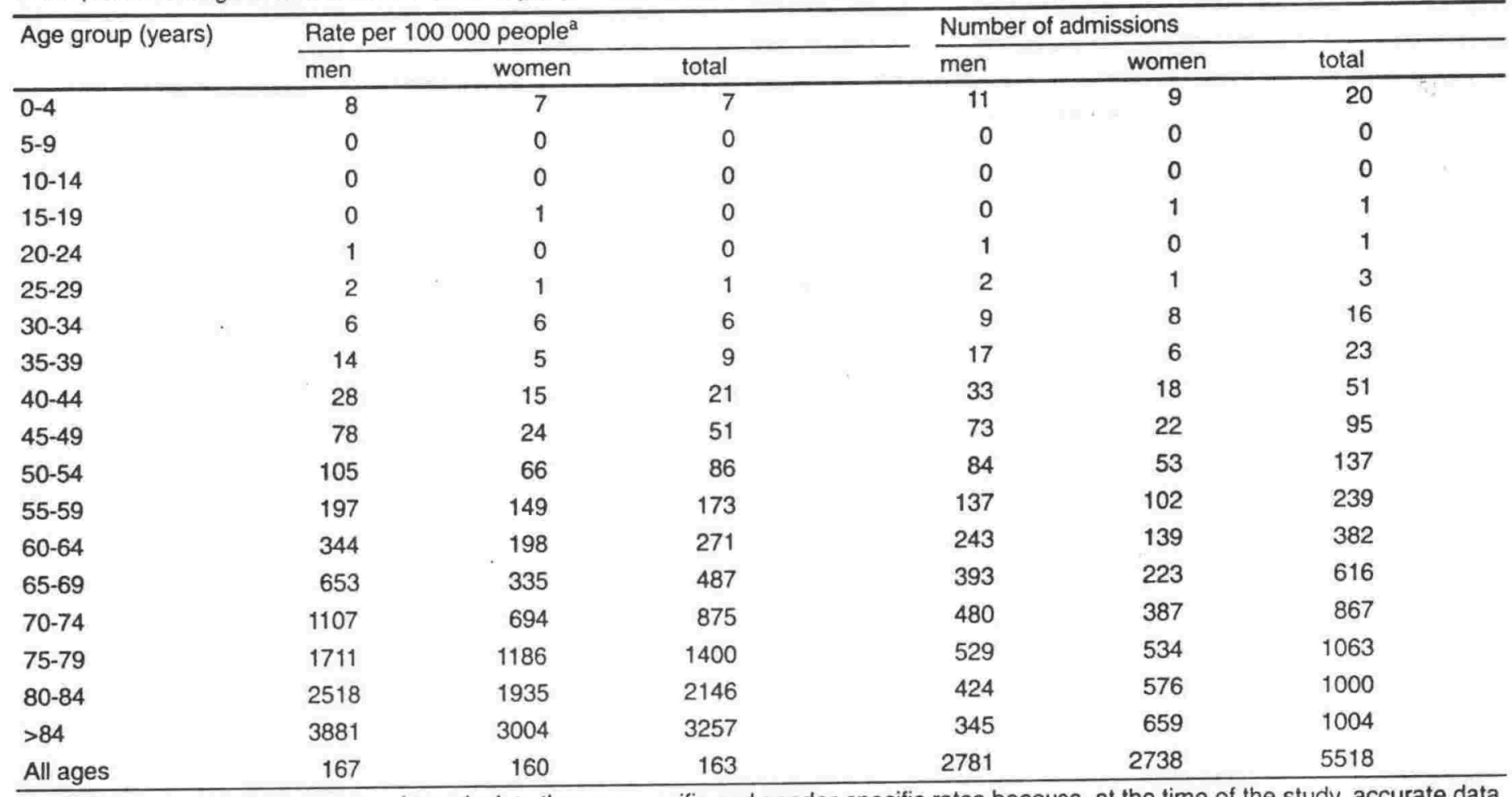

a 1991 population data were used to calculate the age-specific and gender-specific rates because, at the time of the study, accurate data were unavailable for 1992.

increased by $36.7 \%$; from 4038 in 1980 to 5518 in 1992 (128 per 100000 in 1980 to 163 per 100000 in 1992). ${ }^{[7]}$ Figure 2 shows the rates of hospital admissions for HF.

\section{Methods}

The study was performed from a healthcare provider's perspective, and covers both government and privately funded goods and services. All transfer payments such as goods and services tax (GST) were excluded from the analysis. All costs and benefits were estimated incrementally, but the study did not evaluate indirect and intangible costs. The most appropriate and up-to-date unit costs available were used and, where necessary, were adjusted to September 1993 dollars using the health sector cost index (HSCI; see appendix). All unit costs and prices are in 1993 New Zealand dollars (\$NZ1 = \$US0.5509, September 1993).

The decision analytical model enabled data from different sources to be used, and allowed key inputs to be varied in order to evaluate the impact on costs and to test the assumptions made. Monthly survival probabilities were derived from the SOLVD Treatment Trial using parametric failure time survival models assuming underlying Weibull distributions. The enalapril dosages at the final visit of the trial were used to estimate the cost of enalapril. The New Zealand input data for the model were derived from a variety of sources, as described in the following paragraphs. A discount rate of $5 \%$ was used for both costs and benefits as the baseline, and the New Zealand Treasury rate of $10 \%$ for public sector project evaluation was used as the high value for sensitivity analysis.

The Weibull distribution (an accelerated parametric time failure survival method) was used to estimate the medical effect and economic cost of extending enalapril treatment to those patients with HF not currently being treated with an ACE inhibitor. The Weibull distribution was chosen in preference to orúinary least squares or numeric in- 


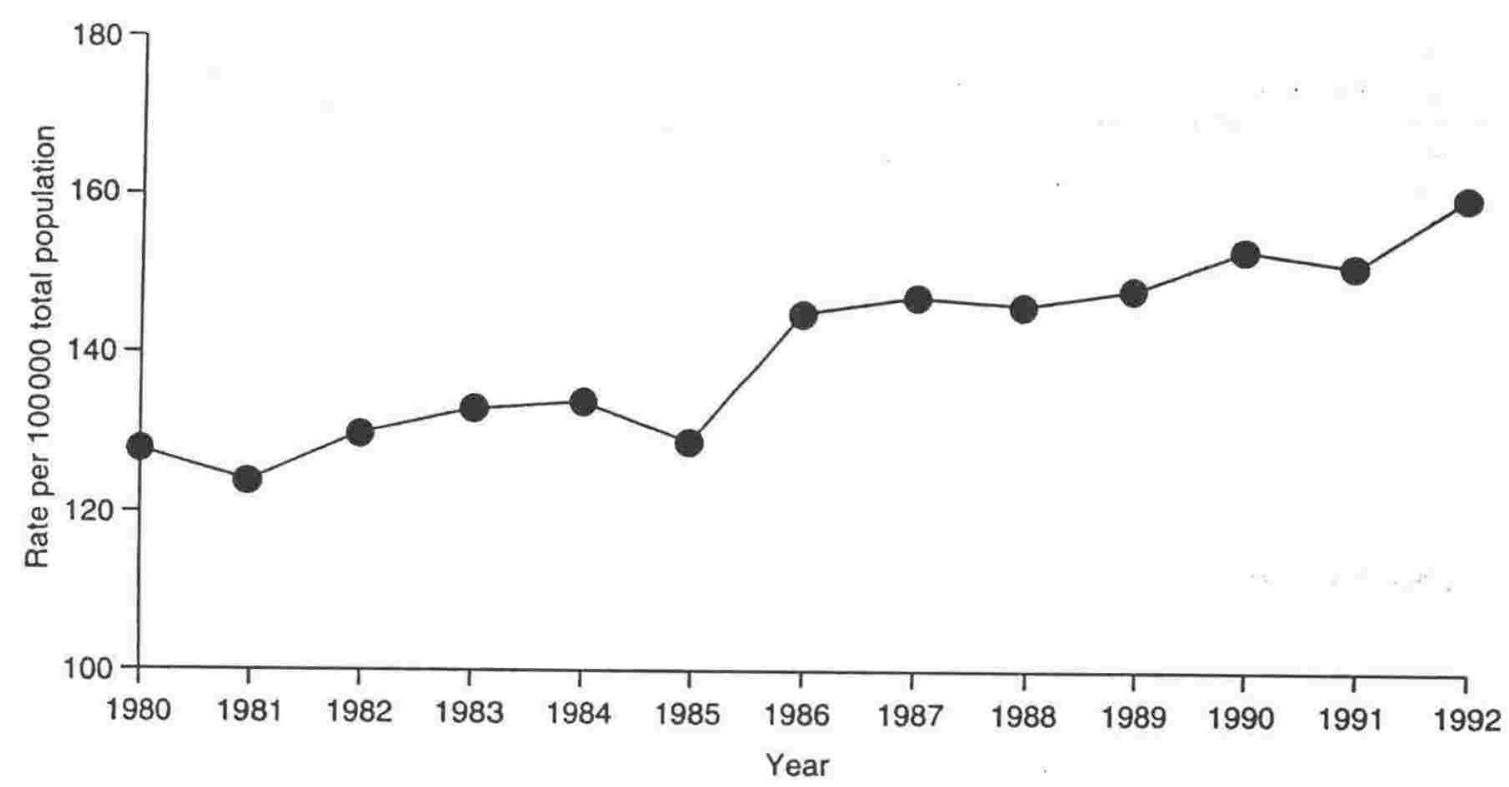

Fig. 2. Hospital admissions for heart failure in New Zealand (total population). ${ }^{[7]}$

egration of linear extrapolation estimates (although all models gave similar results), because unpubished work by the SOLVD investigators indicated hat it provided the best fit to their data.

There are no reliable data on age-specific rates or HF or the numbers of HF patients that would elate to the NYHA classes. In the absence of this lata for New Zealand, age-specific rates from the JS were applied to the New Zealand population. The prevalence rates from Kannel and Belanger, ${ }^{[1]}$ vhen applied, gave a prevalence of 24155 and hose of Schocken et al. ${ }^{[12]}$ a prevalence of 35171. These figures were rounded to the nearest 1000 vhen applied to the model. It should be noted that he rates of Schocken et al., ${ }^{[12]}$ although giving a iigher prevalence, considered only the population ged 25 to 74 years and thus excluded a large group f patients with HF.

Table III shows the application of US agepecific prevalence rates ${ }^{[11,[2]}$ to the New Zealand opulation. A baseline and low value of 24000 and high value of 35000 (representing $1 \%$ of the total opulation) were used for the number of people vith HF. A survey ${ }^{[13]}$ that monitors primary care loctors' prescribing for cardiovascular disease inlicated that, in New Zealand, $43 \%$ of the populaion with $\mathrm{HF}$ were not being treated with an ACE inhibitor. When applied to the population with HF, this percentage gave a range of 10000 to 15000 people who were untreated with ACE inhibitors to whom enalapril treatment could theoretically be extended. A baseline value of $100 \%$ of this potential treatment group was used, with sensitivity analysis ranging from a low of $50 \%$ to a high (and baseline) of $100 \%$.

The cost per tablet of enalapril was $\$ \mathrm{NZ} 0.5558$ for 5mg, \$NZ0.9062 for 10mg and \$NZ1.7212 for $20 \mathrm{mg} \cdot{ }^{[14]}$ As a $2.5 \mathrm{mg}$ tablet was unavailable in New Zealand for the purposes of the model, such a tablet was assumed to be half the price of the $5 \mathrm{mg}$ tablet. Sensitivity analysis was performed on all tablet prices by varying them by $\pm 20 \%$ of the baseline values.

It was assumed that, as enalapril was added to existing treatment, there would be no extra medical practitioner consultations. However, an additional dispensing fee and container charge amounting to $\$ N Z 2.6009$ per 3 months or \$NZ0.87 per month was allocated for enalapril. ${ }^{[14]}$ It was also considered that, as patients with HF would be consulting their medical practitioners on a regular basis, no additional monitoring would be required. This premise was also supported by the published results of 
SOLVD, in which no additional monitoring was reported.

However, as patients treated with enalapril lived an average of 2 months longer than those patients treated with conventional medicines, the incremental routine care costs incurred by enalapril patients during the additional 2 months were incorporated in the model. It was estimated (from discussions with pharmacists), and subsequently verified, ${ }^{[13]}$ that New Zealand patients with $\mathrm{HF}$ routinely visit their medical practitioner on average once every 3 months for monitoring of their condition and a new prescription for a further 3 months' supply of conventional medicine. Thus, two-thirds of the 3-month cost/or 90 days' supply of the following were allocated to the model, to give: medical practitioner consultation ( $\$ N Z 16.09$ ), conventional therapy [digoxin $\$ N Z 2.91$, furosemide (frusemide) $\$ N Z 2.04]$, and dispensing fee and container charge

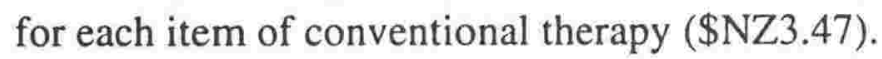

The total of these additional non-enalapril drug costs or routine care costs was $\$ N Z 24.51$. These costs were incorporated in the model as a per-month cost by dividing by the total undiscounted months of enalapril survival ( 38 months) to give a value of $\$ N Z 0.65$. This value was added to the nondrug costs of the model to give a high value (\$NZ0.87 $+\$ N Z 0.65=\$ N Z 1.52$ ) for sensitivity analysis. Medical costs for treatment of conditions other than for HF were not evaluated for these surviving patients.

The proportion of deaths due to arrhythmia unassociated with worsening HF, observed in the treatment arm of the SOLVD trial, was $22.7 \% .^{[2]}$ This proportion of deaths was used for New Zealand, as no other data were available. Sensitivity analysis was applied to the baseline value using $10 \%$ as the lower limit and $35 \%$ as the upper limit. This range was considered to be a reasonable spread around the baseline value, as the SOLVD investigators noted a divergence of opinion in the medical literature regarding mortality in patients with HF.

It was considered that, if a death occurred outside hospital, either an ambulance or a general practitioner (GP), or both, would have been called to attend to the patient. Thus, the cost of an ambulance and the cost of a medical practitioner call-out were used for the estimation of the cost of deaths outside hospital. During the year ending March 1992, the average total cost per patient for the New Zealand Ambulance Board was \$NZ159.74 (New Zealand Ambulance Board, personal communication) and was adjusted to \$NZ162 (September 1993 dollars) per call-out.

A typical total call-out fee for certification of death by a GP as at September 1993 was around $\$ N Z 50$. Rates varied considerably depending on the day (holiday, weekend, weekday), the time of day and geographic location. The range of $\$ N Z 30$

Table III. Prevalence of heart failure in New Zealand (NZ) after application of US age-specific prevalence rates ${ }^{[11.12]}$ to the NZ population. As a result of rounding, individual items may not add exactly to the totals shown

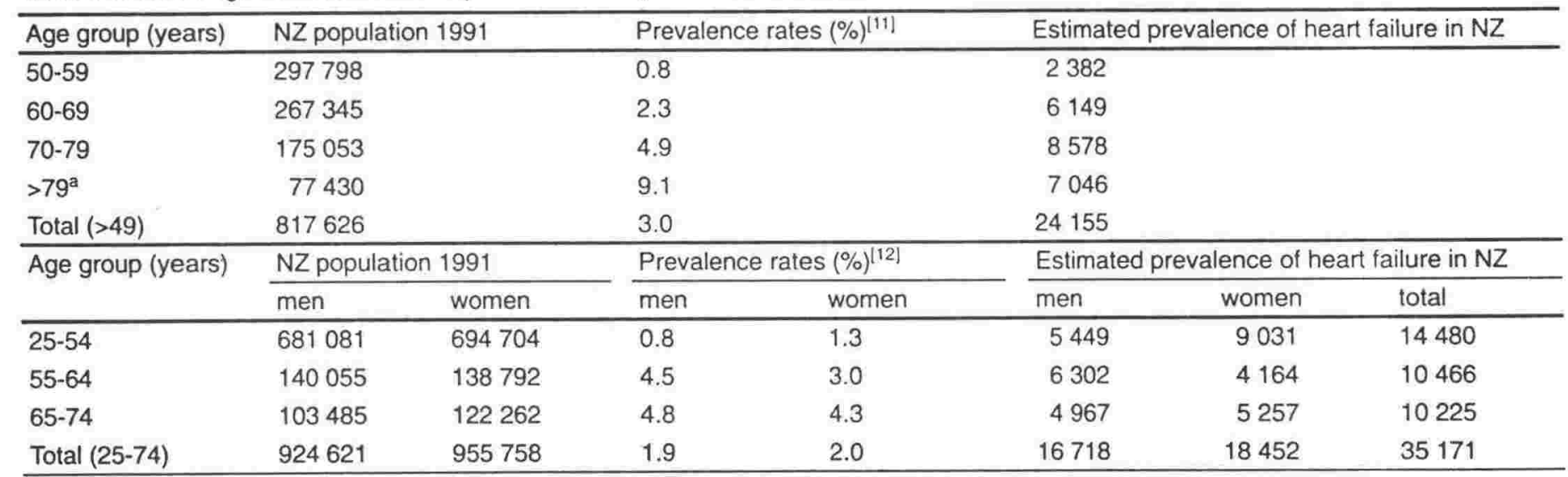

a US data uses the age group 80 to 89 years, but NZ data includes those aged 90 years and over. 
to $\$ N Z 75$ for a total GP fee was used for sensitivity analysis (informal telephone survey of GPs and medical centres in Wellington and Auckland, New Zealand).

Thus, the baseline value (i.e. the cost of an HF death outside hospital) for September 1993 was \$NZ212 (consisting of the ambulance cost of $\$ N Z 162$ plus the typical total GP call-out fee of $\$ N Z 50)$. Sensitivity analysis was not applied to the ambulance fee, as this was estimated from recorded data. As an ambulance would not always be called, the lowest total GP fee of $\$ N Z 30$ was used as the lower limit of the sensitivity analysis. The upper limit was the ambulance fee of $\$ N Z 162$ plus the high total GP fee of $\$ N Z 75$, as both an ambulance and a GP could be called. This gave an upper limit of \$NZ240 (rounded to the nearest \$NZ5).

Sufficiently detailed data were unavailable to enable separate costs to be calculated for fatal and nonfatal hospitalisations. It was therefore assumed that the hospital costs would be identical whether or not a patient was discharged alive or dead. The limited range of diagnosis-related group (DRG) unit cost data available ${ }^{[15]}$ were supplemented by ICD unit cost data from individual cost of illness studies. As $95 \%$ of all hospital inpatients admitted for HF during 1992 were admitted to public hospitals, ${ }^{[7]}$ the unit costs applicable to public hospitals were used for patients in both public and private hospitals.

With the exception of costs for stroke, the DRG and ICD unit costs fully capture the hospital costs. The possibility that some institutional costs associated with stroke have not been captured was taken into account by using data from a cost of illness study, ${ }^{[16]}$ which indicated that the DRG unit cost could be increased by $100 \%$ for sensitivity analysis. The baseline value plus or minus $20 \%$ was used for sensitivity analysis for all hospital unit costs with the exception of stroke. This variation in range was chosen because cost data from the public sector exhibits considerable variation between hospitals. [17]

The average length of stay in a public hospital in 1992 for acute myocardial infarction (ICD-9
410) was 8.6 days for men and 8.5 days for women. ${ }^{[7]}$ An inpatient with myocardial infarction spent on average 2 days in a coronary care unit and the remainder of their hospitalisation in a cardiology ward. ${ }^{18]}$ The cost for a patient's total time in hospital was based on a stay of 2 days in a coronary care unit at $\$ N Z 1053$ per day, and 6.5 days in a cardiology ward at a daily rate of \$NZ592 using prices relevant to 1 January 1994 (Green Lane Hospital, Auckland, personal communication). Using this methodology, the unit cost per patient with a myocardial infarction for their hospital stay was $\$ N Z 5954$. This cost was deflated using the HSCI to establish a baseline value of \$NZ5948 in September 1993 dollars.

The public hospital unit costs for cardiac arrhythmia for the year ending June $1991^{[15]}$ were divided into DRG 138, age over 69 years and/or complicating condition, and DRG 139, age under 70 years without complicating conditions. A weighted average (by patient numbers) of the unit costs applicable to each of these DRGs was calculated and adjusted to give a baseline value of \$NZ2666. The public hospital DRG 127 unit cost for $\mathrm{HF}$ and shock ${ }^{[15]}$ was adjusted to give a baseline value of $\$$ NZ5030.

The public hospital unit cost for DRG 014, [15] specific cerebrovascular disorders except transient ischaemic attack (TIA), was used for stroke and adjusted to provide a baseline and low value of $\$ N Z 10212$. For sensitivity analysis this baseline value was increased by $20 \%$ and by $100 \%$ to obtain 2 high values. The baseline was increased by $100 \%$ to cover the cost of rest home care for stroke, which is approximately equal to the cost of hospital care. ${ }^{[16]}$

The category 'other causes' used in the model represents the average cost for all other non-HF related hospitalisations. Accordingly, the following diagnoses were excluded from the analysis: heart and chest pain, heart attacks, stroke, and HF and shock. A weighted average of the 20 most common kinds of discharge diagnoses for the year ending June $1991^{[15]}$ was calculated and adjusted to a baseline value of $\$ N Z 2403$. The input data for the 
Table IV. Summary of the input data for the decision analytical model used in the study. All costs are in 1993 New Zealand dollars (\$NZ; \$NZ1 = \$US0.5509, September 1993)

\begin{tabular}{|c|c|c|}
\hline Input data & $\begin{array}{l}\text { Baseline } \\
\text { value }\end{array}$ & $\begin{array}{l}\text { Range for } \\
\text { sensitivity analysis }\end{array}$ \\
\hline $\begin{array}{l}\text { Population with heart failure to } \\
\text { be treated with enalapril }\end{array}$ & 10000 & $\begin{array}{l}\text { Low: } 10000 \\
\text { High: } 15000\end{array}$ \\
\hline $\begin{array}{l}\% \text { Population treated with } \\
\text { enalapril }\end{array}$ & 100 & $\begin{array}{l}\text { Low: } 50 \\
\text { High: } 100\end{array}$ \\
\hline \multicolumn{3}{|l|}{ Enalapril cost per tablet (\$NZ) } \\
\hline $2.5 \mathrm{mg}$ & 0.2779 & $\begin{array}{l}\text { Low: }-20 \% \\
\text { High: }+20 \%\end{array}$ \\
\hline $5 \mathrm{mg}$ & 0.5558 & $\begin{array}{l}\text { Low: }-20 \% \\
\text { High: }+20 \%\end{array}$ \\
\hline $10 \mathrm{mg}$ & 0.9062 & $\begin{array}{l}\text { Low: }-20 \% \\
\text { High: }+20 \%\end{array}$ \\
\hline $20 \mathrm{mg}$ & 1.7212 & $\begin{array}{l}\text { Low: }-20 \% \\
\text { High: }+20 \%\end{array}$ \\
\hline Monthly nondrug cost (\$NZ) & 0.87 & $\begin{array}{l}\text { Low: }-20 \% \\
\text { High: }+20 \% \text { and } \\
\$ N Z 1.52\end{array}$ \\
\hline $\begin{array}{l}\text { Annual \% discount rate for } \\
\text { costs and benefits }\end{array}$ & 5 & $\begin{array}{l}\text { Low: } 5 \\
\text { High: } 10\end{array}$ \\
\hline$\%$ Deaths outside hospital & 22.7 & $\begin{array}{l}\text { Low: } 10 \\
\text { High: } 35\end{array}$ \\
\hline $\begin{array}{l}\text { Cost of deaths outside } \\
\text { hospital (\$NZ) }\end{array}$ & 212 & $\begin{array}{l}\text { Low: } 30 \\
\text { High: } 240\end{array}$ \\
\hline \multicolumn{3}{|l|}{ Hospital unit costs (\$NZ) } \\
\hline heart failure & 5030 & $\begin{array}{l}\text { Low: }-20 \% \\
\text { High: }+20 \%\end{array}$ \\
\hline myocardial infarction & 5948 & $\begin{array}{l}\text { Low: }-20 \% \\
\text { High: }+20 \%\end{array}$ \\
\hline arrhythmia & 2666 & $\begin{array}{l}\text { Low: }-20 \% \\
\text { High: }+20 \%\end{array}$ \\
\hline stroke & 10212 & $\begin{array}{l}\text { Low: }-20 \% \\
\text { High: }+20 \% \text { and } \\
+100 \%\end{array}$ \\
\hline other & 2403 & $\begin{array}{l}\text { Low: }-20 \% \\
\text { High: }+20 \%\end{array}$ \\
\hline
\end{tabular}

model as described above have been summarised in table IV.

\section{Results}

\section{Baseline Results}

Analysis of the baseline case for an individual patient treated with enalapril in addition to conventional care over the 4-year period showed that an undiscounted total of 1.88 months of life per individual was gained. That is, for every 1000 patients treated with enalapril, there was a total gain of 157 years. The year-by-year breakdown per individual treated in undiscounted months saved was: 0.25 (year 1), 0.48 (year 2), 0.56 (year 3) and 0.59 (year 4).

Over the 4-year period, 343 of 1000 individuals treated with enalapril would die, compared with 390 deaths in 1000 individuals treated only with conventional medicines. In other words, there were 48 fewer deaths for enalapril recipients over the 4-year treatment period. The year-by-year breakdown showed that virtually all the gain in lives saved were made in the first 2 years of treatment (year 1: 69\%; year 2: 21\%; a cumulative total of $90 \%$ ). There were 33 lives saved per 1000 treated patients in the first year, 10 in year 2, 4 in year 3, and 1 life saved per 1000 patients in year 4 .

The model showed that total hospitalisations were reduced by 347 per 1000 individuals over a 4-year period of treatment with enalapril, compared with conventional treatment alone. Virtually all $(86 \%)$ of the 4-year total for hospitalisations avoided occurred within the first year.

The additional costs of drug treatment and medical practitioner fees (\$NZ1064.51) incurred in the community setting by a patient treated with enalapril were more than offset by hospital costs avoided and medical costs of death outside hospital avoided (\$NZ1716.16). Over the 4-year period, there would be total net costs avoided of \$NZ651.65 for each enalapril-treated patient. In addition, a further 2 months of life per patient would be gained.

If enalapril therapy was extended to $100 \%$ (10 000 people) of the population with $\mathrm{HF}$ who are at present untreated with ACE inhibitors, discounted costs of \$NZ6 517000 could be avoided and, in addition, 1583 undiscounted life-years could be gained (19000 months of life gained). A total of 3465 hospitalisations could be avoided, and 476 deaths could be postponed beyond the 4-year period of the study. The baseline estimates for an individual and for the population to whom enalapril treatment could be extended (New Zealand total) are summarised in table V. 
Table V. Baseline estimates of the effects of enalapril for an individual and for the population in New Zealand to whom enalapril treatment could be extended [all costs discounted to present values - September 1993 New Zealand dollars (\$NZ; \$NZ1 = \$US0.5509, September 1993)]. As a result of rounding, individual items may not add exactly to the totals shown

\begin{tabular}{lll}
\hline Estimate & $\begin{array}{l}\text { Per individual } \\
\text { treated }\end{array}$ & $\begin{array}{l}\text { New Zealand } \\
\text { total }\end{array}$ \\
\hline $\begin{array}{l}\text { Population that could } \\
\text { potentially be treated }\end{array}$ & 1 & 10000 \\
$\begin{array}{l}\text { Undiscounted months of life } \\
\text { gained }\end{array}$ & 1.9 & 19000 \\
$\begin{array}{l}\text { Deaths avoided } \\
\text { Hospitalisations avoided }\end{array}$ & 0.0476 & 476 \\
$\begin{array}{l}\text { Costs (\$NZ) } \\
\text { Hospital costs avoided and } \\
\text { medical cost of death outside } \\
\text { hospital avoided } \\
\begin{array}{l}\text { Additional drug, dispensing } \\
\text { and medical practitioner costs } \\
\text { associated with enalapril }\end{array}\end{array}$ & 1716.16 & 3465 \\
\begin{tabular}{l} 
Total net costs avoided \\
\hline
\end{tabular} & 651.65 & 17161600 \\
\hline
\end{tabular}

\section{Sensitivity Analysis}

Sensitivity analysis, applied by using the high and low limits detailed in table IV, demonstrated that the results, on an individual and on a per population basis, are robust with respect to changes in the input variables (table VI). Four factors in particular were found to be major determinants of the potential net cost savings:

- the population with HF to whom treatment could be extended

- the percentage of this population treated with enalapril

- the price of enalapril

- the hospital costs for nonfatal cases of HF.

If either of the first 2 determinants above is changed by a given percentage from the baseline estimates then the total net costs avoided alter by the same percentage. If the enalapril price is altered by $20 \%$ (holding all other input values at their baseline levels) then the total costs saved alter by $32 \%$. The price of enalapril could be increased by $63 \%$ before the total net costs avoided fall to zero. If the unit cost for nonfatal cases of $\mathrm{HF}$ treated in hospital is altered by $20 \%$, then the total net costs saved change by $47 \%$ in the same direction.
Altering the discount rate makes very little difference to the results, as most of the impact on costs occurs in the first year. It might be argued that a death outside hospital is cost efficient to a healthcare system, and if all deaths $(100 \%)$ occur outside hospital, the net savings in the model reduce by $25 \%$. However, this situation would not occur as there will always be some patients admitted to hospital who die from HF.

Although the results are reported using the Weibull distribution, the model was also run using ordinary least squares and linear extrapolation, both of which gave similar results.

\section{Discussion}

HF is an important cause of mortality and of hospital admissions in New Zealand. The growing number of elderly people (who are most at risk of cardiovascular disease), in combination with improved survival rates for cardiovascular events, will increase the number of those who will eventually have this condition.

The SOLVD decision analytical model was used to simulate changes in key determinants of the costs of HF in New Zealand when enalapril was added to conventional treatment. The population with HF to whom treatment could be extended, the percentage of this population treated with enalapril, the price of enalapril and nonfatal hospital costs were found to be the key determinants of the net benefits of the addition of enalapril to conventional HF therapy.

However, the potential cost savings estimated in dollar terms of adding enalapril to conventional treatment for HF do not include the value to society of lives saved, any production (productivity or output) gains, or the increase in quality of life of those patients treated. Changes in productivity could be considered to be relatively unimportant, as most patients with HF are not of working age. However, from the government perspective, prolonging life could impose additional costs with respect to welfare payments.

It could be considered that enalapril recipients and their families would have achieved higher 
quality-of-life scores than those patients treated with conventional medication, this assumption being based on the difference in usage of healthcare resources (as indicated in this analysis) between the 2 groups. The benefit of enalapril was also underestimated, as the SOLVD Treatment Trial did not take into account the additional cost for the greater number of patients in the placebo group who required additional therapy in the form of vasodilators to treat worsening $\mathrm{HF}$.

It is considered unlikely that enalapril treatment could be extended to all patients untreated with an ACE inhibitor because, as with any intervention, a certain proportion of patients will experience adverse effects and will have to be placed on an alternative treatment regimen. In addition, not all patients (and this may include some patients experiencing adverse effects) will comply with the treatment regimen. The study was unable to evaluate the costs and benefits for men and women separately, or beyond a time horizon of 4 years as these factors were not evaluated in the original trial.
The surviving patients will incur medical costs beyond the 4-year trial period, thereby lowering the net benefits of enalapril treatment.

The results of the study demonstrate that treatment of patients with HF with enalapril was cost effective when compared with conventional medical therapy. Sensitivity analysis indicated that the results were robust with respect to changes in key inputs. Within the ranges of the data evaluated, treatment of such patients with enalapril in addition to conventional therapy would both reduce mortality and lower health sector costs.

Application of the model to 3 other countries has also been reported in the literature. Average net cost savings per patient were \$A1100 in Australia, \$Can574 in Canada and FF8076 in France (all currencies March 1992). ${ }^{[19]}$ Although ACE inhibitors slow the progression of the disease, and treated patients stay longer in less severe HF categories and require less hospitalisation, ${ }^{11]}$ the economic benefits of treating asymptomatic patients with enalapril have not yet been assessed.

Table VI. Results of the sensitivity analyses (see table IV). All costs are in 1993 New Zealand dollars ( $\$ N Z ; \$ N Z 1=\$ U S 0.5509$, September 1993)

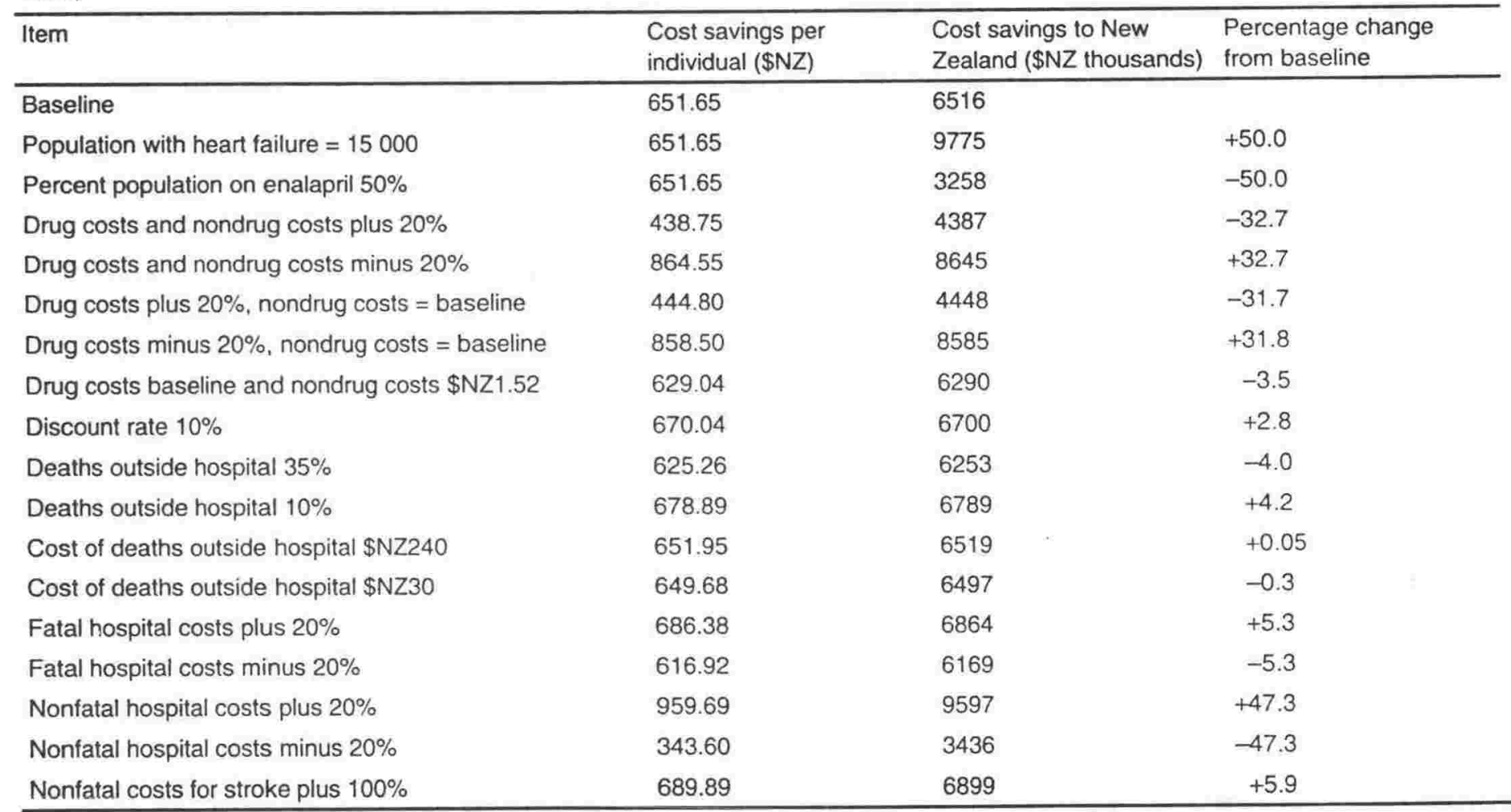


Because healthcare resources are scarce in many countries, the use of health economic models has the potential to provide cost-effective information for policy analysis and decision making. Countryspecific unit costs and epidemiological data should be used if possible as inputs to such health economic models. It is inappropriate to apply the results of a single study to different settings by converting costs and health effects estimated in one country by means of exchange rates and crude population numbers.

\section{Acknowledgements}

We would like to thank Merck Sharp \& Dohme New Zealand Limited for financial assistance for this study, and the developers for their permission to use their decision analytical model.

\section{References}

1. van Hout BA, Wielink G, Bonsel GJ, et al. Effects of ACE inhibitors on heart failure in The Netherlands. PharmacoEconomics 1993 May; 3 (5): 387-97

2. The SOLVD Investigators. Effect of enalapril on survival in patients with reduced left ventricular ejection fractions and congestive heart failure. N Engl J Med 1991; 325: 293-302

3. The CONSENSUS Trial Study Group. Effects of enalapril on mortality in severe congestive heart failure: results of the Cooperative North Scandinavian Enalapril Survival Study (CONSENSUS). N Engl J Med 1987; 316: 1429-35

4. Cohn JN, Johnson G, Ziesche S, et al. A comparison of enalapril with hydralazine-isosorbide dinitrate in the treatment of chronic congestive heart failure. (V-HeFT II). N Engl J Med 1991; 325: 303-10

5. International Classification of Diseases. 9th rev. ed. Clinical Modification (ICD-9CM). Ann Arbor (MI): WHO, 1978

6. Ministry of Health. Mortality and demographic data 1992. Wellington: Ministry of Health, 1994

7. Ministry of Health. Hospital and selected morbidity data 1992. Wellington: Ministry of Health, 1981-93
8. McKendry CG, Muthumala D. Health expenditure trends in New Zealand: update to 1993 [appendix 2A]. Wellington: Ministry of Health, 1994

9. Doughty R, Yee T, Sharpe N, et al. Hospital admissions and deaths due to congestive heart failure in New Zcaland, 1988 1991. N Z Med J 1995; 108 (1012): 473-6

10. Wild CJ, Seber GAF. Introduction to probability and statistics. Auckland: Department of Statistics, Auckland University, 1995

11. Kannel WB, Belanger AJ. Epidemiology of heart failure. Am Heart J 1991; 121: 951-7

12. Schocken DD, Arrieta MI, Leaverton PE, et al. Prevalence and mortality rate of congestive heart failure in the United States. J Am Coll Cardiol 1992; 20: 301-6

13. Taylor Nelson Cardiomonitor - New Zealand, Autumn 1993. Epsom, Surrey: Taylor Nelson Healthcare, 1993

14. Pricing schedules: 1 September 1993. Wellington: Ministry of Health, 1993: 59

15. Core health and disability support services for 1993/94. First report of the National Advisory Committee on Core Health and Disability Support Services [appendix 6]. Wellington: National Advisory Committee on Core Health and Disability Support Services, 1992

16. Scott WG, Scott HM. Ischaemic stroke in New Zealand: an economic study. NZ Med J 1994; 107: 443-6

17. Scott WG, McKendry CG, Scott HM. Methodologies for economic evaluation of high technology in health care. Wellington: Department of Health, 1992

18. Scott WG, White HD, Scott HM. Cost of coronary heart disease in New Zealand. N Z Med J 1993; 106: 347-9

19. Wilde MI, Bryson HM, Goa KL. Enalapril: a review of qualityof-life and pharmacoeconomic aspects of its use in heart failure and mild to moderate hypertension. PharmacoEconomics 1994; 6: 155-82

20. Statistics New Zealand. Personal Computer Information Network for Official Statistics: a computerised database of official statistics for New Zealand and programme for manipulation and analysis of statistical series (PC INFOS). Wellington: Statistics New Zealand, 1993

Correspondence and reprints: Guy and Helen Scott, P.O. Box 14403, Kilbirnie, Wellington 3, New Zealand. 


\section{Appendix}

\section{Unit Costs and Prices in 1993 \\ New Zealand Dollars}

All hospital prices and unit costs were adjusted to September 1993 prices by inflating by a health sector cost index (HSCI). PC INFOS (Personal Computer Information Network for Official Statistics) ${ }^{[20]}$ statistical series and data manipulation routines were used to construct a quarterly HSCI.

The following statistical series were used.

Labour wage rates:

- Prevailing Weekly Wage Rates: Central Government (PWIQ.S4229) December 1977 to June 1993

- Labour Cost Index, Salary and Ordinary Time Wage Rates: Central Government Sector (LCIQ.SA21Z9) December 1992 to September 1993.

Prices for nonlabour inputs:

- Producers Price Index: Inputs Central Government (PPIQ.SIV), December 1977 to September 1993.

All series were converted to a common base. The series 'Prevailing Weekly Wage Rates: Central Government' was discontinued and unavailable from September 1993, and was replaced by a more appropriate series 'Salary and Ordinary Time Wage Rates: Central Government Sector' which commenced in December 1992. A wages index was constructed by splicing the series 'Salary and Ordinary Time Wage Rates: Central Government Sector' on to the discontinued series. Let this series be called 'wages' and the series 'In-
Table A1. Health sector cost index from the March quarter of 1990 to the September quarter of 1993

\begin{tabular}{lc}
\hline Quarter & $\begin{array}{l}\text { Health sector } \\
\text { cost index }\end{array}$ \\
\hline March 1990 & 938 \\
June 1990 & 949 \\
September 1990 & 962 \\
December 1990 & 976 \\
March 1991 & 982 \\
June 1991 & 987 \\
September 1991 & 987 \\
December 1991 & 986 \\
March 1992 & 988 \\
June 1992 & 990 \\
September 1992 & 991 \\
December 1992 & 994 \\
March 1993 & 997 \\
June 1993 & 999 \\
September 1993 & 1000 \\
\hline
\end{tabular}

puts: Central Government' be called 'prices'. The HSCI was constructed by weighting 'prices' as 0.2 , and 'wages' as 0.8 . That is: $\mathrm{HSCI}=(0.8 \times$ wages $)+$ $(0.2 \times$ prices $)$. These weightings were selected because labour costs make up approximately $80 \%$ of the health industry's and individual hospital's total costs.

Table VII details the HSCI from the March quarter 1990 to the September quarter of 1993. 


\section{CASE 9: ISCHAEMIC STROKE}

Discussed in chapter 16

\section{References}

Scott, W. G., and H. M. Scott. "Ischaemic stroke in New Zealand: an economic study." New Zealand Medical Journal 107 (1994): 443-46.

Scott, W. G., and H. M. Scott. "Application of the findings of the European stroke prevention study 2 to a New Zealand ischaemic stroke cost analysis." PharmacoEconomics 12 (1997): 667-74. 


\title{
Ischaemic stroke in New Zealand: an economic study
}

\author{
W Guy Scott, MAgrSc, MNZIAS, ANZIM, Economic Consultant and Lecturer in Health Economics, Wellington \\ Polytechnic, Wellington; Helen Scott, BA, DipNZLS, ANZLA, Research Consultant, Wellington.
}

\begin{abstract}
Aim. To quantify and describe the annual cost of ischaemic stroke to New Zealand society during 1992.

Methods. A prevalence or cross sectional approach employing incremental estimation was used to estimate costs from the perspective of society. The study used unit record hospital and mortality data.

Results. Direct costs ranged from $\$ 93$ million to $\$ 140 \mathrm{~m}$ and loss of production was between $\$ 6 \mathrm{~m}$ and $\$ 14 \mathrm{~m}$. Total quantified costs were estimated to lie between $\$ 99 \mathrm{~m}$ and $\$ 154 \mathrm{~m}$. Hospital and continuing care costs when combined make up $90 \%$ of all quantified costs.

Conclusion. Ischaemic stroke affects mainly older people many of whom are discharged into the community and require costly community support or continuing care. The combination of an ageing population, increasing survival rates and constant incidence rates will result in a substantial rise in the costs of continuing care and community support. Most of these costs are at present obscured because much of the care provided does not involve money payments and/or data are not recorded and collated on a national basis. Incorrect policy decisions will be made if the nonhospital costs of stroke are not taken into account.
\end{abstract}

NZ Med J 1994: 107: 443-6

An economic appraisal was undertaken of the costs of ischaemic stroke to New Zealand society. Cost of illness studies of this nature provide the data that enables different treatment or management options to be evaluated in economic terms.

Stroke has been defined by the World Health Organisation as a rapid onset of disturbances of cerebral function leading to death or lasting for longer than 24 hours with no apparent cause other than vascular. ${ }^{1}$ It results from the interruption of the flow of blood to the brain that can occur with the rupture of an artery wall (cerebral haemorrhage), blood clotting (thrombus), or when a clot lodges in an artery (embolus). It is necessary in clinical practice to differentiate between ischaemic and haemorrhagic strokes as their pathophysiologies and treatment differ. The importance of distinguishing between different types of stroke is discussed by Friedman ${ }^{2}$ who found that $88^{\circ} ;$ of stroke cases of those aged 60 and above admitted to hospital in New Zealand were ischaemic in type. International studies suggest that the proportion of ischaemic stroke to all strokes ranged from $61 \%$ to $83 \%$. $^{3}$

Patients with cerebrovascular disease (of which stroke is a subset) account for the greatest bed day usage in public hospitals (1.5\% of patients and $8.9 \%$ of bed days). ${ }^{4}$ In New Zealand, stroke is the third most common cause of death and both males and females have similar death rates. ${ }^{5}$ In developed countries, stroke is the third ranking cause of death and the most common case of long term disability. ${ }^{6}$ Although there has been no change in the incidence rate for stroke over the last 10 years there has been a decline in the death rate. ${ }^{7}$ This has important consequences for rehabilitation, longterm and community care costs. Ischaemic stroke patients (acute and longstay) are disproportionately high users of hospital resources, accounting for $1 \%$ of all public hospital patients but $5 \%$ of hospital patient days. In comparison, data from Scotland showed that stroke (all forms) accounted for about $4 \%$ of all NHS resources and $5.5 \%$ of hospital resources. ${ }^{8}$

Internationally, age adjusted incidence rates for "first in a lifetime" stroke range between 2 and 7 per thousand people for the age groups 55-64 and 65-74 years. If recurrent strokes (people who have had a stroke) are included, the incidence rates rise by between $20 \%$ and $30 \%$. Prevalence rates (all stroke sufferers) are about 4 to 5 times the incidence rates (new strokes per year). ${ }^{6}$ When data from a Finnish incidence and prevalence study ${ }^{3}$ were used the ratio of the prevalence of stroke survivors dependent on outside help to the incidence of all strokes was calculated to be 1.74. The Perth Community Stroke Study, 1989-90, found that the crude annual event rate for all strokes was 258 per 100000 , and the rate for first strokes was 178 per $100000 .^{9}$ Annual incidence rates per 100000 people (standardised on the world population 15 years and over) for all strokes were 141.5 in Auckland (1991) and 145.6 in Perth (1989-90). ${ }^{10}$ A 27-country study of stroke rates, ranked New Zealand 18th highest for men and 15th highest for women. Australia was ranked 20 for men and 17 for women, England and Wales were ranked 15 for men and 14 for women, and USA was 25 for men and 21 for women. ${ }^{11}$

\section{Methods}

A prevalence or cross section study of the cost of ischaemic stroke from a societal perspective was undertaken. Unit record data for all hospitals in both public and private sectors for the year ended 1992 , and mortality data for the year ended 1990 were analysed. ${ }^{12}$ The following ICD codes were used to define ischaemic stroke; 434 occlusion of cerebral arteries, and 436 acute and ill defined cerebrovascular disease. Longer stay stroke patients may be transferred to another hospital and thus be recorded as a readmission within the same year. To minimise the potential for double counting all hospital stroke patients with stays of more than 90 days were excluded from the hospital data. These patients were not lost to the study as they were captured in the rest home estimates. All identifuble transfer payments, from the perspective of society, were excluded from the analysis of costs which were measured in 1992 New Zealand dollars.

The number of general practitioner consultations (IMS (NZ) Ltd. Data on prescriptions, consultations and costs of medication for the management of stroke. 1993) in combination with the average fee charged $^{13}$ were used to estimate the cost of such consultations. The value of medicine prescribed by general practitioners (IMS (NZ) Ltd, 1993) had the appropriate dispensing fees, container allowances, and markups ${ }^{14}$ added to obtain the cost to society. Transport costs of all ischaemic stroke patients admitted to hospital were evaluated using the average cost of transporting a patient to hospital by ambulance of $\$ 159.74$ (NZ Ambulance Board. Letter, 31 August 1993. Data relating to the financial year 1991/2). Hospital costs were estimated, and then compared, using two 
methods. First, the DRG ${ }^{15} 014$ unit $\cos ^{16}$ of $\$ 10076$ was multiplied by the number of patients. In the second method hospital patient days were multiplied by the daily rate $(\$ 300 / \mathrm{d}$ for public hospitals, ${ }^{17}$ and $\$ 284.44 / d$ for private hospitals). ${ }^{\text {IN }}$

The number of ischaemic stroke events occurring annually was estimated using New Zealand hospital admissions and the assumption that $70 \%$ of stroke cases were admitted to hospital. This was based on a New Zealand study, ${ }^{10,20}$ that found that twothirds of all stroke patients entered hospital, and UK findings that $75 \%$ of patients with acute stroke are admitted to hospital. ${ }^{21}$ This paper constructed a flow chart (Figure 1) of events using a $50 \%$ survival rate and assuming that $50 \%$ of the survivors would require continuing support. The number of patients requiring continuing care or support was based on stroke events, survival rates of $50 \%$ (at 3 to 5 years after an acute stroke event, most dying within the first month), ${ }^{21}$ and the percentages of survivors permanently disabled or needing community support. US data indicated that about half of stroke survivors had major motor problems and up to $30 \%$ of stroke survivors may be permanently disabled. 6.21
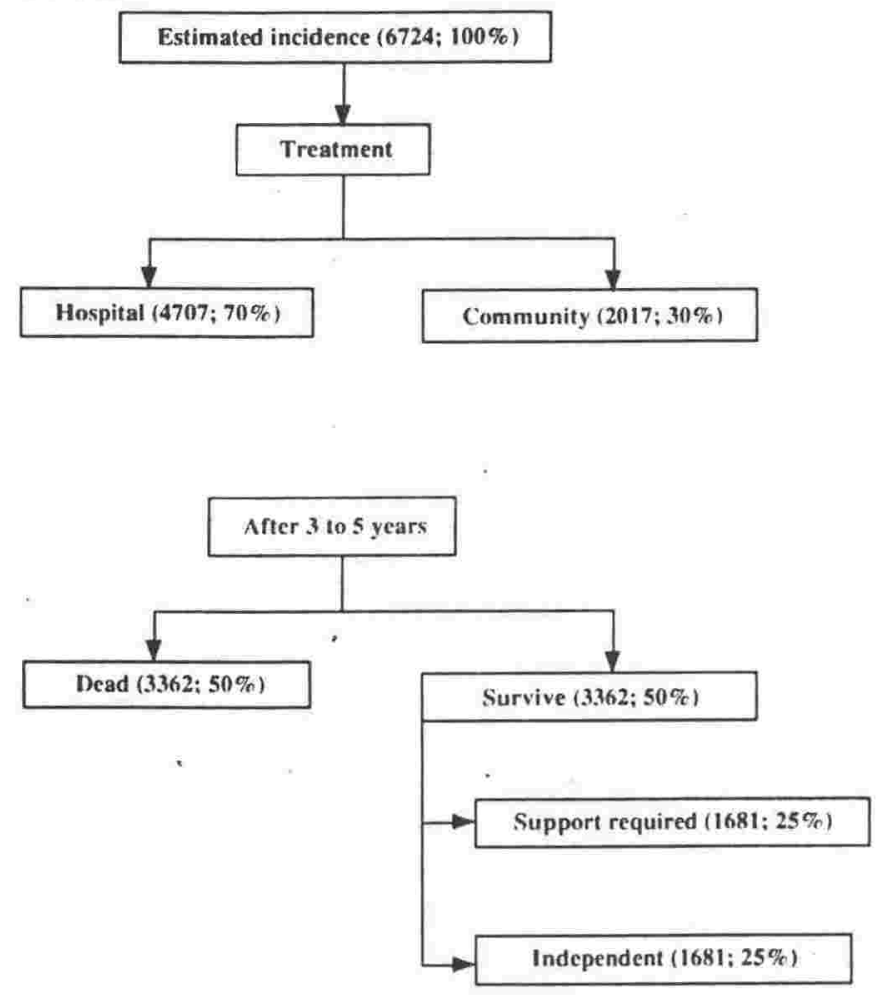

Figure 1. - Annual incidence ischaemic stroke

As the costs of continuing care are unavailable both nationally and in a disease specific form the following methods of measurement were employed. The cost of continuing care in institutions was valued in two ways. The first method evaluated the annual cost of a caregiver (either unpaid or paid) based on the total average weekly earnings for males and females combined of $\$ 579.90{ }^{22}$ The second method used $\$ 401.34$ per week as a proxy for unit cost (Ministry of Health average of stage 1 and stage 2 rest home subsidy relating to $1992 / 3$. Personal communication, 1993) Friedman ${ }^{2}$ found that $48 \%$ of all ischaemic stroke patients 160 years and over) surviving a stroke were discharged to rest homes or geriatric hospitals. This percentage (rounded to $50 \%$ ), with hospital discharge data (live discharges of patients over 60 years) were used to estimate the number of patients discharged to rest homes or geriatric hospitals during 1992

Calculations using data from Finland ${ }^{3}$ indicated that the number of stroke patients dependent on outside help could be estimated by multiplying the annual incidence of stroke by 1.74 . Those permanently institutionalised accounted for $48.8 \%$ of those dependent on outside help, and $74 \%$ of these permanent institution residents were there as a result of the stroke. The incidence data from Finland were used to estimate the requirements for physiotherapy and speech therapy sessions within the first year after stroke. The study found that $52 \%$ of patients were given on average 27 physiotherapy sessions thus, the average stroke patient was given 14 sessions $(1 \times 0.52 \times 27=14.0)$.

In addition the first year after stroke $13.3 \%$ of patients were given on average 14.1 speech therapy sessions thus the average stroke patient was given 1.9 sessions $(1 \times 0.133 \times 14.1=1.9$ ). Our study costed such sessions at a rate of $\$ 20$ per session based on an informal survey of private physiotherapy charges.
Loss of production was valued at the total average weekly earnings for males and females combined over the year ended December 1992. ${ }^{22}$ This valuation was made for those in the working age group 15 to 64 years inclusive, using a lower bound of 12 weeks off work, and an upper bound of 26 weeks off work. These upper and lower bounds were chosen after discussion with health professionals involved in rehabilitation of stroke patients as there was no other national information available. No deduction was made for non-labour force participation or unemployment. It was considered that non-paid output and leisure time had a value to society and lacking more precise alternatives could be valued in the same manner as paid output. Patients discharged dead from hospital were excluded from the analysis.

\section{Results}

The analysis of 1992 unit record data for all hospitals ${ }^{12}$ showed that $98 \%$ of all ischaemic stroke admissions were to public hospitals. Routine and self discharges accounted for most of the discharge types from all hospitals (63\%), but $22 \%$ of ischaemic stroke patients died in hospital.

It was estimated that there were 6724 ischaemic stroke events in 1992; 4707 were admitted to hospital (70\%), and 2017 were treated in the community (30\%). Table 1 shows that the crude hospital admission rate (separations) for all ages was 135 per 100000 people. The age specific rates rise as the age of the cohort increases; 33 for the 40 to 49 age group to 1764 for the age cohort 80 years and over. Figure 1 describes the survival and future continuing care patterns of the estimated 6724 people suffering ischaemic stroke events in 1992.

Table 1. - Hospital separations and mortality 1992: Ischaemic stroke

\begin{tabular}{lccccc}
\hline $\begin{array}{c}\text { Age cohort } \\
\text { Years }\end{array}$ & $\begin{array}{c}\text { NZ } \\
\text { Population } \\
(000)\end{array}$ & $\begin{array}{c}\text { Hospital separations } \\
\text { Total } \\
\text { number }\end{array}$ & $\begin{array}{c}\text { Discharged dead } \\
\text { Rer } 100000\end{array}$ & $\begin{array}{c}\text { Dotal } \\
\text { Tom of } \\
\text { number }\end{array}$ & $\begin{array}{c}\text { separations } \\
\text { to } 9\end{array}$ \\
551370 & 6 & 1 & & \\
10 to 19 & 525480 & 4 & 1 & & \\
20 to 29 & 551280 & 30 & 5 & & $7.27 \%$ \\
30 to 39 & 555370 & 55 & 10 & 4 & $8.28 \%$ \\
40 to 49 & 442520 & 145 & 33 & 12 & $8.03 \%$ \\
50 to 59 & 320170 & 386 & 121 & 31 & $14.70 \%$ \\
60 to 69 & 273040 & 925 & 339 & 136 & $21.67 \%$ \\
70 to 79 & 188460 & 1629 & 864 & 353 & $31.24 \%$ \\
80 and over & 86580 & 1527 & 1764 & 477 & $21.52 \%$ \\
All ages & 3494270 & 4707 & 135 & 1013 & \\
\hline
\end{tabular}

Excludes hospital stays of more than 90 days \# Discharges and deaths

There were 18000 general practitioner consultations for stroke at a total cost of $\$ 0.49 \mathrm{~m}$. General practitioners prescribed medicine valued at $\$ 0.46 \mathrm{~m}$ for stroke patients. Transport for patients is a relatively important aspect of this disease because of the large number of acute admissions required for patients, most of whom were elderly. Acute admissions ${ }^{12}$ to public hospitals accounted for 3959 or $86 \%$ of all admissions for ischaemic stroke, and 17 patients in private hospitals $(15 \%)$. For the purposes of this study all admissions to hospital for ischaemic stroke patients were allotted an ambulance trip estimated to be $\$ 0.75 \mathrm{~m}$ in total $(4707 \mathrm{x} \$ 159.74)$.

Ischaemic stroke patients' hospital costs (based on $\$ 300 / \mathrm{d}$ for public hospitals, and $\$ 284.44$ for private hospitals) amounted to $\$ 27.97 \mathrm{~m}$. Calculation of hospital costs using the DRG 014 unit cost of $\$ 10076$ gave a higher cost of $\$ 47.43$ million. These costs are described in Table 2.

The cost of rehabilitation, that is, physiotherapy and speech therapy $(14+1.9=15.9$ sessions $)$ for each stroke patient (4 $707 \times 15.9 \times \$ 20$ ) was calculated to be $\$ 1.50$ million. If live discharges only are considered this cost falls to $\$ 1.17 \mathrm{~m}$ (3.694 x $15.9 \times \$ 20)$. It was estimated that 2958 $(1.74 \times 4.707 \times 0.488 \times 0.74)$ ischaemic stroke survivors required continuing care in a rest home as a result of the stroke. The annual cost of this continuing care when valued at the average weekly wage was estimated to be $\$ 89.50 \mathrm{~m}$ (\$579.90/7 $\times 365.25 \times 2958)$, and when valued using the rest home subsidy rate was estimated to be $\$ 61.94 \mathrm{~m}(\$ 401.34$ / $7 \times 365.25 \times 2958$ ). 
Table 2. - Hospital costs 1992: ischaemic stroke males and females all ages

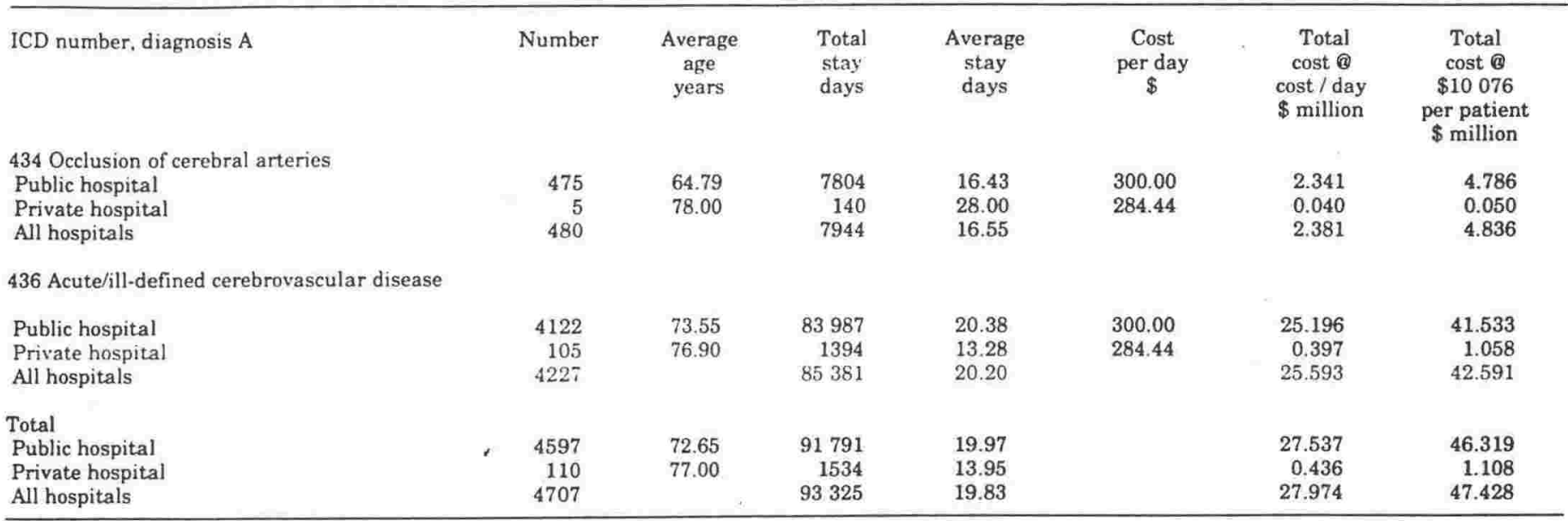

This table excludes patients with stays of over 90 days (47 private patients, average stay 689 days; 307 public patients, average stay 337 days)

Because of rounding individual items may not add exactly to the totals shown.

Table 3. - Lost production 1992: ischaemic stroke males and females, 15 and under 65 years

\begin{tabular}{|c|c|c|c|c|c|c|c|}
\hline ICD number, diagnosis $\mathrm{A}$ & Number & $\begin{array}{l}\text { Average } \\
\text { age } \\
\text { years }\end{array}$ & $\begin{array}{l}\text { Total } \\
\text { stay } \\
\text { days }\end{array}$ & & $\begin{array}{l}\text { Average } \\
\text { stay } \\
\text { days }\end{array}$ & $\begin{array}{l}\text { Lost produ } \\
\$ 579.9 \\
\text { (1) weeks } \\
12 \\
\$ \text { million }\end{array}$ & $\begin{array}{l}\text { on valued at } \\
\text { er week } \\
\text { (1) weeks } \\
26 \\
\$ \text { million }\end{array}$ \\
\hline $\begin{array}{l}434 \text { Occlusion of cerebral arteries } \\
\text { Public hospital } \\
\text { Private hospital } \\
\text { All hospitals }\end{array}$ & $\begin{array}{l}163 \\
163\end{array}$ & 50.04 & $\begin{array}{l}2285 \\
2285\end{array}$ & & $\begin{array}{l}14.02 \\
14.02\end{array}$ & $\begin{array}{l}1.134 \\
1.134\end{array}$ & $\begin{array}{l}2.458 \\
2.458\end{array}$ \\
\hline $\begin{array}{l}\text { 436 Acute/ill-defined cerehrovascular disease } \\
\text { Public hospital } \\
\text { Private hospital } \\
\text { All hospitals }\end{array}$ & $\begin{array}{r}736 \\
13 \\
749\end{array}$ & $\begin{array}{l}54.62 \\
59.77\end{array}$ & $\begin{array}{r}16371 \\
171 \\
16542\end{array}$ & . & $\begin{array}{l}22.24 \\
13.15 \\
22.09\end{array}$ & $\begin{array}{l}5.122 \\
0.090 \\
5.212\end{array}$ & $\begin{array}{r}11.097 \\
0.196 \\
11.293\end{array}$ \\
\hline $\begin{array}{l}\text { Total } \\
\text { Public hospital } \\
\text { Private hospital } \\
\text { All hospitals }\end{array}$ & $\begin{array}{r}899 \\
13 \\
912\end{array}$ & 53.79 & $\begin{array}{r}18656 \\
171 \\
18827\end{array}$ & & $\begin{array}{l}20.75 \\
13.15 \\
20.64\end{array}$ & $\begin{array}{l}6.256 \\
0.090 \\
6.346\end{array}$ & $\begin{array}{r}13.555 \\
0.196 \\
13.751\end{array}$ \\
\hline
\end{tabular}

This table excludes patients who died in hospital

Because of rounding individual items may not add exactly to the totals shown.

Table 3 details lost production for the 912 hospital patients of working age ( 15 and under 65 years) who had an ischaemic stroke. Production loss was evaluated at $\$ 6.35 \mathrm{~m}$ (using 12 weeks off work), and $\$ 13.75 \mathrm{~m}$ (using 26 weeks off work).

Table 4. - Cost summary: ischaemic stroke 1992

\begin{tabular}{lcccc}
\hline \multirow{2}{*}{ Cost } & \multicolumn{2}{c}{ Low } & \multicolumn{3}{c}{ High } \\
& \$ million & \% of total & \$ million & $\%$ of total \\
General practitioner & 0.49 & 0.49 & 0.49 & 0.32 \\
GP prescribed medicines & 0.46 & 0.46 & 0.46 & 0.30 \\
Transport to hospital & 0.75 & 0.76 & 0.75 & 0.49 \\
Hospital & 27.97 & 28.22 & 47.43 & 30.82 \\
Rehabilitation & 1.17 & 1.18 & 1.50 & 0.97 \\
Continuing & 61.94 & 62.48 & 89.50 & 58.16 \\
institutionalised care & & & & \\
& & & & \\
Total direct medical & 92.78 & 93.59 & 140.13 & 91.06 \\
Loss of production & 6.35 & 6.41 & 13.75 & 8.94 \\
Total direct and indirect costs & 99.13 & 100.00 & 153.88 & 100.00 \\
\hline
\end{tabular}

Because of rounding, individual items may not add exactly to the totals shown.

The direct costs of treatment and the indirect cost of lost production as discussed above have been summarised in Table 4. Direct costs ranged from $\$ 93 \mathrm{~m}$ to $\$ 140 \mathrm{~m}$ and loss of production was between $\$ 6 \mathrm{~m}$ and $\$ 14 \mathrm{~m}$. Total quantified costs were estimated to lie between $\$ 99 \mathrm{~m}$ and $\$ 154 \mathrm{~m}$. Hospital and continuing care costs when combined make up $90 \%$ of all quantified costs.

For the year ended 1990 (the latest available data) mortality from ischaemic stroke was 1885 . Although some New Zealanders die at an early age from ischaemic stroke the average age at death was 79 years, with $87 \%$ of those dying aged 70 years and over, and $53 \%$ of those dying aged
80 years and over. The crude all ages death rate for ischaemic stroke was 56 per 100000 with age specific rates ranging from 3 (40 to 49 years) to 1314 ( 80 years and over). Death certificate mortality data is shown in Table 5.

Table 5.-1990 death certificate mortality: Ischaemic stroke

\begin{tabular}{lcrr}
\hline $\begin{array}{l}\text { Age cohort } \\
\text { Years }\end{array}$ & $\begin{array}{c}\text { NZ } \\
\text { Population } \\
\text { number }\end{array}$ & Number & $\begin{array}{c}\text { Mortality } \\
\text { per } 100000\end{array}$ \\
0 to 9 & 521130 & 1 & 0.2 \\
10 to 19 & 552800 & & \\
20 to 29 & 563730 & 3 & \\
30 to 39 & 520310 & 12 & 3 \\
40 to 49 & 414680 & 42 & 74 \\
50 to 59 & 297210 & 635 & 375 \\
60 to 69 & 264270 & 996 & 1314 \\
70 to 79 & 169340 & 1885 & 56 \\
80 and over & 75780 & & \\
All ages & 3379250 & & \\
\hline
\end{tabular}

\section{Discussion}

As in most health economic studies, difficulties were encountered in attempting to quantify costs of ischaemic stroke outside the hospital setting. The cost estimates in this study are conservative as no measurements were made for a reduction in the quality of life of stroke patients and their family and friends. Similarly, the costs of home help, visiting nurses, social workers, of meals on wheels, or the costs for walking aids and modifications to housing or to motor vehicles required to assist mobility were not evaluated. General practitioner consultations and the medicines they prescribed were the only costs able to be evaluated for those people who had an ischaemic stroke but who were not admitted to hospital. 
The level of disability for patients after stroke is dependent upon the severity of the stroke. Disability manifests itself as epilepsy, depression, and cognitive losses (for example, in language and perception) ${ }^{23}$. Studies of surviving stroke patients have shown reduced social contact with those outside immediate family and diminished leisure activity. ${ }^{24}$ Similarly, in an age and sex-matched control population it was found that long term stroke survivors had a decreased level of socialisation outside the home, and a reduced interest in hobbies and activities. ${ }^{25}$ Some of the costs associated with these disabilities have been captured in rehabilitation and continuing care costs but none of the reduction in quality of life has been quantified.

Patients admitted to one hospital and subsequently transferred to another are recorded on separate records and as such all the hospital costs have been captured but numbers may have been double counted as discussed in methods. To minimise double counting, long stay patients (for this study stays of longer than 90 days) were removed from the hospital data and costs for these patients were captured under continuing care. Hospital costs based on the DRG 014 were higher than estimates based on average bed day costs. The actual value probably lies somewhere between the upper and lower estimates.

As diagnosis A (the primary diagnosis for which the patient was admitted) was used to select patient records from the hospital unit data both the numbers of ischaemic stroke patients and hospital costs are conservative. Patients admitted to a public hospital for an unrelated diagnosis A but additionally diagnosed ischaemic stroke in diagnoses B, C or D were not included in the analysis as it was impossible to separate the additional stroke cost component from the cost of the primary diagnosis using the data available. Private hospital data are recorded for primary diagnosis only. Although the Ministry of Health have quality assurance systems, coding errors within hospitals do occur. Jackson and colleagues ${ }^{26}$ found that death certificate coding was accurate to within $10 \%$ and the authors consider that hospital coders are unlikely to exceed this error rate.

Finnish prevalence data ${ }^{3}$ applied to New Zealand were used to evaluate continuing care in institutions and to estimate rehabilitation volumes. Limited data were obtained on the breakdown of consultations with private physiotherapists | One Day in September Study. Wellington: New Zealand Private Physiotherapists Association, 1993] which showed that physiotherapy for hemiplegia formed $1.2 \%$ of their workload but these could not be applied to the public sector in which the majority of stroke patients are treated.

It was calculated that in 1992 there were approximately $1557(0.5 \times 3115)$ patients 60 years and over discharged live to rest homes or geriatric hospitals, but as it was not known how long these patients stayed in care this estimate could not be used to obtain total costs. Although volume and case mix data on rest homes were unavailable the cost of continuing care was estimated to range between $\$ 61.94$ $\mathrm{m}$ and $\$ 89.50 \mathrm{~m}$ based on the estimated 2958 patients permanently institutionalised. Some stroke patients would require additional or more intensive care than was accounted for in the estimates.

Continuing care is an important component of the cost of ischaemic stroke to society. Further research should be undertaken to obtain estimates of the case mix and numbers of patients resident in rest homes and those requiring similar care in private homes. New Zealand rest home/continuing care costs were double the acute hospital costs. USA data ${ }^{27}$ indicated that nursing home costs were equivalent in magnitude to $75 \%$ of inpatient hospital care costs. This difference between the ratios of continuing care to acute hospital costs between USA and New Zealand may be a result of our decision to exclude longstay patients from the New Zealand hospital data and to capture these costs under rest homes.

It could be argued that for an economy with less than full employment, loss of paid output should be omitted from the analysis as this would not reduce the economy's paid output and measured GDP. However, this approach ignores the value of unpaid output and the value of leisure time. Loss of life could have been evaluated using willingness-to-pay criteria or the human capital approach but because of the age of stroke patients neither method yields satisfactory nor plausible results. The human capital method undervalues the lives lost because of the low number of potential working years lost.

Given the average age of the patients ( 73 years, and 77 years, for public and private patients respectively) the willingness-to-pay value of $\$ 2 \mathrm{~m}$ per $\operatorname{life}^{28}$ would result in a total cost that may be considered by some readers to be too high.

This study estimated the costs of ischaemic stroke in 1992 on a prevalence basis. (Prevalence of a disease is the number of cases in the population for the period studied. Incidence of a disease is the number of new cases that occur within a given time period.) Both those patients having stroke events during the current year and the survivors from past events (who still require treatment or support) contribute to costs and are covered in the prevalence estimates. Accurate cost and prevalence data are essential for the economic evaluation of treatment options, policy changes, and the manner in which an ageing population may affect health costs in hospitals and the community. Although this paper provides more information on costs than was previously available, further research is required to define the non hospital costs of stroke that are a large proportion of the total costs of stroke.

Acknowledgments. We thank Bochringer Ingelhem (NZ) l.td for a rese:trch grant to investigate the costs of ischaemic stroke. Terry Auld's comments on the draft paper were appreciated as were those of the referee.

Correspondence. Mr fiuy Scott. PO Box 14403, Wellington 6041

1 Hatano S. Control of stroke in the community - methodological considerations and protocel of WHO stroke register. WHO document no CVD/S73.6 Rev 1,1973.

Friedman P.J. Ischaemic stroke and intracerehral haematoma in the Waikato stroke

regstry. N2 Med J 1992:105:171-3.
Rissinen A Cerchrovascular discase in the Jyvsky la region. central Finland. Kuupio. Universty of Kuopio, 1992 (Department of Neurology Series of Reports No. 23)

Department of Health. Hospital and selected morbidity data 1992. Wellington: Department of Health, 1993

5. Department of Health. New Zealand Health Information Services. Mortality and demographic data 1990. Wellington: Department of Health. 1993

6. Dunbubin D. Cost-effective intervention in stroke. PharmacoEconomics 1992: 2:468-99. The ARCOS Study - ten years of research. PHA News 1993:5 HRC Research News Isard PA. Forbes JF. The cost of stroke to the National Health Service in Scotland. Cerebrovascular Dis 1992:2:409-18.

9. Anderson CS, Jamrozik KD, Burvill PW, Chakera TMH, Johnson GA, StewartWynne EG. Ascertaining the true incidence of stroke: experience from the Perth Community Stroke Study, 1989-1990. Med J Aust 1993; 158:80-4

10. Bonita R. Anderson CS, Broad JB, Jamrozik KD. Stewart-Wynne EG, Anderson NE. Stroke incidence and case fatality in Australasia. A comparison of the Auckland and Perth population-based stroke registers. Stroke 1994: $25: 552.7$.

11. Bonita R. Stewart A. Beaglehole R. International trends in stroke mortality: 1970-85. Stroke 1990; 21 : $989-92$.

12. Ministry of Health. New Zealand Health Information Service. Hospital unit record data 1992. Mortality unit record data 1990. (Electronic data base covering all New Zealand I

13. W Giuy Scott and Associates Lid Survey of General Practitioners Adult Consultation Fees 1992. Wellington. 1992

14. Pricing Schedules. 1 December 1992. Drug tarifl items. Wellington (Department of health publication, 1992 !

15. Culver Ail A glossary of the common terms encountered in health economics. In. Compendum of English course syllabi and textbooks in health economics Cupenhagen. WHO, 1989

16. Core health \& disability support services for 1993/94. First report of the National Advisory Committee on Core Health and Disability Suppor Services. Wellington: the Committee, 1992

17. Stewart RJ. Perry GKS, Bowie RD, O'Dea DJ. An economic evaluation of pentoneal aspiration cytology. Working paper: Wellington School of Medicine 1992

Scot WG White HD. Scott HII Cost of cornary heart disease in 1992 Med J 1993; 106. 171-3. Bonita R, Anderson A.

Ageing 198: $16 \cdot 29-34$

0. Bonita R, Ford MA, Stewart AW. Predicting survival after stroke a three-year follow. up. Stroke 1988:19669-73

1. Kamal A. A colour atlas of stroke. Cerebrovascular disease and its management. London. Wolfe Medical Publications, 1987

22. Department of Statistics. INFOS (Information Network for Official Statistics: Online computerised official statistics for New Zealandi. Total average weekly eamings for males and females combined, year ended December 1992. Department of Statistics: Wellington, 1993.

23. Wade DT, Skilbeck CE, Hewer RL. Selected cognitive losses after stroke: frequency, recovery and prognostic importance. Internat Disability Studies 1989: 11:34-9.

24. Astrom M, Adolfsson R, Asplund $K$, Astrom T. Life before and after stroke: living conditions and life satisfaction in relation to a general elderly population Cerebrovascular Dis1992: 2: 28-34

25. Labi MLC. Phillips TF, Gresham GE. Pyschosocial disability in physically restored long-term stroke survivors. Arch Physical Med Rehabil 1980; 61: 561-5.

26. Jackson R, Graham P, Beaglchole R, De Boer G. Validation of coronary heart disease death certificate diagnoses. NZ Med J 1988; 101: 658.60 .

. Adelman SM. Economic impact. National survey of stroke. Stroke 1981; 12 (Suppl II 169.78.

8. Millar $\mathrm{T}$, Guria J. The value of statistical life in New Zealand. Wellington: Ministry of Transport, 1991. 


\title{
Application of the Findings of the European Stroke Prevention Study 2 (ESPS-2) to a New Zealand Ischaemic Stroke Cost Analysis
}

\author{
Guy Scott ${ }^{1,2}$ and Helen M. Scott ${ }^{1}$ \\ 1 W. Guy Scott and Associates Limited, Wellington, New Zealand \\ 2 Department of Business Studies, Wellington Polytechnic, Wellington, New Zealand
}

\section{Summary}

The aim of this study was to apply the findings of the European Stroke Prevention Study 2 (ESPS-2) to a paper that quantified and described the annual cost of ischaemic stroke in New Zealand, and to compare the cost of alternative drug regimens in the secondary prevention of ischaemic stroke.

Comparisons were made between the costs of low-dosage aspirin (acetylsalicylic acid) monotherapy and a combination of modified-release dipyridamole and low-dosage aspirin. Differences in undiscounted costs were calculated over a 2 -year period. The New Zealand cost per stroke event was multiplied by the ESPS-2 incremental reduction in stroke events to derive the cost of strokes avoided. As the focus of the paper was on direct medical costs, the primary perspective adopted was that of a healthcare provider or funder, but a societal perspective was also considered by evaluation of direct nonmedical and indirect costs.

Compared with aspirin monotherapy, combination therapy generated incremental net direct costs of 18.22 New Zealand dollars (\$NZ) per patient or \$NZ18 223 per 1000 patients. However, individually, each treatment regimen resulted in direct cost savings when compared with placebo: combination therapy \$NZ905.16 per patient; aspirin monotherapy \$NZ923.39 per patient (a difference between the 2 regimens of $\$ N Z 18.22$ per patient). Total direct and indirect incremental cost savings were \$NZ40.96 per patient. and \$NZ40 963 per 1000 patients, for the combination therapy.

The analysis demonstrates that changing patients from low-dosage aspirin to a combination therapy of modified-release dipyridamole plus low-dosage aspirin would result in a small rise in incremental direct costs (using our conservative assumptions relating to hospital and continuing institutional care costs). If less conservative unit cost assumptions were adopted, a more likely outcome would be a saving in direct incremental costs of up to $\$ N Z 400$ per patient treated. 
Preventive therapy is important for patients who ive already had a cerebral ischaemic event as they e at increased risk of further events. [I] One study at assessed this form of preventive medicine was e European Stroke Prevention Study 2 (ESPS-2). The ESPS- 2 was a multicentre, randomised, uble-blind, placebo-controlled study with a $2 \times$ factorial design. Patients were randomised into he of the following treatment regimens: (i) modied-release dipyridamole $200 \mathrm{mg}$ twice daily comned with low-dosage aspirin (acetylsalicylic id) $25 \mathrm{mg}$ twice daily; (ii) modified-release diridamole $200 \mathrm{mg}$ twice daily; (iii) low-dosage asrin $25 \mathrm{mg}$ twice daily; or (iv) a matched placebo vice daily. The study assessed the effects of the eatment regimens in the secondary prevention of chaemic cerebrovascular events. Primary endjints for the study were stroke (fatal and nonfal), death from all causes, and stroke and/or death om any cause. ["]

The 2-year study (the largest such trial to date) volved 6602 patients who had experienced either transient ischaemic attack (TIA) [23.7\%] or an chaemic stroke $(76.3 \%)$ within the previous 3 onths. [1] The age and gender distributions in the udy population reflected both the increase in the idence of TIA and stroke with age and the usual ter occurrence of strokes and TIAs in women ompared with men (the mean age of the men was 5 years, and the mean age of the women was 69 ears). ${ }^{[2]}$

The results of ESPS-2|"1 showed a greater benfit to the patient (in risk reduction of stroke) if a ombination therapy (modified-release dipyridarole and aspirin) was given rather than either gent alone. Although each agent was in itself efective in reducing stroke risk compared with plaebo, when given in combination, their effects ere additive.

These findings have important cost implications or the treatment of ischaemic strokes. In most deeloped countries, strokes are the third most comnon form of death, stroke patients are high users f hospital resources, and strokes often result in isability that has important consequences for re-
Table I. Results of the European Stroke Prevention Study $2^{[1]}$ that are relevant to the cost analysis

\begin{tabular}{ll}
\hline Strategy & $\begin{array}{l}\text { Stroke events avoided } \\
\text { per } 1000 \text { patients } \\
\text { (relative to placebo) }\end{array}$ \\
\hline DYP + ASP & 58 \\
ASP & 29 \\
Difference (DYP + ASP - ASP) & 29 \\
\hline
\end{tabular}

Abbreviations: $\mathrm{ASP}=$ low-dosage aspirin (acetylsalicylic acid); DYP $=$ modified-release dipyridamole.

habilitation, and for long term community-care costs. ${ }^{[3]}$ Friedman ${ }^{[4]}$ observed that $88 \%$ of strokes in patients aged 60 years and above admitted to hospital in New Zealand were ischaemic in type. Scott and $S \operatorname{cott}{ }^{[3]}$ found that (in 1992) the direct medical costs of treating patients with ischaemic stroke ranged from 93 million New Zealand dollars (\$NZ) to $\$ N Z 140$ million.

Therefore, the aim of this study was to apply the findings of ESPS-2 $2^{[1]}$ to the paper that quantified and described the annual cost of ischaemic stroke in New Zealand, ${ }^{[3]}$ and to compare the cost of alternative drug regimens in the secondary prevention of ischaemic stroke. Differences in undiscounted direct medical costs were calculated over a 2-year period from the perspective of a healthcare provider or funder. A societal perspective was also considered by evaluation of direct nonmedical and indirect costs.

\section{Methods}

\section{ESPS-2 Findings}

In ESPS-2, "I] the absolute risk of stroke (fatal or nonfatal events) was reduced by each treatment regimen (compared with placebo): by 26 events per 1000 patients treated for those receiving modified-release dipyridamole; by 29 events for the aspirin group; and by 58 events for those receiving the combination therapy of modified-release dipyridamole and aspirin. "II When the combination (modified-release dipyridamole and aspirin vs placebo) was compared with aspirin (vs placebo) there was a reduction of 29 stroke events per 1000 
patients treated. These findings are summarised in table I.

\section{Cost Data}

The original New Zealand study ${ }^{[3]}$ used unit record discharge data for all hospitals in both public and private sectors for the year ended 1992, and mortality data for the year ended 1990. It was estimated that there were 6724 ischaemic stroke patients in 1992, of whom 4707 were admitted to hospital (70\%) and 2017 were treated in the community $(30 \%)$. $^{[3]}$

The ESPS- 2 results ${ }^{[1]}$ obtained from using treatment with modified-release dipyridamole and aspirin in combination were applied to the New Zealand data to calculate the net costs (or benefits) of treating patients who have experienced a TIA/stroke. The analysis in this current paper was confined to a comparison of combination therapy with aspirin monotherapy, as ESPS-2 found that there was little difference in outcomes between monotherapy with either aspirin or modified-release dipyridamole. Aspirin was chosen as the comparator as it is the drug regimen most likely to be replaced by the compination therapy. Accordingly, incremental costs for he combination therapy were compared with those ff the aspirin regimen.

As the focus of this study was on incremental osts, all resource utilisations (for example, genral practitioner consultations) prior to drug acquiition are irrelevant and thus not evaluated. Costs exclusive of goods and services tax (GST; 12.5\%) which is a transfer payment from the perspective f society as a whole, and from that of healthcare unders and providers] were estimated on both a er patient and per 1000 patient basis for a 2-year eriod with no discounting.

Drug acquisition costs (table II) were the costs or the combination therapy (modified-release ipyridamole plus aspirin) and the cost for aspiin monotherapy. As very low-dosage aspirin $25 \mathrm{mg}$ twice daily), as used in ESPS-2, ${ }^{11]}$ is unvailable in New Zealand, unit costs were based on alf a $300 \mathrm{mg}$ tablet and a price ex-supplier of NZ26.64 per 1000 tablets. ${ }^{|5|}$ This is not to imply that patients would take $150 \mathrm{mg}$ of aspirin, but was a convenient way of arriving at a price proxy. The cost of modified-release dipyridamole was based on the average daily dose (200mg twice daily) from ESPS-2 ${ }^{[1]}$ and a price ex-supplier of $\$ N Z 31.99$ per pack of $60 \times 200 \mathrm{mg}$ tablets. ${ }^{[5]}$ (The cost for 2 years' supply of medicine was calculated as follows: medicine price ex-pharmacy $\times$ daily dose $\times 2 \times 365.25$ ).

Unit costs and total costs for primary care, hospitalisation, rehabilitation, institutional care, transport to hospital and loss of production are given, together with their sources, in table III. The cost per stroke event was calculated by dividing the total cost by the number of stroke events estimated in the article by Scott and Scott. ${ }^{[3]}$ The next step was to take the New Zealand cost per stroke event (table III) and multiply it by the ESPS-2 incremental reduction in stroke events to derive the cost of strokes avoided (table IV).

All costs are in New Zealand dollars $(\$ N Z 1=$ \$US0.6846 mid-rate at the end of June 1996).

Table II. Drug unit costs in 1996 New Zealand dollars (SNZ; \$NZ1 = \$US0.6846; mid-rate June 1996)

\begin{tabular}{|c|c|}
\hline Regimen & $\begin{array}{l}\text { Unit cost } \\
\text { ex-pharmacy } \\
(\mathrm{SNZ})^{\mathrm{a}}\end{array}$ \\
\hline \multicolumn{2}{|l|}{ Low-dosage aspirin (acetylsalicylic acid) [ASP] } \\
\hline $\begin{array}{l}\text { Pack of } 1000 \times 300 \mathrm{mg} \text { tablets } \\
\text { (ex-supplier \$NZ26.64) }\end{array}$ & 32.61 \\
\hline Average dosage: $50 \mathrm{mg} / \mathrm{day}^{\mathrm{b}}$ & 0.0163 \\
\hline Cost for 2 years' supply & 11.91 \\
\hline \multicolumn{2}{|l|}{ Modified-release dipyridamole (DPY) } \\
\hline $\begin{array}{l}\text { Pack of } 60 \times 200 \mathrm{mg} \text { tablets } \\
\text { (ex-supplier } \$ N Z 31.99)^{c}\end{array}$ & 3916 \\
\hline Average dosage: $200 \mathrm{mg}$ twice daily & 1.3053 \\
\hline Cost of 2 years' supply & 953.52 \\
\hline \multicolumn{2}{|l|}{$D P Y+A S P$} \\
\hline $\begin{array}{l}\text { Average dosage: dipyridamole } 200 \mathrm{mg} \text { twice daily } \\
\text { plus aspirin } 25 \mathrm{mg} \text { twice daily }{ }^{\mathrm{b}}\end{array}$ & 1.3216 \\
\hline Cost for 2 years' supply & 965.43 \\
\hline \multicolumn{2}{|c|}{$\begin{array}{l}\text { Ex-pharmacy price }=\text { ex-supplier price }+10 \% \text { wholesale mark- } \\
\text { up }+11.28 \% \text { pharmacy mark-up. }\end{array}$} \\
\hline \multicolumn{2}{|c|}{ b Costed for $150 \mathrm{mg} /$ day, half a $300 \mathrm{mg}$ tablet $^{[5]}$} \\
\hline \multicolumn{2}{|l|}{$\begin{array}{l}\text { C Dipyridamole: price as for } 150 \mathrm{mg} \text { tablets. } \\
\text { Abbreviation: } \$ N Z=\text { New Zealand dollars }\end{array}$} \\
\hline Abbreviation: SNZ = New Zealand dollars. & \\
\hline
\end{tabular}


Table III. Costs associated with ischaemic stroke, assuming a total of 6724 strokes in $1992^{[3]}$ [all costs are in 1996 New Zealand dollars ( $\$ N Z 1$ = \$US0.6846; mid-rate June 1996)]

\begin{tabular}{|c|c|c|c|c|}
\hline Cost factor & Quantity (1992) & $\begin{array}{l}\text { Unit cost } \\
\text { (\$NZ; 1996) }\end{array}$ & $\begin{array}{l}\text { Total cost } \\
\text { (\$NZ; 1996) }\end{array}$ & $\begin{array}{l}\text { Cost per stroke } \\
\text { (\$NZ; 1996) }\end{array}$ \\
\hline Strokes & 6724 & & & \\
\hline \multicolumn{5}{|l|}{$\begin{array}{l}\text { Strokes } \\
\text { Direct costs: }\end{array}$} \\
\hline $\begin{array}{l}\text { general practitioner consultations } \\
\text { general practitioner prescriptions }\end{array}$ & $18000^{[3]}$ & $28.89^{[6]}$ & 520000 & 77.33 \\
\hline $\begin{array}{l}\text { general practitioner prescriptions } \\
\text { hospitalised patients }^{\text {a }}\end{array}$ & & & $460000^{[3]}$ & 68.41 \\
\hline $\begin{array}{l}\text { hospitalised patients } \\
\text { rehabilitation sessions }\end{array}$ & $\begin{array}{l}4707^{[3]} \\
3694^{[3]}\end{array}$ & $5943.06^{[3]}$ & 27974000 & 4160.32 \\
\hline $\begin{array}{l}\text { rehabilitation sessions }^{\mathrm{a}} \\
\text { patients continuing to institutional care }\end{array}$ & $\begin{array}{l}3694^{[3]} \\
2958^{[3]}\end{array}$ & $318.00^{[3]}$ & 1174692 & 174.70 \\
\hline $\begin{array}{l}\text { patients continuing to institutional care } \\
\text { patients requiring transport to hospital }\end{array}$ & $\begin{array}{l}2958^{[3]} \\
4707^{[3]}\end{array}$ & $26216.83^{[7]}$ & 77549393 & 11533.22 \\
\hline $\begin{array}{l}\text { patients requiring transport to hospital }{ }^{2} \\
\text { subtotal }\end{array}$ & & $159.74^{[3]}$ & 751896 & 111.82 \\
\hline \multirow{2}{*}{\multicolumn{5}{|c|}{$\begin{array}{l}\text { subtotal } \\
\text { Indirect costs: }\end{array}$}} \\
\hline & & & & \\
\hline $\begin{array}{l}\text { lost productivity } \\
\text { Total (direct and indirect) costs }\end{array}$ & 10944 weeks $^{[3]}$ & $626.97^{[8]}$ & 6861560 & 1020.46 \\
\hline$\frac{\text { Total (direct and indirect) costs }}{\text { Low estimates used as described in t }}$ & & & 115291541 & 17146.27 \\
\hline
\end{tabular}

a Low estimates used as described in table IV $!^{[3]}$

\section{Sensitivity Analysis}

In order to test the robustness of the results to changes in the assumptions used, a sensitivity analysis was performed.

\section{Results}

Table IV summarises the costs on a per patient and per 1000 patient basis for a 2-year period. Combination therapy (modified-release dipyridamole plus low-dosage aspirin) generated incremental net direct costs of $\$ N Z 18.22$ per patient or $\$ N Z 18223$ per 1000 patients. However, individually, each treatment regimen resulted in direct cost savings compared with placebo: combination therapy \$NZ905.16 per patient; aspirin monotherapy \$NZ923.39 per patient (a difference between the 2 regimens of $\$ N Z 18.22$ ). Total direct and indirect incremental cost savings were $\$ N Z 40.96$ per patient and \$NZ40 963 per 1000 patients for the combination therapy.

Table V summarises the results of the sensitivity analysis. If hospital costs were increased by $20 \%$, the incremental baseline direct cost of $\$ N Z 18.22$ became a cost saving of $\$ N Z 30.04$. If institutional care costs were valued at 'rest home stage II', the incremental direct cost rose to $\$ N Z 103.13$, but if all institutional care costs were valued at 'residen- tial care hospital', the direct cost became a cost saving of \$NZ417.10. Rest home stage II and residential-care hospital represent higher levels of government funding for residents requiring higher levels of care. Modified-release dipyridamole prices needed to fall by $2 \%$ to equate the direct costs of combination therapy with that of aspirin alone. When hospital costs were increased by $20 \%$, the $\$ N Z 18$ cost became a cost saving of $\$ N Z 30$ per patient treated. Lower-estimate continuing institutional care unit costs caused the cost per patient to increase to $\$ N Z 103$, while upper-estimate unit costs resulted in cost savings of \$NZ417.

Sensitivity analysis was also applied to the number of stroke events prevented by the combination therapy. If the combination therapy was $10 \%$ less effective at reducing stroke events than was measured in ESPS-2, 11 the incremental baseline direct cost of \$NZ18.22 increased to \$NZ205.28. However, if combination therapy was $10 \%$ more effective at reducing stroke events, there was a cost saving of \$NZ168.84. Direct costs fell to zero if the combination therapy was $1 \%$ more effective at reducing strokes.

\section{Discussion}

We are aware of concerns relating to problems with data collection from one centre in ESPS-2. 
ener et al., ${ }^{[1]}$ in reporting the results, stated that data from one centre were considered to be uniable and unable to be verified by an audit, they ve been excluded from the analysis and the samsize has been reduced from 7054 to 6602 pants.

When reviewing previous research, Diener et [1] comment that the earlier studies did not show periority of combination therapy over aspirin one. These earlier trials had substantially lower mbers of patients and 'lacked the power to detect ;mall difference'. [1]

Some allowance should be made for patient ncompliance when applying the results of this ady. Compliance is likely to be higher in a clini1 trial than in everyday practice. Estimates of ncompliance vary considerably and depend not ly on the individual patient, but also on the nare of the disease being treated and the type of edication prescribed. The ESPS- 2 results ${ }^{[1]}$ indited a noncompliance rate of up to $16 \%$ for paents on an aspirin, or aspirin combination, regien. In ESPS-2, compliance was evaluated using asma assays that may not have been sensitive enough for the low dosage of aspirin used and, accordingly, actual compliance may have been higher than measured.

Recent studies ${ }^{[9,10]}$ have investigated the appropriate aspirin dosage required to provide efficacy in stroke prevention. These studies have shown that aspirin at any dosage above $30 \mathrm{mg}$ /day prevents $13 \%$ of vascular events, ${ }^{[11]}$ [the UK Transient Ischaemic Attack (UK-TIA) Trial ${ }^{[10]}$ used 300 or $1200 \mathrm{mg} /$ day and the Dutch TIA Group ${ }^{[9]}$ compared $283 \mathrm{mg}$ with $30 \mathrm{mg}$ daily]. Hart and Harrison, ${ }^{[12]}$ in reviewing these studies, concluded that according to the Dutch TIA study, aspirin 283 $\mathrm{mg} /$ day was unlikely to be more than $5 \%$ better than $30 \mathrm{mg} / \mathrm{day}$, and that the UK-TIA study showed that $1200 \mathrm{mg}$ aspirin per day was unlikely to be more than $25 \%$ better than $300 \mathrm{mg} /$ day for stroke prevention. They stated: 'The best existing clinical evidence supports the concept that $75 \mathrm{mg} / \mathrm{d}$ acetylsalicylic acid is effective for stroke prevention and that higher doses may offer no additional protection.' ${ }^{[12]}$

Algra and van Gijn ${ }^{[1]}$ undertook a meta-analysis of 10 randomised trials of aspirin versus a placebo

able IV. Two-year costs [New Zealand dollars (\$NZ; \$NZ1 = \$US0.6846; mid-rate June 1996)] per patient and per 1000 patients treated ith either a combination of modified-release dipyridamole (DPY) plus low-dosage aspirin (acetylsalicylic acid) [ASP] or ASP monotherapy prevent ischaemic stroke. Figures in parentheses indicate negative values (a negative cost $=\mathrm{a}$ cost avoided or a benefit)

\begin{tabular}{|c|c|c|c|c|c|c|}
\hline \multirow[t]{2}{*}{ arameter } & \multicolumn{3}{|l|}{ Per patient } & \multicolumn{3}{|c|}{ Per 1000 patients } \\
\hline & DPY/ASP & ASP & Incremental $^{\mathrm{a}}$ & DPYIASP & ASP & Incremental \\
\hline $\begin{array}{l}\text { lumber of ischaemic stroke events } \\
\text { revented }\end{array}$ & 0.058 & 0.029 & 0.029 & 58 & 29 & 29 \\
\hline \multicolumn{7}{|l|}{ irect costs } \\
\hline drug acquisition & 965.43 & 11.91 & 953.52 & 965431 & 11911 & 953521 \\
\hline \multicolumn{7}{|l|}{ Costs generated by ischaemic stroke events: } \\
\hline primary care & (16.91) & $(8.45)$ & $(8.45)$ & (16 907) & (8453) & $(8453)$ \\
\hline hospital & $(482.60)$ & $(241.30)$ & $(241.30)$ & (482 597) & (241 299) & (241 299) \\
\hline rehabilitation & $(20.27)$ & (10.13) & $(10.13)$ & (20 265) & $\left(\begin{array}{lll}10 & 133\end{array}\right)$ & $\left(\begin{array}{lll}10 & 133\end{array}\right)$ \\
\hline continuing institutional care & $(1337.85)$ & $(668.93)$ & $(668.93)$ & $(1337854)$ & $(668927)$ & $(668927)$ \\
\hline transport to hospital & (12.97) & $(6.49)$ & (6.49) & (12 971) & $(6486)$ & $(6486)$ \\
\hline subtotal of direct costs & $(905.16)$ & (923.39) & 18.22 & (905 163) & (923 386) & 18223 \\
\hline \multicolumn{7}{|l|}{ Indirect costs } \\
\hline Lost productivity ${ }^{b}$ & $(118.37)$ & $(59.19)$ & (59.19) & $(118373)$ & (59 187) & (59 187) \\
\hline Total (direct medical and indirect) costs & $(1023.54)$ & $(982.57)$ & (40.96) & $(1023536)$ & (982 573) & (40 963) \\
\hline
\end{tabular}


Table V. Results of a sensitivity analysis on the total incremental direct cost per patient [New Zealand dollars (\$NZ; $\$ N Z 1=$ \$US0.6846; mid-rate June 1996)] of using modified-release dipyridamole [DPY] plus low-dosage aspirin (acetylsalicylic acid) [ASP] in place of ASP alone

\begin{tabular}{|c|c|}
\hline Parameter and degree of change & $\begin{array}{l}\text { Incremental } \\
\text { cost }(\$ N Z)^{a}\end{array}$ \\
\hline Baseline & 18.22 \\
\hline $\begin{array}{l}\text { Dipyridamole acquisition costs decreased by } \\
2 \%\end{array}$ & 0 \\
\hline Hospital unit costs increased $20 \%$ & $(30.04)$ \\
\hline $\begin{array}{l}\text { Continuing institutional care at rest home } \\
\text { stage II (\$NZ70.50 per day - GST = \$NZ62.67) }\end{array}$ & 103.13 \\
\hline $\begin{array}{l}\text { Continuing institutional care at residential-care } \\
\text { hospital (\$NZ133.30 per day - GST = } \\
\text { \$NZ118.49) }\end{array}$ & $(417.10)$ \\
\hline \multicolumn{2}{|c|}{$\begin{array}{l}\text { Change in efficacy of DPY + ASP in reducing ischaemic stroke } \\
\text { events relative to ESPS-2: }{ }^{[1]}\end{array}$} \\
\hline $10 \%$ less effective & 205.28 \\
\hline $1 \%$ more effective & 0 \\
\hline $10 \%$ more effective & $(168.84)$ \\
\hline
\end{tabular}

a Figures in parentheses indicate cost savings.

Abbreviations: ESPS-2 = European Stroke Prevention Study 2; GST $=$ goods and services tax $(12.5 \%)$.

control involving 6171 patients who had experienced a TIA or a nondisabling stroke. Their intention was to investigate the relative efficacy of low (less than $100 \mathrm{mg} /$ day), medium (300 to $325 \mathrm{mg} /$ day), and high (greater than $900 \mathrm{mg} /$ day) dosages of aspirin in secondary prevention of stroke. They concluded that efficacy in secondary prevention after cerebral ischaemia was similar for any dosage of aspirin between 30 and $1500 \mathrm{mg} /$ day. The results of the ESPS-2 trial have also shown that a lower daily dose of aspirin (25mg twice daily) is clinically effective at reducing secondary strokes.

An increased risk of adverse effects, such as gastrointestinal bleeding, is associated with high dosages of aspirin. The Dutch TIA Group ${ }^{|9|}$ found that adverse events were more frequent in the highdosage group. For example, minor gastrointestinal bleeding occurred twice as often in patients in the group taking $283 \mathrm{mg} / \mathrm{day}$ than in the group taking $30 \mathrm{mg} /$ day. However, the ESPS-2 trial "I) found that although the low aspirin dosage of $25 \mathrm{mg}$ twice daily was associated with a very low incidence of serious gastrointestinal adverse events and bleed- ing, it was not completely free of haemorrhagic complications. About $8 \%$ of patients receiving aspirin-containing regimens (alone, or in combination therapy) were affected by bleeding disorders. The small percentage of patients likely to be unable to tolerate aspirin, even at low dosages, should also be taken into account when applying the results of this study in that not all patients will be able to be transferred to an aspirin-containing regimen.

In the ESPS-2 trial, ${ }^{[1]} 22 \%$ of the placebo group, $22 \%$ of the aspirin group and $29 \%$ of those receiving combination therapy discontinued treatment for medical or nonmedical reasons. Thus, it can be concluded that $78 \%$ of the potential candidates for secondary stroke prevention could be treated with aspirin and $71 \%$ could be treated with the combination therapy of modified-release dipyridamole and aspirin. This would imply that $91 \%$ (71/78) of patients currently treated with aspirin alone, could be changed to the combination therapy.

In the present study, a sensitivity analysis revealed that the results were responsive to assumptions relating to the hospital and continuing institutional care costs. For example, if patients are discharged earlier from hospitals to continuingcare facilities, cost shifting will occur from hospitals to these care facilities. The average cost of patient care in rest homes will rise if the homes are caring for either increased numbers of patients and/or the case mix changes to patients who are more dependent.

To take account of the possible case-mix change, the analysis for continuing institutional care was also run with 'residential care hospital' costs. It is important to note that the Diagnosis Related Group (DRG) hospital cost for stroke, used as the upper bound by Scott and Scott, ${ }^{[3]}$ has almost halved since 1991. The costs used by Scott and Scott ${ }^{|3|}$ were actual hospital costs, while the latest DRG unit $\cos ^{[13]}$ used in this analysis reflects prices paid by New Zealand Regional Health Authorities (RHAs), which do not necessarily reflect the costs of the Crown Health Enterprises (CHEs). A CHE is a government-owned public hospital. For CHEs running deficits, prices are not covering 
costs. Accordingly, in the sensitivity analysis, we ncreased hospital costs by $20 \%$. If indirect costs are evaluated, the cost savings per patient will rise. Continuing unit care costs are more likely to lie jetween the baseline and the upper-bound values. ESPS-2 ${ }^{[1]}$ found differences between treatment options with respect to nonfatal strokes. However, tone of the treatments significantly reduced the isk of death alone from all causes or of fatal stroke. The fatal stroke incidence accounted for about $2 \%$ of all stroke events. The incremental differnce in stroke events avoided per thousand patients etween aspirin and the combination therapy was he same for all stroke events as for nonfatal stroke :vents. At randomisation into ESPS-2, ${ }^{[2]}$ less than $\%$ of patients were classified as severely disabled is a result of stroke, and the 4 treatment groups vere well matched with respect to the type of qualiying event, degree of handicap from stroke and the listribution of risk factors. The published trial reort, ${ }^{[1]}$ while providing information on stroke seerity, did not give specific information on the derrees of dependence of patients at the end of the rial.

The results of ESPS- $2^{[1]}$ demonstrate that with ombination therapy, although the number of leaths remained unchanged, the number of stroke vents was reduced. This means that the number of atients progressing to a state where either contiluing care, or more intensive care, is required vould be reduced. However, this assumes that the listribution of the severity of stroke events is idenical for all treatment groups; if the stroke events of one group were more disabling than those of nother group, the costs per stroke event will be lifferent. Our findings, with respect to potential ost savings, are conservative as the weighted iverage cost for strokes ${ }^{[3]}$ used in this paper covred the cost of all stroke patients (fatal and nonatal and those who recovered with varying derrees of incapacity and dependence), and implicitly issumed that there was no change in the proportion of patients requiring varying levels of care (over he trial period).
If it is assumed that there are 8 additional prescriptions per year (based on double the number of items for combination therapy and a prescription every 3 months), there will be an additional dispensing cost of $\$ N Z 20.80$ over a 2 -year period, or the equivalent of $2 \%$ of the drug acquisition cost of the combination ingredients. However, if monthly dispensing is assumed, additional dispensing costs will rise to $\$ N Z 62.40$, or $6 \%$ of the combination drug regimen ingredient cost. As aspirin is a very low-cost drug, the incremental direct medical costs are sensitive to small changes in the price of dipyridamole.

This analysis demonstrates that changing patients from aspirin to a combination therapy of modified-release dipyridamole and aspirin would result in a small rise in incremental direct costs (using our conservative assumptions relating to hospital and continuing institutional care costs). If less conservative unit cost assumptions were adopted, a more likely outcome would be a saving in incremental direct costs of up to $\$ N Z 400$ per patient treated. Based on ESPS-2 findings, ${ }^{[1]}$ approximately $90 \%$ of patients being treated with aspirin could be switched to the modified-release dipyridamole and aspirin combination. Although this study focused on the cost comparisons between the combination regimen versus aspirin, the combination regimen could replace monotherapy of either drug.

\section{Acknowledgements}

This study was supported by a grant from Boehringer Ingelheim. Opinions expressed in this paper are those of the authors alone.

\section{References}

1. Diener HC. Cunha L, Forbes C, et al. European Stroke Prevention Study 2: dipyridamole and acetylsalicylic acid in the secondary prevention of stroke. J Neurol Sci 1996; 143: 1-13

2. Bertrand-Hardy JM, Cunha L, Forbes C, et al. European Stroke Prevention Study 2: baseline data. J Neurol Sci 1995; 131 Suppl.: $1-58$

3. Scott WG, Scott HM. Ischaemic stroke in New Zealand: an economic study. N Z Med J 1994; 107: 443-6

4. Friedman PJ. Ischaemic stroke and intracerebral haematoma in the Waikato Stroke Registry. N Z Med J 1992; 105: 171-3

5. New Zealand Pharmaceutical Schedule 1996; 3(1): 49 


\section{CASE 10: ASTHMA}

Discussed in chapter 17

\section{References}

Scott, W. G., H. M. Scott, and G. D. Frost. "Pharmacoeconomic evaluation of asthma treatment costs." British Journal of Medical Economics 11 (1997): 87-101.

Scott, W. G., H. M. Scott, A. Penrose, G. D. Frost, J. Hall, and D. I. MacKenzie.

"Economic evaluation of inhalers used in the treatment of Asthma." Journal of Medical Economics 1 (1998): 1-18. 


\title{
Pharmacoeconomic evaluation of asthma treatment costs
}

\author{
Scott WG ${ }^{1,2}$, Scott $\mathrm{HM}^{2}$, Frost GD $^{3}$
}

\section{Wellington Polytechnic \\ 2 Independent Health Economist, \\ 3 3M New Zealand Limited}

Key words: asthma, treatment costs

\section{Summary}

The costs of asthma to New Zealand society were estimated and these costs then used to develop a decision analytic model. The model was designed for evaluating the economic costs of treating asthma using different treatment strategies.

Asthma is an important disease for New Zealand in terms of the number of people affected and the costs, both direct and indirect. Baseline direct medical costs were $\$ 148$ million $(\$ 451$ per treated asthmatic), direct non-medical costs were $\$ 18$ million ( $\$ 54$ per treated asthmatic), indirect costs of lost productivity $\$ 95$ million ( $\$ 291$ per treated asthmatic), and total costs amounted to $\$ 261$ million (\$796 per treated asthmatic). Total costs ranged from $\$ 251$ million to $\$ 340$ million, direct medical $\$ 142$ million to $\$ 205$ million, direct non-medical $\$ 14$ million to $\$ 18$ million, and productivity loss of $\$ 95$ million to $\$ 116$ million. These costs allowed us to validate and develop the model.

A spreadsheet-based deterministic, prevalence decision-analytic model was constructed and tested. A spreadsheet-based system was used to make the underlying assumptions and methodology in the model both explicit and transparent.

A user is able to evaluate the full costs of alternative treatments or, by entering different values in the input screen, look at asthma-treatment cost from the viewpoint of a patient, a general practitioner, a hospital or a specific country. The design is sufficiently flexible to take account

Accepted for publication:

22 July 1997

Address for correspondence: WG Scott, PO Box 14403, Kilbirnie, Wellington, New Zealand. 
of changing treatment options and guidelines. A user is able to calculate total costs, compare data through benchmarking, undertake sensitivity analysis, or consider a defined population, different patient categories, or appropriate medication use.

The development of the model required the skills of a number of professionals and thus mirrors the multidisciplinary nature of health economics. The model is a tool that allows the integration of pharmacoeconomic concepts with clinical decision making and policy analysis in treatment planning for populations with a chronic illness.

\section{Introduction}

The aim of this research was to first estimate the societal costs of asthma to New Zealand and then use this information to develop a decision analytic model. This model was designed for evaluating the economic costs of treating asthma using different treatment strategies.

The development of this model should be seen within the broader context of the cost of asthma to New Zealand society. Asthma is an important disease for New Zealand in terms of the number of people affected and the costs, both direct and indirect. It is generally regarded as a chronic disease requiring continuing treatment to prevent symptoms. As such, asthma has considerable associated morbidity and on-going treatment costs which include the exacerbation of existing and other acute illnesses.

\section{Prevalence}

Asthma is very common in New Zealand.
Crane et al ${ }^{1}$ found that $26 \%$ of a sample of adults aged between 20 and 44 years reported wheezing within the last year, $8 \%$ had experienced an asthma attack, and $9 \%$ had used asthma medication. In 1993, $13 \%$ of New Zealanders had been diagnosed by their doctor as having asthma ${ }^{2}$. A more recent survey ${ }^{3}$ of 2000 adults aged 15 years and over found that $14 \%$ had been told by their medical practitioner that they had asthma, 9\% had experienced an asthma attack during the past year and $11 \%$ were currently taking medication for asthma. Prevalence rates multiplied by total population ${ }^{4}$ indicate that approximately 500,000 people have been diagnosed as asthmatics, about 350,000 are treated with asthma drugs and there are about 150,000 treated asthmatics in the workforce. Table 1 details the prevalence of asthma in New Zealand in 1996.

\section{Cost}

Asthma has important consequences with respect to health care costs, loss of productivity and reduction in quality of life. The costs of asthma may be classified as follows:

- Drug acquisition cost (ingredients, markup and dispensing charge)

- Plus General practitioner, specialist and other health care professional consultations

- Plus Diagnostic tests (e.g. X-ray)

- Plus Secondary care cost (hospital)

$=$ Direct medical (health sector costs)

- Plus Direct non medical cost (non health sector costs: transport to seek treatment and other household costs [e.g. special bedding] of treating or preventing asthma)

$=$ Total direct cost 
- Plus Indirect cost (Lost productivity and leisure time activities of patient, family and friends. That is, time costs of the illness or its treatment)

$=$ Total direct and indirect cost

Where New Zealand information was unavailable, the authors used Australian and UK studies, as treatment guidelines for asthma are similar in all three countries.

All costs quoted in this paper are in 1996 New Zealand dollars exclusive of Goods and Services Tax (GST) which is a transfer payment from the perspective of society. (\$NZ 1 = \$US 0.6846 mid-rate June 1996.)

\section{Drug Acquisition}

In the year ending June 1996, government spending on asthma preventative medicines, bronchodilators and respiratory devices was $\$ 104.16$ million (asthma preventative medicines $\$ 72.44$ million, bronchodilators $\$ 29.53$ million, and respiratory devices $\$ 2.19$ million). This provided the baseline and low estimate for drugs. Asthma medicines and devices accounted for $17 \%$ of total Pharmaceutical Schedule expenditure on government subsidised medicines ${ }^{5}$. A high estimate of $\$ 153.01$ million for drug cost was obtained by taking $17 \%$ of total public and private expenditure on medicaments ${ }^{6}$. This high estimate includes patient co-payments and premiums (patient out-of-pocket payments where the government subsidy does not cover all of the medicine cost) and over-the-counter (OTC) medicines. As different therapeutic groups have different price structures and co-payment amounts and OTC medicine options this estimate must be a interpreted with caution.

\section{General Practitioner}

Asthma is a frequent reason for general practitioner consultations ${ }^{7}$. Studies indicate that asthma accounts for between $2.4 \%{ }^{8}$ and $4.5 \%{ }^{9}$ of general practice encounters. The latter study found that $2 \%$ of general practice consultations that included a referral to a public hospital or to a private specialist were

TABLE 1. Prevalence of asthma in New Zealand 1996.

\begin{tabular}{lccc}
\hline & Rate $\%$ & $\begin{array}{c}\text { Total number } \\
\text { of people }\end{array}$ & $\begin{array}{c}\text { Number in the } \\
\text { employed workforce }\end{array}$ \\
\hline $\begin{array}{l}\text { Diagnosed asthmatics } \\
\quad \text { Baseline }\end{array}$ & $13 \%$ & 473,200 & 218,855 \\
$\quad$ High & $14 \%$ & 509,600 & 235,690 \\
Low & $13 \%$ & 473,200 & 218,855 \\
& & & \\
$\begin{array}{l}\text { Treated asthmatics } \\
\quad \text { Baseline }\end{array}$ & $9 \%$ & 327,600 & 151,515 \\
$\quad$ High & $11 \%$ & 400,400 & 185,185 \\
$\quad$ Low & $9 \%$ & 327,600 & 151,515 \\
& & \\
Total population (mean year ending Dec 1996) & $3,640,000$ & $1,683,500$ \\
Total workforce (June 1996) & & & \\
\hline
\end{tabular}


for asthma and that $4.5 \%$ of consultations that included an admission to a public hospital were for asthma. During the year ending September 1996, 791,000 general practitioner consultations were recorded for asthma (IMS (NZ) Ltd) at a cost of \$23.73 million. Information from (IMS (NZ) Ltd) indicated that consultations for asthma represented $4.0 \%$ of the total number of consultations. The total cost was derived by multiplying the number of consultations by the average adult consultation fee of $\$ 30$ (rounded to the nearest whole dollar) obtained from the Consumers Price Index ${ }^{10}$ (November 1996) and checked against a consumer survey that gave similar results ". A low estimate of $\$ 17.63$ million for expenditure on general practitioner consultations was estimated by multiplying total expenditure on general practitioner consultations ${ }^{12}$ by $3.5 \%$ (average of the high and low estimate of encounters).

\section{Specialist consultations}

The low and baseline estimate for specialist consultations was $\$ 2.72$ million in 1996 (all specialist consultations $\$ 135.88$ million ${ }^{12}$ multiplied by $2.0 \%$ (using the proportion of specialist referrals for asthma)) ${ }^{9}$. A high estimate of $\$ 6.11$ million was obtained ( $\$ 135.88$ million multiplied by $4.5 \%$, using the proportion of general practitioner consultations as a proxy for the proportion of specialist consultations) ${ }^{9}$. If it is assumed that a specialist consultation fee is double that of a general practitioner, a fee of $\$ 60$ is obtained, but if relativity with accident and emergency consultations is assumed, a fee of $\$ 100$ would result. This range of fees was used to calculate the consultation numbers from total expenditure, giving numbers of between 45,000 and 61,000 consultations (rounded to the nearest thousand). Diagnostic imaging, and tests associated with diagnosis and monitoring were unable to be evaluated because of lack of information.

\section{Emergency medical treatment}

A UK study ${ }^{13}$ found that $13 \%$ of asthmatics had attended a hospital accident and emergency department in the last 12 months. Applying this finding to the number of treated and diagnosed asthmatics, and assuming a unit cost of $\$ 100$ per visit ${ }^{14}$ ), the following costs were obtained. Baseline and low estimate (baseline treated asthmatics $\mathrm{x}$ $13 \% \times \$ 100$ ) costs were $\$ 4.26$ million, and high estimate (baseline diagnosed asthmatics x $13 \%$ x $\$ 100$ ) costs were $\$ 6.15$ million.

\section{Hospital inpatient treatment}

Asthma is the most common cause of child admissions to hospital ${ }^{7}$. In 1994, there were 10,558 (5,171 male and 5,387 female, ICD (International Classification of Diseases) 493) inpatient discharges from publicly funded hospitals representing 38,009 days in hospital ${ }^{15}$. The estimated cost was $\$ 12.87$ million (calculated by applying 1994/95 DRG (Diagnostic Related Group) unit prices, DRG codes bronchitis and asthma 096, 097 and $098^{16}$ to the number of asthma inpatients). Asthma accounted for $1.7 \%$ of all inpatient discharges or $0.8 \%$ of all public hospital patient days in 1994. A high estimate of $\$ 16.36$ million for hospital costs was made using 1990/91 DRG prices paid to hospitals ${ }^{17}$ (27\% higher than unit costs in $1994 / 95$ ) to provide a high estimate of inpatient hospital costs.

\section{Transport cost}

It was estimated that transport costs were between $\$ 13.72$ and $\$ 18.48$ million with a 
baseline estimate of $\$ 17.79$ million. This evaluation, at a conservative unit cost of $\$ 20$ a round trip, was made on the basis of one round trip per consultation and hospital visit or admission. An informal survey of ambulance service providers (conducted by the authors) indicated a unit cost of about $\$ 100$ would be justified if an ambulance was used for transport.

\section{Indirect costs}

Indirect costs attributable to lost productivity of those in the paid workforce were estimated to be $\$ 95.17$ million (this calculation takes no account of lost school days, unpaid work or leisure time foregone). The assumptions were; each treated asthmatic in the workforce will lose 5 days or 1 week of work through asthma ${ }^{18}$, the total employed workforce was $1,683,500$ (June 1996) ${ }^{4}$, and the average weekly wage was $\$ 628.11$ (June 1996) ${ }^{4}$. If the average weekly wage is applied to the high estimate of employed treated asthmatics, a high value of $\$ 116.32$ million is obtained. Travel and waiting time (at least one hour per consultation) to attend consultations was assumed to be included in the 5 days' lost output.

If productivity loss was evaluated on the basis of the total labour force (employed and unemployed) the cost would be $6.3 \%$ higher than our estimates. Calculations made using the working age population (employed, unemployed and those not seeking paid employment) would be $62.0 \%$ greater. Reduced productivity of asthmatics while at work, or asthmatics unable to work, was not evaluated because of insufficient data. Similarly, productivity loss by unpaid caregivers or family members unable to work because they were caring for a child or other family member could not be evaluated.

TABLE 2. Summary of the baseline annual costs of asthma from the perspective of New Zealand society 1996

\begin{tabular}{|c|c|c|}
\hline Cost & $\begin{array}{l}\text { Total cost } \\
\text { NZ\$(M) }\end{array}$ & $\begin{array}{c}\text { Cost per } \\
\text { treated } \\
\text { asthmatic } \\
\mathrm{NZ \$}\end{array}$ \\
\hline Drug acquisition (ingredients, markup and dispensing) & 104 & 318 \\
\hline Plus primary care-giver (GP and specialist consultations) & 26 & 81 \\
\hline \multicolumn{3}{|l|}{ Plus secondary care } \\
\hline (hospital inpatient and accident and emergency) & 17 & 52 \\
\hline$=$ Direct medical & 148 & 451 \\
\hline Plus direct non-medical (transport) & 18 & 54 \\
\hline$=$ Total direct & 166 & 505 \\
\hline Plus indirect (lost productivity) & 95 & 291 \\
\hline$=$ Total direct and indirect & 261 & 796 \\
\hline
\end{tabular}

Because of rounding, individual items may not add exactly to the totals shown. 
Figure 1. Asthma cost to New Zealand 1996

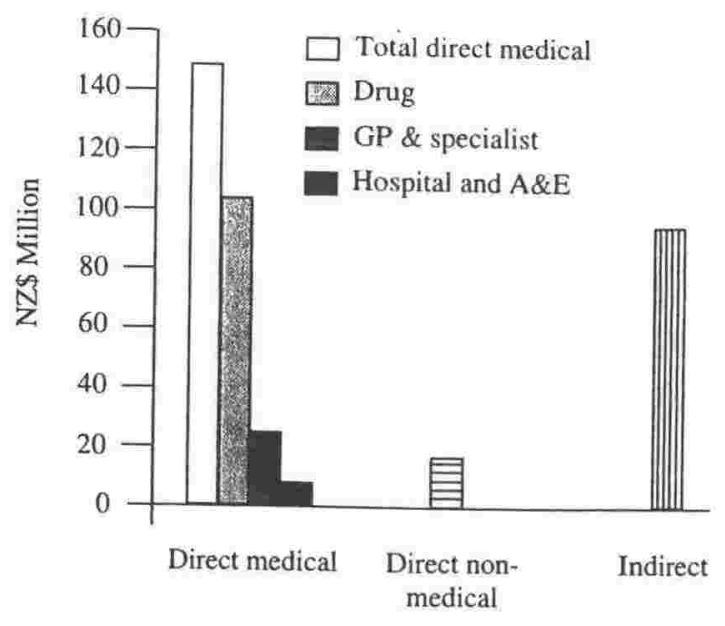

In 1993 there were 126 deaths attributed to asthma which accounted for $0.5 \%$ of all recorded deaths ${ }^{19}$. On a willingness-to-pay basis ${ }^{20}$, these lives would be valued at $\$ 252$ million, but considerably less than this if valued using a human capital and lost productivity approach.

Tables 2 and 3 and Figures 1 and 2 summarise the costs of asthma to New Zealand society as a total cost, and on the basis of the cost per treated asthmatic. Baseline direct medical costs were $\$ 148$ million ( $\$ 451$ per treated asthmatic), direct non-medical costs were $\$ 18$ million ( $\$ 54$ per treated asthmatic), indirect costs of lost productivity $\$ 95$ million (\$291 per treated asthmatic), and total costs amounted to $\$ 261$ million (\$796 per treated asthmatic). Total costs ranged from $\$ 251$ million to $\$ 340$ million, direct medical $\$ 142$ million to $\$ 205$ million, direct non-medical $\$ 14$ million to $\$ 18$ million, and productivity loss of $\$ 95$ million to $\$ 116$ million.

The Asthma and Respiratory Foundation of New Zealand (Inc.) ${ }^{14}$ have higher estimates; direct costs were $\$ 160$ million, indirect costs $\$ 216$ million and total societal costs $\$ 376$
Figure 2. Cost per treated asthmatic 1996

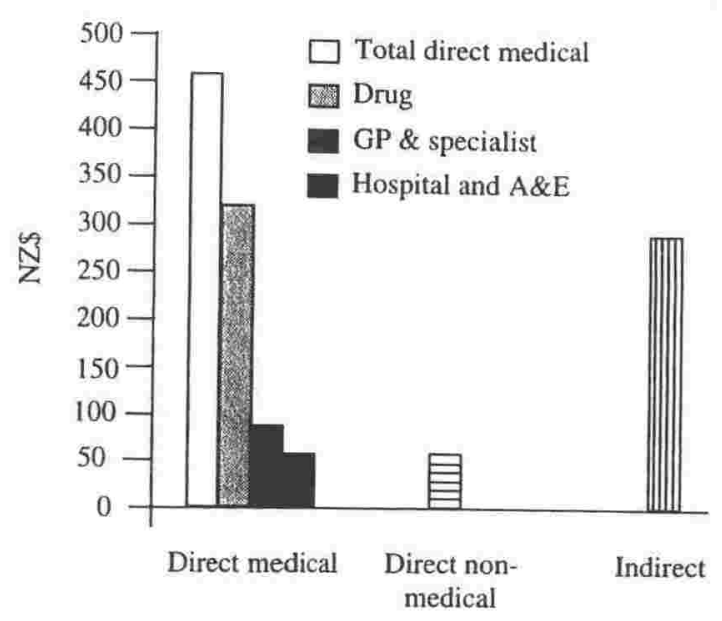

million per annum in $1996^{21}$. The primary difference is that our study took a more conservative approach in estimating indirect costs and excluded all transfer payments. We also focused on treated, rather than diagnosed, asthmatics in the workforce.

This analysis has quantified the important consequences of asthma with respect to resource costs. These costs allowed us to validate and develop the model.

\section{The Asthma Treatment Cost Model}

\section{Background to modelling}

Models are a representation of the relevant aspects of reality. They help in our understanding of the real world. Models are "... an ordered set of assumptions about a complex system" ${ }^{22}$. The origins of computerbased modelling for decision making and forecasting purposes can be traced to the work by Forrester ${ }^{22,23}$ and Meadows in the 1960s and 1970s. Models can be constructed and used without reference to computers but the process is more difficult and considerably slower. 


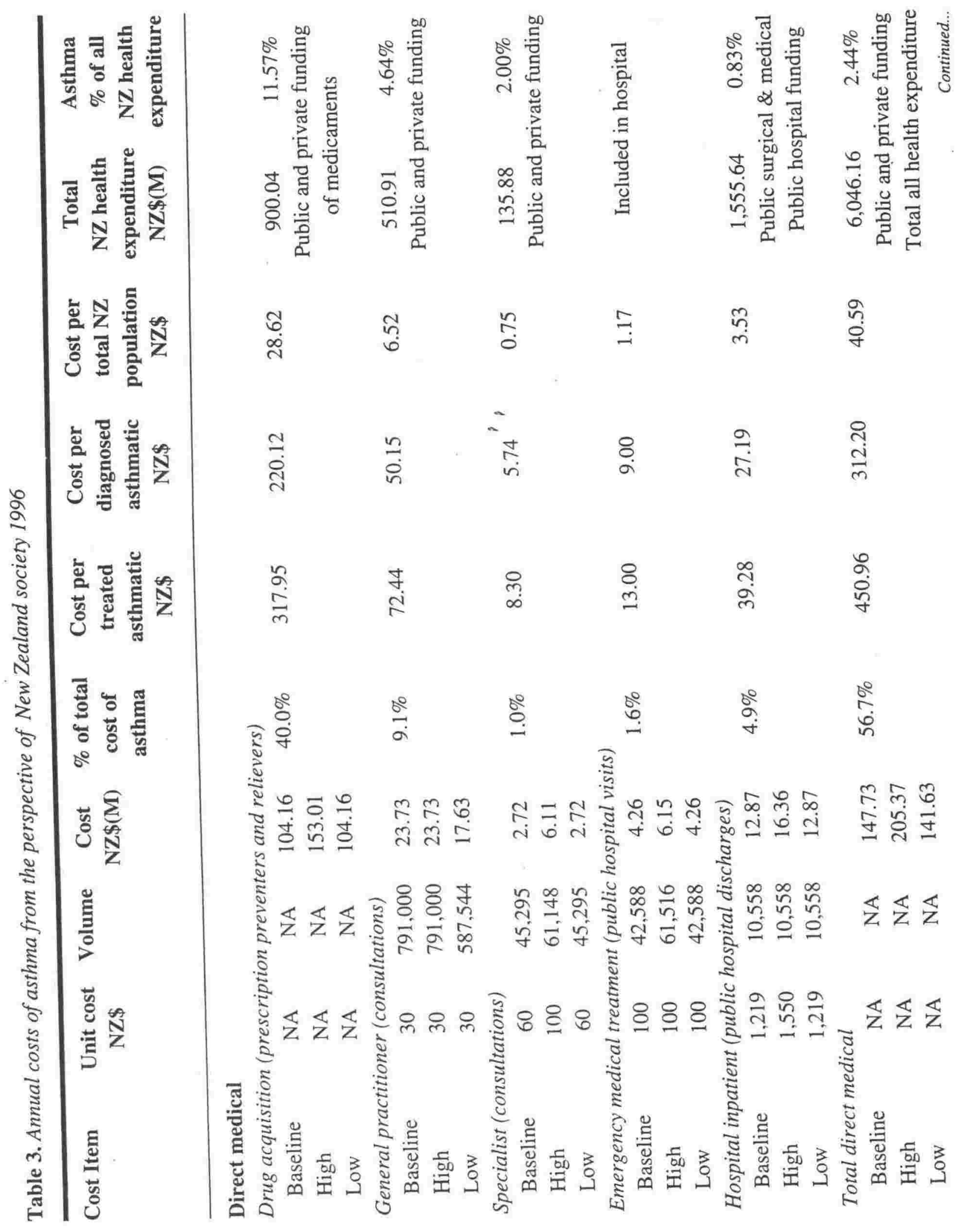




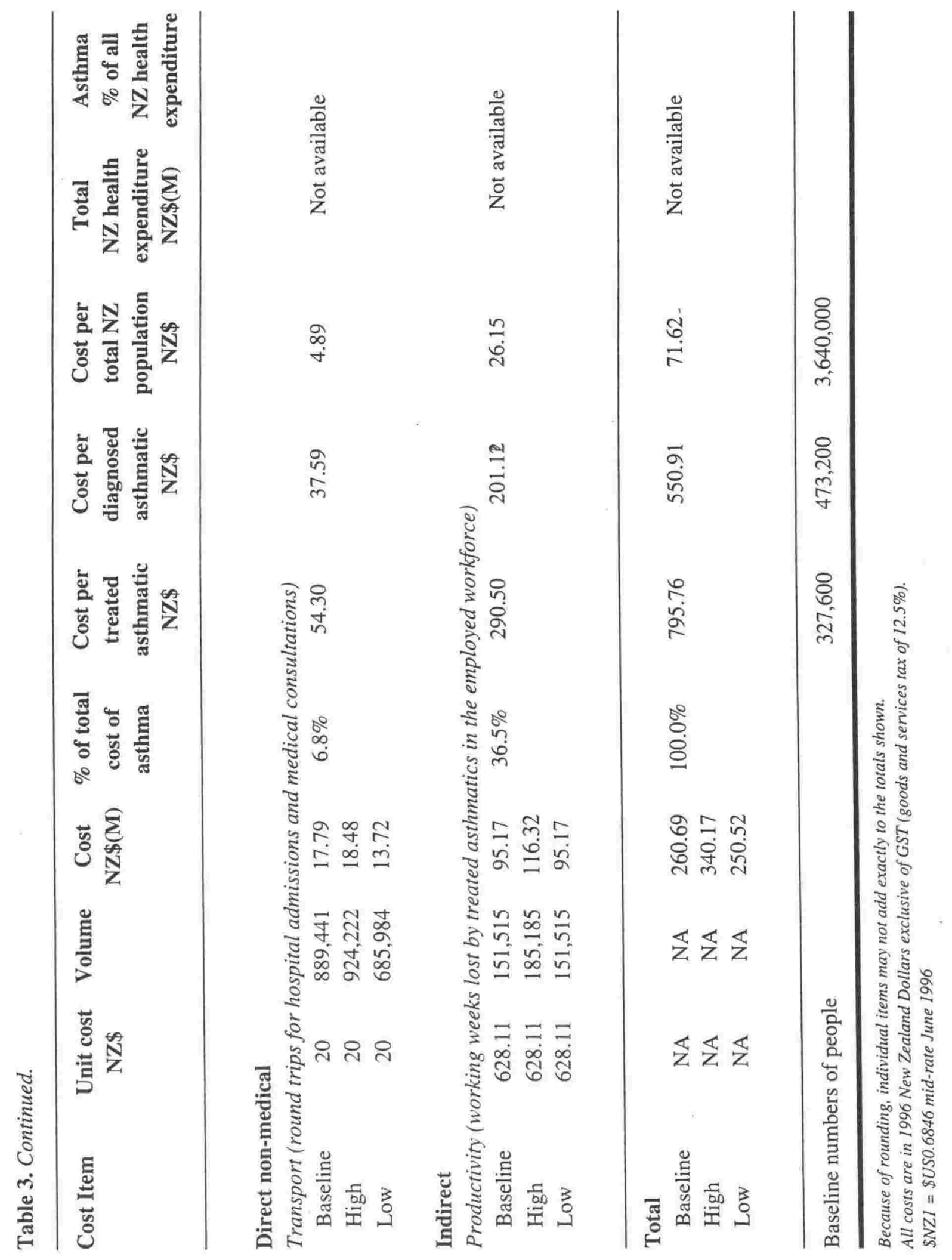


Common criticisms of models and modelling are that they are imperfect, oversimplify the real situation, or are incomplete and unfinished. However, many decisions cannot be postponed until perfect models are developed and a full understanding of the problem is achieved. Models and modelling assist in the decision-making process by helping to assemble and analyse relevant information, but cannot make decisions for clinicians, managers and policy makers.

The types of models of relevance to pharmacoeconomics include the following non mutually exclusive categories.

- Deterministic models give the same result each time the model is run if the same input data are used.

- Stochastic models are based on probabilities and the same inputs will not give identical results for each run.

- Decision tree models track a sequence of events and branching options over time. Each pathway up through the branches of the tree represents one sequence of events.

- Markov or state transition models allot members or groups of the study population to distinct states or categories over time.

- Longitudinal cohort or incidence models consider expected outcomes for groups of people over time.

- Cross-sectional population or prevalence models consider a population at one point in time.

Models designed for use with digital computers were originally created using a general programming language such as Fortran and may still be constructed in this way. However, specific modelling software has been developed and spreadsheets are now more sophisticated, with the ability to create multiple work sheets. These changes have allowed models to be more widely used in a number of disciplines, such as health economics.

\section{Why models are now used in health economics and pharmacoeconomics}

Although health economics has always involved the integration of data from a variety of primary and secondary sources, this process is made more explicit and transparent with the assistance of models.

Modelling of costs and health outcomes -enables the assessment of many factors at the same time and can be used as a tool for "what if analysis' decision making. Users are able to evaluate the effects of changing data and assumptions about unit costs, risks, prevalence, and treatment options. As models may be based on actual clinical practice, rather than randomised controlled clinical trials, they are more realistic in that patients seen in actual clinical practice may differ quite markedly from the artificial, controlled environment of a clinical trial. Thus models are able to utilise evidence-based medicine and management decisions. While all of the above can be done without computer-based models, a modelling system facilitates and speeds up the process.

Pharmacoeconomic analysis of asthma treatment options involves comparing the changes in resource opportunity costs with the health-related outcomes produced. The cost effectiveness ratio is the change in the health related outcome of interest (for example, controlled or treated asthmatics) divided by the changes in cost resulting from the intervention (see Figure 3). 
Figure 3. Pharmacoeconomic analysis of asthma treatment.

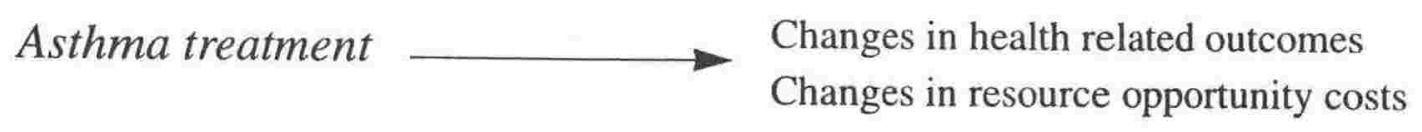

Cost effectiveness ratio $=$

Changes in health related outcomes

Changes in resource opportunity costs

Models and modelling help explore and analyse these changes in costs and healthrelated outcomes.

\section{Type of model developed}

We have constructed and tested a spreadsheet-based, deterministic, prevalence decision-analytic model. A spreadsheet-based system was used to make the underlying assumptions and methodology used in the model both explicit and transparent.

\section{Why we developed the model}

Asthma is an important disease in New Zealand in clinical and resource terms. Clinical decisions have resource implications and therefore any tool that facilitates informed, evidence-based decisions encompassing cost comparisons of treatment is of value.

The authors wished to provide a tool that would enable different stakeholders to evaluate the impact of different unit costs and policy options from their respective perspectives. The model will allow them to focus on overall treatment costs rather than on one particular aspect or cost item.

\section{Considerations in designing the model} The authors took care in designing the model to ensure that baseline data were accurate and that documentation was provided. The model mirrors actual asthma treatment as much as possible and should be able to be used in evaluating the cost of applying asthma guidelines to various at-risk groups. Simplicity, transparency, ability to modify and change and flexibility of use were of underlying importance when building the model. Other design criteria were that the model should be able to be used easily with minimal training and be able to run on a wide range of computing platforms.

\section{Description}

The model has a number of different screens or pages; welcoming pages, instructions for running, background, pre-loaded baseline values and assumptions, inputs, calculations, output tables, chart data and charts. All preloaded values, assumptions and sources have been documented in the model.

The flow of the Model is as follows:

Inputs $\rightarrow$ Calculations $\rightarrow$ Output table $\rightarrow$ Chart data $\rightarrow$ Output charts

\section{Inputs for the Model:}

- Volumes and unit costs

- Drugs and respiratory devices 
Table 4. Asthma treatment cost model inputs.

\section{Cost items by annual volumes and unit costs}

$\begin{array}{ll}\text { Drugs } & \text { Corticosteroid inhalers (assuming no wastage) } \\ \text { Beta-agonist inhalers (assuming no wastage) } \\ \text { Spacers } \\ \text { Other drug 1 } \\ \text { Other drug 2 } \\ \text { Corticosteroid inhaler over/ under use and test-firing } \\ \text { Beta-agonists over/ under use and test firing } \\ \text { Wholesale/ retail markups and dispensing fees } \\ \text { General practitioner consultations } \\ \text { Retraining: additional general practitioner consultations } \\ \text { Specialist consultations } \\ \text { Physiotherapist consultations } \\ \text { Nurse consultations } \\ \text { Retraining: additiōnal nurse consultations } \\ \text { Diagnostic tests }\end{array}$

Secondary care

Emergency medical treatment Hospital outpatient specialist consultations

Hospital admissions for inpatient treatment

Direct non medical Transport to seek treatment

Household costs

Indirect

Paid and unpaid production

Education

Leisure time activities

Epidemiological and Population at risk / study population

demographic data $\%$ of treated asthma patients

$\%$ of treated patients on each inhaler device

- Prescription markups and dispensing costs

- Consultations with general practitioner, specialist, physiotherapist and nurse, retraining in inhaler technique

- Diagnostic tests

- Emergency medical treatment

- Hospital inpatient treatment volumes

- Transport to seek treatment

- Relevant additional household costs
- Paid and unpaid production, education, leisure time activities

- Demographic and epidemiological data

These are described in more detail in Table 4.

The model has been pre-loaded with baseline input data from the perspective of New Zealand society. These baseline assumptions and data sources are described within the 
Figure 4. Demographic data input to the model

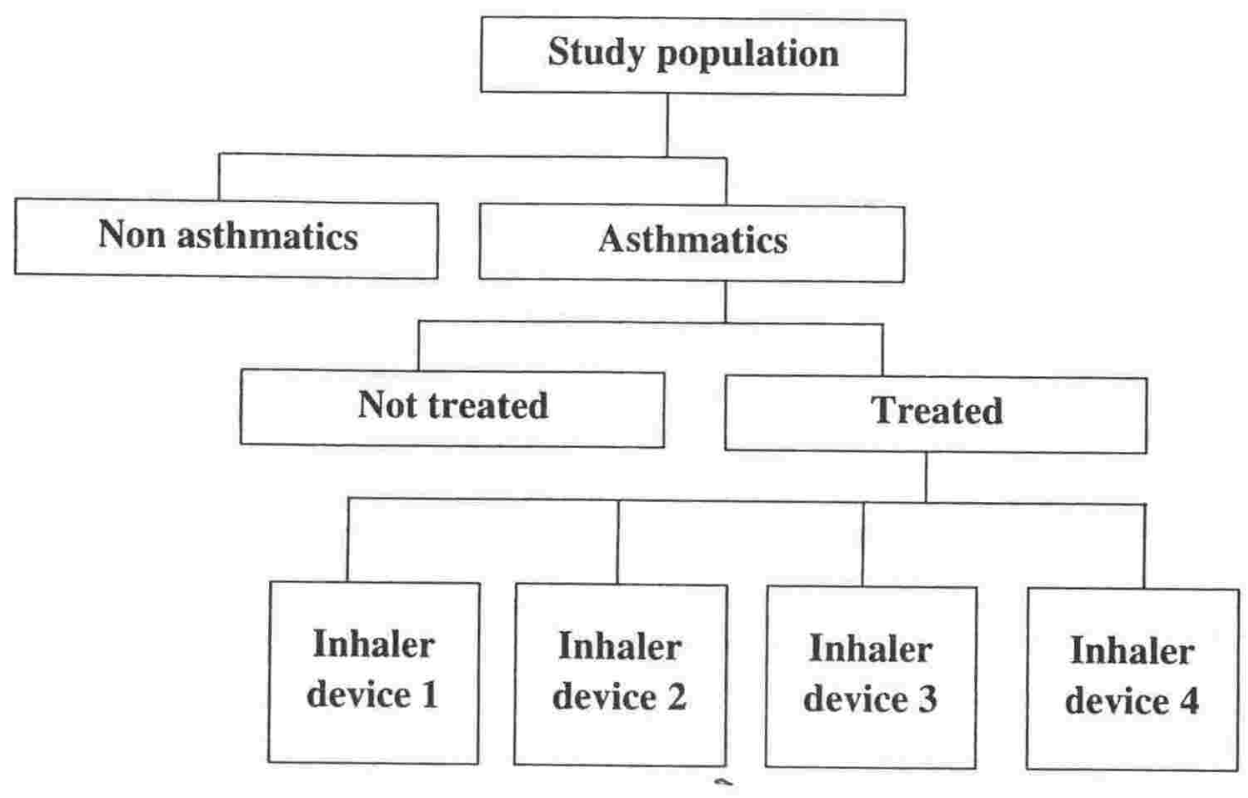

model (pre-loaded baseline values and assumptions) and any of them may be changed to reflect a user's needs, enabling the model to be used from more than one perspective.

The baseline pre-loaded values were derived from the burden of illness section of this paper, the authors' estimates, and from the Royal New Zealand College of General Practitioners, Research Unit technical report: Evaluation of inhalers used in the treatment of asthma (1997). This report provided average medicine unit costs and volumes by inhaler device and numbers of patients treated by each inhaler device.

Figure 4 shows the pathways the model follows in assigning the number of asthmatics treated to each corticosteroid inhaler device.

Calculations involve multiplying numbers of patients by the input unit costs and by the resource volumes for each treatment option.
Output costs in the model are presented (in both tabular and graph form) by inhaler device in terms of per treated patient, per 1000 patients, and per study population (see Table 5 for a listing of the treatment cost outputs).

\section{Discussion}

The model may be used to integrate and aggregate a range of cost data from a variety of sources. A user is then able to evaluate the full costs of alternative treatments. Currently, there are difficulties in determining costs of treatment, and comparisons are only readily available for unit costs of drugs.

Although the model's default settings are pre-loaded from the perspective of New Zealand society, the user can, by entering different values in the input screen, look at asthma treatment cost from the viewpoint of a patient, a general practitioner, a hospital, or a specific country. 
Table 5. Asthma treatment cost model outputs

Asthma annual cost by: Per treated asthma patient, per 1000 members of the study populations $\$(000)$, and by corticosteroid inhaler device

Corticosteroid inhalers

Beta-agonist inhalers

Spacers

Other eg Prednisone

Sub total Ingredients

Wholesale and retail markup

Dispensing

Total Drug acquisition (A)

General practitioner consultations

Specialist consultations

Physiotherapist consultations

Nurse consultations

Diagnostic tests

Total Primary care consultations plus diagnostic tests (B)

Total Primary care $(\mathbf{C}=\mathbf{A}+\mathbf{B})$

Emergency medical care

Hospital inpatient

Total Secondary care (D)

Total Direct medical $(\mathbf{E}=\mathbf{C}+\mathbf{D})$

Transport to seek treatment

Household costs

Total Direct non medical (F)

Total Direct medical and non medical $(\mathrm{G}=\mathrm{E}+\mathbf{F})$

Lost paid and unpaid production

Lost education

Lost leisure time activities

Total Indirect $(\mathbf{H})$

Total Direct and indirect $(\mathrm{I}=\mathbf{G}+\mathbf{H})$

The design is sufficiently flexible to take account of changing treatment options and guidelines. A user is able to calculate total costs, compare data through benchmarking, undertake sensitivity analysis, or consider a defined population, different patient categories, or appropriate medication use.
The model is able to be used as a planning tool, to improve decision making in the clinical management of patients, improve the allocation of resources in the treatment of asthma, and minimise costs of providing high-quality treatment. 


\section{Conclusions}

The development of the model required the skills of a number of professionals (eg clinicians, pharmacists, health economists, and operation researchers), and thus mirrors the multidisciplinary nature of health economics. Ease of use was of particular concern to the authors, who wished to overcome the 'black box syndrome' often encountered in computer applications. It was considered that if the model was difficult to follow it would not be used. The emphasis in the model's development has been on clarity and transparency.

The authors hope that this model will be found to be useful in evaluating the treatment of asthma in New Zealand in particular, and have application to asthma treatment in other countries. The model allows the integration of pharmacoeconomic concepts with clinical decision making and policy analysis in treatment planning for populations with a chronic illness.

\section{Acknowledgements}

We are grateful for permission to use data from the Royal New Zealand College of General Practitioners, Research Unit technical report: Evaluation of inhalers used in the treatment of asthma (1997).

The authors would like to thank those people who evaluated the model at various stages of its development and who made many useful suggestions for its improvement.

This work was supported by a grant from $3 \mathrm{M}$ New Zealand Limited.
Note: Any inquiries regarding the model should be directed to $3 \mathrm{M}$ New Zealand Limited with whom copyright exists. $3 \mathrm{M}$ New Zealand Limited, 250 Archers Road, Glenfield, Auckland, New Zealand.

\section{Postscript}

As this paper was going to press, two publications were issued that have relevance to this study. Hospital inpatient treatment costs using the latest prices ${ }^{24}$ would increase by $1 \%$. A recent survey ${ }^{25}$ of 25,666 adults found the prevalence of asthma to be $15 \%$ rather than the $13 \%$ to $14 \%$ discussed in this paper. The percentage of those surveyed taking asthma medications was $8.5 \%$ compared to our baseline value of $9 \%$. This latest information would have minimal impact on our results and conclusions.

\section{References}

1 Crane J, Lewis S, Slater T, et al. The self reported prevalence of asthma symptoms amongst adult New Zealanders. NZ Med $J$ 1994; 107: 417-21.

2 A picture of health. 1st ed. Wellington: Statistics New Zealand and Ministry of Health, 1993: 41

3 NRB. Research Report; Environmental tobacco smoke study. Prepared for the The Ministry of Health, July 1996.

4 Statistics New Zealand. Personal computer information network for official statistics: a computerised database of official statistics for New Zealand and programme for manipulation and analysis of statistical series (PC INFOS). Wellington, 1996.

5 PHARMAC. Annual review for the year ended 30 June 1996. Wellington:

Pharmaceutical Management Agency Limited, 1996: 22. 
Ministry of Health. Health expenditure trends in New Zealand 1980-1996. Wellington: Sector Analysis Section Ministry of Health, 1997: 47.

7 National Advisory Committee on Core Health and Disability Support Services. Core Services for 1994/95. Wellington: National Advisory Committee on Core Health and Disability Support Services, 1993: 36.

8 Tilyard MW, Dovey SM, Spears GFS. Biases in estimates from the RNZCGP computer research group. NZ Med J 1995; 108: $118-21$.

9 McAvoy B, Davis P, Raymont A, Gribben B. The Waikato Medical Care (WaiMedCa) Survey 1991-1992. NZ Med J 1994; 107 Suppl 986 Part 2: 387-432.

10 Consumers Price Index. Consumers price index weighted average retail prices of selected items in the CPI: general practitioners' services, consultation adult without community services card. Hot Off the Press 1996; December quarter.

11 Consumer. General practitioner consultations: what do you pay your doctor? Consumer 1996; April: 24-5.

12 Ministry of Health. Health expenditure trends in New Zealand 1980-1996. Wellington: Sector Analysis Section Ministry of Health, 1997: 44.

13 National Asthma Campaign. The impact of asthma. Asthma Journal 1996:

(September): 32-3.

14 Asthma and Respiratory Foundation of New Zealand (Inc). New Zealand asthma bill: $\$ 376$ million per year. Media release 30 March 1997.

15 Ministry of Health. Hospital and selected morbidity data 1994. Wellington: Health Information Service, 1996: 63.
16 Ministry of Health. Purchasing for your health: 1994/95. A performance report on the second year of the Regional Health Authorities and the Public Health Commission. Wellington: Ministry of Health, 1996: 291.

17 National Advisory Committee on Core Health and Disability Support Services. Core Services for 1994/95. Wellington: National Advisory Committee on Core Health and Disability Support Services, 1993.

18 Mellis CM, Peat JM, Bauman AE, Woolcock AJ. The cost of asthma in New South Wales. Medical Journal of Australia 1991; 155: 522-8.

19 Ministry of Health. Mortality and demographic data 1993. Wellington: Health Information Service, Ministry of Health, 1995: 7.

20 Miller T, Guria J. The value of statistical life in New Zealand. Wellington: Ministry of Transport, 1991.

21 Crane J. Asthma costs $\$ 376$ million a year report. The Dominion 1997; May 7: 8 .

22 Meadows DH, Meadows DL, Randers J, Behrens III WW. The limits to growth; a report for the Club of Rome's project on the predicament of mankind. New York: Universe Books, 1972: 20

23 Forrester JW. Industrial dynamics. 1st ed. New York: MIT Press and Wiley, 1961.

24 Ministry of Health. Purchasing for your health 1995/96. A performance report on the third year of the Regional Health Authorities. Wellington: Ministry of Health, 1997.

25 Lewis S, Hales S, Slater T et al. Geographical variation in the prevalence of asthma symptoms in New Zealand. NZ Med $J$ 1997; 110: 286-9. 
Erratum: Final sentence page 95 and figure 3 page 96
The cost effectiveness ratio is the change in in cost resulting from the intervention divided by the change in the health related outcome of interest (for example, controlled or treated asthmatics see figure 3 ).

Figure 3. Pharmacoeconomic analysis of asthma treatment
Asthma treatment
Changes in resource opportunity costs
Changes in health related outcomes
Cost effectiveness ratio $=$
Changes in resource opportunity costs
Changes in health related outcomes 


\section{Economic evaluation of inhalers used in the treatment of asthma}

Scott WG ${ }^{1,2}$, Scott $H M^{2}$, Penrose $A^{3}$, Frost GD ${ }^{4}$, Hall $^{3}{ }^{3}$, MacKenzie DI ${ }^{3}$

1 Senior Lecturer and Research Coordinator, Dept of Business Studies, Wellington Polytechnic, Wellington, New Zealand

2 Independent Health Economist, Wellington, New Zealand

3 Junior Research Fellows, RNZCGP Research Unit, Dept of General Practice, University of Otago, Dunedin, New Zealand

4 Manager Medical Affairs, 3M New Zealand Limited, Auckland, New Zealand

Keywords: Asthma, inhalers, economic evaluation, New Zealand, cost

\section{Summary}

Asthma is an important disease for New Zealand in terms of prevalence and costs, both direct and indirect. The objective of this study was to determine if there were any differences in primary care medical costs between treatment of asthma with four corticosteroid inhaler drug delivery systems (Autohaler ${ }^{\mathrm{TM}}$ [AUTO], Diskhaler ${ }^{\mathrm{TM}}$ [DISK], metered dose inhaler [MDI] and Turbuhaler ${ }^{\mathrm{TM}}$ [TURB]) in a general practice setting.

The retrospective observational data-based research completed for this study involved a large population and recorded actual general practitioner prescribing practice. The perspective taken was that of the funder of health care. Data were obtained from the computerised clinical records of 28 New Zealand general practices. Cost data were on a per year per patient basis.

The largest single cost item identified in this study was that of corticosteroid inhalers which ranged from $41 \%$ of total primary care cost for MDI to $52 \%$ for the TURB group. Total drug acquisition cost (inclusive of dispensing costs and wholesale and retail markups) was highest

Accepted for publication:

Address for correspondence:
20 November 1997

Guy Scott, PO Box 14403, Kilbirnie, Wellington, New Zealand. 
for TURB at $\$ 490$ and lowest for MDI at $\$ 265$, a difference of $85 \%$. There was less variation in general practitioner consultations for asthma with total costs ranging from $\$ 104$ for AUTO to $\$ 127$ for DISK. Total primary care costs were lowest for MDI at $\$ 392$ per annum and highest for TURB at $\$ 608$, a difference of $55 \%$. If the MDI total primary care costs are used as the base for an index (MDI total primary care costs set at 100) then AUTO becomes 116, DISK 134 and TURB 155.

The model was robust with respect to conclusions relating to cost differences between the treatment groups. In all but one instance TURB remains the highest cost group.

\section{Introduction}

The objective of this study was to determine if there were any differences in primary care medical costs between treatment of asthma with four corticosteroid inhaler drug delivery systems (Autohaler ${ }^{\mathrm{TM}}$ [AUTO], Diskhaler ${ }^{\mathrm{TM}}$ [DISK], metered dose inhaler [MDI] and Turbuhaler $^{\mathrm{TM}}$ [TURB]) in a general practice setting.

The International Consensus Report on Diagnosis and Treatment of Asthma ${ }^{1}$ defines asthma as "a chronic inflammatory disorder of the airways in which many cells play a role, including mast cells and eosinophils. In susceptible individuals this inflammation causes symptoms which are usually associated with widespread but variable airflow obstruction that is often reversible either spontaneously or with treatment, and causes an associated increase in airway responsiveness to a variety of stimuli."

Asthma is an important disease for New Zealand in terms of prevalence and costs, both direct and indirect. Crane et al. ${ }^{2}$ found that $26 \%$ of a sample of adults aged between 20 and 44 years reported wheezing within the last year, $8 \%$ reported an asthma attack and $9 \%$ reported using asthma medication. In 1993 13\% of New Zealanders (almost 470,000 people) had been diagnosed by their doctor as having asthma ${ }^{3}$.

Asthma is a major reason for consulting a general practitioner and is the most common cause of child admissions to hospital ${ }^{4}$. Studies indicate that asthma accounts for between $2.4 \%{ }^{5}$ and $4.5 \%{ }^{6}$ of general practice encounters. The latter study also found that $2 \%$ of general practice encounters that included a referral to a public hospital or to a private specialist were for asthma and that $4.5 \%$ of encounters that included an admission to a public hospital were for asthma. In 1993 there were 126 deaths attributed to asthma which accounted for $0.5 \%$ of all recorded deaths ${ }^{7}$.

The annual cost of asthma to New Zealand society was estimated to be $\$ 261$ million in $1996^{8}$. This annual cost consisted of publicly funded preventer and reliever drugs of $\$ 104$ million, general practitioner consultations of \$24 million, specialist consultations \$3 million, emergency medical treatment at public hospitals $\$ 4$ million, public hospital inpatient costs of $\$ 13$ million, transport to

- ${ }^{\mathrm{TM}}$ All registered trademarks ${ }^{\mathrm{TM}}$ are the property of their respective owners 
seek treatment $\$ 18$ million and indirect costs of lost productivity of $\$ 95$ million.

The aim of current therapy is to control asthma, with both the patient and health professionals managing treatment. The New Zealand Asthma and Respiratory Foundation has produced an asthma action plan which classifies asthma into a number of categories according to severity ${ }^{9}$ : well-controlled, worsening, severe and dangerous asthma. Asthma is treated mainly with preventer (inhaled corticosteroids) and reliever (inhaled beta agonist) medicines. The action plan suggests that patients with established asthma be treated with an inhaled preventer at an appropriate dose and use a reliever inhaler for symptomatic relief. Preventer dosages are ideally titrated to minimise asthma symptoms and the need for reliever use while maintaining patients on clinically acceptable but low doses of inhaled corticosteroids to optimise efficacy and avoid unnecessary side effects. Acute asthma episodes are treated with increased dosages of preventers and/ or treatment with oral corticosteroids ${ }^{10}$.

There is evidence that the delivery system is an important factor influencing the efficacy of inhaled steroids ${ }^{10-12}$. Accordingly, this study analysed the data by prescribed corticosteroid inhaler as this is considered the primary treatment of asthma.

There are two main types of inhaler device: aerosol (AUTO, MDI), and dry powder (DISK and TURB) inhalers. The dry powder inhalers (DISK, TURB) deliver active ingredient as a powder and require inspiratory effort for drug delivery. Breath actuated inhaler devices such as AUTO overcome the coordination problems commonly associated with MDI use, eliminate the need for spacers, and provide improved medication delivery to the lungs and improved efficacy for patients unable to correctly use MDIs ${ }^{13,14}$.

In New Zealand the general practitioner is regarded as the gate-keeper to the health care system, and hence the decisions they make are major health care cost drivers. Increasingly, the emphasis is on evidencebased medicine which encompasses both the economic and health effects of clinical practice. The retrospective observational data-based research completed for this study involved a large population and recorded (without altering or influencing) actual general practitioner prescribing practice.

\section{Methods}

The setting for the study was general practice. The perspective taken was that of the funder of health care. Regular asthma medication was considered to be inhaled corticosteroids for prevention and/or beta agonists for relief of symptoms, and additional asthma medication (prednisone and Betnesol ${ }^{\mathrm{TM}}$ ) in the event of loss of asthma control ${ }^{9}$. Drug information was classified by preventer, reliever and oral corticosteroid group.

Utilisation of health care resource volumes (drugs, general practitioner consultations and numbers of prescriptions), were obtained from the Royal New Zealand College of General Practitioners (RNZCGP) Research Unit, University of Otago.This was a retrospective population based analysis of data relating to the year ending 30 June 1995 (described in a technical report: Evaluation of 
inhalers used in the treatment of asthma, 1997). Data were obtained from the computerised clinical records of 28 general practices (15 in the North Island and 13 in the South Island) covering 128,585 consulting patients. The data set is maintained to a standard suitable for research and administrative purposes and is considered to be representative of the nature of general practice ${ }^{5}$ in New Zealand and a valid research tool ${ }^{15}$.

All prescribing records (from the 128,585 consulting patients) were electronically searched to identify those patients using AUTO, DISK, MDI or TURB as the sole inhaler device for corticosteroids $(5,704$ patients) over the 12 month study period. These records for patients prescribed inhaled corticosteroids were further examined to identify all beta agonist and oral steroid prescriptions. The medication key words searched were: Autohaler, Becloforte $\mathrm{TM}^{\mathrm{TM}}$, beclomethasone, Becotide ${ }^{\mathrm{TM}}$, Diskhaler, Betnesol $^{\mathrm{TM}}$, Bricanyl ${ }^{\mathrm{TM}}$, budesonide, prednisone, Pulmicort ${ }^{\mathrm{TM}}$, Respocort ${ }^{\mathrm{TM}}$, Respolin ${ }^{\mathrm{TM}}$, salbutamol, terbutaline, Turbuhaler and Ventolin ${ }^{\mathrm{TM}}$. These medication key words were chosen to effectively capture the total market for asthma medication in New Zealand.

Consultations were defined as asthma-related if a prescription for any of these listed medicines was generated, an inquiry about asthma, wheeze or other respiratory condition was noted, or peak flow was recorded in the consultation records. Consultations were actual recorded patient encounters.

Prices for the drugs prescribed, retail and wholesale markups, and dispensing fees and charges were obtained from the New Zealand Pharmaceutical Schedule ${ }^{16}$ (Dispensing fee plus container charge $\$ 2.60$, wholesale markup $10 \%$, retail markup $11.28 \%$.) Asthma inhaler prices in New Zealand are currently established by reference pricing for therapeutic groups. These therapeutic categories are established by grouping the various inhalers according to their label claim dose delivery. As a result, the unit costs for dosing with the different asthma inhalers are generally equivalent as they are reimbursed at the same level. General practitioner consultation fees were obtained from Statistics New Zealand ${ }^{17}$ (\$29.00, May 1996) and checked against a consumer survey ${ }^{18}$. All unit costs and prices were exclusive of Goods and Services Tax (GST). This tax is a transfer payment from the perspective of society, health care providers, government and patients in aggregate and was therefore excluded from this analysis.

The average daily dose of inhaled corticosteroids was calculated from the prescription information recorded in the database and was equivalent to analysing the text of all prescriptions. The average daily dose of beta agonists used was similarly calculated and also standardised by calculation of salbutamol equivalent doses. The dose relationship used for this estimation of terbutaline to salbutamol was $250: 100^{19-22}$.

The data in this analysis did not follow a normal distribution as it exhibited positive skewness in all aspects. Accordingly, Kruskal-Wallis $\mathrm{H}$ tests were used to compare the four samples. The Kruskal-Wallis $\mathrm{H}$ test is a non parametric form of a one-way ANOVA, much the same as the MannWhitney $U$ test is the non parametric form of 
the two sample t-test. The Kruskal-Wallis $\mathrm{H}$ test can be used to test whether two or more independent samples come from the same population ${ }^{23}$.

Skewness also affects the construction of confidence intervals as the classical method is valid only if the data is normally distributed. Skewness adjusted confidence intervals were constructed using an estimate of the skewness of the sample and the appropriate critical value from the standard normal distribution ${ }^{24}$. As a result of the skewness these confidence intervals are not symmetric about the mean.
The analysis by age was undertaken as patterns of asthma treatment differ according to age. Younger patients are generally treated with lower doses and patients over 50 years have a higher prevalence of chronic obstructive airways disease.

Ethical approval for this study was obtained from the Otago Ethics Committee of the Southern Regional Health Authority.

All unit costs and prices used are in 1996 New Zealand dollars $(\$ N Z 1=\$ U S 0.6825$, mid-rate May 1996). 1996 prices were used as these were the most up to date when the analysis was undertaken.

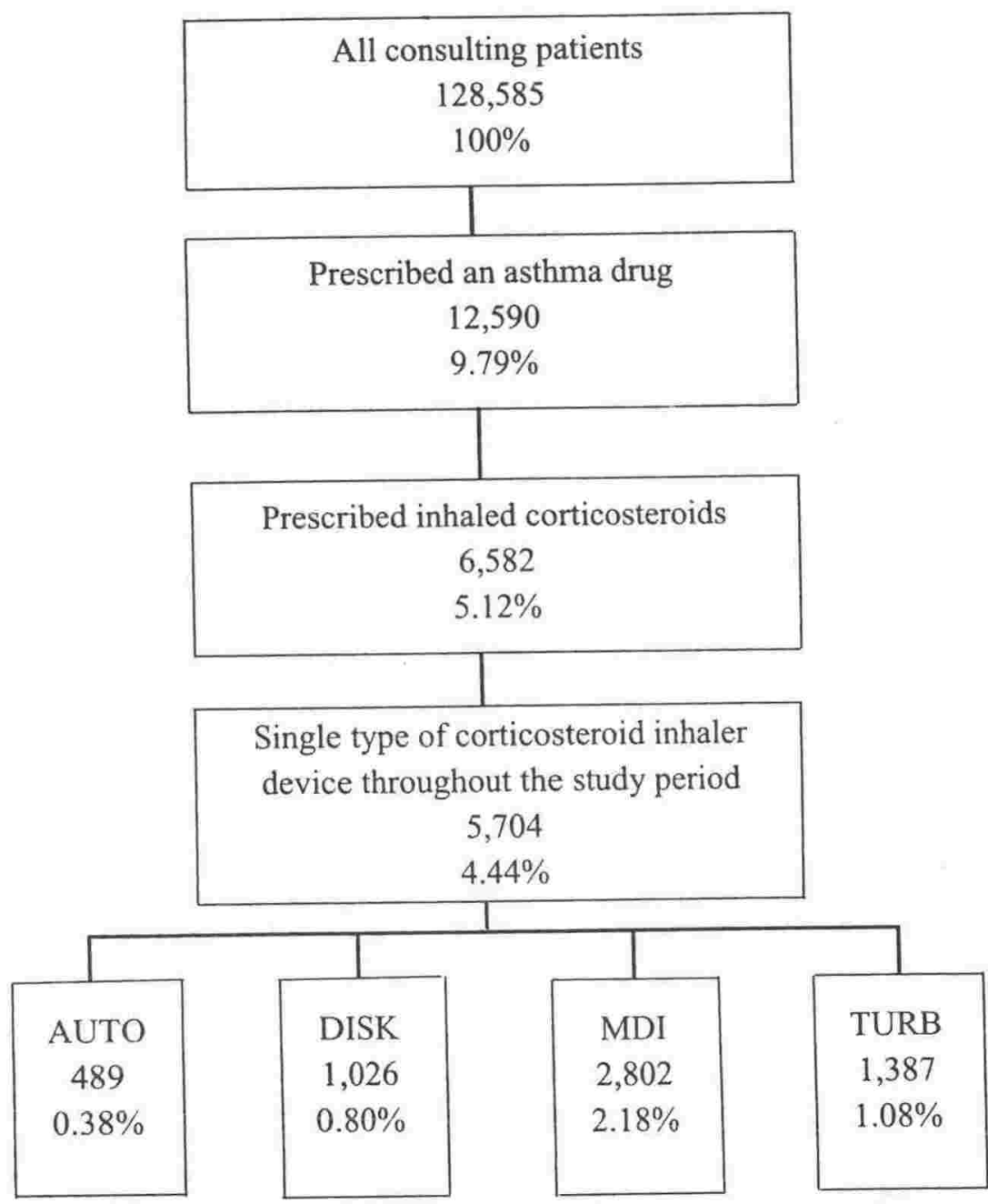




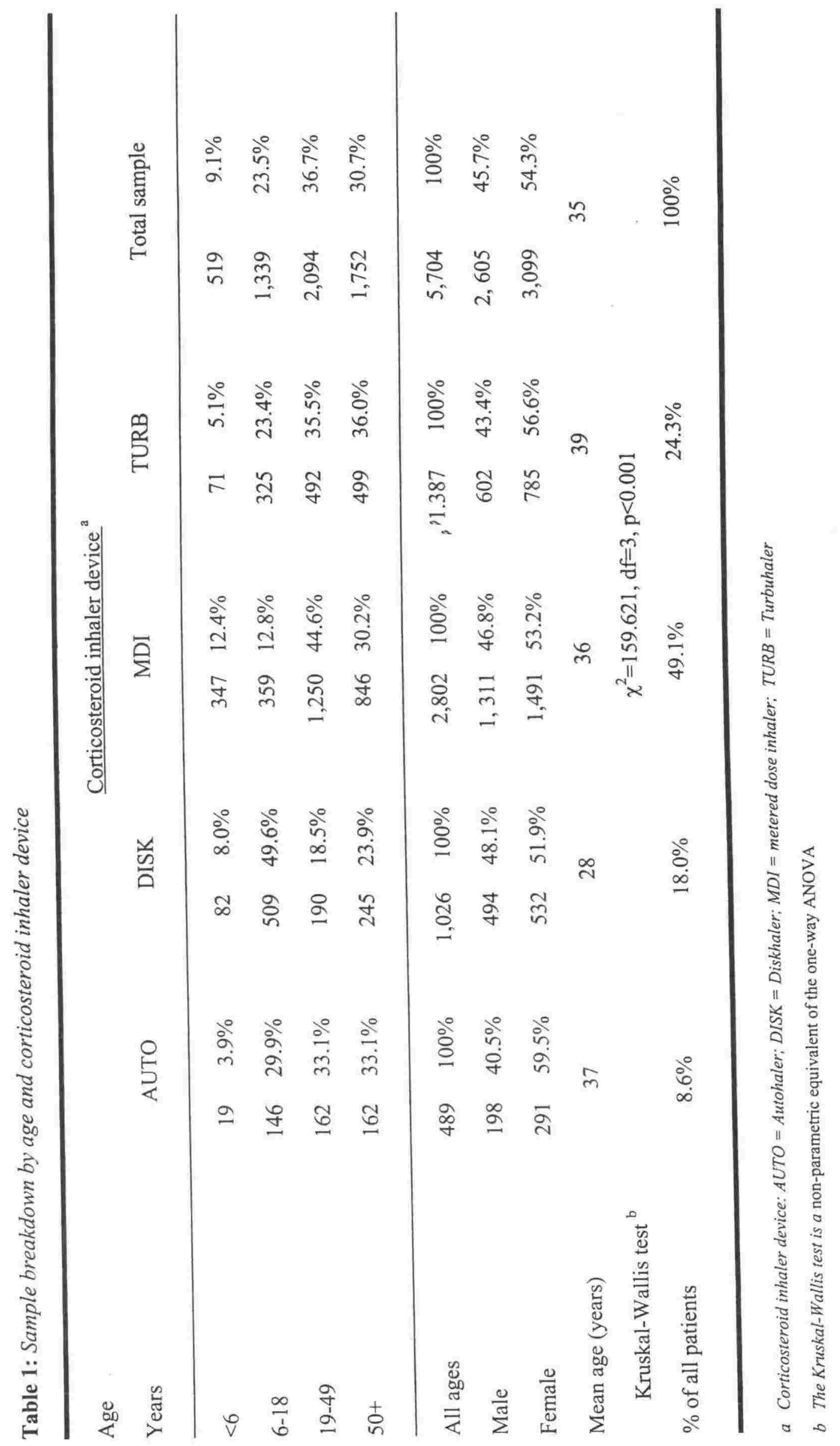


Table 2: General practitioner consultations (mean number per year) by corticosteroid inhaler device

\begin{tabular}{|c|c|c|c|c|c|}
\hline \multirow[t]{2}{*}{ Consultations } & \multicolumn{3}{|c|}{$\underline{\text { Corticosteroid inhaler device }}^{\mathrm{a}}$} & \multirow[b]{2}{*}{ TURB } & \multirow[b]{2}{*}{ Total samp } \\
\hline & AUTO & DISK & MDI & & \\
\hline $\begin{array}{l}\text { Asthma } \\
\text { Kruskal-Wallis test }^{b}\end{array}$ & $3.5944 \%$ & $3.9646 \%$ & $\begin{array}{c}4.3745 \% \\
\chi^{2}=27.913, \mathrm{df}\end{array}$ & $\begin{aligned} & 4.05 \quad 41 \% \\
= & 3, p<0.001\end{aligned}$ & $4.15 \quad 44 \%$ \\
\hline $\begin{array}{l}\text { All consultations } \\
\text { Kruskal-Wallis test }\end{array}$ & $8.26100 \%$ & $8.65100 \%$ & $\begin{array}{c}9.72100 \% \\
\chi^{2}=22.838, \mathrm{df}\end{array}$ & $\begin{aligned} & 9.97100 \% \\
= & 3, \mathrm{p}<0.001\end{aligned}$ & $9.46100 \%$ \\
\hline
\end{tabular}

\section{Results}

From the original 128,585 consulting patients there were 12,590 patients $(9.8 \%)$ prescribed any asthma medication. Of these patients 6,582 received 18,168 prescriptions for inhaled corticosteroids. From this data subset 5,704 patients $(86.7 \%$ of patients prescribed inhaled corticosteroids) were prescribed a single type of inhaled corticosteroid inhaler device throughout the one-year study period. The derivation of the sample is illustrated in Figure 1.

Table 1 describes the sample breakdown by age and corticosteroid inhaler device. Of the four inhaled corticosteroid inhaler groups in this study, DISK patients were the youngest with a mean age of 28 years and TURB patients had the oldest mean age of 39 years. The largest group was MDI with $49.1 \%$ of the total sample, and AUTO was the smallest group with $8.6 \%$ of the sample.

Table 2 shows that the MDI group had the highest average yearly number of asthma consultations ( 4.37 per year) while the
AUTO group had the lowest average number of asthma consultations (3.59 per year). The TURB group had the highest number of total consultations (9.97 per year) and the AUTO group had the lowest average number of total consultations (8.26 per year).

Table 3 presents the components of primary care cost by corticosteroid inhaler device. The average daily dose of prescribed inhaled corticosteroid was lowest for AUTO (569 $\mathrm{mcg}$ ) and highest for the TURB group (990 mcg) - a difference of $74 \%$. Both DISK and MDI recorded higher doses than AUTO but substantially less than TURB. The salbutamol equivalent average daily dose for beta agonists for the treatment groups ranged from AUTO (291 mcg), TURB (332 mcg), MDI ( $358 \mathrm{mcg}$ ) to DISK (407 $\mathrm{mcg}$ ) - a difference between lowest and highest of $40 \%$. The prescribing of a particular corticosteroid inhaler tended to be matched with the same type of inhaler device for inhaled beta agonists.

The largest single cost item identified in this study was that of corticosteroid inhalers 


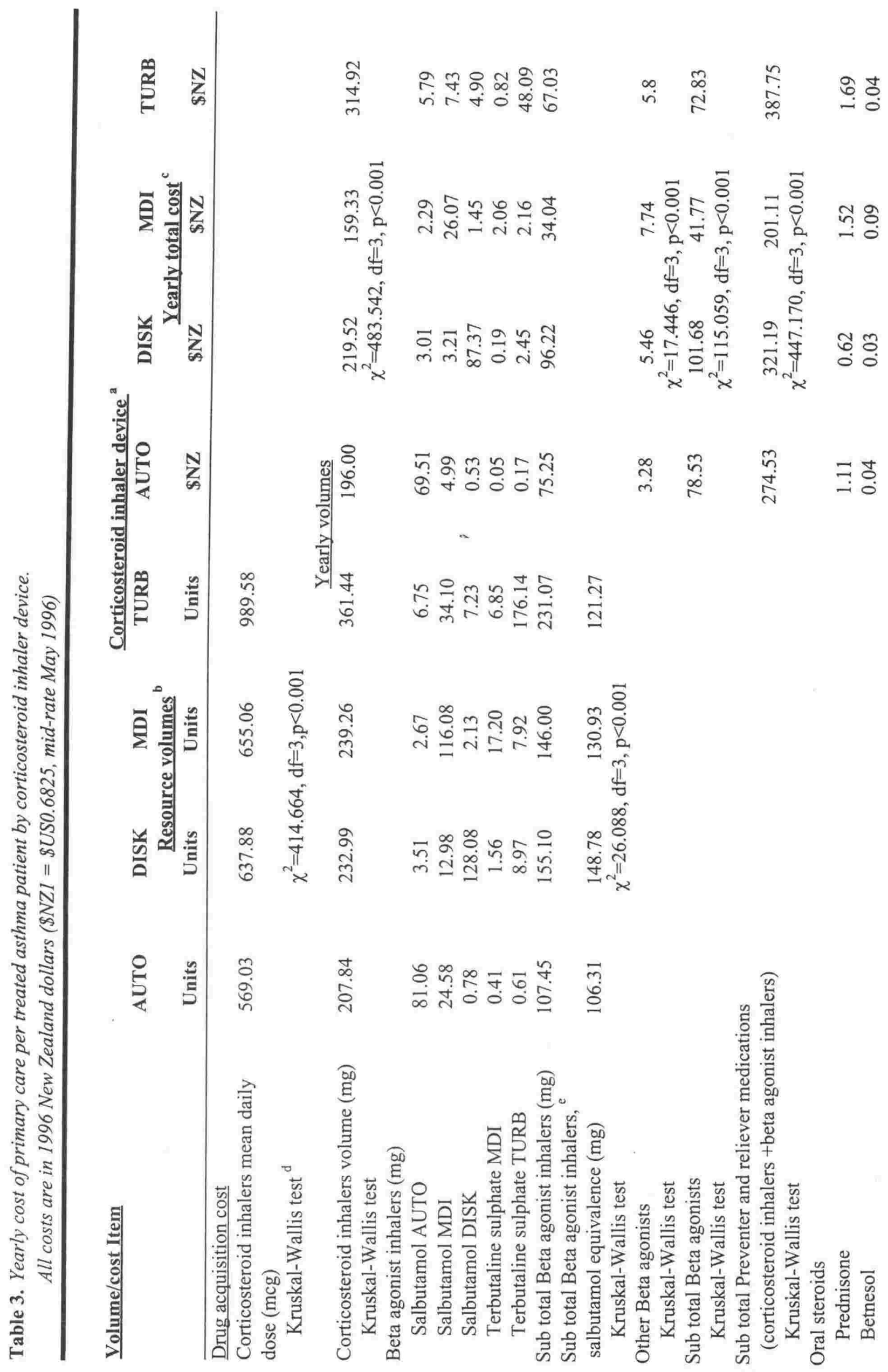




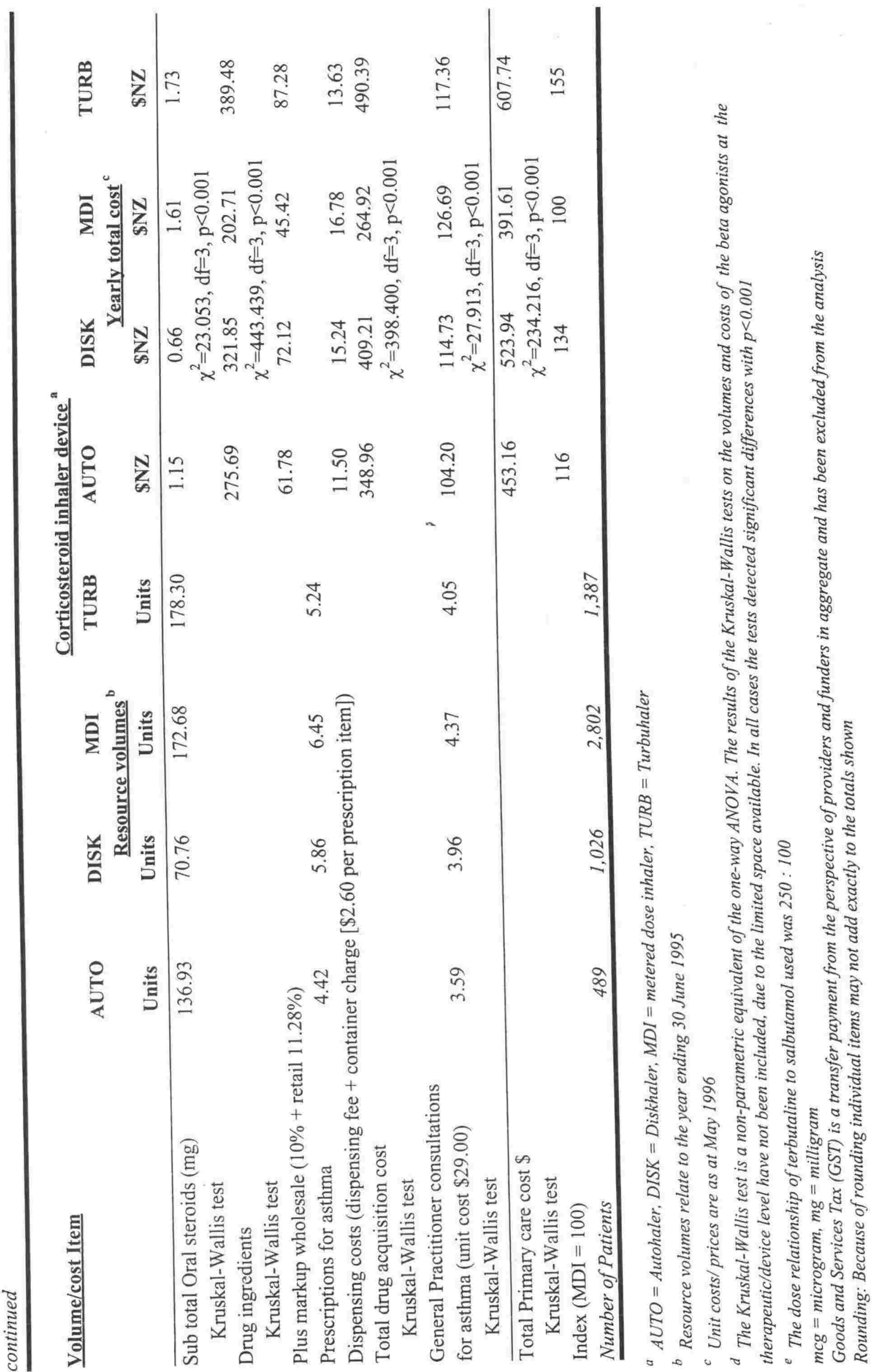


FIGURE 2. Yearly cost of primary care per treated asthma patient by corticosteroid inhaler device ( Corticosteroid inhalers : $\square$ Total drug acquisition; $\square$ General Practitioner consultations for asthma;

圈 Total Primary care)

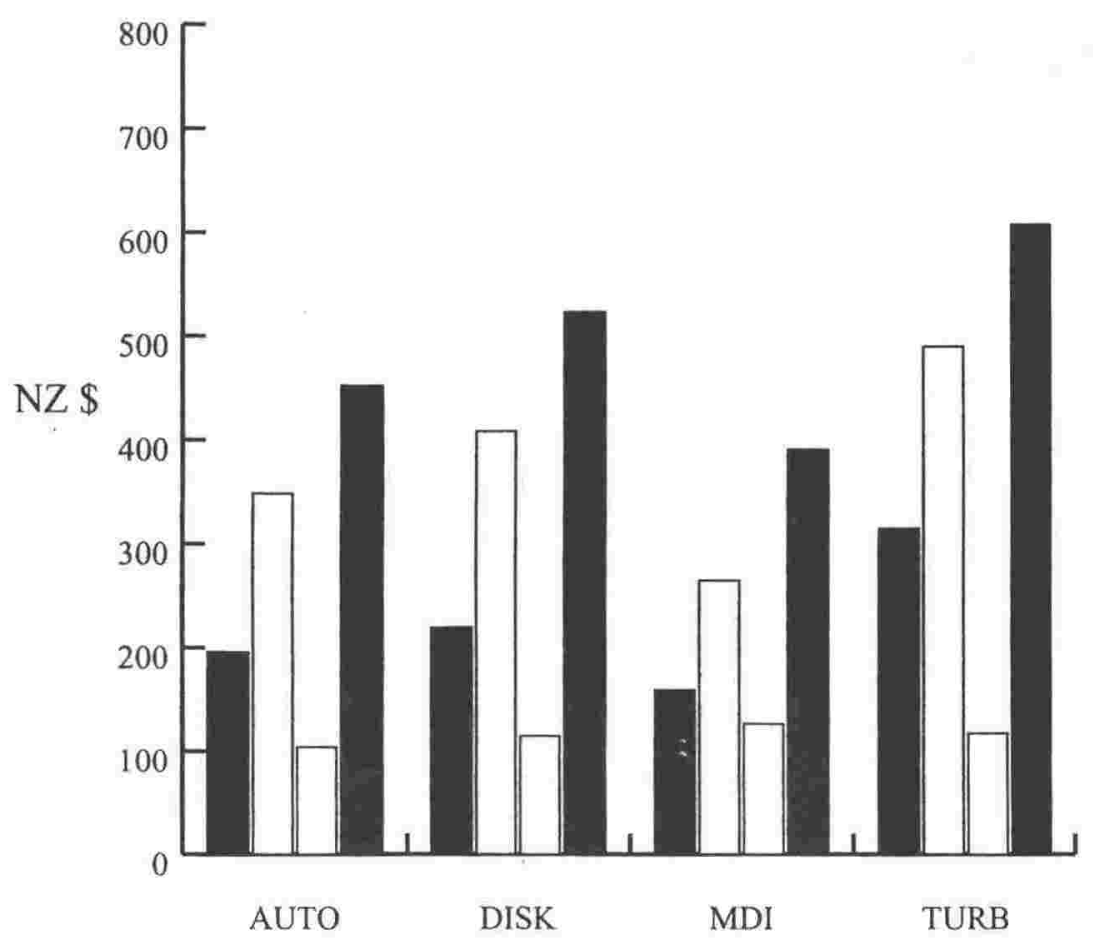

Corticosteroid inhaler device

$A U T O=$ Autohaler,$D I S K=$ Diskhaler, $M D I=$ metered dose inhaler, $T U R B=$ Turbuhaler

which ranged from $41 \%$ of total primary care cost for MDI to $52 \%$ for the TURB group. The acquisition costs for corticosteroid inhalers (exclusive of wholesale and retail markups) ranged from $\$ 159$ for MDI, to $\$ 315$ for TURB, a difference of $98 \%$. Beta agonist acquisition costs (exclusive of wholesale and retail markups) were lowest for MDI at $\$ 42$ and highest for DISK at $\$ 102$, a difference of $143 \%$.

Total drug acquisition cost (inclusive of dispensing costs and wholesale and retail markups) was highest for TURB at $\$ 490$ and lowest for MDI at $\$ 265$, a difference of $85 \%$. There was less variation in general practitioner consultations for asthma with total costs ranging from $\$ 104$ for AUTO to $\$ 127$ for DISK. Total primary care costs were lowest for MDI at $\$ 392$ per annum and highest for TURB at $\$ 608$, a difference of $55 \%$. If the MDI total primary care costs are used as the base for an index (MDI total primary care costs set at 100) then AUTO becomes 116, DISK 134 and TURB 155.

Figure 2 summarises the main cost components by corticosteroid inhaler device. The MDI group had the lowest cost for all major cost components except general practitioner consultations (which were the highest of all groups) and the lowest total primary care costs. 


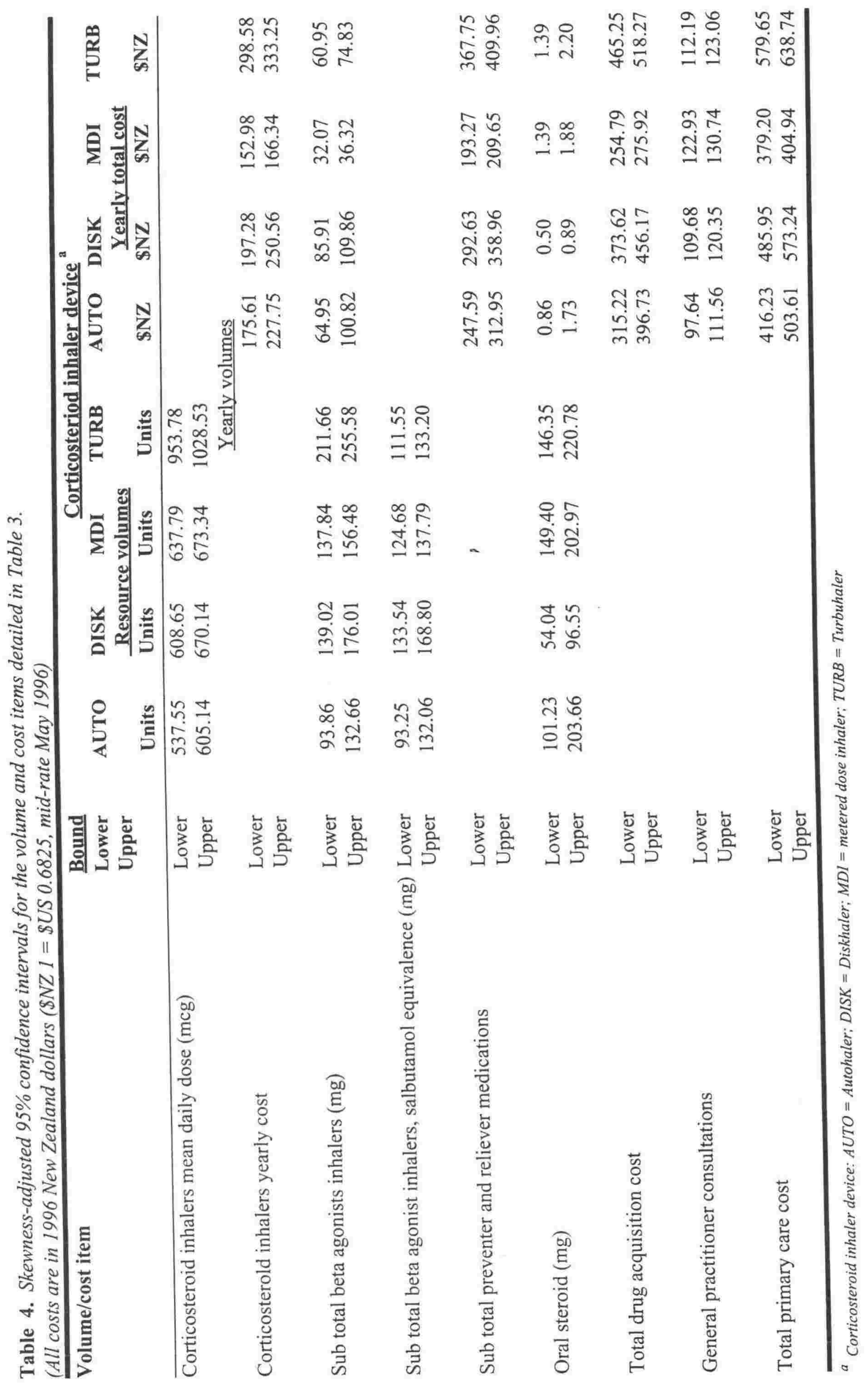


FIGURE 3. Yearly cost of total primary care per treated asthma patient by corticosteroid inhaler device

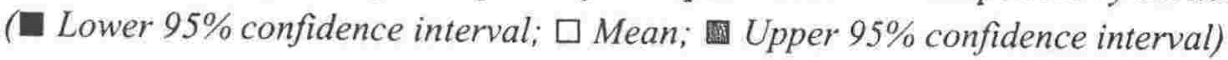

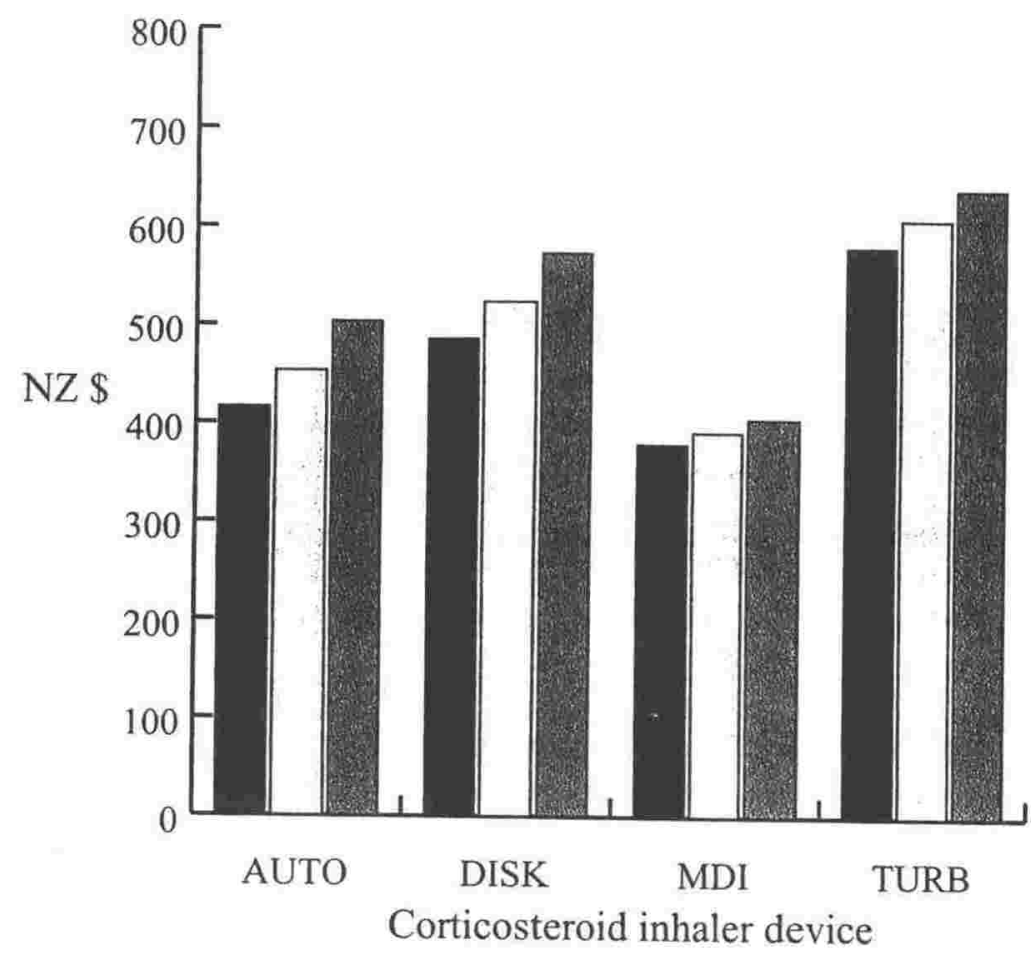

$A U T O=$ Autohaler, DISK $=$ Diskhaler, $M D I=$ metered dose inhaler, $T U R B=$ Turbuhaler

Table 4 presents the skewness adjusted $95 \%$ confidence interval upper and lower bounds for the key cost and volume items described in Table 3.

Figure 3 displays the skewness-adjusted 95\% confidence intervals and means for total primary care cost. This visual presentation clearly shows the statistically significant cost differences between treatment groups.

\section{Discussion}

This study differed from most randomised controlled clinical trials in that it involved a large study population and recorded (without altering or influencing) actual general practitioner prescribing practice. The prescribing information does not include specialist or hospital based prescribing but information from IMS (N.Z.) Limited suggests that these sources form only a small part of the cost of the pharmacological treatment of asthma. The hospital market for asthma medicines is estimated to be less than $4 \%$ by value of the total New Zealand market.

Not all records in the data base used for our study allowed clear identification of the reason for the consultation and thus some asthma-related consultations may not have been counted. As data were actual general practitioner prescribing records and not 
pharmacy dispensing records, failure to present or collect a prescription from the pharmacist was not measured. Estimates for non-dispensing vary but Gardner et al. in a recent study ${ }^{25}$ found that the non-dispensing rate for both bronchodilators and corticosteroids was $10.5 \%$. No information relating to specific devices was available but there was no reason to assume different nondispensing rates within the study groups.

A number of factors may cause wastage of asthma drugs; some patients do not comply with treatment, and inhaler delivery devices may be test fired and used incorrectly. Estimates of patient noncompliance with prescribed medication vary widely. Noncompliance depends upon the individual patient, the nature of the disease being treated, and the type of medication prescribed. The study did not attempt to measure noncompliance but never-the-less the impact on prescriptions and general practitioner consultations has been accounted for in the study. Over-use would have been measured in additional prescriptions and general practitioner consultations. Under-use would have been accounted for by subsequently fewer prescriptions and fewer general practitioner consultations. If under-use resulted in an asthma attack, additional prescriptions and additional consultations may have been generated and measured in the study.

Wastage as a result of test firing, incorrect activation (poor co-ordination) or incorrect usage was not specifically measured in the study but is accounted for as it is included in the volumes of medication prescribed. Gebbie ${ }^{26}$ reported from a survey of asthmatics that test firing was an important cause of wastage of bronchodilator aerosols in pressurised containers (MDIs). Test firing is usually a problem associated with MDIs and is considered to be less of a problem for breath-actuated inhalers.

King et al. ${ }^{27}$ found that $68 \%$ of patients were unable to use their MDI inhalers successfully. Errors in use fell into two main categories; failure to synchronise deep inspiration with depressing the canister (a problem of co-ordination), and failure to hold the breath after inspiration. In a review of asthma literature ${ }^{28}$ Shrestha et al reported that approximately $20 \%$ to $40 \%$ of asthma patients used MDIs correctly and this has important implications for resource usage, morbidity and mortality. McFadden ${ }^{13}$ reviewed the English language literature (20 papers) and found that the error rate per patient in the use of MDIs ranged from $12 \%$ to $89 \%$ with an average of $38 \%$. The proportion of patients misusing MDIs in this review was $36 \%$ (1308 out of 3611 subjects). A French study of the literature came to similar conclusions ${ }^{29}$. Faulty inhaler technique has both clinical and economic consequences in the treatment of asthma.

Prescribing records are then a different but more compound impression of asthma treatment consumption which potentially accommodates a larger number of variables compared to dosing in controlled clinical trials.

As explanantory variables for asthma severity and risk were not sufficiently documented in the database, further investigation such as multivariate analysis (a generic term encompassing multiple regression, cointegration analysis, principal components analysis and factor analysis) was not considered appropriate. However, comment 
Table 5. Patients aged 19 years and over with one or more daily doses of greater than $1500 \mathrm{mcg} /$ day.

\begin{tabular}{lcccc} 
& \multicolumn{4}{c}{ Corticosteroid inhaler device } \\
& AUTO & DISK & MDI & TURB \\
\hline $\begin{array}{l}\text { Number } \\
\text { Proportion of total patients aged } 19\end{array}$ & 9 & 102 & 132 & 339 \\
$\begin{array}{l}\text { and over in inhaler device group } \\
\begin{array}{l}\text { Statistical significance } \\
\text { b }\end{array}\end{array}$ & $2.8 \%$ & $\begin{array}{c}23.4 \% \\
\chi^{2}=470.10, d f=3, p<0.001\end{array}$ & \\
\hline
\end{tabular}

${ }^{a}$ Corticosteroid inhaler device: $A U T O=$ Autohaler, DISK $=$ Diskhaler, $M D I=$ metered dose inhaler, $T U R B=$ Turbuhaler

${ }^{b}$ The Kruskal-Wallis test in a non-parametric equivalent of the one-way ANOVA

has been made on differences in age between treatment groups.

Sensitivity analysis was conducted by referencing or bench marking to MDI costs. If the largest cost component, corticosteroid inhalers, were all set to the cost of the MDI group then total primary care cost would be as follows: AUTO \$416, DISK \$464, MDI unchanged at $\$ 392$ and TURB $\$ 452$. The difference in cost between the highest and lowest cost groups would fall from $55 \%$ to $18 \%$. The MDI group remains the lowest cost but the highest cost group changes from TURB to DISK. This change occurs because the DISK group has the highest cost for beta agonist treatment.

When the beta agonist cost is set to the cost of the MDI group the total primary care cost is as follows: AUTO \$416, DISK \$464, MDI unchanged at \$392, and TURB \$577. The MDI group remain the lowest cost and the TURB group remains the highest cost but the difference in cost between these groups would fall from $55 \%$ to $47 \%$. If the cost for general practitioner consultations is set to the MDI rate costs rise for the other groups and become: AUTO \$476, DISK \$536, MDI unchanged at $\$ 392$, and TURB $\$ 617$. The MDI and TURB groups remain in their positions as the lowest and highest groups but the cost difference increases from $55 \%$ to $58 \%$. The results of the model are robust with respect to conclusions relating to cost differences between the treatment groups. In all but one instance TURB remains the highest cost group.

\section{Economic and Therapeutic Implications}

The average daily dose of inhaled corticosteroids with MDI treatment was significantly higher $(\mathrm{p}<0.001)$ than with AUTO treatment. This possibly represents the effect of high dose treatment with MDI and spacer on average daily dosing. The lower volume of inhaled corticosteroid in the AUTO group may also reflect asthma severity or lower dose treatment for this particular group. Possible reasons for this finding could be: asthma severity was clinically different, or that different efficacy was provided by the different inhalers. Conclusions for the clinical importance of any differences in outcome between study groups must consider that patient asthma severity and treatment was not controlled in this study. While assessment of asthma severity for individual patients was not available, it could be reasonably expected 
that treatment efficacy for the study groups was similar overall. Treatment in this study was not controlled and medical practitioners obviously had an incentive to change prescribing, as occurs in regular medical practice, to optimise individual patient treatment. As a result there are no reasons to suspect that treatment outcome was any different for each treatment group.

The higher dose for TURB for inhaled corticosteroid is difficult to explain as this group of patients did not display the same differences with respect to greater numbers of prescriptions or general practitioner consultations that may have indicated greater severity of asthma. It is surprising to have found patients were being regularly treated with such high daily doses of inhaled corticosteroids considering that there is no evidence to suggest any superior asthma control at high doses from TURB or DISK compared to aerosol treatment (AUTO and MDI) ${ }^{30}$.

Additionally, a possible explanation for the findings from this study is that both the dry powder inhalers (TURB and DISK) are used to treat patients with more severe disease, and so required higher doses of inhaled corticosteroids. The rather surprising high average daily dose for inhaled corticosteroids for the TURB group and the numbers of patients receiving greater than $1500 \mathrm{mcg} /$ day in particular, contrasts with claims for the efficiency of drug delivery with this inhaler (see Table 5). It has previously been suggested that the Turbuhaler has greater lung deposition than aerosols ${ }^{31}$, although the importance of this for efficacy has not been clearly established. If greater efficacy were to be assumed for TURB comparisons with other inhalers this assumption would require that the equivalent dose for this inhaler be increased proportionally.

Definitive conclusions are limited, but considering the recent proposition of advanced delivery from the TURB, the equivalent average daily corticosteroid dose would have been expected to be proportionally less than the doses found in the study for the other inhalers.

AUTO and DISK patients tended to be treated with the matching beta agonist inhaler device. Beta agonists are currently recommended for intermittent or as required use. Volume of beta agonist usage is also considered to reflect asthma control ${ }^{10}$. The average dose of beta agonist used in each of the treatment groups was statistically significantly different between the treatment groups $(\mathrm{p}<0.001)$. As previously discussed the clinical importance of any differences could not be determined as an outcome of this study. However, possible explanations for this finding could relate to differences in asthma severity or efficacy provided by the different inhalers.

Although there were statistically significant differences between the volumes and costs of inhaled beta agonists between groups and the volumes and costs of oral steroids between groups these differences may not be clinically significant. The skewness adjusted confidence intervals for beta agonist volumes show that DISK had a higher mean dose than AUTO and TURB. However, in clinical terms the beta agonist dosages were similar between groups. A finding may be statistically significant but too close to zero to be of clinical relevance ${ }^{32}$.

Although treatment with oral steroid was 
found to be of little economic importance in the cost of asthma treatment, the prescribing pattern does have some implications as an indicator of asthma control and asthma severity.

The skewness adjusted confidence intervals for oral steroids are very similar for all of the treatment groups and do not demonstrate undue differences in the treatment of asthma or severity between the treatment groups. While caution is advised for the interpretation of this information (Betnesol and prednisone have different potencies and the analysis is completed on dose), these oral steroids are used in similar proportions for all the treatment groups in the study.

The lower oral steroid dose range associated with DISK treatment may be explained in part by the more common use of this inhaler treatment for younger patients, who would more likely be treated with lower doses. Further analysis and interpretation is difficult as oral steroids are used infrequently in treatment and the available data are limited.

The total primary care costs for AUTO and MDI were less than the total primary care cost of either of the dry powder inhalers (DISK or TURB). As spacer costs have not been included in this analysis the true cost for MDI would be higher than our estimates. For those MDI patients requiring two spacers per year the additional cost would be $\$ 35.52$. Although spacers should be replaced at six monthly intervals some patients may use less than two per year and others more than this but we had no information from the database on spacer usage. It was not possible to obtain cost data relating to hospital admissions, lost production or reduction in quality of life.
The database information can be analysed in several ways, including further sub-group analysis such as age, gender and dosage rates. Further analysis would be unlikely to further clarify the conclusions of this study. Information derived from this research has also been used to construct a deterministic decision analytic cost of treatment model ${ }^{8}$.

Analysis to determine asthma severity and treatment efficacy as a result of inhaler or corticosteroid type was not attempted in this analysis as separation of the elements of asthma severity and treatment efficacy was not possible with the information in the data base. The importance of this study is that it provides information on total incremental treatment costs in actual clinical practice.

Most other asthma related research has compared specific aspects of inhaler devices and chemical entities rather than difference in costs associated with treatment with particular asthma inhalers. This type of study reported here can provide additional information for evidence-based decisionmaking for clinicians, funders and policy makers. Prospective randomised controlled clinical trials would compliment and could clarify and appropriately further investigate the importance of the findings of this study.

\section{Acknowledgements}

The authors acknowledge a research grant from $3 \mathrm{M}$ New Zealand Limited. Any opinions expressed in this paper are those of the authors alone. 


\section{References}

1. National Heart Lung and Blood Institute National Institutes of Health. International Consensus Report on Diagnosis and Treatment of Asthma. Eur Respir J 1992; 5: 601-41.

2. Crane J, Lewis S, Slater T, et al. The self reported prevalence of asthma symptoms amongst adult New Zealanders. NZ Med J 1994; 107: 417-21.

3. A picture of health. 1st ed. Wellington: Statistics New Zealand and Ministry of Health, 1993.

4. National Advisory Committee on Core Health and Disability Support Services. Core Services for 1994/95. Wellington: National Advisory Committee on Core Health and Disability Support Services, 1993.

5. Tilyard MW, Dovey SM, Spears GFS. Biases in estimates from the RNZCGP computer research group. NZ Med J 1995; 108: $118-21$.

6. McAvoy B, Davis P, Raymont A, Gribben B. The Waikato Medical Care (WaiMedCa) Survey 1991-1992. NZ Med $J$ 1994; 107: 387-432.

7. Ministry of Health. Mortality and demographic data 1993. Wellington: Health Information Service, Ministry of Health, 1995.

8. Scott WG, Scott HM, Frost GD. Pharmacoeconomic model of asthma treatment costs. British Journal of Medical Economics 1997; 11: 87-101.

9. Asthma action plan. New Ethicals Catalogue 1996; 33: C14,C15.

10. The British Guidelines on Asthma Management 1995 Review and Position Statement. Thorax 1997; 52 (Suppl.): S1S21.
11. Barnes PJ, Pedersen S. Efficacy and safety of inhaled steroids in asthma. American Review of Respiratory Disease 1993; 148: S1-26.

12. Lipworth BJ. New perspectives in drug delivery and systemic bioactivity. Thorax 1995; 50: 105-10.

13. McFadden SR. Improper patient techniques with metered dose inhalers: Clinical consequences and solutions to misuse. $J$ Allergy Clin Immunol 1995; 96: 278-83.

14. Newman SP, Weisz AWB, Talaee N, Clarke SW. Improvement of drug delivery with a breath actuated pressurised aerosol for patients with poor inhaler technique. Thorax 1991; 46: 712-6.

15. Dovey SM, Tilyard MW. The computer research network of the Royal New Zealand College of General Practitioners: an approach to general practice research in New Zealand. British Journal of General Practice 1996; 46: 749-52.

16. Respiratory system and allergies. New Zealand Pharmaceutical Schedule 1996; 3: 151-8.

17. Consumers Price Index. Consumers price index weighted average retail prices of selected items in the CPI: general practitioners' services, consultation adult without community services card. Hot Off the Press 1996; September quarter.

18. Consumer. General practitioner consultations: what do you pay your doctor? Consumer 1996; April:24-5.

19. Freedman BJ. Trial of a terbutaline aerosol in the treatment of asthma and a comparison of its effects with those of a salbutamol aerosol. British Journal of Diseases of the Chest 1972; 66: 222-9.

20. Choo-Kang YFJ, MacDonald HL, Horne NW. A comparison of salbutamol and terbutaline aerosols in bronchial asthma. The Practitioner 1993; 211: 801-4. 
21. Hartnett BJS, Marlin GE. Comparison of terbutaline and salbutamol aerosols. Australian and New Zealand Journal of Medicine 1977;7(1):13-15.

22. Persson G, Gruvstad E, Wiren JE. Therapeutic effect of Turbuhaler in comparison with metered dose inhaler in adults. Paper presented at the Proceedings of an international workshop on a new inhaler, 21-22 May 1987, London.

23. Siegel S, Castellan NJ. Non parametric statistics for the behavioural sciences. 2nd ed. New York: McGraw Hill, 1988.

24. Hall P. The Bootstrap and Edgeworth expansion. New York: Springer Verlag, 1992.

25. Gardner TL, Dovey SM, Tilyard WM. Differences between prescribed and dispensed medications. NZ Med J 1996; 109: 69-72.

26. Gebbie T. Aerosols in generalised airway obstruction (Letter). NZ Med J 1971; 74: 115-6.

27. King D, Earnshaw SM, Delaney JC. Pressurised aerosol inhalers: the cost of misuse. British Journal of Clinical Practice 1991; 45(1): 48-9.

28. Shrestha M, Parupia MFH, Andrews B, et al. Metered-dose inhaler technique of patients in an urban ED: prevalence of incorrect technique and attempt at education. Americal Journal of Emergency Medicine 1996; 14(4): 380-4.

29. Chinet T, Huchon G. La mauvaise utilisation des aerosols-doseurs pressurises dans le traitement des maladies bronchiques: Frequence et consequences cliniques. Ann Med Interne 1994; 145: 119 24.

30. Lipworth BJ. Clinical pharmacology of corticosteroids in bronchial asthma.

Pharmacology and Therapeutics 1993; 58: 173-209.
31. Selroos O, Pietinalho A, Riska H. Delivery devices for inhaled asthma medication: Clinical implications of differences in effectiveness. Clinical Immunotherapy 1996; 6: 273-99.

32. O'Brien BJ, Drummond MF. Statistical versus quantitative significance in the socioeconomic evaluation of medicines. PharmacoEconomics 1994; 5: 389-98. 